Simone Frömbling

Zielgruppenmarketing im Fremdenverkehr von Regionen 


\section{Simone Frömbling}

\section{Zielgruppenmarketing im Fremdenverkehr von Regionen}

Seit geraumer Zeit gewinnt die Frage nach einem zielgruppenspezifischen Marketing von Fremdenverkehrsregionen an Bedeutung. Die vorliegende Arbeit hat sich daher zum Ziel gesetzt, auf der Grundlage einer Stichprobe von 936 befragten Bundesbürgern $\mathrm{zu}$ untersuchen, unter Berücksichtigung welcher Markterfassungsansätze und Segmentierungskriterien eine sinnvolle Zielgruppenabgrenzung im Fremdenverkehr von Regionen vorgenommen werden kann. Hierzu wird der Einfluß sozio- und psychographischer Merkmale (Werte, Motive, Einstellungen) auf die Besuchsabsicht und -dauer analysiert. Die Ergebnisse bilden die Grundlage für eine psychographische Marktsegmentierung. Für die identifizierten Segmente werden aus Sicht einer regionalen Fremdenverkehrsorganisation theoriegeleitet Ansatzpunkte für eine zielgruppenspezifische Marktbearbeitung aufgezeigt.

Simone Frömbling wurde 1962 in Osnabrück geboren. Nach der Ausbildung zum Industrie-Kaufmann studierte sie Betriebswirtschaftslehre an den Universitäten Marburg und Münster und absolvierte ein halbjähriges Praktikum im ImportExport-Bereich im Ausland. 1988 erfolgte der Abschluß zum Diplom-Kaufmann. Seither arbeitet sie als wissenschaftliche Mitarbeiterin am Institut für Marketing der Universität Münster. 
Zielgruppenmarketing im Fremdenverkehr von Regionen Ein Beitrag zur Marktsegmentierung auf der Grundlage von Werten, Motiven und Einstellungen 


\section{Schriften zu Marketine und Manasement}

Herausgegeben von Prof.Dr. Heribert Meffert

Band 21

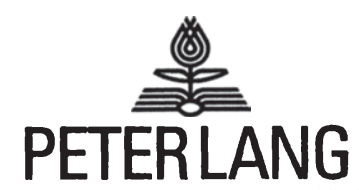

Frankfurtam Main.Berlin. Bern.NewYork.Paris.Wien 


\section{Simone Frömbling}

\section{Zielgruppenmarketing im Fremdenverkehr von Regionen}

Ein Beitrag zur Marktsegmentierung aufder Grundlage von Werten, Motiven und Einstellungen

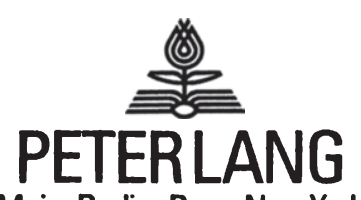

Frankfurtam Main. Berlin · Bern.NewYork·Paris·Wien 
Die Deutsche Bibliothek - CIP-Einheitsaufnahme

Frömbling, Simone:

Zielgruppenmarketing im Fremdenverkehr von Regionen : ein Beitrag zur Marktsegmentierung auf der Grundlage von Werten, Motiven und Einstellungen / Simone Frömbling. - Frankfurt am Main ; Berlin ; Bern ; New York ; Paris ; Wien : Lang, 1993 (Schriften zu Marketing und Management ; Bd. 21) Zugl.: Münster (Westfalen), Univ., Diss., 1993 ISBN 3-631-46422-3

NE: GT

Open Access: The online version of this publication is published on www.peterlang.com and www.econstor.eu under the international Creative Commons License CC-BY 4.0. Learn more on how you can use and share this work: http://creativecommons. org/licenses/by/4.0.

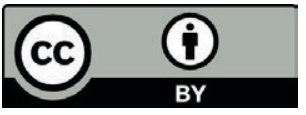

This book is available Open Access thanks to the kind support of ZBW - Leibniz-Informationszentrum Wirtschaft.

\author{
D 6 \\ ISSN 0176-2729 \\ ISBN 3-631-46422-3 \\ ISBN 978-3-631-75102-2 (eBook) \\ (C) Verlag Peter Lang GmbH, Frankfurt am Main 1993 \\ Alle Rechte vorbehalten.
}

Das Werk einschließlich aller seiner Teile ist urheberrechtlich geschützt. Jede Verwertung außerhalb der engen Grenzen des Urheberrechtsgesetzes ist ohne Zustimmung des Verlages unzulässig und strafbar. Das gilt insbesondere für

Vervielfältigungen, Übersetzungen, Mikroverfilmungen und die Einspeicherung und Verarbeitung in elektronischen Systemen.

Printed in Germany 123467 


\section{Vorwort des Herausgebers}

In vielen deutschen Fremdenverkehrsgebieten sind trotz fortschreitender Freizeitorientierung in den westlichen Industriestaaten und gestiegener weltweiter Nachfrage nach touristischen Leistungen seit Jahren stagnationen oder sogar Rückläufe $z u$ beobachten. Die stagnationstendenzen innerhalb der deutschen Fremdenverkehrsgebiete sind im wesentlichen auf eine Sättigung des Marktes mit weitgehend austauschbaren, eher undifferenzierten Reiseangeboten zurückzuführen. Hierdurch wird eine Präferenzbildung gegenüber den Touristen grundsätzlich erschwert. Offensichtlich bestehen in vielen deutschen Fremdenverkehrsgebieten Profilierungsdefizite. Für eine erfolgreiche profilierung ergibt sich zunehmend die Notwendigkeit, den potentiellen Besuchern auf ihre spezifischen Bedürfnisse abgestimmte Urlaubsangebote bereitzustellen.

Gleichzeitig sind angesichts der aufgezeigten Entwicklungstendenzen viele Kommunen immer mehr gefordert, sich durch regionale Zusammenarbeit im Rahmen der Fremdenverkehrsförderung zu profilieren. Dies ergibt sich aus der Erkenntnis, daß eine einzelne Kommune in zukunft kaum mehr in der Lage ist, die notwendige Leistungsfähigkeit aus eigener Kraft zu sichern. Solche, auf der Basis von freiwilligen zusammenschlüssen kommunaler Entscheidungsträger festgelegten Fremdenverkehrsregionen, sehen sich vor die Aufgabe gestellt, aus inrem regionalen Entwicklungspotential heraus und unter Berücksichtigung der heterogenen Erwartungen der Touristen eine Profilierung ihrer Region anzustreben.

Dem zielgruppenmarketing von Fremdenverkehrsregionen wird jedoch erst in jüngster zeit verstärkt Beachtung geschenkt. Allerdings werden hierbei vielfach nur Teilaspekte aufgegriffen und untersucht. In der wissenschaftlichen Forschung 
ist ein Defizit an theoretischen und empirischen Auseinandersetzungen mit Fragestellungen des zielgruppenmarketing im Fremdenverkehr von Regionen $z u$ beklagen. Die Ableitung von Marktbearbeitungskonzepten auf der Grundlage einer systematischen Analyse der Bestimmungsfaktoren des Besuchsverhaltens steht bisher noch aus.

In der vorliegenden Untersuchung wird dieser Problembereich aufgegriffen. Auf der Basis einer Befragung von 940 Bundesbürgern analysiert die Verfasserin, unter Berücksichtigung welcher Markterfassungsansätze und segmentierungskriterien im Fremdenverkehr von Regionen eine zielgruppenabgrenzung vorzunehmen ist und welche Ansatzpunkte sich regionalen Fremdenverkehrsorganisationen für eine zielgruppenspezifische Marktbearbeitung bieten.

Aufbauend auf einer konzeptionellen und empirisch gestützten Systematisierung des Besuchsverhaltens wird im Rahmen der Untersuchung der Frage nachgegangen, welche soziodemographischen und psychographischen Merkmale (Werte, Motive, Einstellungen) die Besuchsabsicht und -dauer in Abhängigkeit von der Entfernung zum zielgebiet determinieren. Darüber hinaus wird ein über die singuläre Einflußanalyse hinausgehender Analyseansatz entwickelt, der Aufschluß über die gemeinsame Erklärungskraft der einbezogenen Bestimmungsfaktoren des entfernungsgruppenspezifischen Besuchsverhaltens gibt. Auf Grundlage dieser Ergebnisse werden innerhalb der zwei in Abhängigkeit von der Entfernung zum zielgebiet abgegrenzten Teilmärkte zentrale zielgruppen abgeleitet, die sich im Besuchsverhalten und in ihren soziodemographischen Merkmalen unterscheiden. Anschließend werden diese im Rahmen einer Homogenitätsanalyse zu fünf zentralen Zielgruppen verdichtet.

Zum AbschluB der Arbeit zeigt die Verfasserin Ansatzpunkte für eine zielgruppenspezifische Marktbearbeitung im Fremden- 
verkehr von Regionen auf. Zunächst werden für die vier Marketing-Mix-Bereiche zahlreiche zielgruppenspezifische Marktbearbeitungsmaßnahmen im Fremdenverkehr von Regionen abgeleitet. Darüber hinaus werden zentrale Ansatzpunkte zur Begegnung von Durchsetzungswiderständen seitens einzelner touristischer Leistungsanbieter oder Meinungsgruppen innerhalb der Bevölkerung erarbeitet.

Mit der vorliegenden Untersuchung greift die Verfasserin einen Problembereich auf, der bisher wissenschaftlich nur wenig bearbeitet worden ist. Die besondere Leistung der Arbeit liegt in der systematischen Übertragung von Meß- und Segmentierungskonzepten aus der Verhaltensforschung im Konsumgüterbereich auf die besonderen Gegebenheiten des Fremdenverkehrsmarketing von Regionen. Dabei ist sich die Verfasserin durchaus der Grenzen der Arbeit bewußt, die aus der kontextspezifischen Interpretation als auch aus dem Meßniveau der erhobenen Verhaltensvariablen resultieren. Trotz dieser Begrenzungsfaktoren leistet die literarisch sorgfältig belegte und methodisch anspruchsvolle Arbeit eine wertvolle Bereicherung der zielgruppenanalyse und -bearbeitung im Bereich des Fremdenverkehrsmarketing von Regionen.

Münster, im Mai 1993

Prof. Dr. Heribert Meffert 
Simone Frömbling - 978-3-631-75102-2

Downloaded from PubFactory at 01/11/2019 08:19:28AM

via free access 


\section{Vorwort}

Angesichts der in einer Vielzahl deutscher Fremdenverkehrsregionen $z u$ beobachtenden Sättigungserscheinungen, gestiegener Anspruchsniveaus deutscher Urlauber sowie zunehmend heterogener Urlaubswünsche gewinnt eine auf die spezifischen Bedürfnisse der Touristen abgestimmte Fremdenverkehrsplanung zunehmend an Bedeutung. Nicht selten wird derzeit von einer "Austauschbarkeitsfalle" deutscher Fremdenverkehrsregionen gesprochen, die bei vielen deutschen Fremdenverkehrsregionen ein Profilierungsdefizit erkennen läßt und eine Präferenzbildung gegenüber dem Touristen erschwert.

Die Frage nach einer zielgruppenspezifischen Ausrichtung von Marketingmaßnahmen stellt daher eine zentrale Herausforderung bei der Vermarktung von Fremdenverkehrsregionen dar. Damit kommt dem Konzept der Marktsegmentierung mit ihren Teilfunktionen der Markterfassung und -bearbeitung im Rahmen des Zielgruppenmarketing im Fremdenverkehr von Regionen eine Schlüsselfunktion $\mathrm{zu}$.

Vor diesem Hintergrund hat sich die vorliegende Arbeit das ziel gesetzt, einen empirisch gestützten Beitrag zum zielgruppenmarketing im Fremdenverkehr von Regionen zu leisten. Im Mittelpunkt der Untersuchung steht zunächst die Analyse soziodemographischer und psychographischer Merkmale (Werte, Motive und Einstellungen) hinsichtlich ihrer Eignung als Segmentierungskriterien. Hierzu werden, bezogen auf eine Untersuchungsregion, die Bestimmungsfaktoren der Besuchsabsicht und -dauer innerhalb von zwei in Abhängigkeit von der Entfernung zum zielgebiet gebildeten Teilmärkten empirisch ermittelt. Auf der Grundlage dieser Ergebnisse wird für jeden Teilmarkt eine zielgruppenabgrenzung vorgenommen. In der sich anschließenden Homogenitätsanalyse können die identifizierten Zielgruppen zu fünf zentralen Besuchersegmenten ver- 
dichtet werden. Unter Berücksichtigung dieser Besuchersegmente werden im zweiten Hauptteil für die vier MarketingMix-Bereiche zahlreiche zielgruppenspezifische Marketingmaßnahmen aus sicht einer regionalen Fremdenverkehrsorganisation aufgezeigt und Ansatzpunkte zur Begegnung von Durchsetzungswiderständen seitens einzelner touristischer Leistungsträger oder ausgewählter Bevölkerungsgruppen abgeleitet.

Die Abfassung der Arbeit war nur mit vielfältiger Unterstützung verschiedener Personen möglich. Mein besonderer Dank gilt an dieser stelle meinem akademischen Lehrer, Herrn Professor Dr. Heribert Meffert, der die Themenstellung anregte und ihre Fertigstellung umfassend förderte. Herrn Professor Dr. Klaus Backhaus sei für die Übernahme des Zweitgutachtens gedankt.

Mein Dank gilt ferner allen Kollegen und Kolleginnen des Institut für Marketing, die mich während der Erstellung der Arbeit von anderweitigen Aufgaben entlastet und vielfältig unterstützt haben. Besonderer Dank gebührt Herrn Dr. Joachim Bolz, der mir stets als geduldiger und motivierter Diskussionspartner zur Verfügung stand. Hervorheben möchte ich ferner Herrn Dr. Manfred Kirchgeorg, Frau Dr. Kerstin Christiane Monhemius und Herrn Dr. Ralf Birkelbach für ihre konstruktiv-kritischen Hinweise bei der Abfassung und Fertigstellung der Arbeit. Frau Marga Bürger verdanke ich die flexible und exzellente Abwicklung der technischen Arbeiten. Herrn oliver Voss habe ich die engagierte Erstellung der Abbildungen $\mathrm{zu}$ verdanken.

Schließlich möchte ich meinen Eltern danken, die in mir das Interesse für betriebswirtschaftliche Fragestellungen weckten und mich in allen phasen meiner Ausbildung unterstützten. 
Inhaltsverzeichnis

Seite

\section{A. Bedeutung des zielgruppenmarketing im Fremden-} verkehr von Regionen

1. Stellenwert einer zielgruppenspezifischen Marktbearbeitung im Fremdenverkehr von Regionen

2. "System Fremdenverkehr" als Untersuchungsgegenstand

3. Ansatzpunkte der Marktsegmentierung als Grundlage des Paradigmas zum Zielgruppenmarketing von Fremdenverkehrsregionen

4. Zielsetzung und Gang der Untersuchung

B. Erfassung und Analyse von soziodemographischen und psychographischen Merkmalen als Kriterien zur Marktsegmentierung im Fremdenverkehr von Regionen

1. Anforderungen an die Marktsegmentierung im Fremdenverkehr von Regionen

2. Ausprägungsformen des regionenspezifischen Besuchsverhaltens auf der Basis einer Makrosegmentierung

2.1 Theoretische Grundlagen zur Erfassung des Besuchsverhaltens und zur Makrosegmentierung

2.2 Kennzeichnung der empirischen Ausprägungsformen der Makro- und Besuchsverhaltenssegmente

3. Erfassung und Analyse soziodemographischer Merkmale als Kriterien zur Marktsegmentierung

3.1 Erfassung soziodemographischer Merkmale als Einflußgrößen des Besuchsverhaltens

3.2 Empirische Analyse der Eignung soziodemographischer Merkmale als Segmentierungskriterien 
4. Erfassung und Analyse psychographischer Merkmale als Kriterien zur Marktsegmentierung

4.1 Erfassung und Analyse von Werten als Kriterien zur Marktsegmentierung

4.11 Theoretische Grundlagen zur Erfassung und Analyse von Werten als Segmentierungskriterien

4.111 Werte als Einflußgrößen des Besuchsverhaltens

4.112 Operationalisierung von Werten

4.12 Empirische Analyse der Eignung von Werten als segmentierungskriterien

4.121 Kennzeichnung der Ausprägungs formen und struktur von Werten

4.122 Analyse der soziodemographischen Identifizierbarkeit und des Einflusses von Werten auf das Besuchsverhalten

4.2 Erfassung und Analyse von Urlaubsmotiven als Kriterien zur Marktsegmentierung

4.21 Theoretische Grundlagen zur Erfassung und Analyse von Urlaubsmotiven als Segmentierungskriterien

4.211 Urlaubsmotive als Einflußgrößen des Besuchsverhaltens

4.212 Operationalisierung von Urlaubsmotiven

4.22 Empirische Analyse der Eignung von Urlaubsmotiven als segmentierungskriterien

4.221 Kennzeichnung der Ausprägungsformen und struktur von Urlaubsmotiven

4.222 Analyse der soziodemographischen Identifizierbarkeit und des Einflusses von Urlaubsmotiven auf das Besuchsverhalten 
4.3 Erfassung und Analyse von Einstellungen als Kriterien zur Marktsegmentierung

4.31 Theoretische Grundlagen zur Erfassung und Analyse von Einstellungen als Segmentierungskriterien

4.311 Einstellungen als Einflußgrößen des Besuchsverhaltens

4.312 Operationalisierung von Einstellungen

4.32 Empirische Analyse der Eignung von Einstellungen als segmentierungskriterien

4.321 Kennzeichnung der Ausprägungsformen und Struktur von Einstellungen

4.322 Analyse der soziodemographischen Identifizierbarkeit und des Einflusses von Einstellungen auf das Besuchsverhalten

4.4 Zusammenfassende Analyse der Eignung psychographischer Merkmale als Kriterien zur Marktsegmentierung

5. Psychographische Marktsegmentierung auf der Grundlage der empirischen Ergebnisse

5.1 Zielgruppenbildung innerhalb der Makrosegmente anhand von Werten, Motiven und Einstellungen

5.2 Homogenitätsanalyse der makrosegmentspezifischen Zielgruppen

C. Ansatzpunkte einer zielgruppenspezifischen Marktbearbeitung im Fremdenverkehr von Regionen

1. Zielgruppenauswahl im Fremdenverkehr von Regionen

2. Ansatzpunkte zielgruppenspezifischer Marketingmaßnahmen im Fremdenverkehr von Regionen 
2.1 Ansatzpunkte für zielgruppenspezifische Maßnahmen im Rahmen der Produktpolitik

2.2 Ansatzpunkte für zielgruppenspezifische Maßnahmen im Rahmen der Kontrahierungspolitik

2.3 Ansatzpunkte für zielgruppenspezifische Maßnahmen im Rahmen der Distributionspolitik

2.4 Ansatzpunkte für zielgruppenspezifische Maßnahmen im Rahmen der Kommunikationspolitik

3. Ansatzpunkte für die Durchsetzung zielgruppenspezifischer Marktbearbeitungskonzepte

D. Zusammenfassung und Ausblick

1. Zusammenfassende Würdigung der Untersuchungsergebnisse

2. Implikationen für weiterführende Forschungen

Anhang I: Tabellen der empirischen Untersuchung

Anhang II: Fragebogen

Literaturverzeichnis 


\section{Abbildungsverzeichnis}

Abb. 1: Marktsegmentierung als Bindeglied zwischen Angebot und Nachfrage als Elemente des Systems Fremdenverkehr

Abb. 2: Paradigma des Zielgruppenmarketing von Fremdenverkehrsregionen

Abb. 3a: Ausgewählte empirische Studien zur Marktsegmentierung im Fremdenverkehr

Abb. 3b: Ausgewählte empirische Studien zur Marktsegmentierung im Fremdenverkehr (Fortsetzung)

Abb. 4: Bezugsrahmen der empirischen Untersuchung

Abb. 5: Konzeptionelle und meBtheoretische Anforderungen an die Marktsegmentierung im Fremdenverkehr von Regionen

Abb. 6: Ubertragung potentiell interessierender Aspekte des Kaufs von Konsumgütern auf das regionenspezifische Besuchsverhalten

Abb. 7: Makrosegmentierung auf der Basis der Entfernung zwischen Wohnort und Zielgebiet

Abb. 8: GröBe der empirisch ermittelten Besuchsverhaltenssegmente

Abb. 9: Zielgebietserfahrung der empirisch ermittelten Besucher- und Nicht-Besucher-Segmente

Abb. 10: Zielgebietsentfernung der empirisch ermittelten Besuchsverhaltenssegmente

Abb. 11: Empirische Ergebnisse der Makrosegmentierung

Abb. 12: Abgrenzung von Lebensphasen in Abhängigkeit von Alter und Kinderanzahl 
Abb. 13: Punktbewertungsverfahren zur Ermittlung der Schichtzugehörigkeit

Abb. 14: Empirische Ergebnisse zum EinfluB von soziodemographischen Merkmalen auf das Besuchsverhalten innerhalb der Makrosegmente

Abb. 15: Ziele der eigenen Lebensfuhrung als Indikatoren zur Erfassung von Werten

Abb. 16: Empirisch ermittelte Wichtigkeit von Werten

Abb. 17: Faktorenanalytische Verdichtung der Werteindikatoren

Abb. 18: Empirische Ergebnisse zum EinfluB soziodemographischer Merkmale auf die Wertefaktoren

Abb. 19: Empirische Ergebnisse zum EinfluB der Wertefaktoren auf das Besuchsverhalten (T-Test) innerhalb der Makrosegmente

Abb. 20: Urlaubsaktivitäten als Indikatoren zur Erfassung von Urlaubsmotiven

Abb. 21: Empirisch ermittelte Wichtigkeit von Urlaubsmotiven

Abb. 22: Faktorenanalytische Verdichtung der Motivindikatoren

Abb. 23: Empirische Ergebnisse zum EinfluB soziodemographischer Merkmale auf die Urlaubsmotivfaktoren

Abb. 24: Empirische Ergebnisse zum EinfluB der Urlaubsmotivfaktoren auf das Besuchsverhalten ( $T$ Test) innerhalb der Makrosegmente

Abb. 25: Indikatoren zur Erfassung von Einstellungen zu einer Fremdenverkehrsregion 
Abb. 26: Empirisch ermittelte Ideal- und Realeinstellungen sowie Ideal- und Realdifferenzen

Abb. 27: Faktorenanalytische Verdichtung von Realeinstellungen

Abb. 28: Empirische Ergebnisse zum EinfluB soziodemographischer Merkmale auf die Real-Einstellungsfaktoren

Abb. 29: Empirische Ergebnisse zum EinfluB soziodemographischer Merkmale auf die Realeinstellungen und Real-Ideal-Differenzen

Abb. 30: Empirische Ergebnisse zum EinfluB der RealEinstellungsfaktoren auf das Besuchsverhalten (T-Test) innerhalb der Makrosegmente

Abb. 31: Empirische Ergebnisse zum EinfluB der Realeinstellungen und Ideal-Real-Differenzen auf das Besuchsverhalten ( $T$-Test) innerhalb der Makrosegmente

Abb. 32: Zusammenfassende Beurteilung der Eignung von Werten, Motiven und Einstellungen als Kriterien zur Marktsegmentierung

Abb. 33: Diskriminanzanalytische Ergebnisse zur Bedeutung psychographischer Merkmale für die Besuchsverhaltenssegmente in der Entfernungsgruppe I

Abb. 34: Diskriminanzanalytische Ergebnisse zur Bedeutung psychographischer Merkmale für die Besuchsverhaltenssegmente in der Entfernungsgruppe II

Abb. 35: Psychographische Zielgruppenbildung und -beschreibung in der Entfernungsgruppe I

Abb. 36: Psychographische Zielgruppenbildung und -beschreibung in der Entfernungsgruppe II 
Abb. 37: Soziodemographische zielgruppenbeschreibung in der Entfernungsgruppe I

Abb. 38: Soziodemographische Zielgruppenbeschreibung in der Entfernungsgruppe II

Abb. 39: Struktur der Urlaubsmotive in den zielgruppen der Entfernungsgruppen I und II

Abb. 40: Zusammenfassung der wesentlichen Merkmale der identifizierten Zielgruppen

Abb. 41: Aufgabenverteilung im zielgruppenmarketing von Fremdenverkehrsregionen

Abb. 42: Beurteilung der Segmentattraktivität anhand der SegmentgröBe und potentieller Ausgabefähigkeit der Segmente

Abb. 43 Zielgruppen-Portfolio als heuristisches Modell zur Zielgruppenbewertung

Abb. 44: Ansatzpunkte einer zielgruppenspezifischen Marktbearbeitung

Abb. 45: Grundtypen von Absatzkanälen im Fremdenverkehr von Regionen

Abb. 46: Zusammenfassende Übersicht zur Prüfung der Untersuchungshypothesen zum EinfluB soziodemographischer Merkmale auf das Besuchsverhalten

Abb. 47: Zusammenfassende Ưbersicht zur Prüfung der Untersuchungshypothesen zur soziodemographischen Identifizierbarkeit von Werten, Motiven und Einstellungen

Abb. 48: Zusammenfassende Übersicht zur Prüfung der Untersuchungshypothesen zum EinfluB psychographischer Merkmale auf das Besuchsverhalten 


\section{Abkürzungsverzeichnis}

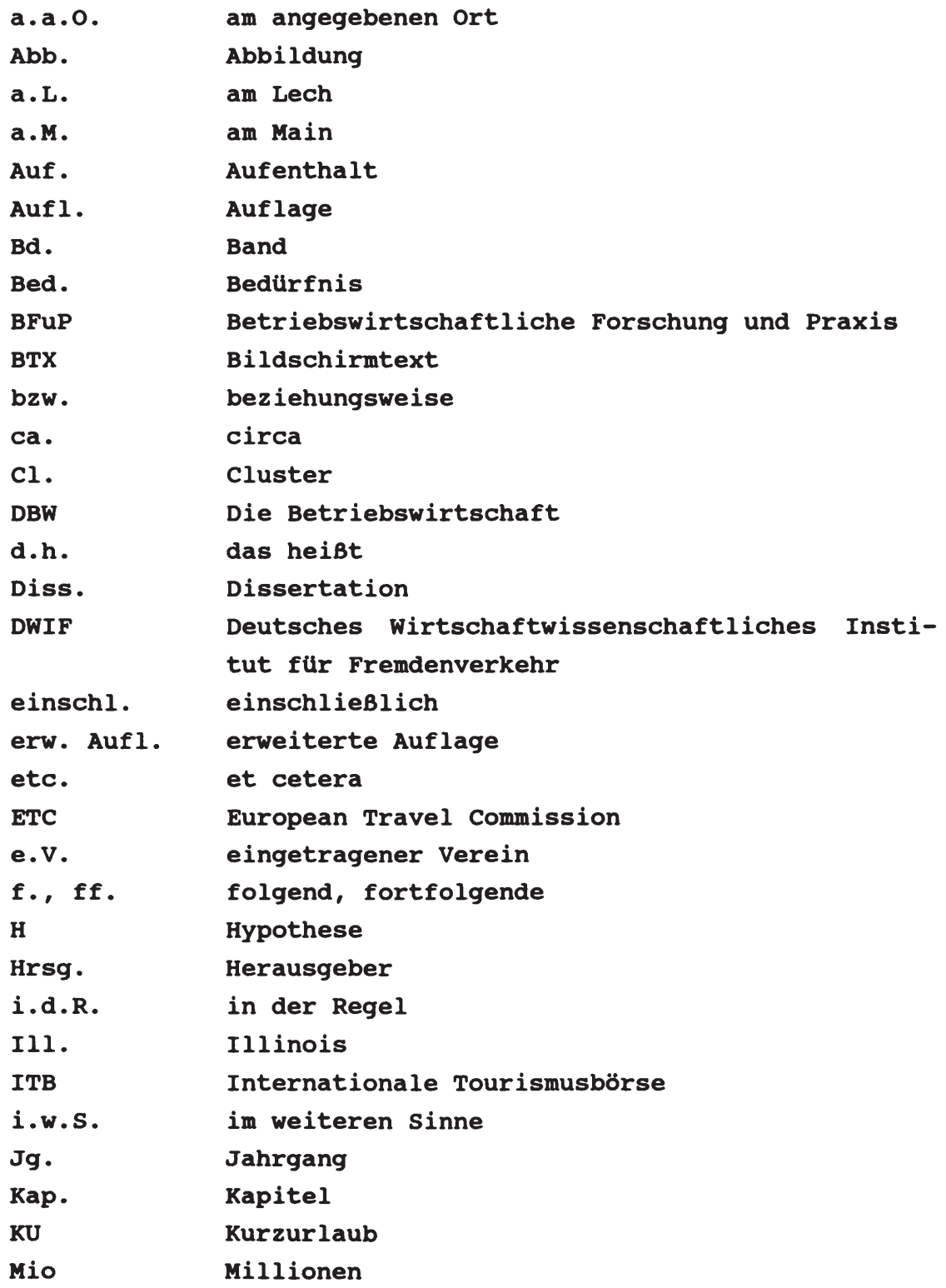




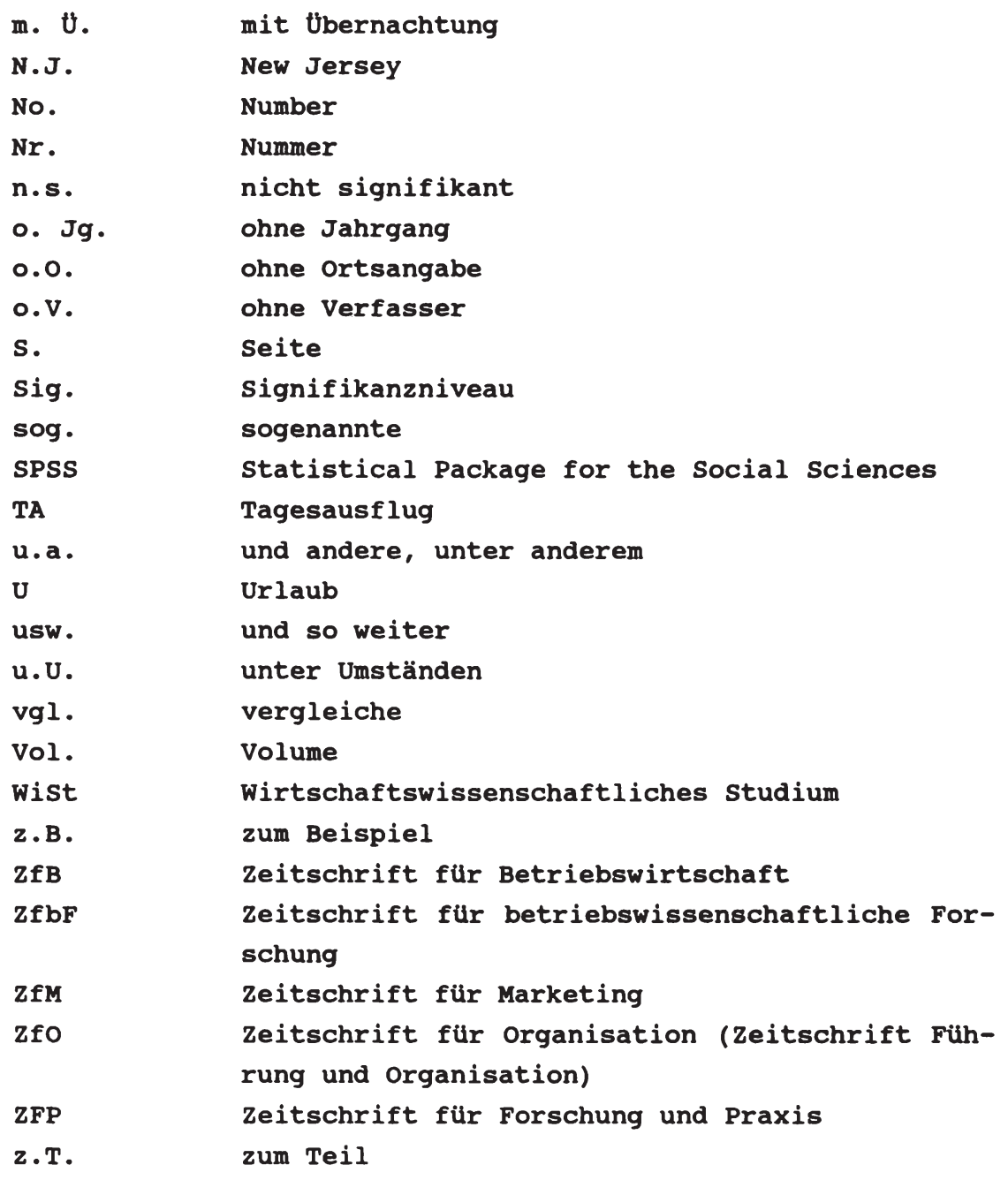


A. Bedeutung des zielgruppenmarketing im Frendenverkehr von Regionen

1. Stellenvert einer zielgruppenspezifischen Marktbearbeitung in Fremdenverkehr von Regionen

In den meisten Industrieländern hat der Trend hin $z u$ einer "Freizeit- und Dienstleistungsgesellschaft"1 zu einer gestiegenen Nachfrage nach touristischen Leistungen gefuhrt. Durchschnittliche Wachstumsraten von zunächst acht Prozent in den 60er und vier prozent in den $80 e r$ Jahren fuhrten dazu, daB derzeit der Fremdenverkehr weltweit zwölf prozent des Bruttosozialprodukts erwirtschaftet. Er steht damit gleichzeitig an vorderer stelle bei der schaffung von Arbeitsplätzen. 2

Ein Vergleich der weltweiten Dynamik des Fremdenverkehrssektors mit der Entwicklung in den alten Bundesländern der Bundesrepublik Deutschland verdeutlicht, daB die Bundesrepublik nur unterdurchschnittlich an den Wachstumsprozessen partizipierte. ${ }^{3}$ Insbesondere in strukturschwachen Gebieten, in denen vor allem der Fremdenverkehr als wirtschaftsbelebendes

1 Vgl. Meffert, H., Birkelbach, R., Freizeitgesellschaft und marktorientierte Unternehmensfuhrung. Thesen $z u$ den Auswirkungen des Freizeitphänomens auf die Marktbearbeitung, in: Marketing-ZFP, 1991, Heft 4, S. 265.

Vgl. Kaspar, C., Die Entwicklung des modernen Tourismus - Ursachen, Erscheinungsformen und Wirkungen, in: Statistisches Bundesamt (Hrsg.), Tourismus in der Gesamtwirtschaft, Ergebnisse des 4. Wiesbadener Gesprächs am 28./29. März 1990, stuttgart 1991, S. 19.

Die Zahl der Inlandsreisenden ist seit Jahren mit ca. 9 Mio konstant. Der Anteil der Inlandsreisen an den Gesamtreisen nahm von 60 in den 60 er Jahren auf derzeit 30 ab. Vgl. Dundler, F., Urlaubsreisen 1954 - 1988. 35 Jahre Erfassung des touristischen Verhaltens der Deutschen durch soziologische stichprobenuntersuchungen, in: studienkreis für Tourismus e.V. (Hrsg.), starnberg 1989, S. $4 \mathrm{f}$. 
Instrument eingesetzt wird, sind seit Jahren stagnationen und sogar Rückläufe zu beobachten. ${ }^{4}$

In Wissenschaft und Praxis herrscht weitgehend Einigkeit ubber die Hintergründe dieser Entwicklungen. Als zentrale Ursache wird die nur unzureichende kooperation zwischen einzelnen regionalen Entscheidungsträgern genannt, die dazu führt, $d a \beta$ ziele und Instrumente einer zentrenorientierten Regionalpolitik der Landesebene auf die regionale Ebene übertragen werden, ohne diese an die spezifischen regionalen Besonderheiten anzupassen. ${ }^{5}$ Hieraus resultierte nicht selten eine auf vornehmlich quantitatives wachstum ausgerichtete Fremdenverkehrsplanung, ${ }^{6}$ wobei sich die Angebotsausweitung an fremdenverkehrsspezifischen Infrastruktureinrichtungen orientierte, ohne jedoch nachfragerspezifische Aspekte zu berücksichtigen. 7

4 Vgl. Koch, A., Zeiner, M., wirtschaftliche wirkungen des Urlaubsreiseverkehrs in der Bundesrepublik Deutschland, in: Akademie für Raumforschung und Landesplanung (Hrsg.), Fremdenverkehr und Regionalpolitik, Forschungsund Sitzungsberichte, Bd. 172, Hannover 1988, S. 41.

5 Vgl. Maier, J., Bewertung eines regionalen Entwicklungsfaktors, in: Akademie für Raumforschung und Landesplanung (Hrsg.), Fremdenverkehr und Regionalpolitik, a.a.o., s. $112 \mathrm{f}$.

6 Das quantitative Wachstum wurde vor allem durch die im Rahmen der Gemeinschaftsaufgabe "Verbesserung der regionalen Wirtschaftsstruktur" zur Verfugung gesteliten strukturfördermittel ermöglicht. Bundesweit hat die Vergabe von strukturfördermitteln den Ausgleich wirtschaftlicher Disparitäten zum Ziel. $\mathrm{zu}$ den zielen der Gemeinschaftsaufgabe vgl. Haneklaus, w., Regionalpolitik in der Europäischen Gemeinschaft. Ziele, Kompetenzen und Instrumente von Gemeinschaft, Bund und Ländern nach Inkrafttreten der Einheitlichen Europäischen Akte, Münster 1991, S. $149 \mathrm{ff.;} \mathrm{Zu}$ den Aufgaben und Instrumenten der regionalen Wirtschaftspolitik vgl. Fürst, D., Klemmer, P., Zimmermann, K., Regionale Wirtschaftspolitik, Tübingen, Düsseldorf, 1976.

7 Vgl. Maier, J., Bewertung eines regionalen Entwicklungsfaktors, a.a.0., s. 112 f. 
Neue Herausforderungen erwachsen den Fremdenverkehrsregionen ferner aus der Einführung des EG-Binnenmarktes ${ }^{8}$ und der daraus resultierenden vergrößerten Mobilität der bundesdeutschen Bevölkerung. Zunehmende Reiseerfahrungen führen zu Änderungen der Anspruchsniveaus, die nur durch auf die spezifischen Bedürfnisse der Urlauber abgestimmte Urlaubsangebote zu befriedigen sind. 9 Dies gilt insbesondere für die wachstumsmärkte "Kurgurlaubs- und Tagesausflugaverkehr". 10

Angesichts dieser Entwicklungen sind Fremdenverkehrsregionen zunehmend gefordert, aus ihrem endogenen Entwicklungspotential heraus und unter Berücksichtigung der heterogenen Erwartungen potentieller Touristen eine Profilierung ihrer Region anzustreben. Vor diesem Hintergrund finden sich in

Zu den Auswirkungen des EG-Binnenmarktes auf den Tourismussektor vgl. Schrand, A., Tourismus 2000: Der Strukturwandel auf den Touristik-Märkten, in: Roth, P., Schrand, A., Touristik-Marketing, Das Marketing der Tourismus-Organisationen, Verkehrsträger, Reiseveranstalter und Reisebüros, München 1992, S. 8 ff. Zusätzlich ist davon auszugehen, daB die einer vielzahl von deutschen Fremdenverkehrsregionen in der Vergangenheit zur Verfügung stehenden Fördermittel aus der genannten Gemeinschaftsaufgabe gekürzt werden oder gänzlich entfallen. Vgl. Haneklaus, W., Regionalpolitik in der Europäischen Gemeinschaft, a.a.0., s. 7 ff.

9 Vgl. RosmeiB-stracke, F., zukunftsperspektiven für den Tourismus - Möglichkeiten und Grenzen einer Freizeitgesellschaft, in: Statistisches Bundesamt (Hrsg.), Tourismus in der Gesamtwirtschaft, a.a.0., s. 29 f.; storbeck, D., Die Entwicklung der Rahmenbedingungen für den Fremdenverkehr in der Bundesrepublik Deutschland, in: Akademie für Raumforschung und Landesplanung (Hrsg.), Fremdenverkehr und Regionalpolitik, a.a.0., S. 95 ff.

10 Die zunehmende Bedeutung des Tagesausflugs- und Kurzurlaubsverkehrs ist auf die im zuge von Arbeitszeitverkürzungen etc. vermehrt zur Verfügung stehende freie zeit zurückzuführen. Vgl. zur detaillierten Begründung der freien Zeit RosmeiB-stracke, F., Die Veränderungen der gesellschaftlichen Rahmenbedingungen für die Touristik, in: Roth, P., Schrand, A. (Hrsg.), Touristik-Marketing, a.a.o., S. 22 f. 
jüngster Zeit zwei zentrale Forderungen im Hinblick auf die Gestaltung des Fremdenverkehrs von Regionen.

Zum einen wird eine auf die regionalen Möglichkeiten angepaste Fremdenverkehrsplanung gefordert. ${ }^{11}$ Dabei sollen die Regionen ihre regionale Entwicklung verstärkt in regionaler Selbstverwaltung übernehmen, Eigeninitiative und Kreativität entfalten, regionale kooperationen festigen und regioneneigene Vorhaben realisieren. 12

Voraussetzung hierfur ist die Festlegung der Regionengrenzen auf der Basis von freivilligen zusammenschlüsen von kommunalen Entscheidungsträgern, da in der Bundesrepublik Regionen nicht normativ festgelegt sind. ${ }^{13}$ Um langfristig eine erfolgreiche Zusammenarbeit $z u$ gewährleisten, ist es sinnvoll, daB die sich in einer Region zusammenschliebenden Kommunen sprachlich-ethische, kulturelle, geographische und historische Gemeinsamkeiten aufweisen. 14 Dies gewährleistet die Wahrnehmung einer Fremdenverkehrsregion als ein einheit-

11 Vgl. Maier, J., Bewertung eines regionalen Entwicklungsfaktors, a.a.0., s. 117 .

12 Diese Forderung findet sich beispielsweise in einem am 16. Oktober 1990 verabschiedeten KabinettsbeschluB der Landesregierung Nordrhein-Westfalen. Vgl. 0.V., Regionales Entwicklungsprogramm Mủnsterland, März 1991.

13 Auf formal-rechtliche Vorgaben, wie z.B. Kreisgrenzen muB dabei kein Bezug genommen werden. Die ZweckmäBigkeit freiwilliger zusammenschlusse hat die Landesregierung Nordrhein-Westfalens erkannt. Im Rahmen des Förderprogramms "Zukunftsinitiative für die Regionen NordrheinWestfalens" (ZIN) wurde daher keine Regionenabgrenzung vorgegeben. Vielmehr soll es den Regionen selbst überlassen bleiben, festzulegen, in welcher Ausdehnung sie sich als eine räumliche Einheit verstehen. Vgl. Behrens, F., ZIN-Zusammenarbeit ist nötig, in: städte- und Gemeinderat, 1990, S. 63 ff.

14 Vgl. Hänni, H.D., Regionales Tourismus-Marketing - Am Beispiel regionaler Verkehrsverbände in der schweiz, zurich 1985, S. 137 ff. 
liches zielgebiet, welches der potentielle Tourist seiner Reiseentscheidung zugrunde legt.

Für die vorliegende Untersuchung werden Fremdenverkehrsregionen als räumliche Einheiten definiert, in denen sich regionale Akteure in Abhängigkeit von wirtschaftlichen und insbesondere fremdenverkehrswirtschaftlichen verflechtungen und sozio-kulturellen Gemeinsamkeiten freiwillig zum zwecke einer, auf die Besonderheiten der räumlichen Einheit abgestimmten Planung des touristischen Angebots im Rahmen einer Kooperation ${ }^{15}$ zusammenschlieBen.

Darüber hinaus ist es notwendig, die Fremdenverkehrsplanung verstärkt den vorhandenen Angebots-, Nachfrage- und Konkurrenzmechanismen anzupassen 16 und auf die Erkenntnisse der Marketing-Wissenschaft zurückzugreifen. In diesem Zusammenhang wird regionales Fremdenverkehrsmarketing als ein ganzheitlicher Denkansatz definiert, der die konsequente Planung, steuerung und Kontrolle aller auf die aktuellen und potentiellen touristischen zielgruppen ausgerichteten Akti-

15 Wesentliches Kennzeichen einer Kooperation, die sich von einem Erfahrungsaustausch bis hin zur rechtlichen Ausgliederung der kooperativen Führung (z.B. Zweckverband, GmbH, etc.) erstrecken kann, ist die Beibehaltung der wirtschaftlichen Selbständigkeit. Vgl. zur Definition von Kooperationen Müller, K., Goldberger, E., Unternehmens-Kooperation bringt Wettbewerbsvorteile, Notwendigkeit und Praxis zwischenbetrieblicher Zusammenarbeit in der Schweiz, Zürich 1986, S, $43 \mathrm{ff}$; vgl. ferner Backhaus, K., Piltz, K., Strategische Allianzen - eine neue Form kooperativen Wettbewerbs?, in: Backhaus, K., Piltz, K. (Hrsg.), Strategische Allianzen, Sonderheft Nr. 27 der ZfbF, Düsseldorf, Frankfurt 1989, S. 2 f.

16 Maier, J., Troeger-Weise, G., Marketing in der räumlichen Planung. Ansätze und Wege zu einer marktorientierten Regional- und Kommunalplanung, in: Beiträge der Akademie für Raumforschung und Landesplanung, Beitrag 117, Hannover 1990, S. 4. 
vitäten von Fremdenverkehrsregionen beinhaltet.17 Bezogen auf Fremdenverkehrsregionen bedeutet der ganzheitliche Denkansatz des Marketing, daß die Integration der Aktivitäten einzelner Kommunen sowie aller touristischen Leistungsträger der Fremdenverkehrsregion $z u$ einem integrierten Problemlösungskonzept anzustreben ist.

Obwohl die Notwendigkeit einer zielgruppenspezifischen Marktbearbeitung in der Tourismus-Praxis erkannt wird, hat sich die wissenschaft im Rahmen des touristischen Regionenmarketing bislang nur ansatzweise mit dieser Fragestellung auseinandergesetzt. Angesichts der herausgearbeiteten Bedeutung dieses Bereichs erscheint es geboten, die Möglichkeiten einer zielgruppenspezifischen Marktbearbeitung im Fremdenverkehr von Regionen zu untersuchen, wobei der Problemstellung der Marktsegmentierung ein zentraler stellenwert zukommt. Die Übertragung des Konzepts der Marktsegmentierung auf den Fremdenverkehr von Regionen erfordert jedoch zunächst die Präzisierung des Begriffs Fremdenverkehr.

\section{2. "System Fremdenverkehr" als Untersuchungsgegenstand}

Die Mehrzahl der neueren Veröffentlichungen zum Bereich des Fremdenverkehrs basieren auf der Definition von Hunziker und Krapf, 18 die dieser Untersuchung zugrunde gelegt wird. Hier

17 vgl. Meffert, H., u.a., Regionenmarketing Münsterland Ansatzpunkte auf der Grundlage einer empirischen Untersuchung, Münster 1991, S. 2 .

18 Der Begriff Fremdenverkehr hat in den verschiedenen Wissenschaftsdisziplinen vielfältige Interpretationen erfahren. Eine einheitliche Terminologie besteht nicht. vielmehr wurden die besonders interessierenden Fragestellungen der jeweiligen Disziplinen (u.a. Volks- und Regionalwirtschaftslehre, Betriebswirtschaftslehre, Psychologie, Soziologie und Sozialpsychologie, Geographie und Raumplanung, Freizeitpädagogik, etc.) in den Vordergrund gestellt. Vgl. zur Entwicklung des Fremdenver- 
wird Fremdenverkehr als ein System von Beziehungen definiert, welche sich aus dem Aufenthalt ortsfremder ergeben, sofern durch den Aufenthalt keine Niederlassung zur Ausübung einer dauernden oder zeitweilig hauptsächlichen Erwerbstätigkeit begründet wird.19 In der Literatur herrscht dabei weitgehend Einigkeit darüber, daB auf der Grundlage dieser und erweiterter Definitionen der Geschäfts- und KongreBtourismus sowie der zeitlich begrenzte Naherholungsverkehr dem Fremdenverkehr zuzuordnen sind. 20

Kaspar 21 versteht den Fremdenverkehr als ein System, dessen Elemente die Nachfrage nach touristischen Leistungen und das Angebot von Fremdenverkehrsleistungen am Aufenthaltsort darstellen, wobei die Reise zum Aufenthaltsort als beziehungsbildendes Element zusätzlich in die Definition aufgenommen wird. 22 Als "Aufenthaltsort" wird dabei im Rahmen der vor-

kehrsbegriffs Berneker, P., Die Wandlungen des Fremdenverkehrsbegriffs, in: Jahrbuch für Fremdenverkehr, 1952/53, Heft 1, s. $31-38$ und Arndt, H., Definitionen des Begriffs "Fremdenverkehr" im Wandel der Zeit, in: Jahrbuch für Fremdenverkehr, 1978, S. 160-174. Zu den einzelnen Fremdenverkehrsbegriffen in den unterschiedlichen Disziplinen vgl. Aderholt, P., Das Marketing von Städtekurzreisen, Rahmenbedingungen und Ansatzpunkte einer Fremdenverkehrspolitik unter beispielhafter Berücksichtigung der Verhältnisse in Kopenhagen, Kopenhagen 1976 , S. 5 sowie die dort angegebene Literatur.

19 Vgl. Hunziker, W., Krapf, K., GrundriB der allgemeinen Fremdenverkehrslehre, Zürich 1942.

20 Vgl. Meyer-Schwickerath, M., Perspektiven des Tourismus in der Bundesrepublik Deutschland - Zur Notwendigkeit eines wirtschaftspolitischen Konzepts, Münster 1990, S. 21; Kaspar, C. Fremdenverkehrslehre im GrundriB, 2 . Aufl., Bern, Stuttgart 1982, S. 17; Freyer, W., Tourismus. Einführung in die Fremdenverkehrsökonomie, 3 . Aufl., München, Wien 1991, S. 17.

21 Vgl. Kaspar, C., Fremdenverkehrslehre im GrundriB, a.a.o., s. 18 .

22 Kaspar spricht an dieser stelle vom Fremdenverkehrssubjekt und -objekt. Vgl. Kaspar, c., a.a.0., S. $15 \mathrm{ff}$. Er greift dabei auf das auf Ulrich zurückzuführende ver- 
liegenden Untersuchung eine in sich geschlossene Fremdenverkehrsregion verstanden. Es wird deutlich, daB Nachfrage und Angebot letztlich erst durch die Reise und den Aufenthalt in die Fremdenverkehrsregion zusammentreffen. 23 In dieser Arbeit werden die beiden Komponenten "Reise" und "Besuch bzw. Aufenthalt" begrifflich unter der Bezeichnung "Besuchsverhalten" subsumiert. Im folgenden Abschnitt werden zunächst beide Systemelemente Angebot und Nachfrage isoliert betrachtet, um darauf aufbauend den Beziehungszusammenhang näher zu erläutern.

Das touristische Leistungsangebot einer Fremdenverkehrsregion besteht aus zwei Hauptkomponenten: dem naturlichen und dem abgeleiteten Angebot. 24 Das natürliche Angebot umfaBt alle Faktoren, die in ihrem Wesensmerkmal keinen Bezug zum Fremdenverkehr aufweisen und erst durch die touristische Inanspruchnahme den charakter einer Fremdenverkehrsleistung annehmen. 25 Folgende drei Elemente des natürlichen Angebotes sind zu unterscheiden:

ständnis der systemtheorie zurück. Ulrich versteht unter einem system eine geordnete Gesamtheit von Elementen, zwischen denen Beziehungen bestehen oder hergestellt werden können. Vgl. Ulrich, H., Die Unternehmung als produktives System, Grundlagen der allgemeinen Unternehmenslehre, Bern, stuttgart 1968, S. $105 \mathrm{ff}$.

23 Dazwischen geschaltet sind sog. beziehungsbildende Unternehmen, wie Reiseveranstalter und Reiseburos, die die Vermittlung von touristischen Leistungen übernehmen. Ferner ist dieses System Fremdenverkehr von der ökonomischen, sozialen, technologischen, politischen und ökologischen Umwelt umgeben. Vgl. hierzu Kaspar, C., Fremdenverkehrslehre im GrundriB, a.a.0., s. 14.

24 Vgl. Kaspar, C., Fremdenverkehrslehre im GrundriB, a.a.o., S. 62 ff.; Tschiderer, F., Ferienortplanung: Eine Anwendung unternehmungsorientierter Planungsmethodik auf den Ferienort, Bern, stuttgart 1980, s. 20 f.

25 Vgl. Kaspar, C., Fremdenverkehrslehre im GrundriB, a.a.o., s. $61 \mathrm{f}$. 
- Natürliche Gegebenheiten wie geographische Lage, Klima, Topographie, Landschaftsbild, Vegetation und Tierwelt;

- 8ozio-kulturelle Verhältnisse wie Tradition, religiöse und historische Bauten, Sprache, Mentalität, Gastfreundschaft, Brauchtum;

- Algemeine Infrastruktur als Grundausstattung der gemeinschaftlich benutzbaren Einrichtungen, welche die Entfaltung umfassender wirtschaftlicher und gesellschaftlicher Aktivitäten überhaupt erst ermöglichen (Basisinfrastruktur wie z.B. Verkehrsanbindung etc.).

Das abgeleitete Angebot umfaBt die Leistungen, welche uberwiegend von rechtlich selbständigen touristischen Anbietern für die touristische Bedürfnisbefriedigung bereitgestellt werden. 26 Hierbei handelt es sich vor allem um die touristische Suprastruktur wie z.B. Einrichtungen der Unterkunft und Verpflegung sowie touristische Freizeit- und Dienstleistungsangebote. Ferner umfaBt das abgeleitete Angebot die speziell für den Tourismus von öffentlichen Institutionen bereitgestellten Infrastruktureinrichtungen und Fremdenverkehrsleistungen. 27 Dieses abgeleitete Angebot wird von einer Vielzahl rechtlich unabhängiger und mit eigenen Entscheidungsbefugnissen ausgestatteten Tourimusanbietern (wie z.B. Hoteliers, Gastronomen) und öffentlichen Institutionen (wie z.B. Verkehrsvereinen) bereitgestellt. Demnach ist das Leistungsangebot einer Fremdenverkehrsregion das Ergebnis einer Vielzahl voneinander unabhängiger, untereinander kaum abgestimmter Teilentscheidungen.

26 vgl. Kaspar, c., Fremdenverkehrslehre im Grundriß, a.a.o., s. 61 ff.

27 In diesem zusammenhang sind die aufgrund des Fremdenverkehrs zusätzlich erforderlichen Infrastruktureinrichtungen der Versorgung und Entsorgung sowie Beratungs- und Vermittlungsleistungen zu nennen. Vgl. Freyer, w., Tourismus, a.a.O., S. 198; Kaspar, C., Fremdenverkehrslehre im GrundriB, a.a.0., s. 62 f. 
Die Nachfrage nach Frendenverkehrsleistungen richtet sich auf eine Vielzahl unterschiedlicher Teilaspekte. Der Nachfrager steht im Rahmen seiner Reiseentscheidung vor der Herausforderung, sowohl ein zielgebiet, eine Unterkunft, eine Anreiseform als auch eine bestimmte organisationsform (Individual- vs. Pauschalreise) auszuwählen. 28 Dies begründet einen komplexen KaufentscheidungsprozeB, wobei sich die touristische vachrrage nicht auf isolierte Fremdenverkehrsleistungen richtet. Vielmehr stellt das Fremdenverkehrsgebiet in seiner Gesamtheit den wesentlichen Anziehungsfaktor der touristischen Nachfrage dar. 29 Die in einer Fremdenverkehrsregion angebotenen Leistungen stehen dabei aus sicht der Nachfrager in einem "Romplementaritätsverhältnis"30 zueinander.

Zur Identifizierung der wesentlichen Bestimmungsfaktoren des Besuchsverhaltens ist auf die Erkenntnisse der Kaufverhal-

Vgl. Mazanec, J., Voraussetzungen rationaler Entscheidungsvorbereitung im Fremdenverkehrs-Marketing unter Rücksicht auf neuere Analyse-Methoden, in: Der Markt, 1979, S. 191.

29 Vgl. Tschiderer, F., Ferienortplanung, a.a.0., s. 30; Krippendorf, J., Marketing im Fremdenverkehr, 2. Aufl., Bern 1980, S. 24; Kaspar, C., Kunz, B.R., Unternehmensführung im Fremdenverkehr. Eine Grundlage für das Management von Hotels und Restaurants, Sportbahnen und -anlagen, Reiseburos, Kur- und Verkehrsbüros, Bern, stuttgart 1982 , S. 35 .

30 Weitere Besonderheiten von Fremdenverkehrsleistungen ergeben sich insbesondere aus den strukturellen Merkmalen von Dienstleistungen. Hierbei sind vor allem die Immaterialität touristischer Leistungen, die Integration des externen Faktors und die Notwendigkeit der Bereitstellung von Leistungsfähigkeiten hervorzuheben. Vgl. zu den generellen Besonderheiten von Dienstleistungen Büker, B., Qualitätsbeurteilung investiver Dienstleistungen. Operationalisierungsansätze an einem empirischen Beispiel zentraler EDV-Dienste, Frankfurt a. M. 1991, S. 28 ff. und die dort angegebene Literatur. Zur ausfuhrlichen Kennzeichnung dieser Besonderheiten bezogen auf den Fremdenverkehr vgl. Krippendorf, J., Marketing im Fremdenverkehr, a.a.0., s. 16 ff. 
tens- und Tourismusforschung zurückzugreifen. Schon in den 60er Jahren wurde in der Tourismusforschung versucht, das Besuchsverhalten anhand soziodemographischer Merknale zu erklären. Aufgrund einer unzureichenden Verhaltensrelevanz 31 wird zur Identifizierung der Bestimmungsfaktoren der touristischen Nachfrage jedoch zunehmend auf psychologische Erklärungsansätze zurückgegriffen. ${ }^{32}$ Diese können in aktivierende und kognitive Determinanten des Besuchsverhaltens sowie in allgemeine Persönlichkeitsdeterminanten unterschieden werden. ${ }^{33} \mathrm{Zu}$ den kognitiven Determinanten werden Informationsverarbeitungsvorgänge wie Wahrnehmung und Lernen gerechnet. $\mathrm{Zu}$ den aktivierenden Variablen zählen Emotionen, Motive und Einstellungen. Persönlichkeitsdeterminanten sind demgegenüber vor allem Involvement, wahrgenommes Risiko sowie Werte. 34 Im Rahmen der psychologischen Tourismusforschung wurden in der Vergangenheit vornehmlich aktivierende Prozesse analysiert. Dabei dominierte insbesondere das Motivkonstrukt zur Erklärung und Prognose des Besuchsverhaltens. ${ }^{35}$ ziel der Analyse von Motiven ist die Identifizierung

31 Vgl. Opaschowski, H.W., Tourismusforschung, Freizeit und Tourismusstudien, Bd. 3, Opladen 1989, S. 107.

32 In den psychologischen Erklärungsansätzen wird versucht, über die Einfürung sog. hypothetischer Konstrukte, die stellvertretend für nicht-beobachtbare Variablen stehen, einen zusätzlichen Erklärungsbeitrag zum Besuchsverhalten zu erzielen. Diese Erklärungsmodelle werden auch als S-O-R-Modelle bezeichnet und bauen auf den neo-behavioristischen sowie den weiterführenden kognitiven Erklärungsansätzen auf. Vgl. hierzu die Ausführungen bei Meffert, $H$., Marketingforschung und Käuferverhalten, 2 . Aufl., Wiesbaden 1992, S. 27 f.

33 Vgl. zur zuordnung der einzelnen Konstrukte zu den aktivierenden und kognitiven Determinanten sowie zu den entsprechenden inhaltlichen Abgrenzungen Meffert, H., Marketingforschung und Käuferverhalten, a.a.0., s. 47 ff.; Kroeber-Riel, W., Konsumentenverhalten, 5. Aufl., München 1992, S. 49 ff.

Vgl. ebenda. 
der wesentlichen Bestimmungsgründe des Besuchsverhaltens. Darüber hinaus hat auch das Einstellungskonstrukt in der Fremdenverkehrsforschung an Bedeutung gewonnen. 36

In den Veröffentlichungen zur Konsumentenverhaltensforschung der letzten Jahre besteht weitgehend Einigkeit darüber, daß komplexe Verhaltensweisen bzw. Verhaltensdispositionen nicht allein mit Hilfe aktivierender Determinanten als Teilkomponenten menschlichen Handelns $z u$ erklären und $z u$ prognostizieren sind. 37 Eine Verbesserung der Erklärung des Besuchsverhaltens ist in diesem Zusammenhang insbesondere durch die Einbeziehung von Perönlichkeitsdeterminanten in die Verhaltensprognosen $\mathrm{zu}$ erwarten. In diesem Zusammenhang kommt werten als relativ stabile, von kurzfristigen Einflussen unab-

35 Zur Dominanz des Motivkonstrukts vgl. den Überblick bei Datzer über Ansätze der psychologischen Tourismusforschung. Datzer, R., Informationsverhalten von Urlaubsreisenden. Ein Ansatz des verhaltenswissenschaftlichen Marketing, starnberg 1983, s. $42 \mathrm{ff}$.

36 Hierbei sind die vom studienkreis für Tourismus durchgeführten Untersuchungen $z u$ Urlaubsangeboten und ausgewählten Feriengebieten hervorzuheben. Vgl. zum Beispiel die Beiträge in: studienkreis für Tourismus e.v. (Hrsg.), Motive-Meinungen-Verhaltensweisen. Einige Ergebnisse und Probleme der psychologischen Tourismusforschung, Starnberg 1969; Hartmann, K.D., Urlaubsland Schleswig-Holstein. Eine motiv- und meinungspsychologische Untersuchung. Kurzfassung, studienkreis für Tourismus (Hrsg.), Starnberg 1979; Wohlmann, R., Lohmann, M. , Urlaub auf dem Bauernhof, Urlaub auf dem Land. Eine empirische Untersuchung der Meinungen, Einstellungen und Verhaltensweisen von Urlaubern, Hrsg.: Studienkreis für Tourismus e.V., starnberg 1986.

37 Vgl. Windhorst, K.-G., Wertewandel und Konsumentenverhalten. Ein Beitrag zur empirischen Analyse der Konsumrelevanz individueller Wertvorstellungen in der Bundesrepublik Deutschland, Münster 1985, S. 3; Meffert, H., Stellenwert der Werteforschung im Marketing, Dokumentation des Workshops vom 29. Juni 1984, Arbeitspapier Nr. 20 der Wissenschaftlichen Gesellschaft fur Marketing und Unternehmensführung e.V., Hrsg.: Meffert, H., Wagner, H. , Münster 1984 , S. 1 f. 
hängige Prädispositionen des Besuchsverhaltens eine Schlüsselrolle $\mathrm{zu}^{38}$

Die zielgobietserfahrung stellt einen weiteren Bestimmungsfaktor des Besuchsverhaltens in bezug auf eine Fremdenverkehrsregion dar. Drei Fünftel der Haupturlaubsreisenden im Jahre 1987 besuchten ein Reiseziel, welches sie bereits von einer früheren Reise her kannten. 39 Eine derartige "Zielgebietstreue" ergibt sich durch positive Erfahrungen aufgrund befriedigter Urlaubsbedürfnisse und wird in das set 40 alternativer Fremdenverkehrsregionen aufgenommen. 41

Ferner ist davon auszugehen, daB das Besuchsverhalten von einer vielzahl situativer BinfluBgrößen bestimmt wird. So konnte in empirischen Untersuchungen nachgewiesen werden, daß z.B. die zielgobietsentfornung einen dominanten EinfluBfaktor insbesondere bei der Auswahl von Kurzurlaubszielen darstellt. 42

38 Vgl. Windhorst, K.-G., Wertewandel und Konsumentenverhalten, a.a.0., s. 142 ff; Trommsdorff, V., Konsumentenverhalten, stuttgart, Berlin, Köln 1989, S. 149.

39 Vgl. Dundler, F., Urlaubsreisen 1987. Einige Ergebnisse der Reiseanalyse 1987. Kurzfassung, studienkreis für Tourismus e.V. (Hrsg.), starnberg 1988, S. 41.

40 In diesem Zusammenhang kann von der Aufnahme der Fremdenverkehrsregion in das Evoked set des Touristen gesprochen werden. Evoked Set wird definiert als die Menge alternativer Fremdenverkehrsgebiete, die ein potentieller Tourist im Rahmen seiner Entscheidung in Betracht zieht. Vgl. Schulte-Frankenfeld, H., Vereinfachte Kaufentscheidungen von Konsumenten. Erklärung psychischer Prozesse kognitiv limitierten Entscheidungsverhaltens von Konsumenten, Frankfurt, Bern, New York 1985, S. 51.

41 Vgl. Goodall, B., How Tourists Choose Their Holidays: An Analytical Framework, in: Goodall, B., Ashworth, G. (Hrsg.), Marketing in the Tourism Industry. The Promotion of Destination Regions, New York 1988 , s. 3 ff.

42 Vgl. DWIF (Hrsg.), Markt- und Motivstudien für den Fremdenverkehrsmarkt Nordrhein-Westfalens, München 1989, S. 
Die wesentlichen Bestimmungsfaktoren der touristischen Nachfrage bzw. des Besuchsverhaltens sind in Abbildung 1 zusammengefaBt. Angesichts der Vielzahl von Bestimmungsfaktoren des Besuchverhaltens wird deutlich, daB die Nachfrage nach Fremdenverkehrsleistungen nicht homogen ist. Vielmehr bestehen unterschiedliche Erwartungen und Anforderungen an eine Fremdenverkehrsregion, die zu einem differenzierten Besuchsverhalten führen. Daher ist davon auszugehen, daB Gruppen von Nachfragern zu identifizieren sind, die in den Bestimmungsfaktoren des Besuchsverhaltens als auch in ihrem Besuchsverhalten bestimmte Ähnlichkeiten aufweisen. Dementsprechend ist es möglich, die Nachfragerseite in verschiedene, in sich homogene und untereinander heterogene vachfragersegmente zu unterteilen.

Für das system Fremdenverkehr bedeutet dies, daB von verschiedenen Nachfragersegmenten auszugehen ist, denen eine Vielzahl, isoliert nicht absetzbarer touristischer Einzelleistungen gegenübersteht. Hieraus folgert, daB das touristische Angebot in seiner Gesamtheit die segmentspezifischen Nachfragerbedürfnisse befriedigen können muB.

Die Fremdenverkehrsregion steht hierbei vor der Herausforderung, Informationen über die wesentlichen Bestimmungsgründe des Besuchsverhaltens verschiedener Nachfragersegmente zu erlangen, um eine auf die Nachfrage abgestimmte Produktbündelung vornehmen $z u$ können. Demnach ist die Markterfassung die grundlegende Voraussetzung für eine zielgruppenspezifi-

103; Lohmann, M., Wohlmann, R., Urlaub in Deutschland. Eine Grundlagenuntersuchung über Verhaltensweisen, Meinungen und Einstellungen von Urlaubern, studienkreis für Tourismus e.V. (Hrsg.), Starnberg 1987, S. 15. 


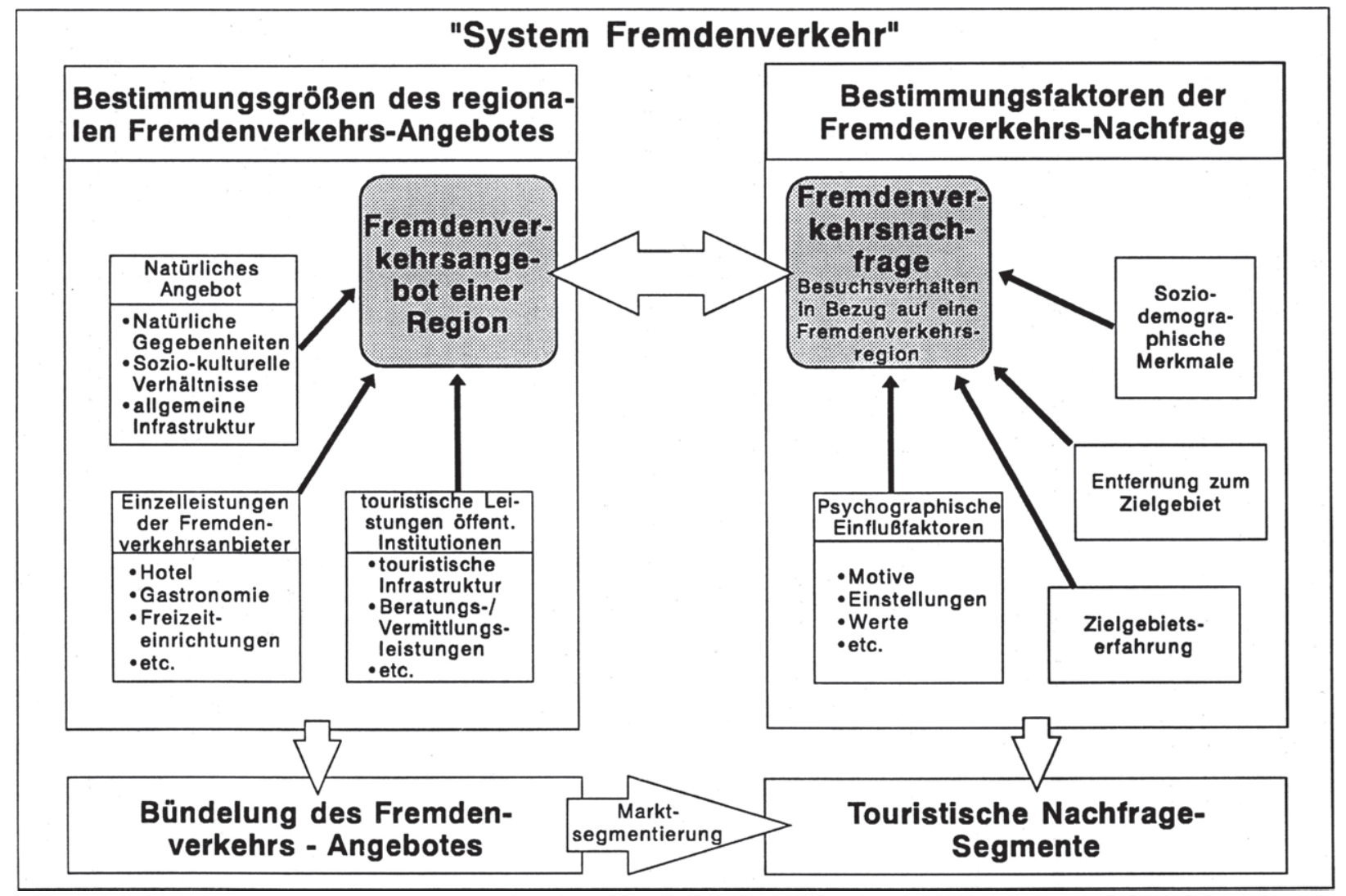

Abb. 1: Marktsegmentierung als Bindeglied zwischen Angebot und Nachfrage als Element des Systems Fremdenverkehr 
sche Ausgestaltung des touristischen Leistungsangebots einer Fremdenverkehrsregion. Dabei erweist sich die Marktsegmentierung als Bindeglied zwischen Angebot und Nachfrage zur Gestaltung eines zielgruppengerechten Marketing im Fremdenverkehr von Regionen.

3. Ansatzpunkte der Marktsegmentierung als Grundlage des Paradigmas zum zielgruppenmarketing von Fremdenverkehrsregionen

Das Konzept der Marktsegmentierung findet sich in der Literatur zum Marketing in unterschiedlichen Interpretationen, die insbesondere hinsichtlich der Einordnung der segmentierung in den GesamtprozeB der Marketingplanung voneinander abweichen. 43 Die Vielzahl von Ansätzen sind auf zwei Basiselemente zurückzuführen. Zum einen steht die Informationsseite im Vordergrund, in deren Rahmen die Marktsegmentierung als reine Marktforschungsmethode aufgefaBt wird. Zum anderen wird eine entscheidungsorientierte sicht zugrunde gelegt, bei der die Marktsegmentierung als Voraussetzung bzw. Mittel für die Durchführung einer differenzierten Marktbearbeitung angesehen wird.

$43 \mathrm{Vgl}$. zu den einzelnen Begriffsabgrenzungen z.B. Winkler, A., Praktische Anwendung der Marktsegmentierung als Methode der Marktforschung, in: Jahrbuch der Absatz- und Verbrauchsforschung, 1972, Heft 2, S. 102 ; Sheth, J.N., Marktsegmentierung als relevante Planungshilfe des Marketing, in: Jahrbuch für Absatz- und Verbrauchsforschung, 1972, Heft 2, S. 129; Angehrn, 0., Marktsegmentierung als Absatzmethode, in: GFM, 1968, Heft 2, S. 38 ; Scheuch, F., Logische strukturen und pragmatische Bedeutung der Marktsegmentierung, in: Die Unternehmung, 1974, Heft 3, S. 213. Ein zusammenfassender Uberblick findet sich bei Kols, P., Bedarfsorientierte Marktsegmentierung auf Produktivgütermärkten, Frankfurt a.M. 1986, S. 24 ff. 
Die Marktsegmentierung als Marketing-8trategie beinhaltet beide Aspekte. 44 Daher werden ihr die voneinander abhängigen Teilaufgaben, die Markterfassung und Marktbearbeitung zugeordnet. Dieser Interpretation folgend wird Marktsegmentierung definiert als die Aufteilung eines heterogenen Gesamtmarktes in homogene segmente, um eine gezielte Bearbeitung eines oder mehrerer segmente mit Hilfe eines segmentspezifischen Marketing-Programms zu ermöglichen. 45

Die vielschichtigen Problembereiche der Marktsegmentierung als Marketing-strategie im Fremdenverkehr lassen sich in einem Paradigma zusammenfassen. Dabei treten 7 zentrale Fragestellungen in den Vordergrund (vgl. Abbildung 2), welche bei einer Marktsegmentierung im Fremdenverkehr von Regionen zu berücksichtigen sind und im folgenden unter dem Begriff zielgruppenmarketing subsumiert werden.

Die erste Dimension bezieht sich auf die zentrale Fragestellung, wer als Träger des zielgruppenmarketing im Fremdenverkehr von Regionen in Betracht kommt. Unterstellt man, daB im Rahmen des Zusammenschlusses von Kommunen eine regionale Fremdenverkehrsorganisation 46 gebildet wird, erscheint es

44 Vgl. Freter, H., Marktsegmentierung, stuttgart u.a., s. 18; Hünerberg, R., Zur Segmentierungsproblematik im Rahmen der Marketingpolitik, in: Marktforschung, 1981, Heft 2 , S. 50; Uebele, H., Marktsegmentierung im Investitionsgüter-Bereich, in: $\mathrm{ZfbF}, 1984$, Heft $2, \mathrm{~S}$. 158 ; Krautter, J., Zum Problem der optimalen Marktsegmentierung, in: $\mathrm{ZfB}, 1975$, Heft 2, S. 114 .

45 Vgl. Meffert, H., Marktsegmentierung und Marktwahl im internationalen Marketing, in: DBW, 1977, Heft 3, $S$. 434; Freter, H., Marktsegmentierung, a.a.O., S. 18.; Backhaus, K., Investitionsgütermarketing, 2. Auf 1., München 1992, S. 158 .

46 Hierbei handelt es sich um öffentlich-rechtliche oder privat-rechtliche Organisationen, die die Teilleistungen einzelner Fremdenverkehrsanbieter und/oder -orte koordinieren. Vgl. Kaspar, C., Fremdenverkehrslehre im GrundriB, a.a.0., s. 85 ff. 


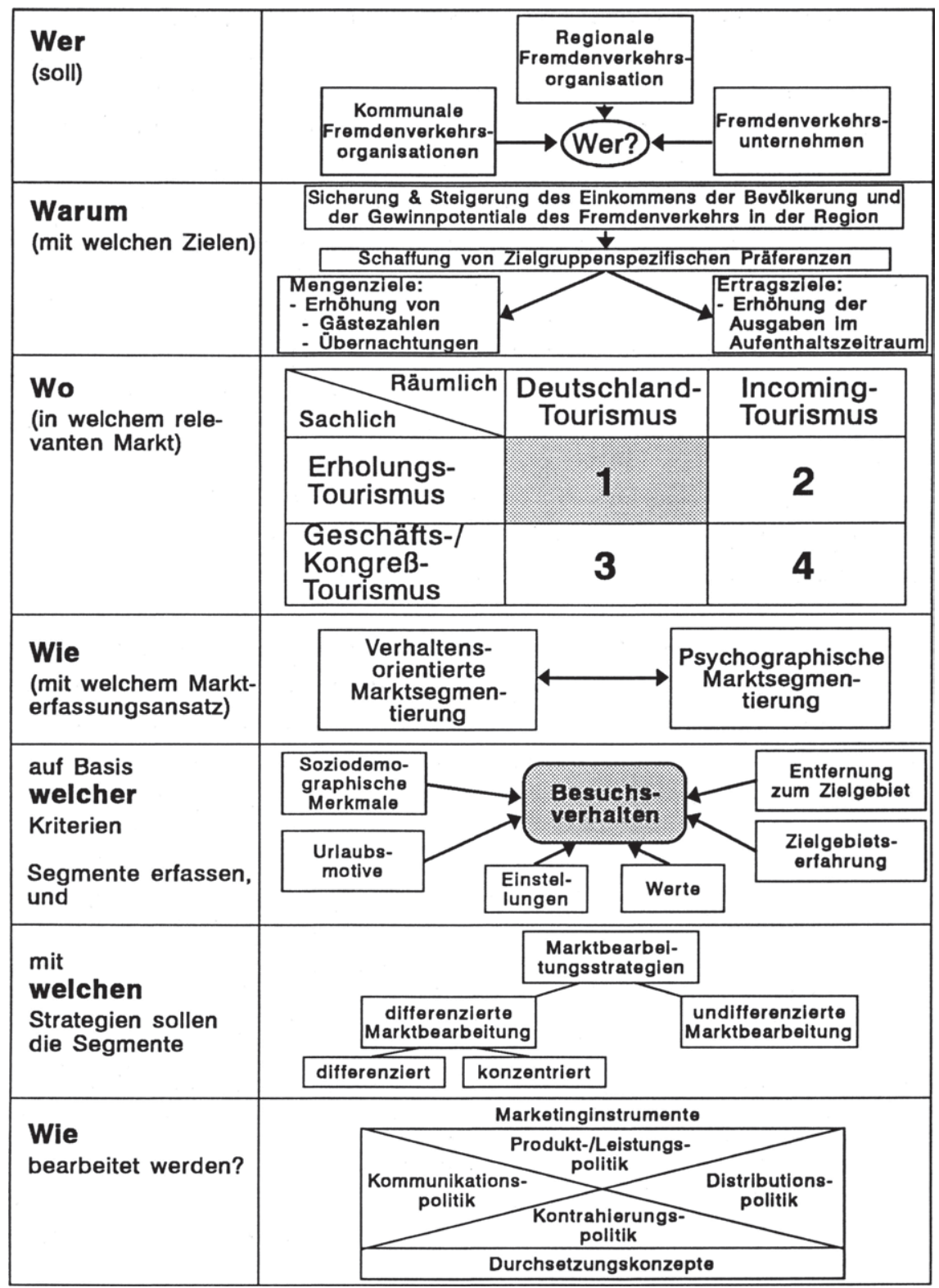

Abb. 2 Paradigma des Zielgruppenmarketing von Fremdenverkehrs-Regionen 
zweckmäßig, dieser die Aufgabe der Erstellung von Marktsegmentierungskonzepten $z u$ übertragen. 47 Andernfalls sind Interessengegensätze und Ressortegoismen vor allem auf der kommunalen Ebene zu erwarten, die auf der Ebene der Fremdenverkehrsanbieter zu störungen bei der Konzepterstellung führen. 48 Trotz zunehmender Forderungen nach einer zielgruppenspezifischen Marktbearbeitung durch Fremdenverkehrsregionen wurden bisher die zentralen Fragestellungen des zielgruppenmarketing aus der sicht von regionalen Fremdenverkehrsorganisationen noch nicht umfassend behandelt. 49 Vor diesem Hintergrund soll im Rahmen dieser Untersuchung die Sichtweise einer regionalen Fremdenverkehrsorganisation eingenommen werden.

Die zweite Dimension betrifft die mit dem zielgruppenmarketing $z u$ verfolgenden ziele. Hier sind die in den einzelnen Kommunen zugrunde gelegten zielformulierungen der kommunalen Fremdenverkehrspolitik ${ }^{50}$ unmittelbar auf Regionen zu übertragen und als oberziele des zielgruppenmarketing im Fremdenverkehr von Regionen $z u$ verstehen. zentrale ziele sind die sicherung bzw. Steigerung der Gewinnpotentiale des Fremdenverkehrs in der Region und der Einkommensverhältnisse der

47 So betonen auch Meffert u.a., daß die grundlegende Voraussetzung für eine erfolgreiche profilierung einer Region die Gründung einer organisation mit klar umrissenen Kompetenz- und Verantwortungsbereichen ist. Vgl. Meffert, H., et al., Regionenmarketing Münsterland, a.a.o., S. $206 \mathrm{ff}$.

48 Vgl. Bleile, G., Gemeinsam in den Wettbewerb. Fremdenverkehrsgemeinschaften - Vorteile und Erfolgsbedingungen, in: Der Fremdenverkehr, 1983, Heft 6, S. 16.

49 Erste Ansatzpunkte finden sich bei Bleile, G., Gemeinsam in den Wettbewerb, a.a.0., S. $14 \mathrm{ff}$.

50 Dabei wird Fremdenverkehrspolitik definiert als die bewuBte Förderung und Gestaltung des Fremdenverkehrs durch Einflußnahme auf die touristisch relevanten Gegebenheiten seitens von Gemeinschaften. Vgl. Kaspar, C., Fremdenverkehrslehre im Grundriß, a.a.0., s. 17 . 
Bevölkerung. ${ }^{51}$ Diese zentralen Ziele sind durch den Aufbau zielgruppenspezifischer Präferenzen zugunsten der jeweiligen Angebote und Leistungsträger in der Fremdenverkehrsregion zu realisieren. Hiermit ist langfristig der Ausbau der Marktstellung der Fremdenverkehrsregion verbunden. 52 In der Fremdenverkehrsforschung werden die Erhöhung der Gästezahlen und die Erhöhung der Ubbernachtungszahlen als gleichberechtigte Teilziele abgeleitet, 53 wobei jedoch die Ertragsziele nur implizit zum Ausdruck kommen. Daher ist diese zielformulierung im Sinne der Oberziele um Deckungsbeitrags- und Erlösziele zu ergänzen. Zum Beispiel könnte statt der Erhöhung der Gästezahlen eine Erhöhung der Ausgaben der Gäste während ihres Aufenthaltes angestrebt werden. 54 Ferner wird bei der zielformulierung nicht beachtet, daB die Verfolgung dieser ziele jeweils unterschiedliche Implikationen für die Ausgestaltung des zielgruppenmarketing haben. Während die zielsetzung der Erhöhung der Gästezahlen die Erhöhung der Tagesausflugsgäste sowie Ubbernachtungsgäste umfaBt, stehen im Hinblick auf das Ziel "Erhöhung der Ubernachtungszahlen" nur letztere im vordergrund. In der vorliegenden Untersuchung werden daher die mit der Besuchsdauer potentieller Touristen in Verbindung stehenden Ziele als Bedingungsrahmen des zielgruppenmarketing im Fremdenverkehr von Regionen berlicksichtigt.

51 Vgl. Krippendorf, J., Marketing im Fremdenverkehr, a.a.0., s. 55 .

52 Vgl. Freyer, w., Tourismus, a.a.0., s. 201; Krippendorf, J., Marketing im Fremdenverkehr, a.a.0., s. $54 \mathrm{ff}$.

53 Vgl. Kirsch, K., Schwerpunktförderung als Instrument regionaler Wirtschaftspolitik unter besonderer Berücksichtigung des Fremdenverkehrs, Diss., Reichenberg 1980, S. 109.

54 Vgl. Krippendorf, J., Marketing im Fremdenverkehr, a.a.0., s. 56 . 
Die dritte Dimension des zielgruppenmarketing bezieht sich auf die Frage, in welchem relevanten karkt eine zielgruppenspezifische Marktbearbeitung erfolgt. Der relevante Markt umfaBt alle für die Besuchs- und Verkaufsentscheidungen bedeutsamen Austauschbeziehungen zwischen touristischen Angeboten in räumlicher, sachlicher und zeitlicher Hinsicht. 55 Bezogen auf den Fremdenverkehrsbereich ist es zweckmäBig in räumlicher Hinsicht zwischen Deutschland- und Incoming-Tourismus 56 und in sachlicher Hinsicht zwischen Erholungs- und Geschäfts-KongreBtourismus $z u$ unterscheiden. Aufgrund der geringen Bedeutung des Incoming-Tourismus für die meisten deutschen Fremdenverkehrsregionen wird dieser ebenso wie der Geschäftstourismus ${ }^{57}$ aus der folgenden Analyse ausgegrenzt.

55 Diese für den Investitionsgüterbereich vorgenommene Abgrenzung des relevanten Marktes läBt sich auch auf den Fremdenverkehr übertragen. Da sich die zeitliche Abgrenzung vor allem auf die Frage bezieht, wie lange Konkurrenz- und Nachfragebeziehungen eines Produzenten bestehen, wenn politische oder wirtschaftliche Umbrüche bevorstehen, soll auf eine Ubbertragung auf den Fremdenverkehr an dieser stelle verzichtet werden. Vgl. hierzu Backhaus, K., Investitionsgütermarketing, a.a.0., s. 151.; In der Literatur sind unterschiedliche Ansätze zur Abgrenzung des relevanten Marktes entwickelt worden, wie z.B. Ubber Kreuz-Preis-Elastizitäten, Konzept der funktionalen Ähnlichkeit, etc. Vgl. hierzu Nieschlag, R., Dichtl, E., Hörschgen, H., Marketing, 15. Aufl., Berlín 1988 , S. 623 f.; Ferner findet sich ein tuberblick bei Backhaus, K., Investitionsgutermarketing, a.a.0., S. 153 ff.

56 Bezogen auf die Bundesrepublik umfaBt der "Incoming-Tourismus" die ausländische Nachfrage nach Fremdenverkehrsleistungen in der Bundesrepublik. Vgl. Meyer-Schwickerath, M., Perspektiven des Tourismus in der Bundesrepublik Deutschland, a.a.0., s. $80 \mathrm{f}$.

57 Beim Geschäftstourismus sind im Vergleich zum privaten Tourismus andere Marktmechanismen $\mathrm{zu}$ vermuten, die eine parallele Abhandlung nicht zweckmäBig erscheinen lassen. Es ist davon auszugehen, daß im Gechäfts-/KongreBtourismus die Existenz und Qualität z.B. von infrastrukturellen Einrichtungen wie Kongreßräumen und KongreBzentren die bedeutsamen Bestimmungsfaktoren des Verhaltens darstellen. Ansatzpunkte für eine auf die Besonderheiten des Geschäftstourismus abgestimmte Marksegmentierung 
Für die vorliegende Untersuchung stellt demnach der Erholungstourismus westdeutscher Bundesbürger den relevanten Markt dar.

Die vierte Dimension des zielgruppenmarketing umfaBt die Fragestellung, unter Berücksichtigung welcher Markterfassungsansätze eine segmentierung durchzufüren ist. Dabei können verhaltensorientierte, soziodemographische und psychographische Markterfassungsansätze unterschieden werden. 58

Verhaltensorientierte Ansätze 59 gehen von dem beobachtbaren Kauf- und Konsumverhalten der Nachfrager aus. In Abhängigkeit von dem beobachtbaren Verhaltensmerkmalen werden die Nachfrager zu Gruppen zusammengefaBt, deren Verhaltensunterschiede durch soziodemographische und psychographische Merkmale erklärt und prognostiziert werden. Auf der Grundlage dieser Ansätze sind segmente des Besuchsverhaltens zu bilden. Das Besuchsverhalten ist jedoch auch anhand der Besuchsdauer zu konkretisieren. Die Berücksichtigung der Be-

finden sich vor allem in der Literatur zur Marktsegmentierung im Investitionsgüterbereich. vgl. hierzu die ausführlichen Utberblicke bei Backhaus, $K$., Investitionsgutermarketing, a.a.0., S. $158 \mathrm{ff}$. und Kols, P., Bedarfsorientierte Marktsegmentierung auf Produktivgutermärkten, a.a.0., S. $93 \mathrm{ff}$.

Bauer spricht in diesem zusammenhang von personen- bzw. persönlichkeits- und einstellungsorientierten Ansätzen. Dieser Unterscheidung soll mit der Bezeichnung psychographischer segmentierungsansatz nicht gefolgt werden. Ferner sollen die von Bauer den persönlichkeitsorientierten Ansätzen zugeordneten soziodemographischen Merkmale hier als eigenständiger segmentierungsansatz aufgefaBt werden. Vgl. zur Abgrenzung der persönlichkeitsund einstellungsorientierten Ansätze Bauer, E., Marktsegmentierung als Maketing-strategie, a.a.0., s. $66 \mathrm{ff}$. $\mathrm{Zu}$ der Dreiteilung von Marktsegmentierungsansätzen vgl. Freter, H., Marktsegmentierung, a.a.O., s. 49 ff.; Meffert, H., Marketing, a.a.0., s. 245 ff.

59 Vgl. Bauer, E., Markt-segmentierung als Marketing-strategie, Berlin 1976, S. 64 und die dort angegebene Literatur. 
suchsdauer erweist sich als erforderlich, um eine den zielsetzungen (Erhöhung der Übernachtungszahlen vs. Gästezahlen) entsprechende Marktbearbeitung vorzunehmen. Der Vorteil der verhaltensorientierten Markterfassung besteht darin, daB damit gleichzeitig die wesentlichen Bestimmungfaktoren des Besuchsverhaltens identifiziert werden. In der Fremdenverkehrsforschung sind verhaltensorientierte Markterfassungsansätze zur Erklärung des regionenspezifischen Besuchsverhaltens insbesondere unter Berücksichtigung der Besuchsdauer bislang vernachlässigt worden. 60

In soziodomographischen Ansätzen 61 erfolgt die segmentbildung über soziodemographische Merkmale. Diese sind relativ leicht erfaBbar und das erforderliche Datenmaterial kann relativ kostengünstig beschafft werden. Dies begründet u.a. die in der Fremdenverkehrspraxis anzutreffende Dominanz der soziodemographischen Ansätze. Da den soziodemographischen Merkmalen eine nur unzureichende Besuchsverhaltensrelevanz zugesprochen wird, 62 ist eine segmentbildung allein auf der Basis von soziodemographischen Merkmalen nicht erfolgversprechend. Daher sollen diese Merkmale im Rahmen der vorliegenden Arbeit als segmentbeschreibende Merkmale zur zielgruppenidentifikation herangezogen werden.

Psychographische Marktsegmentierungsansätze verwenden demgegenüber die psychographischen Merkmale zur Markterfassung.

60 Auch die Untersuchung von wölm, im Rahmen derer auf der Basis eines verhaltensorientierten Markterfassungsansatzes die segmente Alpenurlauber, südreisende und Deutschlandurlauber abgegrenzt werden, gibt keine Hinweise auf die wesentlichen Bestimmungsfaktoren des regionenspezifischen Besuchsverhaltens. Vgl. Wölm, D., Marketing für Deutschlandreisen unter besonderer Berücksichtigung der strategie der Marktsegmentierung, Bamberg 1980 , S. 199 ff.

61 Vgl. Freter, H., Marktsegmentierung, a.a.O., S. 49 ff.

62 Vgl. Kapitel A.2. 
Die Nachfrager werden zunächst hinsichtlich ihrer psychographischen Merkmale zu homogenen Gruppen zusammengefaBt. AnschlieBend wird untersucht, ob die ermittelten Gruppen von Individuen ein in sich gleiches, untereinander aber verschiedenartiges Kauf- und Konsumverhalten zeigen.

Die meisten Markterfassungsansätze in der Fremdenverkehrsforschung verwenden zur segmentbildung psychographische Kriterien. Es wird jedoch in keiner dieser Untersuchungen in Betracht gezogen, eine psychographische Marktsegmentierung erst im AnschluB an eine verhaltensorientierte Markterfassung durchzufuhren. Dies erscheint zweckmäBig, da hierdurch die wesentlichen Bestimmungsfaktoren des Besuchsverhaltens empirisch ermittelt und der psychographischen Marktsegmentierung zugrundegelegt werden können. 63

Die Vernachlässigung dieses Aspektes ist darauf zurückzuführen, daB in der Fremdenverkehrsforschung die Ableitung von Gesamttypologien dominiert. Gesamttypologien geben aber keine Hinweise darauf, inwieweit die identifizierten segmente ein unterschiedliches Besuchsverhalten in bezug auf eine spezifische Fremdenverkehrsregion aufweisen. Daruber hinaus findet in den Gesamttypologien eine Typenbeschreibung auf der Basis von soziodemographischen Merkmalen nur unzureichend Verwendung. Somit lassen sich hieraus keine Anhaltspunkte für eine zielgruppenspezifische, auf die Besonderheiten von Fremdenverkehrsregionen abgestimmte Marktbearbeitung ableiten. 64 Hiermit ist zugleich die Fragestellung angesprochen, welche segmentierungskriterien zur Beschrei-

63 Auch Werner betont die Notwendigkeit, daß in der psychographischen Marktsegmentierung nur die "trennschärfsten Kriterien" verwendet werden sollten. Vgl. Werner, J., Marktsegmentierung für eine erfolgreiche Markt-Bearbeitung, in: Jahrbuch der Absatz- und Verbrauchsforschung, 1987, Heft 4, S. 398.

64 Vgl. Meffert, H., u.a., Regionenmarketing Münsterland, a.a.o., s. $83 \mathrm{f}$. 
bung der Segmente im Rahmen der verhaltensorientierten Segmentierung bzw. zur Markterfassung im Rahmen der psychographischen Marktsegmentierung heranzuziehen sind.

Prinzipiell kommen als segmentierungakriterien alle soziodemographischen und psychographischen Merkmale in Frage. Bestimmungsfaktoren des Besuchsverhaltens stehen hierbei im Mittelpunkt. 65 Vor diesem Hintergrund sind die als Bestimmungsfaktoren der Fremdenverkehrsnachfrage gekennzeichneten Variablen 66 als geeignete segmentierungskriterien im Fremdenverkehr von Regionen zu bezeichnen. Betrachtet man in diesem zusammenhang die in der Fremdenverkehrsforschung angesetzten Kriterien, so zeigt sich, daB

- ein Schwergewicht auf den Urlaubsmotiven liegt,

- nur vereinzelt Einstellungen, und dann mit überwiegend undifferenziertem objektbezug berücksichtigt werden,

- Persönlichkeitsmerkmale nur ansatzweise zur Markterfassung herangezogen werden und

- die zielgebietserfahrung als segmentierungskriterium vernachlässigt wird.

Ferner findet sich in den dargestellten Ansätzen keine explizite Berücksichtigung der zielgobietsentfornung. Angesichts der gestiegen Bedeutung von Kurzurlaubsreisen ist es jedoch notwendig, die zielgebietsentfernung als Makro-EinfluBgröBe auf das Besuchsverhalten zu interpretieren. In Anlehnung an die mehrstufigen Marktsegmentierungsansätze 67 im

65 Vgl. in Anlehnung an Freter, H., Marktsegmentierung, a.a.0., S. 43; Kaiser, A., Die Identifikation von Marktsegmenten, Darmstadt 1978, S. 70 f.; Backhaus, K., Investitionsgütermarketing, a.a.0., s. 158 .

Vgl. Kapitel A.2.

67 Diese Überlegung knüpft an die in der Investitionsgüterbranche bekannte Vorgehensweise der mehrstufigen Marktsegmentierung an, im Rahmen derer versucht wird, in ei- 
Investitionsgütermarketing ${ }^{68}$ ist es im Rahmen der vorliegenden Untersuchung von Interesse, die ZweckmäBigkeit einer auf der Basis der Zielgebietsentfernung durchzufürenden Makrosegmentierung zu überprüfen. 69

Abbildung 3 gibt einen Überblick über die wichtigsten Markterfassungsansätze in der Fremdenverkehrsforschung. Dabei wurden die Ansätze in Abhängigkeit von der Erhebungsart, der zur Typenbildung verwendeten segmentierungskriterien, ihrem zielgebietsbezug (Inland, Ausland, Region, Stadt), der Besuchsdauer sowie den ermittelten Typen näher charakterisiert. Letztere geben zusätzlich Hinweise auf den verwendeten Markterfassungsansatz. Bezogen auf die Markterfassung läßt sich zusammenfassend anführen, daB bisher die systematische Auseinandersetzung mit den zur Marktsegmentierung im Fremdenverkehr von Regionen anzusetzenden segmentierungsansätzen und den dabei zu berücksichtigenden segmentierungskriterien eine noch offene Fragestellung repräsentiert.

nem stufenweisen FilterungsprozeB EinfluBfaktoren des organisationalen Beschaffungsverhaltens für Segmentierungsüberlegungen zu nutzen. Einer der bekanntesten Ansätze stammt von wind/Cardozo, die vorschlagen auf der Basis organisationaler Kriterien (Unternehmensgröße, organisationsstruktur, standort, Kaufsituation) eine Makrosegmentierung durchzuführen. Vgl. Wind, Y., Cardozo, R.N., Industrial Market segmentation, in: Industrial Marketing Management, 1974, Heft 3, S. 156 ff.

68 Einen Überblick über die mehrstufigen Marktsegmentierungsansätze im Investitionsgütermarketing findet sich bei Backhaus, K., Investitionsgutermarketing, a.a.o., s. 162 ff.; Kols, P., Bedarfsorientierte Marktsegmentierung auf Produktivgütermärkten, a.a.0., s. $61 \mathrm{ff}$.

69 Mit der Makrosegmentierung im Investitionsgüterbereich soll den unterschiedlichen Ebenen des industriellen Einkaufverhaltens (z.B. Ebenen der Umwelt, der Organisation, der Kollektive und des Individuums) Rechnung getragen werden. Zum Zwecke der vorliegenden Arbeit soll der Begriff Makrosegmentierung demgegenüber die Existenz von dominanten Bestimmungsfaktoren des Besuchsverhaltens - in diesem Fall der Zielgebietsentfernung - zum Ausdruck bringen. 


\begin{tabular}{|c|c|c|c|c|c|c|c|c|c|}
\hline & \multirow[b]{2}{*}{$\begin{array}{c}\text { Vor- } \\
\text { tahreen }\end{array}$} & \multicolumn{5}{|c|}{ Zlelgebletabezug } & \multirow{2}{*}{ 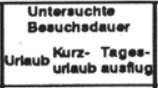 } & \multirow{2}{*}{\begin{tabular}{|c|}
$\begin{array}{c}\text { Sogmen- } \\
\text { terung:- } \\
\text { kritorien } \\
\text { (Sogment- } \\
\text { blloung) }\end{array}$ \\
\end{tabular}} & \multirow{2}{*}{$\begin{array}{c}\text { Identifizierte } \\
\text { Typen }\end{array}$} \\
\hline & & $\mid \begin{array}{l}\text { (Gosamt- } \\
\text { typologie) }\end{array}$ & Ausiand & Inland & Region & Stadt & & & \\
\hline $\begin{array}{c}\text { Meffert } \\
1901\end{array}$ & empirlech & & & & 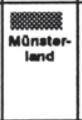 & & & $\begin{array}{l}\text { Roalein- } \\
\text { stellungen }\end{array}$ & 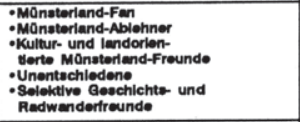 \\
\hline $\begin{array}{l}\text { Studienikrels } \\
\text { fû́r } \\
\text { Tourismus } \\
1991\end{array}$ & empirisch & 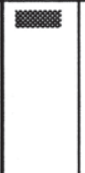 & & & & & & $\begin{array}{c}\text { Lobens- } \\
\text { stil }\end{array}$ & 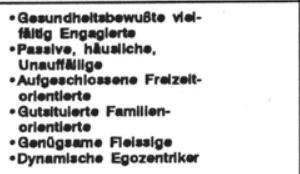 \\
\hline $\begin{array}{c}\text { Wôhler } \\
1990\end{array}$ & empirlech & & & & sodharz & & 桨 & $\begin{array}{l}\text { Uriauba- } \\
\text { erwartung- } \\
\text { on und } \\
\text { anforder- } \\
\text { ungen }\end{array}$ & 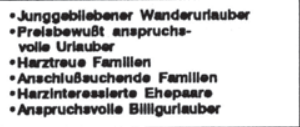 \\
\hline $\begin{array}{c}\text { Bogshard/ } \\
\text { Froy/ } \\
\text { Lambardl/ } \\
\text { Sehonfeld/ } \\
1990\end{array}$ & $\begin{array}{l}\text { a-prlort } \\
\text { Klasain- } \\
\text { katton an- } \\
\text { hand om- } \\
\text { pirischer } \\
\text { Ergobnisas }\end{array}$ & & & & & Basel & 4 & $\begin{array}{l}\text { Touris- } \\
\text { tische } \\
\text { Motive } \\
\text { und } \\
\text {-aktivi- } \\
\text { tilten }\end{array}$ & $\begin{array}{l}\text {-Kultur-Typ } \\
\text {-Kultur-Bummel-Typ } \\
\text {-Shopping-Bummel-Typ } \\
\text {-Zoo-Typ } \\
\text { - Mesec-Typ } \\
\text { - Geschaftaloute }\end{array}$ \\
\hline $\begin{array}{l}\text { DWiF } \\
1989\end{array}$ & $\begin{array}{l}\text { o-priort } \\
\text { Klasaif- } \\
\text { katton an- } \\
\text { hand om- } \\
\text { piriacher } \\
\text { Ergebnisase }\end{array}$ & & & & $\left(\begin{array}{c}\text { Nord- } \\
\text { hein- } \\
\text { Wost- } \\
\text { talon }\end{array}\right)$ & & & $\begin{array}{l}\text { Urlaubs- } \\
\text { motive. } \\
\text {-erwart- } \\
\text { ungen }\end{array}$ & 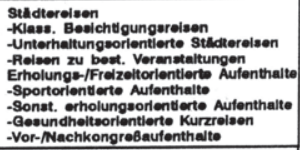 \\
\hline $\begin{array}{l}\text { DWiF } \\
1989\end{array}$ & 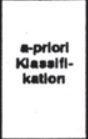 & & & & $\begin{array}{l}\text { Nord- } \\
\text { Whein- } \\
\text { Wost- } \\
\text { falen }\end{array}$ & & & $\begin{array}{l}\text { Uriaube- } \\
\text { motive,- } \\
\text { aktivitition }\end{array}$ & 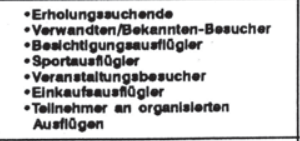 \\
\hline $\begin{array}{l}\text { Opa- } \\
\text { sohowekl } \\
1988\end{array}$ & Konzep & & & & & & 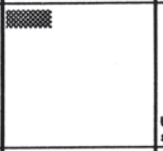 & \begin{tabular}{|c|} 
Lobens- \\
zyklus- \\
phasen \\
(Beschrol- \\
bung \\
anhand \\
von \\
Uriaubavor- \\
stellungen)
\end{tabular} & $\begin{array}{l}\text { - Jugendiliche } \\
\text {-junge Erwecheene } \\
\text { - Singlea } \\
\text { - Paare } \\
\text { - Famillen mit Kndern } \\
\text { - Famillien mit Jugendilichen/ } \\
\text { Erwachaenen } \\
\text { - Jungsenioren } \\
\text { - Ruhestlandier }\end{array}$ \\
\hline $\begin{array}{l}\text { Veon/ } \\
\text { Vorhallion } \\
1986\end{array}$ & empirisch & 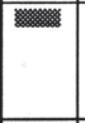 & & & & & 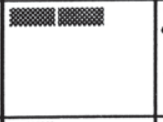 & $\begin{array}{l}\text { Individu- } \\
\text { elle Werte } \\
\text { und } \\
\text { Uriaube- } \\
\text { verhaiten }\end{array}$ & $\begin{array}{l}\text { - Organized Vacation } \\
\text { - Beach Vacation } \\
\text {-Domestic Vacation } \\
\text { - Vaceations with Chilidren } \\
\text {-1-2 person Vencation } \\
\text { - Long Campling Vacation } \\
\text { - Short Vacation } \\
\end{array}$ \\
\hline $\begin{array}{l}\text { Ender } \\
\text { u. a } \\
1983\end{array}$ & empirlech & & & & & & 証 & $\begin{array}{l}\text { Wunsach- } \\
\text { voratel- } \\
\text { lungen an } \\
\text { ein Zlel- } \\
\text { land }\end{array}$ & $\begin{array}{l}\text {-Velseltige Komforturlauber } \\
\text { - Jugendiliche Badeurlauber } \\
\text { - INtere Wanderurlauber }\end{array}$ \\
\hline $\begin{array}{l}\text { Zolles/ } \\
\text { Ferner/ } \\
\text { Müller } \\
1981\end{array}$ & K.A. & & $\begin{array}{l}\text { Ostor- } \\
\text { reich }\end{array}$ & & & & & $\begin{array}{l}\text { Uriaubs- } \\
\text { orwart- } \\
\text { ungen }\end{array}$ & 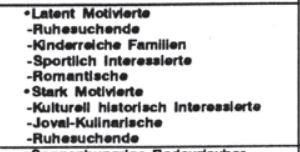 \\
\hline $\begin{array}{c}\text { Wolm } \\
1980\end{array}$ & emplirlsch & 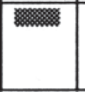 & & & & & & 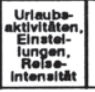 & 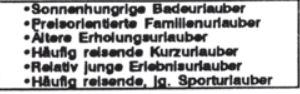 \\
\hline
\end{tabular}

\section{Abb. 3a: Ausgewählte empirische Studien zur Marktsegmentie- rung im Fremdenverkehr}




\begin{tabular}{|c|c|c|c|c|c|c|c|c|c|}
\hline & \multirow[b]{2}{*}{$\begin{array}{c}\text { Vor- } \\
\text { tahron }\end{array}$} & \multicolumn{5}{|c|}{ Zlolgebletebexug } & & \multirow{2}{*}{$\begin{array}{c}\text { Sogmen- } \\
\text { tierungs- } \\
\text { kelterien } \\
\text { (Sogment- } \\
\text { bllidung) }\end{array}$} & \multirow{2}{*}{$\begin{array}{c}\text { Identiflziorte } \\
\text { Typen }\end{array}$} \\
\hline & & 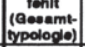 & Ausland & Inland & Reglon & stadt & Untaub Kurz: Tages- & & \\
\hline $\begin{array}{l}\text { Wolm } \\
1980\end{array}$ & omplisiach & & & & & & & 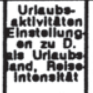 & 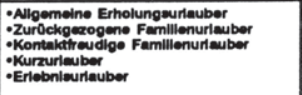 \\
\hline $\begin{array}{l}\text { Wolm } \\
1980\end{array}$ & empirisch & & & & & & & \begin{tabular}{c|} 
Relao- \\
verhalten
\end{tabular} & 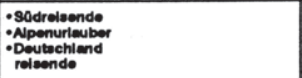 \\
\hline $\begin{array}{c}\text { Mexanec } \\
1979 \mathrm{~b}\end{array}$ & empliriach & & & & & & 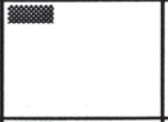 & \begin{tabular}{|c|}
$\begin{array}{c}\text { Rolseo- } \\
\text { verthelition }\end{array}$ \\
\end{tabular} & 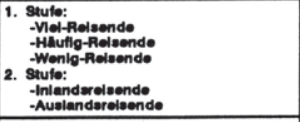 \\
\hline Maranee & emplirioch & & & & & & & 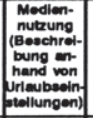 & 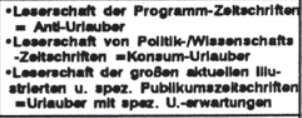 \\
\hline $\begin{array}{l}\text { Mouter } \\
1979\end{array}$ & $\begin{array}{c}\text { Konzop- } \\
\text { tonoill }\end{array}$ & 我 & & & & & 18 & $\begin{array}{c}\text { Frotzell- } \\
\text { akttivitutern } \\
\text { am } \\
\text { Wochen- } \\
\text { onde- }\end{array}$ & 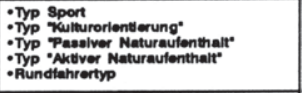 \\
\hline $\begin{array}{c}\text { AQRAR } \\
1977\end{array}$ & empirlech & & & & & & & $\begin{array}{l}\text { Rolso- } \\
\text { motivo: } \\
\text {-aktiv- } \\
\text { thton }\end{array}$ & 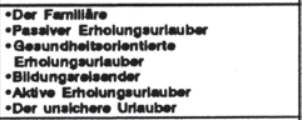 \\
\hline $\begin{array}{l}\text { Perrault/ } \\
\text { Dardon } \\
1977\end{array}$ & empirisch & & & & & & & 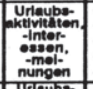 & $\begin{array}{l}\text { - Budget Traveller } \\
\text { - The Adventures } \\
\text { - Homebodiles } \\
\text { - Veceationers } \\
\text { - The moderates } \\
\end{array}$ \\
\hline $\begin{array}{l}\text { Hegeloff } \\
1977\end{array}$ & empirisch & & & & & & 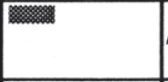 & 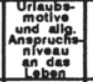 & $\begin{array}{l}\text { Relsotypen: Gesundheit, Blidung. } \\
\text { Status, Konformituk, } \\
\text { Kontakt, Erioben, } \\
\text { Abentover. }\end{array}$ \\
\hline $\begin{array}{c}\text { Gruneresdahr } \\
1975\end{array}$ & empiriach & & & & & & 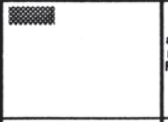 & $\begin{array}{l}\text { allig. Pera. } \\
\text { morkmale: } \\
\text { Froizemitprat } \\
\text { ferenzen }\end{array}$ & 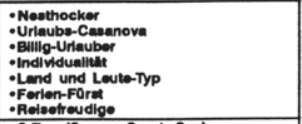 \\
\hline $\begin{array}{l}\text { Hehn } \\
1974\end{array}$ & K.A. & & & & & & & $\begin{array}{l}\text { Uriaubse- } \\
\text { motivo. } \\
\text {-aktivitation }\end{array}$ & 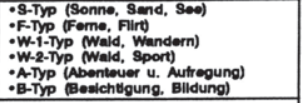 \\
\hline $\begin{array}{l}\text { European } \\
\text { Travel } \\
\text { Commisolon } \\
1974\end{array}$ & emplirisch & & Europe & & & & & $\begin{array}{c}\text { Gronde for } \\
\text { Europen- } \\
\text { Reisen, } \\
\text { Auswahi- } \\
\text { kriterien } \\
\text { (zum } \\
\text { Roisen) }\end{array}$ & 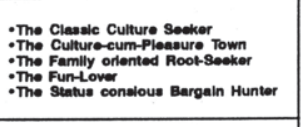 \\
\hline $\begin{array}{l}\text { Fingerhut } \\
\text { u. ef. } \\
1973\end{array}$ & $\begin{array}{l}\text { konzep- } \\
\text { tionell }\end{array}$ & & & & & & & 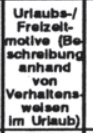 & 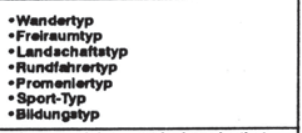 \\
\hline $\begin{array}{l}\text { Naether } \\
1971\end{array}$ & $\begin{array}{l}\text { konzep- } \\
\text { tlonell }\end{array}$ & & & & & & & 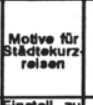 & 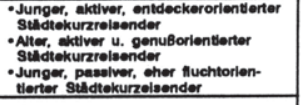 \\
\hline $\begin{array}{l}\text { aelger } \\
1969\end{array}$ & empirisch & & & & & & & 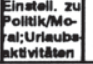 & $\begin{array}{l}\text { - Der altive Urlauber } \\
\text { - Der ortolungasuchende Urlauber } \\
\text { - Der bildungasuchende Urleuber }\end{array}$ \\
\hline
\end{tabular}

\section{Abb. 3b: Ausgewählte empirische studien zur Marktsegmentie- rung im Fremdenverkehr (Fortsetzung)}


Unter zugrundelegung der identifizierten Nachfragersegmente steht die Frage im Mittelpunkt, mit welchen Marktbearbeitungatrategion der relevante Markt zu bearbeiten ist. Dabei ist in Anlehnung an Meffert 70 unter Marktbearbeitungsstrategie ein an den touristischen zielgruppen ausgerichteter bedingter, langfristiger und globaler verhaltensplan zu verstehen, welcher zur Erreichung der ziele der Fremdenverkehrsregion beiträgt. Hier ist das AusmaB der Zielgruppenabdeckung und die Art der Marktbearbeitung festzulegen. Im allgemeinen wird zwischen undifferenzierter und differenzierter Marktbearbeitungsstrategie unterschieden. 71 Weitgehende Utbereinstimmung besteht in der Literatur daruber, daB der differenzierten Marktbearbeitung im Fremdenverkehr von Regionen eine hohe Bedeutung zukommt. 72 Inwieweit die Heterogenität des touristischen Leistungsangebots bzw. die Vielzahl touristischer Leistungsanbieter in einer Fremdenverkehrsregion EinfluB auf die Auswahl der Marktbearbeitungsstrategien und der damit in Verbindung stehenden zielgruppenauswahl hat, geht aus keiner der bisher durchgefuhrten Untersuchungen hervor.

Die siebte und zugleich letzte Dimension umfaBt die Fragestellung, mit welchen Marketing-Instrumenten die touristischen Nachfragersegmente $z u$ bearbeiten sind. Prinzipiell ist davon auszugehen, daB die Instrumente der vier MarketingMix-Bereiche auch auf den Fremdenverkehr von Regionen zu

70 Vgl. Meffert, H., Marketing. Grundlagen der Absatzpolitik, 7. Aufl., s. 55 .

71 Vgl. Meffert, H., Marketing, a.a.0., s. 103 ff. und s. $253 \mathrm{ff}$. Wird die differenzierte Marktbearbeitungsstrategie auf einem Teilmarkt verfolgt, wird von konzentrierter Marktbearbeitung gesprochen. Vgl. ebenda, S. 254.

72 Vgl. Bleile, G., Gemeinsam in den Wettbewerb, a.a.o., s. 14 ff., Müller, W.-H., Der Fremdenverkehr im kommunalen Marketing, in: Der städtetag, 1990, Heft 3, s. 225 ff., Wölm, D., Marketing im Tourismus, in: Marketing ZFP, 1979, Heft 4, S. 230. 
übertragen sind. Dengegenüber ist in der Literatur zum zielgruppenmarketing ein Schwergewicht auf der Kommunikationspolitik, und dabei vor allem auf der Imagepolitik festzustellen. ${ }^{73}$ Eine systematische Auseinandersetzung mit den Möglichkeiten der aktiven, zielgruppenspezifischen Marktbearbeitung durch regionale Fremdenverkehrsorganisationen fehlt bislang.

Ursächlich hierfür ist vor allem der eingeschränkte Handlungsrahmen von regionalen Fremdenverkehrsorganisationen. Die zielgruppenspezifische Ausgestaltung des touristischen Leistungsangebots in einer Fremdenverkehrsregion liegt überwiegend im Kompetenzbereich der rechtlich selbständigen Fremdenverkehrsanbieter und den kommunalen Entscheidungsträgern. Die hieraus resultierende Distanz zwischen der für die Konzeption zuständigen regionalen Fremdenverkehrsorganisation und den für die Umsetzung zuständigen touristischen Leistungsanbietern macht die Notwendigkeit der Entwicklung von Durchsetzungskonzepten deutlich, mit deren Hilfe die Anwendung der zielgruppenspezifischen Marktbearbeitungskonzepte durch die einzelnen touristischen Entscheidungsträger zu fördern ist. Daher überrascht es, daB dieser Aspekt in der Literatur bisher vernachlässigt wurde.

In einer zusammenfassenden würdigung der aufgezeigten Einzelaspekte ist festzuhalten, daß die systematische Auseinandersetzung mit allen relevanten Aspekten des zielgruppenmarketing im Fremdenverkehr von Regionen, insbesondere aber mit der Markterfassung und zielgruppenspezifischen Marktbearbeitung aus sicht von regionalen Fremdenverkehrsorganisationen eine offene Fragestellung repräsentiert. Dabei kommt angesichts der eingeschränkten Möglichkeiten einer aktiven Um-

73 Vgl. hierzu die vielfältigen Fallbeispiele in Roth, P., Schrand, A. (Hrsg.), Touristik-Marketing, a.a.O., S. 215 ff. und Seitz, E., Wolf, J. (Hrsg.), Tourismusmanagement und-marketing, Landsberg a.L. 1991, s. $547 \mathrm{ff}$. 
setzung zielgruppenspezifischer Marktbearbeitungskonzepte durch regionale Fremdenverkehrsorganisationen der Markterfassungsseite eine herausragende Bedeutung $\mathrm{zu}$. Damit ist gleichzeitig das ziel der vorliegenden Untersuchung angesprochen.

\section{4. zielsetzung und Gang der Ontersuchung}

Ausgehend von der vorgenommenen Eingrenzung der Problemstellung liegt die generelle zielsetzung der Arbeit darin, einen empirisch gestützten Beitrag für Marketingentscheidungen im Rahmen des zielgruppenmarketing von Fremdenverkehrsregionen zu leisten. Dabei steht die Frage im Mittelpunkt, unter Berücksichtigung welcher Markterfassungsansätze und segmentierungskriterien im Fremdenverkehr von Regionen eine zielgruppenabgrenzung vorzunehmen ist. Die vorliegende Untersuchung verfolgt deshalb vier Teilziele.

Das erste reilsiel liegt in der Analyse der ZweckmäBigkeit, auf der Basis der zielgebietsentfernung eine Makrosegmentierung durchzuführen. Hierzu sind die Ausprägungsformen des Besuchsverhaltens unter Berücksichtigung der Besuchsdauer zunächst $z u$ systematisieren und $z u$ operationalisieren, um schließlich den Einfluß der zielgebietsentfernung auf das Besuchsverhalten $z u$ untersuchen. Auf der Grundlage dieser Ergebnisse sind entfernungsgrupppenspezifische Verhaltenssegmente zu bilden.

Das zweite Teilziel der Arbeit liegt in der Analyse der wesentlichen Bestimmungsfaktoren des Besuchsverhaltens. Hierbei wird der in Abbildung 4 dargestellte hypothesengestützte Bezugsrahmen zugrunde gelegt. Auf der Basis der identifizierten, entfernungsgruppenspezifischen Verhaltenssegmente ist der EinfluB der zielgebietserfahrung, von sozio-ökonomischen und psychographischen Merkmalen auf das Besuchsverhal- 


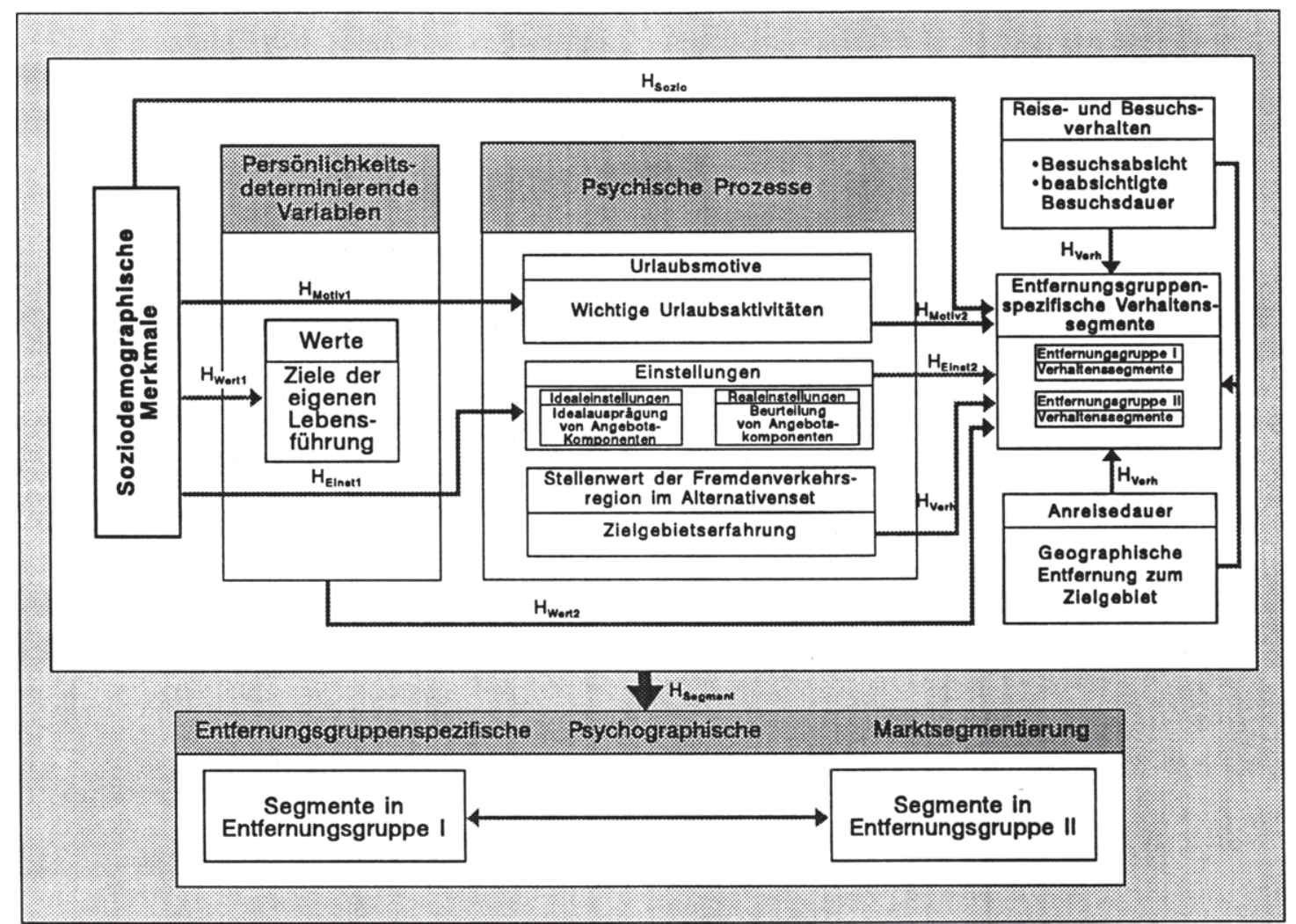


ten im Sinne von Besuch und Besuchsdauer zu analysieren. Dabei stehen Werte, Motive und Einstellungen im Vordergrund der Betrachtung.

Aufbauend auf den jeweiligen theoriegeleiteten Utberlegungen zum EinfluB der Merkmale auf das Besuchsverhalten in bezug auf Fremdenverkehrsregionen und ihrer operationalisierung werden die gewonnenen Erkenntnisse am Beispiel einer Untersuchungsregion empirisch überprüf. Im einzelnen umfaBt dieser Teil der Ausführungen folgende Aspekte:

- Empirische Utberprufung des Einflusses von soziodemographischen Merkmalen auf das Besuchsverhalten,

- Empirische Überprüfung des Einflusses von Werten, Motiven und Einstellungen auf das Besuchsverhalten,

- Integrierte Analyse der als verhaltensrelevant identifizierten Merkmale zur Identifikation der wesentlichen Bestimmungsfaktoren des Besuchsverhaltens.

Das dritte reilgiel der Arbeit liegt in der Entwicklung eines psychographischen Markterfassungsansatzes. Dabei ist zu zeigen, daB auf der Grundlage der wesentlichen Bestimmungsfaktoren des Besuchsverhaltens innerhalb der Makrosegmente Zielgruppen voneinander abzugrenzen sind, die einen höheren Aussagegehalt für die zielgruppenspezifische Marktbearbeitung aufweisen als bei der zugrundelegung von Verhaltenssegmenten.

Das vierte Teilaiel beinhaltet schlieBlich die Ableitung von Ansatzpunkten für eine zielgruppenspezifische Marktbearbeitung im Rahmen einer angebotsorientierten Betrachtung.

Mit den präzisierten schwerpunktsetzungen ist auch gleichzeitig der Gang der Arbeit vorgezeichnet. Kapitel B dient 
der Entwicklung eines Marksegmentierungsansatzes für den Fremdenverkehr von Regionen. Ausgangspunkt ist die Ableitung eines Anforderungskataloges an segmentierungkriterien und Segmentierungsansätze. Anschließend werden die Ausprägungsformen des Besuchsverhaltens und Möglichkeiten einer Makrosegmentierung konzeptionell abgeleitet, in einen operationalisierungsansatz überführt und empirisch überprüft.

Anschließend sind innerhalb der Makrosegmente die wesentlichen Bestimmungsfaktoren des Besuchsverhaltens zu identifizieren. GemäB des Bezugsrahmens der Untersuchung sollen soziodemographische Merkmale sowie Werte, Einstellungen und Motive als psychographische Merkmale im Mittelpunkt der Untersuchung stehen. Diese sind zunächst theoriegeleitet hinsichtlich ihrer Eignung als segmentierungskriterien zu analysieren und $\mathrm{zu}$ operationalisieren. Auf der Grundlage dieser Merkmale werden die in den Verhaltenssegmenten zum Ausdruck kommenden Verhaltensunterschiede zunächst isoliert und abschließend im Rahmen einer integrierten Analyse empirisch untersucht. Die identifizierten Bestimmungsfaktoren des Besuchsverhaltens legen die Grundlage für die Durchführung einer psychographische Marktsegmentierung innerhalb der gebildeten Makrosegmente.

Im Teil c werden Ansatzpunkte einer zielgruppenspezifischen Marktbearbeitung für den Fremdenverkehr von Regionen aufgezeigt. Neben der Formulierung von Marktbearbeitungsstrategien steht die Ableitung von Ansatzpunkten für die zielgruppenspezifische Ausgestaltung des Marketing-Mix im Vordergrund. Ferner ist zu überprüfen, mit Hilfe welcher Maßnahmen die Umsetzung eines solchen Marktbearbeitungskonzeptes auf der Ebene der rechtlich selbständigen Fremdenverkehrsanbieter zu gewährleisten ist. Daher sind Ansatzpunkte für die Durchsetzung zielgruppenspezifischer Marktbearbeitungskonzepte zu erarbeiten. In Kapitel D erfährt das Gesamtkonzept 
des Zielgruppenmarketing im Fremdenverkehr von Regionen eine zusammenfassende würdigung.

Die empirische überprüfung des Bezugsrahmens der Untersuchung erfolgt auf der Grundlage einer im Juni 1990 durchgeführten standardisierten ${ }^{74}$ onnibus-Befragung von 970 Bundesbürgern. Ziel dieser Erhebung war es, die informatorischen Grundlagen für eine Marketing-Konzeption der Fremdenverkehrsregion "Münsterland" zu gewinnen. Dabei wurde der Untersuchungsgegenstand auf den Teilmarkt "Binnentourismus" eingegrenzt. Die Grundgesamtheit wurde definiert als Wohnbevölkerung in Privathaushalten der Bundesrepublik Deutschland. 75

Die Befragungsinhalte basieren auf den Variablengruppen des empirischen Bezugsrahmens. Nach Prüfung auf Vollständigkeit und Konsistenz konnten 936 Fragebögen für die empirische Analyse herangezogen werden. 76 Die statistische Datenauswertung erfolgte mit dem am Rechenzentrum der Universität Münster implementierten softwarepaket SPSS-X 4.1 (Statistical Package for Social sciences). Unter Berücksichtigung des jeweiligen Skalenniveaus wurden zur Auswertung Mittelwertver-

74 Ein standardisierter Fragebogen schreibt dem Interviewer das Vorgehen exakt vor, so daB er genau formulierte Fragen in einer festgelegten Reihenfolge zu stellen hat. Vgl. Meffert, H., Marketingforschung und Käuferverhalten, a.a.0., s. 205 .

75 Die neuen Bundesländer fanden in dieser Untersuchung keine Berücksichtigung. Ferner wurde auf Wunsch der Auftraggeber für die Befragung eine disproportional geschichtete stichprobenziehung gewählt. Die Gebiete im näheren geographischen Umkreis des Münsterlandes sowie der Anteil der Bürger Berlins sind stärker gewichtet.

76 Aktuelle und potentielle Geschäftstouristen wurden aus der Untersuchung ausgeschlossen. 
gleichstests, Faktoren-, Korrelations- Varianz-, Diskriminanz- und clusteranalysen verwendet. 77

77 Vgl. zu den einzelnen Verfahren, auf die im folgenden noch genauer einzugehen ist, Schubö, w., Uehlinger, H.M., U.a., SPSS-X-Handbuch der Programmversion 4.0 und SPSS-X 3.0, Stuttgart, New York 1991, S. 257 ff.; steinhausen, D., Zörkendörfer, D., statistische Datenanlayse mit dem Programmsystem SPSS-X, Software-Information 13 des Universitätsrechenzentrums, Universität Münster, 4. Aufl., Múnster 1987; Backhaus, $K$. et al., Multivariate Analysemethoden, 6. Aufl., Berlin u.a., 1990, S. 1 ff. 
B. Erfassung und Analyse von sosiodonographischen und psychographischen Merknalon als Kriterion sur Marktsegmentierung in Framdenverkehr von Regionen

\section{Anforderungen an die Marktsegmentierung in Frandenverkehr von Regionen}

Die zielgruppenspezifische Marktbearbeitung im Fremdenverkehr von Regionen erfordert die Erfassung von in sich homogenen, untereinander heterogenen zielgruppen. In diesem $\mathrm{Zu}-$ sammenhang sind eine Reihe von Anforderungen $z u$ nennen, die bei der Markterfassung zu berücksichtigen sind. Von dem Erfüllungsgrad dieser Anforderungen hängt $a b$, ob eine differenzierte Marktbearbeitung von Fremdenverkehrsregionen tatsächlich zur Realisierung der angestrebten ziele fuhrt. Insgesamt ergeben die Anforderungen einen Bewertungsrahmen, der eine systematische und vergleichende Beurteilung sowohl von segmentierungskriterien als auch von Marktsegmentierungsansätzen erlaubt. Es ist zu prufen, ob die realisierte Aufteilung des relevanten Marktes bzw. das zugrundeliegende segmentierungskriterium 1

- einen Bezug zum Kauf- bzw. Besuchsverhalten aufweist,

- Hinweise für den Einsatz von Marketing-Instrumenten liefert,

- eine Ansprechbarkeit und Identifizierbarkeit gewährleistet,

- zeitlich stabil ist,

1 Vgl. Freter, H., Marktsegmentierung, a.a.O., S. 43 ff., Frank, R.E., Massay, W.F., Wind, Y., Market Segmentation, Englewood cliffs, N.J., 1972, S. 27 f.; Gröne, A., Marktsegmentierung bei Investitionsgütern. Analyse und Typologie des industriellen Einkaufsverhaltens als Grundlage der Marketingplanung, wiesbaden 1977, s. 43 ff.; Kols, P., Bedarfsorientierte Marktsegmentierung auf Produktivgütermärkten, a.a.0., s. 80 ff., Meffert, H., Marketing, a.a.O., s. 244 f.; Wölm, D., Marketing für Deutschlandreisen, a.a.0., s. $135 \mathrm{ff}$. 
- der Forderung nach Wirtschaftlichkeit entspricht und - meBbar (operationalisierbar) ist.

Die Anforderung der Kauf- bsw. Besuchsverhaltensrelevanz an ein segmentierungskriterium bedeutet, daß dieses einen geeigneten Indikator für das aktuelle und künftige Kauf- bzw. Besuchsverhalten darstellt. ${ }^{2}$ Dabei erweisen sich nicht nur Kriterien als besuchsverhaltensrelevant, die den Besuch in eine Fremdenverkehrsregion erklären, sondern auch diejenigen, welche die Besuchsdauer beeinflussen. Im Rahmen der Markterfassung wird von vornherein versucht, nur solche segmentierungsvariablen $z u$ berücksichtigen, die einen Bezug zum Besuchsverhalten aufweisen und insofern einen Beitrag zur Erklärung des Besuchsverhaltens liefern. Die Hinzuziehung dieser Merkmale zur Markterfassung ermöglicht, Segmente voneinander abzugrenzen, die ein in sich homogenes und untereinander heterogenes Besuchsverhalten aufweisen. Von der Erfullung dieser Anforderung hängt $a b$, ob eine Verhaltensprognose und ein gezielter, segmentspezifischer Einsatz der Marketinginstrumente für die ermittelten segmente möglich ist. $^{3}$

Damit ist zugleich die zweite Anforderung an Segmentierungskriterien und Markterfassungskonzepte im Fremdenverkehr von Regionen angesprochen. Der Markterfassung sind segmentierungskriterien zugrundezulegen, die Anhaltspunkte für den gezielten Einsatz von Marketing-Instrumenten geben. Dabei ist der Bezug zur Marktbearbeitung um so gröBer, je besser es den regionalen Fremdenverkehrsorganisationen gelingt, innerhalb des relevanten Marktes segmente mit einem jeweils honogenen instrument- oder programmspesifischen Reaktions-

2 Vgl. Gröne, A., Marktsegmentierung bei Investitionsgütern, a.a.0., s. 44 .

3 Vgl. Freter, H., Marktsegmentierung, a.a.O., S. 43. 
verhalten $z \mathrm{u}$ bilden. ${ }^{4}$ Das gemeinsame Merkmal von Personen in einem segment besteht darin, daß diese gleiche oder ähnliche Reaktionen im sinne von Wirkungsfunktionen ${ }^{5}$ im Hinblick auf die von der Fremdenverkehrsregion getroffenen MaBnahmen aufweisen.

Allerdings können vor allem aufgrund von Datenbeschaffungsproblemen wirkungsfunktionen nicht für alle denkbaren Gruppen erhoben werden. 6 Daher sind Rückschlüsse für den zielgruppenspezifischen Einsatz der Marketinginstrumente nicht auf der Basis von wirkungsfunktionen, sondern auf der Grundlage von Kenntnissen über das Besuchsverhalten in Verbindung mit den Ausprägungen psychographischer und soziodemographischer Merkmale abzuleiten. 7

Zur Durchführung einer zielgruppenspezifischen Marktbearbeitung ist ferner erforderlich, eine gexielte nnsprache der zielgruppen zu gewährleisten, so daB regionale Fremdenver-

Vgl. Gröne, A., Marktsegmentierung bei Investitionsgütern, a.a.0., s. 47. Das instrumente- bzw. programmspezifische Reaktionsverhalten gibt unmittelbar Auskunft uber die Reaktionen der Touristen auf die Marketing-MaBnahmen einer Fremdenverkehrsregion. Vgl. Dichtl, E., Die Bildung von Konsumententypen als Grundfrage der Marktsegmentierung, in: wirtschaftswissenschaftliches studium, 1974, Heft 2, S. 57 .

5 Unter wirkungsfunktionen (Response-Functions) wird die Form der Abhängigkeit zwischen einer Erfolgsgröße ("Output") und den realisierten bzw. geplanten Marketing-Aktivitäten ("Input") verstanden. Vgl. Dichtl, E., Die Bildung von Konsumententypen als Grundfrage der Marktsegmentierung, a.a.0., s. 57 .

6 Vgl. zu den Problemen bei der Ermittlung solcher wirkungsfunktionen Dichtl, E., Die Bildung von Konsumententypen als Grundfrage der Marktsegmentierung, a.a.0., s. 57.

7 Vgl. Dichtl, E., Müller-Heumann, G., Konsumententypologien und produktorientierte Marktsegmentierung, in: Jahrbuch der Absatz- und Verbrauchsforschung, 1972, Heft 4, S. 251 f. 
kehrsorganisationen den Fremdenverkehrsmarkt selektiv und differenziert uber das touristische Leistungsangebot informieren können. 8 Dies setzt die Identifikation von Kriterien voraus, die zu marketingpolitisch erreichbaren touristischen Nachfragesegmenten füren. ${ }^{9}$ In der vorliegenden Untersuchung wird davon ausgegangen, daB diejenigen segmentierungskriterien der Anforderung der Ansprechbarkeit gerecht werden, die mit Hilfe von sozio-demographischen Merkmalen zu erklären sind. 10 Daher steht die Identifizierbarkeit von segmentierungskriterien im Rahmen dieser Untersuchung im Vordergrund. Dies ermöglicht die Ermittlung von sozio-demographisch beschreibbaren segmenten und damit eine gezielte Ausrichtung der Kommunikationsmaßnahmen, insbesondere im Hinblick auf die Mediaselektion. 11

Eine gezielte Marktbearbeitung setzt weiterhin voraus, daB die in den segmentierungsansätzen identifizierten Beziehungszusammenhänge zwischen den segmentierungskriterien und dem Besuchsverhalten seitlich stabil sind, d.h. zum zeit-

8 Vgl. Rehorn, J., Marktsegmentation - Modeerscheinung oder Orientierungshilfe?, in: Markenartikel, 1973, Heft 8, S. 406 .

9 Vgl. Backhaus, K., Investitionsgutermarketing, a.a.o., S. $158 \mathrm{f}$.

10 Die soziodemographische Identifizierbarkeit der segmente ermöglicht, die strategie der kontrollierten zielung zu verfolgen. Wird die strategie der selbstauswahl verfolgt, so müssen die segmentierungskriterien vor allem einen Bezug zum Medienverhalten und zur Wahrnehmung sowie Bewertung verschiedener Marketingstimuli der Fremdenverkehrsregion aufweisen. Vgl. zur ausführlichen Kennzeichnung dieser strategiealternativen Gröne, A., Marktsegmentierung bei Investitionsgütern, a.a.0., s. 48 f. Im Rahmen dieser Untersuchung liegt der Schwerpunkt auf der strategie der kontrollierten zielung, so daB der soziodemographischen Identifizierbarkeit der segmentierungskriterien und segmente eine besonders hohe Bedeutung zukommt.

11 Vgl. Böhler, H., Methoden und Modelle der Marktsegmentierung, stuttgart 1977 , s. 37. 
punkt der Marktbearbeitung auch tatsächlich Gültigkeit besitzen. Die zeitliche stabilität bestimmt den zeitraum, in dem die identifizierten touristischen Nachfragesegmente für eine Marktbearbeitung zugrunde gelegt werden können. 12

Angesichts einer in den letzten Jahren $z u$ beobachtenden zunehmenden Differenzierung und Polarisierung von Konsumwinschen wird die Möglichkeit der Identifikation zeitlich stabiler Nachfragersegmente zunehmend kritisch beurteilt. Experten sind sich einig, daß Konsum- und damit auch Reisepräferenzen von Konsumenten bzw. Touristen immer häufiger wechseln und somit immer weniger von dem Kauf- bzw. Besuchsverhalten auf die dahinter stehenden Motivstrukturen geschlossen werden kann. ${ }^{13}$ Hybrider werdende Konsumstrukturen lassen darauf schließen, daß Besuchspräferenzen sich immer stärker abwechseln. Beispielsweise steht der Nachfrage nach kostengünstigen Fremdenverkehrsleistungen (z.B. Campingurlaub) nicht selten ein steigendes Bedürfnis nach erlebnisorientiertem Luxuskonsum im Urlaub gegenuber. Der Wunsch nach auf Regeneration gerichteten Urlauben wechselt sich mit dem Wunsch nach erlebnisorientierten Kurzurlaubsreisen oder Tagesausflügen ab. 14 Diese Entwicklungen führen nicht nur zu einer weiteren Zersplitterung von zielgruppen sondern auch und insbesondere $z u$ zeitlich instabiler werdenden zielsegmenten.

12 Vgl. Richter, B., Anmerkungen zur Marktsegmentierung, in: Jahrbuch der Absatz- und Verbrauchsforschung, 1972, Heft 1 , s. 39.

13 Vgl. Meffert, H., Erfolgreiches Marketing in den neunziger Jahren, in: Marketing im Umbruch - Chancen und Gefahren für den Unternehmer, Referate anläBlich der Dolder-Tagung vom 23. Mai 1991, Zürich 1991, S. 18 f.

14 Vgl. zum Trend zum Urlaubsverhalten zwischen Entspannungsbedürnis und Unternehmungslust opaschowski, H.W., Tourismusforschung, a.a.0., s. 162 
Die Gefahr der Identifikation von wenig zeitstabilen segmenten hat unmittelbare Auswirkungen auf die wirtschaftlichkeit der Marktsegmentierung. Die Eignung eines segmentierungskriteriums oder -ansatzes ist grundsätzlich danach zu beurteilen, inwieweit diese $z u$ einer verbesserten Erreichung der ziele der Fremdenverkehrsregion beiträgt. Nur segmente, bei denen der Nutzen der segmentierung gröBer ist als die dafür notwendigen Kosten, sind wirtschaftlich interessant. ${ }^{15}$ Eine Überprüfung dieser Forderung hinsichtlich der segmentierungskriterien erweist sich jedoch als kaum durchführbar, da z.B. erforderlich wäre, für jedes segmentierungskriterium den entsprechenden Nutzen zu präzisieren. ${ }^{16}$ Vor diesem Hintergrund wird im folgenden eine direkte Beurteilung von Einzelkriterien der segmentierung anhand von wirtschaftlichkeitsüberlegungen vernachlässigt.

Allerdings lassen sich durch die Anforderungskriterien "zeitliche Stabilität" und "Gewährleistung der Ansprechbarkeit" indirekt Informationen über die wirtschaftlichkeit eines segmentierungsansatzes ableiten. sind die gefundenen Segmente zeitlich stabil, lassen sich beispielsweise Marktforschungskosten senken, da die Anlässe für Marktforschungsstudien seltener werden. Die Gewährleistung der Ansprechbarkeit von segmenten ermöglicht die Verringerung potentieller streuverluste und damit einen niedrigeren Mitteleinsatz. Wirtschaftlichkeitsüberlegungen äuBern sich ferner in der Anforderung, ausreichend groBe Marktsegmente zu identifizieren. Die mit der Entwicklung und dem Einsatz von segmentspezifischen Marktbearbeitungskonzepten verbundenen hohen Ko-

15 Vgl. Böhler, H., Methoden und Modelle der Marktsegmentierung, a.a.0., s. 37 f., Backhaus, K., Investitionsgutermarketing, a.a.0., s. 158; Meffert, H., Marketing, a.a.0., s. 245 .

16 Vgl. Gröne, A., Marktsegmentierung bei Investitionsgutern, a.a.0., s. $35 \mathrm{f}$. 
sten lassen eine segmentspezifische Marktbearbeitung nur für ausreichend große Marktsegmente zweckmäßig erscheinen. 17

Die Identifikation von Marktsegmenten ist schlieblich nur dann möglich, wenn die einzelnen segmentierungsmerkmale ampirisch neBbar sind. Insbesondere hinsichtlich der psychographischen Merkmale kommt der Forderung nach der MeB- und operationalisierbarkeit von segmentierungskriterien ein hoher stellenwert zu. 18 Unter "Operationalisierung" wird die Ubersetzung eines theoretischen Begriffs oder Sachverhaltes in eine Beobachtungssprache verstanden, die den empirischen Bezug herstellen soll. Das Besuchverhalten und seine Einflußfaktoren werden dabei als nicht direkt beobachtbare GröBen anhand von Korrespondenzregeln mit empirisch meBbaren Begriffen verknüpft. 19

Im folgenden werden die Anforderungen an die operationalisierung insbesondere im engen empirischen sinne untersucht. Hierzu ist es erforderlich, eine MeBvorschrift vorzugeben. Es ist $z u$ beachten, daB hypothetische Konstrukte wie Werte, Motive und Einstellungen nur über sogenannte ronstruktindikatoren erfaßt werden können. Daher sind nicht die eigentlich interessierenden Konstrukte, sondern deren Indikatoren $\mathrm{zu}$ operationalisieren. 20 Bei der Auswahl der Indikatoren ist dabei zu berücksichtigen, welche Kriterien potentielle Touristen üblicherweise bei ihrem AuswahlprozeB von Fremdenverkehrsregionen heranziehen.

17 Vgl. Meffert, H., Marketing, a.a.0., s. 245.

18 Vgl. zu den generellen Gefahren einer unzureichenden operationalisierung Richter, B., Anmerkungen zur Marktsegmentierung, a.a.0., S. 39.

19 Vgl. zur Operationalisierung Andritzky, K., Die Operationalisierbarkeit von Theorien zum Konsumentenverhalten, Berlin 1976, S. $20 \mathrm{ff}$.

20 Vgl. Andritzky, K., Die operationalisierbarkeit von Theorien zum Konsumentenverhalten, a.a.0., S. 23 . 
Neben der Bestimmung der MeBvorschriften gilt es, eine Auswahl der skalierungstechnik vorzunehmen. Dabei sind geeignete Skalen zur Messung der Eigenschaften der psychischen Konstrukte auszuwählen und festzulegen. 21 zur Beurteilung der Qualität der gewählten Skalierungsverfahren werden üblicherweise die folgenden drei Gutekriterien herangezogen.

\section{- Objektivitït}

objektivität eines segmentierungskriteriums ist gegeben, wenn mehrere Anwender in derselben Testsituation dieselben Testwerte ermitteln. Zur Úberprüfung der objektivität ist streng genommen eine wiederholung der Erhebung erforderlich. Da dieser Vorgehensweise wirtschaftlichkeitsüberlegungen gegenuberstehen, wird versucht, durch die standardisierung der Befragung ein ausreichendes MaB an Objektivität zu gewährleisten. 22

\section{- Reliabilitit}

Reliablităt (Zuverlässigkeit) beinhaltet die Forderung nach der formalen Genauigkeit der Merkmalserfassung. Ein MeBinstrument ist dann reliabel, wenn die MeBergebnisse bei wiederholter Messung unter sonst konstanten MeBbedingungen reproduzierbar, d.h. stabil und präzise sind. 23 Dies bedeutet, daB die Ergebnisse von dem einmaligen MeBvorgang unabhängig sein müssen.

21 Vgl. zu den verschiedenen skalierungsverfahren Meffert, H., Marketingforschung und Käuferverhalten, a.a.0., s. $183 \mathrm{ff}$.; Berekoven, L., Eckert, W., Ellenrieder, P., Marktforschung. Methodische Grundlagen und praktische Anwendung, 4. Aufl., Wiesbaden 1989, S. $60 \mathrm{ff}$.

22 Vgl. Berekoven, L., Eckert, w., Ellenrieder, P., Marktforschung, a.a.0., s. 82 .

23 Vgl. Berekoven, L., Eckert, w., Ellenrieder, P., Marktforschung, a.a.o., s. 82 f., Heidenreich, K., Grundbegriffe der MeB- und Testtheorie, in: Roth, E. (Hrsg.), 
- Validität

Validität bringt zum Ausdruck, inwieweit mit Hilfe des Meßinstruments die Merkmale oder Sachverhalte, die gemessen werden sollen, auch tatsächlich erfaßt werden. 24

Die Erfassung psychographischer Kriterien als Bestimmungsfaktoren des Besuchsverhaltens ist unter Berucksichtigung dieser drei Gütekriterien vorzunehmen. Abbildung 5 zeigt zu-

\begin{tabular}{|c|c|c|c|}
\hline & $\begin{array}{c}\text { Generalle Anfordorungen } \\
\text { an Marktsegmentlerungs- } \\
\text { konzepte }\end{array}$ & $\begin{array}{l}\text { Indikatoren zur } \\
\text { Oberprufung dor } \\
\text { Elgnung von seg- } \\
\text { mentlerungskriterien }\end{array}$ & $\begin{array}{c}\text { Indlkatoren zur } \\
\text { Uberprófung dor } \\
\text { Qualitht von Markt- } \\
\text { segmentlerungakonzepten }\end{array}$ \\
\hline \multirow{5}{*}{$\begin{array}{l}\text { Konzep- } \\
\text { tlonelle } \\
\text { Anfor- } \\
\text { derungen }\end{array}$} & $\begin{array}{l}\text { •Kaufverhaltens- } \\
\text { relevanz }\end{array}$ & $\begin{array}{l}\text {-mit dem Besuchs- } \\
\text { verhalten in } \\
\text { Bezlehung stehende } \\
\text { Merkmale }\end{array}$ & $\begin{array}{l}\text {-Segmentspezlifische } \\
\text { Unterschiode Im } \\
\text { Besucheverhalten }\end{array}$ \\
\hline & $\begin{array}{l}\text { - Aussagefahigkelt } \\
\text { for den Elnsatz } \\
\text { der Marketing- } \\
\text { instrumente }\end{array}$ & $\begin{array}{l}\text {-Marketing- } \\
\text { spezlflscho } \\
\text { Merkmale }\end{array}$ & $\begin{array}{l}\text {-Segmentspezlfische } \\
\text { Ahnllchkelten Im } \\
\text { Relse- \& Besuchs- } \\
\text { verhalton und In } \\
\text { psychographlechen } \\
\text { Merkmalen } \\
\end{array}$ \\
\hline & $\begin{array}{l}\text {-Ansprechbarkeit/ } \\
\text { Identifizlerbarkeit }\end{array}$ & $\begin{array}{l}\text {-Sozlodemographische } \\
\text { Identiflzlerbarkelt } \\
\text { der Merkmale }\end{array}$ & $\begin{array}{l}\text {-Sozlodemographische } \\
\text { Identiflzierbarkelt } \\
\text { der Segmente }\end{array}$ \\
\hline & -Zoltliche Stabilitắt & $\begin{array}{l}\text {-Zeitlich stabile } \\
\text { Merkmalo }\end{array}$ & $\begin{array}{l}\text {-Zeltllch stablle } \\
\text { Segmente }\end{array}$ \\
\hline & -Wirtschaftllchkelt & & $\begin{array}{l}\text {-Zeltlliche Stabilltat } \\
\text { der Segmente } \\
\text {-Gewahnelstung der Seg- } \\
\text { ment-Ansprechbarkeit } \\
\text {-Ausrelchend groBe } \\
\text { Segmente }\end{array}$ \\
\hline $\begin{array}{l}\text { MeBtheore- } \\
\text { tische An- } \\
\text { forderungen }\end{array}$ & • Gữte & $\begin{array}{l}\text {-Objektivităt } \\
\text {-Rellabilltăgt } \\
\text {-Valldităt }\end{array}$ & \\
\hline
\end{tabular}

Abb. 5: Konzeptionelle und meBtheoretische Anforderungen an die Marktsegmentierung im Fremdenverkehr von Regionen

Sozialwissenschaftliche Methoden, München, wien 1984, S. 351 .

24 Vgl. Meffert, H., Marketingforschung und Käuferverhalten, a.a.0., S. 180, Berekhoven, L., Eckert, W., Ellenrieder, P., Marktforschung, a.a.0., S. $83 \mathrm{f}$. 
sammenfassend die wichtigsten Anforderungen an Segmentierungskriterien und Markterfassungsansätze und die Kriterien zu ihrer Überprüfung.

Dieser Bewertungsrahmen bietet eine Grundlage für die Überprüfung, ob soziodemographische Merkmale und die psychographischen Merkmale "Werte", "Motive" und "Einstellungen" geeignete segmentierungskriterien im Fremdenverkehr von Regionen darstellen. Im Rahmen dieser Untersuchung wird im Hinblick auf die konzeptionellen Anforderungen lediglich die Besuchsverhaltensrelevanz und die Ansprechbarkeit i.s. der soziodemographischen Identifizierbarkeit empirisch überprüft. 25 Daher stellen beide Kriterien in der vorliegenden Untersuchung die dominanten Kriterien zur Beurteilung der Eignung von soziodemographischen und psychographischen Merkmalen als segmentierungskriterien dar.

Zur Beurteilung der Besuchsverhaltensrelevanz von segmentierungskriterien bietet sich an, auf der Basis einer verhaltensorientierten Marktsegmentierung Ausprägungen soziodemographischer und psychographischer Merkmale hinsichtlich ihrer Differenzierungsfähigkeit von Verhaltenssegmenten $z u$ überprüfen. Hierzu ist zunächst erforderlich, das Besuchsverhalten von Touristen $z u$ erfassen.

25 Zur empirischen Überprüfung der zeitlichen stabilität wäre eine Wiederholung der Erhebung erforderlich. Auf die empirische Überprüfung der Anforderung der Aussagefähigkeit für den Einsatz der Marketing-Instrumente wird aufgrund der mit der Ermittlung von Marktreaktionsfunktionen verbundenen Datenbeschaffungsprobleme verzichtet. 
2. Ausprägungsformen des regionenspezifischen Besuchsverhaltens auf der Basis einer Makrosegmentierung

\subsection{Theoretische Grundlagen zur Erfassung des Besuchsverhal- tens und zur Makrosegmentierung}

Die Durchführung einer verhaltensorientierten Marktsegmentierung erfordert zunächst die Operationalisierung des Besuchsverhaltens. Dabei werden in der vorliegenden Untersuchung weniger generelle Verhaltensweisen erfaBt, als vielmehr das Besuchsverhalten bezüglich einer spezifischen Fremdenverkehrsregion. Generelle Kennziffern über die Reiseintensität und Reisehäufigkeit von potentiellen Touristen, wie sie z.B. vom Statistischen Bundesamt oder der starnberger Reiseanalyse ermittelt werden, geben keine Hinweise für die Markterfassung im Fremdenverkehr von Regionen. 26

Die Notwendigkeit der Erfassung des regionensperifischen Besuchsverhaltens ergibt sich unmittelbar daraus, daß Angebot und Nachfrage erst durch die Reise und den Aufenthalt in einem bestimmten $\mathrm{zielgebiet} \mathrm{in} \mathrm{einen} \mathrm{engeren} \mathrm{Beziehungszusam-}$ menhang gelangen. Die operationalisierung des Besuchsverhaltens hat dabei die beobachtbaren, auf eine spezifische Region bezogenen Ausprägungsformen des Besuchsverhaltens zu berücksichtigen. 27 Die hierbei relevanten Aspekte des Besuchsverhaltens in bezug auf eine Fremdenverkehrsregion sind

26 Die Reiseintensität wird nach der Brutto- und Nettoreiseintensität differenziert. Die Nettoreiseintensität wird definiert als der Anteil an der deutschen Bevölkerung, der jährlich mindestens eine Reise unternimmt. Die Bruttoreiseintensität ist das Verhältnis aus Anzahl getätigter Reisen und Gesamtbevölkerung. Die Reisehäufigkeit ermöglicht Viel- und Wenig-Reisende zu unterscheiden. Vgl. hierzu Freyer, W., Tourismus, a.a.O., S. 102.

27 Vgl. in Anlehnung an Monhemius, K. Ch., Umweltbewußtes Kaufverhalten von Konsumenten. Ein Beitrag zur operationalisierung, Erklärung und Typologie des Verhaltens in der Kaufsituation, Münster 1992, S. 37. 
jedoch in der Fremdenverkehrsforschung bislang nicht systematisiert worden. Daher bietet es sich an, die im Konsumgüterbereich relevanten Kaufdimensionen zur Charakterisierung der Ausprägungsformen des regionenspezifischen Besuchsverhaltens heranzuziehen. Abb. 6 gibt einen Ưberblick über die

\begin{tabular}{|c|c|}
\hline $\begin{array}{l}\text { Aspekte des Kauf- } \\
\text { verhaltens bel } \\
\text { Konsumgūtern }\end{array}$ & $\begin{array}{l}\text { Aspekte des } \\
\text { reglonenspezifischen } \\
\text { Besuchsverhaltens }\end{array}$ \\
\hline $\begin{array}{l}\text { - Qualitativer Aspekt } \\
\text { (Art der Ware; Qualitāts- } \\
\text { eigenschaften) }\end{array}$ & $\begin{array}{l}\text { - Besuch / Nichtbesuch } \\
\text { einer bestimmten } \\
\text { Fremdenverkehrsregion }\end{array}$ \\
\hline $\begin{array}{c}\text { - Mengenmäßiger Aspekt } \\
\text { (Packungsgröße) }\end{array}$ & - Besuchsdauer \\
\hline $\begin{array}{l}\text {-Wertmäßiger Aspekt } \\
\text { (Preis) }\end{array}$ & $\begin{array}{l}\text {-Ausgaben während } \\
\text { des Besuchs }\end{array}$ \\
\hline $\begin{array}{l}\text { - Zeitlicher Aspekt } \\
\text { (Einkäufe im Zeitablauf; } \\
\text { Markentreue; etc) }\end{array}$ & $\begin{array}{l}\text { - Besuchszeitpunkt } \\
\text { - Besuchshäufigkeit } \\
\text { (=Zielgebietserfahrung) }\end{array}$ \\
\hline $\begin{array}{l}\text {-Räumlicher Aspekt } \\
\text { (Nähe zur Einkaufs- } \\
\text { stătte) }\end{array}$ & $\begin{array}{l}\text { - Entfernung zum } \\
\text { Zielgebiet } \\
\text { (Anfahrtsweg/-dauer) }\end{array}$ \\
\hline $\begin{array}{l}\text { - Personeller Aspekt } \\
\text { (z.B. Kaufhandlung in } \\
\text { Begleitung der Familie) }\end{array}$ & $\begin{array}{l}\text {-Besuch allein oder } \\
\text { in Begleitung }\end{array}$ \\
\hline
\end{tabular}

Abb. 6: Ưbertragung potentiell interessierender Aspekte des Kaufs von Konsumgütern auf das regionenspezifische Besuchsverhalten (in Anlehnung an Müller-Hagedorn, L., Das Konsumentenverhalten, a.a.0., s. 47 ff.) 
wichtigsten Kaufdimensionen und deren spezifischen Ausprägungen für den Fremdenverkehr. 28

Von besonderer Bedeutung für die Marktsegmentierung im Fremdenverkehr von Regionen erweist sich der qualitative Aspekt, der im Konsumgüterbereich die Art und relative Beschaffenheit der ware spezifiziert. Im Fremdenverkehr kann die Art der Ware beispielweise nach Kategorien der Reiseart (Bildungsurlaub, Abenteuerlaub, Kururlaub etc.) oder nach Ländern (Inlands-/Auslandsreisen) konkretisiert werden. Eine differenzierte Betrachtung des geographischen Aspektes führt zur Unterscheidung von Fremdenverkehrsregionen. Daher kann der qualitative Aspekt des Besuchsverhaltens auch interpretiert werden als der Besuch einer bestimmten Fremdenverkehrsregion, deren Qualität durch die Angebotskomponenten determiniert ist. Demnach kommt in dem qualitativen Aspekt im Fremdenverkehr von Regionen der Besuch einer spezifischen Fremdenverkehrsregion zum Ausdruck.

Der mengenmäBige Aspekt umfaßt demgegenüber die Besuchsdauer der Touristen. Der wertmäBige Aspekt ist im Hinblick auf den Fremdenverkehr als die Summe der Ausgaben während des Aufenthalts $z u$ interpretieren. Da dieser Bereich bereits implizit in der Besuchsdauer berücksichtigt wird, 29 ist er aus dieser Untersuchung auszugrenzen. Der seitliche Aspekt kann im Fremdenverkehr einerseits als Ausdruck für den Besuchszeitpunkt, andererseits für die Besuchshäufigkeit des zielgebietes angesehen werden. Wird unterstellt, daB Fremdenverkehrsregionen zunächst Ansatzpunkte gewinnnen wollen, wie potentielle Touristen als Erst-Besucher gewonnen werden kön-

28 Vgl. Müller-Hagedorn, L., Das Konsumentenverhalten Grundlagen für die Marktforschung, wiesbaden 1986, S. 47 ff.

29 So kann davon ausgegangen werden, daß mit zunehmender Verweildauer in einer Fremdenverkehrsregion die Gesamtausgaben der Reise steigen. 
nen, so kommt dem zeitlichen Aspekt als Ausdruck für den Besuchszeitpunkt eine im Gegensatz zur Erfassung des Besuches eher nachgeordnete Bedeutung zu. 30 Die Besuchshäufigkeit wird hier als vergangenheitsbezogener Parameter verstanden, mit dessen Hilfe Aussagen über die Zielgebietserfahrung und -treue getroffen werden können. 31 Der personelle Aspekt ist als Besuch oder Reise - allein oder in Begleitung - zu interpretieren. 32 Der räumliche Aspekt im Fremdenverkehr ist als Ausdruck für die Entfernung zum Zielgebiet und damit als Indikator für die Anreisedauer zu sehen. Es ist anzunehmen, daß die in der Entfernung zum Ausdruck kommende Anreisedauer die Besuchsabsicht und -dauer determiniert. Daher ist es notwendig, eine entfernungsgruppenspezifische Analyse zur Identifikation der Bestimmungsfaktoren des Besuchsverhaltens durchzuführen. Die Entfernung zum zielgebiet ist dabei jedoch nicht als Kaufparameter, sondern als dominanter Einflußfaktor auf das Besuchsverhalten $z u$ interpretieren.

Zur Durchführung einer verhaltensorientierten Marktsegmentierung stehen der qualitative und mengenmäßige Aspekt, d.h. der Besuch und die Besuchsdauer, im Vordergrund des Interesses. Die Entfernung zum zielgebiet wird demgegenüber als dominante Bestimmungsgröße des Besuchs und der Besuchsdauer aufgefaßt. Dies wird im Rahmen einer Makrosegmentierung auf

30 Es ist zu betonen, daß dem Aspekt des Besuchszeitpunkts in der Praxis ein besonders hoher stellenwert zukommt, um eine gleichmäßige Auslastung der Fremdenverkehrskapazitäten zu erzielen. Dennoch wird dieser Aspekt in der vorliegenden Untersuchung nicht weiter analysiert.

31 Die zielgebietserfahrung stellt eine vergangenheitsbezogene Verhaltenskomponente dar. Darüber hinaus ist sie als Indikator für die stellung einer Fremdenverkehrsregion im wahrgenommenen Alternativenset von Zielgebieten $\mathrm{zu}$ interpretieren. Aufgrund der Verhaltensdominanz dieses Kriteriums wird die zielgebietserfahrung bei den Ausführungen zum Besuchsverhalten näher betrachtet.

32 Auf die Betrachtung des personellen Aspektes wird im Rahmen dieser Untersuchung verzichtet. 
der Basis der Entfernung zum Zielgebiet analysiert. Bevor auf die Makrosegmentierung näher eingegangen wird, ist vorab zu bestimmen, wie der Besuch und die Besuchsdauer zu operationalisieren sowie welche Methoden der empirischen Erfassung anzuwenden sind.

Für die empirische Messung des Besuchsverhaltens stehen grundsätzlich die Methoden der Beobachtung und Befragung zur Auswahl. 33 Beobachtung wird verstanden als die zielgerichtete und planmässige Erfassung von wahrnehmbaren Sachverhalten durch Personen und oder durch Geräte. 34 Die Beobachtung eignet sich vor allem bei Produktkäufen bestimmter Konsumgüterkategorien, in denen der Beobachter die Konsumenten hinsichtlich ihrer soeben getätigten käufe selektiert. ${ }^{35}$ Hierbei wird deutlich, daB die Beobachtung als Verfahren zur Messung des touristischen Besuchsverhaltens wenig zweckmäBig ist. Die Inanspruchnahme einer Fremdenverkehrsregion ist dabei nicht auf einen bestimmten Kauf-bzw. Inanspruchszeitpunkt, sondern auf die Nutzung von Fremdenverkehrsleistungen über einen längeren Zeitraum (Stunden, Tage, Wochen) zurückzuführen. Vor diesem Hintergrund ist eine Beobachtung während der Leistungsinanspruchnahme offensichtlich mit einem nicht $z u$ vertretenden kosten- und zeitbezogenen Erhebungsaufwand verbunden.

Alternativ erscheint es denkbar, eine Beobachtung bei Reisebüros durchzuführen. Dies erweist sich jedoch zumindest für deutsche Fremdenverkehrsregionen als nicht sinnvoll, da

33 Als weitere Meßmethode ist das Experiment zu nennen, dem jedoch aus operationalisierungsgründen im Fremdenverkehr keine Bedeutung zukommt.

34 Vgl. Meffert, H., Marketingforschung und Käuferverhalten, a.a.O., s. 198, Berekoven, L., Eckert, W., Ellenrieder, P., Marktforschung, a.a.O., S. 88 .

35 Vgl. Monhemius, K. Ch., Umweltbewußtes Käuferverhalten von Konsumenten, a.a.0., S. 44 . 
Deutschlandreisen nur selten über Reisebüros gebucht werden. 36

Die Befragung erweist sich in diesem Rahmen als die zweckmäBigste Methode zur Erfassung des Besuchsverhaltens in bezug auf eine Fremdenverkehrsregion. Dabei kann die operationalisierung des Besuchsverhaltens auf unterschiedliche Weise erfolgen. Da eine Beobachtung des Besuchsverhaltens nicht in Frage kommt, ist anstelle des tatsächlichen Verhaltens ein Indikator anzusetzen.

In der Konsumentenverhaltensforschung finden als Indikator des räuferverhaltens vor allem Einstellungen und Kaufabsichten Verwendung. Dabei wird davon ausgegangen, daB die Kaufabsicht ein besserer Indikator für das tatsächliche Besuchsverhalten darstellt, als die gemessene Einstellung zu einem Produkt. 37

Ferner stellt sich die Frage, ob das vergangenheitsbezogene oder das beabsichtigte Besuchsverhalten als Indikator furr das tatsächliche Besuchverhalten verwendet werden soll. Die Vorteilhaftigkeit dieser Indikatoren ist von den vorliegenden Kaufentscheidungstypen abhängig. Dabei sind auch im Fremdenverkehr habitualisierte, extensive und limitierte Kaufentscheidungen $\mathrm{zu}$ unterscheiden. 38

Vgl. Bleile, G., Marketing-strategien im Fremdenverkehr, in: Der Fremdenverkehr, 1978, Heft 11, S. $26 \mathrm{f}$.

37 Vgl. Kroeber-Riel, w., Konsumentenverhalten, a.a.o., s. 170 .

38 Habituelle und extensive Kaufentscheidungen gehen auf eine Begriffsabgrenzung von Katona zurück. Vgl. Katona, G., Das Verhalten der Verbrauer und Unternehmer, Tubingen 1968; vgl. ferner Kannacker, V.A., Habitualisiertes Kaufverhalten von Konsumenten, München 1982. Die Erweiterung um limitierte Kaufentscheidungen findet sich bei Howard, J.A., Sheth, J.N., The Theory of Buyer Behaviour, a.a.0.,. Ferner werden hier impulsive Kaufentscheidungen genannt, im Rahmen derer ein Konsument spon- 
GewohnheitsmäBiges (habituelles) Verhalten kann als Umsetzung von bereits "vorgefestigten" Entscheidungen in Kaufhandlungen aufgefaßt werden. Das gewohnheitsmäßige Verhalten ist auf die Beibehaltung von Entscheidungen zuruckzufuhren, die sich in der Vergangenheit bewährt haben. In der Konsumentenverhaltensforschung wird dieser Aspekt in Verbindung mit der Produkt- und Markentreue diskutiert und ist im Fremdenverkehr als zielgebietstreue $\mathrm{zu}$ umschreiben. 39 Es ist davon auszugehen, daß bei gewohnheitsmäBigem Kaufverhalten der Kaufabsicht nur eine geringe Erklärungskraft zukommt. 40 Daher wird zur Verhaltensprognose überwiegend das vergangenheitsbezogene Verhalten herangezogen. 41

Hingegen wird bei kognitiv kontrollierten Kaufprosessen (extensive und limitierte Kaufentscheidungen), d.h. Kaufprozessen, die ein stärkeres Ausmaß an gedanklicher Auseinandersetzung mit der Kaufentscheidung aufweisen, davon ausge-

tan auf bestimmte Reize am Point of sale reagiert. Eine Übertragung auf den Fremdenverkehr ist nur eingeschränkt möglich. Es ist zu vermuten, daß vor allem Tagesausflüge den Charakter einer impulsiven Kaufentscheidung annehmen können. Beispielsweise ist es denkbar, daB ein Tagesausflug aufgrund einer Anzeige in einer Zeitung oder aufgrund von Gesprächen mit Freunden spontan unternommen wird. Vgl. zur Kennzeichnung der Ausprägungsformen dieser Kaufentscheidungstypen in bezug auf den Fremdenverkehr Mühlbacher, H., Botschen, G., Benefit-Segmentierung von Dienstleistungsmärkten, in: Marketing ZFP, 1990, Heft 3 , S. $162 \mathrm{f}$.

$39 \mathrm{Zu}$ denken ist beispielsweise an Ostsee-Urlauber, die seit Jahren stets in die gleiche Urlaubsregion fahren.

40 Vgl. Kroeber-Riel, w., Konsumentenverhalten, a.a.o., s. 165.

41 Zur Erfassung des Gewohnheitsverhaltens werden zumeist Angaben über die Häufigkeit, mit der ein Konsument das gleiche produkt (die gleiche Marke) wiederkauft, verwandt. Vgl. Kroeber-Riel, w., Konsumentenverhalten, a.a.O., S. $391 \mathrm{f.i}$ Zur weiteren operationalisierung und Erklärung des gewohnheitsmäBigen Verhaltens vgl. Weinberg, P., Das Entscheidungsverhalten der Konsumenten, Paderborn u.a. 1981, S. 149 ff. 
gangen, daß die Kaufabsicht ein geeigneter Indikator für die Verhaltensprognose darstellt. 42 Bezogen auf den Fremdenverkehr ist anzunehmen, daß überwiegend kognitiv kontrollierte Kaufentscheidungen vorliegen. Dies ist darauf zurückzuführen, daß Touristen bei inrer Urlaubs- oder Reiseplanung in der Regel ein hohes Risiko, beispielsweise in finanzieller Hinsicht wahrnehmen, 43 dem durch intensive Informationsbeschaffung und einer vergleichenden Beurteilung von alternativen Urlaubsangeboten Rechnung getragen wird.

Demnach erweist sich die Kaufabsicht als geeigneter Indikator für das tatsächliche Besuchsverhalten. Das vergangenheitsbezogene Besuchsverhalten soll demgegenüber im Rahmen dieser Untersuchung als Bestimmungsfaktor der Besuchsabsicht herangezogen und als zielgebietserfahrung bezeichnet werden. Diese wird über die Anzahl der getätigten Besuche in den letzten 2 Jahren operationalisiert. Bezogen auf die Besuchsabsicht ist im folgenden der Frage nachzugehen, wie diese operationalisiert werden kann.

Zu den am häufigsten zitierten Veröffentlichungen zur Messung der Kaufabsicht zählen die Modelle von Ajzen und Fishbein. ${ }^{44}$ In diesen Ansätzen wird die Verhaltensabsicht über

Vgl. Kroeber-Riel,w., Konsumentenverhalten, a.a.o., s. 170.

43 Vgl. zur generellen Kennzeichnung des wahrgenommenen Risikos Meffert, H., Marketingforschung und Käuferverhalten, a.a.0., S. 69 ff. Zur Kennzeichnung des wahrgenommenen Risikos im Rahmen des Auswahlprozesses von Fremdenverkehrsregionen vgl. Datzer, R., Informationsverhalten von Urlaubsreisenden, a.a.0., s. 113.

44 Ajzen, I., Fishbein, M., The Prediction of Behaviour from Attitudinal and Normative Variables, in: Journal of Experimental Social Psychology, 1970, Heft 6, S. 466 ff.; dieselben, Attitudinal and Normative Variables as Predictors of Specific Behaviour, in: Journal of Personality and Social Psychology, 1973, S. 41 ff.; beide zitiert aus Kroeber-Riel, W., Konsumentenverhalten, a.a.O., 1992 , s. 170 . 
Einstellungen in Verbindung mit normativen Einflüssen auf das Verhalten bestimmt. Für die vorliegende Untersuchung erweist sich diese Begriffsabgrenzung der Kaufabsicht jedoch als nicht zweckmäBig. Diese Definition geht von einem vermuteten, aber für den Fremdenverkehrssektor bisher nicht nachgewiesenen Zusammenhang zwischen Kaufabsicht und Einstellungen aus.

Vor diesem Hintergrund erscheint es zweckmäBig, die Kaufabsicht über einfache Ratingskalen $z u$ operationalisieren. Dabei kann die Messung der Besuchsabsicht entweder dichotom (Besuch/Nicht-Besuch) oder aber graduell, z.B. in Form einer Besuchswahrscheinlichkeit festgelegt werden. Die dichotome Messung unterstellt, $\mathrm{da} B$ es keine Intensitätsunterschiede in der Besuchsabsicht einer Fremdenverkehrsregion gibt. Die graduelle Begriffsauffassung versucht hingegen, zusätzlich eine Rangordnung nach der Wahrscheinlichkeit des Besuchsverhaltens zu berücksichtigen.

Bei der Auswahl des Skalierungsverfahrens ist zu beachten, daß die Messung der Besuchsabsicht i.d.R. mit systematischen Fehlern verbunden ist. So ist mit einer Divergenz zwischen der Nennung von Besuchsabsicht und dem tatsächlichen Besuch zu rechnen. Für die Marktsegmentierung kommt es jedoch vor allem auf die relativen Unterschiede der Kaufabsichtsnennungen zwischen Teilstichproben an. Weisen identifizierte Zielgruppen signifikante Unterschiede in der Nennung der Besuchsabsicht auf, so kann die genannte Besuchsabsicht als Indikator für eine gezielte zielgruppenansprache verwendet werden. 45 Um den systematischen Fehler möglichst gering $z u$ halten und die Befragten nicht zu einer bloßen Absichtserklärung zu verleiten, wurde die Besuchsabsicht in das Fremdenverkehrsgebiet durch die Vorgabe eines zeitlichen Hori-

45 Vgl. Böhler, H., Methoden und Modelle der Marktsegmentierung, a.a.0., s. 111 . 
zonts von 6 Monaten eingeschränkt. 46 Ferner soll nicht die Besuchswahrscheinlichkeit, sondern die tatsächliche Besuchsabsicht ermittelt werden.

Um dem zeitlichen Aspekt des Besuchsverhaltens Rechnung zu tragen, ist die Besuchsabsicht nach der Besuchsdauer zu differenzieren. In der Fremdenverkehrsforschung wird üblicherweise folgende operationalisierung der Besuchsdauer vorgenommen, die auch für diese Untersuchung $z u$ verwenden ist. 47 Ein Tagesausflug wird als ein Aufenthalt in einer Fremdenverkehsregion ohne Übernachtung definiert. Der Kurzurlaub umfaBt mindestens eine Übernachtung und dauert zwischen zwei und vier Tage. Von Urlauben wird gesprochen, wenn die Besuchsdauer mindestens fünf Tage beträgt. Aus Marktbearbeitungssicht kommt der Unterscheidung der Besuchsdauer eine besondere Bedeutung $z u$, da die Kenntnis ihrer Bestimmungsfaktoren die Ableitung von strategien und Maßnahmen zur Erhöhung der Utbernachtungszahlen in den Fremdenverkehrsregionen ermöglicht.

Bisher wurden die Besuchsabsicht sowie die beabsichtigte Besuchsdauer als Indikatoren für das Besuchsverhalten identifiziert und operationalisiert. Anhand dieser Merkmale sind Verhaltenssegmente $z u$ bilden, wobei Besucher von Nicht-Besuchern unterschieden werden. Ferner werden Besucher in $\mathrm{Ta}-$ gesausflügler, Kurzurlauber und Urlauber unterteilt. Darüber hinaus wurde auf die Notwendigkeit der entfernungsgruppenspezifischen Analyse zur Identifikation der Bestimmungsfak-

46 Vgl. Fragebogen im Anhang II.

47 Vgl. zu dieser Operationalisierung von Urlauben, Kurzurlauben und Tagesausflügen Meffert, H., et. al., Regionenmarketing Münsterland, a.a.0., s. 76 ; Gilbrich, M., Urlaubsreisen 1991. Kurzfassung der Reiseanalyse 1991, studienkreis für Tourismus e.V. (Hrsg.), Starnberg 1992, S. 9 f.; O.V., Marken-Profile 4: Touristik, Fluggesellschaften, Mietwagen, in: Die stern Bibliothek, Hamburg 1992 , S. 24 und S. 56. 
toren des Besuchsverhaltens hingewiesen. Diesem soll durch die Durchführung einer Makrosegmentierung auf der Basis der Entfernung Rechnung getragen werden.

Die Makrosegmentierung erfolgt mit dem Ziel, auf der Basis der Entfernung Gruppen von Personen voneinander abzugrenzen, die sich in ihrem Besuchsverhalten unterscheiden. Zur Erfassung der Entfernung zum zielgebiet werden im folgenden die straßenkilometer herangezogen. 48 Die Entfernungsgruppen sollen so gebildet werden, $d a B$ sie signifikante Unterschiede in der Besuchsdauer aufweisen. Dies basiert auf der Annahme, daß $a b$ einer bestimmten Entfernung eine Fremdenverkehrsregion als Tagesausflugsziel von Touristen aufgrund einer zu langen Anreisedauer nicht mehr in Betracht gezogen wird. Es ist daher erforderlich, einen in den Entfernungskilometern zum Ausdruck kommenden Grenzwert zu ermitteln, der es ermöglicht, zwei Entfernungsgruppen zu bilden, die sich in ihrem Anteil an Tagesausflüglern signifikant unterscheiden. Dabei sollte die eine Entfernungsgruppe möglichst viele bzw. alle Tagesausflügler, die andere möglichst keine oder nur wenige Tagesausflügler enthalten. Gelingt diese Makrosegmentierung, können drei nach der Besuchsdauer abgegrenzte Verhaltenssegmente den Entfernungsgruppen, wie in Abbildung 7 dargestellt, zugeordnet werden.

48 Die Entfernungskilometer wurden unter Zuhilfenahme des Programms Eurotour VVF/CPA 1.1 ermittelt. In diesem Zusammenhang erweist sich die Festlegung des zielortes als problematisch. Angesichts der geographischen Größe der hier zu untersuchenden Fremdenverkehrsregion wurde als zielgebietsort das oberzentrum in der Region angesetzt. Das Programm bietet auch die Möglichkeit, Schienenkilometer (Bahn) als Indikator für die Entfernung zum Zielgebiet anzusetzen. Die Berücksichtigung der Bahn als Anreisemittel erweist sich angesichts der Dominanz des Autos als Anreisemittel im deutschen Fremdenverkehr jedoch als unzweckmäBig. Bei der Berechnung der straBenkilometer zum zielgebiet wird davon ausgegangen, da $B$ der potentielle Tourist den schnellsten Weg für seine Anreise wählt. 


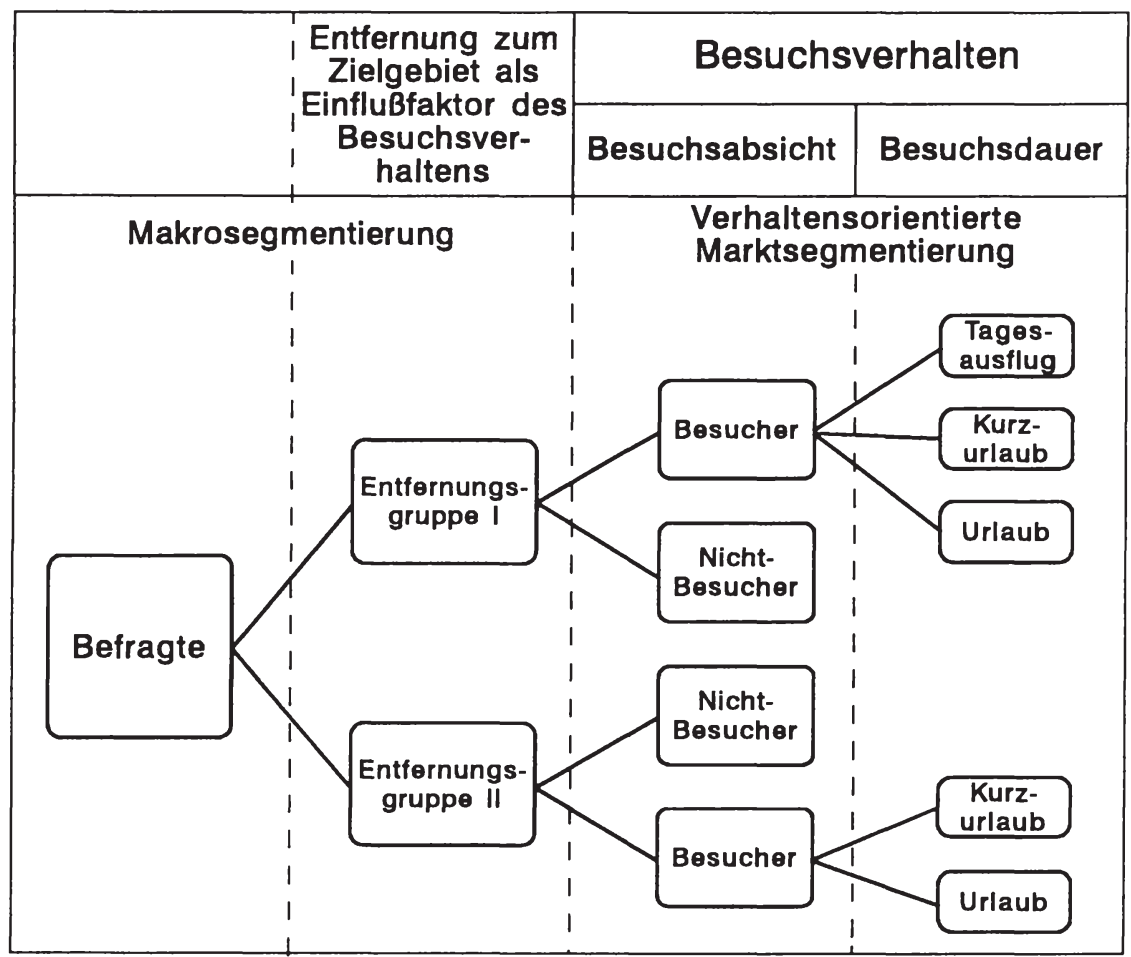

Abb. 7: Makrosegmentierung auf der Basis der Entfernung zwischen Wohnort und Zielgebiet

Die Einteilung in die zwei Entfernungsgruppen ermöglicht unmittelbar, eine der zielsetzung "Erhöhung der Übernachtungszahlen" Rechnung tragende Fokussierung der Analyse der Bestimmungsfaktoren des Besuchsverhaltens vorzunehmen. In der Entfernungsgruppe I interessiert vor allem, durch welche gezielte Marktbearbeitung Tagesausflügler zu einem Aufenthalt mit Übernachtung zu gewinnen sind. Demgegenüber ist in Hinblick auf die Entfernungsgruppe II von Bedeutung, wie Kurzurlauber $\mathrm{zu}$ einem längeren Aufenthalt $\mathrm{zu}$ motivieren sind. Es ist daher erforderlich, die Bestimmungsgründe für die Be- 
suchsdauer in den beiden Entfernungsgruppen separat zu analysieren.

Unter Bezugnahme auf die bisherigen Ausführungen lassen sich folgende Untersuchungshypothesen $\mathrm{zu}$ den Ausprägungsformen des Besuchsverhalten und $z u$ der Entfernung als Bestimmungsfaktor des Besuchsverhaltens formulieren.

Basishypothese ${ }^{49}$ :

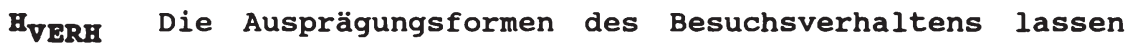
sich durch die Besuchsabsicht und die beabsichtigte Besuchsdauer bestimmen.

Auf der Grundlage dieser Basishypothese werden folgende Hypothesen zum EinfluB der Entfernung auf das Besuchsverhalten überprüft.

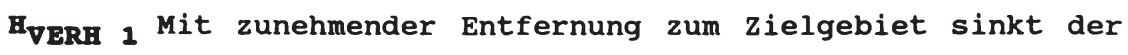
Anteil der Befragten, die eine Besuchsabsicht äuBern.

49 Im Rahmen der Generierung von Untersuchungshypothesen können zwei Typen von Hypothesen, die Basishypothesen und die Tendenzhypothesen unterschieden werden. Sofern Aussagen nur rudimentär formuliert werden können und dadurch AnlaB zur Mehrdeutung gegeben ist, werden Basishypothesen $\mathrm{zu}$ einer Orientierung im Untersuchungsverlauf gegeben. Lassen sich konkrete Beziehungsrichtungen zwischen unabhängigen und abhängigen Variablen der Untersuchung angeben, sind Tendenzhypothesen $\mathrm{zu}$ formulieren. Im folgenden wird zwischen Basishypothesen und Hypothesen differenziert. Vgl. Schanz, G., Zwei Arten von Empirismus, in: $\mathrm{ZfbF}, 1975$, S. 325; Bruhn, M., Das soziale Bewußtsein von Konsumenten. Erklärungsansätze und Ergebnisse einer empirischen Untersuchung in der Bundesrepublik Deutschland, Wiesbaden 1978, S. 104; Kopp, M., Hypothesenformulierung in der Absatzforschung, Berlin 1972, S. 537 ff.; Kirchgeorg, M., Ökologieorientiertes Unternehmensverhalten, Typologien und Erklärungsansätze auf empirischer Grundlage, Wiesbaden 1990, S. 50. 
HVRH $_{2}$ Mit zunehmender Entfernung zum zielgebiet verlängert sich die beabsichtigte Besuchsdauer.

HVRR $_{3}$ Die auf der Basis der Entfernung zum Zielgebiet vorzunehmende Makrosegmentierung führt zu zwei Entfernungsgruppen, die sich in ihrem Anteil an Tagesausflüglern signifikant unterscheiden. Dabei werden der Entfernungsgruppe II kaum bzw. keine Tagesausflügler zugeordnet.

Darüber hinaus soll der EinfluB der zielgebietserfahrung auf das Besuchsverhalten auf der Basis folgender Hypothese überprüft werden.

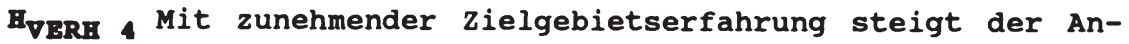
teil der Befragten, die eine Besuchsabsicht in die Untersuchungsregion äußern.

\subsection{Kennzeichnung der empirischen Ausprägungsformen der Ma- kro- und Besuchsverhaltenssegmente}

Im folgenden werden die Ausprägungsformen des Besuchsverhaltens für die Untersuchungsregion zunächst deskriptiv dargestellt. Der Anteil der jeweiligen Besucher bzw. Nicht-Besucher sowie eine Differenzierung in Abhängigkeit von der Besuchsdauer ist Abbildung $8 \mathrm{zu}$ entnehmen. Es fällt auf, daB Befragte, die keine Besuchsabsicht in das Fremdenverkehrsgebiet aufweisen, am häufigsten auftreten. Allerdings äuBern immerhin $40,8 \%$ eine Besuchsabsicht in den nächsten 6 Monaten. 


\begin{tabular}{|c|c|c|c|c|c|c|}
\hline \multirow{2}{*}{\multicolumn{2}{|c|}{$\begin{array}{l}\text { Verhaltens- } \\
\text { segmente }\end{array}$}} & \multirow{2}{*}{\multicolumn{2}{|c|}{$\begin{array}{c}\text { Anzahl } \\
\text { der } \\
\text { Nennungen }\end{array}$}} & \multicolumn{2}{|c|}{$\begin{array}{l}\text { Anteil der Besucher } \\
\text { an der Gesamtzahl: }\end{array}$} & \multirow{2}{*}{$\begin{array}{c}\text { Anteil an } \\
\text { Besuchern } \\
\text { nach der } \\
\text { Besuchs- } \\
\text { dauer }\end{array}$} \\
\hline & & & & insgesamt & $\begin{array}{c}\text { nach der } \\
\text { Besuchs- } \\
\text { dauer }\end{array}$ & \\
\hline \multicolumn{2}{|c|}{ Nicht-Besucher } & \multicolumn{2}{|c|}{554} & $59,2 \%$ & & \\
\hline \multirow{3}{*}{ 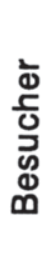 } & Tagesausflũgler & \multirow{3}{*}{382} & 148 & \multirow{3}{*}{$40,8 \%$} & $15,8 \%$ & $38,8 \%$ \\
\hline & $\begin{array}{l}\text { Kurz- } \\
\text { urlauber }\end{array}$ & & 141 & & $15,1 \%$ & $36,9 \%$ \\
\hline & Urlauber & & 93 & & $9,9 \%$ & $24,3 \%$ \\
\hline
\end{tabular}

Abb. 8: Größe der empirisch ermittelten Besuchsverhaltenssegmente

Es zeigt sich ferner, daB die Fremdenverkehrsregion vor allem als Tagesausflugs- und Kurzurlaubsziel genutzt wird. Dies wird durch den hohen Anteil von 75,7 if der Besucher deutlich, die einen Tagesausflug oder einen Kurzurlaub beabsichtigen. Allerdings deutet der mit 24,3 \%och relative hohe Anteil der Urlauber an den Besuchern darauf hin, daB die Untersuchungsregion auch für einen Aufenthalt von länger als fünf Tagen ausreichend Attraktivitätspotentiale bietet. Deshalb werden alle Verhaltenssegmente in die Analyse einbezogen.

Die Charakterisierung dieser Verhaltenssegmente anhand der zielgobietserfahrung findet sich in Abbildung 9. Dabei wird deutlich, daB Befragte mit einer Besuchsabsicht eine signi- 
fikant höhere zielgebietserfahrung aufweisen, als diejenigen ohne Besuchsabsicht. Immerhin die Hälfte der Personen mit Besuchsabsicht haben in den letzten 2 Jahren schon einmal die Untersuchungsregion besucht. Bei den Personen ohne Besuchsabsicht sind dies hingegen nur $36,2 \%$. Die Hypothese Horrh4, die zum Ausdruck bringt, daß die zielgebietserfahrung EinfluB auf die Besuchsabsicht hat, ist zu bestätigen.

\begin{tabular}{|c|c|c|c|c|c|c|}
\hline $\begin{array}{l}\text { Besuchs- } \\
\text { verhaltenssegmente }\end{array}$ & $\begin{array}{l}\text { ielgebiets- } \\
\text { erfahrung }\end{array}$ & keine & einmal & 2mal & \begin{tabular}{|c|} 
3mal \\
und \\
häufiger
\end{tabular} & \\
\hline \multirow{2}{*}{ Besucher } & $\begin{array}{c}\text { \%-Anteil } \\
\text { nach } \\
\text { Besuchern }\end{array}$ & $50,5 \%$ & $11,5 \%$ & $14,2 \%$ & $23,8 \%$ & $100 \%$ \\
\hline & \begin{tabular}{|c|}
$\%-A n t e i l$ \\
nach der \\
Zielgebiets- \\
erfahrung
\end{tabular} & $35,3 \%$ & $24,7 \%$ & $18.1 \%$ & $67.5 \%$ & \\
\hline \multirow{2}{*}{$\begin{array}{l}\text { Nicht- } \\
\text { Besucher }\end{array}$} & $\begin{array}{c}\text { \%-Anteil } \\
\text { nach } \\
\text { Nicht- } \\
\text { Besuchern }\end{array}$ & $63,8 \%$ & $24,2 \%$ & $4,2 \%$ & $7,8 \%$ & $100 \%$ \\
\hline & \begin{tabular}{c|} 
\%-Anteil \\
nach der \\
Zielgebiets \\
erfahrung
\end{tabular} & $64.7 \%$ & $75,3 \%$ & $20,9 \%$ & $32,1 \%$ & \\
\hline Chi²-Test: $\alpha<0,01$ & & $100 \%$ & $100 \%$ & $100 \%$ & $100 \%$ & \\
\hline
\end{tabular}

Abb. 9: Zielgebietserfahrung der empirisch ermittelten Besucher- und Nicht-Besucher-Segmente

Eine differenziertere Betrachtung der zielgebietserfahrung in Abhängigkeit von der Häufigkeit des Besuches der Fremdenverkehrsregion macht deutlich, daB eine Besuchsabsicht signifikant um so häufiger genannt wird, je häufiger das zielgebiet in der Vergangenheit aufgesucht wurde. So äuBerten mehr als zwei Drittel der Befragten eine Besuchsabsicht, die in den letzten 2 Jahren mindestens zweimal die Fremdenver- 
kehrsregion besuchten. Interessanterweise ist der zusammenhang zwischen einem einmaligen Besuch in der Vergangenheit und der geäußerten Besuchsabsicht umgekehrt. So gaben 75,3 q der Befragten an, die in den letzten 2 Jahren das Fremdenverkehrsgebiet schon einmal aufsuchten, keinen Besuch in den nächten 6 Monaten $z$ u beabsichtigen. Dies zeigt, daß das vergangenheitsbezogene Besuchsverhalten kein ausreichender Indikator für die Besuchsabsicht darstellt und vielmehr uber die Besuchsabsicht das Besuchsverhalten zu erfassen ist. Die Basishypothese $\mathbf{B}_{\mathbf{V E R H}}$ ist somit zu bestätigen.

Der Zusammenhang zwischen Besuchsabsicht und -dauer sowie der Entfernung zum Zielgebiet ist Abbildung $10 \mathrm{zu}$ entnehmen. Mit Hilfe des T-Tests wurde überprüf, ob sich die Besucher von den Nicht-Besuchern signifikant in ihrer Entfernung zum zielgebiet unterscheiden. Es zeigt sich, daß die Entfernung zur Fremdenverkehrsregion keinen EinfluB auf die Besuchsabsicht hat. Daher ist die Hypthese $\mathbf{B}_{\text {VERH }} \mathrm{zu}$ verwerfen. Allerdings kann hieraus nicht die SchluBfolgerung gezogen werden, daß eine Makrosegmentierung nicht zweckmäßig ist.

\begin{tabular}{|c|c|c|c|}
\hline $\begin{array}{l}\text { Besuchs- } \\
\text { verhaltens- } \\
\text { segmente }\end{array}$ & $\begin{array}{c}\text { Durchschnitt- } \\
\text { liche } \\
\text { Entfernung }\end{array}$ & \multicolumn{2}{|c|}{$\begin{array}{c}\text { Signifikanz- } \\
\text { niveau }\end{array}$} \\
\hline Nicht-Besucher & 261,01 & \multicolumn{2}{|c|}{ n.s. } \\
\hline 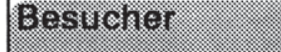 & 256.518 & & \\
\hline $\begin{array}{l}\text { Thagesaustimgler } \\
\text { (TI A }\end{array}$ & 180,8, & 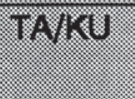 & 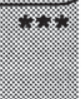 \\
\hline 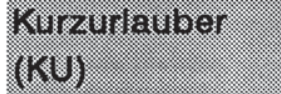 & 28.8 & 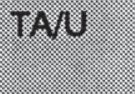 & 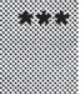 \\
\hline 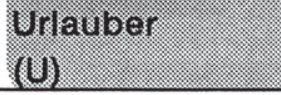 & 366,28 & $\sqrt{10} / 10$ & 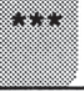 \\
\hline $\begin{aligned} & \text { Legende: } \text { Mittelwe } \\
& \text { mit eine } \\
& \alpha<0,01=\star \star \star \\
& \alpha<0,05=\star \star\end{aligned}$ & $\begin{array}{c}\text { tunterschiede } \\
\text { Signifikanzniv } \\
\alpha<0,1 \\
\text { nicht Signi }\end{array}$ & $\begin{array}{l}\text { lest) } \\
\begin{array}{l}\text { u von: } \\
\text { ant }=\text { * }\end{array}\end{array}$ & \\
\hline
\end{tabular}

Abb. 10: Zielgebietsentfernung der empirisch ermittelten Besuchsverhaltenssegitiente römbling - 978-3-631-75102-2 
Eine Analyse der nach der Besuchsdauer abgegrenzten Verhaltenssegmente auf der Basis von T-Tests zeigt, daB Tagesausflügler, Kurzurlauber und Urlauber sich in der Entfernung zum zielgebiet voneinander jeweils signifikant unterscheiden (vgl. Abbildung 10). Zur Überprüfung dieses Ergebnisses wurde eine Korrelationsanalyse duchgeführt, im Rahmen derer die Besuchsdauer als ordinal skalierte Variable interpretiert wurde. 50 Der so ermittelte spearmannsche Korrelationskoeffizient 51 in Höhe von - 0,492 zwischen Entfernung und Besuchsdauer bestätigt, daß zwischen der Besuchsdauer und der Entfernung ein hoher Zusammenhang besteht. Vor diesem Hintergrund ist die Hypothese H $_{\text {VBRH2 }}$, die von einem positiven Zusammenhang von Entfernung und Besuchsdauer ausgeht, zu bestätigen. Es ist anzunehmen, $\mathrm{daB}$ es sich hierbei um eine kausale Beziehung zwischen Entfernung und Besuchsdauer handelt.

Angesichts des hohen Einflusses der Entfernung auf die Besuchsdauer wird die Notwendigkeit der Durchführung einer Makrosegmentierung auf der Basis der Entfernung zum zielgebiet offensichtlich. Dabei wurde die Vorgabe abgeleitet, einen Grenzwert in Entfernungskilometern zugrundezulegen, der dazu führt, daB der Entfernungsgruppe II möglichst keine Tagesausflügler zugeordnet werden. Diese Anforderung ist wei-

50 Die Klassifizierung in Tagesausflügler, Kurzurlauber und Urlauber kann in eine Rangordnung gebracht werden, die als ordinal skalierte variable interpretiert werden kann.

51 Der Spearmannsche Korrelationskoeffizient erweist sich als ein geeignetes $M a B$ zur Identifizierung des Zusammenhangs zwischen zwei Variablen, wenn eine der Variablen nur Ordinalskalenniveau aufweist. Vgl. Bauer, F., Datenanalyse mit SPSS, Berlin u.a. 1984 , S. $167 \mathrm{ff}$. Das negative Vorzeichen ergibt sich dadurch, daB Besuchsdauer und zielgebietsentfernung gegenläufig kodiert sind. Ein negatives Vorzeichen bedeutet, daB mit zunehmender zielgebietsentfernung die Besuchsdauer steigt. 
testgehend erfüllt, wenn folgende Gruppeneinteilung vorgenommen wird:

Entfernungsgruppe I: 0 bis $300 \mathrm{~km}$

Entfernungsgruppe II: mehr als $300 \mathrm{~km}$

In Abbildung 11 ist die Verteilung der Befragten auf diese Entfernungsgruppen, differenziert nach der Besuchsabsicht und -dauer wiedergegeben. Der zusätzlich durchgeführte ChiSquare Test macht deutlich, daß sich in den beiden Entfer-

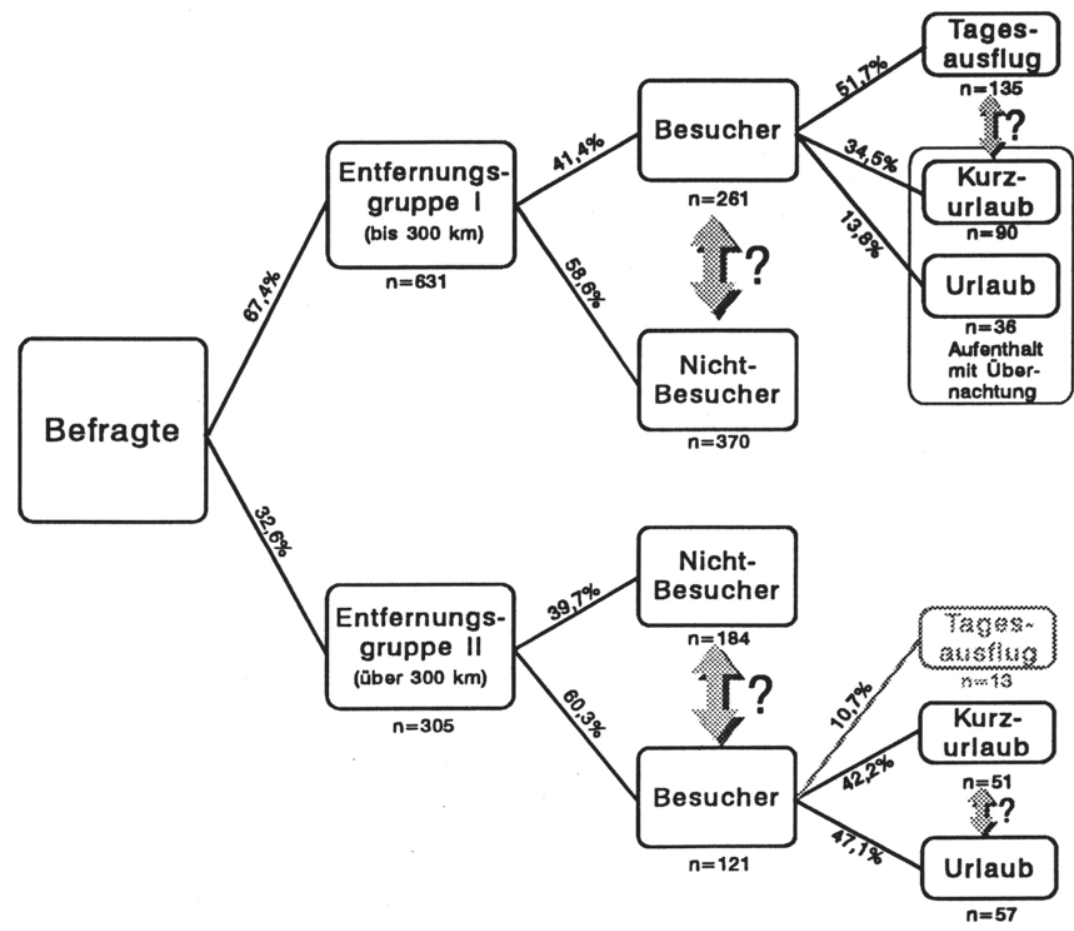

Abb. 11: Empirische Ergebnisse der Makrosegmentierung 
nungsgruppen die Anteile an Tagesausflüglern, Kurzurlaubern und Urlaubern signifikant unterscheiden. Dabei kommt in der Entfernungsgruppe I den Tagesausflüglern die größte und den Urlaubern mit 13,8\% nur eine untergeordnete Bedeutung $z u$. Vor diesem Hintergrund erscheint es zweckmäßig, die Urlauber und Kurzurlauber in dieser Entfernungsgruppe zusammenzufassen und als Besucher mit übernachtung zu charakterisieren. ziel der folgenden Ausführungen ist es dabei, in der Entfernungsgruppe I die Gruppen "Tagesausflügler" und "Besucher mit Übernachtung" hinsichtlich ihrer Unterschiede in den soziodemographischen und psychographischen Merkmalen zu analysieren, wobei die Entfernungsgruppe I den Naherholungsverkehr umfaßt.

In der Entfernungsgruppe II kommt den Tagesausflüglern mit $10,7 \%$ eine nur geringe Bedeutung $\mathrm{zu}$. Im Rahmen einer weiterführenden Analyse wurden diese Personen auf ihre Entfernung zum zielgebiet untersucht. Es zeigte sich, daß diese Personen zwischen 361 und $710 \mathrm{~km}$ von der Untersuchungsregion entfernt wohnen. Diese Gruppe wird im folgenden aus der Untersuchung ausgeschlossen, da sie für die Fremdenverkehrsregion angesichts ihrer segmentgröße nur geringe Attraktivitätspotentiale bietet. Daher werden in der Entfernungsgruppe II den weiteren Analyseschritten die Besuchsverhaltenssegmente "Kurzurlauber" und "Urlauber" zugrunde gelegt.

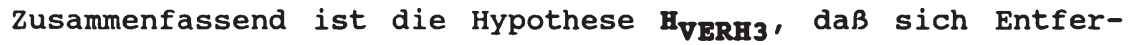
nungsgruppen bilden lassen, die signifikante Unterschiede in der Verteilung der nach der Besuchsdauer abgegrenzten Verhaltenssegmente aufweisen, zu bestätigen. Auf der Grundlage der so ermittelten entfernungsgruppenspezifischen Besuchsverhaltenssegmente gilt es im folgenden $z u$ überprüfen, ob sie die Anforderung der Identifizierbarkeit erfüllen. Gegenstand der folgenden Ausführungen sind daher die soziodemographischen Merkmale. 


\section{Erfassung und Analyse soziodemographischer Merkmale als Rriterien zur Marktsegmentierung}

\subsection{Erfassung soziodemographischer Kerkmale als EinfluBgrö- Ben des Besuchsverhaltens}

Bei der Analyse der Eignung soziodemographischer Merkmale als segmentierungskriterien für den Fremdenverkehr von Regionen ist zwischen demographischen (Alter, Geschlecht, Familienstand, Haushaltsgröße) und sozioökonomischen Merkmalen (Einkommen, Bildungsgrad, Beruf) zu differenzieren. 52 Dabei wird den Merkmalen Alter, Bildung und Einkommen tendenziell die größte Erklärungskraft für das Besuchsverhalten zugesprochen. 53

Die Fremdenverkehrsforschung beschäftigt sich überwiegend mit dem Einfluß soziodemographischer Merkmale auf die Reiseintensität und häufigkeit. Ferner wird versucht, Unterschiede zwischen Inlands- und Auslandsreisenden soziodemographisch zu beschreiben. Inwieweit das regionenspezifische Besuchsverhalten anhand von soziodemographischen Merkmalen zu erklären ist, wurde bislang nur ansatzweise überprüft. 54 In diesem Zusammenhang ist $z u$ berücksichtigen, daB allgemeine Erkenntnisse über die Beziehung zwischen Reiseintensität sowie -häufigkeit und soziodemographischen Merkmalen keine Auskunft darüber geben, ob diese zusammenhänge auch das regionenspezifische Besuchsverhalten erklären. So unterschieden sich in Deutschland die Besucherstrukturen verschiedener Fremdenverkehrsregionen z.T. stark in ihren so-

52 Vgl. Böhler, H., Methoden und Modelle der Marktsegmentierung, a.a.0., s. $68 \mathrm{ff}$.

53 Vgl. z.B. Gilbrich, M., Urlaubsreisen 1991, a.a.O., s. $17 \mathrm{f}$.

54 Vgl. z.B. Wölm, D., Marketing für Deutschlandreisen, a.a.o., S. 180 ff. 
ziodemographischen Merkmalen. 55 Dies bedeutet, $\mathrm{daB}$ Aussagen zum Einfluß soziodemographischer Merkmale auf die Besuchsabsicht und -dauer nur unter Kenntnis der spezifischen Eigenarten der einzelnen Fremdenverkehrsregion abzuleiten sind.

Dem soziodemographischen Merkmal Alter kommt eine besondere Bedeutung zu. Dies ergibt sich dadurch, daB das Alter in einem engen Zusammenhang mit spezifischen Urlaubsbedürnissen $\mathrm{zu}$ sehen ist. 56 Beispielsweise bevorzugen jüngere Personen im Urlaub eher sportbezogene Aktivitäten oder einen strandBadeurlaub, während ältere Personen eher den Wunsch nach Spazierengehen, Wandern etc. haben. 57 Geht man in diesem Zusammenhang davon aus, daß eine Fremdenverkehrsregion nur ausgewählte Bedürfnisse befriedigen kann, ist auch der Besuch einer bestimmten Fremdenverkehrsregion auf das Alter der Touristen zurückzuführen.

Empirische Untersuchungen zeigen, daß vor allem altersspezifische Unterschiede in der Reiseintensität und -häufigkeit bestehen. Jüngere Personen weisen eine größere Reiseintensität auf als ältere Personen, 58 wobei diese vor allem Inlandsreisen bevorzugen. 59 Allerdings deuten aktuelle studien darauf hin, daß zunehmend auch jüngere Personen Inlandsrei-

55 Vgl. Datzer, R., Informationsverhalten von Urlaubsreisenden, a.a.0.; S. $10 \mathrm{ff}$.

56 Vgl. Freter, H., Marktsegmentierung, a.a.0., s. 51.

57 Vgl. Wachenfeld, w., Freizeitverhalten und Marketing. Grundlagen des Marketing für Freizeitangebote, Heidelberg 1987 , S.182.

58 Vgl. Dundler, F., Urlaubsreisen 1987 , a.a.0., s. 18; o.V., Marken Profile 4: Touristik, Fluggesellschaften, Mietwagen, a.a.0., s. 24 f.

59 Vgl. Wölm, D., Marketing für Deutschlandreisen, a.a.o., S. 200. Auch Hachmann konnte für Deutschlandreisende im Vergleich zu Auslandsreisenden ein signifikant höheres Durchschnittsalter feststellen. Vgl. Hachmann, H., Reisebefragung 1977, Starnberg 1978, S. 16. 
sen unternehmen, 60 wenngleich nicht von einer Umkehr des $\mathrm{Zu-}$ sammenhangs gesprochen werden kann. Unter der Annahme, daB sich der Zusammenhang zwischen Alter und der Durchfuhrung von Inlandsreisen auch in dem regionenspezifischen Besuchsverhalten äußert, sind die Besucher der Untersuchungsregion tendenziell älteren Jahrgangs. Dies erscheint plausibel, da das touristische Angebot der Untersuchungsregion überdurchschnittlich viele Angebote (z.B. Rundwanderwege, Wasserschlösser etc.) für ältere Personen umfaBt.

Ferner ist anzunehmen, daB das Angebot der Untersuchungsregion vor allem für Kurzurlaubsreisende Attraktivitätspotentiale bietet. 61 Eine Analyse von aktuellen Untersuchungen zu Kurzurlaubsreisen zeigt in diesem zusammenhang, daß eine hohe Übereinstimmung zwischen Kurzurlaubsreisenden und der Gesamtheit aller Reisenden im Hinblick auf die Merkmale Alter sowie andere demographische Kriterien besteht. 62 Dies bedeutet z.B., daß sich die hohe Reiseintensität von jüngeren Personen auch in einer hohen Intensität von Kurzurlaubsreisen äußert.

Die Ausführungen machen deutlich, daß der Einfluß des Alters auf das regionenspezifische Besuchsverhalten vor allem in Verbindung mit der Besuchsdauer $\mathrm{zu}$ betrachten ist. Angesichts der Vielzahl von Beweggründen zum Reisen und der heterogenen Zusammenhänge zwischen Alter und dem Besuchsverhalten in Abhängigkeit von der Besuchsdauer ist anzunehmen, daß die Reiseabsicht nicht anhand des Alters zu erklären

60 Vgl. Lohmann, M., Wohlmann, R., Urlaub in Deutschland, a.a.0., s. 13 .

61 Vgl. Expertengespräch, auszugsweise dokumentiert in Meffert, u.a., Regionenmarketing Münsterland, a.a.O., s. 19 ff.

62 Vgl. o.v., Marken-Profile 4: Touristik, Fluggesellschaften, Mietwagen, a.a.0., S. 56 f.; Lohmann, M., Wohlmann, R., Urlaub in Deutschland, a.a.0., s. 6 . 
ist. Demgegenüber ist ein positiver Zusammenhang zwischen Alter und Besuchsdauer anzunehmen.

Bezüglich anderer demographischer Merkmale wie z.B. Anzahl der Kinder, Geschlecht, Familienstand finden sich in der Fremdenverkehrsforschung kaum Hinweise auf einen Zusammenhang mit dem generellen oder regionenspezifischen Besuchsverhalten. In Abhängigkeit von dem Geschlecht wird in der Konsumentenverhaltensforschung von einer zunehmenden Angleichung der Verhaltensweisen gesprochen, 63 so daß auch bezogen auf den Fremdenverkehr nicht von einem Zusammenhang mit dem Besuchsverhalten auszugehen ist. Der Variablen Familienstand (ledig, verheiratet) kommt angesichts der Bedeutungszunahme von single-Haushalten und Nicht-Ehelichen Partnerschaften immer weniger Erklärungskraft zum Kauf- und Besuchsverhalten $z u$, und wird daher in dieser Untersuchung nicht weiter betrachtet.

Die Kinderanzahl hingegen scheint im Fremdenverkehr durchaus dazu geeignet zu sein, das Besuchsverhalten zu erklären. Es wird vermutet, $d a \beta$ das Vorhandensein von Kindern unter 18 Jahren tendenziell einen hemmenden EinfluB auf die Reisehäufigkeit ausübt. 64 Dies bezieht sich vor allem auf Kurzurlaubsreisen und Tagesausflüge. Bezogen auf Urlaubsreisen ist hingegen festzustellen, daß gerade Familien mit Kindern überdurchschnittlich häufig einen Urlaub in Deutschland verbringen. Überträgt man diese Erkenntnisse auf die Untersuchungsregion, ist anzunehmen, daß in der Entfernungsgruppe I Personen ohne Kinder häufiger eine Besuchsabsicht äuBern als Personen mit Kindern. Demgegenüber ist für die Entfernungsgruppe II $z u$ vermuten, daß Personen mit Kindern tendenziell

63 Vgl. Wachenfeld, w., Freizeitverhalten und Marketing, a.a.0., S. 187 .

64 Vgl. Aderhold, P., Das Marketing von städtekurzreisen, a.a.o., S. 103 . 
eher eine Besuchsabsicht äußern und dabei eine längere Aufenthaltsdauer in Betracht ziehen.

Anstelle der isolierten Betrachtung der demographischen Merkmale wird zunehmend eine Kombination von demographischen Merkmalen in Form des Lebens- oder Familienzyklus zur Erklärung des Besuchsverhaltens herangezogen. Der Lebenszyklus beschreibt die Phasen, die ein Mensch in zeitlicher Folge in einem eigenständigen Haushalt durchläuft. ${ }^{65}$ Er läßt sich über eine Kombination von demographischen Merkmalen ermitteln, wobei vor allem das Alter, der Familienstand und die Zahl der Kinder herangezogen werden. 66 Eine in Abhängigkeit vom Alter und der Kinderanzahl durchgeführte Abgrenzung von Lebenszyklusphasen ist in Abbildung 12 dargestellt. 67 Jeder Lebensphase sind spezifische Vorstellungen von einem Urlaub zuzuordnen. Hieraus ergibt sich, daß in Abhängigkeit von der Lebenszyklusphase auch das Besuchsverhalten $z u$ erklären ist. 68 Unter Berücksichtigung der Aussagen zum Alter und zur Kinderzahl ist anzunehmen, daß in der Entfernungsgruppe I und II zwischen der stellung im Lebenszyklus und dem Besuchsverhalten ein Zusammenhang besteht.

65 Vgl. Freter, H., Marktsegmentierung, a.a.o., s. 54.

66 Vgl. ebenda, S. 54.

67 Gegenüber älteren Konzepten wird auf eine starre Trennung zwischen Lebens- und Familienzyklus verzichtet, um den aktuellen soziodemographischen Entwicklungen (mehr Single-Haushalte, mehr zusammenlebende unverheiratete Paare) Rechnung zu tragen. Vgl. Wachenfeld, H., Freizeitverhalten und Marketing, a.a.0., S. 193.

68 Vgl. Opaschowski, Tourismusforschung, a.a.0., s. $107 \mathrm{ff}$. 


\begin{tabular}{|c|c|c|}
\hline $\begin{array}{c}\text { Lebens- } \\
\text { phase }\end{array}$ & Bezelchnung & Indlkatorenausprăgungen \\
\hline | & $\begin{array}{c}\text { Jugendliche } \\
\text { und junge } \\
\text { Erwachsene }\end{array}$ & -Altersgruppe von 14-24 Jahren \\
\hline II & $\begin{array}{l}\text { Singles } \\
\& \text { Paare }\end{array}$ & $\begin{array}{l}\text {-1-2 Personen-Haushalte } \\
\text { im Alter von 25-49 Jahren }\end{array}$ \\
\hline III & $\begin{array}{c}\text { Familien } \\
\text { mit Kindern \& } \\
\text { Jugendlichen }\end{array}$ & $\begin{array}{l}\text { - Mehr-Personen-Haushalte } \\
\text { im Alter von 25-49 Jahren } \\
\text { mit Kindern/Jugendlichen }\end{array}$ \\
\hline IV & $\begin{array}{l}\text { Jung- } \\
\text { senioren }\end{array}$ & -Altersgruppe von 50-64 Jahren \\
\hline V & $\begin{array}{l}\text { Ruhe- } \\
\text { ständler }\end{array}$ & $\begin{array}{l}\text { - Altergruppe von } 65 \text { Jahren } \\
\text { und älter }\end{array}$ \\
\hline
\end{tabular}

Abb. 12: Abgrenzung von Lebensphasen in Abhängigkeit von Alter und Kinderanzahl (in Anlehnung an opaschowski, H.W., Tourismusforschung, a.a.O., S. 108)

Angesichts der aufgezeigten zusammenhänge sollen in bezug auf die demographischen Merkmale folgende Hypothesen überpruft werden:

Bozio 1 In beiden Entfernungsgruppen besteht kein zusammenhang zwischen dem Alter und der Besuchsabsicht.

$\mathbf{H}_{\text {8OzIO } 2}$ In beiden Entfernungsgruppen steigt mit zunehmendem Alter die Besuchsdauer.

$\mathbf{H}_{\text {8OzIO } 3}$ In beiden Entfernungsgruppen besteht kein Zusammenhang zwischen dem Geschlecht und dem Besuchsverhalten. 


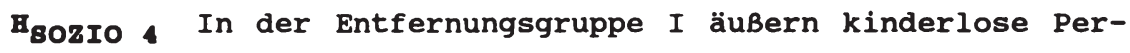
sonen häufiger eine Besuchsabsicht in die Untersuchungsregion als Personen ohne Kinder.

HozIo $5_{5}$ In der Entfernungsgruppe II äuBern Personen mit Kindern häufiger eine Besuchsabsicht und planen eine längere Aufenthaltsdauer als Personen ohne Kinder.

Bezüglich des Einflusses der stellung im Lebenszyklus auf das Besuchsverhalten werden folgende Basishypothesen uberprüft.

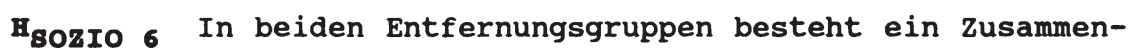
hang zwischen Besuchsabsicht und der stellung im Lebenszyklus.

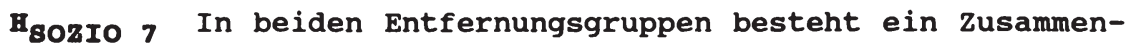
hang zwischen Besuchsdauer und der stellung im Lebenszyklus.

Mit Blick auf sozioökonomische Merkmale wird vor allem dem Bildungsgrad zur Erklärung des Besuchsverhaltens eine hohe Erklärungskraft zugesprochen. 69 Der Bildung kommt insbesondere bei solchen Kaufentscheidungen eine hohe Bedeutung $z u$, die ein Problemlösungsverständnis von Konsumenten verlangen. Daher trägt der Bildungsgrad vor allem zur Unterscheidung von Inlands- und Auslandsreisenden unter der Annahme bei, daB die Reiseplanung von Auslandsreisen ein höherers Problemverständnis von Touristen erfordert. Ferner steht der Bildungsgrad in einem hohen zusammenhang mit spezifischen Urlaubsbedürfnissen. Personen mit hohem Bildungsgrad werden

Vgl. Gilbrich, M., Urlaubsreisen 1991, a.a.0., S. 21. 
tendenziell häufiger in ihrem Urlaub Fremdenverkehrsregionen aufsuchen, die sich durch ein umfangreiches Kulturangebot auszeichnen.

Empirische Untersuchungen zeigen ferner, daß mit zunehmendem Bildungsniveau die Reiseintensität und -häufigkeit sowie die Reisezielentfernung bei Haupturlaubsreisen steigt. 70 zudem weisen Deutschlandurlauber einen geringeren Bildungsgrad auf als "Südreisende". 71 Aktuelle Untersuchungen zeigen jedoch, daß sich der Anteil von Personen mit höherem Bildungsgrad an den Inlandsreisen stark erhöht hat. 72 Hierbei handelt es sich überwiegend um zusätzliche Reisen in Form von Kurzreisen, mit Hilfe derer höher Gebildete ihr Interesse an Inlandsreisen realisieren. Bezogen auf die Untersuchungsregion ist angesichts der hohen Reiseintensität höher gebildeter Personen in Verbindung mit dem vorhandenen breiten kulturellen Angebot $z u$ vermuten, daß sich die Besucher der Untersuchungsregion von den Nicht-Besuchern im Bildungsgrad unterscheiden. Da längere Aufenthalte von höher gebildeten Personen vornehmlich in das Ausland unternommen werden, wird in der Entfernungsgruppe II vermutlich die Besuchsdauer mit abnehmendem Bildungsgrad steigen.

Als weiteres sozioökonomisches Merkmal ist der Beruf zu nennen, dem jedoch nur dann eine Erklärungskraft zum Kaufverhalten zugesprochen wird, wenn die Nachfrage nach der relevanten Produktgruppe in einem engen Zusammenhang zum Beruf steht. Da diese Beziehung im Erholungstourismus nicht be-

70 Vgl. Zucker, W.H., Urlaubsreisen 1985. Kurzfassung einiger Ergebnisse der Reiseanalyse 1985, Studienkreis für Tourismus e.V. (Hrsg.), Starnberg 1986, S. 19, Dundler, F., Urlaubsreisen 1987, a.a.0., S. 19.

71 Vgl. hierzu und zur Abgrenzung von "Südreisenden" Wölm, D., Marketing für Deutschlandreisen, a.a.0., S. 202 .

72 Vgl. Lohman, M., Wohlmann, R., Urlaub in Deutschland, a.a.0., s. 15 . 
steht, ist von keinem unmittelbaren zusammenhang mit dem Besuchsverhalten auszugehen. So weist auch uttitz ${ }^{73}$ darauf hin, daß nicht der Beruf, sondern der im Beruf zum Ausdruck kommende Bildungsgrad und das Einkommen die wesentlichen sozio-ökonomischen Einflußfaktoren auf das Besuchsverhalten darstellen.

Dem Kriterium Einkommen kommt als Bestimmungsfaktor des Besuchsverhalten eine zentrale Bedeutung $z u$, da es einen Indikator für die Kaufkraft unterschiedlicher Einkommensgruppen darstellt. Es ist anzunehmen, daß die Reisehäufigkeit mit zunehmenden Einkommen steigt. Übertragen auf das regionenspezifische Besuchsverhalten ist $z u$ vermuten, daB eine Besuchsabsicht um so eher geäußert wird, je größer das verfügbare Einkommen ist. Inwieweit das Einkommen EinfluB auf die Besuchsdauer hat, ist differenziert $\mathrm{zu}$ betrachten. Es ist einerseits möglich, daß angesichts finanzieller Restriktionen eine Reise nicht durchgeführt wird. Andererseits könnte auch die Besuchsdauer verkürzt oder eine möglichst günstige Fremdenverkehrsregion aufgesucht werden.

Der Zusammenhang zwischen Einkommen und Besuchsverhalten hat im Zuge der wachsenden Einkommensnivellierung an Bedeutung verloren. Vor allem das Einkommen aus Erwerbstätigkeit stellt angesichts hoher Kapitalvermögen und -einkünfte, welche unter dem stichwort "Erbengeneration" zum Ausdruck kommen, keinen geeigneten Indikator für die zur Verfügung stehenden finanziellen Mittel dar. ${ }^{74} \mathrm{Je}$ mehr das Einkommen zur

73 Vgl. Uttitz, P., Von den vertikalen Spannungslinien zu den horizontalen Gegensätzen: Wandel des Freizeitverhaltens als Indikator, Köln 1984, S. $116 \mathrm{ff}$.

74 Dies bedeutet, daß zur operationalisierung des Einkommens nicht das Einkommen aus Erwerbstätigkeit, sondern das gesamte Einkommen inclusive Kapitaleinkommen herangezogen werden müßte. Es ist jedoch $z u$ vermuten, daß entsprechende Fragen auf eine geringe Anwortbereitschaft seitens Probanden stoßen. 
Differenzierung unterschiedlicher Verbrauchergruppen an Bedeutung verliert, um so häufiger wird das Kriterium der sozialen schichtung zur Erklärung des Kaufverhaltens eingesetzt. Soziale schichten sind relativ dauerhafte, homogene Gesellschaftsgruppen mit ähnlichen Wertvorstellungen, Interessen, Lebensstilen und Verhaltensmustern. ${ }^{75}$ In Abbildung 13 ist ein Punktbewertungsverfahren zur Ermittlung der schichtzugehörigkeit dargestellt. Dieses Verfahren ermöglicht auf der Basis von Bildung, Beruf und Einkommen, die Zugehörigkeit von Personen zur Ober-, Mittel- und Unterschicht zu bestimmen. 76

Da sich mittlerweile die Hälfte der Bundesbevölkerung eine jährliche Haupturlaubsreise leistet, wird allerdings das Reisen als sozial weitgehend indifferent bezeichnet. 77 Dies

75 Vgl. Wachenfeld, H., Freizeitverhalten und Marketing, a.a.O., S. 196

76 Eine objektive Punktvergabe zur Ermittlung des Berufsindex ist kaum möglich. Häufig wird den Hausfrauen und Rentnern nur ein geringer Punktwert zugesprochen. Dies erscheint jedoch nicht zweckmäßig, da Hausfrauen auch in Familien anzutreffen sind, die der Mittel- oder oberschicht angehören. Die Schichtzugehörigkeit von Rentnern ist abhängig von der Berufsausübung und dem Einkommen während der Berufstätigkeit. Daher erscheint es wenig zweckmäßig, einer während ihrer Berufstätigkeit mit einem hohen Wert des Berufsindex gekennzeichneten Person nur aufgrund des Eintritts in das Rentenalter einen geringeren Punktwert zuzuordnen. Vor diesem Hintergrund sollen Hausfrauen und Rentner im Rahmen des Berufsindex einen dem Durchschnitt entsprechenden Punktwert erhalten. Vgl. zu dem hier modifizierten Punktbewertungsverfahren Koerdt, W., Das Anspruchsniveau von Urlaubsreisenden. Ein empirischer Beitrag zur Konsumentenforschung, Heidelberg 1988 , S. 147 ff.

77 Vgl. Hörning, K.H., Sozialökonomische schichtdifferenzierung und Konsumstandards, in: Specht, K.G., Wiswede, G., Marketing Soziologie. Soziale Interaktionen als Determinanten des Marktverhaltens, Berlin 1976, S. 123 ff.; Wachenfeld, H., Freizeitverhalten und Marketing, a.a.O., S. 198 . 


\begin{tabular}{|l|c|}
\hline \multicolumn{2}{|c|}{ Schulolidungsinder } \\
\hline Bildungsgrad & Punkte \\
\hline •Volkschule ohne Lehre & 1 \\
•Volksschule mit Lehre & 3 \\
•Weiterbildende Schulen & 4 \\
•Abitur & 7 \\
•Studium & $\mathbf{8}$ \\
\hline
\end{tabular}

\begin{tabular}{|c|c|}
\hline \multicolumn{2}{|l|}{ Eerutsindex } \\
\hline Beruf & Punkte \\
\hline $\begin{array}{l}\text { - nicht erwerbstätig } \\
\text { - in Ausbildung (Schüler, Student, Azubi) } \\
\text { - Hausfrauen, Rentner, Arbeiter } \\
\text { - Angestellte, Beamte } \\
\text { - Selbstständige }\end{array}$ & $\begin{array}{l}1 \\
2 \\
4 \\
6 \\
8\end{array}$ \\
\hline
\end{tabular}

\begin{tabular}{|l|c|}
\hline \multicolumn{2}{|c|}{ Elnkommensindex } \\
\hline Monatliches Netto-Haushaltseinkommen & Punkte \\
\hline$\cdot$ bis 1000 DM & 1 \\
$\cdot 1000-1500 \mathrm{DM}$ & 2 \\
$\cdot 1500-2000 \mathrm{DM}$ & 3 \\
$\cdot 2000-2500 \mathrm{DM}$ & 4 \\
$\cdot 2500-3000 \mathrm{DM}$ & 5 \\
$\cdot 3000-3500 \mathrm{DM}$ & 6 \\
$\cdot 3500-4000 \mathrm{DM}$ & 7 \\
$\cdot 4000-5000 \mathrm{DM}$ & 8 \\
$\cdot>5000 \mathrm{DM}$ & 9 \\
\hline
\end{tabular}

\begin{tabular}{|l|r|}
\hline & \\
\hline & Schlohtindex \\
\hline & Punkte \\
\hline - Unterschicht & $3-10$ \\
- Mittelschicht & $11-17$ \\
Oberschicht & $18-25$ \\
\hline
\end{tabular}

Abb. 13: Punktbewertungsverfahren zur Ermittlung der Schichtzugehörigkeit (in Anlehnung an Koerdt, U.,

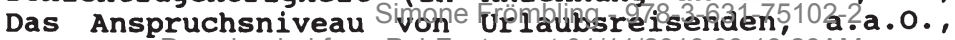
S. 149) Downloaded from PubFactory at 01/11/2019 08:19:28AM 
bedeutet, daß die soziale Schichtung keinen geeigneten Bestimmungsfaktor für das regionenspezifische Besuchsverhalten darstellt. Unter der Annahme, daß die Reisehäufigkeit einen zusammenhang mit der schichtzugehörigkeit aufweist, ist zu vermuten, daB um so eher eine Besuchsabsicht in die Untersuchungregion geäußert wird, je eher eine Person einer höheren sozialen schicht zugeordnet werden kann.

Zusammenfassend sind bezogen auf die sozioökonomischen Merkmale folgende Untersuchungshypothesen $\mathrm{zu}$ überprüfen.

H8OzI0 8 Je höher der Bildungsgrad, desto stärker wird in beiden Entfernungsgruppen eine Besuchsabsicht in die Untersuchungsregion geäußert.

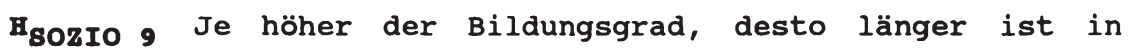
beiden Entfernungsgruppen die Besuchsdauer.

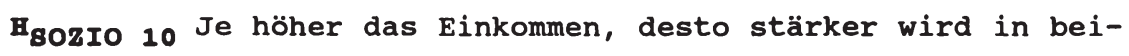
den Entfernungsgruppen eine Besuchsabsicht geäuBert.

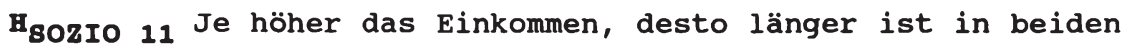
Entfernungsgruppen die beabsichtigte Besuchsdauer.

H8OzIo 12 Je höher die soziale Schichtzugehörigkeit, desto stärker wird in beiden Entfernungsgruppen eine Besuchsabsicht geäußert.

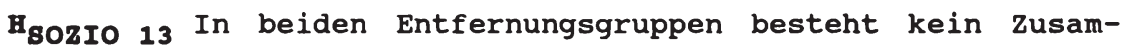
menhang zwischen dem Beruf und der Besuchsabsicht.

$\mathbf{H}_{\text {gozIo } 14}$ In beiden Entfernungsgruppen besteht kein zusammenhang zwischen dem Beruf und der Besuchsdauer. 


\subsection{Empirische Analyse der Eignung soziodemographischer Merkmale als segmentierungskriterien}

Die Ausprägungen der soziodemographischen Merkmale, differenziert nach den Entfernungsgruppen und den dort abgegrenzten Verhaltenssegmenten, sind in Abbildung 14 dargestellt. Eine Analyse der domographischen Merkmale zeigt hierbei, daß diese nur z.T. signifikante Unterschiede in den abgegrenzten Verhaltenssegmenten aufweisen.

In der Entfernungsgruppe I können zwischen Besuchern und Nicht-Besuchern der Untersuchungsregion als auch hinsichtlich der beabsichtigten Besuchsdauer (Tagesausflügler, Besucher mit Übernachtung) keine signifikanten Unterschiede mit Blick auf Geschlecht, Alter, Kinderanzahl und der stellung im Lebenszyklus ${ }^{78}$ identifiziert werden. Für die Entfernungsgruppe I kann festgehalten werden, daB demographische Merkmale nicht dazu geeignet sind, im Naherholungsverkehr das Besuchsverhalten $z u$ erklären. 79 Daher sind die Hypothesen $\mathbf{H}_{\mathbf{8 O z I O 2}}$ und $\mathrm{H}_{\mathbf{8 O z I O 6}}$ in bezug auf die Entfernungsgruppe I und die Hypothese $\mathbf{H}_{\text {8OzIO4 }} \mathrm{zu}$ verwerfen. Die Hypothese $\mathbf{B}_{\text {8OzIOI }}$ und $\mathbf{H}_{\text {8OzI03 }}$, die keinen Zusammenhang zwischen dem Alter und dem Geschlecht mit der Besuchsabsicht unterstellen, sind für die Entfernungsgruppe I zu bestätigen.

Ähnliche Ergebnisse zeigen sich auch in der Entfernungsgruppe II. Auch hier besteht kein Zusammenhang zwischen Besuchsabsicht und den Merkmalen Geschlecht und Alter, so daß sich die Hypothesen $\mathbf{H}_{80 z I 01}$ und $\mathbf{H}_{802 I 03}$ in bezug auf die

78 Die Einteilung in Lebenszyklusphasen erfolgte auf der Basis der in Kapitel 3.1 in Abbildung 12 dargestellten Merkmale.

79 Signifikante Abweichungen bei den Ausprägungen soziodemographischer Merkmale der einzelnen Verhaltenssegmente können als Hinweis dafür gewertet werden, daß sie einen Einluß auf das regionenspezifische Besuchsverhalten ausüben. 


\begin{tabular}{|c|c|c|c|c|c|c|c|c|c|c|c|c|c|}
\hline \multirow{3}{*}{ (1) } & \multirow{3}{*}{ 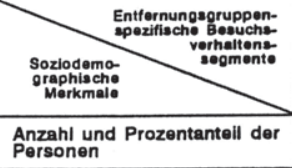 } & \multicolumn{6}{|c|}{ Entromungsgrupiog ! } & \multicolumn{6}{|c|}{ Entiomungsoruppo it } \\
\hline & & \begin{tabular}{|c|} 
Besu \\
ja
\end{tabular} & nein & $\begin{array}{l}\text { bsicht } \\
\text { Sig. }\end{array}$ & $\begin{array}{l}\text { Besu } \\
\text { Tages } \\
\text { aushiug }\end{array}$ & $\begin{array}{l}\text { Auchsda } \\
\text { Aut } \\
\text { mit U. }\end{array}$ & $\begin{array}{l}\text { auer } \\
\text { Sig. } \\
\end{array}$ & \begin{tabular}{|} 
Besuc \\
ja
\end{tabular} & nein & $\begin{array}{l}\text { Ssicht } \\
\text { Sig. }\end{array}$ & $\begin{array}{c}\text { Bes: } \\
\text { Kurz- } \\
\text { uriaub }\end{array}$ & Urlaub & $\begin{array}{l}\text { lauer } \\
\text { Sig. }\end{array}$ \\
\hline & & $\begin{array}{r}261 \\
41,3 \% \\
\end{array}$ & $\begin{array}{r}370 \\
58,6 \% \\
\end{array}$ & & $\begin{array}{r}135 \\
57,7 \% \\
\end{array}$ & $\begin{array}{c}128 \\
48,3 \% \\
\end{array}$ & & $\begin{array}{r}121 \\
39,7 \% \\
\end{array}$ & $\begin{array}{r}184 \\
60,2 \% \\
\end{array}$ & & $\begin{array}{r}51 \\
47,2 \% \\
\end{array}$ & $\begin{array}{c}57 \\
52,8 \% \\
\end{array}$ & \\
\hline \multirow{4}{*}{ 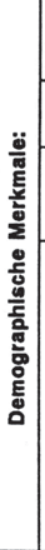 } & $\begin{array}{ll}\text {-Geschlecht } & \text {-mãnnlich } \\
\text { (in } \mathbf{x}) & \text {-weiblich }\end{array}$ & $\begin{array}{l}47,1 \\
52,9\end{array}$ & $\begin{array}{l}47,0 \\
53,0\end{array}$ & n.s. & $\begin{array}{l}43,0 \\
57,0\end{array}$ & $\begin{array}{l}51,6 \\
48,4\end{array}$ & n.s. & $\begin{array}{l}46,3 \\
53,7\end{array}$ & $\begin{array}{l}46,7 \\
53,3\end{array}$ & n.s. & $\begin{array}{l}41,2 \\
58,8\end{array}$ & $\begin{array}{l}47,4 \\
52,6\end{array}$ & n.s. \\
\hline & - Durchschnittsalter & 43,81 & 44,55 & n.s. & 44,69 & 42,87 & n.s. & 43,61 & 42,55 & n.s. & 43,33 & 43,70 & n.s. \\
\hline & $\begin{array}{l}\left.- \text { Kinderanzahl (in } x^{\prime}\right) \\
\text {-Keine Kinder } \\
-1 \text { und mehr Kinder }\end{array}$ & $\begin{array}{l}64,3 \\
35,7\end{array}$ & $\begin{array}{l}67,6 \\
32,5\end{array}$ & n.s. & $\begin{array}{l}65,9 \\
34,1\end{array}$ & $\begin{array}{l}62,7 \\
37,3\end{array}$ & n.s. & $\begin{array}{l}70,0 \\
30,0\end{array}$ & $\begin{array}{l}81,0 \\
19,0\end{array}$ & $*$ & $\begin{array}{l}78,4 \\
21,6\end{array}$ & $\begin{array}{l}62,5 \\
37,5\end{array}$ & * \\
\hline & $\begin{array}{l}\text { - Lebenszyklusphase (in x) } \\
\text { I: Jugendliche und } \\
\text { Junge Erwachsene } \\
\text { II: Singles und Paare } \\
\text { III:Familien mit Kindern } \\
\text { und Jugendlichen } \\
\text { IV: Jungsenioren } \\
\text { V: Ruheständler }\end{array}$ & $\begin{array}{c}16,3 \\
23,7 \\
23,3 \\
23,7 \\
13,1\end{array}$ & \begin{tabular}{|c|}
17,6 \\
20,6 \\
23,8 \\
18,8 \\
19,1 \\
\end{tabular} & n.s. & \begin{tabular}{|l|}
14,4 \\
23,2 \\
24,0 \\
24,0 \\
11,4
\end{tabular} & $\begin{array}{r}22,5 \\
23,3 \\
11,7\end{array}$ & n.s. & $\begin{array}{l}14,0 \\
32,5 \\
16,7 \\
21,1 \\
15,8\end{array}$ & $\begin{array}{l}14,9 \\
18,8 \\
16,0\end{array}$ & n.s. & $\begin{array}{l}10,4 \\
35,4 \\
14,6 \\
27,1 \\
12,5\end{array}$ & $\begin{array}{r}16,4 \\
29,1 \\
20,0 \\
18,2 \\
16,4\end{array}$ & n.s. \\
\hline \multirow{5}{*}{ 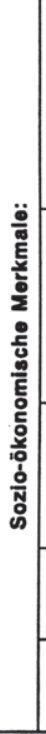 } & $\begin{array}{l}\text { - Bildung (in x) } \\
\text {-Volksschule ohne } \\
\text { abgeschlossene Lehre } \\
\text {-Volksschule mit } \\
\text { abgeschlossener Lehre } \\
\text {-Weiterbildende Schulen } \\
\text {-Abitur/Fachhochschule/ } \\
\text { Studium }\end{array}$ & \begin{tabular}{l|}
10,0 \\
48,5 \\
23,1 \\
18,5
\end{tabular} & $\begin{array}{l}16,1 \\
45,2 \\
24,4 \\
14,4\end{array}$ & n.S. & $\begin{array}{l}12,7 \\
45,5 \\
25,4 \\
16,4\end{array}$ & \begin{tabular}{|r|}
7.1 \\
51,6 \\
20,6 \\
20,6
\end{tabular} & n.s. & $\begin{array}{r}5,0 \\
38,7 \\
26,1 \\
30,3\end{array}$ & $\begin{array}{l}11,0 \\
39,6 \\
28,6 \\
20,9\end{array}$ & n.s. & $\begin{array}{r}4,0 \\
42,0 \\
30,0 \\
24,0\end{array}$ & $\begin{array}{r}5,4 \\
35,7 \\
21,4 \\
37,5\end{array}$ & n.s. \\
\hline & $\begin{array}{l}\text {-Berufsgruppen (inx) } \\
\text {-Selbständige } \\
\text {-Angestellte } \\
\text {-Beamte } \\
\text {-Arbeiter } \\
\text {-Hausfrau } \\
\text {-Schūler/Student/Azubi } \\
\text {-Rentner } \\
\end{array}$ & $\begin{array}{r}7,0 \\
30,0 \\
5,4 \\
19,8 \\
14,0 \\
9,7 \\
14,0 \\
\end{array}$ & $\begin{array}{r}6,0 \\
22,4 \\
6,3 \\
24,0 \\
13,4 \\
10,9 \\
16,9 \\
\end{array}$ & n.s. & \begin{tabular}{r|r|}
6,8 \\
27,3 \\
6,8 \\
18,9 \\
17,4 \\
9,1 \\
13,6 \\
\end{tabular} & $\begin{array}{r}7,2 \\
32,8 \\
4,0 \\
20,8 \\
10,4 \\
10,4 \\
14,4 \\
\end{array}$ & n.s. & $\begin{array}{r}12,2 \\
27,8 \\
8,7 \\
12,2 \\
10,4 \\
18,3 \\
10,4 \\
\end{array}$ & $\begin{array}{r}7,7 \\
39,9 \\
4,4 \\
19,7 \\
4,9 \\
10,9 \\
12,6 \\
\end{array}$ & $* *$ & $\begin{array}{l}14,0 \\
16,0 \\
12,0 \\
18,0 \\
14,0 \\
16,0 \\
10,0 \\
\end{array}$ & \begin{tabular}{|r|}
11,5 \\
42,3 \\
3,8 \\
3,8 \\
7,7 \\
21,2 \\
9,6 \\
\end{tabular} & $\cdots$ \\
\hline & \begin{tabular}{|l}
-HH-Nettoeinkommen (in *) \\
- bis 1500,- DM \\
- $1500-2500,-$ DM \\
- 2500-3500,- DM \\
- 3500-5000,- DM \\
- über 5000,- DM \\
\end{tabular} & \begin{tabular}{r|}
9,5 \\
30,7 \\
27,6 \\
25,4 \\
7,3 \\
\end{tabular} & \begin{tabular}{|l|}
15,7 \\
29,1 \\
28,1 \\
16,7 \\
10,5 \\
\end{tabular} & $*$ & \begin{tabular}{|r|}
11,1 \\
29,1 \\
28,2 \\
26,5 \\
5,1 \\
\end{tabular} & $\begin{array}{r}7,8 \\
31,3 \\
27,0 \\
24,3 \\
9,6 \\
\end{array}$ & n.s. & $\begin{array}{l}23,9 \\
23,9 \\
19,3 \\
21,1 \\
11,9\end{array}$ & \begin{tabular}{|r|}
16,3 \\
27,9 \\
29,7 \\
20,3 \\
5,8 \\
\end{tabular} & * & $\begin{array}{l}25,0 \\
25,0 \\
22,7 \\
13,6 \\
13,6 \\
\end{array}$ & $\begin{array}{l}19,2 \\
21,2 \\
19,2 \\
26,9 \\
13,5 \\
\end{array}$ & $*$ \\
\hline & $\begin{array}{l}\text { Pers. Netto-Einkom. (in x) } \\
\text { - bis 1500,- DM } \\
\text { - } 1500-3000,- \text { DM } \\
\text { - über } 3000,- \text { DM } \\
\end{array}$ & \begin{tabular}{|l|}
36,3 \\
48,0 \\
15,7 \\
\end{tabular} & \begin{tabular}{|r|}
41,7 \\
48,5 \\
9,8 \\
\end{tabular} & n.s. & \begin{tabular}{|l|}
39,0 \\
44,8 \\
16,2 \\
\end{tabular} & \begin{tabular}{l|}
33,9 \\
50,8 \\
15,3 \\
\end{tabular} & n.s. & $\begin{array}{l}43,4 \\
38,7 \\
17,9 \\
\end{array}$ & $\begin{array}{l}37,7 \\
50,9 \\
11,4 \\
\end{array}$ & * & $\begin{array}{l}43,2 \\
40,9 \\
15,9 \\
\end{array}$ & $\begin{array}{l}44,0 \\
36,0 \\
20,0 \\
\end{array}$ & n.S. \\
\hline & $\begin{array}{l}\text {-Schicht (in x) } \\
\text {-Unterschicht } \\
\text {-Mittelschicht } \\
\text {-Oberschicht } \\
\end{array}$ & \begin{tabular}{|l|}
17,5 \\
61,0 \\
21,5 \\
\end{tabular} & \begin{tabular}{|l|}
26,5 \\
60,7 \\
12,8 \\
\end{tabular} & $\cdots$ & \begin{tabular}{|l|}
20,2 \\
60,5 \\
19,3 \\
\end{tabular} & $\begin{array}{l}14,9 \\
61,4 \\
23,7 \\
\end{array}$ & n.s. & $\begin{array}{l}14,7 \\
63,7 \\
21,8 \\
\end{array}$ & \begin{tabular}{|l|}
15,4 \\
66,3 \\
18,3 \\
\end{tabular} & n.s. & \begin{tabular}{|l|}
11,9 \\
76,2 \\
11,9 \\
\end{tabular} & $\begin{array}{r}8,5 \\
61,7 \\
29,8 \\
\end{array}$ & n.s. \\
\hline & Chi" & Signifilk & kanzenh & . & $0,00=$ & $=\cdots$ & & nicht S & Signin & & $\begin{aligned} 0.1 & = \\
& =\end{aligned}$ & $=\dot{n}$ n.s. & \\
\hline
\end{tabular}

Abb. 14: Empirische Ergebnisse zum EinfluB von soziodemographischen Merkmalen auf das Besuchsverhalten innerhalb der Makrosegmente 
Entfernungsgruppe II bestätigen. Deutliche Beziehungszusammenhänge lassen sich jedoch in Abhängigkeit von der $\mathbf{k i n}-$ deranzahl ermitteln (vgl. Abbildung 14). So äuBern Befragte, die keine Kinder haben, eine signifikant geringere Besuchsabsicht als Personen mit Kindern. Offensichtlich bietet die Fremdenverkehrsregion Familien der Entfernungsgruppe II ein höheres Attraktivitätspotential als kinderlosen Personen. Dies bestätigt sich auch in der Besuchsdauer. So beabsichtigen kinderlose Personen, die Fremdenverkehrsregion nur für einen Kurzurlaub aufzusuchen, während Familien häufiger einen Urlaubsaufenthalt planen. Demnach ist die Hypothese

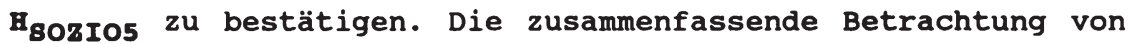
Alter und Kinderzahl in Form von der zugehörigkeit zu den Lebensphasen erbrachte demgegenüber keine signifikanten Unterschiede zwischen den Verhaltenssegmenten. H80zI06 und $\mathrm{H}_{\mathbf{8 O z I 0 7}}$ sind in bezug auf die Entfernungsgruppe II $\mathrm{zu}$ verwerfen.

Eine Analyse der sozioökonomischen Variablen zeigt, daß diese in beiden Entfernungsgruppen die Besuchsabsicht und -dauer beeinflussen. In beiden Entfernungsgruppen besteht ein signifikanter Zusammenhang zwischen Besuchsabsicht und monatlichem Netto-Haushaltseinkommen. In der Entfernungsgruppe I sind Personen, die eine Besuchsabsicht äuBerten, durch ein deutlich höheres Netto-Haushaltseinkommen gekennzeichnet. offensichtlich sind Reisen im Naherholungsverkehr von der Existenz eines ausreichenden Finanzbudgets abhängig, so daß sich die Hypothese $\mathbf{H}_{80 z I 010}$ bezogen auf die Entfernungsgruppe I bestätigt. Diese Interpretation wird dadurch bestätigt, daB die Besuchsabsicht von der Schichtzugehörigkeit beeinflußt wird. 80 Daher ist auch die Hypothese $\mathbf{H}_{\mathbf{8 0 z I 0 1 2}} \mathrm{zu}$

Die Schichtzugehörigkeit wurde gemäß dem in Abbildung 13 (Kapitel B.3.1) dargestellten Punktbewertungsverfahren ermittelt. Eine überprüfung dieses Verfahrens hinsichtlich seines Realitätsgehaltes erfolgte über einen Vergleich mit der Schichtaufteilung in der Grundgesamtheit. Dabei wurde davon ausgegangen, daB ca. 20 \& der Unter- 
bestätigen. Die Besuchsdauer ist hingegen unabhängig von sozio-ökonomischen Merkmalen, so daß die in den Hypothesen

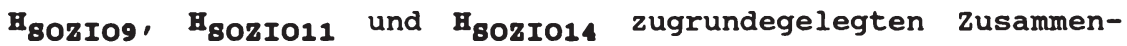
hänge zwischen sozio-ökonomischen Merkmalen und der Besuchsdauer zu verwerfen sind.

Bei der Betrachtung der Entfernungsgruppe II sind unter Bezugnahme auf Beruf, monatliches Netto-Haushaltseinkommen und persönliches Netto-Einkommen signifikante Unterschiede zwischen Besuchern und Nicht-Besuchern hervorzuheben. Dabei äuBerten Personen mit einem geringeren Netto-Haushaltseinkommen und persönlichem Netto-Einkommen, aber auch Personen mit einem hohen Einkommen eine überdurchschnittlich hohe Besuchsabsicht. Daher kann zwar von einem Zusammenhang zwischen Einkommen und Besuchsabsicht gesprochen werden. Eine Richtungszuweisung ist jedoch nicht möglich.

Betrachtet man zunächst die Personen mit niedrigen Einkommen, ist anzunehmen, daB diese geringe Finanzkraft insbesondere auf die berufliche stellung zurückzuführen ist. So äuBern Befragte, die sich beruflich noch in der Ausbildungsphase (Studenten, Schüler, Auszubildende) befinden oder als Hausfrau tätig sind, eine überdurchschnittlich hohe Besuchsabsicht. Offenbar ist die mit dieser Berufsausübung verbundene, vermehrt zur Verfügung stehende freie zeit ursächlich für die überdurchschnittlich hohe Besuchsabsichtserklärung. Demnach ist davon auszugehen, daß sich das zunehmende Interesse jüngerer Personen an Inlandsreisen auch in den Ergebnissen der vorliegenden Untersuchung widerspiegelt.

schicht, $65 \%$ der Mittelschicht und $15 \%$ der oberschicht zugeordnet werden können. Vgl. hierzu Wachenfeld, H., Freizeitverhalten und Marketing, a.a.0., S. 196; Kroeber-Riel, Konsumentenverhalten, a.a.0., 1984 , S. 574. In der vorliegenden Stichprobe konnte unter Zugrundelegung des Punktbewertungsmodells folgende Verteilung ermittelt werden: Unterschicht: 20,1 ; ; Mittelschicht: $62,4 \%$; Oberschicht: $17,6 \%$. Dies entspricht einer guten Annäherung an die tatsächliche schichtaufteilung der Grundgesamtheit. 
Gleichzeitig deutet die stärkere Besuchsabsicht der höher verdienenden Personen darauf hin, daß bei bestimmten Personengruppen mit steigendem Finanzbudget vermehrt Inlandsreisen - vermutlich in Form zusätzlicher Reisen - vorgenommen werden. Dabei ist die beabsichtigte Besuchsdauer vor allem vom Netto-Haushaltseinkommen abhängig. Personen mit höherem Haushaltseinkommen planen häufiger einen Urlaub in die Region. Ein Blick auf den Beruf zeigt in diesem Zusammenhang, $\mathrm{da} B$ es sich hierbei offensichtlich um Angestellte handelt. Bezieher mittlerer und niedrigerer Einkommen bevorzugen hingegen Kurzurlaube. Wenngleich ein signifikanter zusammenhang zwischen dem Einkommen und der Besuchsabsicht und -dauer besteht, können vor allem bezüglich der Besuchsabsicht aufgrund der fehlenden Richtungsbeziehung die Hypothesen $\mathbf{H}_{\mathbf{8 0 2 I 0 1 0}}$ und $\mathbf{H}_{\mathbf{8 0 2 I 0 1 1}}$ nur eingeschränkt bestätigt werden. Da der Beruf einen signifikanten Zusammenhang mit der Besuchsabsicht und -dauer aufweist, sind entgegen der oben abgeleiteten Annahme die Hypothesen $\mathrm{H}_{80 z I 013}$ und $\mathrm{H}_{80 z I 014}$ bezogen auf die Entfernungsgruppe II $\mathrm{zu}$ verwerfen.

Die gefundenen Zusammenhänge bezüglich des Einkommens und des Berufes bestätigen sich nicht in dem Bildungsgrad und in der Schichtzugehörigkeit. Dies überrascht, weil diese Merkmale Interdependenzen aufweisen, und somit ein gleichgerichteter Zusammenhang vermutet werden konnte. Zwar weisen Personen mit einem studium sowie der Angehörigkeit zur oberschicht eine tendenziell höhere Besuchsabsicht auf als Personen der Unterschicht mit geringerem Bildungsgrad, sie können aber nicht als statistisch signifikante Abweichungen gewertet werden. Daher sind die Hypothesen HozIos' H8OzIO9 und $\mathbf{H}_{\mathbf{8 O z I} 012}$ auch für die Entfernungsgruppe II zu verwerfen.

In einer Gesamtbetrachtung ist bezüglich der Entfernungsgruppe I festzuhalten, daß die Besuchsabsicht ausschließlich anhand des monatlichen Haushaltseinkommens und der Schichtzugehörigkeit zu erklären ist. Zur Erklärung der Besuchs- 
dauer geben die soziodemographischen Merkmale keine Hinweise. Daher ist eine der zielsetzung "Erhöhung der Übernachtungszahlen" entsprechende gezielte Ansprache von zielgruppen auf der Basis dieser Ergebnisse nicht möglich. Für die Entfernungsgruppe I ist daher zusammenfassend festzuhalten, $\mathrm{daB}$ eine verhaltensorientierte Markterfassung nicht in ausreichendem Maße die Identifizierbarkeit der segmente gewährleistet.

In der Entfernungsgruppe II ist hingegen die Identifizierbarkeit der Verhaltenssegmente weitaus besser gewährleistet. Sowohl die Besuchsabsicht als auch die Besuchsdauer lassen sich anhand des Einkommens, des Berufes und der Kinderanzahl beschreiben. Allerdings können auch hier die Verhaltenssegmente nicht anhand des Alters und der Bildung als wichtigste soziodemographische Merkmale beschrieben werden.

Insgesamt stellt sich daher die Frage, ob unter Umständen eine psychographische Marktsegmentierung besser dazu geeignet ist, die Identifizierbarkeit von Zielgruppen zu gewährleisten. Vor diesem Hintergrund wird im folgenden zunächst die Besuchsverhaltensrelevanz von psychographischen Merkmalen überprüft. 
4. Erfassung und Analyse psychographischer Kerkmale als Kriterien zur Marktsegmentierung

Zur Durchführung einer psychographischen segmentierung sind eine Vielzahl unterschiedlicher Merkmale heranzuziehen. Bei der Analyse der Eignung psychographischer Merkmale stehen im folgenden Werte, Motive und Einstellungen im Mittelpunkt. Diese Überprüfung ist von der Grundannahme geleitet, daß eine psychographische Marktsegmentierung besser als die verhaltensorientierte Marktsegmentierung geeignet ist, die Anforderung der Identifizierbarkeit von segmenten zu gewährleisten.

Ausgangspunkt der Analyse von Werten, Einstellungen und Motiven ist das hierarchische Verhältnis dieser Konstrukte zueinander, wonach Werte als übergeordnetes Konstrukt Motive und Einstellungen determinieren. 81 Es wird ferner unterstellt, daß die Beziehung zwischen Motiven und dem Besuchsverhalten von Einstellungen als intervenierende Variable geprägt wird.82 Demnach sind Einstellungen als "Bindeglied" zwischen dem tatsächlichen Verhalten und dem Handlungsmotiv anzusehen.

Im folgenden wird der Einfluß der Konstrukte auf das Besuchsverhalten zunächst theoretisch abgeleitet. Darauf aufbauend sind die Konstrukte $z u$ operationalisieren und anschließend hinsichtlich ihrer soziodemographischen Identifizierbarkeit und Besuchsverhaltensrelevanz zu überprüfen.

81 Vgl. Windhorst, K.-G., Wertewandel und Konsumentenverhalten, a.a.0., s. 32 ff.; Dahlhoff, H.-D., Individuelle Wertorientierungen - Analyse und Aussagewert personenspezifischer Werthierarchien im Marketing, Arbeitspapier Nr. 23 des Instituts für Marketing, Hrsg.: H. Meffert, Münster 1980, S. 22 ff.; Trommsdorff, V., Konsumentenverhalten, a.a.o., s. 21 f.

82 Vgl. Wiswede, G., Motivation und Verbraucherverhalten. Grundlagen der Motivforschung, München 1973, S. 32 f. 
4.1 Erfassung und Analyse von werten als Rriterien aur Marktsegmentierung

\subsection{Theoretische Grundlagen zur Erfassung und Analyse von werten als segmentierungskriterien}

\subsection{1 werte als EinfluBgröBen des Besuchsverhaltens}

Werte als persönlichkeitsdeterminierende Variable 83 wurden in der Fremdenverkehrsforschung bislang nicht näher untersucht. Werte als "Wünschenswertes", die explizit oder implizit für ein Individuum oder für eine Gruppe kennzeichnend sind und welche die Auswahl der zugänglichen Weisen, Mittel und ziele des Handelns beeinflussen, 84 determinieren als persönlichkeitsbildende Determinanten sowohl die Urlaubspräferenzen als auch die Einstellungen $z u$ ausgewählten Fremdenverkehrsregionen. 85 Insofern sind werte als relativ stabile,

83 Meffert weist explizit darauf hin, daß ein Wert als verhaltensbeeinflussendes Element der Persönlichkeit interpretiert werden kann. Vgl. Meffert, H., Änderungen und Entwicklungstendenzen im Konsumentenverhalten, in: Meffert, H., Strategische Unternehmensführung und Marketing. Beiträge zur marktorientierten Unternehmenspolitik, Wiesbaden 1988, s. 303.

84 Vgl. Kluckhohn, C., The study of Values, in: Values in America, Barret, D.N. (Hrsg.), Notre Dame 1961, S. 17 ff. Andere Autoren heben zusätzlich die Generalisierbarkeit und die kognitive Komponente von Werten hervor. Vgl. Windhorst, G.-G., Wertewandel und Konsumentenverhalten, a.a.O., S. 32 ff.; Dahlhoff, H.-D., Wertorientierungen von Verbrauchern, in: Haase, H., Molt, $w$. (Hrsg.), Handbuch der angewandten Psychologie, Bd. 3: Markt und Umwelt, Landsberg a.L. 1981, S. 130-152.

85 Eine Analyse des Beziehungszusammenhangs von Werten und anderen verhaltensbeeinflussenden Variablen sowie des Besuchsverhaltens fehlt bislang in der Fremdenverkehrsforschung. Im Konsumgüterbereich ist hingegen der Zusammenhang zwischen Werten und verhaltensbeeinflussenden Variablen und dem Kaufverhalten empirisch nachgewiesen worden, so daß auch im Fremdenverkehr von diesen Beziehungszusammenhängen auszugehen ist. Vgl. zu den empirischen Ergebnissen bezüglich des Beziehungszusammenhangs 
von kurzfristigen Einflüssen unabhängige Prädispositionen des Besuchsverhaltens anzusehen.

Wenngleich Werte bisher noch nicht hinsichtlich ihres Einflusses auf das Besuchsverhalten untersucht wurden, finden sich in jüngeren Beiträgen zunehmend Hinweise auf den zusammenhang zwischen verändertem Besuchsverhalten und dem seit einigen Jahren viel diskutierten Wertewandel. 86

Die Ursachen dieses wertewandels liegen vor allem im Entstehungsproze $\beta$ von Werten begründet. Werte sind das Ergebnis von Sozialisationsprozessen, die wesentlich durch die Faktoren Familie, Umwelt, Schichtzugehörigkeit und Ausbildung beeinfluBt werden. 87 Demnach sind Veränderungen von Werthaltungen im sozialen Wandel der Lebensverhältnisse begründet, wie z.B. im Massenwohlstand, im stand der sozialen sicherung, in demographischen Veränderungen (z.B. Verringerung der Kinderanzahl), in der zunehmenden Technologisierung so-

von werten und anderen verhaltensbeeinflussenden Variablen Lessing, P.V., A Measurement of Dependencies Between Values and other Levels of the Consumer's Belief Space, in: Journal of Business Research, 1975, Heft 5, S. 234 ff.; Windhorst, K. -G., Wertewandel und Konsumentenverhalten, a.a.0., S. $143 \mathrm{ff}$.

86 Vgl. Kaspar, C., Die Entwicklungen des modernen Tourismus, a.a.o., S. 20 ff.; RosmeiB-Stracke, F., zukunftsperspektiven für den Tourismus, a.a.o., s. $32 \mathrm{ff}$. Die Diskussion um den Wertewandel setzte Ender der 70er Jahre mit der These von Inglehart ein, die besagt, da $\beta$ materialistische Werte zunehmend durch postmaterialistische Werte überlagert oder verdrängt werden. Vgl. Inglehart, R., The silent Revolution, Princeton 1977. Zitiert nach Seyfarth, C., Wertewandel und gesellschaftliche Rationalisierung: Eine theoretische Diskussion aktueller Tendenzaussagen, in: Luthe, H.O., Meulemann, H. (Hrsg.), Wertewandel - Faktum oder Fiktion?, Bestandsaufnahme und Diagnosen aus kultursoziologischer sicht, Frankfurt, New York 1988, S. 165 .

87 Vgl. Windhorst, H.-G., Wertewandel und Konsumentenverhalten, a.a.0., s. 48 . 
wie in der Beschäftigungslage, aber auch in den Erziehungsstilen. 88

Eine kritische Auseinandersetzung mit den Ausprägungen dieses Wertewandels ${ }^{89}$ macht deutlich, daß den postmaterielien werten 90 eine zunehmende Bedeutung zuzusprechen ist. Die hieraus abgeleitete und in empirischen studien teilweise sich bestätigende Schlußfolgerung der Freizeitforschung, daß die "Arbeit zunehmend als Lebensunterhalt" und die "Freizeit als Lebensinhalt" betrachtet wird,91 hat zweifellos auch Auswirkungen auf das regionenspezifische Besuchsverhalten.

So sieht Opaschowski angesichts der zunehmenden Bedeutung postmaterieller Werte den Wandel der Freizeit in Richtung auf einen neuen "freizeitkulturellen Lebensstil".92 Dabei

88 Vgl. Strümpel, B., Scholz-Ligma, J., BewuBtseins- und sozialer Wandel: Wie erleben Menschen die Wirtschaft? in: Luthe, H.O., Meulemann, H. (Hrsg.), Wertewandel Faktum oder Fiktion?, a.a.0., s. 21.

89 Auf die kontroverse Diskussion in der Werteforschung über die These des Wertewandels und den damit in Verbindung stehenden Ursachen wird hier nicht näher eingegangen. Vgl. hierzu seyfarth, C., Wertewandel und gesellschaftliche Rationalisierung, a.a.0., S. 163 ff.; Bürklin, W., Wertewandel oder zyklische Wertaktualisierung?, in: Luthe, H.O., Meulemann, H. (Hrsg.), Wertewandel - Faktum oder Fiktion?, a.a.O., s. 193 ff. und die dort angegebenen Literaturhinweise.

90 Materialistische Werte umfassen vor allem das streben nach materiellen Gütern und ökonomischer Wohlfahrt, die Betonung von Leistung und Disziplin sowie sicherheit. Demgegenüber wird unter postmaterialistischen Werten das streben nach Partizipation, Solidarität und Selbstverwirklichung, nach Freiheit und Gleichheit sowie die Artikulation intellektueller, sozialer und ethischer Bedürfnisse verstanden. Vgl. Seyfarth, c., Wertewandel und gesellschaftliche Rationalisierung, a.a.0., s. 165.

91 Vgl. Kaspar, C., Die Entwicklung des modernen Tourismus, a.a.o., s. 20 .

92 Vgl. Opaschowski, H.W., Raddatz, G., Freizeit im Wertewandel. Die neue Einstellung zu Arbeit und Freizeit, 
bildet der Lebensstil eine zusammenfassung aus übernommenen Rollen, Interessen und Aktivitäten, die eine bestimmte Art der Lebensführung dokumentieren. Werte und Lebenstile stehen in einem hierarchischen Verhältnis zueinander, so daB Lebensstile auch als sichtbarer Ausdruck bestimmter Wertestrukturen interpretiert werden.93 Daher können die von opaschowski abgeleiteten, den freizeitkulturellen Lebensstil determinierenden Lebensorientierungen auch als Werthaltungen interpretiert werden, die für das Besuchsverhalten von Bedeutung sind. $\mathrm{zu}$ nennen sind hier eine zunehmende

- Freizeitorientierung,

- Erlebnisorientierung,

- GenuBorientierung,

- Gegenwartsorientierung,

- Natur- und Umweltorientierung. 94

Unter Berücksichtigung dieser veränderten Werthaltungen ist eine wachsende Bedeutung von Kurzreisen und die Ablösung der klassischen Erholungsreisen durch sogenannte Erlebnisreisen abzuleiten. 95

B.A.T. Freizeit-Forschungsinstitut (Hrsg.), Bd. 4 der Schriftenreihe zur Freizeitforschung, Hamburg 1982.

93 Vgl. Windhorst, H.-G., Wertewandel und Konsumentenverhalten, a.a.0., s. 35 .

94 Vgl. Opaschowski, H.W., Raddatz, G., Freizeit im Wertewandel, a.a.0., s. $1 \mathrm{ff}$.

95 Vgl. Opaschowski, H.W., Urlaub 1986/87 - Was Reiseziele bieten müssen, B.A.T. Freizeit-Forschungsinstitut (Hrsg.), Hamburg 1987, S. 1 ff.; Kaspar, C., Die Entwicklungen des modernen Tourismus, a.a.0., s. 21. Ferner wird aus diesen Werthaltungen, speziell aus der zunehmenden Natur- und Umweltorientierung abgeleitet, daB Touristen zukünftig die Umweltsituation des Fremdenverkehrsgebietes bei der Auswahl eines zielgebietes stärker berücksichtigen werden. Empirische Untersuchungen bestätigen diesen Zusammenhang. Vgl. Venth, O., Freyer, M., Lebensziele - UmweltbewuBtsein - Urlaubsverhalten, in: Studienkreis für Tourismus (Hrsg.), Tourismus und Umwelt. Bericht über eine Fachtagung des studienkreises 
Die postulierten Zusammenhänge zwischen Werthaltungen und Besuchsverhalten sind jedoch nicht zu pauschalieren. So weist z.B. Opaschowski darauf hin, daß sich die veränderten Freizeitauffassungen und Anforderungen an einen Urlaub oder eine Reise vorrangig bei der jüngeren Generation finden lassen. Vor diesem Hintergrund wird die Notwendigkeit einer differenzierten Betrachtung des Zusammenhangs zwischen Werten und dem Besuchsverhalten offenbar.

Auch RosmeiB-stracke betont ${ }^{96}$, daB verschiedene Werthaltungen $z u$ unterschiedlichen Freizeitstilen und damit zu anderen Auswirkungen auf das Besuchsverhalten führen. Aus der Verknüpfung von werthaltungen und Besuchsverhalten leitet sie folgende vier Urlaubertypen ab: 97

\section{- "Nur-Erholer"}

Dieser Gruppe sind überwiegend ältere Menschen zuzuordnen, deren Lebenseinstellung sich darin äuBert, daß Freizeit und Urlaub vornehmlich der Erholung von der Arbeit dient.

\section{- "Familiäre"}

Die Lebenseinstellung dieser Gruppe besteht in einer familienorientierten Gestaltung der Freizeit und des Urlaubs.

- "Hedonisten bzw. aktive GenieBer"

Schwerpunkt dieser Lebenseinstellung ist, daß Freizeit und Urlaub aktiv mit Spaß und Konsum zu verbringen sind.

für Tourismus e.v. im Rahmen der 21. Internationalen Tourismus-Börse am 11. März 1987 in Berlin, Starnberg 1987 , S. 17 .

96 Vgl. Rosmeiß-stracke, F., Zukunftsperspektiven für den Tourismus, a.a.0., s. 33 .

97 Vgl. ebenda. 


\section{- "Alternative"}

Diese Gruppe strebt insbesondere nach Selbstverwirklichung sowohl bei der Arbeit als auch im Urlaub. Arbeitsfreie zeit wird außer zur Erholung auch für neue Erfahrungen mit sich selbst, der Umwelt und der Arbeit genutzt ("Einheit des Alltags").

Wenngleich diese Typologie nicht empirisch abgesichert ist, lassen sich dennoch Hinweise auf eine mögliche differenzierte Marktbearbeitung ableiten. Beispielweise stellen "Nur-Erholer" keine sehr hohen Ansprüche an die TourismusInfrastruktur, während die "aktiven GenieBer" speziell auf ihre Genußorientierung abgestimmte Freizeitangebote fordern. 98

Zusammenfassend ist festzuhalten, daB der Berücksichtigung von Werthaltungen im Rahmen der Markterfassung im Fremdenverkehr ein hoher stellenwert zukommt, da sich hierdurch zusätzliche Hinweise für die Ausgestaltung zielgruppenspezifischer Marketingprogramme ableiten lassen.

In diesem Zusammenhang stellt sich die Frage, welche Wertekategorien zur Analyse hinsichtlich ihres Einflusses auf das Besuchsverhalten heranzuziehen sind. Generell lassen sich Werte in globale und produkt- und bereichsspezifische werte kategorisieren. Globale Werte stellen überdauernde Überzeugungen dar, die sich auf gewünschte Verhaltensweisen, wie z.B. Selbstverwirklichung, Freiheit, etc. beziehen. Sie haben für das gesamte Spektrum des menschlichen Verhaltens Gültigkeit. 99 Demgegenüber gelten spezifische Werte nur für bestimmte Lebensbereiche, wie zum Beispiel Politik, Konsum,

98 Vgl. Rosmeiß-stracke, F., Zukunftsperspektiven für den Tourismus, a.a.0., S. 33 .

99 Vgl. Vinson, D.E., scott, J.E., Lamont, L.M., The Role of Personal Values in Marketing and Consumer Behaviour, in: Journal of Marketing, 1977, Heft 4, S. 46. 
Freizeit oder Urlaub. Auch diese Werte weisen keinen Bezug zu bestimmten Produkten oder Produktklassen auf.

Die bereichsapesifischen, in der anglo-amerikanischen Literatur als "domain specific values" bezeichneten Werte, beinhalten bereits konkrete Anforderungen an Produkte und Hersteller bzw. Fremdenverkehrsangebote und -regionen. 100 Aufgrund ihres engen Beziehungszusammenhangs mit den Einstellungen wird in der Werteforschung davon ausgegangen, da $B$ sie gegenüber den Einstellungen einen nur geringen zusätzlichen Erklärungsbeitrag liefern. 101

Zur Erfassung der dominanten Werthaltungen von Individuen und zur Erklärung des menschlichen Verhaltens sind die globalen Werte heranzuziehen. 102 sollen zusätzlich Aussagen für bestimmte Lebensbereiche getroffen werden, sind die globalen Werte durch spezifische Werte zu ergänzen. 103 Im Rahmen dieser Untersuchung werden nur globale Werte betrachtet.

Eine Segmenterfassung auf der Basis globaler Werte ermöglicht angesichts ihrer relativ hohen seitlichen stabilität

$100 \mathrm{Vgl}$. Windhorst, W., Wertewandel und Konsumentenverhalten, a.a.0., s. 43 f. Interessanterweise basiert die einzige, dem Verfasser bekannte empirische studie über Wertesegmentierung im Fremdenverkehr auf bereichsspezifischen Werten. Vgl. van Veen, W.M.O., Verhallen, T.W.M., Vacation Market Segmentation. A Domain-Specific Value Approach, in: Annals of Tourism Research, Vol. 13, 1986 , S. 37-58.

101 Vgl. Banning, T.E., Lebenstilorientierte Marketing-Theorie. Analyse und Weiterentwicklung modelltheoretischer und methodischer Ansätze der Lebensstil-Forschung im Marketing, Heidelberg 1987 , S. 177 .

102 Vgl. windhorst, K.-G., Wertewandel und Konsumentenverhalten, a.a.0., s. 44 .

$103 \mathrm{Vgl}$. ebenda. 
eine kontinuierliche Marktbearbeitung. 104 Die zeitliche stabilität von Werten ergibt sich dadurch, daß sich Werteveränderungen über einen längeren zeitraum erstrecken 105 und somit Zeitperioden existieren, in denen keine signifikanten veränderungen von Werten erfaBbar sind.

Es ist davon auszugehen, daß Werte anhand von soziodemographischen Merkmalen gut erklärt werden können und somit relativ gut die Anforderung der soziodemographischen Identifizierbarkeit an segmentierungskriterien erfüllen. So wurde in verschiedenen Untersuchungen das Alter und die Schulbildung als wesentliche Bestimmungsfaktoren der Ausprägungsformen von Werthaltungen identifiziert. 106 windhorst stellt beispielsweise fest, daB ältere Personen den Werten "Gesundheit", "Familienleben" und "Orientierung am Bewährten" eine höhere Bedeutung beimessen, während jüngere Personen vermehrt nach Freizeit streben. 107 Daher ist zu vermuten, daß bei älteren Personen die traditionellen Wertorientierungen, wie z.B. Sicherheit und Tradition die dominanten Werte darstellen, während jüngere Personen zunehmend nach postmateriellen Werten (wie z.B. Freiheit, Selbstverwirklichung) streben. Ferner wird in der Werteforschung davon ausgegangen, daB mit zunehmendem Bildungsgrad und steigendem Einkommen postmaterielle werte an Bedeutung gewinnen.108 Demgegenüber wird angenommen, daß Familien mit Kindern dem

104 Vgl. Windhorst, K.-G., Wertewandel und Konsumentenverhalten, a.a.0., s. 39 .

$105 \mathrm{Vgl}$. Windhort, K.-G., Wertewandel und Konsumentenverhalten, a.a.0., s. 51 .

$106 \mathrm{Vgl}$. Scholz, J., Wertewandel und wirtschaftskultur, München 1987, S. 178 ff.; Windhorst, K.-G., Wertewandel und Konsumentenverhalten, a.a.0., s. $120 \mathrm{ff}$.

107 Vgl. Windhorst, K.-G., Wertewandel und Konsumentenverhalten, a.a.0., s. $120 \mathrm{ff}$.

$108 \mathrm{Vgl}$. Scholz, J., Wertewandel und Wirtschaftskultur, a.a.o., S. $175 \mathrm{ff}$. 
Streben nach sicherheit eine besonders hohe Bedeutung zusprechen.

Bezugnehmend auf die dargestellten Zusammenhänge zum Einfluß soziodemographischer Merkmale auf die Ausprägungen von Werten sollen im Rahmen der empirischen Analyse folgende Untersuchungshypothesen überprüt werden.

Basishypothese zum Einfluß soziodemographischer Merkmale auf die Ausprägungen von Werten:

BWRTE 1 Soziodemographische Merkmale bestimmen die Wichtigkeit einzelner Werthaltungen.

Diese Basishypothese ist angesichts der aufgezeigten zusammenhänge durch folgende Tendenzhypothesen zu konkretisieren:

H materielle Werte.

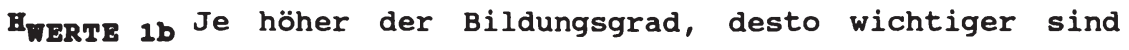
postmaterielle werte.

B HERTE 1c Je höher das Einkommen, desto wichtiger sind postmaterielle Werte.

HERTE 1d Kinderlosen Personen sind postmaterielle Werte wichtiger als Personen mit Kindern.

Die dargestellten zusammmenhänge zwischen Besuchsverhalten und Werten weisen schließlich darauf hin, daß auch von einem Beziehungszusammenhang zwischen Werten und einem regionenspezifischen Besuchsverhalten auszugehen ist. So vermutet auch storbeck, daB aus einer Analyse der Werteveränderungen wichtige Rückschlüsse auf die regionale Verteilung des Frem- 
denverkehrs in der Bundesrepublik und im Ausland $z u$ ziehen sind. 109

In diesem zusammenhang ist $z u$ berücksichtigen, daB die Ausgabebereitschaft für den Urlauber in einem engen Beziehungszusammenhang mit Werten steht. So wurde nachgewiesen, daB die Ausgabebereitschaft für den Urlauber um so höher ist, je wichtiger die Werteindikatoren "Selbstverwirklichung", "genußreiches Leben" sowie "Bildung" und je unwichtiger die Indikatoren "Sparsamkeit" und "Tradition" sind. 110

Diese Ergebnisse deuten darauf hin, daB mit zunehmender wichtigkeitseinschätzung von postmateriellen Werten eine generell höhere Reiseintensität verbunden ist. Unter der Annahme, daß sich diese erhöhte Reiseintensität auch im regionenspezifischen Besuchsverhalten niederschlägt, weisen Besucher der Fremdenverkehrsregion eine höhere Wichtigkeitseinschätzung postmaterieller Werte auf als Nicht-Besucher. In diesem Zusammenhang sind vor allem die postmateriellen Werte "Selbstverwirklichung", "Freiheitsstreben" und "Umwelt und Gesundheit" zu nennen. 111

Der EinfluB der Werte auf die Besuchsdauer ist hingegen in Abhängigkeit von der Entfernung $z u$ beurteilen. Aufgrund des mit einem längeren Aufenthalt verbundenen höheren wahrgenommenen Risikos ist anzunehmen, da $B$ in der Entfernungsgruppe I die Tagesausflügler eher traditionelle Werte verfolgen. Dem-

$109 \mathrm{Vgl}$. Storbeck, D., Die Entwicklung der Rahmenbedingungen für den Fremdenverkehr in der Bundesrepublik Deutschland, Arbeitsberichte "Regional- und Raumplanung", Heft 9, Bielefeld 1987, S. 19.

$110 \mathrm{Vgl}$. Windhorst, K.-G., Wertewandel und Konsumentenverhalten, a.a.0., s. 163 .

111 Vgl. zur Bedeutung postmaterieller Werte Seyfarth, c., Wertewandel und gesellschaftliche Rationalisierung, a.a.0., S. 165 . 
gegenüber werden Deutschlandurlaube vermutlich vor allem von traditions- und sicherheitsbetonten Personen unternommen. 112 Daher ist für die Entfernungsgruppe II davon auszugehen, daB nach Tradition und nach sicherheit strebende Personen eher einen Urlaub in der Fremdenverkehrsregion bevorzugen als einen Kurzurlaub.

Insgesamt kann festgehalten werden, daB globale Werte vermutlich dazu geeignet sind, das Besuchsverhalten zu erklären. Dabei ist davon auszugehen, daB in den einzelnen Entfernungsgruppen unterschiedliche werthaltungen sowohl die Besuchsabsicht als auch die Besuchsdauer erklären. Folgende Basis- und Tendenzhypothesen sind zum EinfluB von Werten auf das regionenspezifische Besuchsverhalten empirisch $z u$ überprufen:

Basishypothese zum EinfluB von Werten auf die Besuchsabsicht:

BERTE 2 Besucher und Nicht-Besucher sind in beiden Entfernungsgruppen durch unterschiedliche Werteausprägungen gekennzeichnet.

Diese Basishypothese läßt sich unter Berücksichtigung der aufgezeigten Zusammenhänge durch folgende Tendenzhypothesen konkretisieren:

HERTE 2a Je wichtiger das streben nach selbstverwirklichung, um so stärker wird in beiden Entfernungsgruppen eine Besuchsabsicht geäußert.

112 Vgl. hierzu auch die Charakterisierung von Deutschlandreisenden bei Lohmann, M., Wohlmann, R., Urlaub in Deutschland, a.a.0., s. $37 \mathrm{ff}$. 


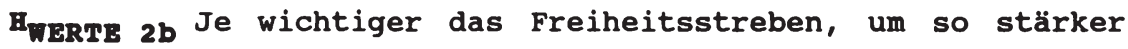
wird in beiden Entfernungsgruppen eine Besuchsabsicht geäußert.

Basishypothese zum EinfluB von Werten auf die Besuchsdauer:

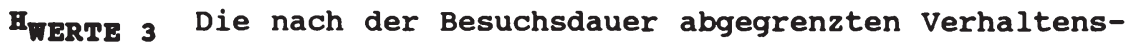
segmente sind in beiden Entfernungsgruppen durch unterschiedliche Werteausprägungen gekennzeichnet.

Diese Basishypothese läßt sich durch folgende Tendenzhypothesen konkretisieren:

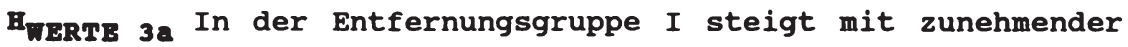
wichtigkeitseinschätzung des Wertes "Selbstverwirklichung" die Besuchsdauer.

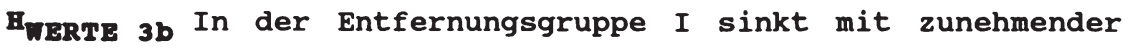
wichtigkeitseinschätzung traditioneller Werte die Besuchsdauer.

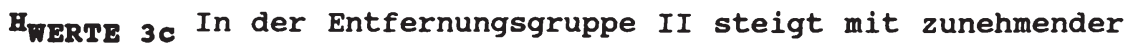
wichtigkeitseinschätzung traditioneller Werte die Besuchsdauer.

\subsection{Operationalisierung von Werten}

Die Operationalisierung von werten setzt eine Auswahl von Einzelwerten bzw. Indikatoren, die im Rahmen der Wertemessung erfaBt werden, voraus. Den Ausgangspunkt bilden die in der Literatur in einer Vielzahl von Forschungsansätzen zu- 
grundegelegten Werteindikatoren. ${ }^{113}$ Für die vorliegende Untersuchung wird auf eine Indikatorenbatterie von windhorst zurückgegriffen, bei der die Werte über die ziele der eigenen Lebensführung operationalisiert sind. Als skalierungsverfahren 114 wird eine fünf-stufige Ratingskala verwendet. 115 In Abbildung 15 sind die zur operationalisierung von Werten verwendeten Werteindikatoren wiedergegeben. 116 Durch einen Vergleich der Werteausprägungen dieser studie mit denen aus der windhorst-Untersuchung lassen sich Aussagen über veränderte Werteprioritäten ableiten. Haben sich die Werteprioritäten seit 1985 nicht bzw. nur schwach verändert, ist dies ein Beurteilungsmaßstab für die zeitliche stabilität von Werten.

113 Ein Überblick über operationalisierungsansätze zur Werteerfassung findet sich bei windhorst, H., Wertewandel und Konsumentenverhalten, a.a.0., S. 58 ff., Dahlhoff, H.-D., Individuelle Wertorientierungen - Analyse und Aussagewert personenspezifischer Werthierarchien im Marketing, Arbeitspapier $\mathrm{Nr} .23$ des Instituts für Marketing, Hrsg.: H. Meffert, Münster 1980, S. 52 f.

114 Für die Messung von werteindikatoren werden in der Literatur unterschiedliche skalierungsverfahren diskutiert. Zentrale Verfahren sind Paarvergleiche, Rangreihen, Ratingskalen und das semantische Differential. Vgl. Jacob, P.E., Singh, K.K., Measuring Values, in: Jacob, P.E., u.a. (Hrsg.), The International studies in Politics. Values and the Active Community, New York 1971, S. 385. Einen zusammenfassenden Überblick ubber die Diskussion der Eignung der verschiedenen skalierungsverfahren findet sich bei windhorst, K.-G., Wertewandel und Konsumentenverhalten, a.a.0., s. 71 ff. sowie die dort angegebene Literatur.

115 Entsprechend wurden auch in der vorliegenden Untersuchung die Befragten aufgefordert, die jeweilige Wertefrage anhand von vorgegebenen Antwortkategorien auf einer Fünf-Punkte-Skala zu beantworten.

116 Die Übertragung dieses operationalisierungsansatzes auf die vorliegende Untersuchung ist zweckmäBig, da dieser aus den theoretischen Erkenntnissen der Werteforschung abgeleitet wurde und sich im Rahmen der empirischen Analyse als reliabel und valide erwies. Vgl. Windhorst, K.G., Wertewandel und Konsumentenverhalten, a.a.O., S. 124 ff. 
- Gesellschaftliche Anerkennung

- Umwelt- und energiebewußtes Leben

- Sich selbst entfalten können

- Ein bequemes Leben führen

- Persönliche und finanzielle Sicherheit

- Persönliche Freiheit

- Viel Freizeit

- Ein ausgeprägtes Familienleben führen

- Gesundheitsbewußtes Leben

- "Alternativ" leben

- Ein genußreiches Leben führen

- Sich an "Bewährtem" orientieren

- Aufgaben für die Gesellschaft übernehmen

- Hoher Bildungsstand

- Kulturelles Interesse

Abb. 15: Ziele der eigenen Lebensfühung als Indikatoren zur Erfassung von Werten

Im Rahmen seiner Untersuchung differenzierte windhorst zwischen unterschiedlichen Wertedimensionen. Neben den aktuell realisierten und zukünftig $z u$ realisierenden Werten wurden wichtige und ideale Werte erfaßt.117 Es wurde nachgewiesen,

117 Die Frage nach den idealen Werten bezog sich dabei auf die Werte, die idealerweise in unserer Gesellschaft verwirklicht sein sollten. Demgegenuber gibt die wichtigkeit der Werte Auskunft darüber, welchen stellenwert bestimmte Werte für die eigene Lebensführung darstellen. Die aktuellen und zukünftigen Werte geben Auskunft darüber, in welchem Ausmaß diese werte tatsächlich reali-

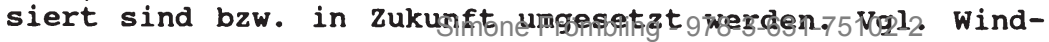


daB insbesondere den wichtigen und idealen Werten eine vergleichsweise hohe Besuchsverhaltensrelevanz zukommt. 118 Die Analyse des Zusammenhangs zwischen Werten und bereichsspezifischen Konsumstrukturen i.s, der zukunftigen Verwendung des Einkommens für unterschiedliche Konsumbereiche zeigte zudem, $\mathrm{daB}$ die Ausgabebereitschaft für Urlaube besser anhand wichtiger Werte als durch ideale Werte zu erklären ist. 119 Daher wird in der vorliegenden Untersuchung die operationalisierung von Werten über die wahrgenommene wichtigkeit vorgenommen. Durch die faktoranalytische Verdichtung der Werteindikatoren ist es möglich, die hinter den zielen der Lebensführung stehenden Werte $z u$ erfassen. 120 Hieraus leiten sich folgende meBtheoretische Basishypothesen ab:

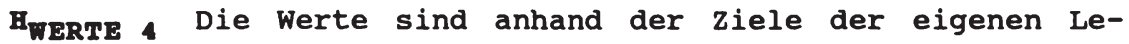
bensführung erfaBbar.

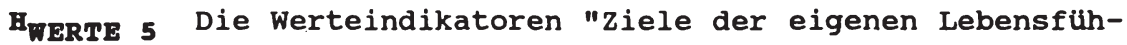
rung" sind auf Wertefaktoren zurückzuführen.

horst, K.-G., Wertewandel und Käuferverhalten, a.a.o., S. $255 \mathrm{ff}$.

$118 \mathrm{Vgl}$. Windhorst, K.-G., Wertewandel und Konsumentenverhalten, a.a.0., s. 222 .

119 Vgl. Windhorst, G.-G., Wertewandel und Konsumentenverhalten, a.a.0., s. 175 ff.

120 Die Faktorenanalyse unterstellt, daB die Beziehungen der Ausgangsdaten durch einen oder mehrere hinter den Variablen stehenden Faktoren beschreibbar sind und sucht diese Faktoren durch mathematisch-statistische Behandlung der Ausgangsinformationen aufzudecken. Vgl. Backhaus, K., u.a., Multivariate Analysemethoden, a.a.o, s. 68. Vgl. zur Beschreibung der Vorgehensweise ebenda, $S$. $67 \mathrm{ff}$. 


\subsection{Bmpirische Analyse der Bignung von Nerten als segmen- tierungakriterien}

\subsection{Kennselchnung der Ausprägungsformen und struktur von werten}

Die Mittelwerte der Werteindikatoren sind in Abbildung 16 wiedergegeben. 121 Es zeigt sich, daB alle vorgegebenen Werteindikatoren eine mittlere bis hohe wichtigkeitseinschätzung erfahren. Am wichtigsten erachten die Befragten die Werte "persönliche Freiheit", "persönliche und finanzielle sicherheit", "gesundheitsbewußtes Leben", "sich entfalten können" und "umwelt- und energiebewuBtes Leben". Hingegen wird den Werten "alternative Lebensformen", "genuBreiches Leben" und "Übernahme von Aufgaben für die Gesellschaft" eine deutlich geringere wichtigkeitseinschätzung zugeordnet.

Es wird deutlich, daB die postmateriellen werte angesichts der hohen wichtigkeitseinschätzung von Freiheit und Freizeit, sowie Gesundheit und Umwelt in der Wertehierarchie der Konsumenten einen hohen stellenwert einnehmen. Allerdings kann nicht von einem "Absterben" traditioneller Werte gesprochen werden. Dies ergibt sich vor allem aus der hohen wichtigkeitseinschätzung des Wertes "finanzielle und persönliche Sicherheit". Auch die hohe Bedeutung der "Familien-

121 Die Kodierung der Einzelausprägungen der Indikatoren erfolgte dergestalt, daB niedrige Punktwerte eine starke wichtigkeitseinschätzung anzeigen. In diesem Zusammenhang ist $z u$ beachten, daB die über Ratingskalen erfaBten Werteausprägungen strenggenommen ordinalskalenniveau aufweisen. Im folgenden wird unterstellt, daB Unterschiede in zwei MeBwerten über die ganze skala hinweg immer die gleichen Unterschiede in den Werteausprägungen ausdrücken. Nur unter dieser Annahme können die Werteausprägungen, als auch die später zu betrachtenden Motive und Einstellungen als metrisch skalierte Variablen interpretiert werden. Vgl. Kroeber-Riel, W., Konsumentenverhalten, a.a.O., S. 186; Meffert, H., Marketingforschung und Käuferverhalten, a.a.0., S. 185. 
Gesellschaftliche Anerkennung

Umwelt- und energiebewußtes Leben

Sich selbst entfalten können

Ein bequemes Leben führen

Persönliche und finanzielle Sicherheit

Persönliche Freiheit

Viel Freizeit

Ein ausgeprägtes Familienleben führen

Gesundheitsbewußtes Leben

"Alternativ" leben

Ein genußreiches Leben führen

Sich an "Bewährtem" orientieren

Aufgaben für die Gesellschaft übernehmen

Hoher Bildungsstand

Kulturelles Interesse

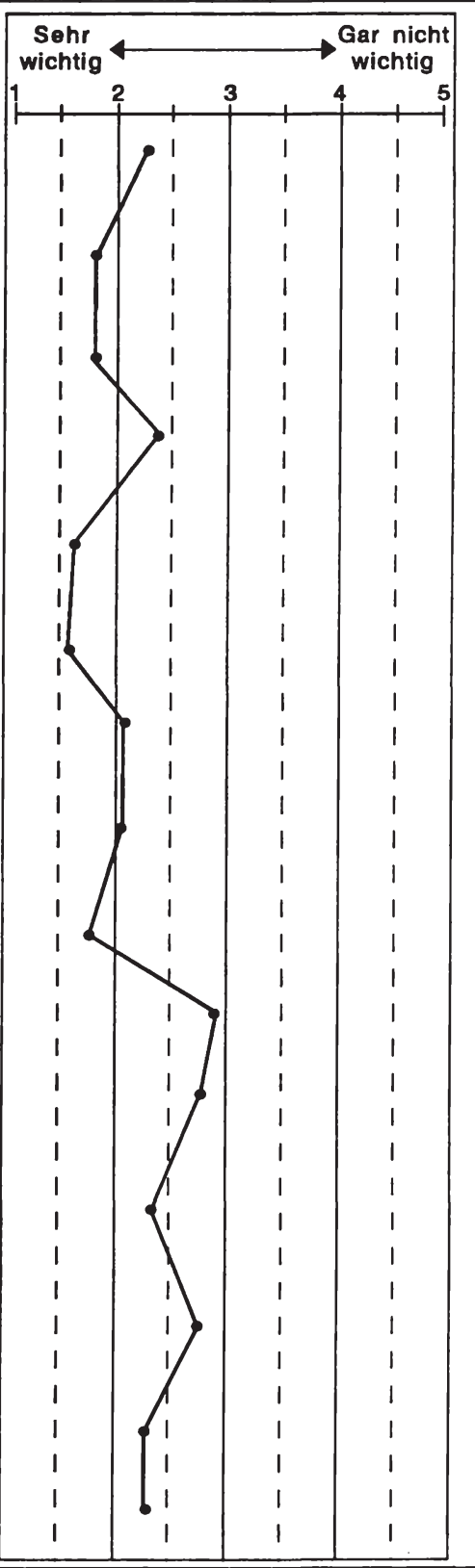

Abb. 16: Empirisch ermittelte wichtigkeit von Werten 
orientierung" offenbart, daß den traditionellen Werten weiterhin eine starke Gewichtung zukommt. Daher kann diesen im Rahmen der Markterfassung im Fremdenverkehr von Regionen weiterhin ein besonderer stellenwert zugeordnet werden.

Ein Vergleich zu den von Windhorst im Jahre 1985 ermittelten wichtigkeitseinschätzungen 122 von Werten zeigt insgesamt nur geringe Verschiebungen in den Profilausprägungen. Demnach haben sich seit 1985 keine erheblichen Verschiebungen in den Werteprioritäten der Bevölkerung eingestellt. 123 offensichtlich handelt es sich bei den werten um relativ zeitstabile Ronstrukte, die sich in bezug auf dieses Anforderungskriterium in besonderem Maße für die segmentierung eignen.

Gemäß der Hypothese HwERTE5 wurde angenommen, daß sich die Werteindikatoren der Befragten auf wertefaktoren zurückführen lassen. Zur Überprüfung wurde anhand einer explorativen Faktorenanalyse eine Verdichtung der Einzelvariablen vorgenommen. Die Abbildung 17 zeigt das aufbereitete Ergebnis einer nach dem Kaiser-Kriterium ermittelten fünf-faktoriellen Lösung. 124 Die hohen Faktorladungen 125 des ersten Faktors

122 Eine unmittelbare Vergleichbarkeit ist nicht gegeben, da in der vorliegenden Untersuchung eine disproportional geschichtete stichprobenziehung vorgenommen wurde. Vgl. Kapitel A.4.

123 Demgegenüber kommt den Werten "Umwelt und Gesundheit" in der Untersuchung von Balderjahn in der Wertehierarchie eine nur untergeordnete Bedeutung $\mathrm{zu}$. Die von ihm durchgeführte Auswertung basiert auf einem Datensatz aus dem Jahre 1980. Der Unterschied der wichtigkeitseinschätzung der Werte "Umwelt und Gesundheit" aus dem Jahre 1980 und 1985 läßt demnach darauf schließen, daß sich die zentralen Veränderungen im Wertewandel $\mathrm{zu}$ Beginn der 80er Jahre vollzogen haben. Vgl. Balderjahn, J., Das umweltbewußte Konsumentenverhalten - Eine empirische studie, Berlin 1986, S. 12 und S. 174 ff.

124 Nach dem Kaiser-Kriterium werden nur Faktoren mit einem Eigenwert größer als 1 generiert. Der Eigenwert eines Faktors erklärt dessen Anteil an der Varianz aller Einzelvariablen. Als Schätzverfahren kam in der vorliegen- 

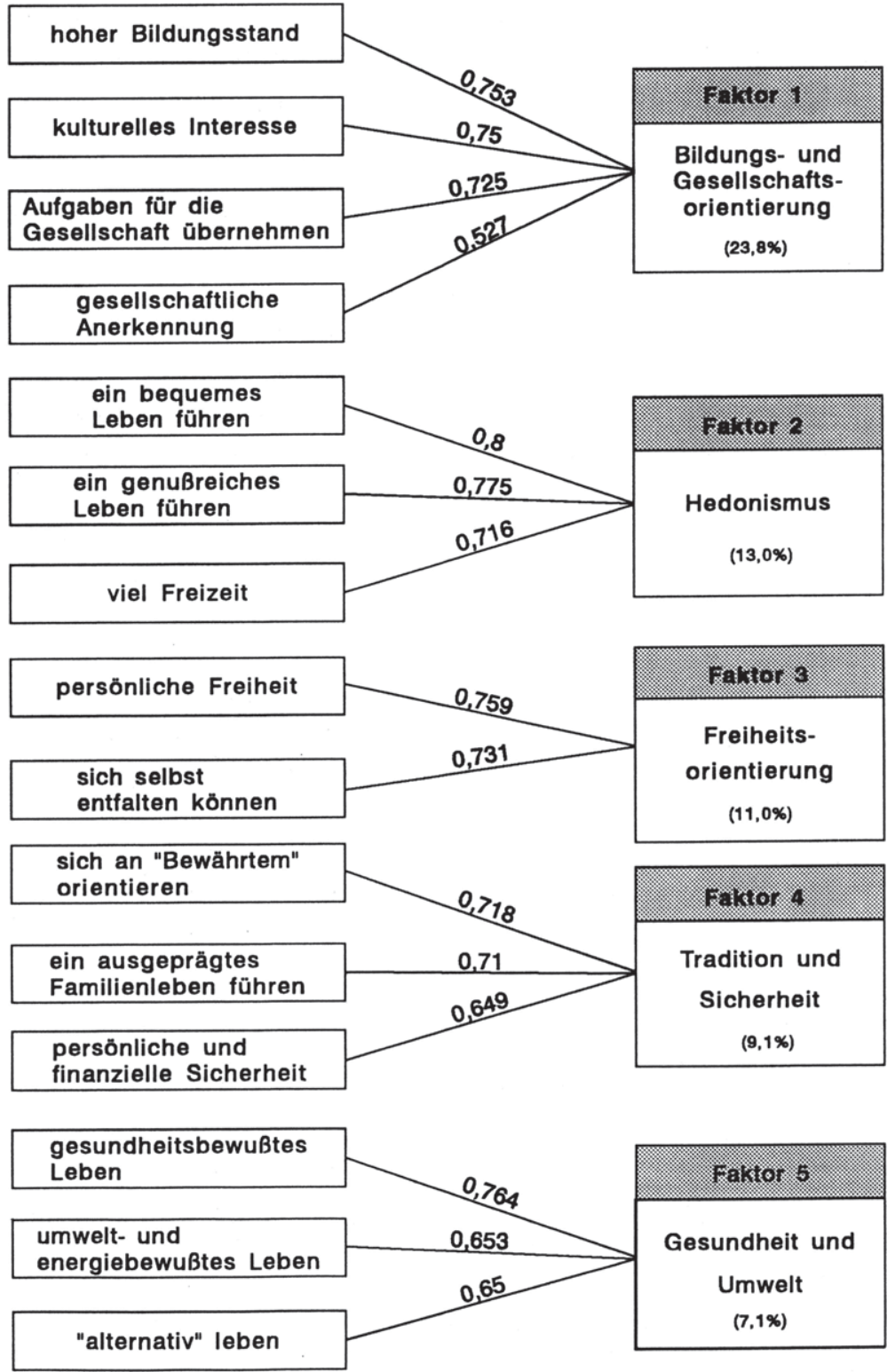

Erklărter Varianzanteil: $64,0 \%$

Abb. 17 : Faktorenanabytiogche frohepdifchtumgt oler/2 Wertgeinddikatoren 
bei den Wertevariablen "Bildungsstand", "kulturelles Interesse", "Übernahme von Aufgaben für die Gesellschaft" und "gesellschaftliche Anerkennung" kennzeichnen deutlich die Dimension "Bildungs- und Gesellschaftsorientierung".126 Es ist $z u$ vermuten, $\mathrm{daB}$ sich in diesem Faktor ferner das streben nach selbstverwirklichung widerspiegelt. In Anlehnung an die Wertetypologie von RosmeiB-Stracke gibt dieser Faktor offenbar Hinweise auf das streben nach sinnvollen Tätigkeiten in Arbeit und Freizeit. Insgesamt werden durch diesen Faktor 23,8 \& der Gesamtvarianz erklärt.

Der zweite Faktor kennzeichnet deutlich eine hedonistische Grundorientierung. Zentrale Dimensionen dieses Wertefaktors sind "bequemes Leben", "genuBreiches Leben" und "viel Freizeit". Der dritte Wertefaktor läBt sich als Freiheitsorientierung kennzeichnen. Er zeichnet sich durch hohe Faktorladungen bei den Variablen "persönliche Freiheit" und "Entfaltung" aus. Der erklärte Varianzanteil an der Gesamtvarianz beträgt $11,0 \%$. Die Werteorientierung "sicherheit und Tradition" wird durch den vierten Faktor repräsentiert. Er läBt sich gut anhand der Werteindikatoren "Orientierung an Bewährtem", "Streben nach einem ausgeprägten Familienleben" und "persönliche und finanzielle sicherheit" umschrei-

den Analyse die Hauptkomponentenmethode zur Anwendung. Zur besseren Interpretation der Faktorenstruktur wurde eine Varimax-Rotation durchgeführt. Vgl. hierzu Backhaus, K., u.a., Multivariate Analysemethoden, a.a.o., s. 90 ff.; Meffert, H., Marketingforschung und Käuferverhalten, a.a.0., S. 257 ff.

125 Bei der Interpretation der Faktorladungen werden nur diejenigen Werteindikatoren herangezogen, die eine hohe Faktorladung aufweisen. Dabei gehen Backhaus und Weiber davon aus, daB eine hohe Faktorladung gegeben ist, wenn diese mindestens 0,5 beträgt. Ferner sind Doppelladungen bei der Interpretation der Ergebnisse zu berücksichtigen. Vgl. Backhaus, K., Weiber, R., Entwicklung einer Marketingkonzeption mit SPSS/PC ${ }^{+}$, Berlin u.a., S. 50 .

$126 \mathrm{Vgl}$. zur vollständigen Dokumentation der Faktorenstruktur Anhang $I$. 
ben. In diesem Faktor kommt die traditionelle Werthaltung besonders gut zum Ausdruck. Der erklärte Varianzanteil an der Gesamtvarianz beträgt $9,1 \%$.

Der Faktor "Gesundheit und Umwelt" erklärt den geringsten Varianzanteil. Er läßt sich durch die Lebensziele "gesundheitsbewußtes Leben", "umwelt- und energiebewußtes Leben" und "alternativ Leben" kennzeichnen. Der geringe Varianzanteil in Höhe von $7,1 \%$ an der Gesamtvarianz zeigt jedoch, daB diesem Wertefaktor zur Erklärung der Wertestrukturen der Befragten die geringste Bedeutung zukommt.

Insgesamt erklären die fünf Wertefaktoren 64,0 \% der Gesamtvarianz aller Werteindikatoren. 127 Demnach erweist sich die ermittelte Faktorenstruktur als gute Ausgangsbasis für wei-

127 Die statistische Weiterverwendung der Wertefaktoren erfordert die Berechnung der Faktorwerte. Die Schätzung der Faktorwerte wurde mit Hilfe der multiplen Regressionsrechnung vorgenommen. Bei den Faktorwerten handelt es sich um standardisierte Größen, die einen Mittelwert von 0 und eine standardabweichung von 1 aufweisen. Bei der Interpretation ist die Ausgangskodierung, die hohen wichtigkeitseinschätzungen kleine Punktwerte zuwies, zu beachten. Demnach besagt ein negativer (positiver) Faktorwert, daß ein objekt in bezug auf diesen Wertefaktor im Vergleich $\mathrm{zu}$ anderen betrachteten objekten überdurchschnittlich (unterdurchschnittlich) ausgeprägt ist. Ein Faktorwert von 0 besagt, daB ein objekt in bezug auf diesen Faktor eine dem Durchschnitt entsprechende Ausprägung besitzt. Vgl. zur Interpretation der Faktorwerte Backhaus, K., Multivariate Analysemethoden, a.a.0., s. $105 \mathrm{f.;}$ vgl. zu unterschiedlichen Verfahren der Faktorwertberechnung. Brosius, G., SPSS/PC+ Advanced Statistics and Tables. Einführung und praktische Beispiele, Hamburg, New York u.a. 1989, S. 166. 
terführende Berechnungen. 128 Daher ist die Hypothese HWETE5 zu bestätigen. 129

\subsection{Analyse der soziodemographischen Identifizierbarkeit} und des Einflusses von Werten auf das Besuchsverhalten

Die Analyse der Identifizierbarkeit von Werten bzw. der zusammenhänge zwischen Werten und soziodemographischen Merkmalen ermöglicht, Personengruppen mit bestimmten Werthaltungen anhand objektiv meßbarer Kriterien $z u$ beschreiben und $z u$ identifizieren.

Zur Überprüfung dieser Beziehungen werden die oben abgeleiteten demographischen und sozioökonomischen Variablen herangezogen. 130 In der Abbildung 18 sind die Zusammenhänge

128 Bei einer Weiterberechnung auf der Basis von Faktoren kommt es $z u$ einem Verlust an erklärter Varianz in Höhe von 36 . Dennoch ist eine Weiterberechnung auf der $\mathrm{Ba}-$ sis von den Wertefaktoren akzeptabel. Vgl. Backhaus, K., Weiber, R., Entwicklung einer Marketing-Konzeption mit SPSS $/ \mathrm{PC}^{+}$, a.a.0., S. $55 \mathrm{ff}$.

129 Ein Vergleich mit der windhorst-studie, bei der keine eindeutigen Wertestrukturen identifiziert werden konnten, zeigt, daß sich offensichtlich die Wertestrukturen seit 1985 verfestigt haben. Allerdings ist bei dieser Interpretation die nur bedingt gegebene Vergleichbarkeit beider Untersuchungen $\mathrm{zu}$ beachten.

$130 \mathrm{Vgl}$. zu den soziodemographischen Merkmalen Kapitel B. 3 . Aufgrund der unterschiediichen skalenniveaus wurden verschiedene Verfahren zur Ermittlung der Zusammenhänge herangezogen. Bei den Variablen Bildung, monatliches Netto- und persönliches Haushaltseinkommen wurde aufgrund ihrer ordinalen skalierung der Spearmannsche Korrelationskoeffizient angesetzt. Für die Variablen Alter und Anzahl der Kinder unter 18 Jahren konnte angesichts ihres metrischen Skalenniveaus der Produkt-Moment-Korrelationskoeffizient angewendet werden. Die Zusammenhänge zwischen Werteausprägungen und den nominal skalierten Variablen Geschlecht und Beruf wurden mit Hilfe von Kreuztabellierungen ermittelt. Dabei gibt der Chi-square Wert Hinweise auf signifikante Zusammenhänge. Vgl. zu 


\begin{tabular}{|c|c|c|c|c|c|}
\hline \multirow{2}{*}{$\begin{array}{l}\text { Sozlodemo- } \\
\text { graphlsche Merkmale }\end{array}$} & $\begin{array}{l}\text { Blldungs-1 } \\
\text { und Gosoll- } \\
\text { ochafts- } \\
\text { oriontiorung }\end{array}$ & $\begin{array}{l}\text { Hedo- } \\
\text { nismus }\end{array}$ & $\begin{array}{l}\text { Froihoits- } \\
\text { orien- } \\
\text { tierung }\end{array}$ & $\begin{array}{l}\text { Sichor- } \\
\text { holt \& } \\
\text { Tradl- } \\
\text { tlon }\end{array}$ & $\begin{array}{l}\text { Umwolt \& } \\
\text { Gosundhoit }\end{array}$ \\
\hline & 1 & 2 & 3 & 4 & 5 \\
\hline $\begin{array}{l}\text { Demographische Merkmale } \\
\text { - Geschlecht }\end{array}$ & n.S. & $++t$ & n.s. & +++ & $++t$ \\
\hline - Alter & n.S. & 0,178 & 0,265 & $\begin{array}{c}-0,367 \\
\star \star \star\end{array}$ & n.S. \\
\hline - Kinderanzahl & n.S. & n.S. & n.S. & $-0,087$ & $\underset{\star \star \star \star}{-0,08}$ \\
\hline - Lebenszyklusphase & 0,06 & $0 \underset{\star \star \star \star}{0,184}$ & $\underset{\star \star \star \star}{0,232}$ & $\underset{\star \star \star}{-0,391}$ & n.s. \\
\hline -Bildung & $\underset{\star \star \star \star \star \star}{-0,284}$ & 0,15 & 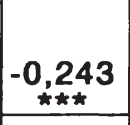 & 0,197 & n.S. \\
\hline -Beruf & +++ & +++ & n.s. & +++ & +++ \\
\hline - Haushalt-Nettoeinkommen & $-\underset{\star \star \star \star}{0,096}$ & 0,054 & $\underset{\star \star \star}{-0,081}$ & $-\underset{\star \star \star \star}{0,152}$ & n.S. \\
\hline •Persönl. Nettoeinkommen & 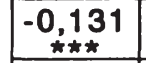 & n.S. & n.s. & n.S. & n.S. \\
\hline • Schichtzugehörigkeit & $\underset{\star \pi \star \star}{-0,231}$ & $0, \substack{\star \star 108 \\
\vdots \star \star}$ & $-0,179$ & n.S. & n.S. \\
\hline \multicolumn{6}{|c|}{ Korrelationskoeffizienten $\left({ }^{*}\right)$ und F-Test $(+)$ mit: } \\
\hline \multicolumn{6}{|c|}{$\begin{array}{lll}\text { Signifikanzniveau } & \alpha<0,01=* * & (+++) \\
\text { Signifikanzniveau } & \alpha<0,05=* & (++)\end{array}$} \\
\hline
\end{tabular}

Abb. 18: Empirische Ergebnisse zum EinfluB soziodemographischer Merkmale auf die Wertefaktoren

zwischen den Wertefaktoren und den soziodemographischen Merkmalen dargestellt. Es zeigt sich, daB der Wertefaktor

den Anwendungsvoraussetzungen der genannten Verfahren Brosius, G., SPSS/PC+. Basics and Graphics. Einführung und praktische Beispiele, Hamburg, New York 1988, S. 217 ff.; Bauer, F., Datenanalyse mit SPSS, a.a.0., S.174 f. 
"Bildungs- und Gesellschaftsorientierung" einen starken Bezug zu sozioökonomischen Variablen, insbesondere zu der Bildung, der Schichtzugehörigkeit und dem Beruf sowie abgeschwächt zum persönlichen Nettoeinkommen aufweist. Je höher das Bildungsniveau, die soziale Schichtzugehörigkeit und das persönliche Nettoeinkommen ist, desto wichtiger wird der Wert "Bildungs - und Gesellschaftsorientierung" eingeschätzt.

Anhand dieser Beziehungszusammenhänge wird deutlich, daß erst $a b$ einem bestimmten Lebensstandard Werte, die eher das Streben nach Selbstverwirklichung zum Ausdruck bringen, an Bedeutung gewinnen. So steigt auch die wichtigkeitseinschätzung des Wertefaktors "Freiheitsorientierung" mit zunehmenden Bildungsgrad und höherer schichtzugehörigkeit. Demgegenüber erfährt der Wert "sicherheit und Tradition" eine um so höhere wichtigkeitseinschätzung, je geringer der Bildungsgrad ist. Angesichts des hohen Einflusses der Bildung und der Schichtzugehörigkeit auf die wichtigkeitseinschätzung von Werten bestätigt sich die in der Literatur vertretene Ansicht, daß Werte aus Sozialisationsprozessen heraus resultieren. 131 Die Hypothesen HWERTE1b $_{\text {WE }}$ HERTE1C' die davon ausgingen, daß mit zunehmender Bildung und höherer Schichtzugehörigkeit postmaterielle Werte an Bedeutung gewinnen, sind daher zu bestätigen.

Desweiteren zeigt sich, daß die Wertefaktoren "Hedonismus" und "Freiheitsorientierung" sowie "Sicherheit und Tradition" in besonderem Maße von demographischen Merkmalen abhängen. Je älter die Befragten und je fortgeschrittener die stellung im Lebenszyklus ist, desto wichtiger wird der wert "sicherheit und Tradition" bzw. desto unwichtiger werden die Werte "Hedonismus" und "Freiheitsorientierung" eingeschätzt. Hier wird unmittelbar deutlich, daß Werte einem Generatio-

131 Vgl. Strümpel, B., Scholz-Ligma, J., Bewußtseins- und sozialer Wandel, a.a.0., S. 21 sowie die dort angegebene Literatur. 
nenwandel unterliegen. Während ältere Personen an den traditionellen Werthaltungen festhalten, streben jüngere Altersgruppen vermehrt nach Freiheit, Freizeit und Genußorientierung. Daher ist die Hypothese H WERTE1a' die von einem negativen Zusammenhang zwischen Alter und der wichtigkeitseinschätzung postmaterieller Werte ausging, zu bestätigen.

Auch in Abhängigkeit vom Geschlecht ergeben sich signifikante Unterschiede in den Werthaltungen. Es zeigt sich, daß Frauen eher nach traditionellen Werten streben, während Männer vermehrt hedonistische Wertorientierungen aufweisen. Demgegenüber hat die Kinderanzahl nur einen geringen Einfluß auf die Werteausprägungen. Angesichts des geringen Korrelationskoeffizienten kann die Hypothese HWRTE1d jedoch nicht bestätigt werden.

Der Wertefaktor "Omwelt und Gesundheit" ist nur eingeschränkt anhand soziodemographischer Merkmale zu erklären. offensichtlich haben die zunehmende Umweltverschmutzung und regelmäßige Berichterstattungen über Umweltprobleme in allen Gesellschaftskreisen $z u$ einer umweltorientierten Werthaltung geführt. Signifikante Unterschiede in der wichtigkeitseinschätzung dieses Faktors ergeben sich nur auf der Basis der Variable Geschlecht.

Insgesamt kommt den demographischen Merkmalen "Alter" und "Stellung in der Lebenszyklusphase" und dem sozioökonomischen Merkmal "Bildung" die gröBte Bedeutung bei der Erklärung der Werteausprägungen zu. Ferner zeigt sich deutlich, daß der Wertewandel von materialistischen werten hin zu postmateriellen Werten vor allem bei den jüngeren Altersgruppen und Personen mit höherem Bildungsgrad zu beobachten ist.

In einer Gesamtbewertung bleibt festzuhalten, daB werte in hohem Maße die in dem zusammenhang mit den soziodemographi- 
schen Merkmalen zum Ausdruck kommende Anforderung nach Identifizierbarkeit erfüllen. Daher ist die Basishypothese HERTE1 insgesamt zu bestätigen.

Die Wertefaktoren haben jedoch auch das segmentierungskriterium der verhaltensrelevanz zu erfüllen. Hierzu wird mit Hilfe des T-Tests untersucht, ob die Verhaltenssegmente in den beiden Entfernungsgruppen in ihren Werthaltungen signifikante Mittelwertunterschiede aufweisen.132 Abbildung 19 gibt einen Überblick über die zusammenhänge zwischen den Wertefaktoren und den verschiedenen Ausprägungsformen des Besuchsverhaltens, getrennt nach Entfernungsgruppen.

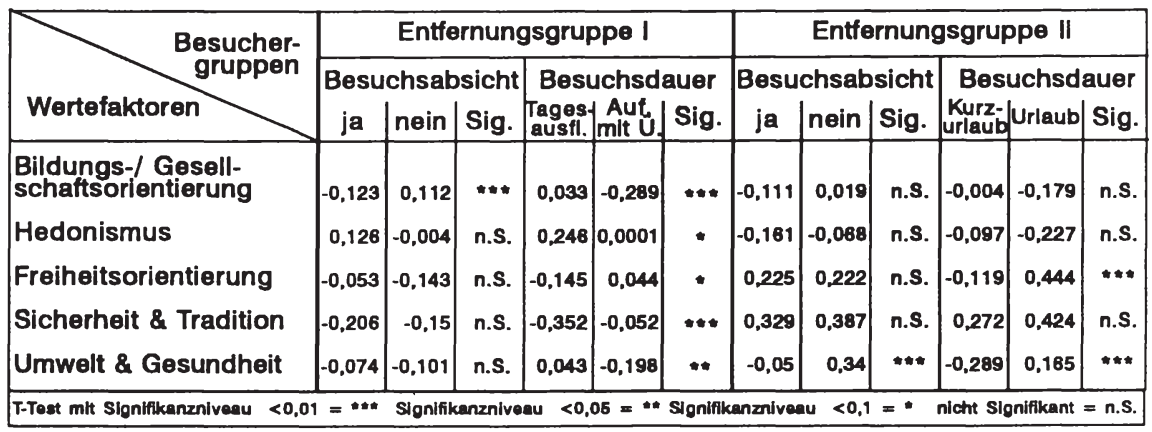

Abb. 19: Empirische Ergebnisse zum Einfluß der Wertefaktoren auf das Besuchsverhalten (T-Test) innerhalb der Makrosegmente

In der Entfernungsgruppe I zeigt sich, daß Besucher und Nicht-Besucher der Fremdenverkehrsregion nur hinsichtlich

132 Der T-Test eignet sich als Verfahren immer dann, wenn überprüft werden soll, ob in 2 unabhängigen stichproben signifikante Mittelwertuntschiede bestehen, bzw. ob von einer beobachteten Differenz der stichprobenmittelwerte auf eine Differenz der Mittelwerte in der Grundgesamtheit zu schließen ist. Vgl. Schubö, W., Uehlinger, H.-M. u.a., Handbuch der Programmversion 4.0 und SPSS-X 3.0, a.a.0., S. 491 ; Brosius, G., SPSS/PC+. Basics and Graphics, a.a.0., s. $263 \mathrm{ff}$. 
des Wertefaktors "Bildungs- und Gesellschaftsorientierung" signifikante Mittelwertunterschiede aufweisen. Dabei ordnen Personen mit Besuchsabsicht dem Faktor "Bildungs- und Gesellschaftsorientierung" eine signifikant höhere wichtigkeitseinschätzung $z u$ als Nicht-Besucher. Es ist anzunehmen, $\mathrm{da} B$ das in einer hohen Gesellschafts- und Bildungsorientierung zum Ausdruck kommende streben nach Selbstverwirklichung zu einer generell höheren Besuchsintensität im Rahmen des Naherholungsverkehrs führt. Wenn sich der Wertefaktor "Bildungs- und Gesellschaftsorientierung" in bildungsorientierten Urlaubswünschen manifestiert, ist $z u$ vermuten, daß Personen der Entfernungsgruppe I eine Besuchsabsicht in die Fremdenverkehrsregion vor allem aufgrund des attraktiven kulturellen Angebots äußern. Daher ist für die Entfernungsgruppe I zu schließen, daß vor allem Personen mit einer hohen Bildungs- und Gesellschaftsorientierung als potentielle Besucher der Fremdenverkehrsregion in Frage kommen. Hypo-

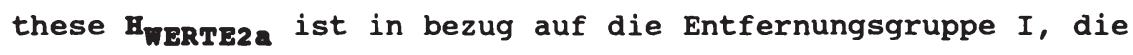
den entsprechenden Zusammenhang unterstellte, zu bestätigen.

Postmaterielle Werte wie "Freiheitsorientierung" haben keinen EinfluB auf die Besuchsabsicht. Dies überrascht, da ein streben nach Freiheit $z u$ einer vergrößerten Mobilität und damit $z u$ einem intensiveren Besuchsverhalten im Naherholungsverkehr führen müßte. Hypothese $\mathbf{H}_{\text {WERTE2b }}$ ist somit zu verwerfen.

In der Entfernungsgruppe II sind signifikante Unterschiede zwischen Besuchern und Nicht-Besuchern hinsichtlich des Wertefaktors "Omwelt und Gesundbeit" festzustellen. Diesem weisen potentielle Besucher eine signifikant höhere wichtigkeitseinschätzung $z u$. Dies ist nicht zuletzt deshalb nachvollziehbar, da sich die Untersuchungsregion durch eine relativ natürliche, weitgehend unversehrte Landschaft auszeichnet. Anscheinend bietet die Fremdenverkehrsregion vor allem gesundheits- und umweltorientierten Personen der Ent- 
fernungsgruppe II hohe Attraktivitätspotentiale. Im Hinblick auf die anderen Werte sind keine signifikanten Mittelwertunterschiede $z u$ identifizieren. Die Hypothesen HERTTE2a und HTERTE2b sind in bezug auf die Entfernungsgruppe II zu verwerfen.

Insgesamt zeigt die Analyse der Mittelwertunterschiede zwischen Personen mit und ohne Besuchsabsicht in beiden Entfernungsgruppen, daß Werte zur Erklärung der Besuchsabsicht geeignet sind. Daher ist die Basishypothese MERTE3 $_{\text {w }}$ bestätigen.

Betrachtet man ferner die Mittelwertausprägungen der Wertefaktoren bei den nach der Besuchsdauer abgegrenzten verhaltenssegmenten, so zeigen sich hier z.T. hoch signifikante Unterschiede. In der Entfernungsgruppe I unterscheiden sich die nach der Besuchsdauer abgegrenzten Verhaltenssegmente signifikant in allen Wertefaktoren. Personen, die die Wertefaktoren "Freiheitsorientierung" und "Sicherheit und Tradition" als sehr wichtig einstufen, bevorzugen einen Tagesausflug in die Untersuchungsregion. Demgegenüber wird mit zunehmender wichtigkeitseinschätzung der Wertefaktoren "Bildungs- und Gesellschaftsorientierung", "Hedonismus" und "Umwelt und Gesundheit" ein Aufenthalt mit Übernachtung angegeben. Daher sind die Hypothesen $\mathbf{H}_{\text {WERTE3a }}$ und HERTE3b $_{\text {w }}$ bestätigen.

In der Entfernungsgruppe II ergeben sich signifikante Mittelwertunterschiede zwischen Kurzurlaubern und Urlaubern hinsichtlich der Wertefaktoren "Freiheitsorientierung" sowie "Umwelt und Gesundheit". So führt eine geringe wichtigkeitseinschätzung des Wertefaktors "Freiheitsorientierung" aufgrund der längeren Anfahrtsdauer in die Fremdenverkehrsregion zu einer Bevorzugung von Aufenthaltsdauern von länger

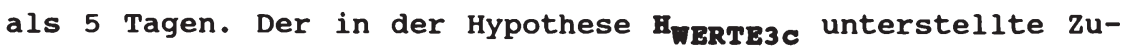
sammenhang, daß mit zunehmender wichtigkeitseinschätzung 
traditioneller Werte die Besuchsdauer steigt, ist nicht zu bestätigen. Die Hypothese ist demnach $z u$ verwerfen.

Zusammenfassend zeigt die Analyse der Besuchsverhaltensrelevanz von Wertefaktoren, daß diese zur Erklärung der Besuchsabsicht und der Besuchsdauer geeignet sind. Die Basishypo-

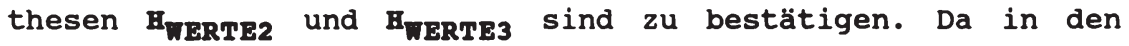
beiden Entfernungsgruppen jeweils unterschiedliche Wertefaktoren zur Erklärung der Besuchsabsicht und -dauer identifiziert wurden, bestätigt sich die Vorteilhaftigkeit der hier zugrundegelegten Vorgehensweise, einer Analyse der psychischen Prozesse eine Makrosegmentierung in Abhängigkeit von der Entfernung vorzuschalten.

Insgesamt erfüllen die Wertefaktoren die Anforderungen der Besuchsverhaltensrelevanz und soziodemographischen Identifizierbarkeit. Allerdings deuten die überwiegend nicht signifikanten Mittelwertunterschiede zwischen Besuchern und Nicht-Besuchern darauf hin, $\mathrm{daB}$ eine segmentierung ausschlieBlich auf der Basis von Wertefaktoren nicht erfolgversprechend ist. Vor diesem Hintergrund werden im folgenden die Urlaubsmotive als Segmentierungskriterium einer eingehenderen Analyse unterzogen.

\subsection{Erfassung und Analyse von Urlaubsmotiven als Rriterien zur Marktsegmentierung}

4.21 Theoretische Grundlagen zur Erfassung und Analyse von Orlaubsmotiven als segmentierungskriterien

\subsection{Urlaubsmotive als EinfluBgröBen des Besuchsverhaltens}

Den Reisemotiven wird in der Fremdenverkehrsforschung eine besonders hohe Erklärungskraft zugesprochen, da sie das reisebezogene Handeln aktivieren und mit einer bestimmten 
Zielorientierung des Handelns verbunden sind. Die Zielorientierung ergibt sich dadurch, daß Motive neben den grundlegenden aktivierenden Vorgängen ${ }^{133}$ zusätzlich kognitive wirkungen der Verhaltenssteuerung umfassen. Da Urlaubsmotive die Beweggründe des Reisens angeben, gelten sie als wesentliche Bestimmungsfaktoren der Besuchsabsicht und der Wahl von Fremdenverkehrsgebieten sowie Urlaubsangeboten. 134

\section{Zur Identifikation der generellen Beweggründe des Reisens} wird in der Fremdenverkehrsforschung der Beziehungszusammenhang zwischen Alltag und Freizeit herangezogen, wobei sich die zahlreichen Reisemotive auf zwei Grundpositionen reduzieren. Dies sind die Komplementär- und die Konträrhaltung, in denen mit den Urlaubsmotiven zugleich das Verhältnis von Urlaub und Alltag enthalten ist. 135

Die Konträrhaltung wird durch eine "Weg-von-Motivation"136 bestimmt, die den wunsch der Realitätsflucht impliziert und sich durch den Wunsch nach Entspannung, Erholung, Ausgleich,

133 wichtigste Antriebskräfte sind Emotionen. Sie können definiert werden als innere Erregungszustände, die angenehm oder unangenehm empfunden werden. Emotionen unterscheiden sich von den Motiven dadurch, das letztere bereits mit einer bestimmten zielorientierung verbunden sind. Vgl. Kroeber-Riel, w., Konsumentenverhalten, a.a.0., 1992, S. 50 .

134 Vgl. Wolf, K., Jurczek, P., Geographie der Freizeit und des Tourismus, stuttgart $1986, \mathrm{~S} .76 \mathrm{f}$.

$135 \mathrm{Vgl}$. Steinecke, A., Gesellschaftliche Grundlagen der Fremdenverkehrsentwicklung, in: Haedrich, G., Kaspar, c., u.a., Tourismus-Management. Tourismusmarketing und Fremdenverkehrsplanung, Berlin, New York 1983, S. 47 ; opaschowski, H.W., Tourismusforschung, a.a.O., S. $84 \mathrm{ff}$.

136 Motive und Motivationen werden in der Literatur uneinheitlich gebraucht. Im folgenden kennzeichnen Motivationen die aktuelle Handlungsausrichtung, während Motive durch den überdauernden Aspekt gekennzeichnet sind. Vgl. Meffert, H., Marketingforschung und Käuferverhalten, a.a.0., s. 55 . 
Ablenkung, Freizeit und Herauskommen aus dem Alltag ausdrückt. Die Romplementärhaltung zeichnet sich hingegen durch eine "Hin-zu-Motivation" aus, in der der Urlaub als Zeitraum der selbstverwirklichung wahrgenommen wird. Im Mittelpunkt stehen Aktivitäten, die den Alltag sinnvoll ergänzen. Hierin kommen Urlaubsmotive wie der Wunsch nach Sozialkontakt, Geselligkeit und kreativer Erlebnisentfaltung zum Ausdruck.

Die aufgezeigte Reduzierung der vielfältigen individuellen Reisemotive auf die Dichotomie der Konträr- und Komplementärhaltung gibt jedoch nur die struktur von Reisemotivationen wieder. Für die Marktsegmentierung erweist sich eine derartige strukturierung als wenig zweckmäBig, da sich identische Reisemotive wie zum Beispiel das Geselligkeitsbedürnis 137 sowohl der Komplementär- als auch Konträrhaltung zuordnen lassen. Ferner ist davon auszugehen, daB die unter der Konträrhaltung abgeleiteten Reisemotive, wie zum Beispiel das Erholungsbedürfnis auch bei Personen, die den Urlaub als sinnvolle Ergänzung des Alltags ansehen, eine hohe Bedeutung einnehmen. Daher bedarf es vor allem hinsichtlich der operationalisierung dieser Urlaubsmotive einer weitergehenden Differenzierung.

Anstelle der strukturierung der Reisemotive in Abhängigkeit von ihrem Verhältnis zum Alltagsleben ist es zweckmäBig, auf die in der Motivationsforschung gängige Unterteilung in pri-

137 Ein Bedürfnis kennzeichnet als Motivauslöser einen empfundenen Mangelzustand, der zunächst nicht auf ein ziel gerichtet ist. Erst durch die emotionale oder gedankliche Verarbeitung dieser Empfindung erlangt ein Bedürfnis Motivqualität. Trommsdorff, V., Konsumentenverhalten, a.a.0., s. 98. Da jedoch Motive durch einen überdauernden Aspekt gekennzeichnet sind, können Motive auch als Beweggründe des Handelns oder als Bedürfnisse bezeichnet werden. Vgl. Meffert, H., Marketing, a.a.0., s. 150 . 
märe und sekundäre Bedürfnisse Bezug zu nehmen. 138 Bereits 1930 wies Häussler darauf hin, daß der Mensch infolge des Arbeitsprozesses ein "biologisches Bedürfnis" nach Entapannung aufweist. 139 Daraus ist zu folgern, daß Menschen, die das Jahr über physisch und psychisch angestrengt tätig sind, mit einem Urlaub oder einer Reise primär die Befriedigung ihrer biologischen Bedürfnisse anstreben.

Bestätigung findet diese Annahme in zahlreichen empirischen Untersuchungen. Schon im Rahmen einer Untersuchung aus dem Jahr 1962 wurde festgestellt, daß dem Bedürfnis nach Erholung und Entspannung mit $83 \%$ der Nennungen aller Befragten die weitaus größte Bedeutung zukam. 140 Aber auch aktuelle Untersuchungen bestätigen die Dominanz des Erholungsbedürfnisses. So wurde in einer Untersuchung zum Reiseverhalten der deutschen Bundesbürger aus dem Jahre 1991 das Bedürfnis nach Entspannung und Erholung als das dominante Urlaubsmotiv festgestellt. 141 Interessanterweise hat dieses Motiv mit Blick auf Urlaubsreisen sogar seit 1985 an Bedeutung gewon-

138 Die Einteilung in primäre und sekundäre Bedürfnisse findet sich bei Meffert, H., Marketing, a.a.O., S. 150. Anstelle der Einteilung in primäre und sekundäre Motive ist häufig auch eine Einteilung in "niedere", biologisch bedingte und "höhere", psychisch bedingte Motive zu finden. Vgl. Kroeber-Riel, W., Konsumentenverhalten, a.a.o., S. 141 .

139 Vgl. Häussler, X., Der Fremdenverkehr, Leipzig 1930, s. 18, zitiert aus Freyer, W., Tourismus, a.a.0., s. 65 .

140 Vgl. Schmidt-Scherzer, R., Rudinger, G., Motive - Erwartungen - Wünsche in bezug auf Urlaub und Verreisen, in: Studienkreis für Tourismus (Hrsg.), Motive - Meinungen Verhaltensweisen, Einige Ergebnisse und Probleme der psychologischen Tourismusforschung, starnberg 1969, S. 12 .

141 Vgl.: Gilbrich, M., Urlaubsreisen 1991, a.a.0., s. 23 f.; Desweiteren kommt dem Motiv "Aus dem Alltag herauskommen, Tapetenwechsel" eine hohe Bedeutung zu. Vgl. ebenda. Dies entspricht der aufgezeigten Konträr-Haltung und ist daher an dieser stelle nicht weiter auszuführen. 
nen. Während 1985 knapp zwei Drittel der Nennungen auf das Motiv "Abschalten/sich entspannen" entfiel, waren es im Jahre 1987 schon $70,9 \%$ und im Jahre $199080,3 \% .142$

In diesem Zusammmenhang ist $z u$ beachten, daß mit den Begriffen "Erholung" und "Entspannung" von Touristen unterschiedliche Inhalte assoziiert werden. Einerseits kann unter Erholung viel Ruhe und Nichtstun im sinne von physischer und psychischer (mentaler) Regeneration verstanden werden. Andererseits können mit Erholung Aktivitäten assoziiert werden, wie zum Beispiel aktiv sein im sinne körperlicher Bewegung oder Aktivitäten wie Gespräche mit Einheimischen, die mehr den wissensdrang befriedigen. 143 Letztere Aktivitäten sind jedoch den sekundären Bedürfnissen zuzuordnen. Dies bedeutet, daß das primäre Reisemotiv "Erholung" im Verbund mit den sekundären Motiven zu betrachten ist. Zur näheren Erläuterung dieses zusammenhangs ist es zunächst erforderlich, die sekundären Bedürfnisse näher zu charakterisieren.

Zur Kennzeichnung möglicher sekundärer, psychisch bedingter Reisemotive kann auf die Hierarchie menschlicher Bedürfnisse nach Maslow 144 zurückgegriffen werden, die auch eine Übertragung auf den Fremdenverkehr erfahren hat. ${ }^{145}$ Auf der ersten stufe finden sich die physiologischen Bedürfnisse, die mit den eben beschriebenen primären Bedürfnissen bzw. Regenerationsbedürfnissen gleichzusetzen sind. Die sekundären

142 Vgl. ebenda sowie Dundler, F., Urlaubsreisen 1987, a.a.0., S. 29 .

$143 \mathrm{Vgl}$. Lohmann, M., Wohlmann, R., Urlaub in Deutschland, a.a.0., s. 32 .

144 Vgl. Maslow, A.H., Motivation and Personality, in: Levine, F.M. (Hrsg.), Theoretical Readings in Motivation: Perspectives on Human Behaviour, Chicago 1975, S. 370 ff.

145 Vgl. Mill, R.Ch., Morrison, A.M., The Tourism System, An Introducing Text, Englewood Cliffs 1985, S. 6 ff. 
Urlaubsmotive kommen in den nächsten Bedürfnisstufen zum Ausdruck.

Das Streben nach Erholung im Urlaub als auch das aktive Gesundheitsstreben - zum Beispiel durch die Durchführung eines Kururlaubes - dient vor allem der Befriedigung des sicherheitsbedürfnisses im sinne von Erhaltung der Gesundheit und Sicherung der Erwerbstätigkeit für die zukunft. Demgegenüber ist der Wunsch nach Geselligkeit und sozialen Kontakten im Urlaub dem streben nach sozialen Bedürfnissen zuzuordnen. Dient der Urlaub vornehmlich als Prestige- und statusobjekt, so kommt es hierdurch zur Befriedigung von Anerkennungsbedürfnissen (z.B. Kreuzfahrten). Der Wunsch nach selbstverwirklichung im Urlaub äuBert sich beispielsweise in Motiven wie Erleben, Spontanität, Aktivität und GenuB von Naturnähe. Dabei sind unterschiedlichste, sich ergänzende oder sich ausschließende Urlaubsmotive, wie z.B. Urlaub als Chance zur Selbsterkennung, Urlaub zur Befriedigung von Wissensstreben, Urlaub zur kreativen Erlebnisentfaltung etc. zu identifizieren.

Zur Erklärung des Besuchsverhaltens wird davon ausgegangen, daß die übergeordneten Motive der Hierarchie erst dann aktiviert werden, wenn die untergeordneten Bedürfnisse einem bestimmten Anspruchsniveau entsprechend erfüllt sind. Dies ist lediglich als ein idealtypisches Reaktionsmuster zu verstehen.

Das Maslowsche Modell macht jedoch deutlich, daB es im Fremdenverkehr offensichtlich gewisse Grundbedürfnisse gibt, die für alle zielgruppen bis zu einem gewissen Grad durch das touristische Angebot befriedigt werden müssen. Erst wenn die Befriedigung dieser auf Erholung und Regeneration gerichteten Bedürfnisse gewährleistet ist, werden die höherstehenden Bedürfnisse wirksam. Diese sekundären Bedürfnisse können durch eine Vielzahl von Urlaubsaktivitäten während des Auf- 
enthalts in einer Fremdenverkehrsregion befriedigt werden und geben damit unmittelbar Auskunft über gewünschte Urlaubserlebnisse. Um diesem Erlebnisaspekt begrifflich verstärkt Rechnung zu tragen, können die oberhalb der Sicherheitsbedürfnisse 146 angesiedelten Bedürfnisebenen als sog. Erlebnisbedürfnisse bezeichnet werden. Verwendet man die zur Befriedigung der Urlaubsbedürfnisse durchzuführenden Urlaubsaktivitäten als Raster zur strukturierung von Erlebnisbedürfnissen, so können diese in Bewegungs-, Sport-, Kulturund sonstige Freizeitbedürfnisse untergliedert werden. 147

Der enge Verbund zwischen Regenerations- und Erlebnisbedürfnissen ergibt sich dadurch, daß die Erlebnisbedürfnisse direkt oder indirekt der Befriedigung der primären Motive dienen. 148 Es ist davon auszugehen, daß die Art und Weise der Realisierung von unterschiedlichsten Erlebnisbedürfnissen den Grad der Befriedigung der Regenerationsbedürfnisse determiniert. Daher kommt den Erlebnisbedürfnissen im Rahmen des Auswahlprozesses von Fremdenverkehrsregionen eine besondere Bedeutung $\mathrm{zu}$.

146 Das Sicherheitsbedürfnis umfaßt im wesentlichen den Wunsch nach Erholung und Regeneration, wobei die Gesundheitskomponente im Vordergrund steht. Daher sind mit diesem Bedürfnis nicht unmittelbar Erlebniswirkungen verbunden. Das Sicherheitsbedürfnis soll im Rahmen dieser Untersuchung nicht weiter analysiert werden. Die Begründung hierfür ist, daB der Kur- und Gesundheitstourismus eine sonderform des Fremdenverkehrs darstellt, der vom hier vorliegenden Untersuchungsgegenstand des Erholungstourismus abzugrenzen ist. Vgl. zu den Besonderheiten im Kur- und Gesundheitstourismus Fehrlin, P., Marketing-Konzeption für einen Badekurort am Beispiel von Bad Ragaz, Heerbrugg 1983, S. 11 ff.

147 Vgl. zu ähnlichen strukturierungen schmid, J.-M., Kundenstamm-Marketing - aus der Sicht des Reiseveranstalters, Gais 1989, S. 48; Gilbrich, M., Urlaubsreisen 1991, a.a.0., S.24 f.

148 Vgl. Meffert, H., Marketing, a.a.O., s. 150. 
Ein Tourist steht nach dem Entschluß, einen Urlaub oder eine Reise durchzuführen, zahlreichen Fremdenverkehrsgebieten und Urlaubsangeboten gegenüber. Diese ähneln sich vermutlich stark in ihren grundnutzenstiftenden Eigenscharten 149 und können insofern als homogen bezeichnet werden. Die grundnutzenstiftenden Eigenschaften ergeben sich aus der "Grundausstattung" einer Fremdenverkehrsregion, die im wesentlichen das natürliche Angebot und die sog. "Suprastruktur" umfaßt. 150 Diese Eigenschaften finden sich in den meisten Prospekten als Hinweise auf die natürliche Landschaft, Ruhe, saubere Luft etc. wieder, die den Erholungswert einer Region und damit den Grundnutzen "Regeneration" umschreiben. 151

Generell stellt diese Homogenität den potentiellen Touristen vor das Problem einer erschwerten Angebotsauswahl. Er aktiviert deshalb weitere ziele, die er mit dem Urlaub oder der

149 Unter Nutzen wird ein nach subjektiven Maßstäben bewertbares $M a B$ an Bedürfnisbefriedigung verstanden. Vgl. Nieschlag, R., Dichtl, E., Hörschgen, H., Marketing, 15. Aufl., Berlin 1988, S. 3. Der Grundnutzen eines Produktes resultiert aus der technisch-funktionalen seite und verkörpert damit gewissermaßen die Qualität i.e.s.

150 Die Homogenität von Fremdenverkehrsregionen ist sehr gut am Beispiel der Urlaubsgebiete von Spanien, Frankreich und Italien $z u$ verdeutlichen. Das natürliche Angebot, welches vor allem den Grundnutzen "Regeneration" in Verbindung mit Sonne und strand befriedigen kann, unterscheidet sich zwischen den Fremdenverkehrsregionen kaum. Die Fremdenverkehrsgebiete sind in diesen grundnutzenstiftenden Eigenschaften homogen. Vgl. Goodall, B., How Tourists choose their Holidays, a.a.0., s. 13.

151 Die Austauschbarkeit von Fremdenverkehrsangeboten und regionen bezieht sich einerseits auf das tatsächliche Angebot und andererseits auf die werbepolitischen Aktivitäten. In der Wahrnehmung der Touristen ähnen sich Fremdenverkehrsregionen vor allem aufgrund von stereotypen, austauschbaren Prospekten. Da die Auswahl von Fremdenverkehrsregionen in der Regel auf der Basis von Prospekten erfolgt, ist eine differenzierte Beurteilung von Fremdenverkehrsregionen durch den Touristen damit nicht möglich. Vgl. Maier, J., Bewertung eines regionalen Entwicklungsfaktors, a.a.0., s. 117 . 
Reise erreichen möchte. Zu denken ist beispielsweise an Touristen mit bildungsorientierten Bedürfnissen, die sie mit dem Besuch einer Fremdenverkehrsregion befriedigen wollen. Andere Personen streben hingegen nach der Befriedigung sportbezogener Bedürfnisse, so daß diese dem Auswahlprozess einer Fremdenverkehrsregion zugrundegelegt werden. Die Befriedigung der, über den Grundnutzen der Regeneration hinausgehenden Urlaubsbedürfnisse, können daher in Anlehnung an die allgemeine Nutzentheorie ${ }^{152}$ als zusatznutzen umschrieben werden, der mit einer Reise oder einem Urlaub angestrebt wird. 153 Erst diese sekundären Bedürfnisse (Erlebnisbedürfnisse) führen $z u$ einer differenzierten wahrnehmung des an sich homogenen auf Regeneration und Erholung ausgerichteten Angebots von Fremdenverkehrsregionen.

Die Herausbildung sekundärer Motive läßt den Schluß zu, daß Angebotskomponenten einer Fremdenverkehrsregion, die auf die Befriedigung touristischer Grundbedürfnisse abzielen, kaum Differenzierungspotentiale bieten. Somit ist davon auszugehen, $d a B$ der Wunsch nach Regeneration und Entspannung zwar das Motiv des Reisens an sich darstellt, aber kaum Einfluß auf die Wahl einer Urlaubsregion hat.

Daher ergeben sich Ansatzpunkte einer zielgruppenspezifischen Marktbearbeitung vor allem aufgrund des Inhaltes und der Intensität der hierarchisch höher angesiedelten Bedürfnisse, d.h. sekundäre Motive in Gestalt von Erlebnisbedürfnissen. Allerdings ist $\mathrm{zu}$ beachten, $\mathrm{da \beta}$ der stellenwert der Erlebnisbedürfnisse in der Bedürfnishierarchie von Touristen in Abhängigkeit von der Besuchsdauer variiert. Sowohl Kaspar als auch Opaschowski sind der Ansicht, daß dem Erlebnisbe-

152 Vgl. Vershoven, W., Die Marktentnahme als Kernstück der wirtschaftsforschung, Berlin 1959, S. 111.

153 Vgl. zur Kennzeichnung des Zusatznutzens Nieschlag, R., Dichtl, E., Hörschgen, H., Marketing, a.a.0., S. 4 . 
dürfnis im Rahmen von Kurzurlaubsreisen und Tagesausflügen eine dominante Bedeutung zukommt. 154 Demnach ist davon auszugehen, daß mit abnehmender Aufenthaltsdauer in einer Fremdenverkehrsregion das Erholungsbedürfnis abnimmt, und anstelle dessen das Erlebnisbedürfnis deutlich an stellenwert gewinnt. Dabei sieht Kaspar diesen zusammenhang vor allem in bezug auf Bildungsreisen. 155

Der stellenwert der Erlebnisbedürfnisse im Motivsystem eines potentiellen Touristen gibt Auskunft über den subjektiven ziel-Mittel-zusammenhang. 156 Mit ihm wird abgebildet, ob der Tourist ein bestimmtes Erlebnisziel anstrebt, und ob er den Besuch einer bestimmten Fremdenverkehrsregion als geeignetes Mittel ansieht, dieses ziel zu realisieren.

Inwieweit sich ein Zusammenhang zwischen Urlaubsmotiven und dem Besuch einer bestimmten Fremdenverkehrsregion feststellen läßt, hängt demnach von dem touristischen Angebot einer Fremdenverkehrsregion ab. So kann beispielsweise das Erlebnisbedürfnis nach Kultur nur befriedigt werden, wenn die Fremdenverkehrsregion tatsächlich auch ein Angebot an Kultureinrichtungen und historischen sehenswürdigkeiten bereithält. Die dieser Untersuchung zugrundeliegende Fremdenverkehrsregion zeichnet sich vor allem durch ein breites Ange-

154 Vgl. Kaspar, C., Die Entwicklung des modernen Tourismus, a.a.O., S. 21 ff., Opaschowski, H.w., Urlaub 1986/87, a.a.0., s. 9 .

155 Vgl. Kaspar, C., Die Entwicklung des modernen Tourismus, a.a.0., s. 21 .

156 Der ziel-Mittel-Zusammenhang ist eine zentrale Annahme der Motivtheorie. Dabei ist es in der Literatur jedoch strittig, ob diese kognitive und willentliche zielorientierung noch zum Motivvorgang gehört. Es soll hier der Ansicht von Kroeber-Riel gefolgt werden, daß sowohl die aktivierenden Antriebskräfte als auch die kognitiven Komponenten dem Motivationsbegriff zugrundezulegen sind. Vgl. Kroeber-Riel, w., Konsumentenverhalten, a.a.o., s. 137 . 
bot an historischen und kulturellen sehenswürdigkeiten, einer natürlichen Landschaft und zahlreichen sportmöglichkeiten aus. 157 Daher ist davon auszugehen, daB ein Besuch der Fremdenverkehrsregion auf Reisemotive zurückgeführt werden kann, die das Naturerleben, Kulturstreben und das Verlangen nach körperlicher Bewegung beinhalten.

Berücksichtigt man ferner die aufgezeigten Zusammenhänge zwischen Besuchsdauer und Erlebnisbedürfnissen, so ist zu vermuten, daB das Regenerationsbedürfnis mit zunehmender Besuchsdauer relativ zunimmt und dem Bildungsbedürfnis bei Kurzurlaubsreisen ein höherer stellenwert zukommt. Demnach müßte in der Entfernungsgruppe I mit zunehmendem Bildungsbedürfnis die Besuchsdauer steigen und demgegenüber in der Entfernungsgruppe II sinken.

Ferner ist bei der Analyse der Besuchsverhaltensrelevanz von Reisemotiven die Entfernung zum zielgebiet zu berücksichtigen. Es ist davon auszugehen, daß in Abhängigkeit von der Entfernung unterschiedliche Motive zum Besuch einer Fremdenverkehrsregion aktiviert werden. So sind mit einer Reise, die eine längere Anfahrtsdauer impliziert, vermutlich andere Erlebnisinhalte verbunden als mit einem Kurzurlaub im nahegelegenen Erholungsgebiet.

Insgesamt erweist sich die Analyse von Urlaubsmotiven zum Zwecke der Marktsegmentierung als brauchbarer Ansatz, da sie die Identifizierung der Beweggründe des Besuchs einer Fremdenverkehrsregion und somit die Ableitung von Ansatzpunkten einer zielgruppenspezifischen Marktbearbeitung ermöglicht.

Dieser $\mathrm{zu}$ vermutende Eignungsgrad von Urlaubsmotiven als segmentierungskriterien erfährt allerdings dadurch eine Ein-

157 Vgl. zu den Charakteristika der Untersuchungsregion Meffert, H., Regionenmarketing Münsterland, a.a.0., S. 19 ff. 
schränkung, daß i.d.R. von einer geringen zeitlichen stabilität des Motivaystems ausgegangen werden muß. So ist die Verhaltenswirksamkeit von Motivsystemen in besonderem MaBe dem intervenierenden Einfluß situativer und zeitlich instabiler Variablen unterworfen. 158 Intra- und interpersonelle Motivkonflikte können generell zu einer verunsicherung in Handlungssituationen und $z u$ einer Verlängerung des Entscheidungsprozesses führen. 159 Ferner haben aufgrund der zeitlichen Instabilität einmal aufgefundene Beziehungen zwischen Motivationen und Verhaltensweisen i.d.R. nur eine zeitlich begrenzte Gültigkeit.

$158 \mathrm{Vgl}$. Wachenfeld, H., Freizeitverhalten und Marketing, a.a.0., s. 210. Beispielsweise erweist sich die Verfügbarkeit von zeit als ein wesentlicher Einflußfaktor auf den Zusammenhang zwischen Motiven und Besuchsverhalten. steht z.B. nur wenig freie zeit für einen Urlaub oder eine Reise zur Disposition, so ist davon auszugehen, da $B$ durch diesen Einflußfaktor die Zielgebietswahl in besonderem Maße determiniert wird und die mit der Reise angestrebte Befriedigung der Erlebnisbedürfnisse im Rahmen des Auswahlprozesses in den Hintergrund tritt. Vgl. zu den situativen Variabeln Mill, R. Ch., Morrison, A.M., The Tourism System, a.a.0., S. 39 ff.

159 Personelle Motivkonflikte entstehen, wenn zwei Verhaltensweisen zueinander in widerspruch geraten. So kann ein Tourist bei der Auswahlentscheidung für eine Fremdenverkehrsregion zum Beispiel folgende divergierende Motive aufweisen. Aufgrund seiner Bildungsmotivation ist er bestrebt, eine Kulturreise (Verhaltenstendenz 1) und aufgrund seiner sicherheitsmotivation einen Kururlaub (Verhaltenstendenz 2) durchzuführen. Interpersonelle Konflikte entstehen, wenn an der Reiseentscheidung mehrere Personen, zum Beispiel bei einem Familienurlaub die Familienmitglieder, beteiligt sind. Weisen diese Personen unterschiedliche Urlaubsbedürfnisse auf, so kommt es $\mathrm{zu}$ interpersonellen Konflikten. Vgl. Opaschowski, H., Urlaub 91/92, Trendziele und Trendsetter im Tourismus der 90er Jahre, B.A.T-Freizeit-Forschungsinstitut (Hrsg.), Hamburg 1992, S. 16. Zur Kennzeichnung der unterschiedlichen motivationalen Konfliktarten vgl. die Ausführungen bei Kroeber-Riel, w., Konsumentenverhalten, a.a.0., S. $153 \mathrm{ff}$. und die dort angegebene Literatur. 
Die Motivstruktur wird darüber hinaus auch vom Entwicklungsstand des Individuums beeinflußt. So lassen sich in der $\mathrm{Zu}-$ sammensetzung des Motivgefüges erhebliche Unterschiede bezüglich der Alters-, Bildungs- und Berufssitution der betrachteten Personengruppen feststellen. 160 so ist davon auszugehen, daß jüngere Personen in höherem Maße sportbezogene Bedürfnisse aufweisen als ältere Personen. Darüber hinaus ist anzunehmen, daß bei älteren Personen das Bedürfnis nach Naturerleben in den Vordergrund tritt, 161 welches durch Aktivitäten wie Wandern und spazierengehen befriedigt wird. Darüber hinaus werden sich Personen mit kindern von kinderlosen Personen in ihren familienorientierten Erlebnisbedürfnissen unterscheiden.

Ferner wird in der Literatur davon ausgegangen, daß mit zunehmendem Bildungsgrad sowie zugehörigkeit $z u$ einer höheren sozialen Schicht auch im Urlaub das kulturelle Bedürfnis an Bedeutung gewinnt. 162 zudem ist $z u$ vermuten, daß höher gebildete Personen mit i.d.R. geringen physischen Belastungen im Berufsumfeld ein höheres sport- und Bewegungsbedürfnis aufweisen als Personen mit niedrigerem Bildungsgrad. Geht man davon aus, da $\beta$ sich der Bildungsgrad in dem Einkommen widerspiegelt, müßten auch mit zunehmendem Einkommen die Befriedigung der vergleichsweise teureren bildungs- und sportbezogenen Aktivitäten an Bedeutung gewinnen. Können tatsächlich solche zusammenhänge zwischen soziodemographischen Merkmalen und Urlaubsmotiven nachgewiesen werden, so bedeutet dies, daß Urlaubsmotive in hohem Maße die Anforderung der Identifizierbarkeit an segmentierungskriterien gewährleisten.

160 Vgl. Opaschowski, H.W., Urlaub 91/92, a.a.O., S. 15 ff.

161 Vgl. Opaschowski, H., Tourismusforschung, a.a.O., s. 109.

162 Vgl. Schmid, J.-M., Kundenstamm-Marketing - aus der sicht des Reiseveranstalters, a.a.0., S. 58 . 
Mit Blick auf die diskutierten Reisemotive als Einflußfaktoren auf das regionenspezifische Besuchsverhalten sollen im Rahmen der empirischen Analyse folgende Untersuchungshypothesen überprüft werden:

Basishypothese zum Einfluß soziodemographischer Merkmale auf die Ausprägungen von Orlaubsmotiven:

HOTIV 1 Soziodemographische Merkmale bestimmen generell die Ausprägungsformen der Urlaubsmotive.

Diese Basishypothese ist angesichts der aufgezeigten zusammenhänge durch folgende Hypothesen zu konkretisieren:

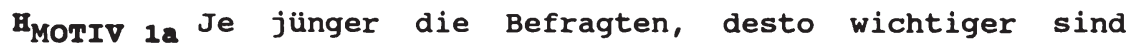
sportbezogene Urlaubsbedürfnisse.

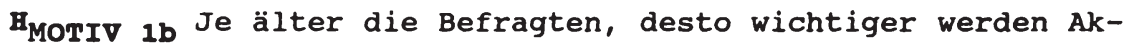
tivitäten in der freien Natur.

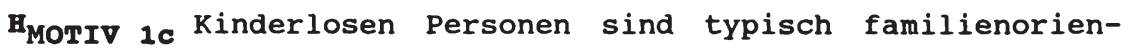
tierte Bedürfnisse unwichtiger als Personen mit Kindern.

HOTIV 1d Je höher der Bildungsgrad und die Schichtzugehörigkeit, desto wichtiger sind kulturelle Urlaubsbedürfnisse.

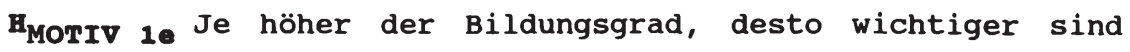
sportbezogene Urlaubsbedürfnisse. 
Basishypothese zum EinfluB von Urlaubsmotiven auf die Besuchsabsicht :

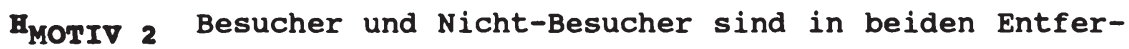
nungsgruppen durch unterschiedliche Motivstrukturen gekennnzeichnet.

Aus dieser Basishypothese leiten sich folgende Hypothesen $a b$ :

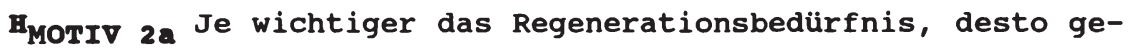
ringer wird in der Entfernungsgruppe I eine Besuchsabsicht geäußert.

ByorIv 2b Je wichtiger das kulturelle Bedürfnis, um so stärker wird in beiden Entfernungsgruppen eine Besuchsabsicht geäußert.

Horrv 2c Je wichtiger das Sportbedürfnis, um so stärker wird in beiden Entfernungsgruppen eine Besuchsabsicht geäußert.

Basishypothese zum EinfluB von Urlaubsmotiven auf die Besuchsdauer :

Hotrv 3 Die nach der Besuchsdauer abgegrenzten Besuchsverhaltenssegmente sind in beiden Entfernungsgruppen durch unterschiedliche Motivausprägungen gekennzeichnet.

Hieraus leiten sich folgende Hypothesen $a b$ :

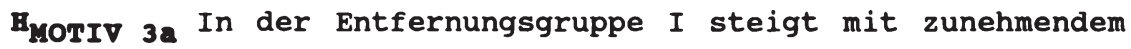
Regenerationsbedürfnis die Besuchsdauer. 
HoTIV 3b In der Entfernungsgruppe II steigt mit zunehmendem Regenerationsbedürfnis die Besuchsdauer.

HoTIV 3c In der Entfernungsgruppe I steigt mit zunehmendem kulturellen Bedürfnis und sportbedürfnis die Besuchsdauer.

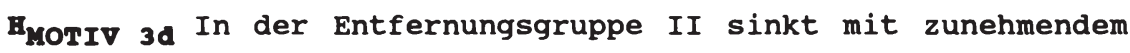
kulturellen Bedürfnis die Besuchsdauer.

\subsection{Operationalisierung von Urlaubsmotiven}

Hinsichtlich der Operationalisierung von Urlaubsmotiven ist zu beachten, daß eine valide Messung gleichermaßen die Antriebskomponenten und die kognitiven Komponenten der Motive erfassen muß. Zur Messung der Antriebskomponenten von Motiven lassen sich die Intensität, die Richtung und die Qualität von Reisemotiven unterscheiden. 163

Mit der Intensität wird die objektiv vorhandene, physiologische Aktivierung zum Ausdruck gebracht. Die Richtung zeigt dagegen an, inwieweit durch das Motiv ein Annäherungsverhalten oder ein Vermeidungsverhalten in bezug auf das ziel der Reise zum Ausdruck kommt. Schließlich bezieht sich die qualität auf den mit einem Reisemotiv verbundenen Erlebnisinhalt.

Die skizzierten Komponenten stellen jeweils unterschiedliche Anforderungen an die operationalisierung bzw. Messung. Daher existieren in der Literatur zur Motivforschung eine Vielzahl von verfahren sur Motivessung. Wird überwiegend die An-

163 Mit der Bestimmung von Intensität, Richtung und qualität der Motive wird analog zu Vorgehensweise der Operationalisierung von Emotionen verfahren. Vgl. Kroeber-Riel, W., Konsumentenverhalten, a.a.O., S. 103 . 
triebsseite gemessen, so bieten sich die Verfahren der Emotionsmessung, d.h. psychobiologische Messungen, Messung des Ausdrucksverhaltens sowie der Erlebnismessung an. Werden überwiegend die kognitiven Komponenten gemessen, so wird i.d.R. auf die Verfahren der Einstellungsmessung zurückgegriffen. 164

Die psychobiologische Messung ${ }^{165}$ und auch die Messung des Ausdrucksverhaltens 166 erweisen sich jedoch für den Zweck der Marktsegmentierung als wenig sinnvoll. Diese Verfahren sind sehr aufwendig, so daß i.d.R. eine Beschränkung auf kleinere stichproben erfolgen muß. Für die Marktsegmentierung werden jedoch größere stichproben benötigt, um im Bereich der Motive Unterschiede zwischen Touristen feststellen und zugleich verläßliche Angaben über die Größe der einzelnen segmente erhalten zu können. 167

Die subjektive Erlebnismessung eignet sich sowohl zur Erfassung der Intensität und Richtung als auch der Qualität der aktivierenden Motivationskomponenten. Dabei sind verbale und nichtverbale Meßverfahren zu unterscheiden. Für die Ermittlung von unbewußten Motiven bieten sich nonverbale Meßverfahren an. Mit Hilfe von Bilder- und Farbskalen sollen die

Vgl. Kroeber-Riel, W., Konsumentenverhalten, a.a.0., 1984 , S. 145 .

165 Psychobiologische Messungen bedienen sich unterschiedlicher physiologischer Indikatoren wie z.B. Lidschlagfrequenz, elektrischer Hautwiderstand, Atmung, Pulsfrequenz, Blutdruck, Hirnströme etc., die mittels apparativer Verfahren erfaßt werden. Vgl. zu einem tuberblick über diese Verfahren Meffert H., Marketingforschung und Käuferverhalten, a.a.0., s. 222 ff.

166 Die Messung des Ausdrucksverhaltens bezieht sich auf sichtbare Verhaltensweisen wie z.B. Mimik, Gestik und Körpersprache. Vgl. Konert, F.-J., Vermittlung emotionaler Erlebniswerte, Heidelberg 1986, S. 109 ff.

167 Vgl. Freter, H., Marktsegmentierung, a.a.o., S. 63. 
"inneren Bilder", d.h. die visuellen Vorstellungen eines Menschen sichbar gemacht werden. 168

Hinsichtlich der verbalen Messung finden überwiegend Ratingskalen Verwendung, bei denen unterschiedliche Items die inhaltliche Qualität der Motive beschreiben. In der Emotionspsychologie liegen Erklärungsansätze vor, nach denen das Erlebnis von Emotionen in erheblichen AusmaB auch sprachlich bewußt wahrgenommen wird und von daher verbal mitgeteilt werden kann. 169 Mithin können Motive über Ratingskalen erfaßt werden. Durch die Frage nach der wichtigkeit von Motiven kann zusätzlich der kognitiven Motivationskomponente Rechnung getragen werden, da hierdurch implizit das bewuBte Anstreben von zielen berücksichtigt wird.

Für die operationalisierung der Reisemotive wird daher im folgenden ein verbaler MeBansatz gewählt. Unter der Verwendung einer fünf-stufigen, nach der wichtigkeit abgestuften Ratingskala finden dabei die Komponenten von Urlaubsmotiven Berücksichtigung. Hierdurch besteht die Möglichkeit, ein den wirtschaftlichkeitsanforderungen entsprechendes standardisiertes Erhebungsverfahren einzusetzen.

Die Güte der Messung wird im wesentlichen von der Itemmessung bestimmt. 170 Daher kommt der Indikatorenauswahl eine besondere Bedeutung zu. Da es sich bei dem AuswahlprozeB von Fremdenverkehrsregionen, insbesondere wenn Urlaubsentschei-

$168 \mathrm{Vgl}$. zu den nonverbalen Meßverfahren Vgl. Kroeber-Riel, w., Konsumentenverhalten, a.a.O., S. 107.

169 Heinemann, G., Betriebstypenprofilierung und Erlebnishandel. Eine empirische Analyse des textilen Facheinzelhandels, Wiesbaden 1989, S. $168 \mathrm{ff}$, Schmidt-Atzert, L., Emotionspsychologie, stuttgart, Berlin, u.a. 1981, s. 32 ff.

$170 \mathrm{Vgl.} z u$ den Gütekriterien Reliabilität und Validität die in Kapitel B.1 gemachten Ausführungen. 
dungen $z u$ treffen sind, um einen komplexen Kaufentscheidungsproze $B$ handelt, 171 erscheint die Erfassung bestimmter Antriebskräfte wie z.B. Prestige, Geselligkeit, Geborgenheit, Abwechslung 172 nur wenig geeignet, das Besuchsverhalten in bezug auf eine Fremdenverkehrsregion zu erklären. zudem liefert solch eine operationalisierung von Urlaubsmotiven zum Zwecke der Marktsegmentierung einer Fremdenverkehrsregion nur unzureichend Anhaltspunkte für die zielgruppenspezifische Ausgestaltung ihrer Marketinginstrumente.

Stattdessen erweist sich als sinnvoll, die in den Urlaubsmotiven zum Ausdruck kommenden Erlebnisinhalte in den Vordergrund zu stellen. Es interessiert hier vor allem, welche Erlebnisinhalte mit einem Urlaub oder einer Reise verbunden werden. Daher ist es zweckmäßig, als Indikatoren der Urlaubsmotive konkrete Urlaubsaktivitäten heranzuziehen, die in hohem Maße die mit einem Besuch einer Fremdenverkehrsregion in Verbindung stehenden Erlebnisinhalte widerspiegeln. Ferner kann über Urlaubsaktivitäten, wie zum Beipiel "Sich ausruhen" oder "Faulenzen" das Regenerationsbedürfnis erfaßt werden. 173 In der vorliegenden Untersuchung sollen daher die Erlebnis- und Regenerationsbedürfnisse über die in Abbildung 20 dargestellten, gewünschten Urlaubsaktivitäten erfolgen. $\mathrm{zu}$ beachten ist jedoch, daß eine Motivation gleichzeitig hinter vielen Urlaubsaktivitäten, aber auch umgekehrt viele Motivationen hinter ein und derselben Freizeittätigkeit ste-

171 Vgl. zur Komplexität des Kaufentscheidungsprozesses bei der Auswahl von Fremdenverkehrsregionen in Abhängigkeit von Kaufentscheidungstypen und deren tibertragung auf den Fremdenverkehr die Ausführungen in Kapitel B.21

$172 \mathrm{Vgl}$. Kroeber-Riel, Konmsumentenverhalten, a.a.0., s. 148

173 Kaspar, C., Fremdenverkehrslehre im Grundriß, a.a.0., s. 40; McIntosh, R.W., Tourism. Principles, Practices, Philosophies, 3. Aufl., Columbus 1980 , S. 65; Lohmann, M., Wohlmann, R., Urlaub in Deutschland, a.a.0., S. 29 ff., Aderhold, P., Das Marketing von Städtekurzreisen, a.a.0., S. 125 ff. 
hen können. Das bedeutet, daß erst durch die faktoranalytische Verdichtung der Indikatoren Hinweise auf die hinter den Urlaubsaktivitäten stehenden Urlaubsmotive gewonnen werden können.

\begin{tabular}{|c|c|c|}
\hline $\begin{array}{l}\text { Basis- } \\
\text { Motive }\end{array}$ & $\begin{array}{l}\text { Motiv- } \\
\text { Spezifi- } \\
\text { zierung }\end{array}$ & $\begin{array}{c}\text { Urlaubsaktivi- } \\
\text { täten als Indi- } \\
\text { katoren für } \\
\text { Urlaubsmotive }\end{array}$ \\
\hline $\begin{array}{l}\text { Regenerations- } \\
\text { bedürfnis }\end{array}$ & $\begin{array}{l}\text { Entspannungs-/ } \\
\text { Erholungs- } \\
\text { bedūinnis }\end{array}$ & $\begin{array}{l}\text { - Faulenzen } \\
\text { - Sich ausruhen }\end{array}$ \\
\hline \multirow{3}{*}{$\begin{array}{l}\text { Erlebnis- } \\
\text { bedürfnis }\end{array}$} & Kulturbedūrfnis & $\begin{array}{l}\text { - Sehenswūrdigkeiten } \\
\text { besichtigen } \\
\text { - Kulturelle Veran- } \\
\text { staltungen } \\
\text { - Kontakt mit } \\
\text { Einheimischen }\end{array}$ \\
\hline & $\begin{array}{l}\text { Bewegungs-/ } \\
\text { Sportbedürnis }\end{array}$ & $\begin{array}{l}\text { - Spazieren gehen } \\
\text { - Wandern } \\
\text { - Sport treiben } \\
\text { - Radfahren }\end{array}$ \\
\hline & $\begin{array}{l}\text { sonstige } \\
\text { Freizeit- } \\
\text { bedürfnisse }\end{array}$ & $\begin{array}{l}\text { - Tier- und Freizeitparks } \\
\text { besuchen } \\
\text { - Volksfeste/ Veran- } \\
\text { staltungen besuchen } \\
\text { - Aktiv sein } \\
\text { - Schlemmen } \\
\text { - Gaststätten/ Kneipen } \\
\text { besuchen } \\
\text { - Mit den Kindern spielen } \\
\text { - Einkaufen gehen }\end{array}$ \\
\hline
\end{tabular}

Abb. 20: Urlaubsaktivitäten als Indikatoren zur Erfassung von Urlaubsmotiven 
Zur Analyse der Qualität des Operationalisierungsansatzes ist folgende meßtheoretische Basishypothese empirisch zu überprüfen:

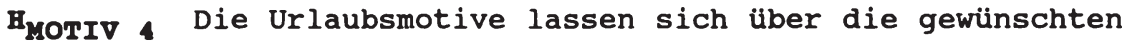
Urlaubsaktivitäten erfassen. Dabei lassen sich die Urlaubsaktivitäten auf faktoranalytischem Wege zu Urlaubsmotiven (Supervariablen) verdichten.

\subsection{Empirische Analyse der Eignung von orlaubsmotiven als segmentierungskriterien}

\subsection{Kennzeichnung der Ausprägungsformen und struktur von orlaubsmotiven}

Die Messung der Urlaubsaktivitäten als Indikatoren für die Urlaubsmotive ergab das in Abbildung 21 dargestellte Motivprofil. Es zeigt sich, daß der Aktivität "sich ausruhen" die größte Bedeutung bei der Durchführung eines Urlaubs oder einer Reise zukommt, gefolgt von den Urlaubsaktivitäten "Spazierengehen" und "Faulenzen". Auch den Aktivitäten "Begegnung mit Einheimischen", "Besichtigung von Sehenswürdigkeiten", "Wandern" und "Schlemmen" erfahren noch eine hohe Wichtigkeitseinschätzung. Hierin bestätigt sich insgesamt, daß dem Naturaufenthalt in Verbindung mit Regeneration eine hohe Bedeutung im Rahmen von Urlauben und Reisen zukommt.

Interessanterweise werden sportaktivitäten und Radfahren nur unterdurchschnittlich wichtig eingestuft. Die geringe wichtigkeitseinschätzung entspricht den Ergebnissen anderer Untersuchungen. 174 Es ist demnach $z u$ vermuten, daß nur ein

174 Vgl. Gilbrich, M., Urlaubsreisen 1991, a.a.O., s. 26 f., O.V., Marken Profile 4: Touristik, Fluggesellschaften, Mietwagen, a.a.0., S. 60 . 
Sich ausruhen

Spaziergänge machen

Tier-, Freizeitparks besuchen

Begegnungen mit Einheimischen

Mit den Kindern spielen

Aktiv sein

Volksfeste, Veranstaltungen besuchen

Hobbies betreiben

Sehenswürdigkeiten besichtigen

Kulturelle Veranstaltungen besuchen

Schwimmen, baden, am Strand liegen

Wandern

Radfahren

Sport treiben

Einkaufen gehen

Gaststätten/ Kneipen besuchen

Faulenzen

Schlemmen

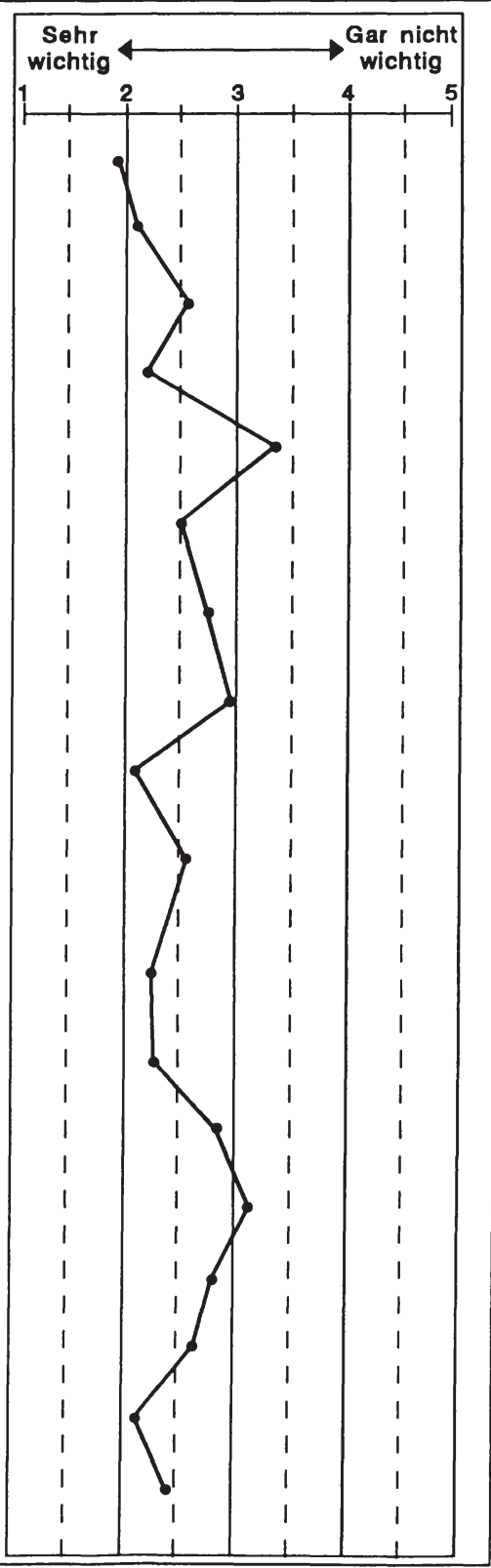

Abb. 21: Empirisch ermittelte wichtigkeit von Urlaubsmotiven 
kleiner Prozentsatz potentieller Touristen mit einem sportangebot $z u$ einer Reise bewegt werden kann.

Auch der Urlaubsaktivität "mit den Kindern spielen" kommt für ein Wohlbefinden im Urlaub nur eine stark unterdurchschnittliche Rolle zu. Offensichtlich ist ein Familienurlaub nicht das dominante Urlaubsmotiv.

Unter Berücksichtigung dieses Profils sind im folgenden Schritt die einzelnen Aktivitäten zu sog. Aktivitäts- bzw. Motivfaktoren zusammenzufassen. Hierzu wurde eine explorative Faktorenanalyse durchgeführt. Die Abbildung 22 zeigt das aufbereitete Ergebnis einer nach dem Kaiser-Kriterium ermittelten 5-faktoriellen Lösung, die zu einem erklärten Varianzanteil an der Gesamtvarianz in Höhe von 61,4 \% fuhrte. 175

Die hohen Faktorladungen der Indikatoren des ersten Faktors kennzeichnen deutlich die Dimension eines sportbezogenen $\mathrm{Ak}-$ tivitätsbedürfnisses. Dieser Faktor erklärt 23,8 $\%$ der Gesamtvarianz, so daß ihm bei der Erklärung der Motivstruktur die größte Bedeutung zukommt.

Der zweite Faktor zeichnet sich durch hohe Faktorladungen bei den Urlaubsaktivitäten "Faulenzen", "Schlemmen", "Gaststätten und Kneipen besuchen" und "sich ausruhen" aus. Diese Urlaubsaktivitäten deuten unmittelbar auf den Wunsch nach

175 Vgl. zur vollständigen Dokumentation der Faktorenstruktur Anhang $I$. 


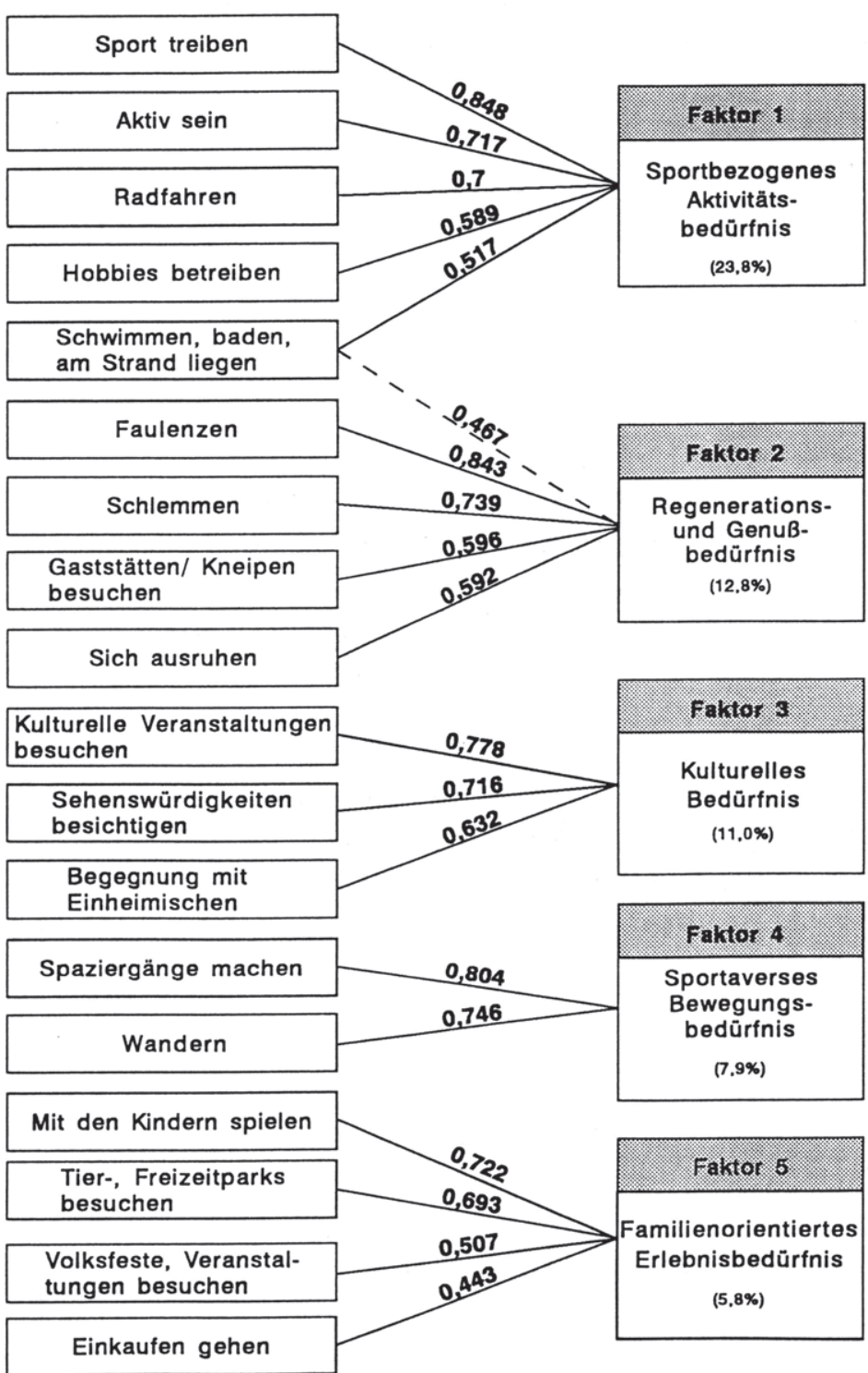

Erklärter Varianzanteil: $61,4 \%$

Abb. 22: Faktorenanalytische Verdichtung der Motivindikatoren 
Erholung, Entspannung und Genuß hin und können demnach als Regenerations- und Genußbedürfnis umschrieben werden. 176

Der dritte Faktor (11\% Varianzanteil) läßt sich als kulturelles Bedürfnis kennzeichnen. Er zeichnet sich durch hohe Faktorladungen bei den Aktivitäten "Besuch kultureller Veranstaltungen", "Besichtigung von Sehenswürdigkeiten" und "Begegung mit Einheimischen" aus. Offensichtlich kommt hier das streben nach Bildung, Weiterbildung und Wissen zum Ausdruck.

Der vierte Faktor kennzeichnet deutlich das in der Literatur vielfach als "Wanderbedürfnis" gekennzeichnete Reisemotiv. Es zeichnet sich durch hohe Faktorladungen bei den Aktivitäten Wandern und Spazierengehen aus. Daher kann dieser Faktor auch als sportaverses Bewegungsbedürfnis in der freien Natur umschrieben werden. Dieser Faktor erklärt 7,9\% der Gesamtvarianz.

Der fünfte Faktor läßt sich als familienorientiertes Erlebnisbedürfnis interpretieren. Sowohl die Urlaubsaktivität "mit den Kindern spielen" als auch der Besuch von Tier- und Freizeit-Parks stellen die zentralen Dimensionen dieses Fak-

176 Interessanterweise weist die Urlaubsaktivität "Schwimmen" bzw. "Am Strand liegen", die dem Faktor 1 zugeordnet wurde, auch bei dem Faktor 2 eine relativ hohe Faktorladung in Höhe von 0,467 auf. Offensichtlich ist diese Urlaubsaktivität personenspezifisch mit unterschiedlichen Inhalten verbunden. Einerseits wird die mit dieser Urlaubsaktivität verbundene sportkomponente betont, andererseits scheint die Erholungskomponente dieser Aktivität, in Form von "in der Sonne bzw. am Strand liegen", zu dominieren. Daher ist diese Variable bei der Interpretation beider Faktoren heranzuziehen. Anhand der unterschiedlichen Interpretationsmöglichkeiten der Aktivität "Schwimmen, baden, am strand liegen" wird deutlich, daß hinter verschiedenen Urlaubsaktivitäten offensichtlich unterschiedliche Reisemotive stehen können. Dies ist bei der Interpretation der Ergebnisse $z u$ berücksichtigen. 
tors dar. offensichtlich sind in diesem Faktor diejenigen Aktivitäten zusammengefaßt, die der Befriedigung des Bedürfnisses nach gemeinsamen Aktivitäten mit der Familie dienen. Die Urlaubsaktivität "Einkaufen gehen" kann angesichts ihrer geringen Faktorladung nicht zur Interpretation herangezogen werden. Insgesamt kommt diesem Faktor aufgrund seines geringen Varianzerklärungsanteils in Höhe von 5,8 \& zur Erklärung der Motivstruktur der Befragten nur eine untergeordnete Bedeutung zu.

Da mit Hilfe der Faktoren immerhin noch $61,4 \%$ der Gesamtvarianz erklärt und die einzelnen Faktoren inhaltlich in ausreichendem Maße voneinander abgegrenzt werden können, ist die Basisypothese $\mathbf{H}_{\text {KOTIV4 }}$ zu bestätigen. Daher soll im folgenden die Analyse der soziodemographischen Identifizierbarkeit und Besuchsverhaltensrelevanz auf der Basis der Motivfaktoren durchgeführt werden.

\subsection{Analyse der soziodemographischen Identifizierbarkeit und des Einflusses von Orlaubsmotiven auf das Besuchs- verhalten}

Zur Analyse der Identifizierbarkeit der Reisemotive ist der Zusammenhang zwischen soziodemographischen Merkmalen und den Ausprägungsformen der Motivfaktoren $\mathrm{zu}$ ermitteln. Dieser geht aus Abbildung 23 hervor.

In Abhängigkeit von den demographischen Merkmalen zeigen sich deutliche Unterschiede in den Motivstrukturen. Dabei kann allen vier demographischen Merkmalen eine vergleichsweise hohe Erklärungskraft zugesprochen werden. Die Motivfaktoren "sportbezogenes Aktivitätsbedürfnis" sowie "Regenerations- und Genußbedürfnis" werden um so wichtiger eingeschätzt, je jünger die potentiellen Touristen sind und je 


\begin{tabular}{|c|c|c|c|c|c|}
\hline \multirow{2}{*}{$\begin{array}{l}\text { Sozlodemo- } \\
\text { graphlsche Merkmale }\end{array}$} & $\begin{array}{l}\text { Sportbe-1 } \\
\text { zogenes } \\
\text { Aktlul- } \\
\text { tatsbe- } \\
\text { dürnnis } \\
\end{array}$ & 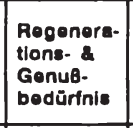 & $\begin{array}{l}\text { Kulturelles } \\
\text { Bedürtnis }\end{array}$ & $\begin{array}{l}\text { Sport- } \\
\text { averses } \\
\text { Bowegungs } \\
\text { bodürnie }\end{array}$ & $\begin{array}{l}\text { Famillon- } \\
\text { orientiont. } \\
\text { Erlebnisbo } \\
\text { dÖrfnis }\end{array}$ \\
\hline & 1 & 2 & 3 & 4 & 5 \\
\hline \multicolumn{6}{|l|}{ Demographische Merkmale } \\
\hline $\begin{array}{l}\text { - Geschlecht } \\
\text { - Alter }\end{array}$ & $\begin{array}{c}0,448 \\
\star \star \star\end{array}$ & 0,217 & 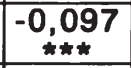 & $\underset{\substack{* \star 2 \\
\star \star \star \star}}{-0,254}$ & n.S. \\
\hline -Kinderanzahl & $\begin{array}{c}-0,142 \\
\star \star \star \\
\end{array}$ & n.S. & 0,19 & n.S. & $-\underset{\hbar \star \star}{-0,363}$ \\
\hline - Lebenszyklusphase & 0,39 & 0,212 & n.S. & $\underset{\hbar \star \star}{-0,253}$ & $\underset{\substack{\vdots \star \\
\vdots \star}}{-0,068}$ \\
\hline -Bildung & $-\underset{\star \star \star \star \star}{-0,235}$ & n.S. & $-0,0,099$ & $\begin{array}{c}0,07 \\
\star \star \\
\end{array}$ & 0,215 \\
\hline -Beruf & +++ & + & n.S. & ++ & +++ \\
\hline \multirow{2}{*}{$\begin{array}{l}\text { - Haushalts-Nettoeinkommen } \\
\text {-Persönl. Nettoeinkommen }\end{array}$} & $\underset{\star \star \star}{-0,075}$ & n.S. & n.S. & n.S. & $-0,055$ \\
\hline & n.S. & n.S. & $\underset{\hbar \star \star}{-0,092}$ & $-0,049$ & n.S. \\
\hline - Schichtzugehörigkeit & $\underset{\substack{-0,19 \\
\star \star \star}}{-0,19}$ & n.S. & $-0,104$ & n.S. & $\underset{\star \star \star \star \star}{0,096}$ \\
\hline \multicolumn{6}{|c|}{ Korrelationskoeffizienten ( $\left.{ }^{\star}\right)$ und F-Test $(+)$ mit: } \\
\hline \multicolumn{2}{|c|}{$\begin{array}{lll}\text { Signifikanzniveau } & \alpha<0,01=* \star \star & (+++) \\
\text { Signifikanzniveau } & \alpha<0,05=* \star & (++)\end{array}$} & \multicolumn{2}{|c|}{$\begin{array}{l}\text { Signifikanzniveau } \\
\text { nicht Signifikant }\end{array}$} & \multicolumn{2}{|c|}{$\begin{aligned} \alpha<0,1 & =*(+) \\
& =\text { n.s. }\end{aligned}$} \\
\hline
\end{tabular}

Abb. 23: Empirische Ergebnisse zum Einfluß soziodemographischer Merkmale auf die Urlaubsmotivfaktoren

geringer ihre stellung im Lebenszyklusmodell ist. Demgegenüber steigt die wichtigkeitseinschätzung des "sportaversen Bewegungsbedürfnisses" mit zunehmendem Alter und fortgeschrittener Lebenszyklusphase. Die Hypothesen $\mathbf{H}_{\text {MOTIV1a }}$ und HOTIV1b, die einen negativen Zusammenhang zwischen sportbezogenen Urlaubsbedürfnissen und dem Alter und einen positiven Zusammenhang zwischen dem Bewegungsbedürfnis in der freien Natur und dem Alter unterstellen, sind demnach zu bestätigen. 
Interessanterweise besteht zwischen dem "familienorientierten Erlebnisbedürfnis" und dem Alter kein Zusammenhang. Auch die stellung im Lebenszyklus trägt angesichts des geringen Korrelationskoeffizienten von 0,07 kaum zur Erklärung dieses Bedürfnisses bei. Demgegenüber steigt das Bedürfnis nach familienorientierten Erlebnissen im Rahmen eines Urlaubs signifikant mit der Kinderanzahl, so daB sich die Hypothese HorIV1C bestätigt.

Betrachtet man die sozioökomomischen Merkmale so zeigt sich, daß vor allem zwischen den Merkmalen Bildung und Beruf Zusammenhänge zu Ausprägungen der Urlaubsmotive bestehen. Mit steigendem Bildungsgrad wird das "sportbezogene Aktivitätsbedürfnis" zunehmend wichtiger eingeschätzt. Vermutlich ist dies darauf zurückzuführen, daß sich mit zunehmenden Bildungsgrad verstärkt die Erkenntnis durchsetzt, daß zur Erhaltung der Gesundheit das Betreiben von sport unabdingbar ist. Horrvie ist somit $z u$ bestätigen. Das Regenerationsbedürfnis ist hingegen unabhängig von Bildungsgrad, Einkommen sowie der Schichtzugehörigkeit.

Betrachtet man den Einfluß der sozio-ökonomischen Merkmale auf das kulturelle Bedürfnis, so zeigt sich überraschenderweise nur ein sehr schwacher Zusammenhang mit dem Bildungsgrad, persönlichem Nettoeinkommen und der sozialen Schichtung. Kein signifikanter Zusammenhang besteht mit dem Beruf und dem Netto-Haushaltseinkommen. Bildung, Einkommen sowie die soziale Schichtung sind demnach nicht geeignet, das Bildungs- und Wissensstreben während des Urlaubs oder einer Reise zu erklären. H MOTIV1d ist somit zu verwerfen.

Der geringe Einfluß der sozioökonomischen Merkmale "Einkommen" und "Bildung" auf das sportaverse Bewegungsbedürfnis kann kaum überraschen. Demgegenüber besteht ein $\mathrm{Zu}-$ sammenhang dieses Bedürfnisses mit dem Beruf der Befragten. Dabei schätzen vor allem Beamte und Hausfrauen das sport- 
averse Bewegungsbedürfnis signifikant wichtiger ein als Schüler und Studenten, Auszubildende sowie Rentner.

Das familienorientierte Erlebnisbedürfnis wird vor allem vom Bildungsgrad determiniert. Dabei sinkt mit steigendem Bildungsgrad die wichtigkeiteinschätzung des familienorientierten Erlebnisbedürfnisses. Es ist $z u$ vermuten, daß bei hohem Bildungsgrad das Bedürfnis nach Aktivitäten mit der Familie von anderen Reisemotiven überlagert wird.

Insgesamt zeigt die Analyse des Einflusses von soziodemographischen Merkmalen auf die Urlaubsmotive, daß diese in hohem Maße der Anforderung der Identifizierbarkeit entsprechen. Dabei erweisen sich vor allem die demographischen Merkmale Alter, Kinderanzahl und stellung im Lebenszyklus sowie das sozioökonomische Merkmal Bildung als geeignete Bestimmungsgrößen der Urlaubsmotive. Daher ist die Basishypothese HorIv1, daß soziodemographische Merkmale die Ausprägungen von Urlaubsmotiven beeinflussen, tendenziell zu bestätigen. Ferner deuten die insgesamt plausiblen Ergebnisse auf eine hohe inhaltliche validität der Konstruktmessung hin.

Zur Analyse der Verhaltensrelevanz der Reisemotive wurden TTests 177 mit dem ziel durchgeführt, die abgegrenzten Verhaltenssegmente nach Besuchsverhaltensunterschieden $z u$ überprüfen und damit motivationale Bestimmungsfaktoren des Besuchsverhaltens $z u$ identifizieren. Die Ergebnisse sind in Abbildung 24 dargestellt.

In der Entfernungsgruppe I zeigt sich, daB den potentiellen Besuchern das "sportbezogene Aktivitätsbedürfnis" als auch das "kulturelle Bedürfnis" signifikant wichtiger ist als den

$177 \mathrm{Vgl}$. zu den Anwendungsvoraussetzungen die in Kapitel B. 4.122 gemachten Anmerkungen. 


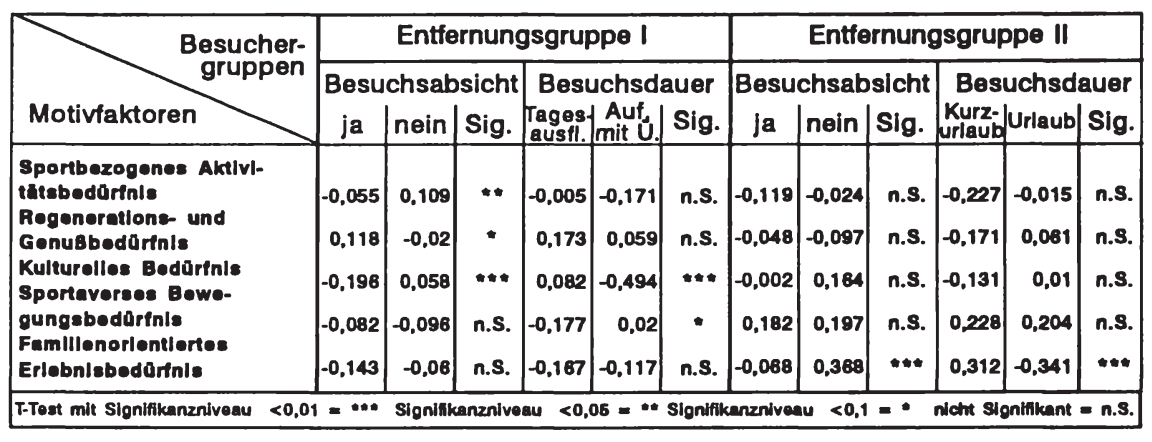

Abb. 24: Empirische Ergebnisse zum EinfluB der Urlaubsmotivfaktoren auf das Besuchsverhalten (T-Test) innerhalb der Makrosegmente

Nicht-Besuchern. Hierin scheint sich zu bestätigen, daß Motive in einem ziel-Mittel-Zusammenhang zu sehen sind, denn die Untersuchungsregion zeichnet sich vor allem durch das Angebot an Sehenswürdigkeiten und sportmöglichkeiten in der freien Natur aus. Offensichtlich wird der Besuch der Fremdenverkehrsregion zur Befriedigung des sportbezogenen Aktivitätsbedürfnisses und des kulturellen Bedürfnisses beabsichtigt. Vor diesem Hintergrund sind die Hypothesen $\mathbf{H}_{\text {MOTIV2b }}$ und $\mathbf{H}_{\text {MOTIV2 }}$ für die Entfernungsgruppe I zu bestätigen.

Ferner unterscheiden sich Besucher von den Nicht-Besuchern der Entfernungsgruppe I signifikant in ihrem Regenerationsund GenuBbedürfnis. Da den Nicht-Besuchern dieses Bedürfnis signifikant wichtiger ist als den potentiellen Touristen, ist die Hypothese H MOTIV2a zu bestätigen. Unter der Annahme, daß sich das Regenerationsbedürfnis nicht nur im Urlaub und auf Reisen, sondern im generellen Freizeitverhalten niederschlägt, liegt die vermutung nahe, daß die Nicht-Besucher generell eine geringere Neigung zur Durchführung von Ta- 
gesausflügen oder Kurzurlaubsreisen im Naherholungsverkehr aufweisen.

Bei der Betrachtung der Motivausprägungen in Abhängigkeit von der Besuchsdauer zeigt sich, daß Tagesausflügler vor allem nach einem familienorientierten Erlebnisbedürfnis streben. Allerdings bestehen keine Unterschiede zu den Personen, die einen längeren Aufenthalt beabsichtigen. Signifikante Unterschiede zwischen diesen beiden Besuchergruppen ergeben sich nur bei dem "kulturellen Bedürfnis" sowie dem "sportaversen Bewegungsbedürfnis". Während Tagesausflügler das sportaverse Bewegungsbedürfnis signifikant wichtiger einschätzen, streben Personen mit längerer Aufenthaltsdauer vor allem nach der Befriedigung des kulturellen Bedürfnisses. Dieses stellt zugleich das wichtigste Reisemotiv in diesem Verhaltenssegment dar. Die Hypothese H Motiv3c ist daher in bezug auf das kulturelle Bedürfnis zu bestätigen. Das Regenerationsbedürfnis und sportbezogene Aktivitätsbedürfnis haben offensichtlich keinen Einfluß auf die Besuchsdauer. Die Hypothese HorIv3b, die unterstellte, daß die Besuchsdauer mit zunehmendem Regenerationsbedürfnis steigt, und die

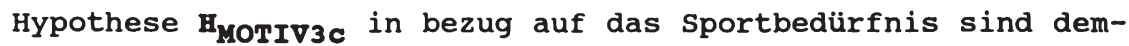
nach $z u$ verwerfen.

In der Entfernungsgruppe II unterscheiden sich die nach der Besuchsabsicht und -dauer abgegrenzten Verhaltensegmente jeweils nur in Abhängigkeit von dem familienorientierten Erlebnisbedürfnis. Dabei schätzen Besucher dieses Bedürfnis signifikant wichtiger ein als Nicht-Besucher. Ferner weisen Urlauber diesem Motiv eine signifikant höhere Bedeutung $z u$ als Kurzurlauber. Offensichtlich wird die Untersuchungsregion vor allem von denjenigen Befragten der Entfernungsgruppe II als attraktives Reiseziel angesehen, die einen Familienurlaub planen. 
Allerdings ist der geringe Einfluß der anderen Reisemotive auf das Besuchsverhalten überraschend. Daher sind die Hypothesen H HOTIV2b und HorIV2C bezogen auf die Entfernungsgruppe II, die einen Zusammenhang zwischen kulturellem und sportbezogenem Bedürfnis sowie der Besuchsabsicht unterstellen, $z u$ verwerfen. Auch die Hypothesen $\mathbf{H}_{\text {MOTIV3b und }}$ Hotrv3d, die davon ausgingen, daß die Besuchsdauer mit zunehmendem Regenerationsbedürfnis steigt und mit zunehmendem kulturellen Bedürfnis sinkt, sind $z u$ verwerfen. In diesem Zusammenhang liegt die Vermutung nahe, daß eine relative Unkenntnis der Befragten über die Angebotsstruktur in der hier untersuchten Fremdenverkehrsregion vorliegt, so daß sich unterschiedliche Motivstrukturen bei den Befragten der Entfernungsgruppe II nicht auf das Besuchsverhalten auswirken.

Zusammenfassend kann festgehalten werden, daß Urlaubsmotive in der Entfernungsgruppe I durchaus in der Lage sind, unterschiedliches Besuchsverhalten zu erklären. Dabei kommt dem kulturellen Bedürfnis die größte Erklärungskraft zu. In der Entfernungsgruppe II sind hingegen Urlaubsmotive nur stark eingeschränkt als besuchsverhaltensrelevant $z u$ bezeichnen. offensichtlich erweist sich die Entfernung zum zielgebiet und die damit in Verbindung stehende Unkenntnis der Angebotsstrukturen der Fremdenverkehrsregion als verantwortlich dafür, daß die Urlaubsmotive mit Ausnahme des familienorientierten Erlebnisbedürfnisses keinen Einfluß auf das Besuchsverhalten ausüben. Diese Erkenntnis bestätigt erneut die Vorteilhaftigkeit einer Analyse der Besuchsverhaltensrelevanz in Abhängigkeit von der Entfernung zum Zielgebiet.

Angesichts des geringen Einflusses von Motiven auf das Besuchsverhalten in der Entfernunsgruppe II ist $z u$ vermuten, daß der Nicht-Besuch auf Unkenntnis der Fremdenverkehrsregion und damit in Verbindung stehenden Vorurteilen bzw. negativen Einstellungen beruht. Daher stehen Einstellungen im Mittelpunkt der folgenden Betrachtung. 
4.3 Erfassung und Analyse von Einstellungen als Kriterien zur Marktsegmentierung

\subsection{Theoretische Grundlagen zur Erfassung und Analyse von Einstellungen als segmentierungskriterien}

\subsection{Einstellungen als EinfluBgrößen des Besuchsverhaltens}

Einstellungen unterscheiden sich von den oben dargestellten Motiven dadurch, daB in diesem Konstrukt zusätzlich eine "Gegenstandsbeurteilung" enthalten ist. Daher können sie als erworbene und relativ stabile Bereitschaften (Prädispositionen) definiert werden, auf bestimmte Stimuli der Umwelt konsistent positiv oder negativ zu reagieren. 178 sie spiegeln damit die subjektiv wahrgenommene Eignung einer Fremdenverkehrsregion zur Befriedigung einer Motivation wieder, 179 wobei die Beurteilung einer Fremdenverkehrsregion auf verfestigte (gespeicherte) Ansichten zurückgeht. Zur Analyse des Einflusses von Einstellungen auf das Besuchsverhalten sind Einstellungskomponenten und -arten differenzierter $\mathrm{zu}$ betrachten. 180

$178 \mathrm{Vgl}$. Meffert, H., Marketingforschung und Käuferverhalten, a.a.0., S. 54 .

179 Vgl. Kroeber-Riel, w., Konsumentenverhalten, a.a.o., s. 158; Der Imagebegriff wird nahezu deckungsgleich zum Einstellungsbegriff verwendet. Images beschreiben die Summe aller Einstellungen $z u$ einem Meinungsgegenstand und können insofern in der vorliegenden Untersuchung als differenziertes, aber ganzheitliches Bild einer Fremdenverkehrsregion begriffen werden. Vgl. Trommsdorff, V., Konsumentenverhalten, a.a.0., s. 123 .

180 Vgl. Kroeber-Riel, w., Konsumentenverhalten, a.a.O., s. $171 \mathrm{f}$. 
Zur Interpretation von Einstellungen werden in der Literatur die affektive und kognitive Komponente unterschieden. ${ }^{181}$ Die affektive Romponente beinhaltet die gefühlsmäBige Einschätzung einer Fremdenverkehrsregion. Ein potentieller Tourist, der einer bestimmten Fremdenverkehrsregion positiv gegenübersteht (gefühlsmäBige Bewertung), gibt damit seinen gefühlsmäßigen Gesamteindruck von der Fremdenverkehrsregion wieder. Liegt eine hohe Intensität positiver gefühlsmäBiger Gesamteinstellung $\mathrm{zu}$ einer Fremdenverkehrsregion vor, so kann davon ausgegangen werden, daß auch ihre Teileigenschaften positiv wahrgenommen werden. 182

Die kognitive Romponente beinhaltet die mit der Einstellung verbundenen Gedanken bzw. das subjektive wissen über die Fremdenverkehrsregion. Beispielweise könnte eine Fremdenverkehrsregion als eine Region wahrgenommen werden, die sich durch ein breites Angebot an Unterhaltungsmöglichkeiten, Ho-

181 Ferner wird im Rahmen der Dreikomponententheorie als dritte Komponente die Handlungstendenz (konative Komponente) aufgegriffen. Im Hinblick auf die Zugehörigkeit dieser Komponente zum Einstellungsbegriff besteht in der Einstellungsforschung Uneinigkeit. Überwiegend wird die Verhaltenskomponente als ein vom objektbezogenen Einstellungsbegriff $\mathrm{zu}$ trennendes, eigenständiges Konstrukt angesehen. Da in dieser Untersuchung die Operationalisierung des Besuchsverhaltens über die Besuchsabsicht erfolgt, soll im folgenden der dualen Einstellungstheorie gefolgt werden, die den Einstellungsbegriff auf die kognitiven und affektiven Bestandteile reduziert. Vgl. zur Kennzeichnung und Diskussion der Zweckmäßigkeit einer Berücksichtigung der konativen Komponente Meffert, H., Steffenhagen, H., Freter, H., Entwicklungen und Schwerpunkte der Käuferverhaltensforschung, in: Meffert, H., Steffenhagen, H., Freter, H. (Hrsg.), Konsumentenverhalten und Information, Wiesbaden 1979, S. 29; Triandis, H.C., Einstellungen und Einstellungsänderungen, Weinheim, Basel 1975, S.15 ff.

182 Vgl. McDougall, G.H.G., Munro, H., Scaling and Attitude Measurement in Tourism and Travel Research, in: Ritchie, J.R.B., Goeldner, C.R., Travel, Tourism and Hospitality Research. A Handbook for Managers and Researchers, New York u.a. 1987 , S. 88 . 
tel- und Gastronomiefazilitäten sowie einer ökologisch intakten Landschaft auszeichnet. Jede dieser Meinungen spiegelt das individuelle gegenwärtige wissen über die Charakteristika der Fremdenverkehrsregion wider. 183

Eine besonders hohe Bedeutung kommt vor allem der kognitiven Komponente für die zielgruppenspezifische Marktbearbeitung zu. Durch ihre Erfassung können Fehlwahrnehmungen und wissenslücken von Personen ermittelt werden, die unmittelbar Ansatzpunkte für die Ausgestaltung beispielsweise der Kommunikationspolitk liefern. ${ }^{184}$ Allerdings ist $z u$ berücksichtigen, daB die affektiven und kognitiven Einstellungskomponenten nicht voneinander unabhängig sind. Vielmehr ist die gefühlsmäBige Gesamteinstellung gegenüber einer Fremdenverkehrsregion mit der objektspezifischen Beurteilung der relevanten Angebotskomponenten einer Fremdenverkehrsregion verbunden. 185

Die hohe Bedeutung der Einstellungen für die Marktsegmentierung ergibt sich aus dem konsistenten Beziehungszusammenhang zwischen Verhalten und Einstellung. Dieser Zusammenhang wird vor allem bei stark involvierten Käufen vermutet.186 Das hohe Involvement 187 bezüglich Urlauben und Reisen ergibt

183 Vgl. McDougall, G.H.G., Munro, H., Scaling and Attitude Measurement in Tourism and Travel Research, a.a.o., S. 88 .

184 Vgl. Kook, W., Einstellungen zur Universität, a.a.o., s. 137; Freter, H., Marktsegmentierung, a.a.0., s. 77 f.

185 Vgl. Freter, H., Marktsegmentierung, a.a.0., s. 64. In diesem Zusammenhang ist davon auszugehen, da $\beta$ das Wissen über eine Fremdenverkehrsregion in besonderem MaBe auch die gefühlsmäßige Bewertung dieser Fremdenverkehrsregion beeinflußt.

186 Vgl. Kroeber-Riel, W., Konsumentenverhalten, a.a.O., s. 168 .

187 Involvement wird dabei als der individuelle Grad an innerlicher Beschäftigung mit und Betroffenheit durch ein 
sich vor allem dadurch, daß angesichts festgelegter Urlaubsansprüche (i.d.R. 30 Tage) insbesondere bei Arbeitern und Angestellten permanent eine Notwendigkeit besteht, sich mit Reisezielen auseinanderzusetzen und dabei der geeigneten Auswahl von Fremdenverkehrsgebieten eine hohe Bedeutung zugesprochen wird. Das hohe Involvement bezüglich Reisen und Urlauben bestätigt sich auch in der das ganze Jahr über andauernden Informationsbereitschaft potentieller Touristen. 188 Durch das mit einer Reise oder einem Urlaub in Verbindung stehende hohe Involvement ist demnach die grundsätzliche Voraussetzung für eine hohe Besuchsverhaltensrelevanz von Einstellungen erfüllt. Daher müste mit zunehmender Stärke positiver (negativer) Einstellungen eines Konsumenten gegenüber einer Fremdenverkehrsregion die Wahrscheinlichkeit des Besuches steigen (sinken).

Der dargestellte Zusammenhang zwischen Einstellungen und Besuchsverhalten ist jedoch davon abhängig,

- welche Arten von Einstellungen

- bei welchen Personen

- welches Verhalten vorhersagen sollen. 189

Objekt (Reiz) definiert, woraus unterschiedliche individuelle Niveaus von Interesse, Erregung und Antrieb resultieren. Meffert, H., Marketingforschung und Käuferverhalten, a.a.0., s. 66 f.; Schnetkamp, G., Einstellungen und Involvement als Bestimmungsfaktoren des sozialen Verhaltens, a.a.0., s. 23.

$188 \mathrm{Vgl}$. Datzer, Informationsverhalten von Urlaubsreisenden, a.a.0., S. 106 .

189 Kroeber-Riel unterscheidet ferner situative Bedingungen in der Kaufsituation. Diesen situativen Bedingungen kommt im Fremdenverkehr eine nur untergeordnete Bedeutung $z u$, weil sich der Kaufentscheidungsprozess über einen längeren Zeitraum erstreckt. Vgl. zu dem Einfluß situativer Bedingungen auf den Zusammenhang von Einstellungen und Kaufverhalten Kroeber-Riel, W., Konsumentenverhalten, a.a.0., S. 169 . 
Im Vordergrund dieser Untersuchung steht sowohl der Einfluß von Einstellungen auf die Besuchsabsicht als auch die geplante Besuchsdauer in einer spezifischen Fremdenverkehrsregion. Dabei unterscheidet sich die Besuchsverhaltensrelevanz von Einstellungen deutlich in Abhängigkeit von ihrer Art. Es ist somit zwischen spezifischen und unspezifischen Einstellungen zu unterscheiden.

Unsperifische Einstellungen 190 umfassen beispielweise generelle Einstellungen $z u$ Urlaubsreisen, Kurzurlaubsreisen oder - im speziellen Falle - zu Deutschlandreisen. Aus ihnen lassen sich Hinweise ableiten, inwieweit eine bestimmte Reise überhaupt in Frage kommt, welche Formen von Reisen generell bevorzugt werden sowie ob ein Deutschlandaufenthalt als mögliche Alternative überhaupt in Betracht gezogen wird. Für die Marktsegmentierung einer spezifischen Fremdenverkehrsregion sind solche unspezifischen Einstellungen jedoch wenig geeignet, weil sie keine Anhaltspunkte über spezifische Besuchsabsichten wiedergeben. Vielmehr kann das Besuchsverhalten nur anhand spezifischer Einstellungen, die einen Bezug zu den Ausprägungsformen einer Fremdenverkehrsregion aufweisen, analysiert werden. 191 In diesem Zusammenhang ist davon auszugehen, daß erst eine positive Einstellung zu einer

190 während die hier vorgestellten unspezifischen Einstellungen noch einen Urlaubs-/Reisebezug aufweisen, finden sich in der Konsumentenverhaltensforschung zudem allgemeine persönlichkeitsbezogene Einstellungen, die sich aufgrund ihrer übergeordneten Bedeutung zur Erklärung des Kaufverhaltens in einer vielzahl von Produktarten einsetzen lassen. Angesichts einer Vielzahl empirischer Belege, daß diese generellen Einstellungen kaum eine Kaufverhaltensrelevanz aufweisen, soll auf ihre Betrachtung verzichtet werden. Vgl. zur Kritik an der Verwendung genereller Einstellungen im Rahmen der Einstellungsforschung und den empirischen Belegen Freter, H., Marktsegmentierung, a.a.0., s. 65 .

191 Vgl. Kroeber-Riel, w., Konsumentenverhalten, a.a.o., 1984 , S. 171 . 
Fremdenverkehrsregion die grundlegende Voraussetzung für ihre Wahrnehmung als Alternative darstellt.

Neben der Differenzierung in spezifische und unspezifische Einstellungen können diese hinsichtlich ihres Entstehungsprosesses eingeteilt werden. Demnach sind durch direkte oder indirekte Erfahrungen gelernte Einstellungen $z u$ unterscheiden, die sich in ihrem Einfluß auf das Besuchsverhalten in eine spezifische Fremdenverkehrsregion unterscheiden. 192

Indirekte Erfahrungen entstehen durch Kommunikation, beispielsweise im Rahmen von Gesprächen mit Freunden und Bekannten, durch Berichterstattungen über Fremdenverkehrsthemen in den Medien oder durch Informationsbeschaffung uber Prospekte von Reiseveranstaltern. Angesichts der das ganze Jahr über andauernden Bereitschaft zur Informationsaufnahme von Fremdenverkehrsthemen, 193 erwirbt der Tourist laufend neue Erfahrungen über Fremdenverkehrsangebote und -regionen, so daß es kontinuierlich zu Einstellungsveränderungen kommt. Dieses ist als Grund dafür zu sehen, daß auf der Basis von Einstellungen nur eingeschränkt Hinweise auf das Besuchsverhalten abgeleitet werden können.194 In diesem Zusammenhang ist allerdings zu berücksichtigen, daß sich nur ein Teil der indirekten Erfahrungen in den Einstellungen verfestigt. Handelt es sich nur um flüchtige Eindrücke, so werden diese nicht als Einstellungen abgespeichert, so daß ihnen keine Besuchsverhaltensrelevanz zuzusprechen ist. Daher dürfen diese flüchtigen Eindrücke nicht zum Zwecke der Markterfassung angesetzt werden. Vielmehr ist darauf $z u$ achten, daß

192 Vgl. Krober-Riel, w., Konsumentenverhalten, a.a.0., s. 171.

193 Vgl. Datzer, R., Informationsverhalten von Urlaubsreisenden, a.a.0., S. 106 .

194 Vgl. McDougall, G.H.G., Munro, H., Scaling and Attitude Measurement in Tourism and Travel Research, a.a.0., S. 97. 
zur Markterfassung nur die aus umfangreichen Informationsverarbeitungsprozessen gelernten, zeitlich relativ stabilen Einstellungen herangezogen werden.

Auch den aus direkten Erfahrungen gelernten Einstellungen ist generell eine hohe Besuchsverhaltensrelevanz zuzusprechen. Empirische Untersuchungen der Konsumentenverhaltensforschung konnten in diesem Zusammenhang nachweisen, daB durch derartige Einstellungen das Kaufverhalten besser prognostiziert werden kann als durch aus indirekten Erfahrungen resultierende Einstellungen.195 Entgegen dieser Erkenntnis gehen einige Autoren davon aus, daB auf der Basis von positiven, durch eigene Erfahrungen gelernte Einstellungen zu einer spezifischen Fremdenverkehrsregion kaum auf eine erneute Besuchsabsicht geschlossen werden kann. 196 sie begründen dies damit, daB Touristen in der Regel bestrebt sind, neue Fremdenverkehrsregionen kennenzulernen. Somit steht die Realisierung neuer Erfahrungen im Vordergrund und zwar auch dann, wenn stabile Präferenzen für bestimmte Urlaubsformen bestehen, wie z.B. ein "typischer" Sommerurlaub (faulenzen, am Strand liegen). Demgegenüber zeigen jedoch empirische Ergebnisse, daß ein überdurchschnittlich hoher prozentsatz deutscher Touristen eine hohe zielgebietstreue aufweist. 197

195 Vgl. Fazio, R.N., Zanna, M.P., Direct Experience and Attitude-Behaviour-Relationship", in: Journal of Experimental Social Psychology, 1981, S. 398-408; zitiert aus Kroeber-Riel, W., Konsumentenforschung; a.a.O., S. 171; Marks, L.J., Kamins, M.A., The Use of Product Sampling and Advertising: Effects of Sequence of Exposure and Degree of Advertising Claim Exaggeration on Consumer's Belief Strength, Belief Confidence and Attitudes, in: Journal of Marketing Research, 1983, Heft 4, S. $266 \mathrm{ff}$.

196 Vgl. McDougall, G.H.G., Munro, H., Scaling and Attitude Measurement in Tourism and Travel Research, a.a.0., s. $97 \mathrm{f}$.

197 Im Jahre 1987 gaben immerhin 60 \% der Haupturlaubsreisenden an, ihr Reiseziel bereits von einer früheren Reise her zu kennen. Vgl. Dundler, F., Urlaubsreisen 1987, a.a.O., S. 41; Vgl. auch den in Kapitel B.2 iden- 
Es kann daher davon ausgegangen werden, daß der wiederholte Besuch der gleichen Fremdenverkehrsregion vor allem auf aus eigenen Erfahrungen gelernte Einstellungen zurückzuführen ist.

Inwieweit sich die aus direkten Erfahrungen resultierenden Einstellungen als verhaltenswirksam erweisen, hängt, so wird vermutet, von den persönlichkeitsfaktoren des jeweiligen Touristen ab. 198 Dazu zählt die generelle Risikoneigung und das hieraus resultierende bezüglich einer Reise wahrgenommene Risiko. Dieses entsteht dadurch, daß der Tourist aufgrund ihm verfügbarer Informationen Abweichungen zwischen seinen Erwartungen an eine Reise und den voraussehbaren Folgen eines Besuches wahrnimmt. 199 Da eine Fremdenverkehrsregion nicht vorab getestet werden kann, sind die Folgen eines Besuches nur eingeschränkt abzuschätzen. Daher werden vermutlich risikoaverse Personen, die mit einer Fremdenverkehrsregion eigene positive Erfahrungen gemacht haben, diese stärker für einen erneuten Besuch in Betracht ziehen als risikofreudige Personen. Ferner ist davon auszugehen, daß soziodemographische Merkmale den Zusammenhang zwischen Einstellungen und Besuchsverhalten erklären können, so daß von einer ausreichenden Identifizierbarkeit von Einstellungen ausgegangen werden kann. 200

tifizierten zusammenhang zwischen zielgebietserfahrung und Besuchsabsicht für die vorliegende Untersuchungsregion.

198 Vgl. Kroeber-Riel, W., Konsumentenverhalten, a.a.O., s. 173.

199 Vgl. zur begrifflichen Abgrenzung des wahrgenommenen Risikos Meffert, H., Marketingforschung und Käuferverhalten, a.a.O., S. 69 f.; Bauer, R.A., Konsumentenentscheidungen als Risikoverhalten, in: Specht, K.G., Wiswede, G. (Hrsg.), Marketing-Soziologie - Soziale Interaktionen als Determinanten des Marktverhaltens, Berlin 1976, S. $209 \mathrm{ff}$.

200 Dieser Zusammenhang ergibt sich aus den zahlreichen Ergebnissen zur Einstellungsforschung. vgl. z.B. Schnet- 
Neben dem bisher aufgezeigten Zusammenhang zwischen Einstellungen und Besuchsverhalten stellt sich die Frage, welchen Teileinstellungen bei der Auswahl von Fremdenverkehrsregionen eine besondere Bedeutung zukommt. In der Fremdenverkehrsforschung wird davon ausgegangen, $\operatorname{da} \beta$ dem natürlichen Angebot die gröBte Bedeutung im Rahmen des Auswahlprozesses von Fremdenverkehrsregionen zuzusprechen ist. 201 tuberträgt man diese Erkenntnis auf die Untersuchungsregion, so ist zu vermuten, daß Besucher eine positivere Einstellung zum natürlichen Angebot aufweisen als Nicht-Besucher. Dabei ist von einem entsprechenden Zusammenhang auch bezüglich der anderen Angebotskomponenten einer Fremdenverkehrsregion, wie z.B. der suprastruktur sowie sonstiger sport- und Freizeitangebote auszugehen.

Ferner ist $z u$ vermuten, daß Einstellungen $z u$ einer Fremdenverkehrsregion einen EinfluB auf die Besuchsdauer ausüben. Da mit Urlauben, Kurzurlauben und Tagesausflügen die Befriedigung unterschiedlicher Bedürfnisse angestrebt wird, ist von einer Heranziehung unterschiedlicher Entscheidungskriterien auszugehen. Dies legt die schluBfolgerung nahe, daB sich in Abhängigkeit von der Besuchsdauer unterschiedliche Teileinstellungen als verhaltensrelevant erweisen. Dabei kann generell vermutet werden, daß mit einer positiveren Einstellung gegenüber Angebotskomponenten einer Fremdenverkehrsregion auch die Besuchsdauer steigt. Es ist zu vermuten, daß mit längeren Aufenthaltsdauern den Übernachtungs-

kamp, G., Einstellungen und Involvement als Bestimmungsfaktoren des sozialen Verhaltens, a.a.O., S. 123 ff.; Kook, w., Einstellungen zur Universität, a.a.0., s. 71 ff., Meffert, H. (Hrsg.), Das Meinungsbild einer stadtbevölkerung über öffentliche Institutionen - Empirische Studie von Einstellungen der Münsteraner Bevölkerung gegenüber Universität, Stadttheater und Landesmuseum, Münster 1979, S. 21 ff.

201 Vgl. Kaspar, C., Fremdenverkehrslehre im GrundriB, a.a.0., S. 78 f.; Tschiderer, F., Ferienortplanung, a.a.o., s. $12 \mathrm{ff}$. 
möglichkeiten eine zunehmend größere Bedeutung zugesprochen wird.

Die aufgeführten zusammenhänge sollen anhand der folgenden Hypothesen zum Einfluß von Einstellungen auf das Besuchsverhalten empirisch überprüft werden.

Basishypothese zum Einfluß soziodemographischer Merkmale auf die Ausprägungen von Einstellungen: 202

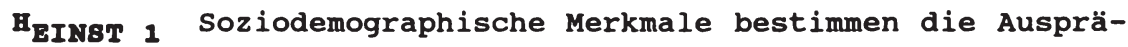
gungsformen von Einstellungen.

Basishypothese zum EinfluB von Einstellungen auf die Besuchsabsicht:

HEIN8T 2 Es besteht ein positiver Zusammenhang zwischen den Teileinstellungen zur Fremdenverkehrsregion und der Besuchsabsicht.

Aus dieser Basishypothese leiten sich folgende Hypothesen $a b$ :

HEN8T 2a Je positiver das natürliche Angebot eingeschätzt wird, desto stärker wird in beiden Entfernungsgruppen eine Besuchsabsicht geäuBert.

202 Auf eine Ableitung von Hypothesen soll an dieser stelle verzichtet werden. Da dieser Untersuchung eine spezifische Region zugrundeliegt, können allgemeine Tendenzaussagen $z u$ dem Zusammenhang zwischen Einstellungen und soziodemographischen Merkmalen nicht abgeleitet werden. So ist dieser Zusammenhang sowohl von dem touristischen Angebot der Fremdenverkehrsregion, von dem Wissen der Touristen über die Angebotskomponenten als auch von ihren Urlaubsmotiven abhängig. 


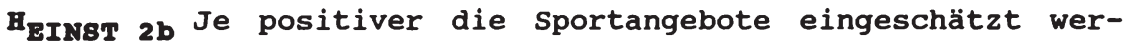
den, desto stärker wird in beiden Entfernungsgruppen eine Besuchsabsicht geäußert.

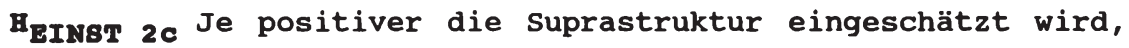
desto stärker wird in beiden Entfernungsgruppen eine Besuchsabsicht geäuBert.

Basishypothese zum EinfluB von Einstellungen auf die Besuchsdauer:

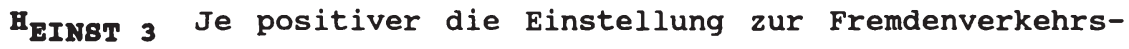
region, um so länger ist die Besuchsabsicht.

Hieraus leiten sich folgende Hypothesen ab:

HeINgT 3a Je positiver die Einstellung zum natürlichen Angebot, desto länger ist in beiden Entfernungsgruppen die beabsichtigte Besuchsdauer.

HEIN8T 3b Je positiver die Einstellung zum Freizeitangebot, desto länger ist in beiden Entfernungsgruppen die beabsichtigte Besuchsdauer.

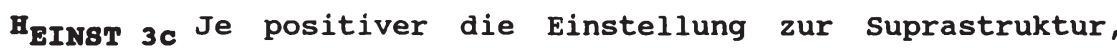
desto länger ist in der Entfernungsgruppe II die beabsichtigte Besuchsdauer. 


\subsection{Operationalisierung von Binstellungen}

Einstellungen stellen nicht beobachtbare Größen und damit theoretische Konstrukte dar. Daher müssen Indikatoren gefunden werden, die Aussagen über deren Existenz, Inhalt und Ausprägungen ermöglichen. Ferner gilt es im Rahmen der operationalisierung von Einstellungen, die Dimension der Messung sowie das skalenniveau festzulegen. 203

Bei der Festlegung der Dimensionen von Einstellungen können eindimensionale und mehrdimensionale Messungen unterschieden werden. Die eindimensionalen Messungen beziehen sich nur auf einen Aspekt der Einstellung, die sich in einer zustimmenden oder ablehnenden Haltung zur Fremdenverkehrsregion äuBert. 204 Hierbei wird i.d.R. nur die affektive Komponente der Einstellung in Form eines Gesamteindrucks gemessen.

Die eindimensionale Einstellungsmessung ist jedoch zum Zweck der Marktsegmentierung eher ungeeignet, weil sie keine Aussagen über die Einstellungen zu den Teileigenschaften einer Fremdenverkehrsregion liefert. Um ausreichend Ansatzpunkte zur zielgruppenspezifischen Marktbearbeitung ableiten zu können, erweist sich demnach die mehrdimensionale Messung von Einstellungen als das zweckmäßigere Vorgehen.

In der Einstellungsforschung sind eine Vielzahl unterschiedlicher mehrdimensionaler Modelle entwickelt worden. Im folgenden sollen die sog. direkten Verfahren im Vordergrund der

203 Vgl. Kroeber-Riel, w., Konsumentenverhalten, a.a.o., s. 183.

204 Die bekanntesten eindimensionalen skalierungsverfahren sind die Methode der gleicherscheinenden Intervalle von Thurstone, die Methode der summierten Ratings von Likert sowie die Guttmann-Technik. Einen ausführlichen Überblick über die eindimensionalen skalierungsverfahren findet sich bei Triandis, H.C., Einstellungen und Einstellungsveränderungen, a.a.0., S. $58 \mathrm{ff}$. 
Betrachtung stehen, in denen die Touristen bei einer expliziten Vorgabe relevanter Eigenschaften die wahrgenommenen Eigenschaftsausprägungen der Fremdenverkehrsregion auf vorgegebenen skalen bewerten müssen. 205

Die Vielzahl der in der Einstellungsforschung entwickelten Modelle zur mehrdimensionalen Messung von Einstellungen unterscheiden sich vor allem darin, daB die affektiven und kognitiven Komponenten in unterschiedlicher Weise miteinander verknüpft werden.206 In Anlehnung an Freter können zwei Gruppen von Ansätzen unterschieden werden: Modelle, die kein "Idealprodukt" berücksichtigen und Modelle, welche die Eigenschaften eines solchen hypothetischen Idealproduktes in die Analyse einbeziehen. 207

205 Ferner finden in der Einstellungsforschung indirekte Verfahren Verwendung. Bei den indirekten, auch als dekompositorische Modelle bezeichneten Verfahren wird versucht, aufgrund von Ähnlichkeits- oder Präferenzangaben der Befragten die relevanten Dimensionen eines Eigenschaftsraumes indirekt $z u$ bestimmen. Hierzu werden die Verfahren der Multidimensionalen skalierung und der conjoint-Analyse herangezogen. Vgl. zu den beiden Verfahren Backhaus, K. U.a., Multivariate Analysemethoden, a.a.o., S. $317 \mathrm{ff}$. und S. $345 \mathrm{ff}$.

206 Diese lassen sich ferner in kompensatorische oder nichtkompensatorische Modelle differenzieren. Eine zusammenfassende Beurteilung dieser Modelle findet sich bei Kook, W., Einstellungen zur Universität, a.a.0., S. 137 ff.

207 Vgl. Freter, H., Interpretation und Aussagewert mehrdimensionaler Einstellungsmodelle im Marketing, in: Meffert, H., steffenhagen, H., Freter, H. (Hrsg.), Konsumentenverhalten und Information, Wiesbaden 1979, S. 164 ; Freter, H., Mehrdimensionale Einstellungsmodelle im Marketing. Interpretation, Vergleich und Aussagewert, Arbeitspapier $\mathrm{Nr}$. 12 des Instituts für Marketing, Hrsg.: H. Meffert, Münster 1976, S. $3 \mathrm{f}$. 
Das bekannteste Modell, welches kein Idealprodukt enthält, stammt von Fishbein. 208 Das wichtigste der Idealpunkt-Modelle stammt von Trommsdorff.209 Im folgenden sollen zur Charakterisierung der Grundstruktur von Einstellungsmodellen diese beiden Modelle kurz dargestellt werden.

Im Modell von Pishbein werden subjektive Wahrscheinlichkeiten über das Vorhandensein von Produkteigenschaften, die die kognitiven Elemente der Einstellung darstellen, mit den korrespondierenden subjektiven (affektiven) Bewertungen dieser Eigenschaften multiplikativ verknüpft. 210 Dabei wird davon ausgegangen, daß sich die Motive in der subjektiven Bewertung der Eigenschaften äußern. Der hierdurch gebildete Eindruckswert gibt an, inwieweit eine Eigenschaft wahrgenommen und positiv oder negativ eingeschätzt wird. 211

208 Ein weiteres bekanntes Modell ohne Idealprodukt stammt von Rosenberg. Vgl. Rosenberg, M.J., Cognitive structure and Attitudinal Affect, in: Journal of Abnormal and Social Psychology, 1956, S. 367 ff; Rosenberg, M.J., Inconsistency Arousal and Reduction in Attitude Change, in: Steiner, I.D., Fishbein, M. (Hrsg.), Current studies in Social Psychology, New York 1965, S. $121 \mathrm{ff}$; beide zitiert aus Freter, H., Interpretation und Aussagewert mehrdimensionaler Einstellungsmodelle im Marketing, a.a.0., s. 164 .

209 Die Übernahme dieser Grundmodelle in die Käuferverhaltensforschung hat zu einer Fülle von Varianten geführt. Für einen zusammenfassenden Überblick vgl. Freter, H., Mehrdimensionale Einstellungsmodelle im Marketing, a.a.0., s. 4 ff.

$210 \mathrm{Vgl}$. Fishbein, M., A Behaviour Theory Approach to the Relations between Beliefs about an objekt and the Attitude toward the object, in: Readings in Attitude Theory and Measurement, Hrsg.: Fishbein, M., New York, u.a. 1967 , S. 389 ff.

211 In formaler Schreibweise hat das Modell folgendes Aussehen: $A_{i j}=$ Summe aus $B_{i j k}{ }^{\prime} a_{j j k}$ mit $B_{i j k}$ als Wahrscheinlichkeit, mit der Person'l Elgenschaftjk an objekt $j$ für vorhanden hält und $a_{i j k}$ für die Bewertung von Eigenber-Riel, W., Konsumentenverhalten, a.a.O., S. $194 \mathrm{f}$. und die dort angegebene Literatur. 
Der Ansatz von Fishbein hat in der Folge zu zahlreichen Modellvarianten geführt, die sich zwar in der Grundstruktur ähneln, aber durch andere Operationalisierungen der Modellkomponenten gekennzeichnet sind. Am häufigsten fand dabei das "adequacy-importance-Modell" in der Konsumentenverhaltensforschung Anwendung. Es stellt darauf $a b$, in welchem Ausmaß (mengenmäßige Ausprägung) vorgegebene Eigenschaften bei einem objekt vorhanden sind. Diese einzelnen Eigenschaften sind nach ihrer Bedeutung zu gewichten. 212

Eine Reihe empirischer Untersuchungen in unterschiedlichen Produktfeldern hat insgesamt $z u$ dem Ergebnis geführt, daß die prognostische Relevanz mehrdimensionaler Einstellungsmodelle durch die Einbeziehung von Bedeutungsgewichten keineswegs immer verbessert wird.213 Im Hinblick auf den eingeschränkten Aussagewert insbesondere der Bedeutungskomponente wird in der Literatur unter anderem angeführt, daB bei der Beurteilung der wahrgenommenen Eigenschaftsausprägungen implizit die Bedeutungsgewichte berücksichtigt werden. Dies führt im Ansatz von Fishbein zu einer doppelten Berücksichtigung von Bedeutungsgewichten. 214 Um die Vor- und Nachteile

212 Vgl. Freter, H., Mehrdimensionale Einstellungsmodelle im Marketing, a.a.0., s. 6 f. Übertragen auf eine Fremdenverkehrsregion ist damit z.B. die Fragestellung angesprochen "wieviele Hotels gibt es in einer Fremdenverkehrsregion" und "welche Bedeutung wird den Hotels zugesprochen".

213 Vgl. Trommsdorff, V., Die Messung von Produktimages für das Marketing. Grundlagen und operationalisierung, Köln u.a. 1975, S. $63 \mathrm{ff}$, Sheth, J.N., Talarzyk, W.W., Perceived Instrumentality and Value Importance as Determinants of Attitudes, in: Journal of Marketing Research, Vol. 9, 1972, S. 6 ff., Bass, F.M., Talarzyk, W.W., An Attitude Model for the study of Brand Preference, in: Journal of Marketing Research, Vol. 9, 1972, s. 93 ff., Lehmann, D.R., O'Shaughnessy, J., Difference in Attribute Importance for Different Industrial Products, in: Journal of Marketing, Vol. 38, 1974, No. 2, S. $36 \mathrm{ff}$.

$214 \mathrm{Vgl}$. Freter, H., Mehrdimensionale Einstellungsmodelle im Marketing, a.a.0., s. 33 . 
einer multiplikativen Verknüpfung der Eigenschaftsausprägungen mit entsprechenden Bedeutungsgewichten hat sich in diesem Zusammenhang ein Meinungsstreit entwickelt, der als "Gewichtungskontroverse" Eingang in die Literatur fand.215 Angesichts der sehr kritischen Bewertung dieser Ansätze soll auf eine Analyse der Übertragbarkeit von Modellen, die Bedeutungsgewichte berücksichtigen, auf den vorliegenden Untersuchungsgegenstand verzichtet werden. 216

Zur Vermeidung der meßtechnischen Nachteile hat Trommsdorff einen Ansatz entwickelt, der hypothetische Idealeinstellungen berücksichtigt. 217 Das Modell beruht auf der Annahme, daß sich Konsumenten bei der Bewertung eines Produktes an einem produktart-typischen Idealbild orientieren. Anstelle einer multiplikativen Verknüpfung der affektiven und kognitiven Komponente werden bei Trommsdorff Distanzen zwischen Real- und Idealeindruck von objekteigenschaften ermittelt. Das Modell hat folgende Formalstruktur:

$$
E_{i j}=\sum_{k}\left|B_{i j k}-I_{i k}\right|
$$

215 Vgl. z.B. Trommsdorff, V., Die Messung von Produktimages für das Marketing, a.a.o., S. $63 \mathrm{ff}$.

216 Vgl. zur ausführlichen Kritik an den Meßmodellen von Rosenberg und Fishbein sowie den hinter diesen Modellen stehenden Prämissen Trommsdorff, V., Die Messung von Produktimages für das Marketing, a.a.0., S. 48 ff.; ferner soll auch das Lehman-Modell keine weitere Berücksichtigung erfahren, bei dem die Eigenschaftsbedeutung mit der Distanz zwischen Real- und Idealausprägung multipliziert wird. Vgl. hierzu Freter, H., Interpretation und Aussagewert mehrdimensionaler Einstellungsmodelle im Marketing, a.a.0., S. 169 ff.

$217 \mathrm{Vgl}$. Trommsdorff, V., Die Messung von Produktimages für das Marketing, a.a.0., s. $72 \mathrm{ff}$. 
$\mathrm{E}_{\mathrm{ij}}$ ist die geschätzte Einstellung von einer Person (einem Touristen) $i$ gegenuber einer Marke (Fremdenverkehrsregion) $j$.

$B_{i j k}$ ist die von der Person (dem Touristen) $i$ wahrgenommene Ausprägung der Marke (Fremdenverkehrsregion) j mit dem einstellungsrelevanten Merkmal k.

I ik ist die von der Person (dem Touristen) i bei Fremdenverkehrsregionen des Typs $j$ als ideal empfundene Ausprägung des Merkmals k.

Es ist in diesem Zusammenhang jedoch $z u$ berücksichtigen, da $B$ Idealeinstellungen keinen eigenständigen Ansatzpunkt zur Erfassung von Einstellungen darstellen. Die Einbeziehung von Idealeinstellungen führt vielmehr durch die Differenzenbildung zur Erfassung der motivationalen Komponente von Einstellungen. Dabei wird davon ausgegangen, daB die Einstellung eines Konsumenten gegenüber einem Einstellungsobjekt um so positiver ist, je kleiner die Distanz zwischen Ideal- und Realeinstellungen ist. 218

Die gleiche Interpretation negativer und positiver Abweichungen ist jedoch kritisch zu beurteilen.219 Es erscheint sehr unwahrscheinlich, daß im Fremdenverkehr eine negative Abweichung vom Realeindruck (Untererfüllung) zum Beispiel bezogen auf das natürliche Angebot einer Fremdenverkehrsregion genauso bewertet wird wie eine gleich groBe Abweichung in positiver Richtung (Übererfüllung).

218 Bedingt durch die Absolutdarstellung werden negative und positive Abweichungen gleich behandelt.

219 Es ist $z u$ vermuten, $d a \beta$ aus diesem Grunde in der Qualitäts- und Zufriedenheitsforschung nicht-kompensatorische Modelle verwendet werden. Vgl. Parasuraman, A., Zeithaml, V.A., Berry, L.L., A Conceptual Model of Services Quality and its Implications for Future Research, in: Journal of Marketing, 1985, Heft 4, S. 48 f.; Büker, B., Qualitätsbeurteilung investiver Dienstleistungen, a.a.0., s. 80 . 
Der Verzicht auf eine Absolutdarstellung ist jedoch mit dem Nachteil verbunden, daB sich positive und negative Abweichungen kompensieren können. 220 Daher ist die Berechnung eines Gesamteinstellungswertes wenig zweckmäBig, allerdings zum Zwecke der Marktsegmentierung auch nicht erforderlich. Das globale EinstellungsmaB summativer Einstellungsmodelle gibt weder AufschluB über den Inhalt noch über die Beiträge der Einstellungen $z u$ den einzelnen Angebotskomponenten einer Fremdenverkehrsregion. 221 Diese Informationen werden jedoch sowohl für die Markterfassung als auch vor allem für die Ableitung von Ansatzpunkten einer zielgruppenspezischen Marktbearbeitung benötigt.

Der Vorteil dieses Modells sieht Trommsdorff darin, daß unabhängig voneinander Erkenntnisse über kognitiv und motivational geprägte Einstellungsaspekte gewonnen werden, die Ansatzpunkte zur Einstellungsbeeinflussung liefern. 222 Die Bewertung der Eigenschaften erfolgt nicht direkt. Vielmehr wird eine indirekte Bewertung der Produkteigenschaften vorgenommen, indem die tatsächlich wahrgenommenen Ausprägungen eines Produktmerkmals zur Ausprägung dieses Merkmals bei einem idealen Produkt in Beziehung gesetzt werden.

Die Anwendbarkeit dieses Modells hängt demnach davon ab, inwieweit der Konsument in der Lage ist, tatsächlich wahrgenommene Ausprägungen eines Produktes (real) anzugeben, ohne eine Bewertung vorzunehmen. Gerade in dieser Prämisse kommt

220 In diesem Zusammenhang erscheint es zum Beispiel denkbar, im Rahmen des MeBmodells Utbererfüllungen und Erfüllungen gleichzusetzen. Allerdings wird dann davon ausgegangen, daB eine Übererfüllung gegenüber der Erfüllung keinen zusätzlichen EinfluB auf das Besuchsverhalten ausubt.

221 In Anlehnung an Böhler, H., Methoden und Modelle der Marktsegmentierung, a.a.0., s. 94 .

222 Vgl. Trommsdorff, V., Die Messung von Produktimages für das Marketing, a.a.0., s. 75 . 
die Schwierigkeit der Übertragung des Modells auf den Fremdenverkehrbereich zum Ausdruck.

So besteht die Besonderheit im Fremdenverkehr darin, daß eine Fremdenverkehrsregion vor dem eigentlichen Besuch nicht gesehen, und damit nicht getestet werden kann. Dies bedeutet, daß Touristen keine objektiven Aussagen über das Vorhandensein von spezifischen Ausprägungen einer Fremdenverkehrsregion - sofern diese nicht früher schon einmal besucht wurden - treffen können. Daher kann vermutet werden, daß bei der Angabe der Ausprägungsformen einer Fremdenverkehrsregion insbesondere gefühlsmäßige Bewertungen mit einfließen. Ist dies tatsächlich der Fall, so würde bei der übertragung des Modells auf den Fremdenverkehr die affektive Komponente überbetont: einerseits durch die Erfassung wahrgenommener Ausprägungen von Produkt-bzw. Regioneneigenschaften, andererseits über die Ideal-Realdifferenzen. Hieraus folgt, da die Kritik an den Modellen, die Bedeutungsgewichte berücksichtigen, im Fremdenverkehr auch für die Ideal-Real-Differenzen zutrifft. Somit müßten die auf der Basis von IdealReal-Differenzen ermittelten Teileinstellungen einen geringeren Erklärungsbeitrag zum Besuchsverhalten liefern, als wenn nur die Realeinstellungen Berücksichtigung fänden.223 Kann in der empirischen Untersuchung tatsächlich nachgewiesen werden, daß Realeinstellungen eine höhere Verhaltensre-

223 Auch neueste Ergebnisse in der Zufriedenheits- und Qualitätsforschung weisen darauf hin, daß die Messung der Qualität eines Produktes über die Differenz von erwarteter und wahrgenommener Qualität nicht zwingend zur Verhaltensprognose geeignet ist. So konnte nachgewiesen werden, daB die Distanzbildung aus erwarteter und wahrgenommener Qualität sich als schlechterer Indikator für die Erklärung des Kaufverhaltens erweist, als wenn nur die wahrgenommene Qualität berücksichtigt wird. Vgl. Berry, L.L., Parasuraman, A., Zeithaml, V.A., Moving Forward in Quality Research. Presentation Handouts for Marketing science Institute Conference, supporting service Quality, Boston 1992 (unveröffentlichtes Manuskript). 
levanz als Ideal-Real-Differenzen aufweisen, so sind einer psychographischen Marktsegmentierung die Realeinstellungen zugrunde zu legen.

Zur Erfassung der Einstellungen ist es erforderlich eine geeignete Auswahl von Indikatoren vorzunehmen. Dabei müssen die anzusetzenden Eigenschaften folgenden Anforderungen genügen: 224 sie sollten insbesondere

- aus Konsumentensicht relevante Kriterien darstellen

- eine Diskriminierungsfähigkeit zwischen verschiedenen Fremdenverkehrsregionen aufweisen 225

- vollständig und unabhängig voneinander sein

- einen Bezug zum Einsatz des absatzpolitischen Instrumentariums aufweisen.

Um einen Bezug zum Einsatz der absatzpolitischen Instrumente zu gewährleisten, erscheint es zweckmäßig, als Indikatoren für die Einstellungen die Teileinstellungen zu den Angebotskomponenten der Fremdenverkehrsregion heranzuziehen. Da während des Aufenthalts in einer Region deren Angebotskomponenten von den Touristen in Anspruch genommen werden, kann davon ausgegangen werden, $\mathrm{da} \beta$ es sich um konsumrelevante Eigenschaften handelt.

$224 \mathrm{Vgl}$. Waltermann, B., Internationale Markenpolitik und Produktpositionierung. Markenpolitische Entscheidungen im europäischen Automobilmarkt, Wien 1989, S. 99 f.; Böhler, H., Beachtete Produktalternativen und ihre relevanten Eigenschaften im Kaufentscheidungsproze $B$ von Konsumenten, in: Meffert, H., Steffenhagen, H., Freter, H. (Hrsg.) Konsumentenverhalten und Information, a.a.0., s. $265 \mathrm{f}$.

225 Auf die Diskriminierungsfähigkeit soll im folgenden nicht weiter eingegangen werden, da in der vorliegenden Untersuchung die Einstellungen nur zu der Untersuchungsregion ermittelt wurden. 
Um die genannten Aspekte überprüfen zu können, ist es erforderlich, die Ideal- und Realeinstellungen anhand der gleichen Indikatoren $\mathrm{zu}$ erfassen und eine Differenzenbildung vorzunehmen. In der Einstellungsforschung dominiert hinsichtlich der dargestellten Einstellungsmodelle die Erfassung der Einstellung über Ratingskalen. 226 Im folgenden soll eine fünf-stufige Ratingskala zur Erfassung der Indikatoren herangezogen werden. Die Idealeinschätzungen werden ubber die Frage, aufgrund welcher Eigenschaften sich die Befragten besonders im Urlaub wohlfühlen, ermittelt. Demgegenüber werden die Realeinstellungen über die Einschätzung vorgegebener Eigenschaften der Fremdenverkehrsregion erfaBt. 227

Das Fremdenverkehrsangebot einer Fremdenverkehrsregion läBt sich in das natürliche und das abgeleitete Angebot unterteilen. 228 In der Fremdenverkehrsforschung wird davon ausgegangen, daB das natürliche Angebot den "Kristallisationspunkt" der touristischen Nachfrage darstellt. Deshalb ist vor allem die Einstellung zum natürlichen Angebot dazu geeignet, die Besuchsabsicht und die Besuchsdauer zu erklären.

Als Indikatoren für die Einstellung $z u$ einer Fremdenverkehrsregion sollen die in Abbildung 25 dargestellten, den Hauptangebotskomponenten zugrundeliegenden Teileigenschaften dieser Fremdenverkehrsregion herangezogen werden.

Inwieweit diese Eigenschaften der Anforderung der Unabhängigkeit entsprechen, ist allerdings kritisch zu beurteilen. Angesichts des Komplementaritätsverhältnisses touristischer Leistungen ist davon auszugehen, daß die Unabhängig-

226 Vgl. Kroeber-Riel, W., Konsumentenverhalten, a.a.O., s. $186 \mathrm{f}$.

$227 \mathrm{Vgl}$. Fragebogen im Anhang II

228 Vgl. Kapitel A.2 dieser Arbeit. 
- Natürliches Angebot

-Ökologisch intakte Landschaft

-Abwechslungsreiche Landschaft

-Freundlichkeit der Menschen

-Wetter

\section{- Suprastruktur}

-Vielfältige Gastronomie

-Qualität der Beherbergungsstätten

\section{- Sonstige fremdenverkehrsspezifische Infrastruktur (Freizeitangebote)}

-Kulturelles Angebot

-Erholungs- und Freizeitparks

-Historische Sehenswürdigkeiten

-Wandermöglichkeiten

-Einkaufsmöglichkeiten

-Sportmöglichkeiten

-Radwandermöglichkeiten

Abb. 25: Indikatoren zur Erfassung von Einstellungen zu einer Fremdenverkehrsregion

keitsprämisse im Rahmen der Einstellungsmessung im Fremdenverkehr nur zum Teil erfüllt werden kann.229 zur Gewährleistung der Unabhängigkeit der in die segmentierung eingehenden Einstellungen ist es daher erforderlich, die Einstellungsindikatoren $\mathrm{zu}$ Faktoren $\mathrm{zu}$ verdichten. 230

229 Waltermann weist jedoch darauf hin, daB die Auswahl der Indikatoren in erster Linie den Erfordernissen der Relevanz aus Konsumentensicht entsprechen muB. Vgl. Waltermann, B., Internationale Markenpolitik und Produktpositionierung, a.a.0., s. 100 .

230 In diesem Zusammenhang ist mit Blick auf die Verwendung von Ideal-Real-Differenzen als mögliche segmentierungskriterien zu berücksichtigen, daß genaugenommen die Fak- 
In diesem Zusammenhang gilt es zu prüfen, ob sich diese aus Anbietersicht vorgenommene Klassifizierung möglicher Bezugsobjekte der Einstellung auch als Dimensionen der Einstellungen wiederfinden lassen. Hieraus leiten sich folgende Hypothesen $\mathrm{ab}$ :

HEIN8T \& Die Einstellungen lassen sich über die Teileinstellungen $\mathrm{zu}$ den Angebotskomponenten einer Fremdenverkehrsregion messen. Dabei lassen sich diese Teileinstellungen auf faktoranalytischem Wege zu Einstellungsfaktoren verdichten.

\subsection{Empirische Analyse der Eignung von Einstellungen als segmentierungskriterien}

\subsection{Kennzeichnung der Ausprägungsformen und struktur von Einstellungen}

Die Ausprägungen der Ideal- und Realeinstellungen sowie der Ideal-/Real-Differenzen sind in Abbildung 26 wiedergegeben. Betrachtet man zunächst nur die Idealeinstellungen gegenüber einer Fremdenverkehrsregion, so zeigt sich, daß den Angebotskomponenten des natürlichen Angebots und den Wandermöglichkeiten aus sicht der Touristen die größte Bedeutung zukommt. Hierin scheint sich die in der Literatur vertretene

toren der Ideal-Real-Eindruckswerte gebildet werden müßten. Eine Faktorbildung auf der Basis von Ideal-RealDifferenzen gibt jedoch keine Auskunft darüber, welche Angebotskomponenten einer Fremdenverkehrsregion von den Touristen gemeinsam wahrgenommen werden. Auf diese Weise können nur Muster von Abweichungen identifiziert werden. Vor diesem Hintergrund soll an entsprechender stelle lediglich eine Verdichtung der Realeinstellungen vorgenommen werden. Diese gibt Auskunft darüber, welche Angebotskomponenten der vorliegenden Untersuchungsregion im Verbund wahrgenommen werden. 


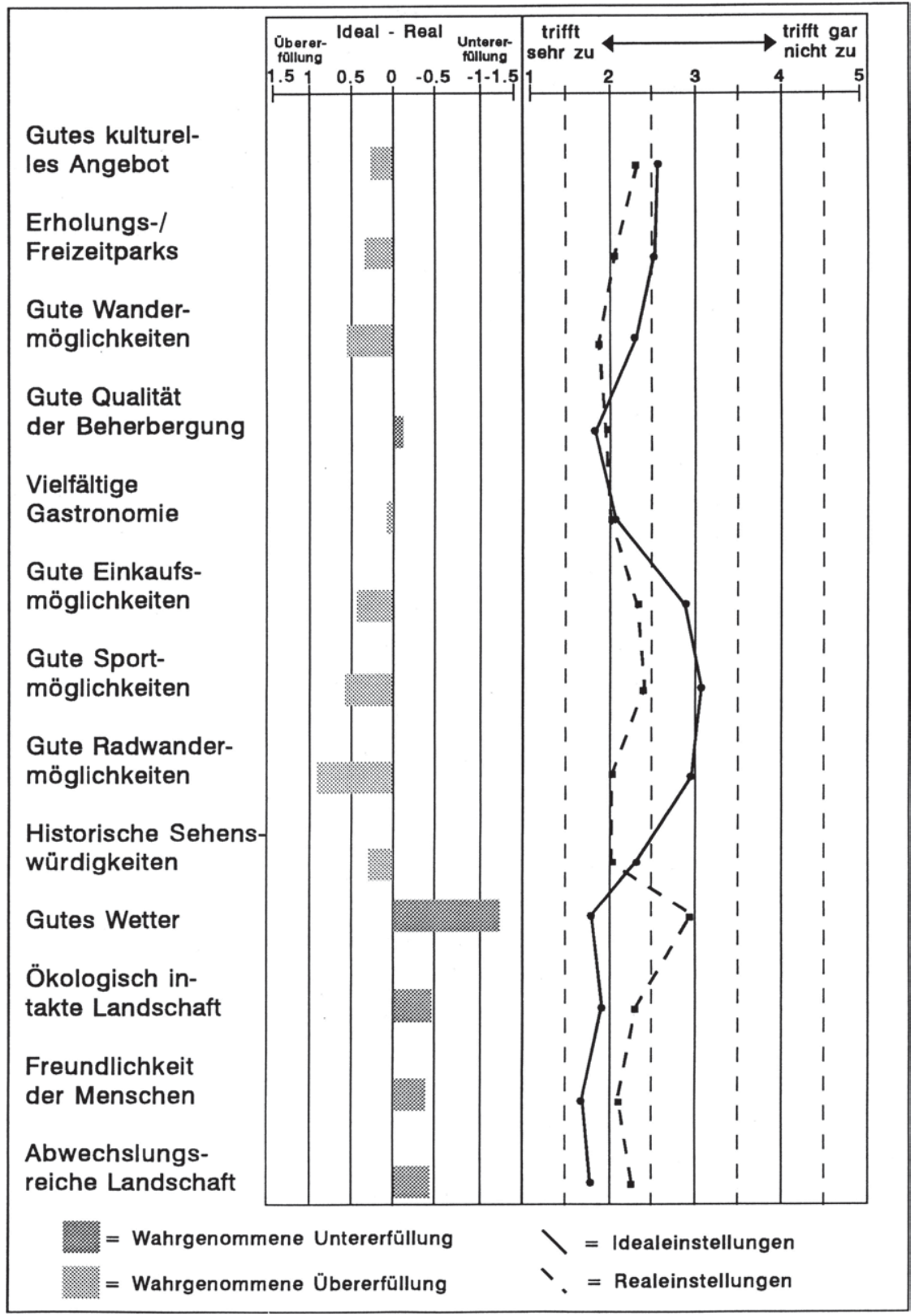

Abb. 26: Empirisch ermittelte Ideal- und Realeinstellungen 
These $z u$ bestätigen, daB das natürliche Angebot den "Kristallisationspunkt" der touristischen Nachfrage darstellt. Das Angebot an sport- und Radwandermöglichkeiten spielt in den Einstellungen von einer idealen Fremdenverkehrsregion demgegenüber eine nur untergeordnete Rolle. offensichtlich äuBert sich die in der Motivanalyse identifizierte unterdurchschnittliche Bedeutung von sportbedürfnissen auch in entsprechend geringeren Anforderungen an eine Fremdenverkehrsregion.

Die Realeinstellungen geben Auskunft über die relativen Stärken und Schwächen der Fremdenverkehrsregion. Die besten Bewertungen hinsichtlich der Untersuchungsregion erfahren dabei die Wandermöglichkeiten, die Qualität der Beherbergungsstätten, gefolgt von den historischen Sehenswürdigkeiten, der vielfältigen Gastronomie sowie den Radwandermöglichkeiten. Die Hauptschwäche der Untersuchungsregion liegt in den klimatischen Bedingungen (Wetter).

Allerdings lassen die geringen Mittelwertunterschiede in den Bewertungen der einzelnen Angebotskomponenten darauf schlieBen, daß die Befragten ein eher undifferenziertes Bild von der Untersuchungsregion haben. Es werden weder ausgeprägte stärken noch Schwächen wahrgenommen.

Die Betrachtung der Ideal-/Real-Differensen erlaubt Rückschlüsse darüber, inwieweit die von den Touristen gestellten Anforderungen an eine Fremdenverkehrsregion auch tatsächlich von der Untersuchungsregion erfüllt werden. Es zeigt sich, daB alle infrastrukturellen Angebotskomponenten der Fremdenverkehrsregion eine den Idealeinstellungen entsprechende bzw. übertreffende Bewertung erfahren. Auch die geringe wahrgenommene Abweichung zwischen Ideal- und Realeinstellung hinsichtlich der Beherbergungsqualität ist dahingehend $z u$ interpretieren, daB das Angebot den gestellten Anforderungen der Touristen entspricht. 
Demgegenüber zeigen sich deutlich negative Abweichungen hinsichtlich des natürlichen Angebots der Fremdenverkehrsregion. Hierbei handelt es sich um diejenigen Angebotsbestandteile, an die die höchsten Anforderungen gestellt werden.

In einem nächsten schritt sollen die Realeinstellungen zu einer Fremdenverkehrsregion auf Einstellungsfaktoren zurückgeführt werden. Abbildung 27 zeigt das aufbereitete Ergebnis einer nach dem Kaiser-Kriterium ermittelten drei-faktoriellen Lösung.

Auf den ersten Faktor entfallen sechs, vordergrünig recht heterogene Teileinstellungen zur Untersuchungsregion. In diesem Faktor werden sowohl die Einstellungen zu den kulturellen Angeboten, $z u$ den Einkaufsmöglichkeiten als auch $z u$ der suprastruktur zusammengefaBt. Die Teileinstellung zu den Erholungs- und Freizeitparks kann angesichts ihrer relativ geringen Faktorladung von 0,462 nur bedingt zur Interpretation dieses Faktors herangezogen werden. Insgesamt wird deutlich, daB infrastrukturelle Angebotskomponenten, die keinen Bezug zu sportangeboten aufweisen, von den Touristen im Verbund wahrgenommen werden. Offensichtlich wird die sportunabhängige touristische Infrastruktur in ihrer Gesamtheit bewertet. Angesichts eines erklärten Varianzanteils an der Gesamtvarianz in Höhe von 42,8\% kommt diesem Faktor zur Erklärung der Wahrnehmungsstruktur der Untersuchungsregion eine dominante Bedeutung zu.

Der zweite Faktor zeichnet sich durch hohe Faktorladungen bei den Produkteigenschaften "ökologisch intakte Landschaft", "gutes Wetter", "Freundlichkeit der Menschen" und "abwechslungsreiche Landschaft" aus. Er gibt Auskunft über die Einstellung sum natürlichen Angebot der Fremdenverkehrsregion. Allerdings entfällt auf diesen Faktor nur noch ein 

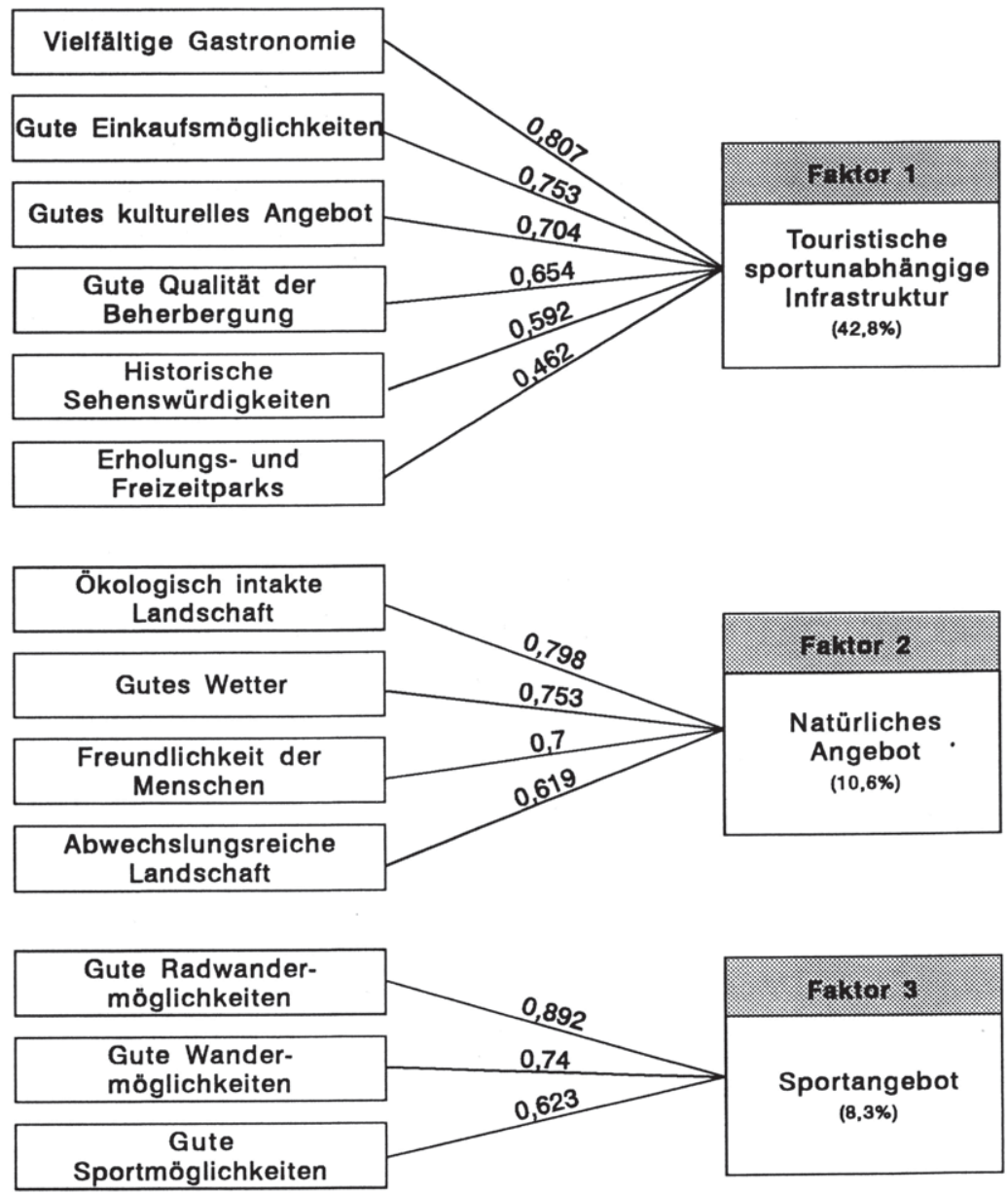

Erklärter Varianzanteil: $61,7 \%$

Abb. 27: Faktorenanalytische Verdichtung von Realeinstellungen 
erklärter Varianzanteil an der Gesamtvarianz in Höhe von $10,6 \%$.

Der geringste erklärte Varianzanteil $(8,6 \%)$ entfällt auf den dritten Faktor, der die Einstellung sum sportangobot in der Fremdenverkehrsregion wiedergibt. Er zeichnet sich durch hohe Faktorladungen bei den Teileinstellungen zu den Radwander-, Wander- und sportmöglichkeiten aus.

Die Faktorenstruktur macht deutlich, daß das in der Theorie dargestellte Komplementaritätsverhältnis touristischer Teilleistungen vor allem auf die touristische, sportunabhängige Infrastruktur zutrifft. Dabei werden zur Beurteilung einer Fremdenverkehrsregion die Infrastruktur und das natürliche Angebot jeweils in ihrer Gesamtheit beurteilt. Interessanterweise bestätigt diese Faktorlösung die aus angebotsorientierter sicht vorgenommene Einteilung in das natürliche Angebot und die touristische Infrastruktur. Unterschiede ergeben sich lediglich dadurch, daß nicht die suprastruktur, sondern das sportangebot einen eigenständigen Einstellungsfaktor darstellt. Insgesamt zeigt sich, daß sich die Realeinstellungen auf zentrale Einstellungsfaktoren zurückführen lassen, so daß die Basishypothese $\mathbf{H}_{\text {EINBT4 }}$ in ihrer Tendenz bestätigt werden kann.

In einem nächsten schritt ist zu überprüfen, inwieweit diese Einstellungen sich soziodemographisch erklären lassen und ihnen eine Besuchsverhaltensrelevanz zugesprochen werden kann. Dabei soll die Analyse auf Basis der Einstellungsindikatoren erfolgen. Nur so kann festgestellt werden, ob den Realeinstellungen oder den Ideal-/Real-Differenzen eine bessere Eignung als Segmentierungskriterium zugesprochen werden kann. Darüber hinaus sollen im Rahmen der Analyse der soziodemographischen Identifizierbarkeit und Besuchsverhaltensrelevanz die ermittelten Einstellungsfaktoren herangezogen werden. 
4.322 Analyse der soziodemographischen Identifizierbarkeit und des Einflusses von Einstellungen auf das Besuchsverhalten

Die Zusammenhänge zwischen den Einstellungsfaktoren und den soziodemographischen Merkmalen finden sich in Abbildung 28. Dabei zeigt sich, daB die Beurteilung der sportunabhängigen touristischen Infrastruktur keinen zusammenhang mit den demographischen und sozioökonomischen Merkmalen aufweist. Auch die geringen Korrelationskoeffizienten hinsichtlich Alter, Bildung und Netto-Haushalts-Einkommen deuten eher auf einen fehlenden Zusammenhang hin.

\begin{tabular}{|c|c|c|c|}
\hline \multirow[b]{2}{*}{ Merkmale } & \begin{tabular}{|c|} 
Touristische \\
sportunab- \\
hangige日 \\
Infrastruktur
\end{tabular} & $\begin{array}{l}\text { Natürliches } \\
\text { Angebot }\end{array}$ & $\begin{array}{l}\text { Sport- } \\
\text { angebot }\end{array}$ \\
\hline & 1 & 2 & 3 \\
\hline \multirow{4}{*}{$\begin{array}{l}\text { Demographische Merkmale } \\
\text { - Geschlecht } \\
\text { - Alter } \\
\text { - Kinderanzahl } \\
\text { - Lebenszyklusphase }\end{array}$} & n.S. & n.S. & n.S. \\
\hline & $\begin{array}{c}-0,074 \\
\star \star \\
\end{array}$ & & \\
\hline & n.S. & & n.s. \\
\hline & $-0,0$ & -0, & \\
\hline \multirow{5}{*}{$\begin{array}{l}\text { Sozioökonomische Merkmale } \\
\text {-Bildung } \\
\text {-Beruf } \\
\text { - Haushalt-Nettoeinkommen } \\
\text {-Persönl. Nettoeinkommen } \\
\text {-Schichtzugehörigkeit }\end{array}$} & 0,0 & $\begin{array}{c}0,107 \\
\star \star \star \star \\
\end{array}$ & -0 \\
\hline & +++ & + & n. \\
\hline & $-\underset{\star \star \star x}{-0,067}$ & $-0,06$ & $-0,106$ \\
\hline & n.S. & $-\underset{\star \star \star \star}{-0,113}$ & n.S. \\
\hline & n.S. & n.s. & $-0,151$ \\
\hline \multicolumn{4}{|c|}{ 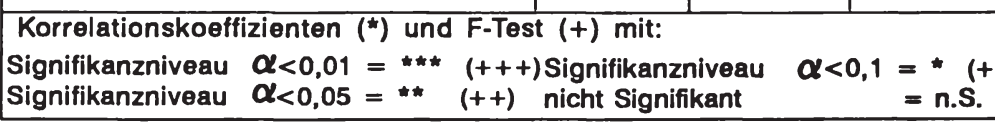 } \\
\hline
\end{tabular}

Abb. 28: Empirische Ergebnisse zum EinfluB soziodemographischer Merkmale auf die Real-Einstellungsfaktoren 
Bezüglich der Beurteilung des natürlichen Angebots und des sportangebots wird deutlich, daß diese sowohl von demographischen als auch sozioökonomischen Merkmalen beeinfluBt werden. Das natürliche Angebot wird signifikant besser und das sportangebot signifikant schlechter beurteilt, je älter die Touristen, je fortgeschrittener ihre stellung im Lebenszyklus und je geringer ihr Bildungsgrad ist. Ferner erhält das sportangebot der Untersuchungsregion mit zunehmendem Netto-Einkommen und höherer Schichtzugehörigkeit bessere Bewertungen.

Neben der Analyse des Einflusses soziodemographischer Merkmale auf die ermittelten Einstellungsfaktoren ist es ferner von Interesse, ihren EinfluB auf die Ideal-/Real-Differensen $z u$ ermitteln. In der Abbildung 29 sind die zusammenhänge zwischen den soziodemographischen Merkmalen, den Realeinstellungen sowie den Real-Ideal-Differenzen dargestellt. Um die Unterschiede in der Identifizierbarkeit von Realeinstellungen und Ideal-Real-Differenzen vergleichen $z u$ können, muBte die Analyse auf der Basis von Einzelindikatoren durchgeführt werden.

Dabei zeigt sich insgesamt, daß Einstellungen unabhängig von ihrem operationalisierungsansatz nur in eingeschränktem MaBe die Anforderung der soziodemographischen Identifizierbarkeit

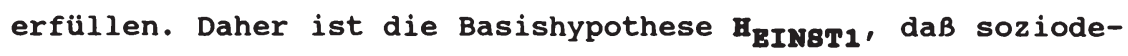
mographische Merkmale die Ausprägungsformen von Einstellungen bestimmen, nur tendenziell zu bestätigen.

Neben der Identifizierbarkeit von Einstellungen ist ihr EinFlub auf das Besuchsverhalten von Interesse. In Abbildung 30 sind die zusammenhänge zwischen den Einstellungsfaktoren und dem Besuchsverhalten dargestellt. 


\begin{tabular}{|c|c|c|c|c|c|c|c|c|c|c|c|c|c|c|c|c|c|c|c|c|c|c|c|c|c|c|c|}
\hline \multirow{2}{*}{\multicolumn{2}{|c|}{$\begin{array}{l}\text { Elnstel- } \\
\text { Sozio- lungen } \\
\text { demo- } \\
\text { graphisecho } \\
\text { Morkmale }\end{array}$}} & \multicolumn{12}{|c|}{ Sportunabhănglge tourlstische Infrastruktur } & \multicolumn{8}{|c|}{ Natũrliches Angebot } & \multicolumn{6}{|c|}{ Sportangebot } \\
\hline & & \multicolumn{2}{|c|}{$\begin{array}{c}\text { Kulturelles } \\
\text { Angobot }\end{array}$} & \multicolumn{2}{|c|}{$\begin{array}{c}\begin{array}{c}\text { Erholungs-1 } \\
\text { Fioizoit- } \\
\text { parks }\end{array} \\
\end{array}$} & \multicolumn{2}{|c|}{\begin{tabular}{|c|}
$\begin{array}{c}\text { Hlstorische } \\
\text { Sehonswür- } \\
\text { digkelton }\end{array}$ \\
\end{tabular}} & \multicolumn{2}{|c|}{\begin{tabular}{|c|} 
Qualfit dor der \\
Beher- \\
bergung
\end{tabular}} & \multicolumn{2}{|c|}{\begin{tabular}{|l|} 
Velfaitige \\
Gestronomic \\
\end{tabular}} & \multicolumn{2}{|c|}{\begin{tabular}{|c|}
$\begin{array}{c}\text { Elnkeuts- } \\
\text { mbglich- } \\
\text { kelten }\end{array}$ \\
\end{tabular}} & \multicolumn{2}{|c|}{ Wotter } & \multicolumn{2}{|c|}{$\begin{array}{c}\text { Okologisch } \\
\text { ntakte Lend } \\
\text { scheft }\end{array}$} & \multicolumn{2}{|c|}{\begin{tabular}{|c|} 
Froundlich- \\
kolt der \\
Menschen
\end{tabular}} & \multicolumn{2}{|c|}{$\begin{array}{l}\text { Abwechs- } \\
\text { lungereiche } \\
\text { Lendschant }\end{array}$} & \multicolumn{2}{|c|}{$\begin{array}{c}\text { Sport- } \\
\text { moglich- } \\
\text { ketten }\end{array}$} & \multicolumn{2}{|c|}{$\begin{array}{c}\text { Aadwandor- } \\
\text { moglieh- } \\
\text { ketten }\end{array}$} & \multicolumn{2}{|c|}{$\begin{array}{c}\text { Wander- } \\
\text { mbglich- } \\
\text { keiten }\end{array}$} \\
\hline \multirow{4}{*}{ 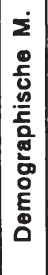 } & Geschlecht & n.6. & ++ & n.s. & n.s. & n.s. & n.6. & n.s. & n.6. & n.8. & n.6. & + & ++ & n.6. & n.6. & n.8. & n.6. & n.8. & n.8. & n.s. n & n.6. & n.8. & +++ & + & ++ & + & n.6. \\
\hline & Alter & -0.11 & n.s. & n.s. & n.8. & $\mid-0.07$ & $-0,08$ & -0.08 & n.8. & $-0.08:$ & 0.07 & n.s. & n.8. & 0.05 & 0.08 & 0,09 & 0,06 & $\mid \begin{array}{c:c}-0.1 \\
\cdots\end{array}$ & 0,08 & $\because-0,08\}$ & n.6. & 0.16 & 0.20 & 0,08 & 0.15 & n.8. & \begin{tabular}{lll}
-0.16 \\
\hdashline-16
\end{tabular} \\
\hline & Kinderzahl & n.8. & 0,06 & n.6. & \begin{tabular}{ccc}
-0.11 \\
\hdashline$-\bullet$
\end{tabular} & n.s. & n.6. & n.6. & n.8. & $-0,05$ & 0,05 & n.s. & n.s. & {$[-0,07$} & 0.08 & $-0,07$ & n.8. & $\mid$\begin{tabular}{c|c}
$-0,11$ \\
$\cdots$
\end{tabular} & 0,11 & $-0.05\}$ & 0.07 & n.8. & $-0,07$ & 0,06 & $\begin{array}{l}-0.12 \\
\cdots\end{array}$ & n.8. & n.8. \\
\hline & $\begin{array}{l}\text { Lebenszyklus- } \\
\text { phase }\end{array}$ & $\mid-0.08$ & $\begin{array}{l}\text { n.s. } \\
\end{array}$ & n.8. & n.8. & $|-0,08|$ & -0.05 & -0.07 & $-0,05$ & $-0,05$ & 0.06 & n.8. & n.6. & $\mid$\begin{tabular}{c|}
$-0,09$ \\
$\cdots$
\end{tabular} & 0.13 & -0.1 & 0.05 & $\mid-0.11$ & 0.1 & $-0,08 \mathrm{n}_{\mathrm{n}}$ & n.8. & $\begin{array}{l}0.14 \\
\cdots\end{array}$ & 0.16 & 0.08 & \begin{tabular}{ll}
0.11 \\
\hdashline..
\end{tabular} & n.s. & $\begin{array}{l}-0.14 \\
\cdots\end{array}$ \\
\hline \multirow{4}{*}{ 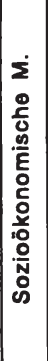 } & Bildung & n.s. & $\begin{array}{c}-0,13 \\
\cdots\end{array}$ & n.6. & $\begin{array}{l}-0,1 \\
-\cdots\end{array}$ & $\left|\begin{array}{c}-0.12 \\
\because \cdots\end{array}\right|$ & n.6. & $\mid$ & n.6. & $\begin{array}{lll}0.06 \\
00\end{array}$ & $-0,06$ & $\mid \begin{array}{ll}0.12 \\
\cdots\end{array}$ & n.8. & $\mid 0,07$ & $\begin{array}{l}-0.1 \\
\cdots\end{array}$ & $\begin{array}{ll}0,05 \\
\cdots\end{array}$ & $\begin{array}{c}-0,12 \\
\cdots\end{array}$ & 0.12 & $\begin{array}{l}-0,16 \\
-.80\end{array}$ & 0,07 & $-0,08$ & n.8. & $\left\{\begin{array}{l}-0.11 \\
\cdots\end{array}\right.$ & $\mid$\begin{tabular}{c|}
-0.1 \\
$\cdots$
\end{tabular} & n.8. & $\mid \begin{array}{c:c}-0,08 & 0\end{array}$ & 0 \\
\hline & \begin{tabular}{|l|} 
Persōnliches- \\
einkommen
\end{tabular} & n.s. & \begin{tabular}{c}
-0.07 \\
\hdashline $0^{-1}$
\end{tabular} & n.s. & n.s. & n.s. & $-0,00$ & n.6. & n.s. & n.s. & $-0,06$ & n.8. & n.8. & $\mid$\begin{tabular}{l|l}
-0.00 \\
\hdashline$\cdots$
\end{tabular} & 0.11 & $\because 0.1$ & 0 & $\mid-0.06$ & 0.06 & $-0,08 \mathrm{n}$ & n.6. & $-0,08\}$ & n.8. & n.8. & n.6. & n.s. & \begin{tabular}{l}
$-0,08$ \\
\hdashline-10
\end{tabular} \\
\hline & Beruf & ++ & ++ & n.8. & n.8. & n.8. & + & + & ++ & ++ & n.8. & +++ & n.8. & n.8. & n.8. & n.8. & ++ & +++ & ++ & +++1 & ++ & n.8. & +++ & n.8. & n.8. & n.8. & +++ \\
\hline & Schicht & n.8. & \begin{tabular}{c}
$-0,08$ \\
\hdashline \\
\end{tabular} & n.s. & n.s. & $\left|\begin{array}{c}-0,09 \\
\cdots\end{array}\right|$ & n.6. & n.s. & n.s. & n.s. & 0.00 & 0.05 & $\stackrel{-0,05}{0}$ & n.s. & 0.08 & n.6. & $\begin{array}{c}-0,08 \\
\cdots \cdots\end{array}$ & n.6. & 0.06 & n.8. & -0.05 & -0.06 & 0.13 & $\mid$\begin{tabular}{c|}
-0.19 \\
$\cdots$
\end{tabular} & $\begin{array}{l}\text { n.8. } \\
\end{array}$ & $\mid$\begin{tabular}{c|}
-0.11 \\
$\cdots \cdots$
\end{tabular} & n.8. \\
\hline
\end{tabular}

Abb. 29: Empirische Ergebnisse zum Einfluß soziodemographischer Merkmale auf die Realeinstellungen und Real-Ideal-Differenzen 


\begin{tabular}{|c|c|c|c|c|c|c|c|c|c|c|c|c|}
\hline \multirow{2}{*}{$\begin{array}{l}\text { Real- } \\
\text { Einstel- } \\
\text { lungsfaktoren }\end{array}$} & \multicolumn{6}{|c|}{ Entfernungsgruppe I } & \multicolumn{6}{|c|}{ Entfernungsgruppe II } \\
\hline & \begin{tabular}{|c|} 
Besuc \\
ja \\
\end{tabular} & $\begin{array}{l}\text { ichsab } \\
\text { nein } \\
\end{array}$ & \begin{tabular}{|l|} 
Sicht \\
Sig.
\end{tabular} & \begin{tabular}{|l} 
Besu \\
Tages \\
ausfl.
\end{tabular} & $\begin{array}{l}\text { uchsd } \\
\text { Auff. } \\
\text { mit U. }\end{array}$ & \begin{tabular}{|l|} 
Suer \\
Sig.
\end{tabular} & \begin{tabular}{|c|} 
Besuc \\
ja
\end{tabular} & $\begin{array}{l}\text { ichsab } \\
\text { nein } \\
\end{array}$ & $\begin{array}{l}\text { sicht } \\
\text { Sig. }\end{array}$ & $\begin{array}{c}\text { Best } \\
\text { Kurz- } \\
\text { urlaub }\end{array}$ & $\begin{array}{l}\text { Uchsde } \\
\text { Urlaub }\end{array}$ & $\begin{array}{l}\text { lauer } \\
\text { Sig. }\end{array}$ \\
\hline $\begin{array}{l}\text { Touristische } \\
\text { sportunabhängige } \\
\text { Infrastruktur }\end{array}$ & $|-0,283|$ & $\mid-0,023$ & $\cdots$ & $-0,320 \mid$ & $-0,246$ & n.s. & 0,095 & 0,419 & $"$ & 0,030 & 0,120 & n.s. \\
\hline Natūrliches Angebot & $-0,147$ & $-0,059$ & n.s. & 0,099 & $-0,393$ & $*$ & $-0,117$ & 0.427 & $\cdots$ & 0,180 & $-0,398$ & $\cdots$ \\
\hline Sportangebot & $-0,050$ & 0,078 & n.s. & -0.193 & 0,093 & $*$ & $-0,261$ & 0.101 & $\cdots$ & -0.143 & $-0,375$ & n.s. \\
\hline iveau & & & & & & & & & $=$ & & & n.s. \\
\hline
\end{tabular}

Abb. 30: Empirische Ergebnisse zum EinfluB der Real-Einstellungsfaktoren auf das Besuchsverhalten (T-Test) innerhalb der Makrosegmente

Die Analyse auf Basis der Einstellungsfaktoren zeigt, daB sich Besucher und Nicht-Besucher in der Entfernungsgruppe I signifikant in ihrer Beurteilung der touristischen sportunabhängigen Infrastruktur unterscheiden. Dabei bewerten potentielle Besucher diese Hauptangebotskomponente signifikant besser als Nicht-Besucher. Da in der touristischen sportunabhängigen Infrastruktur die suprastruktur mit erfaßt ist,

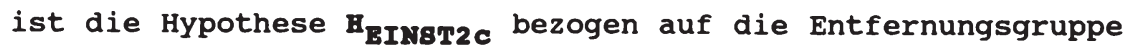
I zu bestätigen. Demgegenüber haben die Einstellungen zum natürlichen Angebot und sportangebot offensichtlich keinen Einfluß auf die Besuchsabsicht bei den Personen der Entfernungsgruppe $I$. Daher sind die Hypothesen HEstra und

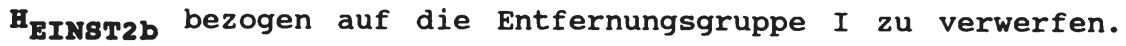
Es kann vermutet werden, $d a \beta$ die Untersuchungsregion vor allem aufgrund ihrer positiv wahrgenommenen touristischen sportunabhängigen Infrastruktur als zielgebiet ausgewählt wird.

Interessanterweise hat die Einstellung zur touristischen sportunabhängigen Infrastruktur keinen Einfluß auf die Besuchsdauer in der Entfernungsgruppe $I$. Demgegenüber können 
signifikante Unterschiede in den Einstellungen zum natürlichen Angebot und Sportangebot in Abhängigkeit von den nach der Besuchsdauer abgegrenzten Besuchergruppen identifiziert werden. Während das natürliche Angebot von den Besuchern mit einer längeren Aufenthaltsdauer relativ positiv beurteilt wird, schätzen die Tagesausflügler das sportangebot besser ein. Daher ist davon auszugehen, daB der Einstellung zum Sportangebot bei der Entscheidung für einen längeren Aufent-

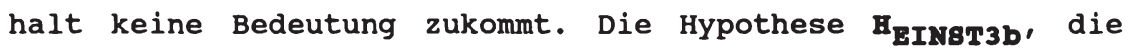
einen positiven zusammenhang $z$ wischen sportangebot und Besuchsdauer unterstellte, ist demnach $\mathrm{zu}$ verwerfen. Demgegenüber ist die Einstellung zum natürlichen Angebot of fensichtlich der herausragende Bestimmungsfaktor für eine längere

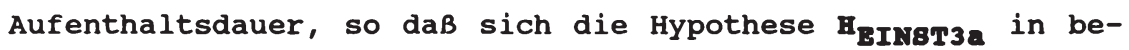
zug auf die Entfernungsgruppe I bestätigt.

In der Entfernungsgruppe II unterscheiden sich die Besucher von den Nicht-Besuchern hinsichtlich ihrer Einstellungen $z u$ allen Angebotskomponenten. Dabei schätzen potentielle Besucher die touristische Infrastruktur, das natürliche Angebot und das sportangebot signifikant besser ein als die NichtBesucher. Daher sind die Hypothesen HErst2a, HEIM8T2b und

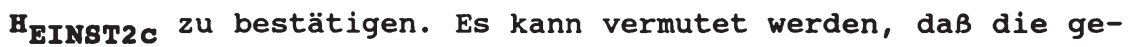
genüber der Entfernungsgruppe I zusätzlich verhaltenswirksam werdenden Einstellungen zum natürlichen Angebot und Sportangebot auf die mit der Entfernung in Verbindung stehende Anreisedauer und der mehrtägigen Aufenthaltsdauer zurückzuführen sind. Die hohe Bedeutung einer positiven Beurteilung des natürlichen Angebots für die Besuchsabsicht ist auch für die Besuchsdauer festzustellen. Personen, die das natürliche Angebot besser beurteilen als diejenigen mit einer kürzeren Aufenthaltsdauer, beabsichtigen einen längeren Aufenthalt in der Region. Demgegenüber hat die Einstellung zur touristischen Suprastruktur keinen Einfluß auf die Besuchsdauer, so daß die Hypothese HINsT3c $_{\text {Eu verwerfen ist. }}$ 
Zusammenfassend kann festgehalten werden, daß den über die Realeinstellungen gebildeten Einstellungsfaktoren eine hohe Bedeutung bei der Erklärung des Besuchsverhaltens zugesprochen werden muß. Aus dem Blickwinkel der Marktsegmentierung erfüllen sie in besonderem Maße die Anforderung der Besuchsverhaltensrelevanz. Dabei kommt der Einstellung zum natürlichen Angebot die größte Bedeutung zur Erklärung der Besuchsdauer zu. Hierin bestätigt sich die in der Theorie genannte Vermutung, daß eine positive Einschätzung des natürlichen Angebots offensichtlich die Grundvoraussetzung dafür ist, daß Touristen einen längeren Aufenthalt in einer Fremdenverkehrsregion verbringen.

Wenngleich den Realeinstellungsfaktoren eine hohe Besuchsverhaltensrelevanz zuzusprechen ist, interessiert darüber hinaus, ob die Ideal-Real-Differenzen besser dazu geeignet sind, das Besuchsverhalten zu erklären. Die Ergebnisse der auf der Einzelindikatorenebene durchgeführten Untersuchung finden sich in Abbildung 31. Es wird unmittelbar deutlich, daß die Realeinstellungen in der Entfernungsgruppe I einen deutlich höheren Erklärungsbeitrag für das Besuchsverhalten erbringen als die Ideal-Real-Differenzen. Während die Realeinstellungen nahezu bei allen vorgegebenen Angebotskomponenten des touristischen sportunabhängigen Infrastrukturangebots signifikante Unterschiede in den nach der Besuchsabsicht abgegrenzten Besuchergruppen aufweisen, haben die Ideal-Real-Differenzen keinen EinfluB auf die Besuchsabsicht. Offensichtlich führt die Differenzenbildung zwischen Ideal- und Realeinstellungen dazu, daß Einstellungsunterschiede in den Besuchergruppen nivelliert werden.

Bei der Betrachtung der Mittelwertunterschiede in den nach der Besuchsdauer abgegrenzten Besuchergruppen bestätigt sich, daß den Realeinstellungen eine höhere Verhaltensrelevanz als den Ideal-Real-Differenzen zukommt. Dabei wird deutlich, daß Personen der Entfernungsgruppe I, die einen 


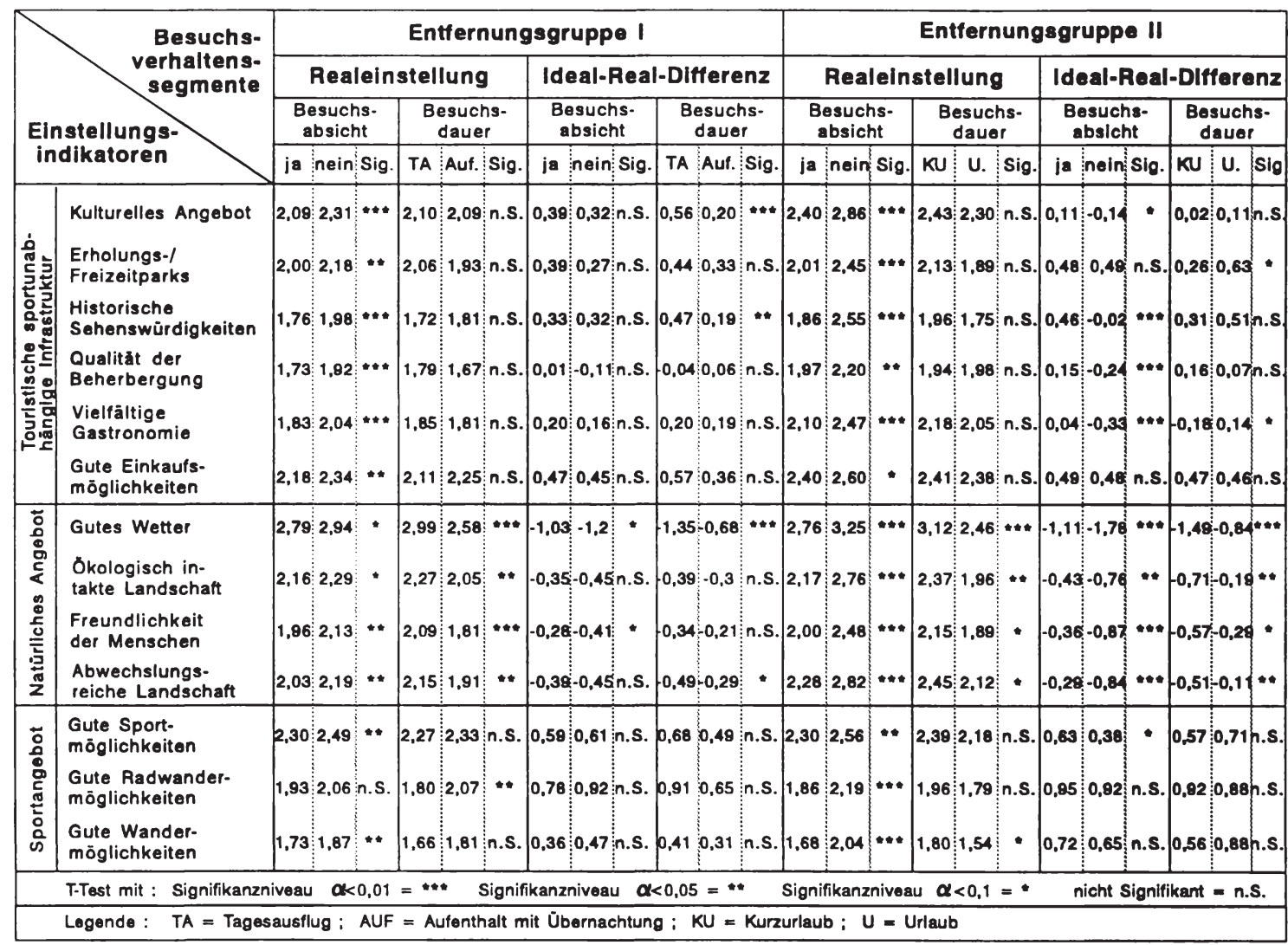

Abb. 31: Empirische Ergebnisse zum EinfluB der Realeinstellungen und RealIdeal-Differenzen auf das Besuchsverhalten innerhalb der Makrosegmente 
Aufenthalt mit Übernachtung beabsichtigen, die Angebotskomponenten des natürlichen Angebots signifikant besser beurteilen als die Tagesausflügler. Die Ideal-Real-Differenzen sagen hingegen nur aus, daß Personen mit einem längeren Aufenthalt gegenüber dem Wetter und der abwechslungsreichen Landschaft eine signifikant geringere Untererfüllung durch die Untersuchungsregion wahrnehmen als die Tagesausflügler.

Entgegen den ermittelten Ergebnissen auf der Basis von Realeinstellungen lassen sich auf der Basis von Ideal-RealDifferenzen signifikante Einstellungsunterschiede bezüglich des kulturellen Angebots und der historischen Sehenswürdigkeiten zwischen den nach der Besuchsdauer abgegrenzten Verhaltenssegmenten feststellen. So nehmen Personen mit einer längeren Aufenthaltsdauer eine signifikant geringere Übererfüllung dieser Angebotskomponenten wahr als Tagesausflügler. Es kann davon ausgegangen werden, daB Personen mit einer längeren Aufenthaltsdauer das kulturelle Angebot und die historischen Sehenswürdigkeiten für einen idealen Urlaub als wichtiger erachten als Tagesausflügler. Demnach ist nicht die Einstellung $\mathrm{zu}$ den Angebotskomponenten, sondern allein die motivationale Komponente ursächlich für die Unterschiede im Besuchsverhalten. Dieses Ergebnis deckt sich mit den im Rahmen der Motivanalyse identifizierten Mittelwertunterschieden zwischen den nach der Besuchsdauer abgegrenzten Besuchsverhaltenssegmenten der Entfernungsgruppe I.231

In der Entfernungsgruppe II ergeben sich auf der Basis der Realeinstellungen bei allen Angebotskomponenten signifikante Unterschiede in den Realeinstellungen zwischen den nach der Besuchsabsicht abgegrenzten Besuchergruppen. Dabei weisen Besucher signifikant positivere Einstellungen $z u$ den Angebotskomponenten auf als Nicht-Besucher. Ferner zeigt sich ebenso wie bei der Entfernungsgruppe I, daß Personen, die

$231 \mathrm{Vgl}$. Kapitel B.4.222. 
eine längere Aufenthaltsdauer in der Untersuchungsregion beabsichtigen, die Komponenten des natürlichen Angebots signifikant besser beurteilen.

Allerdings liefert eine Betrachtung der Ideal-Real-Differenzen ähnliche Ergebnisse. Besucher nehmen nahezu über alle Angebotskomponenten hinweg signifikant geringere Untererfülungen bzw. höhere Überfüllungen durch die Untersuchungsregion als die Nicht-Besucher wahr. Ferner wird bezüglich der Eigenschaften des natürlichen Angebots von den Urlaubern eine signifikant geringere Untererfüllung wahrgenommen als von den Kurzurlaubern. Es ist daher $z u$ vermuten, daB die Beurteilung einer Fremdenverkehrsregion in Abhängigkeit von der Kontaktzeit (Aufenthaltsdauer) vorgenommen wird.

Die Ergebnisse auf der Basis der Ideal-Real-Einstellungen verdeutlichen, daß sich die im Rahmen des Trommsdorff-Modells unterstellte Annahme, wonach wahrgenommmene Unter- und Übererfüllungen den gleichen EinfluB auf das Besuchsverhalten ausüben, im Rahmen dieser Untersuchung nicht bestätigt. Die Ergebnisse bestätigen somit die gewählte Vorgehensweise, auf eine Absolutbetrachtung bei der Ermittlung der Ideal-Real-Differenzen zu verzichten.

Interessanterweise finden sich bei der Beurteilung des Sportangebots auf der Basis der Ideal-Real-Differensen im Gegensatz zu den Realeinstellungen kaum signifikante Unterschiede zwischen Besuchern und vicht-Besuchern. Offensichtlich werden Unterschiede in den Realeinstellungen durch die Einbeziehung der Idealeinstellungen eliminiert. Dies bedeutet, daB die Ideal-Real-Differenzen weder Auskunft über die tatsächliche Beurteilung dieser Angebotskomponente noch ubber die motivationale Komponente widerspiegeln.

Zusammenfassend kann daher festgehalten werden, daB Realeinstellungen offenbar besser dazu geeignet sind, die Beurtei- 
lung einer Fremdenverkehrsregion wiederzugeben als IdealReal-Differenzen. Vor diesem Hintergund sollen für die weiterführenden Analysen die Realeinstellungsfaktoren Berücksichtigung finden.

\subsection{Zusammenfassende Analyse der Eignung psychographischer Merkmale als Kriterien zur Marktsegmentierung}

Auf der Grundlage der bisher im Rahmen univariater Analysen identifizierten Bestimmungsfaktoren des Besuchsverhaltens ist im folgenden ihr Gesamterklärungsbeitrag zu ermitteln. Dabei ist den bisher theoretisch und empirisch ermittelten Ergebnissen der untersuchten psychographischen Variablen Rechnung $z u$ tragen, die in Abbildung 32 zusammengefaBt sind. Als Beurteilungskriterien werden die in Kapitel B.1 abgeleiteten Anforderungen an Segmentierungskriterien herangezogen.

Die Beurteilung der Besuchsverhaltensrelevanz sowie der Identifizierbarkeit der betrachteten Konstrukte leitet sich unmittelbar aus den empirischen Ergebnissen ab. Die Beurteilung der Besuchsverhaltensrelevanz der untersuchten Kriterien ergibt sich aus den identifizierten signifikanzniveaus der Mittelwertunterschiede zwischen den abgegrenzten Verhaltenssegmenten.

Die Bewertung der Identifizierbarkeit der psychographischen Merkmale erfolgt durch eine zusammenfassende Bewertung ihrer Zusammenhänge mit den soziodemographischen Merkmalen. Mit Ausnahme der Realeinstellung zur touristischen, sportunabhängigen Infrastruktur und der Wertorientierung "Gesundheit und Umwelt" gewährleisten die untersuchten Merkmale die Identifizierbarkeit.

Ferner fließen die im Rahmen der theoretischen Auseinandersetzung abgeleiteten Aussagen zur zeitlichen stabilität in 


\begin{tabular}{|c|c|c|c|c|c|c|c|c|c|c|}
\hline & & \multicolumn{6}{|c|}{ Konzeptionelle Anforderungen } & \multirow{2}{*}{\multicolumn{3}{|c|}{$\begin{array}{l}\text { Meßtheoretlsche } \\
\text { Anforderungen }\end{array}$}} \\
\hline & & \multicolumn{4}{|c|}{ Besuchsverhaltensrelevanz } & \multirow{3}{*}{$\begin{array}{l}\text { Identi- } \\
\text { fizier- } \\
\text { barkeit }\end{array}$} & \multirow{3}{*}{$\begin{array}{l}\text { Zeitliche } \\
\text { Stabilität }\end{array}$} & & & \\
\hline & & \multicolumn{2}{|c|}{ Entfernungsgruppe I } & \multicolumn{2}{|c|}{ Entfernungsgruppe \| } & & & \multirow{2}{*}{ Objektivitāt } & \multirow{2}{*}{ Validitåt } & \multirow{2}{*}{$\begin{array}{l}\text { Reliabilitiăt } \\
\text { rerombeones alones } \\
\end{array}$} \\
\hline & & $\begin{array}{c}\text { Besuchs- } \\
\text { absicht }\end{array}$ & $\begin{array}{c}\text { Besuchs- } \\
\text { dauer }\end{array}$ & $\begin{array}{l}\text { Besuchs- } \\
\text { absicht }\end{array}$ & $\begin{array}{c}\begin{array}{c}\text { Besuchs- } \\
\text { dauer }\end{array} \\
\end{array}$ & & & & & \\
\hline \multirow{5}{*}{$\stackrel{8}{\frac{8}{0}}$} & $\begin{array}{l}\text { - Bildungs- und Gesell- } \\
\text { schaftsorientierung }\end{array}$ & $\star \star \star \star$ & $\star \star \star \star$ & n.S. & n.s. & ++ & \multirow{5}{*}{+++} & \multirow{5}{*}{++} & \multirow{5}{*}{++} & 0,70 \\
\hline & -Hedonismus & n.s. & $\star$ & n.s. & n.s. & + & & & & 0,73 \\
\hline & - Freiheitsorientierung & n.s. & $\star$ & n.s. & $\star \star \star$ & +++ & & & & 0,61 \\
\hline & \begin{tabular}{|l|} 
- Tradition \& \\
Sicherheit
\end{tabular} & n.s. & $\star \star \star$ & n.s. & n.S. & +++ & & & & 0,56 \\
\hline & $\begin{array}{l}\text { Gesundheit \& } \\
\text { Umwelt }\end{array}$ & n.S. & $\star \star$ & $\star \star \star \star$ & 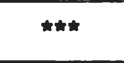 & 0 & & & & 0,55 \\
\hline \multirow{5}{*}{$\begin{array}{l}0 \\
\frac{0}{5} \\
0 \\
\frac{5}{0} \\
0 \\
\frac{0}{2} \\
\frac{0}{5}\end{array}$} & $\begin{array}{l}\text {-Sportbezogenes } \\
\text { Aktivitătsbedūrfnis }\end{array}$ & $\star \star$ & n.s. & n.S. & n.S. & +++ & \multirow{5}{*}{+} & \multirow{5}{*}{++} & \multirow{5}{*}{+} & 0,75 \\
\hline & \begin{tabular}{|l|} 
Regenerations- \& \\
GenuBbedūrfnis \\
\end{tabular} & $\star$ & n.s. & n.S. & n.s. & $++t$ & & & & 0,69 \\
\hline & -Kulturelles Bedūrfnis & $\star \star \star$ & $\hbar \star \star$ & n.s. & n.s. & ++ & & & & 0,71 \\
\hline & \begin{tabular}{|l|} 
- Sportaverses \\
Bewegungsbedūrfnis \\
\end{tabular} & n.s. & $\star$ & n.S. & n.s. & +++ & & & & 0,74 \\
\hline & $\begin{array}{l}\text {-Familienorientiertes } \\
\text { Erlebnisbedūrfnis }\end{array}$ & n.s. & n.S. & $\star \star \star \star$ & $\| \star \star$ & $+t+$ & & & & 0,60 \\
\hline \multirow{3}{*}{ 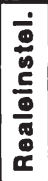 } & $\begin{array}{l}- \text { Touristische } \\
\text { Infrastruktur }\end{array}$ & $\star \star \star \star$ & n.S. & $\star \star$ & n.s. & -10 & \multirow{3}{*}{++} & \multirow{3}{*}{++} & \multirow{3}{*}{+++} & 0,84 \\
\hline & $\begin{array}{l}\text { - Natūrliches } \\
\text { Angebot }\end{array}$ & n.s. & $\hbar \star \star$ & $\hbar \star \star \star$ & $\star \star \star \star$ & + & & & & 0,79 \\
\hline & -Sportangebot & n.s. & $\star \star$ & $\star \star \star$ & n.s. & + & & & & 0,72 \\
\hline
\end{tabular}

Abb. 32: Zusammenfassende Beurteilung der Eignung von werten, Motiven und Einstellungen als Kriterien zur Marktsegmentierung 
die Eignungsbeurteilung der untersuchten Merkmale ein. Dabei ist sowohl bei den Werten und den Urlaubsmotiven als auch den Realeinstellungen von einer hinreichenden zeitlichen stabilität auszugehen. 232

Die Objektivität der Konstrukterfassung ist aufgrund des verwendeten Erhebungsverfahrens einer standardisierten Befragung gewährleistet. 233 Ferner ist von einer hinreichenden inhaltlichen validität der Konstruktmessung auszugehen.234 Um die Bestimmung relevanter Indikatoren der psychographischen Konstrukte abzusichern, werden üblicherweise Expertenurteile eingeholt. 235 Diesem Erfordernis wird dadurch entsprochen, daB in der vorliegenden Untersuchung bei der Auswahl der Indikatoren für die Urlaubsmotive und Realeinstellungen Experten befragt wurden. Bezüglich der Werte wurde auf eine Untersuchung zurückgegriffen, die auf umfangreiche Forschungsergebnisse der Werteforschung aufbaute und sich als äußerst valide erwies. ${ }^{236}$ Die Reliabilität der ermittel-

232 Vgl. hierzu im Detail die in den Kapiteln B.41, B.42 und B.43 abgeleiteten Aussagen.

$233 \mathrm{Vgl}$. hierzu auch Kapitel B.1.

234 Neben der inhaltlichen Validität sind ferner die Konstrukt- und Kriteriumsvalidität $\mathrm{zu}$ unterscheiden. Die Überprüfung der Kriteriumsvalidität erfolgt i.d.R. dadurch, daß ein Prüfkriterium entwickelt wird, das mit dem zu messenden Konstrukt in einer theoretisch engen Beziehung steht. Die Konstruktvalidität beschreibt die Eignung des Meßkonzeptes, ein nicht beobachtbares theoretisches Konstrukt weitestgehend $z u$ erfassen sowie Erklärungsansätze liefern zu können. Vgl. zu den unterschiedlichen Verfahren der Überprüfung der Konstruktvalidität Hilke, R., Grundlagen normorientierter und kriterienorientierter Tests, Bonn 1980, S. 47 f.; Holm, K., Die Gültigkeit des sozialwissenschaftlichen Messens, in: K. Holm (Hrsg.), Die Befragung 4, München 1976, S. 126 ff.

$235 \mathrm{Vgl}$. Büker, B., Qualitätsbeurteilungen investiver Dienstleistungen, a.a.0., S. $112 \mathrm{f}$.

236 Vgl. Kapitel B. 4.1. 
ten Werte-, Motiv- und Einstellungsfaktoren, die zusätzlich auf der Basis des Crombach's alpha überprüft wurde, ist gewährleistet. 237

Zusammenfassend verdeutlicht die Übersicht die Eignung unterschiedlicher psychographischer Merkmale als segmentierungskriterien. Es ist jedoch $\mathrm{zu}$ berücksichtigen, daB die psychographischen Merkmale jeweils nur isoliert hinsichtlich ihres Einflusses auf das Besuchsverhalten analysiert wurden. Dabei wurden die Beziehungen zwischen den einzelnen Konstrukten nicht berücksichtigt. Im folgenden ist daher zu überprüfen, inwieweit die als besuchsverhaltensrelevant identifizierten Merkmale im Rahmen einer integrierten Analyse geeignet sind, die innerhalb der Entfernungsgruppen (Makrosegmente) nach Besuchsabsicht und -dauer abgegrenzten Verhaltenssegmente voneinander $\mathrm{zu}$ unterscheiden. Hierdurch ist es möglich, die wesentlichen Bestimmungsfaktoren des Besuchsverhaltens über alle Konstruktebenen hinweg zu identifizieren.

237 Der Crombach's alpha ist ein Maß zur Beurteilung der Zuverlässigkeit der Konstruktmessung. Dieses Zuverlässigkeitsmaß kann Werte zwischen 0 und 1 annehmen. Bei einem Koeffizienten von 1 ist kein Meßfehler gegeben. In diesem Zusammenhang ist $z u$ beachten, daB bei der Ermittlung des Crombach's Alpha die Indikatoren gleichgewichtet in die Bildung des "Faktor-Gesamtwertes" eingehen. Daher kann im Rahmen dieser Untersuchung der Crombach's alpha nur als Indikator für die Reliabilität der Faktoren verwendet werden. Zur Messung der Reliabilität vgl. Heidenreich, K., Grundbegriffe der Meß- und Testtheorie, in: Sozialwissenschaftliche Methoden, Roth, E. (Hrsg.), München, wien 1984, S. 365 f.; vgl. zur Berechnung des Zuverlässigkeitsmaßes die spss-Prozedur Reliability in: Schubö, W., Uehlinger, H.-M., Handbuch der Programmversion 4.0 und SPSS $-\mathrm{X} 3.0, \mathrm{a} . \mathrm{a} .0 ., \mathrm{S} .564 \mathrm{ff}$. Die hier ermittelten Werte in Höhe von 0,55 bis 0,84 deuten auf eine ausreichende Reliabilität hin. Vgl. Dahlhoff, H.D., Individuelle Wertorientierungen - Analyse und Aussagewert personenspezifischer Werthierarchien im Marketing, Arbeitspapier Nr. 23 des Instituts für Marketing der Universität Münster, Hrsg.: Meffert, H., Münster 1980, S. $39 \mathrm{ff}$. 
In die integrierte Analyse werden vor allem diejenigen Merkmale einbezogen, die sowohl Relevanz für das Besuchsverhalten besitzen als auch die spätere Identifizierbarkeit der Segmente gewährleisten. Wenngleich die Realeinstellung zur "touristischen, sportunabhängigen Infrastruktur" und die Wertorientierung "Gesundheit und Umwelt" nur bedingt soziodemographisch beschrieben werden können, sind sie aufgrund ihrer hohen Besuchsverhaltensrelevanz aus der folgenden Untersuchung nicht auszuschließen. Zusätzlich zu diesen psychographischen Merkmalen ist im Rahmen der integrierten Analyse die zielgebietserfahrung $\mathrm{zu}$ berücksichtigen, da ihr ein hoher Einfluß auf die Besuchsabsicht zuzusprechen ist.

Die integrierten Analysen werden mit der Diskriminanzanalyse durchgeführt, in deren Rahmen zwei oder mehrere Gruppen simultan hinsichtlich einer vielzahl von Merkmalsvariablen analysiert werden können. 238 Dabei müssen die in die Diskriminanzanalyse einzubeziehenden Variablen möglichst unabhängig voneinander sein.

Anhaltspunkte für bestehende Multikollinearität sind anhand der Korrelationen zwischen den, in die Analyse einzubeziehenden, Variablen zu gewinnen. 239 Aufgrund der relativ hohen Korrelation $(0,431)^{240}$ zwischen dem kulturellen und soziokulturellen Bedürfnis und der Wertorientierung "Bildungsund Gesellschaftsorientierung" wurde letzteres Merkmal in

$238 \mathrm{Vgl}$. Backhaus, K., Multivariate Analysemethoden, a.a.0., S. $162 \mathrm{ff.;}$ Norusis, M.J., SPSS/PC+. Advanced Statistics 4.0, Chicago 1990, S. B-1 ff.

$239 \mathrm{Vgl}$. Backhaus, Multivariate Analysemethoden, a.a.o., s. 35 .

$240 \mathrm{Vgl}$. zu den Korrelationen zwischen den psychographischen Merkmalen die im Anhang I abgebildete Korrelationstabelle. 
der Entfernungsgruppe I vorab aus der Diskriminanzanalyse ausgeschlossen. 241

Für beide Entfernungsgruppen (Makrosegmente) werden sowohl für die nach der Besuchsabsicht als auch nach der Besuchsdauer abgegrenzten Verhaltenssegmente Diskriminanzanalysen durchgeführt. Die wichtigsten Ergebnisse der Diskriminanzanalysen sind in der Abbildung 33 für die Entfernungsgruppe I und in der Abbildung 34 für die Entfernungsgruppe II zusammenfassend dargestellt. Die innerhalb der vier Diskriminanzanalysen ermittelten Diskriminanzfunktionen weisen jeweils ein Signifikanzniveau von 0,001 auf. Sie geben Auskunft über die Diskriminierungsfähigkeit der einzelnen psychographischen Merkmale sowie über ihre Bedeutung für die Erklärung der Besuchsverhaltenssegmente. 242

Für die nach der Besuchsabsicht abgegrenzten segmente der Entfernungsgruppe I zeigt sich, daß zur Trennung der Besucher von den Nicht-Besuchern der zielgebietserfahrung die größte Bedeutung zukommt, da allein auf diese Dimension $50,65 \%$ der erklärten Varianz entfällt. Weiterhin weisen die erklärten Varianzanteile der Faktoren "kulturelles Bedürfnis" $(15,44 \%)$ und "Realeinstellung zur touristischen Infrastruktur" $(14,91 \%)$ auf ihre hohe Bedeutung bei der Trennung der Besuchsverhaltenssegmente hin. Der Faktor "Regenerationsbedürfnis" leistet mit einem Gewicht von 8,58 \& einen

241 Backhaus weist darauf hin, daß bei Korrelationen zwischen 0,1 und 0,44 kaum Gefahren der Multikollinearität bestehen. Vgl. Backhaus, K., Multivariate Analysemethoden, a.a.0., s. 36. In dieser Untersuchung wurde als Grenze eine Korrelation in Höhe von 0,4 angesetzt.

242 Die standardisierten Diskriminanzfunktionskoeffizienten geben angesichts des hier jeweils zugrundeliegenden Zwei-Gruppen-Falls unmittelbar Auskunft über das relative Bedeutungsgewicht der einzelnen Merkmale bei der Unterscheidung der abgegrenzten Verhaltenssegmente. Vgl. Backhaus, K., Multivariate Analysemethoden, a.a.o., s. 201. 


\begin{tabular}{|c|c|c|c|c|c|c|c|}
\hline \multicolumn{2}{|c|}{$\begin{array}{l}\text { Besuchs- } \\
\text { verhaltens- } \\
\text { sogmente }\end{array}$} & \multirow{2}{*}{$\mid \begin{array}{c}\text { Konstrukt- } \\
\text { obone }\end{array}$} & \multirow{2}{*}{\begin{tabular}{|l|}
$\begin{array}{c}\text { Bestimmungsfaktoren } \\
\text { des Bosuchs- } \\
\text { vorhalten }\end{array}$ \\
- Sportbezogenes \\
Aktivitătsbeürfnis \\
- Regenerations- \\
bedürfnis \\
- Kulturelles \\
Bedürfnis
\end{tabular}} & \multirow{2}{*}{$\begin{array}{c}\begin{array}{c}\text { Standardisiorter } \\
\text { Diskriminanz- } \\
\text { funktions- } \\
\text { koofflzient }\end{array} \\
-0,176 \\
0,145\end{array}$} & \multicolumn{2}{|c|}{$\begin{array}{l}\text { Rolative Bodoutung } \\
\text { dos mittleren } \\
\text { Diskriminanzfunktions- } \\
\text { kooffizionten }\end{array}$} & \multirow{2}{*}{$\begin{array}{c}\begin{array}{c}\text { Rang in der } \\
\text { rolativen Bo- } \\
\text { doutung }\end{array} \\
4 \\
5 \\
2\end{array}$} \\
\hline \multirow{8}{*}{ 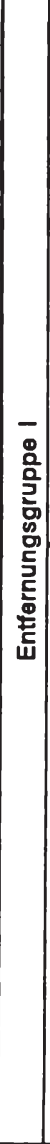 } & \multirow{4}{*}{ 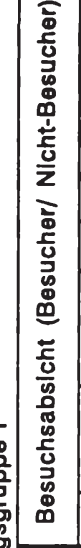 } & & & & $\begin{array}{r}10,42 \% \\
8,58 \% \\
15,44 \%\end{array}$ & $34,44 \%$ & \\
\hline & & $\begin{array}{l}\text { Realein- } \\
\text { stel- } \\
\text { lungen }\end{array}$ & $\begin{array}{l}\text { - Touristische sport- } \\
\text { unabhängige } \\
\text { Infrastruktur }\end{array}$ & $-0,252$ & $14,91 \%$ & $14,91 \%$ & 3 \\
\hline & & $\begin{array}{c}\text { Ziel- } \\
\text { gebiets- } \\
\text { erfahrung }\end{array}$ & $\begin{array}{l}\text { - Zielgebiets- } \\
\text { erfahrung }\end{array}$ & 0,856 & $50,65 \%$ & $50,65 \%$ & 1 \\
\hline & & \multicolumn{2}{|r|}{ Summe } & 1,69 & \multicolumn{3}{|c|}{$100 \%$} \\
\hline & \multirow{4}{*}{ 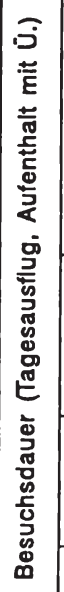 } & Werte & $\begin{array}{l}\text { - Hedonismus } \\
\text { - Freiheitsorientierung } \\
\text { - Tradition \& } \\
\text { Sicherheit } \\
\text { - Gesundheit \& } \\
\text { Umwelt } \\
\end{array}$ & $\begin{array}{l}-0,193 \\
-0,613\end{array}$ & $\begin{array}{r}8,78 \% \\
27,90 \%\end{array}$ & $36,68 \%$ & $\begin{array}{l}5 \\
2\end{array}$ \\
\hline & & $\begin{array}{l}\text { Urlaubs- } \\
\text { motive }\end{array}$ & $\begin{array}{l}- \text { Kulturelles } \\
\text { Bedūrfnis } \\
\text {-Sportaverses } \\
\text { Bewegungsbedūrfnis }\end{array}$ & $\begin{array}{l}0,466 \\
-0,271\end{array}$ & $\begin{array}{l}21,21 \% \\
12,34 \%\end{array}$ & $33,55 \%$ & 3 \\
\hline & & $\begin{array}{l}\text { Real- } \\
\text { ein- } \\
\text { stellung }\end{array}$ & $\begin{array}{l}\text { - Natürliches } \\
\text { Angebot } \\
\text { - Sportangebot }\end{array}$ & $\begin{array}{l}0,654 \\
-\end{array}$ & $29,77 \%$ & $29,77 \%$ & 1 \\
\hline & & & Summe & 2,197 & & $100 \%$ & \\
\hline
\end{tabular}

Abb. 33: Diskriminanzanalytische Ergebnisse zur Bedeutung psychographischer Merkmale für die Besuchsverhaltenssegmente in der Entfernungsgruppe I 


\begin{tabular}{|c|c|c|c|c|c|c|c|}
\hline \multicolumn{2}{|c|}{$\begin{array}{l}\text { Besuchs- } \\
\text { voritatens- } \\
\text { sogmente }\end{array}$} & \multirow{2}{*}{$\begin{array}{c}\begin{array}{c}\text { Konstrukt- } \\
\text { ebene }\end{array} \\
\text { Werte }\end{array}$} & \multirow{2}{*}{$\begin{array}{c}\begin{array}{c}\text { Bestimmungsfaktoren } \\
\text { des Besuchs- } \\
\text { verhalten }\end{array} \\
\begin{array}{c}\text {-Gesundhelt \& } \\
\text { Umwelt }\end{array}\end{array}$} & \multirow{2}{*}{$\begin{array}{c}\begin{array}{c}\text { Standardisierter } \\
\text { Diskriminanz- } \\
\text { funktions- } \\
\text { kooffizlent }\end{array} \\
0,326\end{array}$} & \multicolumn{2}{|c|}{$\begin{array}{l}\text { Relative Bedeutung } \\
\text { des mittleren } \\
\text { Diskriminanzfunktions- } \\
\text { koeffizienten }\end{array}$} & \multirow{2}{*}{$\begin{array}{c}\begin{array}{c}\text { Rang in der } \\
\text { relativen Be- } \\
\text { deutung }\end{array} \\
4\end{array}$} \\
\hline \multirow{10}{*}{ 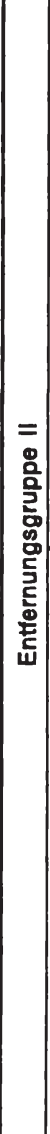 } & \multirow{5}{*}{ 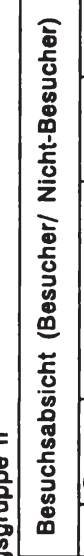 } & & & & $14,05 \%$ & $14,05 \%$ & \\
\hline & & $\begin{array}{l}\text { Urlaubs- } \\
\text { motive }\end{array}$ & $\begin{array}{l}\text {-Famillenorientiertes } \\
\text { Erlebnlsbodürfnls }\end{array}$ & & & & \\
\hline & & $\begin{array}{l}\text { Realein- } \\
\text { stel- } \\
\text { lungen }\end{array}$ & $\begin{array}{l}\text { - Tourlatische sport- } \\
\text { unabhånglge } \\
\text { Intrastruktur } \\
\text { - Natūrliches Angebot } \\
\text { - Sportangebot }\end{array}$ & $\begin{array}{l}0,491 \\
0,617 \\
0,655\end{array}$ & $\begin{array}{l}21,15 \% \\
26,58 \% \\
28,22 \%\end{array}$ & $75,95 \%$ & $\begin{array}{l}3 \\
2 \\
1\end{array}$ \\
\hline & & \begin{tabular}{|c|} 
Ziel- \\
gebiets- \\
erfahrung
\end{tabular} & $\begin{array}{l}\text { - Zielgeblets- } \\
\text { erfahrung }\end{array}$ & 0,232 & $10 \%$ & $10 \%$ & 5 \\
\hline & & \multicolumn{2}{|r|}{ Summe } & 2,321 & \multicolumn{3}{|c|}{$100 \%$} \\
\hline & \multirow{5}{*}{ 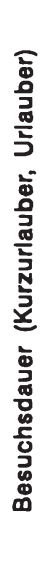 } & Werte & $\begin{array}{l}\text { - Freie Entfaltung } \\
\text { - Gesundheit \& } \\
\text { Umwolt }\end{array}$ & 0,543 & $22,69 \%$ & $22,69 \%$ & 3 \\
\hline & & $\begin{array}{l}\text { Urlaubs- } \\
\text { motive }\end{array}$ & $\begin{array}{l}\text {-Famillenorientiertes } \\
\text { Eriebnisbedürfnis }\end{array}$ & $-0,745$ & $31,13 \%$ & $31.13 \%$ & 1 \\
\hline & & $\begin{array}{c}\text { Real- } \\
\text { ein- } \\
\text { stellung }\end{array}$ & $\begin{array}{l}\text { - Natūrliches } \\
\text { Angebot }\end{array}$ & $-0,444$ & $18,56 \%$ & $18,56 \%$ & 4 \\
\hline & & $\begin{array}{c}\text { Ziel- } \\
\text { gebiets- } \\
\text { erfahrung }\end{array}$ & $\begin{array}{l}\text {-Zielgebiets- } \\
\text { erfahrung }\end{array}$ & 0,661 & $27,62 \%$ & $27,62 \%$ & 2 \\
\hline & & & Summe & 2,393 & & $100 \%$ & \\
\hline
\end{tabular}

Abb. 34: Diskriminanzanalytische Ergebnisse zur Bedeutung psychographischer Merkmale für die Besuchsverhaltenssegmente in der Entfernungsgruppe II 
relativ geringen Beitrag zur Diskriminierung. Angesichts der hohen Bedeutung der zielgebietserfahrung für die Besuchsabsicht ist $z u$ schließen, daß gerade im Naherholungsverkehr Fremdenverkehrsregionen präferiert werden, die den Touristen aus früheren Besuchen bekannt sind.

Insgesamt beträgt der Anteil korrekter Klassifikationen jedoch nur 67,70 \%. Darüber hinaus weist der Canonische Korrelationskoeffizient von 0,373 auf eine nur mittlere Trennfähigkeit der Gruppen mit Hilfe der ermittelten Diskriminanzfunktion hin. 243 Ein Vergleich mit der Zufallswahrscheinlichkeit von 56,64 \& zeigt jedoch, daB durch die Berücksichtigung der psychographischen Merkmale die Zufallstrefferquote um 19,53 \& (11,06 Prozentpunkte) verbessert werden konnte.

Zur Trennung der nach der Besuchsdauer abgegrenzten Verhaltenssegmente kommt insbesondere der "Realeinstellung zum natürlichen Angebot" mit einem erklärten Varianzanteil von $29,77 \%$, dem Wert "Tradition und Sicherheit" $(27,9 \%)$ sowie dem Motiv "kulturelles Bedürfnis" $(21,21$ \%) die größte Bedeutung zu. Bei der Bildung der Diskriminanzfunktion werden die Wertefaktoren "Hedonismus", "Gesundheit und Tradition" sowie die Einstellungen zum sportangebot aus der Analyse ausgeschlossen. Sie weisen demnach im Verbund mit den anderen Variablen keine ausreichende Trennfähigkeit auf. Die Betrachtung der relativen Bedeutung der Diskriminanzfunktionskoeffizienten auf der Konstruktebene verdeutlicht, daß die in der Analyse verbleibenden Werte, Motive und Einstellungen

243 Die Diskriminanzfunktion und ihre signifikanz-, Wilk's Lamda- sowie Eigenwerte sind dem Anhang zu entnehmen. Auch der durch Wilk's Lambda generierte Chi-Quadrat-Test zeigt ein hohes signifikanzniveau hinsichtlich der einbezogenen Variablen. Allerdings sind die werte des Wilk's Lambda als relativ hoch zu beurteilen. Vgl. die im Anhang dokumentierten Ergebnisse der Diskriminanzfunktion. 
einen relativ gleichbedeutenden Einfluß auf die Besuchsdauer ausuiben.

Der Canonische Korrelationskoeffizient $(0,446)$ und der hohe Anteil korrekter Klassifikationen $(70,34$ \%) verdeutlichen eine gute Trennfähigkeit der nach der Besuchsdauer abgegrenzten Verhaltenssegmente. Damit konnte gegenüber der zufälligen Zuordnung eine um 20,34 Prozentpunkte bessere Klassifikation erzielt werden. Dies entspricht einer steigerung um 40,68 \%.

In der Entfernungsgruppe II kommt zur Diskriminierung der Besucher von den Nicht-Besuchern der "Einstellung zum sportangebot", gefolgt von der "Einstellung zum natürlichen Angebot" und zur "touristischen sportunabhängigen Infrastruktur" die größte Bedeutung zu. 75,95 \& der Diskriminanzinformationen entfallen auf diese Realeinstellungen. Urlaubsmotive sind hingegen nicht geeignet, Besucher und Nicht-Besucher der Entfernungsgruppe II voneinander $z u$ unterscheiden. So ist $z u$ vermuten, daß die Nicht-Besucher entweder aufgrund von Fehlwahrnehmungen der Fremdenverkehrsregion oder wegen bestehender Wissenslücken einen Besuch in die Untersuchungsregion erst gar nicht in Betracht ziehen.

Interessanterweise kommt der zielgebietserfahrung angesichts des geringen Anteils erklärter Varianz (10 ₹) lediglich eine geringe Bedeutung zu. Es ist anzunehmen, daß bei Reisen, die mit einer längeren Anfahrtsdauer verbunden sind, neue, noch nicht aufgesuchte Fremdenverkehrsregionen präferiert werden. Ferner verdeutlicht der hohe Einfluß der Einstellungen auf die Besuchsabsicht, daß Reiseentscheidungen, die eine längere Anfahrtsdauer voraussetzen, einem komplexen Kaufentscheidungsprozeß unterliegen.

Die Klassifikationsergebnisse mit einer "Trefferquote" von 68,46 \% deuten auf eine mittlere Diskriminierungsfähigkeit 
der Diskriminanzfunktion hin. Allerdings beträgt die $\mathrm{Zu}-$ fallswahrscheinlichkeit $60,24 \%$, so daß die Berücksichtigung der psychographischen Merkmale nur zu einer Ergebnisverbesserung um 8,22 Prozentpunkte (bzw. 13,65 ₹) führt.

Die nach der Besuchsdauer abgegrenzten Segmente der Entfernungsgruppe II unterscheiden sich vor allem in ihrem "familienorientierten Erlebnisbedürnis" und in der zielgebietserfahrung. Allein auf diese Variablen entfallen 31,13 \& bzw. 27,62 \% der insgesamt erklärten Varianz. Ferner kommt der Wertorientierung "Umwelt und Gesundheit" mit einem Varianzanteil von 22,69 sowie der "Einstellung zum natürlichen Angebot" $(18,56 \%)$ eine hohe Bedeutung zur Erklärung dieser Verhaltenssegmente $\mathrm{zu}$. Der mit 0,578 vergleichsweise hohe Canonische Korrelationskoeffizient und das Klassifikationsergebnis mit 75,56 \% richtiger zuordnung deuten auf eine gute Trennfähigkeit der nach der Besuchsdauer abgegrenzten Verhaltenssegmente hin.

Unterzieht man die Ergebnisse der empirischen Analyse zur Besuchsverhaltensrelevanz der dieser Untersuchung zugrundeliegenden Konstrukte einer abschließenden würdigung, so ist festzuhalten, daß in den beiden Makrosegmenten zentrale Bestimmungsfaktoren des Besuchsverhaltens identifiziert werden konnten. Bereits die univariaten signifikanzprüfungen zeigten, daB sich die in den Enfernungsgruppen abgegrenzten Besuchsverhaltenssegmente anhand von psychographischen Merkmalen gut differenzieren lassen. Im Rahmen der simultanbetrachtung aller bis dahin als trennscharf ermittelten variablen konnten darüber hinaus die über alle Konstruktebenen hinweg relevanten Bestimmungsfaktoren des Besuchsverhaltens festgestellt werden.

Insgesamt verdeutlichen die vorliegenden Ergebnisse, daß die in der Fremdenverkehrsforschung vorherrschende einseitige Ausrichtung auf Urlaubsmotive als Grundlage zur Erklärung 
des Besuchsverhaltens als nicht ausreichend zu würdigen ist. Erst die Kombination unterschiedlicher psychographischer Merkmale und Werte ermöglicht eine hinreichend präzise Erklärung des Besuchsverhaltens. Dabei kommt der zielgebietserfahrung zur Erklärung der Besuchsabsicht in der Entfernungsgruppe I sowie den Einstellungen in der Entfernungsgruppe II ein besonders hoher stellenwert zu. Werte bringen vor allem in bezug auf die Besuchsdauer einen zusätzlichen Erklärungsbeitrag.

Allerdings deuten die nur durchschnittlichen Klassifikationsergebnisse darauf hin, daß das Besuchsverhalten neben den hier untersuchten Werten, Motiven und Einstellungen von zusätzlichen Einflußfaktoren abhängt. So ist anzunehmen, daß zum Beispiel durch die Berücksichtigung von Präferenzen für andere Fremdenverkehrsregionen das Besuchsverhalten noch besser erklärt werden könnte.

\section{Psychographische Marktsegmentierung auf der Grundlage der empirischen Ergebnisse}

\subsection{Zielgruppenbildung innerhalb der Makrosegmente anhand von werten, Motiven und Einstellungen}

Auf der Grundlage der ermittelten Bestimmungsfaktoren des Besuchsverhaltens ist im folgenden eine psychographische Marktsegmentierung durchzuführen. Ziel ist es dabei, die Eignung einer psychographischen Marktsegmentierung auf der Grundlage dieser Bestimmungsfaktoren gegenüber der verhaltensorientierten Marktsegmentierung mit Blick auf die Anforderung der soziodemographischen Identifizierbarkeit der segmente zu überprüfen. Die Eignung der psychographischen Marktsegmentierung als geeigneterer Markterfassungsansatz erfordert darüber hinaus, daß sich die identifizierten Seg- 
mente in ihrem Besuchsverhalten unterscheiden. Dabei ist die psychographische Marktsegmentierung für beide Entfernungsgruppen getrennt durchzuführen, da in diesen Makrosegmenten jeweils unterschiedliche Bestimmungsfaktoren des Besuchsverhaltens identifiziert werden konnten. In diesem zusammenhang ist folgende Basishypothese zu überprüfen:

HEGMENT Auf der Basis der Bestimmungsfaktoren des Besuchs-
verhaltens lassen sich in beiden Entfernungsgrup-
pen touristische Nachfragersegmente bilden, die
sich in ihrem Besuchsverhalten unterscheiden und
die soziodemographische Identifizierbarkeit ge-
währleisten.

Allerdings stellt sich die Frage, ob die zweistufige, nach der Besuchsabsicht und -dauer getrennt durchgeführte Analyse zur Ermittlung der Bestimmungsfaktoren des Besuchsverhaltens im Rahmen der psychographischen Marktsegmentierung beizubehalten ist. Im Rahmen einer zweistufigen psychographischen Marktsegmentierung müBte je Entfernungsgruppe zunächst eine Segmentierung auf der Basis der Bestimmungsfaktoren der Besuchsabsicht durchgeführt werden. Erst in einem zweiten Schritt würden die Bestimmungsfaktoren der Besuchdauer herangezogen, um eine weiterführende segmentierung der ermittelten psychographischen segmente durchzuführen. Wenngleich dieses Vorgehen $z u$ besser voneinander abgrenzbaren Verhaltenssegmenten je Entfernungsgruppe führen kann, besteht ein schwerwiegender meßtheoretischer Nachteil in den zu geringen Fallzahlen pro segment. Übertragen auf den Gesamtmarkt bedeutet dies, daß die segmente vermutlich keine ausreichende Größe für eine eigenständige Marktbearbeitung aufweisen würden. 
Vor diesem Hintergrund sind im folgenden bei der Segmentbildung sowohl die erklärenden Merkmale der Besuchsabsicht als auch die der Besuchsdauer simultan zu berücksichtigen.

Unter Verwendung des Hill-climbing-Clusteranalyseverfahrens $^{244}$ werden die befragten Personen in der Entfernungsgruppe I in vier segmente und in der Entfernunsgruppe II in drei segmente aufgeteilt. Zur Beurteilung der Trennschärfe der ermittelten Zielgruppen wurde eine Diskriminanzanalyse auf der Grundlage der zielgruppenbildenden Variablen durchgeführt. Anhand der Klassifikationsmatrix wurden in der Entfernungsgruppe I 95,6 und in der Entfernungsgruppe II $96,39 \%$ der Befragten richtig zugeordnet, wodurch die sehr gute Trennschärfe der psychographischen Marktsegmente bestätigt wird.

Entsprechend der Zugehörigkeit einzelner psychographischer Merkmale $z u$ den Konstruktebenen zeigen die Abbildungen 35 und 36 die clusterspezifischen Mittelwertunterschiede in den jeweiligen Entfernungsgruppen. Neben den clusterbildenden Variablen werden in der Betrachtung die bei der Zielgruppenbildung nicht verwendeten Werte-, Motiv- und Einstellungsfaktoren als zusätzliche clusterbeschreibende Variablen aufgenommen. Letztere sind bei der segmentbeschreibung zu berücksichtigen.

244 In der vorliegenden Untersuchung wurde im Rahmen der Clusteranalyse die "Hill-Climbing-Methode" als Algorithmus zur Gruppenbildung verwendet. Sie beruht auf einer Verbesserung des Varianzkriteriums durch eine iterative Veränderung der Cluster. Anhand des sog. Elbow-Kriteriums wurde die "optimale" Anzahl der cluster festgelegt. Die optimale clusteranzahl ist dann erreicht, wenn eine weitere Verringerung der clusterzahl einen relativ starken Heterogenitätszuwachs nach sich ziehen wïrde. Vgl. Meffert, H., Käuferverhalten und Marktforschung, a.a.o., S. 267 ff.; Backhaus, K., Multivariate Analyseverfahren, a.a.O., S. S. $147 \mathrm{ff}$. 


\begin{tabular}{|c|c|c|c|c|c|c|c|}
\hline \multirow{2}{*}{\multicolumn{2}{|c|}{$\begin{array}{l}\text { Psycho- } \\
\text { graphische } \\
\text { Merkmale }\end{array}$}} & \multicolumn{6}{|c|}{ Entfernungsgruppe I: } \\
\hline & & \multirow{2}{*}{\begin{tabular}{|c|}
$\sigma_{(n=546)}$ \\
\\
$-0,023$ \\
0,011 \\
\end{tabular}} & \multirow{2}{*}{$\begin{array}{c}1_{\text {(n= 122) }} \\
\\
-- \\
-\end{array}$} & \multirow{2}{*}{\begin{tabular}{|c|}
$2_{(n=131)}$ \\
\\
0 \\
0
\end{tabular}} & \multirow{2}{*}{$\begin{array}{c}3_{(n=154)} \\
\\
+++ \\
0\end{array}$} & \multirow{2}{*}{\begin{tabular}{|c|}
$4(n=139)$ \\
\\
--- \\
+ \\
\end{tabular}} & \multirow{2}{*}{$\begin{array}{l}\text { Sig. } \\
\star \star \star \star \\
\text { n.S. }\end{array}$} \\
\hline \multirow{3}{*}{ 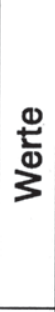 } & $\begin{array}{l}\text {-Bildungs- \& Gesell- } \\
\text { schaftsorientierung } \\
\text {-Hedonismus }\end{array}$ & & & & & & \\
\hline & 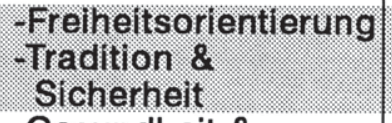 & $\mid \begin{array}{l}-0,042 \\
-0,165\end{array}$ & ( & : & t+ +4 & 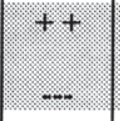 & : \\
\hline & $\begin{array}{l}\text {-Gesundheit \& } \\
\text { Umwelt }\end{array}$ & $-0,097$ & 0 & $0 /-$ & + & 0 & n.S. \\
\hline \multirow[t]{2}{*}{ 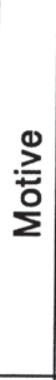 } & 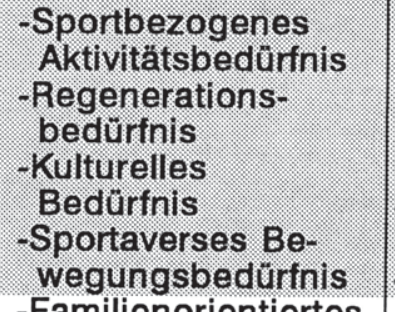 & $\begin{array}{l}0,043 \\
0,001 \\
-0,078 \\
-0,106\end{array}$ & 01 & $\begin{array}{l}0 \\
0 \\
0\end{array}$ & $\begin{array}{l}0 \\
+ \\
+ \\
+\end{array}$ & : & : \\
\hline & $\begin{array}{c}\text {-Familienorientiertes } \\
\text { Erlebnisbedürfnis }\end{array}$ & $-0,134$ & 0 & ++ & + & - & $\star \star \star \star$ \\
\hline \multirow[t]{4}{*}{ 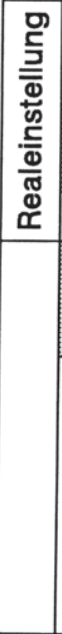 } & $\begin{array}{l}\text { - Touristische sport- } \\
\text { unabhängige Infra- } \\
\text { struktur } \\
\text { - Naturliches } \\
\text { Angebot }\end{array}$ & $\mid \begin{array}{ll}-0,123 \\
-0,1 & 1 \\
0 & 010\end{array}$ & + & 0 & 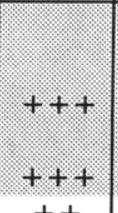 & -3 & 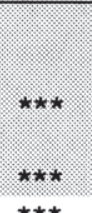 \\
\hline & -Sportangebot & 0,019 & -- & ++ & ++ & 0 & $\star \star \star \star$ \\
\hline & $\begin{array}{l}\text {-Zielgebiets- } \\
\text { erfahrung: }\end{array}$ & 0,12 & $-r$ & $+t+$ &.- & - & $* * *$ \\
\hline & \multicolumn{2}{|c|}{$\begin{array}{ll}\text { Cl.1: } & \begin{array}{l}\text { Traditionelle Spazier- } \\
\text { gängerfreunde }\end{array} \\
\text { Cl.2: } & \begin{array}{l}\text { Sportaktive Familien- } \\
\text { urlauber }\end{array} \\
\mathrm{Cl} .3: & \begin{array}{l}\text { Land- und Kulturorien- } \\
\text { tierte Urlauber }\end{array} \\
\mathrm{Cl} .4: & \begin{array}{l}\text { Regenerations- \& Genuß- } \\
\text { orientierte Strandurlauber }\end{array} \\
\end{array}$} & $\begin{array}{l}\text { Signifil } \\
\alpha<0,01 \\
\alpha<0,05 \\
\alpha<0,1 \\
\text { nicht } S\end{array}$ & $\begin{array}{l}\text { kanznive } \\
1 \\
5 \\
\text { Signifikar } \\
\text { Zielg } \\
\text { Varia }\end{array}$ & 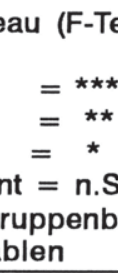 & Idende & \\
\hline
\end{tabular}

Abb. 35: Psychographische Zielgruppenbildung und -beschreibung in der Entfernungsgruppe I I ling - 978-3-631-75102-2 


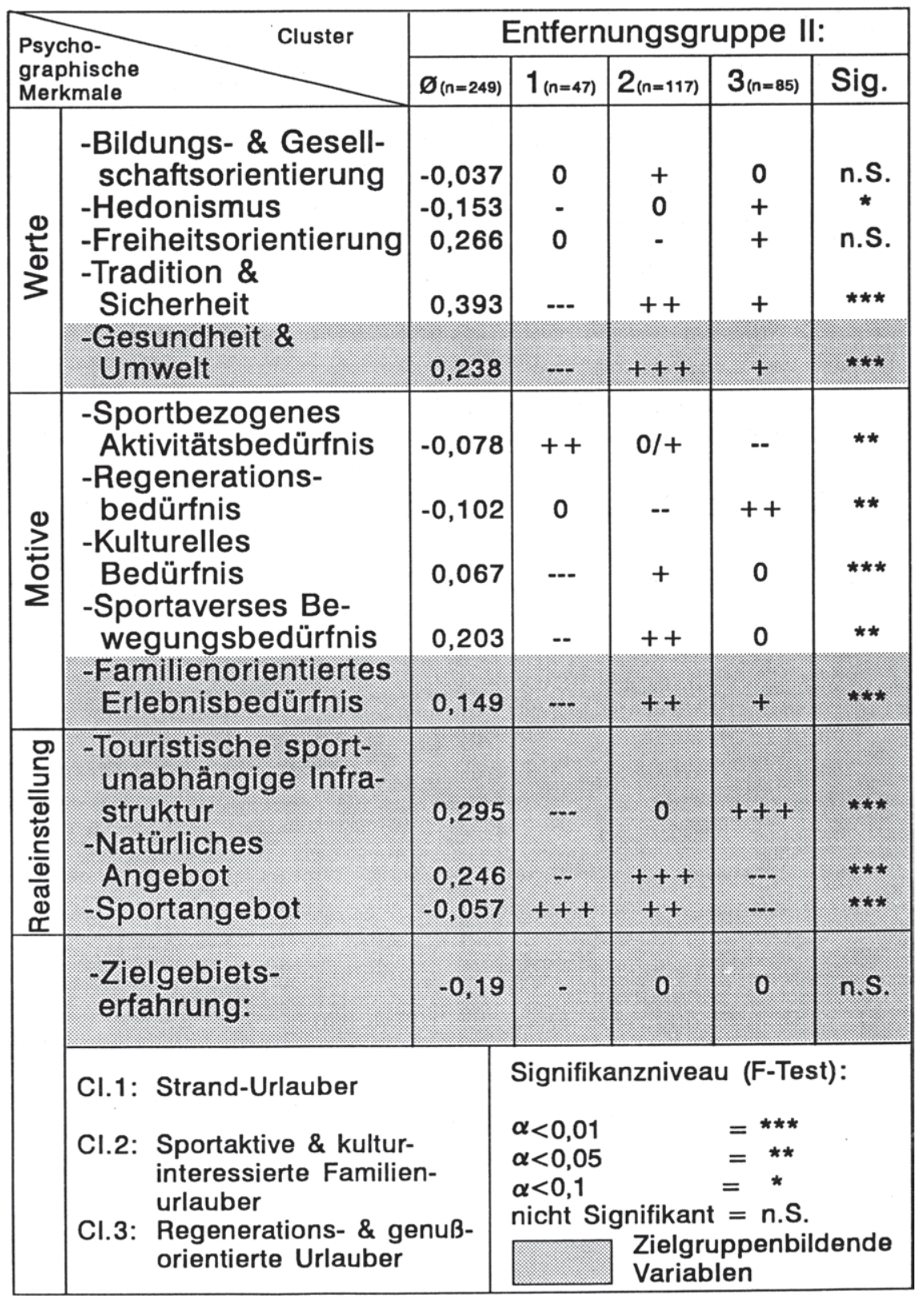

Abb. 36: Psychographische Zielgirupperibüibdung 97 and63-beschrei-

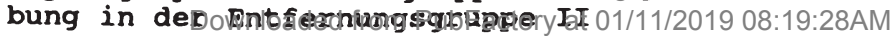


Zur Interpretation der cluster wurden im Rahmen von Detailanalysen ferner die Einzelindikatoren der psychographischen Konstrukte herangezogen, 245 um bei Vorliegen besonders interessanter Einzelergebnisse eine differenzierte Beschreibung der segmente zu ermöglichen. Ferner sind in Abbildung 37 und 38 die clusterspezifischen Ausprägungen der soziodemographischen Merkmale wiedergegeben. Im einzelnen lassen sich die cluster der Entfernungsgruppe I und II somit wie folgt beschreiben.

\section{Entfernungsgruppe I:}

\section{Cluster 1: Traditionelle spaziergängerfreunde}

Die dem ersten Cluster angehörenden Touristen $(22,34 \%$ der Befragten in der Entfernungsgruppe $I)$ erweisen sich als überdurchschnittlich stark traditions- und sicherheitsorientiert. Ferner ordnen sie dem Wertefaktor "Umwelt und Gesundheit" einen vergleichsweise hohen stellenwert $z u$, wenngleich die wichtigkeitseinschätzung weitgehend dem Durchschnitt aller segmente entspricht. Diese eher konservativ sicherheitsbetonte und gesundheitsorientierte Werthaltung äuBert sich im Urlaub vor allem durch die Befriedigung des sportaversen Bewegungsbedürfnisses. Sportbezogene Aktivitäten sowie "Faulenzen" und "Genuß" sind den Befragten dieses segments im Urlaub demgegenüber eher unwichtig.

Eine Detailanalyse hinsichtlich der Einzelindikatoren im Rahmen von Urlaubsmotiven 246 verdeutlicht in diesem zusammenhang, daß spazierengehen die wichtigste Aktivität für einen angenehmen Urlaub darstellt. Dabei ist die positive Bewertung des natürlichen Angebots hervorzuheben. offen-

245 Vgl. zu den Detailanalysen Anhang I.

$246 \mathrm{Vgl}$. Anhang I 


\begin{tabular}{|c|c|c|c|c|c|c|c|c|}
\hline \multirow{2}{*}{\multicolumn{3}{|c|}{$\begin{array}{l}\text { Sozio- } \\
\text { demographische } \\
\text { Merkmale }\end{array}$}} & \multicolumn{6}{|c|}{ 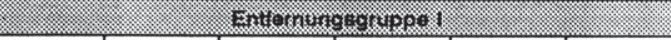 } \\
\hline & & & $\boldsymbol{\varnothing}$ & 1 & 2 & 3 & 4 & Sig. \\
\hline \multicolumn{3}{|c|}{$\begin{array}{ll}\text {-Besuchsabsicht: } \\
\text { (Inx) }\end{array}$} & $\begin{array}{l}43,2 \\
56,8\end{array}$ & $\begin{array}{l}29,5 \\
70,5\end{array}$ & $\begin{array}{l}75,6 \\
24,4 \\
\end{array}$ & $\begin{array}{l}42,9 \\
57,1\end{array}$ & $\begin{array}{l}25,2 \\
74,8\end{array}$ & $\star \star \star \star$ \\
\hline \multicolumn{3}{|c|}{$\begin{array}{l}\text { - Besuchsdauer: (n x) } \\
\text {-Tagesausflug } \\
\text {-Aufenthalt mit Ũ. }\end{array}$} & $\begin{array}{l}50,0 \\
50,0\end{array}$ & $\begin{array}{l}52,8 \\
47,2\end{array}$ & $\begin{array}{l}54,5 \\
45,5\end{array}$ & $\begin{array}{l}48,5 \\
51,5\end{array}$ & $\begin{array}{l}37,1 \\
62,9\end{array}$ & n.S. \\
\hline \multirow{4}{*}{ 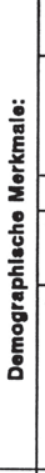 } & \multicolumn{2}{|c|}{\begin{tabular}{|ll} 
- Geschlecht & -mănnlich \\
(n x) & -weiblich \\
\end{tabular}} & $\begin{array}{l}46,0 \% \\
54,0 \%\end{array}$ & $\begin{array}{l}41,0 \% \\
59,0 \%\end{array}$ & $\begin{array}{l}50,4 \% \\
49,6 \%\end{array}$ & $\begin{array}{l}42.9 \% \\
57,1 \%\end{array}$ & $\begin{array}{l}49.6 \% \\
50.4 \%\end{array}$ & n.s. \\
\hline & \begin{tabular}{|l}
-Alter: \\
(nn x) \\
-Durch: \\
\end{tabular} & $\begin{array}{l}\text {-bis } 20 \text { Jahre } \\
-21-30 \text { Jahre } \\
-31-45 \text { Jahre } \\
-45-64 \text { Jahre } \\
-65 \text { Jahre ălter } \\
\text { schnittsalter: }\end{array}$ & $\begin{array}{r}8,8 \\
18,8 \\
25,9 \\
31,7 \\
14,7 \\
-44,4 \\
4\end{array}$ & $\begin{array}{r}2,5 \\
10,9 \\
17.6 \\
37.0 \\
31,9 \\
-54,25 \\
\end{array}$ & $\begin{array}{r}5,5 \\
18,1 \\
26,0 \\
38,5 \\
11,8 \\
45,41 \\
\end{array}$ & $\begin{array}{r}7,9 \\
13,2 \\
29,8 \\
35,1 \\
13,9 \\
-45,46 \\
\end{array}$ & $\begin{array}{r}18,0 \\
32,4 \\
28,8 \\
17,3 \\
-3,6 \\
33,62\end{array}$ & $-\underset{\star \star \star}{-1}$ \\
\hline & \multicolumn{2}{|c|}{\begin{tabular}{|l|} 
- Kinderanzahl on \\
-Keine Kinder \\
-1 und mehr Kinder \\
\end{tabular}} & $\begin{array}{l}66.5 \\
33,5 \\
\end{array}$ & $\begin{array}{l}73.0 \\
27.0 \\
\end{array}$ & $\begin{array}{l}60.3 \\
39,7 \\
\end{array}$ & $\begin{array}{l}70.6 \\
29.4 \\
\end{array}$ & $\begin{array}{l}62.3 \\
37.7 \\
\end{array}$ & * \\
\hline & \multicolumn{2}{|c|}{$\begin{array}{l}\text {-Lebenszyklusphase } \\
\text { I: Jugendliche und } \\
\text { Junge Erwachsene } \\
\text { II: Singles und Paare } \\
\text { III:Familien mit Kindern } \\
\text { und Jugendlichen } \\
\text { IV:Jungsenioren } \\
\text { V: Ruheständler } \\
\end{array}$} & $\begin{array}{l}17,2 \\
22,1 \\
23,3 \\
21,9 \\
15,6\end{array}$ & $\begin{array}{r}7,9 \\
9,6 \\
20,2 \\
28,9 \\
33,3 \\
\end{array}$ & $\begin{array}{l}12,9 \\
20,2 \\
25,8 \\
29,0 \\
12,1\end{array}$ & $\begin{array}{l}14,2 \\
29,1 \\
20,6 \\
21,3 \\
14,9\end{array}$ & $\begin{array}{r}32,8 \\
27,3 \\
\\
26,6 \\
9,4 \\
3,9 \\
\end{array}$ & $\star \star \star \star *$ \\
\hline \multirow{5}{*}{ 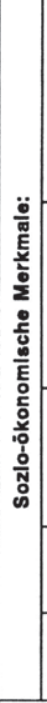 } & \multicolumn{2}{|c|}{$\begin{array}{l}\text {-Bildung (in \%) } \\
\text {-Volksschule ohne } \\
\text { abgeschlossene Lehre } \\
\text {-Volksschule mit } \\
\text { abgeschlossener Lehre } \\
\text {-Weiterbildende Schulen } \\
\text {-Abitur/Fachhochschule/ } \\
\text { Studium }\end{array}$} & $\begin{array}{l}12,8 \\
49,3 \\
22,5 \\
15,4\end{array}$ & $\begin{array}{r}16,7 \\
61,7 \\
12,5 \\
9,2\end{array}$ & $\begin{array}{r}7,6 \\
55,0 \\
18,3 \\
19,1\end{array}$ & $\begin{array}{l}15,8 \\
42,8 \\
28,9 \\
12,5\end{array}$ & $\begin{array}{l}11,1 \\
40,0 \\
28,1 \\
20,7\end{array}$ & $\star \star \star \star *$ \\
\hline & \multicolumn{2}{|c|}{$\begin{array}{l}\text {-Berufsgruppen (in\%) } \\
\text {-Selbständige } \\
\text {-Angestellte } \\
\text {-Beamte } \\
\text {-Arbeiter } \\
\text {-Hausfrau } \\
\text {-Schūler/Student/Azubi } \\
\text {-Rentner } \\
\end{array}$} & $\begin{array}{r}6,9 \\
25,4 \\
5,6 \\
24,4 \\
13,7 \\
9,4 \\
14,6 \\
\end{array}$ & $\begin{array}{r}4,9 \\
16,4 \\
3,3 \\
23.0 \\
17.2 \\
2.5 \\
32,8 \\
\end{array}$ & $\begin{array}{r}7.0 \\
31,8 \\
7,0 \\
19,4 \\
14,7 \\
7.8 \\
12,4 \\
\end{array}$ & $\begin{array}{r}8,6 \\
23,0 \\
6,6 \\
29,6 \\
12,5 \\
7.2 \\
12,5 \\
\end{array}$ & $\begin{array}{r}6,6 \\
29,9 \\
5,1 \\
24,8 \\
10,9 \\
19,7 \\
2,9 \\
\end{array}$ & $\star \star \star \star$ \\
\hline & \multicolumn{2}{|c|}{\begin{tabular}{|l|} 
- Monatl. HH-Nettoeinkom. \\
- bis $1500,-\mathrm{DM}$ \\
- $1500-2500,-\mathrm{DM}$ \\
$-2500-3500,-\mathrm{DM}$ \\
- 3500-5000,- DM \\
- über 5000,- DM \\
\end{tabular}} & $\begin{array}{r}12,5 \\
29,6 \\
28,3 \\
20,6 \\
9,0\end{array}$ & $\begin{array}{r}16,8 \\
36,4 \\
27.1 \\
13.1 \\
6,5 \\
\end{array}$ & $\begin{array}{r}10.1 \\
30.3 \\
27.5 \\
22,9 \\
9.2 \\
\end{array}$ & $\begin{array}{r}5.8 \\
28.8 \\
28.8 \\
26.6 \\
10.1 \\
\end{array}$ & $\begin{array}{r}18,4 \\
24,0 \\
29,6 \\
18,4 \\
9.6 \\
\end{array}$ & $\star$ \\
\hline & \multicolumn{2}{|c|}{\begin{tabular}{|l}
-Persōnl. Netto-Elinkom. \\
- bis $1500,-$ DM \\
- $1500-3000,-$ DM \\
- ûber $3000,-$ DM \\
\end{tabular}} & $\begin{array}{l}39.7 \\
48,6 \\
11,7 \\
\end{array}$ & $\begin{array}{r}40.6 \\
53.5 \\
5.9 \\
\end{array}$ & $\begin{array}{l}33.7 \\
43.3 \\
23.1\end{array}$ & $\begin{array}{l}34.1 \\
54.1 \\
11,9 \\
\end{array}$ & $\begin{array}{r}50.4 \\
43.0 \\
6.6 \\
\end{array}$ & 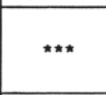 \\
\hline & \multicolumn{2}{|c|}{$\begin{array}{l}\text {-Schicht } \\
\text {-Unterschicht } \\
\text {-Mittelschicht } \\
\text {-Oberschicht } \\
\end{array}$} & $\begin{array}{l}22.1 \\
62.1 \\
15.7 \\
\end{array}$ & $\begin{array}{r}37.1 \\
57.1 \\
5.7 \\
\end{array}$ & $\begin{array}{l}18.7 \\
60.7 \\
20.6 \\
\end{array}$ & $\begin{array}{l}13.1 \\
68.6 \\
18.2 \\
\end{array}$ & $\begin{array}{l}22.3 \\
60.3 \\
17.4 \\
\end{array}$ & $\star \star \star \star$ \\
\hline \multicolumn{2}{|r|}{$\begin{array}{l}\text { Cl.1: } \\
\text { Cl.2: } \\
\text { Cl.3: } \\
\text { Cl.4: }\end{array}$} & $\begin{array}{l}\text { Traditionelle Spa } \\
\text { Sportaktive Fami } \\
\text { Land- \& Kulturori } \\
\text { Regenerations- \& }\end{array}$ & $\begin{array}{l}\text { kiergāngerf } \\
\text { ienurlaube } \\
\text { entierte Url } \\
\text { GenuBorie }\end{array}$ & $\begin{array}{l}\text { eunde } \\
\text { auber } \\
\text { ntierte Urla }\end{array}$ & & \multicolumn{3}{|c|}{$\begin{array}{ll}\text { Signifikanzniveau } & \text { (Chi }{ }^{2} \text {-Test): } \\
\alpha<0,01 & =* * \star \\
\alpha<0,05 & =\star * \\
\alpha<0,1 & =\star \\
\text { nicht Signifikant } & =\text { n.S. }\end{array}$} \\
\hline
\end{tabular}

Abb. 37: Soziodemographische Zielgruppenbeschreibung in der
Entfernungsgruppe I Simone Frömbling-978-3-631-75102-2 


\begin{tabular}{|c|c|c|c|c|c|c|}
\hline \multirow{2}{*}{\multicolumn{2}{|c|}{$\begin{array}{l}\text { Sozio- } \\
\text { demographische } \\
\text { Merkmale }\end{array}$}} & \multicolumn{5}{|c|}{ 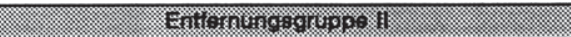 } \\
\hline & & $\boldsymbol{\theta}$ & 1 & 2 & 3 & Sig. \\
\hline \multicolumn{2}{|r|}{$\begin{array}{l}\text { - Besuchsabsicht: }{ }_{\text {(in } \mathbf{x})}^{\text {ja }} \text { nein } \\
\text {. }\end{array}$} & $\begin{array}{l}39,8 \\
60,2\end{array}$ & $\begin{array}{l}25,5 \\
74,5\end{array}$ & $\begin{array}{l}52,1 \\
47,9\end{array}$ & $\begin{array}{l}30,6 \\
69,4\end{array}$ & 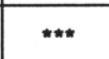 \\
\hline \multicolumn{2}{|r|}{$\begin{array}{c}\text {-Besuchsdauer: (in } \mathbf{x} \text { ) } \\
\text {-Kurzurlaub } \\
\text {-Urlaub }\end{array}$} & $\begin{array}{l}\mathbf{5 0}, 0 \\
50,0\end{array}$ & $\begin{array}{l}54,5 \\
45,5\end{array}$ & $\begin{array}{l}45,5 \\
54,5\end{array}$ & $\begin{array}{l}58,3 \\
41,7\end{array}$ & n.s. \\
\hline \multirow{4}{*}{ 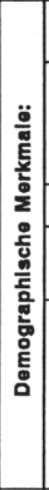 } & 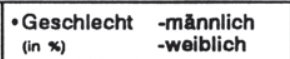 & $\begin{array}{l}45,4 \% \\
54,6 \%\end{array}$ & $\begin{array}{l}55,3 \% \\
44,7 \%\end{array}$ & $\begin{array}{l}42,7 \% \\
57,3 \%\end{array}$ & $\begin{array}{l}43,5 \% \\
56,5 \%\end{array}$ & n.s. \\
\hline & \begin{tabular}{|ll}
-Alter: & -bis 20 Jahre \\
(in x) & $-21-30$ Jahre \\
& $-31-45$ Jahre \\
& $-45-64$ Jahre \\
& -65 Jahre alter \\
& -
\end{tabular} & $\begin{array}{r}7,7 \\
23,8 \\
27,8 \\
25,8 \\
14,9 \\
-42,76\end{array}$ & $\begin{array}{r}8,5 \\
36,2 \\
27,7 \\
25,5 \\
2,1 \\
36,57\end{array}$ & $\begin{array}{r}4,3 \\
24,1 \\
21,6 \\
25,9 \\
24,1 \\
-46,80\end{array}$ & $\begin{array}{r}11,8 \\
16,5 \\
36,5 \\
25,9 \\
-\quad-9,4 \\
40,61\end{array}$ & $\overline{m * \pi}$ \\
\hline & $\begin{array}{l}\text { - Kinderanzahi (in x) } \\
\text {-Keine Kinder } \\
-1 \text { und mehr Kinder }\end{array}$ & $\begin{array}{l}75,9 \\
24,1\end{array}$ & $\begin{array}{r}93,6 \\
6,4\end{array}$ & $\begin{array}{l}65,8 \\
34,2\end{array}$ & $\begin{array}{l}80,0 \\
20,0\end{array}$ & $\star \star \star \star$ \\
\hline & $\begin{array}{l}\text { - Lebenszyklusphase } \\
\text { I: Jugendliche und } \\
\text { Junge Erwachsene } \\
\text { II: Singles und Paare } \\
\text { III:Familien mit Kindern } \\
\text { und Jugendlichen } \\
\text { IV: Jungsenioren } \\
\text { V: Ruhestăndler } \\
\end{array}$ & $\begin{array}{l}15,8 \\
33,2 \\
16,2 \\
19,5 \\
15,4\end{array}$ & $\begin{array}{r}19,1 \\
57,4 \\
2,1 \\
19,1 \\
2,1\end{array}$ & $\begin{array}{l}12,6 \\
19,8 \\
22,5 \\
19,8 \\
25,2\end{array}$ & $\begin{array}{l}18,1 \\
37,3 \\
15,7 \\
19,3 \\
19,6\end{array}$ & $\star \star \star \star$ \\
\hline \multirow{5}{*}{ 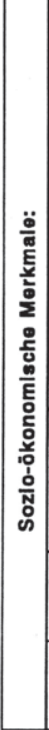 } & $\begin{array}{l}\text {-Bildung (in \%) } \\
\text {-Volksschule ohne } \\
\text { abgeschlossene Lehre } \\
\text {-Volksschule mit } \\
\text { abgeschlossener Lehre } \\
\text {-Weiterbildende Schulen } \\
\text {-Abitur/Fachhochschule/ } \\
\text { Studium }\end{array}$ & $\begin{array}{r}8,9 \\
42,3 \\
26,4 \\
22,4\end{array}$ & $\begin{array}{r}8,5 \\
19,1 \\
25,5 \\
46,8\end{array}$ & $\begin{array}{r}9,6 \\
42,6 \\
29,6 \\
18,3\end{array}$ & $\begin{array}{r}8,3 \\
54,8 \\
22,6 \\
14,3\end{array}$ & $\star \star \star \star$ \\
\hline & $\begin{array}{l}\text {-Berufsgruppen (in \%) } \\
\text {-Selbstāndige } \\
\text {-Angestellte } \\
\text {-Beamte } \\
\text {-Arbeiter } \\
\text { - Hausfrau } \\
\text {-Schūler/Student/Azubi } \\
\text {-Rentner }\end{array}$ & $\begin{array}{r}9,4 \\
34,0 \\
5,7 \\
18,0 \\
7,4 \\
13,5 \\
11,9\end{array}$ & $\begin{array}{r}17,0 \\
34,0 \\
4,3 \\
21,3 \\
21,3 \\
2,1\end{array}$ & $\begin{array}{r}8,9 \\
30,4 \\
8,0 \\
17,0 \\
9,8 \\
9,8 \\
16,1\end{array}$ & $\begin{array}{r}5,9 \\
38,8 \\
3,5 \\
17,6 \\
8,2 \\
14,1 \\
11,8\end{array}$ & * \\
\hline & $\begin{array}{l}\text {-Monatl. HH-Nettoeinkom. } \\
\text { - bis 1500,- DM } \\
\text { - } 1500-2500,- \text { DM } \\
\text { - 2500-3500,- DM } \\
\text { - 3500-5000,- DM } \\
\text { - über 5000,- DM }\end{array}$ & $\begin{array}{r}18,9 \\
28,1 \\
24,6 \\
21,5 \\
7,0\end{array}$ & $\begin{array}{r}26,7 \\
24,4 \\
26,7 \\
20,0 \\
2,2\end{array}$ & $\begin{array}{r}13,7 \\
29,4 \\
23,5 \\
25,5 \\
7,8\end{array}$ & $\begin{array}{r}21,0 \\
28,4 \\
24,7 \\
17,3 \\
8,6\end{array}$ & n.S. \\
\hline & $\begin{array}{l}\text {-Persönl. Netto-Einkom. } \\
\text { - bis 1500,- DM } \\
\text { - 1500-3000,- DM } \\
\text { - über } 3000,- \text { DM }\end{array}$ & $\begin{array}{l}39,4 \\
49,3 \\
11,3\end{array}$ & $\begin{array}{l}37,8 \\
51,1 \\
11,1\end{array}$ & $\begin{array}{l}37,4 \\
47,5 \\
15,2\end{array}$ & $\begin{array}{r}42,9 \\
50,6 \\
6,5\end{array}$ & n.S. \\
\hline & $\begin{array}{l}\text {-Schicht } \\
\text {-Unterschicht } \\
\text {-Mittelschicht } \\
\text {-Oberschicht } \\
\end{array}$ & $\begin{array}{l}14,9 \\
68,3 \\
16,7\end{array}$ & $\begin{array}{l}13,3 \\
57,8 \\
28,9\end{array}$ & $\begin{array}{l}11,5 \\
72,9 \\
15,6\end{array}$ & $\begin{array}{l}20,0 \\
68,8 \\
11,3 \\
\end{array}$ & * \\
\hline & $\begin{array}{ll}\text { Cl.1: } & \text { Strand-Urlauber } \\
\text { Cl.2: } & \text { Sportaktive \& Kult } \\
& \text { Familienurlauber } \\
\text { Cl.3: } & \text { Regenerations- \& }\end{array}$ & Urinteressi & e Urla & $\begin{array}{l}\text { Signifi } \\
\alpha<0,0 \\
\alpha<0,0 \\
\alpha<0,1 \\
\text { nicht }\end{array}$ & ignifikant & $\begin{array}{l}\text { (Chi } i^{2} \text {-Test): } \\
=* \star * \\
=* * \\
={ }^{*} \\
=\text { n.S. }\end{array}$ \\
\hline
\end{tabular}


sichtlich ist die Untersuchungsregion durchaus dazu geeignet, mit ihrem natürlichen Angebot das zentrale Urlaubsbedürfnis dieser zielgruppe $z u$ befriedigen. Allerdings bestehen negative Einstellungen sowohl gegenüber dem sportangebot als auch der touristischen sportunabhängigen Infrastruktur. Dabei ist jedoch davon auszugehen, daB angesichts des schwach ausgeprägten sportbezogenen Aktivitätsbedürfnisses der negativen Einstellung zum sportangebot keine Verhaltensrelevanz zukommt. Die relativ schlechte Beurteilung der touristischen sportunabhängigen Infrastruktur ist angesichts der niedrigen zielgebietserfahrung vor allem ein Resultat der fehlenden eigenen Erfahrungen mit der Untersuchungsregion. Die geringe zielgebietserfahrung in der Vergangenheit äußert sich auch in einem mit 29,5\% geringen Anteil an Touristen, die eine Besuchsabsicht in die Fremdenverkehrsregion äußern.

Es ist davon auszugehen, daß das eher negative Bild von der Untersuchungsregion auf Informationsdefizite zurückzuführen ist. Falls dieses Bild durch entsprechende kommunikative Aktivitäten in ein positives Image überführt werden kann, ist dieses segment jedoch als attraktive Zielgruppe der Untersuchungsregion zu bewerten.

Die soziodemographische Analyse zeigt, daß dieses segment durch einen hohen Anteil an Personen im Alter von über 45 Jahren geprägt ist. Angesichts des mit 54,25 Jahren höchsten Durchschnittsalters aller zielgruppen sind in diesem segment vergleichsweise wenig Personen mit Kindern unter 18 Jahren anzutreffen. Knapp zwei Drittel dieser zielgruppe gehören der Lebenszyklusphase IV (Jungsenioren) und V (Ruheständler) an. Das Ausbildungsniveau fällt infolge des von allen zielgruppen niedrigsten Anteils an Personen mit Abitur bzw. studium oder weiterbildenden schulen tendenziell gering aus. Immerhin drei viertel dieser zielgruppe haben nur eine Volksschule mit bzw. ohne abgeschlossener Lehre absolviert. 
Die Altersstruktur findet in der Berufsstruktur ihren Niederschlag. So ist der Anteil an Rentnern und Hausfrauen mit $32,8 \%$ bzw. 17,2 \& relativ hoch. Ein Blick auf das Einkommen zeigt darüber hinaus, daß die Befragten dieser zielgruppe zu den eher niedrig Verdienenden gehören. Über die Hälfte der Befragten dieses segments weisen nur ein monatliches NettoHaushaltseinkommen bis zu 2500,-- DM auf. Daher ist zu vermuten, daß diese zielgruppe nur über relativ preiswerte Urlaubsangebote als Besucher der Untersuchungsregion zu gewinnen ist.

\section{Cluster 2: 8portaktive Familienurlauber}

Als zweites Segment $(23,99 \%$ der Befragten in der Entfernungsgruppe I) wurde eine zielgruppe identifiziert, die ein eher undifferenziertes Bild in ihren Werteausprägungen aufweist. Demgegenüber liegen im Hinblick auf den Urlaub differenzierte Bedürfnisse vor. Dominante Urlaubsmotive sind das "sportbezogene Aktivitäts-" und das "familienorientierte Erlebnisbedürfnis". Letzteres drückt sich vor allem darin aus, daß der Urlaubsaktivität "mit den Kindern spielen" von allen Segmenten die größte wichtigkeitseinschätzung erfährt. Ferner werden sämtliche sportaktivitäten als überdurchschnittlich wichtig eingestuft. 247

Betrachtet man in diesem Zusammenhang die Einstellung zur Untersuchungsregion, ist vor allem die positive "Einstellung zum Sportangebot" hervorzuheben. Die Untersuchungsregion ist offenbar in der Wahrnehmung der Befragten gut geeignet, die sportspezifischen Bedürnisse dieser zielgruppe zu befriedigen. Auch die "touristische sportunabhängige Infrastruktur" erfährt eine überdurchschnittlich positive Beurteilung. Die hohe Zielgebietserfahrung in dieser zielgruppe macht weiter-

247 Vgl. Anhang I 
hin deutlich, daß das positive Meinungsbild der Untersuchungsregion vor allem aus eigenen Erfahrungen resultiert. Diese positive Einstellung äußert sich in einem sehr hohen Anteil an Personen, die in den nächsten sechs Monaten einen Besuch in die Untersuchungsregion beabsichtigen $(75,7 \%)$.

Die Charakterisierung anhand soziodemographischer Merkmale zeigt, daß dieses segment eine relativ ausgewogene Altersstrukur mit einem leicht überdurchschnittlichen Anteil an Personen der Altersklasse 45-65 Jahre aufweist. Entsprechend der hohen Bedeutung der Familienorientierung im Urlaub findet sich in diesem segment ein hoher Anteil an Personen mit Kindern unter 18 Jahren $(40 \%)$. Die sozioökonomischen Merkmale dieser zielgruppe weisen zunächst auf den uberdurchschnittlichen Anteil an Angestellten hin. Das Bildungsniveau dieser Zielgruppe ist relativ heterogen. So ist der Anteil an Personen mit Abitur und studium zwar als relativ hoch zu bezeichnen. Dennoch findet sich in diesem segment mit 55,0 \% auch ein hoher Anteil an Personen, die einen Volkschulabschluß mit abgeschlossener Lehre aufweisen. Die Einkommenssituation dieses segments ist als relativ gut zu bezeichnen, da ein hoher Teil an Personen ein hohes persönliches Nettoeinkommen aufweist. Dementsprechend sind immerhin 20,6 \% der Befragten dieses Segments der oberschicht zuzurechnen.

\section{cluster 3: Land- und kulturorientierte orlauber}

Die dritte und zugleich personenstärkste zielgruppe $(28,21$ \% der in der Entfernungsgruppe I Befragten) beurteilt mit Ausnahme des "Hedonismus" alle Werte als überdurchschnittlich wichtig für ihre eigene Lebensführung. Dabei kommt der "traditionellen und sicherheitsbetonten Werthaltung" sowie der "Freiheitsorientierung" ein besonders hoher stellenwert zu. Diese relativ undifferenziert hohe wichtigkeitseinschätzung von Werten spiegelt sich auch in den Urlaubsmotiven wieder. 
Allein dem "sportbezogenen Aktivitätsbedürfnis" kommt ein etwas geringerer stellenwert zu. Ein Blick auf einzelne Urlaubsaktivitäten macht jedoch deutlich, daB in diesem segment der "Besichtigung von historischen sehenwirdigkeiten" und dem "Besuch kultureller Veranstaltungen" eine besonders hohe Bedeutung im Urlaub zukommt. 248 Diese kulturellen Aktivitäten stehen offenbar in einem engen Verbund mit dem "sportaversen Bewegungsbedürfnis". "Besichtigungen von Sehenswürdigkeiten" und "Spaziergänge" bzw. "Wanderungen" sind miteinander verknüpft, so daß dieses segment trotz des relativ undifferenziert hohen Anspruchsniveaus als Land- und kulturorientierte Urlauber gekennzeichnet werden kann.

Ein Blick auf die Realeinstellungen zur Untersuchungsregion zeigt, daß alle Angebotskomponenten der Untersuchungsregion von den Mitgliedern dieses segments sehr positiv beurteilt werden. Allerdings ist diese positive Einstellung aufgrund einer geringen zielgebietserfahrung mit der Untersuchungsregion wohl nicht auf aktuelle eigene Erfahrungen zurückzuführen. Darüber hinaus äußert sich die positive Einstellung überraschenderweise nicht in einer überdurchschnittlich hohen Nennung von Besuchsabsichten. Es liegt daher die vermutung nahe, daß ein Teil der Mitglieder dieses Segments generell eine geringe Neigung zu Ausflügen und Urlauben im Naherholungsverkehr aufweist.

Als charakteristisches demographisches Merkmal dieser Zielgruppe ist zunächst der relativ hohe Anteil an Personen der Alterklasse 31-64 Jahre zu nennen. Zusammen mit dem geringen Anteil an Personen mit Kindern $(29,4 \%)$ äußert sich dies in einem überdurchschnittlichen Anteil an Personen der Lebenszyklusphase II (Singles \& Paare). Die Analyse der Bildungsstruktur zeigt einen relativ hohen Anteil an Personen, die weiterbildende schulen besucht haben. Darüber hinaus sind 
aber auch relativ viele Personen mit Volkschulabschluß ohne abgeschlossener Lehre in diesem segment vertreten. Letzteres resultiert in einen hohen Anteil an Arbeitern. Dennoch weist dieses Segment von allen Zielgruppen den höchsten Anteil an Personen auf, denen ein Netto-Haushaltseinkommen von über 3500,- DM zur Verfügung steht. Der Vergleich mit dem persönlichen Netto-Einkommen deutet darauf hin, daB das hohe Haushaltseinkommen auf zweit-Verdiener zurückzuführen ist.

\section{Cluster 4: Regenerations- und genuBorientierte strand- urlauber}

Die dem vierten Segment angehörenden Befragten $(25,46 \%$ der Befragten der Entfernungsgruppe I) räumen der hedonistischen Wertorientierung und dem Wert "Freiheitsorientierung" einen besonders hohen stellenwert zu. Diese Werthaltungen äußern sich im Urlaub in stark ausgeprägten sportbezogenen Aktivitäts- und Regenerationsbedürfnissen. Betrachtet man in diesem Zusammenhang die einzelnen Urlaubsaktivitäten, so zeigt sich, daß das sportbezogene Aktivitätsbedürfnis in diesem Segment auf "Schwimmen gehen", "am strand liegen" und "Baden" einzugrenzen ist. 249 offensichtlich handelt es sich bei dieser Zielgruppe um den typischen strand- und Badeurlauber. Dieses einseitige Anforderungsprofil äußert sich bezüglich der vorliegenden Fremdenverkehrsregion in einer stark unterdurchschnittlichen Bewertung sowohl der "touristischen sportunabhängigen Infrastruktur" als auch des "natürlichen Angebots". Ferner deutet die geringe zielgebietserfahrung und Besuchsabsichtsnennung auf nur geringe Akquisitionspotentiale hin.

Diese Zielgruppe ist durch einen überdurchschnittlichen Anteil an Personen im Alter von bis zu 45 Jahren und Personen 
mit Kindern gekennzeichnet. Daher befinden sich drei viertel der Befragten in den ersten drei Lebenszyklusphasen. Das geringe Durchschnittsalter von 33,62 Jahren äußert sich in dem mit $20,7 \%$ recht hohen Anteil an Studenten, Schülern und Auszubildenden. Hierin begründet sich auch das niedrige Einkommensniveau dieser Zielgruppe. Aufgrund der geringen Kaufkraft dieser zielgruppe und dem von der Untersuchungsregion kaum $\mathrm{zu}$ befriedigenden Urlaubswünschen ist eine gezielte Bearbeitung dieser Zielgruppe nur bedingt sinnvoll.

\section{Entfernungsgruppe II}

\section{Cluster I: strand-Urlauber}

Die erste Zielgruppe der Entfernungsgruppe II weist generell ein starkes Desinteresse an Werten auf. Vor allem die werte "Tradition und Sicherheit" und "Gesundheit und Umwelt" werden als unwichtig erachtet. Im Urlaub strebt diese zielgruppe vor allem nach der Befriedigung ihres "sportbezogenen Aktivitätsbedürfnisses". Ähnlich wie bei der in der Entfernungsgruppe I identifizierten Zielgruppe "Badeurlauber" ist auch bei diesem segment das sportbezogene Aktivitätsbedürfnis auf die Urlaubsaktivität "sonnen, baden, am Strand liegen" einzugrenzen. 250 Angesichts dieses einseitigen Anforderungsprofils an einen Urlaub, ist die schlechte Bewertung der touristischen sportunabhängigen Infrastruktur und des natürlichen Angebots nicht überraschend. Allerdings besteht gegenüber dem sportangebot eine relativ positive Einstellung. Das einseitige Anforderungsprofil und die unterdurchschnittliche Zielgebietserfahrung äußern sich in der von allen Zielgruppen dieser Entfernungsgruppe niedrigsten Besuchsabsichtsnennung. Nur ein Viertel dieser Zielgruppe beabsichtigt in den nächsten sechs Monaten einen Besuch der 
Region. Insgesamt stellt dieses segment daher eine vergleichsweise unattraktive zielgruppe für die Untersuchungsregion dar.

Bei der soziodemographischen Beschreibung dieser zielgruppe fällt zunächst der relativ hohe Anteil an Männern sowie das niedrige Durchschnittsalter $(36,57$ Jahre) auf. Ferner haben weniger als zehn Prozent dieser Befragten keine Kinder, so daß die Lebenszyklusphasen I und II entsprechend dominieren. Die Berufsstruktur zeigt konsequenterweise einen niedrigen Anteil an Rentnern. Demgegenüber sind studenten, Auszubildende und Schüler $(21,3 \%)$ sowie Selbständige $(17,0 \%)$ in diesem segment überdurchschnittlich stark vertreten. Daher ist auch das Bildungsniveau dieser Zielgruppe sehr hoch. Fast die Hälfte der dieser Zielgruppe angehörenden Personen weisen ein Abitur, FH-AbschluB oder Studium auf.

\section{cluster 2: sportaktive und kulturinteressierte Familienur- lauber}

Dieses Segment ordnet insbesondere den Werten "Gesundheit und Umwelt", "Tradition und Sicherheit" sowie "Bildungs- und Gesellschaftsorientierung" eine überdurchschnittliche Wichtigkeit zu. Diese Werthaltungen schlagen sich unmittelbar in den Urlaubsmotiven nieder. Den Befragten dieses Segments sind das "kulturelle Bedürfnis", das "sportaverse Bewegungsbedürfnis" sowie das "familienorientierte Erlebnisbedürfnis" überdurchschnittlich wichtig, wobei das "sportaverse Bewegungsbedürfnis" im Vergleich $z u$ den anderen Motiven als deutlich unwichtiger eingestuft wird. Offensichtlich strebt diese Zielgruppe im Urlaub nach der Verwirklichung ihrer Werthaltungen. Betrachtet man in diesem Zusammenhang die Urlaubsaktivitäten, so zeigt sich, daß die Personen dieser Zielgruppe den Urlaubsaktivitäten "Besichtigung von kulturellen sehenswürdigkeiten" aber auch den "sportbezogenen 
Aktivitäten" eine relativ hohe Bedeutung einräumen. 251 Der in den Werthaltungen zum Ausdruck kommende Wunsch nach einem ausgeprägten Familienleben 252 spiegelt sich in der als uberdurchschnittlich wichtig erachteten Urlaubsaktivität "mit den Kindern spielen" wieder. Daher werden diese Personen als "sportaktive und kulturinteressierte Familienurlauber" bezeichnet. Da die Urlaubsbedürfnisse gut mit dem touristischen Angebot der Untersuchungsregion befriedigt werden können, ist dieses segment für die Untersuchungsregion als attraktive zielgruppe $z u$ bezeichnen. Dies bestätigt sich auch in der Einstellung $z u$ den Angebotskomponenten der Untersuchungsregion. Vor allem das "natürliche Angebot" und das "Sportangebot" werden positiv beurteilt. Dieses positive Meinungsbild äußert sich in einem mit über $50 \%$ sehr hohen Anteil an Personen, die in den nächsten sechs Monaten einen Besuch in die Untersuchungsregion beabsichtigen.

Hinsichtlich der soziodemographischen Merkmale ist zunächst der hohe Altersdurchschnitt festzustellen. Ferner weist dieses Segment den von allen Zielgruppen der Entfernungsgruppe II höchsten Anteil an Personen mit Kindern auf. Die Altersstruktur und die Kinderanzahl äußern sich erwartungsgemä $B$ in einem hohen Anteil an Mitgliedern, die sich in den Lebenszyklusphasen III (Familien mit Kindern) und $V$ (Rentner) befinden. Sozioökonomische Merkmale weisen demgegenüber nur geringe Abweichungen vom Durchschnitt auf. Das Einkommensniveau dieser zielgruppe liegt leicht über dem Durchschnitt, wobei insgesamt davon auszugehen ist, daß diese zielgruppe über ein ausreichendes Finanzbudget für Urlaube verfügt.

\footnotetext{
251 Vgl. Anhang I

252 Vgl. hierzu vor allem die im Anhang I dargestellte wichtigkeitseinschätzung der Werteindikatoren
} 


\section{Cluster 3: Regenerations- und genuBorientierte orlauber}

Die dritte und zugleich letzte zielgruppe der Entfernungsgruppe II beurteilt mit Ausnahme des Wertes "Bildungs- und Gesellschaftsorientierung" alle Werte als uberdurchschnittlich wichtig. Im Urlaub dominiert das "Regenerations- und Genußbedürnis". Betrachtet man in diesem zusammenhang die Urlaubsaktivitäten, 253 so zeigt sich, daB neben dem Wunsch nach "Ruhe" und "sich ausruhen", die Aktivität "Einkaufen gehen" noch als überdurchschnittlich wichtig eingestuft wird. Die Einstellungen zur Untersuchungsregion sind demgegenüber differenziert $z u$ betrachten. Während die "touristische sportunabhängige Infrastruktur" sehr positiv beurteilt wird, bestehen deutlich negative Einstellungen zum "natürlichen Angebot" und zum "Sportangebot". Da diese Angebotskomponenten für einen angenehmen Urlaub für die Mitglieder dieser zielgruppe nur von untergeordneter Bedeutung sind, ist davon auszugehen, daß hierdurch die Besuchsabsicht in die Fremdenverkehrsregion nicht entscheidend beeinfluBt wird. Dies bedeutet, daB der geringe Anteil an Personen, die einen Besuch in die Untersuchungsregion in den nächsten sechs Monaten beabsichtigen, nicht auf diese negativen Einstellungen zurückzuführen ist. Eine gezielte Bearbeitung dieses segmentes scheint erfolgsversprechend.

Gekennzeichnet ist diese zielgruppe durch einen überdurchschnittlichen Anteil an Personen der Altersklasse 31-45 Jahre. Da nur ein geringer Teil der Befragten Kinder hat, findet sich tendenziell eine ausgewogene Verteilung der Lebenszyklusphasen, wobei entsprechend der Altersstruktur die Phasen I (Junge Erwachsene) und II (Singles und Paare) einen leicht überdurchschnittlichen Anteil aufweisen. Charakteristisches Merkmal der Bildungsstruktur dieses segments ist der mit 54,8 $\%$ hohe Anteil an Personen mit abgeschlossener

253 Vgl. Anhang I 
Lehre. Darüber hinaus ist eine im Vergleich zum Durchschnitt ausgewogene Berufsstruktur dieser zielgruppe festzustellen.

Die zielgruppenbeschreibungen zeigen, daB sich die in den beiden Entfernungsgruppen ermittelten Zielgruppen in Abhängigkeit von ihren Werten, Urlaubsmotiven sowie Einstellungen zur Untersuchungsregion stark voneinander unterscheiden. Ferner deuten die signifikanten Unterschiede in den Besuchsabsichtsnennungen darauf hin, daß die psychographische Marktsegmentierung die Anforderung der Besuchsverhaltensrelevanz erfüllt. Demgegenüber ist kritisch anzumerken, daß zwischen den segmenten keine signifikanten Unterschiede in der beabsichtigten Besuchsdauer festzustellen sind. Dies bedeutet, $d a \beta$ bei der Ableitung von Ansatzpunkten für eine Verlängerung der Aufenthaltsdauer auf die Ergebnisse der verhaltensorientierten Marktsegmentierung zurückzugreifen ist.

Darüber hinaus verdeutlicht die soziodemographische Beschreibung der segmente, daß eine auf der Basis zentraler sozziodemographischer Bestimmungsfaktoren des Besuchsverhaltens durchgeführte psychographische Marktsegmentierung die Anforderung der Identifizierbarkeit der segmente gut er-

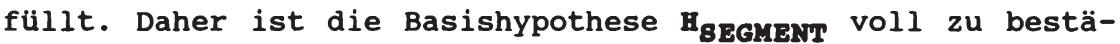
tigen.

Insgesamt bleibt festzuhalten, daß die hier gewählte Vorgehensweise, auf der Basis der wesentlichen Bestimmungsfaktoren des Besuchsverhaltens eine psychographische Marktsegmentierung durchzuführen, dazu geeignet ist, den Anforderungen an Marktsegmentierungskonzepten zu entsprechen. Die Segmentbeschreibungen deuten ferner darauf hin, daß die psychographische Marktsegmentierung besser eine soziodemographische Identifizierbarkeit gewährleistet als eine verhaltensorientierte Marktsegmentierung. 
Da für eine Fremdenverkehrsregion eine differenzierte Marktbearbeitung von sieben segmenten vor allem aufgrund von Wirtschaftlichkeitsüberlegungen wenig zweckmäßig erscheint, stellt sich im folgenden die Frage, welche zielgruppen die Untersuchungsregion für eine gezielte Marktbearbeitung auswählen soll. Die einzelnen zielgruppen sollten eine ausreichende Größe aufweisen, um eine effiziente Marktbearbeitung zu ermöglichen. In diesem zusammenhang muß überprüft werden, ob segmente der Entfernungsgruppe I und II aufgrund ähnlicher Ausprägungsformen der psychographischen Merkmale mit dem gleichen Marktbearbeitungskonzepten bearbeitet werden können.

\subsection{Homogenitätsanalyse der makrosegmentspezifischen ziel- gruppen}

Zur Überprügung der in den Entfernungsgruppen I und II identifizierten zielgruppen auf mögliche Gemeinsamkeiten ist es erforderlich, einen Vergleichsmaßstab auszuwählen. Eine Überprüfung aller psychographischen Merkmale hinsichtlich ihrer Homogenität zwischen allen Zielgruppen der Entfernungsgruppen soll an dieser stelle aufgrund der damit verbundenen Komplexität unterbleiben. Auch die alleinige überprüfung auf Basis von Werten oder Einstellungen erscheint wenig sinnvoll, da diese in ihrer isolierten Betrachtung nur unzureichend Anhaltspunkte für die Ausgestaltung von Marktbearbeitungsprogrammen geben. Unterstellt man, daB negative Einstellungen zur Untersuchungsregion überwiegend auf wissens- und Informationsdefizite zurückgeführt werden können, geben diese vor allem Hinweise auf die Notwendigkeit einer verstärkten Informationspolitik. Die inhaltliche Ausgestaltung der Informationspolitik sollte sich jedoch nicht nur an möglichen wissensdefiziten oder Fehlwahrnehmungen orientieren, sondern insbesondere auf die Beweggründe des Besuchsverhaltens Bezug nehmen. Daher ist es zweckmäBig, die Homo- 
genitätsanalyse zwischen den identifizierten zielgruppen der beiden Entfernungsgruppen auf der Basis von Urlaubsmotiven vorzunehmen.

Abbildung 39 stellt die Struktur der Urlaubsmotive in den ermittelten Zielgruppen dar. Auf der Basis einer Analyse dieser Motivstrukturen ist es möglich, Aussagen über Ähnlichkeiten zwischen den zielgruppen abzuleiten.

Es zeigt sich, daß die sportaktiven Familienurlauber der Entfernungsgruppe I eine ähnliche Motivstruktur aufweisen wie die sportaktiven und kulturinteressierten Familienurlauber der Entfernungsgruppe II. Der Unterschied zwischen diesen zielgruppen besteht nahezu ausschlieblich in der Höhe der Wichtigkeitseinschätzungen der Urlaubsmotive. Die Rangfolge der Urlaubsmotive ist weitgehend identisch. Vor diesem Hintergund ist eine unterschiedliche Marktbearbeitung dieser zielgruppen offensichtlich nicht erforderlich. Für die weitere Untersuchung werden sie daher zusammengefaBt und als zielgruppe der "sportaktiven Familienurlauber" gekennzeichnet.

Eine hohe Übereinstimmung in der Motivstruktur findet sich auch zwischen den regenerations- und genuBorientierten strandurlaubern und den 8trand-Urlaubern der Entfernunsgruppen I und II. Beide Zielgruppen weisen als dominantes Urlaubsmotiv das sportbezogene Aktivitätsbedürfnis auf, welches vor allem den Wunsch nach "Baden", "Schwimmen" und "am strand liegen" umfaßt. Das Regenerationsbedürfnis ist bei beiden zielgruppen das zweitwichtigste Urlaubsmotiv. Daher können beide Zielgruppen zum Zwecke der Markbearbeitung zusammengefaßt werden. Im folgenden sind sie als "strand/Badeurlauber" bezeichnet. 


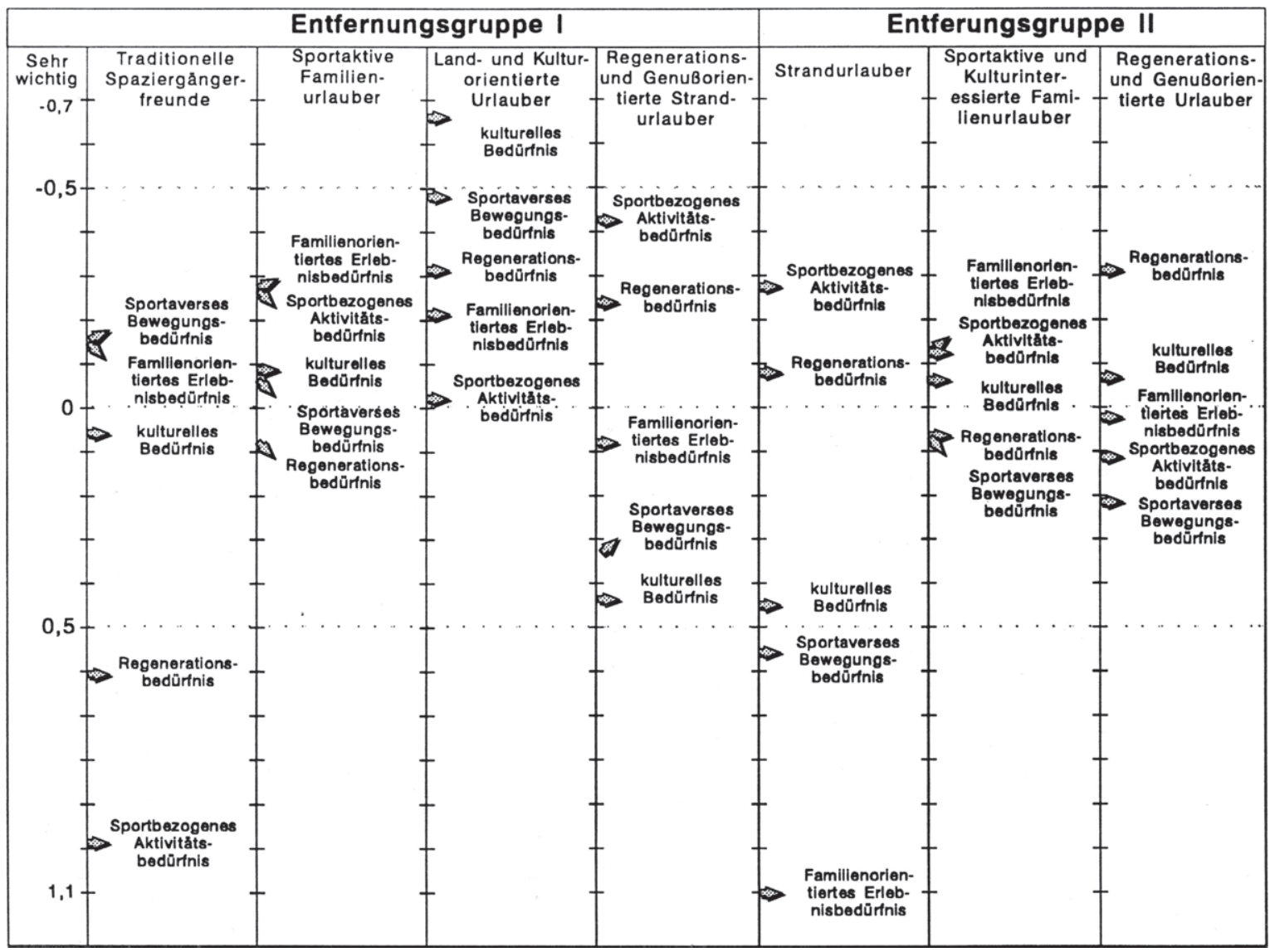

Abb. 39: Struktur der Urlaubsmotive in den Zielgruppen der Entfernungsgruppen I und II 
Die "land- und kulturorientierten Urlauber" sowie die "traditionellen Spaziergängerfreunde" der Entfernungsgruppe I und die "Regenerations- und genußorientierten Urlauber der Entfernungsgruppe II weisen erhebliche Unterschiede in ihren Motivstrukturen auf. Daher sind für diese zielgruppen jeweils eigenständige Marktbearbeitungsprogramme $z u$ entwickeln.

Zusammenfassend bedeutet dies, daß die Zahl der zu bearbeitenden segmente um zwei auf fünf zielgruppen reduziert werden konnte. Im folgenden wird daher überprüft, wie die Fremdenverkehrsregion eine zielgruppenauswahl und eine zielgruppenspezifische Marktbearbeitung durchführen kann. 


\section{c. Ansatzpunkte einer zielgruppenspezifischen Marktbearbei-} tung im Fremdenverkehr von Regionen

Im vorherigen Untersuchungsabschnitt wurden auf der Basis einer psychographischen Marktsegmentierung innerhalb entfernungsgruppenspezifischer Makrosegmente voneinander abgrenzbare zielgruppen identifiziert, die sich sowohl in ihren psychographischen (werte-, motiv- und einstellungsbezogenen) und soziodemographischen Merkmalen als auch in ihrem Besuchsverhalten unterscheiden. Die wesentlichen Merkmale dieser Zielgruppen sind in Abbildung 40 zusammengefaBt. Mit Hilfe dieser Informationen ist es der Marketing betreibenden regionalen Fremdenverkehrsorganisation möglich, eine Auswahl geeigneter zielgruppen vorzunehmen und entsprechende zielgruppenspezifische Marktbearbeitungskonzepte zu erstellen.

Bei der zielgruppenspezifischen Ausgestaltung der Marketingmaßnahmen für Fremdenverkehrsregionen kann grundsätzlich auf das bekannte Marketinginstrumentarium des Konsumgüter- und Dienstleistungsbereichs zurückgegriffen werden. ${ }^{1}$ Die Besonderheit im Marketing von Fremdenverkehrsregionen besteht darin, daß in der Umsetzungsphase ${ }^{2}$ heterogene und nur be-

1 So hat Meffert für den analogen Anwendungsfall des städtemarketing den grundsätzlichen Eignungsgrad des Marketing-Mix als Gestaltungsinstrumentarium nachgewiesen. Vgl. Meffert, H., städtemarketing - Pflicht oder Kür?, in: Symposium Stadtvisionen 1989, Dokumentation, Ideenschmiede Münster (Hrsg.), Münster 1989, S. 1 ff. Vgl. zur ausführlichen Darstellung der Marketing-MixMaßnahmen im Konsumgüterbereich Meffert, H., Marketing, a.a.0., S. 259 ff.; Nieschlag, R., Dichtl, E., Hörschgen, H., Marketing, a.a.0., S. 93 ff.; zur Darstellung der Marketing-Mix-Maßnahmen im Dienstleistungsbereich vgl. Maleri, R., Grundlagen der Dienstleistungsproduktion, 2. Aufl., Berlin, Heidelberg 1991, S. 176 ff.

2 Die Umsetzungsphase umfaßt vor allem die Aspekte der Spezifizierung von strategien in ein konkretes Maßnahmenpaket. Vgl. Meffert, H., Grundlagen der Strategieimplementierung, in: Marketingmanagement - Analyse, strategie, Umsetzung, Wiesbaden 1993 (im Druck), s.6. 


\begin{tabular}{|c|c|c|c|c|c|c|c|}
\hline & \begin{tabular}{|c|} 
Traditionelle \\
Spazler- \\
gänger- \\
freunde
\end{tabular} & $\begin{array}{l}\text { Land- und } \\
\text { Kulturorien- } \\
\text { tlerte } \\
\text { Urlauber }\end{array}$ & \multicolumn{2}{|c|}{$\begin{array}{c}\text { Sportaktive } \\
\text { Familien- } \\
\text { urlauber }\end{array}$} & $\begin{array}{c}\text { Regenera- } \\
\text { tions- und } \\
\text { GenuBorien- } \\
\text { tierte } \\
\text { Urlauber }\end{array}$ & \multicolumn{2}{|c|}{$\begin{array}{l}\text { Bade- } \\
\text { urlauber }\end{array}$} \\
\hline Markt & $\begin{array}{c}\text { Entfernungs- } \\
\text { gruppe I }\end{array}$ & $\begin{array}{c}\text { Entfernungs- } \\
\text { gruppe I }\end{array}$ & \multicolumn{2}{|c|}{$\begin{array}{c}\text { Gesamt- } \\
\text { markt }\end{array}$} & $\begin{array}{c}\text { Entfernungs } \\
\text { gruppe II }\end{array}$ & \multicolumn{2}{|c|}{$\begin{array}{c}\text { Gesamt- } \\
\text { markt }\end{array}$} \\
\hline \begin{tabular}{|l|} 
Segment- \\
gröBe: \\
-bezogen auf \\
Teilmarkt \\
-bezogen auf \\
Gesamtmarkt \\
\end{tabular} & $\begin{array}{l}22,34 \% \\
15,35 \% \\
\end{array}$ & $\begin{array}{l}28,21 \% \\
19,37 \% \\
\end{array}$ & \multicolumn{2}{|c|}{$\begin{array}{c}\text { I: } 23,99 \% \\
\text { II: } 46,99 \% \\
31,19 \% \\
\end{array}$} & $\begin{array}{l}34,14 \% \\
10,69 \% \\
\end{array}$ & \multicolumn{2}{|c|}{$\begin{array}{c}\text { I: } 25,46 \% \\
\text { II: } 18,89 \% \\
23,40 \% \\
\end{array}$} \\
\hline Werte & $\begin{array}{c}\text { konservativ, } \\
\text { sicherheits- } \\
\text { betont, ge- } \\
\text { sundheits- } \\
\text { orientiert }\end{array}$ & $\begin{array}{l}\text { Wertefan, } \\
\text { insb. } \\
\text { traditions- } \\
\text { und sicher- } \\
\text { heitsorien- } \\
\text { tiert und } \\
\text { freiheits- } \\
\text { orientiert }\end{array}$ & $\begin{array}{c}\text { undiff. } \\
\text { Werte- } \\
\text { aus- } \\
\text { prā- } \\
\text { gung- } \\
\text { en }\end{array}$ & \begin{tabular}{|l|} 
tradi- \\
tions- \\
und \\
ge- \\
sund- \\
heits- \\
orien- \\
tiert
\end{tabular} & Wertefan & $\begin{array}{l}\text { Hedo- } \\
\text { nis- } \\
\text { tisch } \\
\text { und } \\
\text { frei- } \\
\text { heits- } \\
\text { orien- } \\
\text { tiert }\end{array}$ & $\begin{array}{c}\text { Werte- } \\
\text { geg- } \\
\text { ner }\end{array}$ \\
\hline Motive & $\begin{array}{l}\text { sportaverses } \\
\text { Bewegungs- } \\
\text { bedurfnis: } \\
\text { insb. spa- } \\
\text { zleren-- } \\
\text { gehen }\end{array}$ & $\begin{array}{l}\text { hohes An- } \\
\text { spruchsniveau; } \\
\text { mil schwerpunkt } \\
\text { auf kulturellem } \\
\text { Bedürfis und } \\
\text { sportaversem } \\
\text { Bewegungsbe- } \\
\text { durfnis }\end{array}$ & \multicolumn{2}{|c|}{ 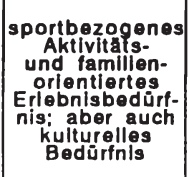 } & $\begin{array}{l}\text { Regenera- } \\
\text { tions-- } \\
\text { und Genup- } \\
\text { bedúfnis }\end{array}$ & \multicolumn{2}{|c|}{ 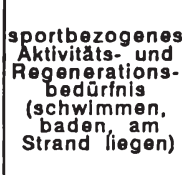 } \\
\hline $\begin{array}{l}\text { Einstell- } \\
\text { ungen }\end{array}$ & 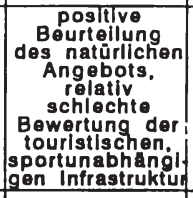 & $\begin{array}{c}\text { positive } \\
\text { Beurreilung } \\
\text { aller } \\
\text { Angebots. } \\
\text { komponenten }\end{array}$ & \multicolumn{2}{|c|}{$\begin{array}{c}\text { positive } \\
\text { Beurteilung } \\
\text { aller } \\
\text { Angebots. } \\
\text { komponenten }\end{array}$} & \begin{tabular}{|c|} 
positive Be- \\
wertung der \\
touristischen \\
Infrastruktur. \\
negative Ein- \\
stellungen \\
zum natürlichen \\
Angebot \\
\end{tabular} & \multicolumn{2}{|c|}{ 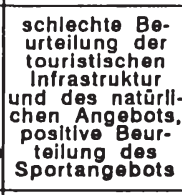 } \\
\hline $\begin{array}{c}\text { Zielgebiets- } \\
\text { erfahrung }\end{array}$ & gering & gering & \multicolumn{2}{|c|}{ hoch } & mittel & \multicolumn{2}{|c|}{ gering } \\
\hline $\begin{array}{l}\text { Sozio- } \\
\text { demo- } \\
\text { graphie }\end{array}$ & $\begin{array}{c}\text { alter: viele } \\
\text { Rentrer. gae- } \\
\text { ringeres. Aus- } \\
\text { bildungsniveau; } \\
\text { geringeres Ein- } \\
\text { kommen }\end{array}$ & $\begin{array}{l}31-64 \text { jahrige: } \\
\text { singles \& Paare: } \\
\text { mitteres } \\
\text { Bildungs- } \\
\text { niveau: h8heres } \\
\text { Einkommens- } \\
\text { niveau durch } \\
\text { Zweitverdiener }\end{array}$ & \multicolumn{2}{|c|}{$\begin{array}{c}\text { Personen mit } \\
\text { Kindern: } \\
\text { Angestellie: } \\
\text { hoheres Ein. } \\
\text { kommen }\end{array}$} & \begin{tabular}{|c|}
$31-45$ \\
Singles ahrige: \\
Paare: \\
mittleres \\
Bildungs- \\
niveau:
\end{tabular} & \multicolumn{2}{|c|}{$\begin{array}{l}\text { jünger: hohes } \\
\text { Bildungs:- } \\
\text { niveau: } \\
\text { geringeres } \\
\text { Einkommen }\end{array}$} \\
\hline $\begin{array}{c}\text { Besuchs- } \\
\text { absicht }\end{array}$ & gering & $\begin{array}{c}\text { durch- } \\
\text { schnittlich }\end{array}$ & \multicolumn{2}{|c|}{ sehr hoch } & gering & \multicolumn{2}{|c|}{ sehr gering } \\
\hline
\end{tabular}

Abb. 40: Zusammenfassung der wesentlichen Merkmale der identifizierten zielgruppen 
dingt kooperierende Leistungsanbieter die eigentliche Leistungserstellung übernehmen. Da die Marktbearbeitungskonzeption der regionalen Fremdenverkehrsorganisation nicht kongruent mit den Marktbearbeitungsprogrammen der Einzelanbieter sein muB, sind zwischen beiden Ebenen vielfältige interne Durchsetzungskonflikte ${ }^{3} \mathrm{zu}$ erwarten.

Ferner kann die regionale Fremdenverkehrsorganisation selbst nur wenige direkte absatzpolitische Maßnahmen ergreifen. Diese variieren zudem in Abhängigkeit von der organisatorischen Gestaltung der Fremdenverkehrsorganisation und der ihr zugewiesenen Kompetenzbereiche. 4 Die Hauptfunktion der Fremdenverkehrsorganisation besteht daher zunächst in der Planung eines sielgruppenspesifischen Marktbearbeitungskonzopts. Dieses ist als ein umfassender gedanklicher Entwurf zur Marktbearbeitung einer Region zu verstehen, der sich an bestimmten Richtgrößen (zielen) orientiert und grundlegende Handlungsrahmen (Strategien) und notwendige operative Handlungen der verschiedenen regionalen Leistungsträger gegenüber touristischen zielgruppen in einem schlüssigen durch die regionale Fremdenverkehrsorganisation formulierten plan zusammenfaBt. 5

3 Durchsetzungskonflikte entstehen vor allem aus zielkonflikten zwischen den touristischen Leistungsträgern und der regionalen Fremdenverkehrsorganisation und aus Erwartungsdivergenzen aufgrund unterschiedlicher Erfolgseinschätzungen der prognostizierten strategiewirkungen. Ferner können diese Konflikte aus persönlichkeitsbezogenen und/oder qualifikationsbezogenen Einstellungen resultieren. Diese Konflikte können eine einheitliche Durchsetzung zielgruppenspezifischer Marktbearbeitungskonzepte durch alle in der Fremdenverkehrsregion operierenden touristischen Leistungsanbieter verhindern. Vgl. Meffert, H., Grundlagen der strategieimplementierung, a.a.0., s. 8 .

4 Vgl. hierzu Klemz, w., Interkommunale zusammenarbeit im Fremdenverkehrsbereich, a.a.0., s. $34 \mathrm{ff}$.

Vgl. dazu in Anlehnung an Meffert, H., Marketing, a.a.o., s. 54 . 
Vor diesem Hintergrund besteht ein erster zentraler Bestandteil eines zielgruppenspezifischen Marktbearbeitungskonzeptes darin, daß die Fremdenverkehrsorganisation aus einer leistungsträgerunabhängigen sicht globale Ansatzpunkte der Leistungs-, Kontrahierungs-, Distributions- und Kommunikationspolitik für die jeweiligen zielgruppen aufzeigt.

In einem zweiten Schritt gilt es, die entwickelten Marktbearbeitungskonzepte an die eigentlichen touristischen Entscheidungsträger ${ }^{6}$ heranzutragen und somit indirekt für ihre Umsetzung Sorge zu tragen. Regionale Fremdenverkehrsorganisationen sind nicht wie Unternehmen durch einen hierarchisch gestalteten Aufbau gekennzeichnet. Deshalb stehen ihnen i.d.R. keinerlei Sanktionsmechanismen zur Verfügung. Einen Überblick über die Aufgabenverteilung im Zielgruppenmarketing von Regionen auf die regionale Fremdenverkehrsorganisation und die touristischen Leistungsträger findet sich in Abbildung 41 .

Die Umsetzung einer differenzierten Marktbearbeitungsstrategie setzt zunächst die Auswahl von zielgruppen voraus. Im folgenden ist $z u$ analysieren, welche zielgruppensegmente zweckmäBigerweise für die Marktbearbeitung auszuwählen sind.

\section{Zielgruppenauswahl im Fremdenverkehr von Regionen}

Die durchgeführte Markterfassung führte zunächst nur zu einer zusammenfassung aktueller und potentieller Touristen in homogene segmente. Die Umsetzung einer differenzierten Marktbearbeitungstategie erfordert dagegen eine Entscheidung darüber, welche und wieviele zielgruppen in welcher Intensi-

6 Dabei sind als touristische Entscheidungsträger einerseits die einzelnen Fremdenverkehrsunternehmen und andererseits die politischen und verwaltungsmäBigen Gremien der kommunalen Ebene zu nennen. 


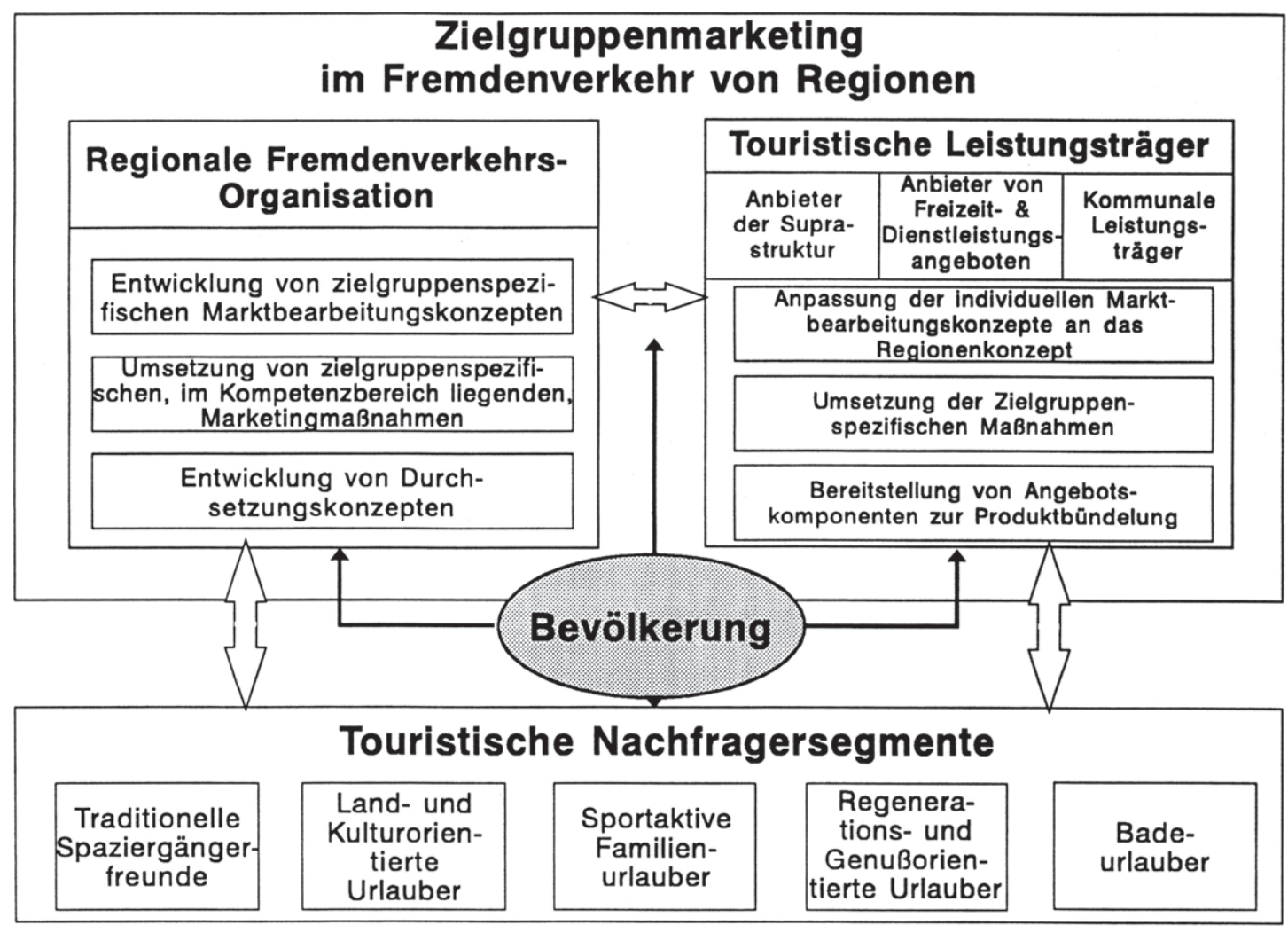

Abb. 41: Aufgabenverteilung im Zielgruppenmarketing von Fremdenverkehrsregionen 
tät mit segmentspezifischen Marketingprogrammen bearbeitet werden sollen. 7

Die Auswahl von Zielgruppen steht dabei in unmittelbarem Zusammenhang mit der $\mathrm{zu}$ verfolgenden Marktbearbeitungsstrategie. Hierbei ist die differenzierte und die konzentrierte Marktbearbeitungsstrategie zu unterscheiden. 8 Von einer konzentrierten Marktbearbeitungsstrategie wird gesprochen, wenn in einem Teilmarkt nur eine oder einige zielgruppen mit einem einheitlichen Marketingprogramm bearbeitet werden und das ziel verfolgt wird, in diesem zielsegment eine starke Marktstellung $z \mathrm{u}$ erreichen. Die Umsetzung einer solchen strategie im Fremdenverkehr von Regionen erscheint jedoch vergleichsweise schwierig. So lassen es die Heterogenität im touristischen Leistungsangebot einer Fremdenverkehrsregion und die rechtliche selbständigkeit der einzelnen Leistungsanbieter wenig sinnvoll erscheinen, nur eine einzige spezifische zielgruppe in einer Fremdenverkehrsregion zu bearbeiten. Deshalb bieten sich für Fremdenverkehrsregionen vor allem differenzierte Marktbearbeitungsstrategien an, im Rahmen derer eine differenzierte Ansprache aller oder vieler zielgruppen vorgenommen wird.

Im Rahmen der zielgruppenauswahl sind die zielgruppen dahingehend zu beurteilen, inwieweit sie zur zielerreichung einer Fremdenverkehrsregion beitragen. 9 In Anlehnung an die zielsetzungen des zielgruppenmarketing von Fremdenverkehrsregionen kann die zielgruppenspezifische Marktbearbeitung direkt

7 Vgl. Bauer, E., Markt-Segmentierung als Marketing-Strategie, a.a.0., S. $119 \mathrm{ff}$.

8 Vgl. Meffert, H., Marketing, a.a.O., S. 254 f.; Kotler, Ph., Marketing Management. Analysis, Planning, Implementation, and Control, 6. Aufl., Englewood Cliffs, S. 302 f.

9 Vgl. Bauer, E., Markt-Segmentierung als Marketing-strategie, a.a.o., s. 121 . 
auf die Erhöhung ökonomischer zielerreichungsgrade (Erhöhung der Gäste- und Übernachtungszahlen, Erhöhung der Ausgaben während des Aufenthalts, etc.) ${ }^{10}$ gerichtet sein. Die Realisierung psychographischer ziele, wie zum Beispiel Erhöhung des Bekanntheitsgrades sowie Imageverbesserung steht dabei in einem Mittel-Zweck-Zusammenhang zu den ökonomischen zielen. Aus den psychographischen zielen lassen sich Soll-Positionierungen für die einzelnen zielgruppen ableiten. zum Beispiel kann für die Zielgruppe der "sportaktiven Familienurlauber" das Imageziel "Profilierung der Fremdenverkehrsregion als familienfreundliches Urlaubsparadies" vorgegeben werden.

Zur Zielgruppenauswahl gibt es in der Marktsegmentierungsliteratur eine vielzahl von Bewertungsverfahren und -kriterien. ${ }^{11}$ Bewertungsverfahren können in heuristische und analytische Verfahren unterteilt werden. ${ }^{12}$ Dabei finden in der Unternehmenspraxis vor allem heuristische Verfahren Verwendung, die sich in Checklist-Verfahren, Punktbewertungsver-

Vgl. Kapitel A.3.

11 Vgl. Bauer, E., Markt-Segmentierung als Marketing-strategie, a.a.O., S. 121 ff.; Kollat, D.T., Blackwell, R.D., Robeson, J.F., Strategic Marketing, New York 1972, S. $199 \mathrm{ff}$.

12 Heuristische Methoden umfassen qualitative Verfahren, die im Vergleich $z u$ den analytischen Methoden geringere Anforderungen an die Informationsbeschaffung stellen. Analytische Methoden unterstellen, daB eine Quantifizierung der relevanten Alternativen, Umweltsituationen und Handlungskonsequenzen möglich ist. Da sie von einem guten Informationsstand und geschlossenen Entscheidungsproblemen ausgehen, sollen sie im Rahmen dieser Untersuchung nicht weiter betrachtet werden. Vgl. zur Abgrenzung heuristischer und analytischer Verfahren Meffert, H., Althans, J., Internationales Marketing, stuttgart, u.a. 1982 , s. 71 ff. 
fahren, sequentielle Bewertungsverfahren und die PortfolioAnalyse weiter differenzieren lassen. 13

Im folgenden soll als Bewertungsverfahren die Portfoliomethode 14 zugrunde gelegt werden. Wenngleich die PortfolioTechnik in ihrer ursprünglichen Funktion eine ganzheitliche Methode zur Analyse des Ertrags- und Risikoausgleichs von Geschäftseinheiten einer Unternehmung darstel1t ${ }^{15}$, sind

13 Der Zweck der Checklist-Verfahren besteht vor allem in einer Grobauswahl von Zielgruppen, um die detaillierte Analyse auf eine überschaubare Zahl von Zielgruppen zu beschränken und den unnötigen Einsatz von Ressourcen für aufwendige Marktforschungsmaßnahmen $z u$ vermeiden. Im Rahmen von Punktbewertungsverfahren werden anhand geeigneter Beurteilungskriterien die einzelnen zielgruppen beurteilt, verglichen und ausgewählt. Entsprechend der wichtigkeit von Kriterien können Gewichtungen vorgegeben werden. Es lassen sich Gesamtpunktwerte berechnen, anhand derer eine Auswahl von Zielgruppen erfolgt. Das sequentielle Bewertungsverfahren ist eine Weiterentwicklung des Punktbewertungsverfahrens, bei dem die Punktbewertung der einzelnen Zielgruppen in mehreren Unterstufen erfolgt. Am Ende der einzelnen stufen entscheidet sich, ob eine zielgruppe aus der weiteren Betrachtung ausscheidet. Die bisher aufgezeigten Verfahren eignen sich insbesondere für eine Grobauswahl von Zielgruppen. Die Portfolio-Analyse wird hingegen teilweise der Feinauswahl zugeordnet und soll daher im folgenden im Mittelpunkt der Betrachtung stehen. Sie baut auf den Punktbewertungsverfahren auf. Der Vorteil der Portfolio-Analyse besteht vor allem darin, daB zwei voneinander unabhängige Beurteilungskriterien i.d.R. auf Basis von Punktbewertungsverfahren ermittelt und zur zielgruppenbewertung einander gegenübergestellt werden. Vgl. zu den aufgezeigten heuristischen Verfahren ebenda, s. $73 \mathrm{ff}$.

14 Vgl. zur ausführlichen Darstellung der Portfolio-Theorie Kreilkamp, E., strategisches Management und Marketing. Markt- und Wettbewerbsanalyse, strategische Frühaufklärung, Portfolio-Management, Berlin, New York 1987, S. 445 ff.; Meffert, H., Marketing, a.a.O., S. 72 ; Kotler, $\mathrm{Ph}$., Bliemel, F., Marketing-Management. Analyse, Planung, Umsetzung und Steuerung, 7. Aufl., Stuttgart 1992, s. $51 \mathrm{ff}$.

15 Der Grundgedanke der Portfolio-Analyse basiert darauf, unter Berücksichtigung von umweltbezogenen und unternehmensbezogenen Erfolgsfaktoren eine gesamtunternehmensbe- 
zahlreiche Varianten entwickelt worden, sie als vereinfachte heuristische Bewertung von Analyseobjekten in einem zweidimensionalen Raum zu nutzen. ${ }^{16}$ In dieser Eignung soll sie im vorliegenden Kontext Anwendung finden.

Im Rahmen der Portfolio-Analyse erfolgt die objektbewertung bzw. in diesem Fall die zielgruppenbewertung auf Basis markt- und wettbewerbsbezogener Kriterien. 17 Es bietet sich demnach an, die durch die Fremdenverkehrsregion nicht beeinflussbare segmentattraktivität der jeweiligen touristischen zielgruppen als marktbezogenes Kriterium und die von der Fremdenverkehrsregion direkt beeinflubbare Nettbewerbsstärke als zweites Beurteilungskriterium heranzuziehen.

Die Einordnung der Zielgruppen in das zweidimensionale Beurteilungsraster gibt Aufschluß darüber, welche zielgruppen für ein übergreifendes zielgruppenmarketing von Fremdenverkehrsregionen besonders geeignet sind. Zielgruppen, die aus sicht der Fremdenverkehrsregion eine nur geringe segmentattraktivität aufweisen, sollten bei der Bearbeitung solchen Leistungsträgern vorbehalten sein, die mit ihren spezifischen Kompetenzen die Bedürfnisse dieser Zielgruppen befriedigen können. 18

zogene Betrachtung von Geschäftsfeldern zu ermöglichen. Vgl. Kreilkamp, E., Strategisches Management und Marketing, a.a.0., s.446.

16 Vgl. Meffert, H., Althans, J., Internationales Marketing, a.a.0., s. 85 .

17 Da in der vorliegenden Untersuchung nicht die Bestimmung eines optimalen Zielgruppenbündels für die Fremdenverkehrsregion ermittelt werden soll, handelt es sich bei diesem modifizierten Portfolio um eine zwei-dimensionale Darstellung von mit Hilfe von Scoring-Modellen ermittelten Beurteilungskriterien.

18 Beispielsweise kann der Anbieter eines Freizeiterholungsheimes für behinderte Kinder diese Zielgruppe sinnvoll und effektiv bearbeiten, ohne daB selbige zielsegment einer ganzen Region sind. 
Als wesentliche Kriterien zur Bestimmung der segmentattraktivität im Fremdenverkehr von Regionen sind beispielhaft zu nennen: 19

- Segmentgröße

- Ausgabenpotential der Mitglieder der Segmente

- Ansprechbarkeit der segmente

- Zeitliche stabilität der segmente

- generelle Reisebereitschaft der Mitglieder der Segmente

- etc.

Um die zur Auswahl stehenden Segmente in eine Attraktivitätsrangfolge einordnen zu können, ist es sinnvoll, die einzelnen Beurteilungskriterien mittels eines Scoring-Modells zur Portfolio-Dimension "Segmentattraktivität" $\mathrm{zu}$ verdichten. 20 Durch eine differenzierte Gewichtung kann die unterschiedliche Bedeutung der einzelnen Kriterien für die individuelle situation der Fremdenverkehrsregion berücksichtigt werden. Eine Beurteilung der Kriterien hinsichtlich ihres Beitrags zur segmentattraktivität ist dabei von Experten der Fremdenverkehrsregion vorzunehmen. Die genaue Darstellung einer detaillierten Ermittlung der Segmentattraktivität soll an dieser stelle aufgrund der dieser Methode anhaftenden subjektivität und nur geringen Verallgemeinerungsfähigkeit

Vgl. zu ähnlichen Bestimmungsfaktoren Bauer, E., MarktSegmentierung als Marketing-Strategie, a.a.0., s.121 ff.

Vgl. zur Kennzeichnung von Scoring-Modellen Meffert, H., Marketing, a.a.0., s. 386 ff.; Becker, J., MarketingKonzeption. Grundlagen des strategischen Marketing-Management, 4. Aufl., München 1992, S. 361 f.; Als Kritikpunkte an solchen Scoring-Modellen ist vor allem die subjektive Festlegung der Gewichtungsfaktoren und Merkmalsausprägungen sowie die subjektive Formulierung von Entscheidungsregeln $z u$ nennen. Ferner sind die Kriterien i.d.R. nicht überschneidungsfrei. Darüber hinaus werden weitere Einflußgrößen, vor allem Umwelteinflußgrößen in diesem Bewertungsmodell, nicht erfaBt. Vgl. zur Kritik an Scoring-Modellen Meffert, H., Althans, J., Internationales Marketing, a.a.0., s. 75 
unterbleiben. Statt dessen gibt Abbildung 42 einen stark vereinfachten Einblick in die grundsätzliche Methodik einer Bewertungsverdichtung.

Die wettbewerbsstärke einer Fremdenverkehrsregion ergibt sich vor allem aus der relativen, im vergleich zu Konkurrenzregionen gegebenen Kompatibilität der segmentspezifi-

\begin{tabular}{|c|c|c|c|c|}
\hline Zlelgruppe & $\begin{array}{l}\text { Markt- } \\
\text { bezug }\end{array}$ & $\begin{array}{c}\text { Segmentgr } \mathrm{OB} \\
\text { In Bezug auf den } \\
\text { Gesamtmarkt }\end{array}$ & \begin{tabular}{|c|} 
Beurtellung dor \\
Ausgabofhigkelt \\
dor Segmento far \\
Urlauber \& Relsen \\
\end{tabular} & $\begin{array}{l}\text { Segmont- } \\
\text { aftraktlvitut }\end{array}$ \\
\hline $\begin{array}{l}\text { Sportaktlve } \\
\text { Famillen- } \\
\text { urlauber }\end{array}$ & $\begin{array}{l}\text { Gesamt- } \\
\text { markt }\end{array}$ & $31,19 \%$ & $\begin{array}{l}\text { mittel bis } \\
\text { hoch }\end{array}$ & hoch \\
\hline $\begin{array}{l}\text { Land- und } \\
\text { Kulturorlen- } \\
\text { tlerte Uriauber }\end{array}$ & $\begin{array}{l}\text { Entfernungs- } \\
\text { gruppo I }\end{array}$ & $19,37 \%$ & $\begin{array}{l}\text { mittel bls } \\
\text { hoch }\end{array}$ & hoch \\
\hline $\begin{array}{l}\text { Traditlonelle } \\
\text { Spazlorglinger- } \\
\text { froundo }\end{array}$ & $\begin{array}{l}\text { Entfornungs- } \\
\text { gruppo I }\end{array}$ & $15,35 \%$ & gerlnger & mittel \\
\hline $\begin{array}{l}\text { Regeneratlons- } \\
\text { und GenuBorion- } \\
\text { tlerte Urlauber }\end{array}$ & $\begin{array}{l}\text { Entfornungs- } \\
\text { gruppe II }\end{array}$ & $10,69 \%$ & mittel & mittel \\
\hline Badeurlauber & $\begin{array}{l}\text { Gesamt- } \\
\text { markt }\end{array}$ & $23,4 \%$ & gerlnger & mittel \\
\hline
\end{tabular}

Abb. 42: Beurteilung der Segmentattraktivität anhand der Segmentgröße und potentieller Ausgabefähigkeit der Segmente

schen Anforderungen potentieller Touristen an eine Fremdenverkehrsregion mit den entsprechenden regionalen Angebotskomponenten. Da in dieser Untersuchung Konkurrenzregionen nicht in die Analyse einbezogen wurden, kann die Bestimmmung der Wettbewerbsstärke hier nur mit einer groBen Unschärfe 
vorgenommen werden. Offensichtlich bietet sich auch hier die Beurteilung der Wettbewerbsstärke im Hinblick auf die einzelnen zielgruppen auf der Grundlage von Expertenurteilen an.

Für die vorliegende Untersuchung sollen als Indikatoren für die Wettbewerbsstärke die Anteile an Besuchsabsichtsnennungen innerhalb der segmente und die $\mathrm{zu}$ vermutende Kompatibilität zwischen Anforderungen und Angebot 21 verwendet werden. Von einer relativ hohen (niedrigen) Wettbewerbsstärke ist in der vorliegenden Untersuchung dann auszugehen, wenn die zentralen segmentspezifischen Anforderungen an eine Fremdenverkehrsregion mit den tatsächlichen Angebotskomponenten übereinstimmen (abweichen) und gleichzeitig ein hoher (niedriger) Anteil an Besuchsabsichtsnennungen je segment vorliegt.

Unter Berücksichtigung der segmentspezifischen Wettbewerbsstärken und der segmentattraktivität kann ein zielgruppenPortfolio erstellt werden, auf dessen Grundlage die zielgruppenprioritäten abzuleiten sind. In Abbildung 43 sind die für die Untersuchungsregion identifizierten zielgruppen in ein solches Zielgruppen-Portfolio eingeordnet worden. Es zeigt sich, daB die "sportaktiven Familienurlauber" offenbar die attraktivste Zielgruppe für die Untersuchungsregion darstellen. Dies ergibt sich sowohl aus einer wahrgenommenen Kompatibilität zwischen Anforderungen und tatsächlicher Ausprägung der Angebotskomponenten der Fremdenverkehrsregion

21 Die Beurteilung der Kompatibilität zwischen zielgruppenspezifischen Anforderungen an eine Fremdenverkehrsregion und den tatsächlichen Angebotskomponenten basieren auf Expertengesprächen. Zur Beurteilung der Angebotskomponenten der dieser Untersuchung zugrundeliegenden Fremdenverkehrsregion vgl, auch Meffert, H., u.a., Marketing für das Münsterland. Eine Bestandsaufnahme auf der Grundlage sekundärstatistischer Analysen, Münster 1989, S. $8 \mathrm{ff.;}$ vgl. ferner die knappe Zusammenfassung der im Rahmen von Experten-Workshops ermittelten stärken der Untersuchungsregion in Meffert, H., Regionenmarketing Münsterland, a.a.0., S. $19 \mathrm{ff}$. 
als auch aus dem hohen Besucheranteil in diesem segment. Auch den "land- und kulturorientierten orlaubern" ist eine hohe Segmentattraktivität bei gleichzeitig etwas schwächerer Wettbewerbsstärke zuzusprechen. Angesichts der Übereinstimmung von Anforderungen an eine Fremdenverkehrsregion und der Wahrnehmung der Ausprägungen der Untersuchungsregion sollte diesen beiden zielgruppen im Rahmen der Marktbearbeitung die höchste Priorität zugeordnet werden.

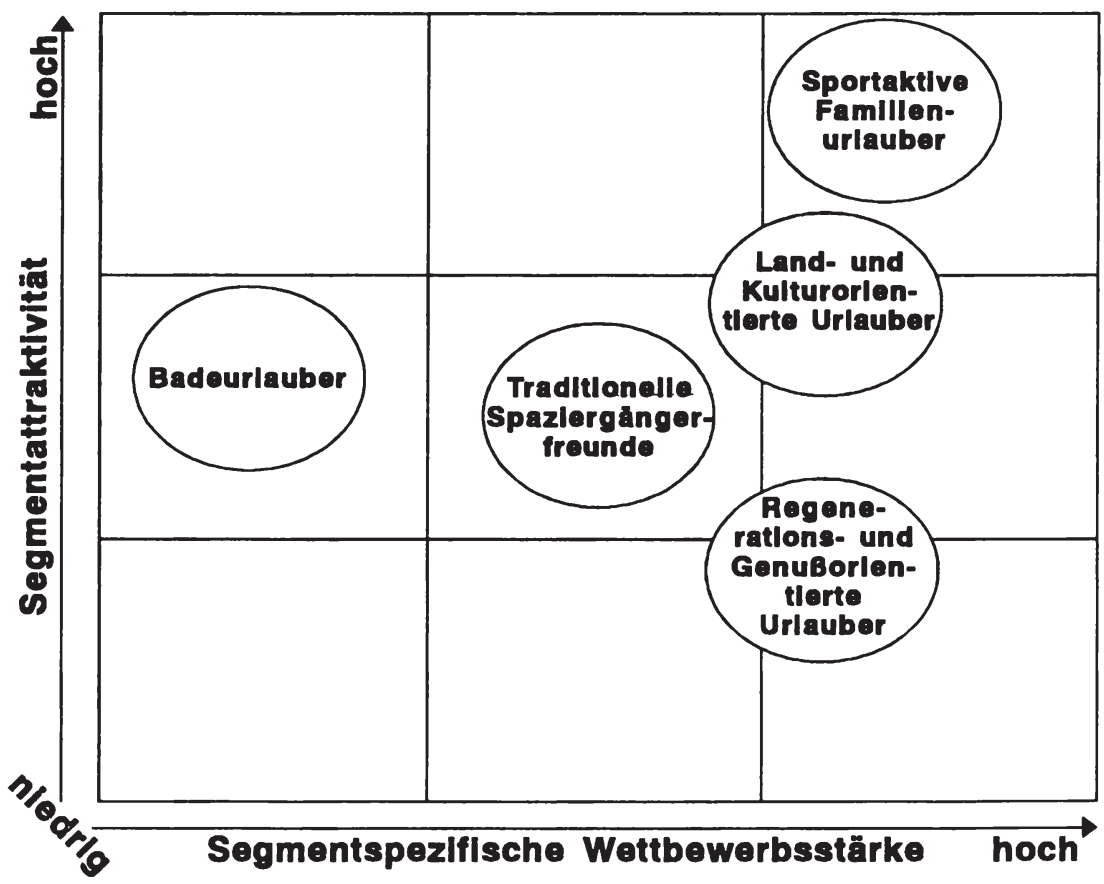

Abb. 43: Zielgruppen-Portfolio als heuristisches Modell zur Zielgruppenbewertung

Den "traditionellen spaziergängerfreunden" und den "regenerations- und genuBorientierten orlaubern" ist nur eine mittlere Segmentattraktivität zuzusprechen. Ferner ist angesichts des geringen Besucheranteils in diesen segmenten von 
einer nur mittleren Wettbewerbsstärke der Untersuchungsregion auszugehen. Die "regenerations- und genuBorientierten orlauber" erweisen sich allerdings von daher als interessante Zielgruppe, da sie innerhalb der Entfernungsgruppe II das zweitbedeutendste segment darstellen und ihre regionenspezifischen Anforderungen hohe übereinstimmungen mit dem tatsächlichem Angebotsspektrum der Untersuchungsregion aufweisen. Eine Erhöhung der Besuchsintensität dieser zielgruppe würde unmittelbar zu einer Erhöhung der tibernachtungszahlen in der Untersuchungsregion führen.

Bezüglich den "8trand-Badeurlaubern" weist die Untersuchungsregion eher segmentspezifische Wettbewerbsnachteile auf. Angesichts des dominanten Urlaubsmotives dieser Zielgruppe nach "sonnen", "baden", und "am strand liegen" ist eine Bearbeitung dieser zielgruppe trotz der relativ hohen Segmentgröße für die Untersuchungsregion wenig erfolgversprechend. Die Bearbeitung dieses Segments sollte solchen touristischen Leistungsträgern vorbehalten bleiben, die in der Lage sind, die natürlichen Angebotsnachteile durch Ersatzangebote zu kompensieren, wie zum Beispiel durch das Angebot von Erlebnisbädern. Daher kommt den Badeurlaubern aus sicht der regionalen Fremdenverkehrsorganisation im Rahmen der Marktbearbeitung eine nur untergeordnete Priorität zu.

Die isolierte Bewertung einzelner zielgruppen vernachlässigt jedoch 22 die zwischen den zielgruppen bestehenden Interdependenzen. Im vorliegenden Anwendungsfall sind dies vor allem Verhaltensinterdependenzen der zielgruppen während ihres Aufenthalts in der Fremdenverkehrsregion. Der Kontakt unterschiedlicher zielgruppen kann positive, negative oder neu-

Vgl. zu weiteren Nachteilen solcher Portfolios, Meffert, H., Marketing, a.a.O., S. 71 f.; Aaker, D.A., Strategisches Marketing-Management. Wetbewerbsvorteile erkennen. Märkte erschließen - strategien entwickeln, wiesbaden 1989 , S. 179 ff. 
trale Auswirkungen auf die Zufriedenheit während des Aufenthalts und damit auf die Gesamtbeurteilung der Fremdenverkehrsregion haben. 23 Daher sollten nur solche zielgruppen ausgewählt werden, die sich bei einem möglichen Kontakt positiv oder zumindest neutral gegenüberstehen.

Die global identifizierten zielgruppenprioritäten wirken sich in der Praxis über die Intensität der zu ihrer Bearbeitung eingesetzten Marketingmaßnahmen aus. Die zielgruppenspezifische Bearbeitungsintensität ist ein komplexer Umsetzungs- und Durchsetzungsproze $B$ bei den touristischen Leistungsträgern. Ihn gilt es zunächst mit der Darstellung zielgruppenspezifischer Umsetzungsmaßnahmen zu erläutern.

2. Ansatzpunkte gielgruppenspezifischer MarketingmaBnahmen im Fremdenverkehr von Regionen

2.1 Ansatzpunkte für zielgruppenspezifische Maßnahmen im Rahmen der Produktpolitik

Die Produktpolitik umfaBt alle Entscheidungstatbestände, welche sich auf die marktgerechte Gestaltung der einzelnen Angebotskomponenten einer Fremdenverkehrsregion als auch der Gestaltung der Fremdenverkehrsregion in ihrer Gesamtheit beziehen. 24 Ferner umfaBt die Produktpolitik Markierungsentscheidungen sowie die Festlegung von zusatg-(8ervice-)1ei-

23 Vgl. Krippendorf, J., Marketing im Fremdenverkehr, a.a.0., S. 78. Zum Beispiel ist davon auszugehen, daB eine forcierte Ansprache der eher aktions- und erlebnisorientierten "Bade-Urlauber" sich nicht mit den Erwartungen der "traditionellen spaziergängerfreunde" an Ruhe und Beschaulichkeit eines zielgebietes vertragen.

24 Vgl. Kaspar, C., Kunz, B.R., Unternehmensführung im Fremdenverkehr, a.a.0., s. 132 f.; Haedrich, H., Angebotspolitik, in: Haedrich, G., Kaspar, C., u.a. (Hrsg.), Tourismus-Management. Tourismusmarketing und Fremdenverkehrsplanung, Berlin, New York 1983, S. 241 f. 
stungen. 25 Die genannten Entscheidungstatbestände unterscheiden sich dabei offensichtlich in ihrer Möglichkeit einer zielgruppenspezifischen Ausgestaltung.

Bei der Ableitung von Ansatzpunkten einer sielgruppenspesifischen Angebotsgestaltung im Fremdenverkehr von Regionen ist es zunächst zweckmäBig, das natürliche und das abgeleitete Angebot zu unterscheiden. 26 Bezüglich des natürlichen Angebots ist $z u$ beachten, daB dieses von einer Fremdenverkehrsregion bzw. den dort vertretenen touristischen Leistungsanbietern nur in stark eingeschränktem Maße variiert und gestaltet werden kann. 27 Es besteht kaum die Möglichkeit, neue natürliche Angebotselemente zu entwickeln und ungünstige Angebotselemente (wie zum Beispiel nachteilige Wettereinflüsse) $z u$ eliminieren. 28 Die Gestaltungsaufgabe bezüglich des natürlichen Angebots besteht damit vor allem in der Erhaltung der schützenswerten ursprünglichen Angebotsfaktoren. 29 Möglichkeiten der zielgruppenspezifischen Gestaltung des natürlichen Angebots sind damit i.d.R. nicht gegeben. Vielmehr determiniert das natürliche Angebot - wie die empirische Analyse aufgezeigt hat 30 -, welche Zielgruppen überhaupt als potentielle Besucher der Fremdenverkehrsregion in Betracht gezogen werden können.

$25 \mathrm{Zu}$ den Bestandteilen der Produktpolitik vgl. Meffert, H., Marketing, a.a.O., S. $361 \mathrm{ff}$; Nieschlag, R., Dichtl, E., Hörschgen, H., Marketing, a.a.0., s. 177 ff.

26 Vgl. zur begrifflichen Abgrenzung von natürlichem und abgeleiteten Angebot Kapitel A. 2 .

27 Vgl. Kaspar, C., Kunz, B.R., Unternehmensführung im Fremdenverkehr, a.a.0., S. 133; Krippendorf, J., Marketing im Fremdenverkehr, a.a.0., s. 117 .

28 Vgl. Kapar, C., Kunz, B.R., Unternehmensführung im Fremdenverkehr, a.a.0., S. 35 .

29 Eine weitere Gestaltungsmöglichkeit besteht in der Erschließung des natürlichen Angebots. Vgl. ebenda.

Vgl. Kapitel B.4.322. 
Ansatzpunkte einer zielgruppenspezifischen Angebotsgestaltung bieten sich damit vor allem im Rahmen des abgeleiteten Angebots. Gestaltungsparameter lassen sich aus den bei der Motivanalyse ermittelten Motivfaktoren in Verbindung mit den im Rahmen der Einstellungsanalyse zur Beurteilung einer Fremdenverkehrsregion herangezogenen Angebotskomponenten ermitteln. Demnach sind zu unterscheiden:

- Einrichtungen der Suprastruktur (Unterkunft und Verpflegung)

- Sportangebote

- Wanderangebote

- Kulturangebote

- sonstige Freitzeitangebote. 31

Abbildung 44 gibt beispielhaft einen Ưberblik über mögliche zielgruppenspezifische Ausgestaltungsalternativen dieser Angebotskomponenten für die in dieser Untersuchung identifizierten zielgruppen. Beispielsweise sind für die zielgruppe der "sportaktiven Familienurlauber" ausreichend sportmöglichkeiten zur Verfügung zu stellen, die gemeinsam mit der Familie betrieben werden können. $\mathrm{zu}$ denken ist hierbei an Radfahrwege, Fitness-Wege, Kanufahrten etc. Um der Familienorientierung Rechnung zu tragen, sind ferner kinderfreundliche Hotels, Spielplätze etc. einzurichten. Demgegenuber sollte das Angebot an die "traditionellen spaziergängerfreunde" vor allem relativ preisgünstige Unterkünfte und gut ausgebaute Wander- und Gehwege umfassen. Für die in ihrer zielgruppenspezifischen Bedeutung nachrangig attraktiven "Badeurlauber" muB ein Mindestangebot an Freibädern in Verbindung mit Liegewiesen aufrecht erhalten werden. Angesichts der klimatischen Bedingungen könnten Erlebnisbäder als kompensatorisches Leistungsangebot bereitgestellt werden. 


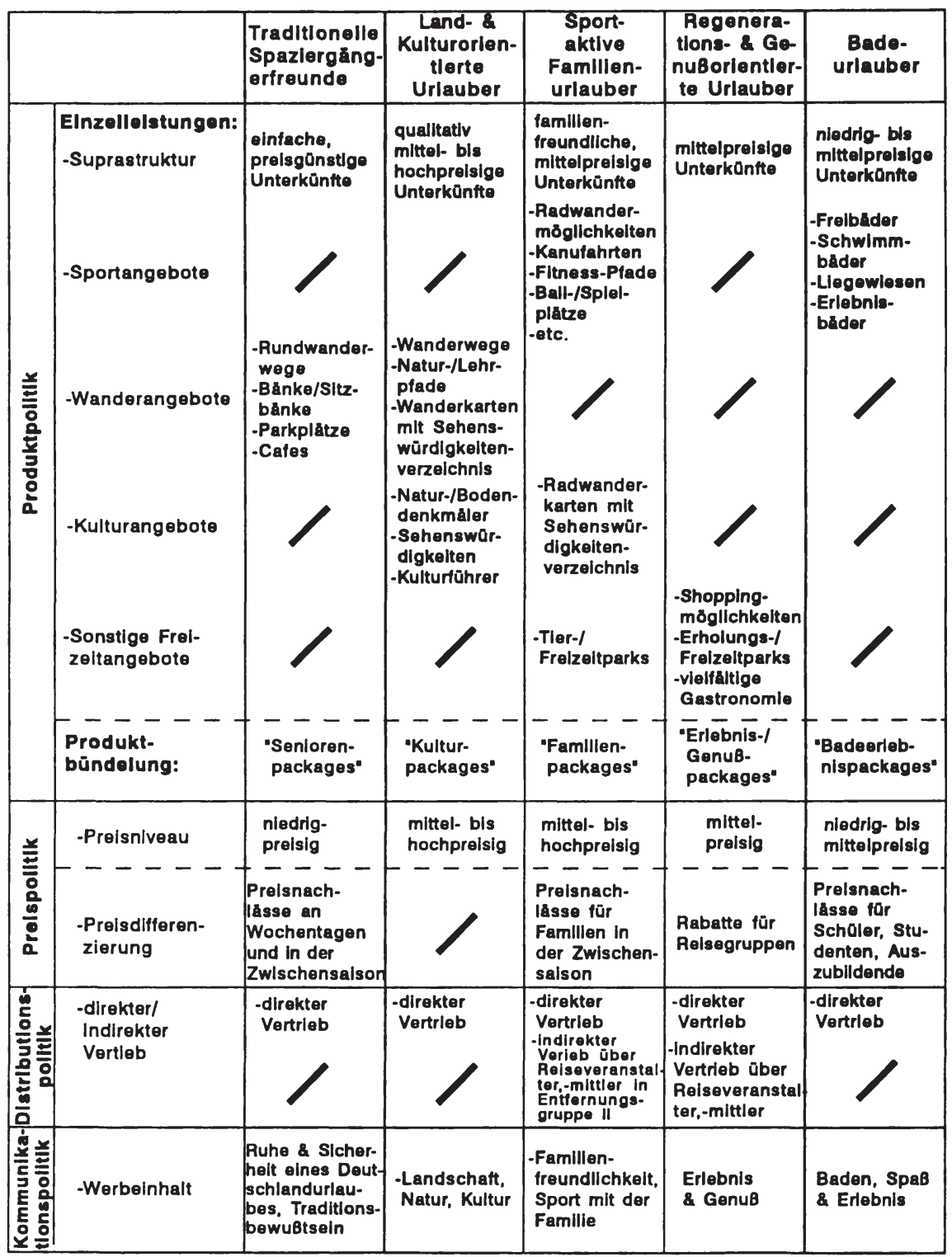

Abb. 44: Ansatzpunkte einer zielgruppenspezifischen Marktbearbeitung 
Die isolierte Bereitstellung von touristischen Einzelangeboten ist allerdings allein nicht ausreichend für eine zielgruppenspezifische Profilierung. Vielmehr ist es angesichts des Komplementaritätsverhältnisses zwischen touristischen Leistungen 32 zweckmäßig, die Vielzahl von Einzelleistungen einer Fremdenverkehrsregion in Abhängigkeit von den Bedürfnissen der zielgruppen in einem integrierten Konzept aufeinander abzustimmen. ${ }^{33}$ Beispielsweise ist ein einzelnes Hotel, auch wenn es sein Leistungsprogramm an allen oder ausgewählten, von der Fremdenverkehrsorganisation aufgezeigten zielgruppen orientiert, kaum in der Lage, unabhängig von den anderen, in der Region anzutreffenden Angebotsbestandteilen seine touristische Leistung erfolgreich $\mathrm{zu}$ vermarkten. Daher erscheint es für Fremdenverkehrsregionen zweckmäBig, eine Zusammenstellung der vielen Einzelangebote zu vermarktungsfähigen Gesamtprodukten vorzunehmen. Hierunter ist die in der Fremdenverkehrsliteratur unter dem stichwort "systemSelling" diskutierte Ableitung von Pauschalangoboten $z u$ verstehen. 34 Ein Pauschalangebot umfaßt dabei ein Dienstleistungspaket, welches mindestens zwei aufeinander abgestimmte Reisedienstleistungen umfaBt. ${ }^{35}$ Dieses wird im voraus auf der Grundlage segmentspezifischer Merkmale erstellt und zu einem Gesamtpreis angeboten.

Grundlegende Voraussetzung für die Zusammenstellung solcher Produktbündel ist die Kooperation mehrerer touristischer

Vgl. Kapitel A.2.

33 Vgl. Bleile, G., Marketing-strategien im Fremdenverkehr, a.a.0., s. 77 .

34 Vgl. Bleile, G., Marketing-strategien im Fremdenverkehr, a.a.0., S. 77; wölm, D., Marketing für Deutschlandreisen, a.a.o., s. 88 .

35 Vgl. Hebestreit, D., Touristik-Marketing. Ziele, strategien, Instrumentarium, organisation und Planung des Marketing von Reiseveranstaltern, Berlin 1979, S. 16 f.; Schmid, J.-M., Kundenstamm-Marketing, a.a.O., s. 3 . 
Leistungsanbieter. Um sämtliche Angebotskomponenten einer Fremdenverkehrsregion der Programmgestaltung zugrundelegen zu können, bietet es sich an, diese Bündelungsfunktion der regionalen Fremdenverkehrsorganisation $z u$ übertragen. Die Zusammenfassung der ortsgebundenen touristischen Teilleistungen zu Programmaketen hat ferner den Vorteil, daB hierdurch ein Produktsurrogat geschaffen wird, welches dem Touristen in seiner Gesamtheit die Entscheidung für den Besuch einer bestimmmten Fremdenverkehrsregion erleichtert. ${ }^{36}$ Dabei ist eine Zusammenstellung der Pauschalangebote offensichtlich in Abhängigkeit von den zielgruppenspezifischen Bedürfnissen der zielgruppe vorzunehmen.

Angesichts der hohen Bedeutung der Individualreisen im Deutschlandtourimus ${ }^{37}$, erscheint es zweckmäßig, zusätzlich zu kompletten Programmpaketen (bestehend aus Unterkunft, Verplegung, Transport und Freizeitangeboten) zielgruppenspezifische Programmmodule anzubieten. Diese können vom jeweiligen Touristen selbständig zu einem, den spezifischen Bedürfnissen entsprechenden, individuellen Gesamtpaket zusammengestellt werden. Beispielsweise könnten zielgruppenspezifische Übernachtungs-/Verpflegungsmodule, sport- sowie Besichtigungsmodule bereitgestellt werden. Für die "sportaktiven Familienurlauber" sind Angebotsmodule konzipierbar, die speziell auf Familien mit Kindern abgestimmte Übernachtungsmöglichkeiten, kindergerechte Verpflegung und Kinderhorts, besichtigungsorientierte sportmodule, einen Fahrradverleih, Radwanderkarten und Eintrittskarten für Besichtigungen umfassen.

Damit eine Fremdenverkehrsregion als spezifische Marktleistung erkannt, präferiert und in Anspruch genommen werden

36 Vgl. Wölm, D., Marketing für Deutschlandreisen, a.a.o., S. $90 \mathrm{ff}$.

37 Vgl. Freyer, w., Tourismus, a.a.O., s. 106. 
kann, erscheint es notwendig, durch die karkierung der Fromdenverkehrsregion die Nutzenvorteile in einer regionenspezifischen Identität zusammenzufassen. 38 Hierbei handelt es sich zunächst um eine zielgruppenubergreifende MaBnahme. Durch die Markierung einer Fremdenverkehrsregion wird den Touristen ein psychologischer zusatznutzen angeboten, der eine Differenzierung von Konkurrenzregionen ermöglicht. Die psychologische Alleinstellung kann vor allem durch eine entsprechende werbliche Botschaftsgestaltung im sinne einer Unique Advertising Proposition (UAP) angestrebt werden.

Als Elemente der Markierung stehen der Markenname, das Markenzeichen und - sofern es sich um tangible Angebote handelt - das Markendesign zur Verfügung. 39 Für Fremdenverkehrsregionen ist es im Hinblick auf einen hohen Bekanntheitsgrad und wiedererkennungseffekt zweckmäBig, den geographischen Namen der Region als Markennamen $z u$ verwenden. Demnach würde der Name der Fremdenverkehrsregion den Charakter einer Dachmarke erhalten. 40

Um eine zielgruppenspezifische Ausgestaltung der Markierung zu erreichen, bietet es sich an, die Dachmarke um die Bezeichnung zielgruppenspezifischer Angebotskomponenten zu ergänzen. $41 \mathrm{Zu}$ denken ist beispielsweise an "Radwandern in der Region X", "Familienurlaub in der Region X". Hierdurch ist

38 So weist auch Meffert darauf hin, daB die Markierung ein Identifikations- und Differenzierungsmittel darstellt. Vgl. Meffert, Marketing a.a.0., S. 409.

39 Vgl. Meffert, H., Marketing, a.a.O., s. 410; Kotler, Ph., Bliemel, F., Marketing-Management, a.a.0., s. 641.

40 Vgl. zur näheren Kennzeichnung von Dachmarkenstrategien und deren Unterscheidung in Einmarken-, Mehrmarken- und Markenfamilienstrategien Meffert, H., Markenstrategien im Wettbewerb, in: Meffert, H. (Hrsg.), strategische Unternehmensfuhrung und Marketing, a.a.o., s. $123 \mathrm{ff}$.

41 Vgl. Scheuch, F., Dienstleistungsmarketing, Munchen 1982 , S. 163 . 
es möglich, den einzelnen zielgruppen über die Markierung hinaus einen ihren Bedürfnissen entsprechenden psychologischen zusatznutzen zu vermitteln. Die Dachmarke fungiert dabei als Gütesiegel für das zielgruppenspezifische Programmangebot.

Neben der Markierung kann ein Zusatznutzen auch über das Angebot zielgruppenspezifischer zusatzleistungen erzielt werden. Hierbei handelt es sich um Servive-Leistungen, die von den einzelnen touristischen Leistungsanbietern zusätzlich angeboten werden, um über die eigentliche Bereitstellung touristischer Leistungen hinausgehende Kundenprobleme zu lösen. 42 Hierdurch kann ein Vertrauensverhältnis zwischen touristischen Anbietern bzw. der Fremdenverkehrsregion und den Touristen geschaffen werden, welches eine wichtige Grundlage für die Bildung von Marken- bzw. Zielgebietstreue darstellt. 43 Dabei können die zusatzleistungen in Abhängigkeit von dem Zeitpunkt der Leistungserstellung in Angebote vor, während und nach dem Aufenthalt in einer Fremdenverkehrsregion strukturiert werden. 44 Mögliche zusatzleistungen vor dem Aufenthalt sind insbesondere die Beratung und Informa-

42 Vgl. Meffert, H., Der Kundendienst als Marketinginstrument - Einführung in die Problemkreise des KundendienstManagement, in: Meffert, H. (Hrsg.), Kundendienst-Management. Entwicklungsstand und Entscheidungsprobleme der Kundendienstpolitik, Frankfurt 1982, S. 2; Krippendorf, J., Marketing im Fremdenverkehr, a.a.o., s. 124 .

43 Vgl. Meffert, H., Der Kundendienst als Marketinginstrument, a.a.0., s. 4 .

44 Eine ähnliche allerdings nur zwei Zeitpunkte umfassende Strukturierung von Kundendienstleistungen für produzierende Unternehmen findet sich bei Meffert, H., Der Kundendienst als Marketinginstrument, a.a.0., s. 7. Ferner wird hier eine Einteilung in technische Leistungen (Hardware) und nicht-technische Leistungen (Software) vorgenommen. Eine direkte tibertragung auf den Fremdenverkehr ist angesichts des Dienstleistungscharakters von Fremdenverkehrsleistungen nicht möglich. Vgl. ebenda, $\mathrm{S}$. 8 . 
tion, Ausarbeitung von Reiserouten in der Region, kostenlose Zusendung von Prospekten, Buchungsabwicklung etc.. Als $\mathrm{Zu}-$ satzleistungen während des Aufenthaltes können z.B. die Reisebetreuung, kostenlose zur Verfügungstellung von Fahrrädern oder Busfahrdiensten sowie touristische Rahmenprogramme angeführt werden. Die zusatzleistungen nach dem Aufenthalt umfassen zum Beispiel zentralisierte Reklamationsabwicklungen und sonstige das touristische Leistungsangebot der Fremdenverkehrsregion betreffende Informationsdienste.

Die Zusatzleistungen sind zielgruppenspezifisch bereitzustellen. Während beispielsweise die Reisebetreuung bei den "traditionellen Spaziergängerfreunden" überwiegend auf ältere Altersgruppen abgestimmte Wanderrundgänge beinhalten könnte, sind bei den "land- und kulturorientierten Urlaubern" organisierte Besichtigungstouren anzubieten. Für die "familienorientierten Urlauber" könnten demgegenüber Radwandertouren mit der gesamten Familie zusammengestellt werden.

\subsection{Ansatzpunkte für zielgruppenspezifische MaBnahmen im Rahmen der Kontrabierungspolitik}

Die Kontrahierungspolitik umfaßt im Marketing für Regionen vor allem Entscheidungen über die Festlegung von Preisen und Rabatten. 45 zur Preisfindung können prinzipiell kostenorientierte und marktbezogene Verfahren sowie marginalanalytische

45 Vgl. Roth, P., Grundlagen des Touristik-Marketing, in: Roth, P., Schrand, A. (Hrsg.), Touristik-Marketing, a.a.0., S. 164; Krippendorf, J., Marketing im Fremdenverkehr, a.a.0., S. $127 \mathrm{ff}$. Die Gewährung von Rabatten bezieht sich im Fremdenverkehr weniger auf Einzelpersonen sondern vor allem auf den Sonderfall von Gruppenreisen. 
optimierungsmodelle unterschieden werden. 46 In der Fremdenverkehrspraxis dominiert bislang eine kostenorientierte Preisfestsetzung, die vielfach $z u$ einer wenig nachfragerkonformen Preisfestsetzung und letztlich zu einer nur unzureichenden Auslastung der Kapazitäten im Fremdenverkehr führte. 47 Hieran wird unmittelbar deutlich, daß sich die Preisfindung daran orientieren sollte, wieviel der potentielle Tourist für eine bestimmte Produktqualität auszugeben bereit ist. 48 Dabei gibt die kostenorientierte Preisfestsetzung Hinweise darauf, welche Preise langfristig nicht unterschritten werden dürfen. In diesem Zusammenhang ist $z u$ beachten, daß bezogen auf Teilangebotskomponenten durchaus die Möglichkeiten einer zielgruppenspezifischen Festlegung der Preise gegeben ist. Entsprechend sind die Fremdenverkehrsorganisationen und die Leistungsträger gefordert, unterschiedlichste Vorstellungen und Nutzenerwartungen der zielsegmente an eine Fremdenverkehrsregion in adäquate Preisforderungen umzusetzen.

Bezogen auf die für die Untersuchungsregion ermittelten Zielgruppen ist beispielsweise davon auszugehen, daß "traditionelle spaziergängerfreunde" aufgrund ihres geringen Einkommensniveaus und ihres relativ schwach ausgeprägten Anforderungsprofils an die ideale Fremdenverkehrsregion nur über relativ preisgünstige Urlaubsangebote als Besucher gewonnen werden können. Demgegenüber erscheint es plausibel, die "land- und kulturorientierten Urlauber" angesichts ihres höheren Einkommensniveaus auch mit höherpreisigen Angeboten anzusprechen.

46 Vgl. zur ausführlichen Kennzeichnung dieser Preisfindungsverfahren Diller, H., Preispolitik, stuttgart u.a. 1985 , S. 150 .

47 Vgl. Krippendorf, Marketing im Fremdenverkehr, a.a.o., S. 128 .

48 Vgl. Krippendorf, J., Marketing im Fremdenverkehr, a.a.o., s. 128 . 
Eine sielgruppenspesifische preisfestlegung muß mit einer preiskonformen Angebotsdifferenzierung einhergehen, da während des Aufenthalts unterschiedlicher zielgruppen in der Fremdenverkehrsregion ein Zusammentreffen und Erfahrungsaustausch über Preis-/Leistungsverhältnisse einzelner Angebotskomponenten $z u$ erwarten ist. Eine möglicherweise diskriminierte Gruppe könnte Kenntnis von der niedrigeren Bepreisung leistungsgleicher Angebote für andere Touristen erlangen, wodurch es $\mathrm{zu}$ Konflikpotentialen und Vertrauensverlusten gegenüber der Fremdenverkehrsregion kommen kann. 49

Dies macht deutlich, daB die zielgruppenspezifische Preisfestlegung nicht als isolierte segmentspezifische MaBnahme zu verstehen ist. Ein zusätzlicher zielgruppeneffekt mittels der Preispolitik kann erreicht werden, wenn die zielgruppenspezifische Preissetzung mit einer zeitlichen Preisdifferenzierung ${ }^{50}$ kombiniert wird, um so ein zeitgleiches Aufeinandertreffen Angehöriger verschiedener Segmente $z u$ verhindern. So ist bezogen auf die Untersuchungsregion zu überlegen, die potentiell für Wochendurlaube und Urlaube in den Ferienzeiten in Betracht kommenden "sportaktiven Familienurlauber" mit höherpreisigen Konditionen anzusprechen. Demgegenüber könnten an den Wochentagen im Rahmen einer zeitlichen Preis-

49 Mit diesem Kritikpunkt ist die Voraussetzung einer möglichst geringen Markttransparenz für eine erfolgreiche Preisdifferenzierung angesprochen. Vergleiche $\mathrm{zu}$ weiteren Voraussetzungen der Preisdifferenzierung Meffert, H., Marketing, a.a.0., S. $336 \mathrm{f}$.

50 Preisdifferenzierung bedeutet, daB bei einem gleichen Produkt bzw. gleichem touristischen Angebot von verschiedenen Touristen aufgrund bestimmter Kriterien verschieden hohe Preise gefordert werden. Vgl. Meffert, H., Marketing, a.a.0., s. 336. Zu den verschiedenen Arten der Preisdifferenzierung vgl. Simon, H., Preismanagement. Analyse - Strategie - Umsetzung. 2. Aufl., Wiesbaden 1992, S. 361 ff. Zur Preisdifferenzierung im Fremdenverkehr vgl. Krippendorf, H., Marketing im Fremdenverkehr, a.a.O., s, 129 f.; Wölm, D., Marketing im Tourismus, in: Marketing ZFP, Heft 4, 1979, s. 232 . 
differenzierung Preirabatte gewährt werden, durch die vor allem die ältere zielgruppe der "traditionellen Spaziergängerfreunde" gezielt akquiriert wird. Neben der besseren und damit kostengünstigeren Auslastung der Kapazitäten kann zusätzlich ein höheres, zielgruppenadäquates Serviceniveau in den vorgesehenen Zeitkorridoren realisiert werden.

\subsection{Ansatzpunkte für zielgruppenspesifische MaBnahmen im Rahmen der Distributionspolitik}

Im Rahmen der Distributionspolitk sind die Vertriebswege für den Absatz der touristischen Leistungen festzulegen. Dabei können die Entscheidungstatbestände der Distributionspolitik nach der Frage der Gestaltung von Absatzkanälen einerseits und dem logistischen system andererseits getrennt werden. 51 Die Distributionslogistik hat die räumlichen und zeitlichen Differenzen zwischen der Güterproduktion und der Güterkonsumption zu überbrücken. 52 Da im Fremdenverkehr von Regionen ein Zusammenfallen von Leistungserstellung und -Inanspruchnahme vorliegt, kommt der Distributionslogistik im Fremdenverkehr von Regionen keine Bedeutung zu. Mithin steht im Fremdenverkehr nicht die Frage im Mittelpunkt, wie die Leistung den Endabnehmer erreicht, sondern wie der übergang von Leistungsversprechen erfolgen kann. 53

Wesentliche Entscheidungstatbestände im Rahmen der Distributionspolitik von Fremdenverkehrsregionen sind daher vor allem die Wahl der Absatzwege (direkter, indirekter Absatz), die Festlegung der Zahl und Art der einzuschaltenden Absatz-

51 Vgl. Meffert, H., Marketing, a.a.O., s. 423.

52 Vgl. Specht, G., Distributionsmanagement, stuttgart, u.a. 1988 , S. 83 .

53 Vgl. Krippendorf, J., Marketing im Fremdenverkehr, a.a.0., S. 132 . 
mittler sowie die Ausgestaltung der vertraglichen Bindungen zwischen dem Erstanbieter und den Mittlern. 54

Die möglichen Alternativen zur Gestaltung der Absatzwege im Fremdenverkehr sind in einer vereinfachten Grundstruktur in Abbildung 45 dargestellt. In Kanal I ist der direkte Absatz von einem touristischen Leistungsträger an potentielle Tou-

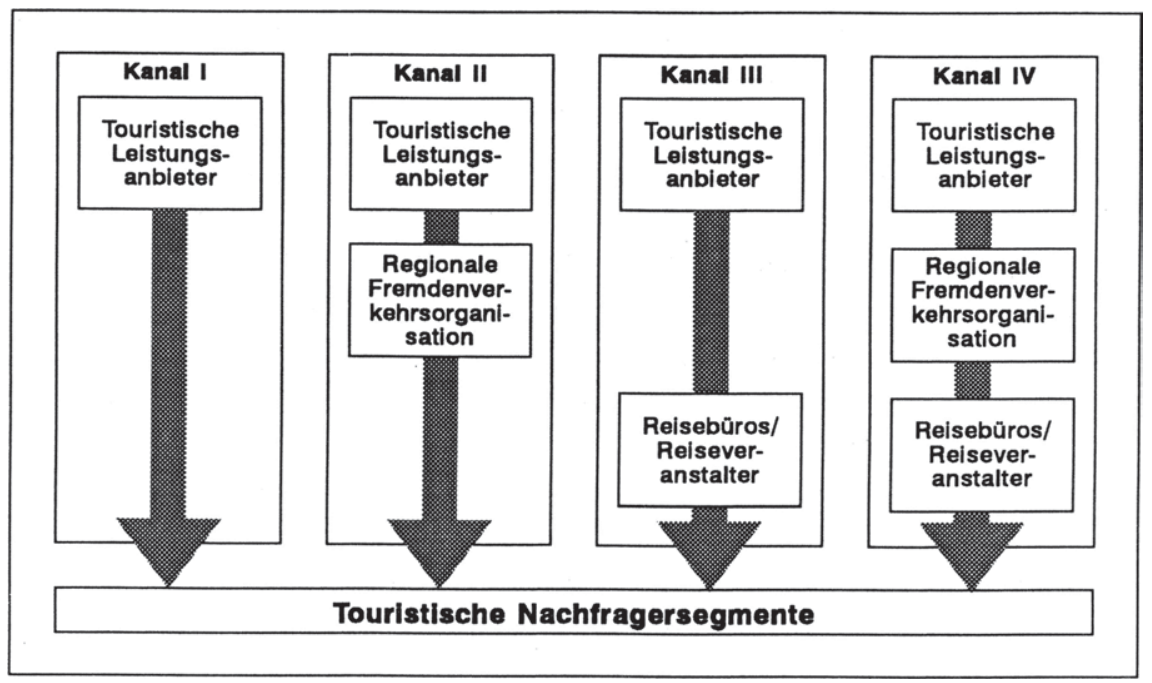

Abb. 45: Grundtypen von Absatzkanälen im Fremdenverkehr von Regionen

risten wiedergeben. Von direktem Absatz wird immer dann gesprochen, wenn ein Fremdenverkehrunternehmen alle Distributionsfunktionen selbst übernimmt (Kanal I). 55 Erfolgt der

54 Vgl. Meffert, H., Marketing, a.a.O., S. 423. Die Analyse der Ausgestaltung der vertraglichen Bindungen soll an dieser stelle unterbleiben.

55 Vgl. Meffert, H., Marketing, a.a.O., s. 426. 
Absatz von originären Leistungen über den Mittler "regionale Fremdenverkehrsorganisation" (Kanal II), so handelt es sich aus sicht der Einzelanbieter strenggenommen um einen indirekten Absatz. Unter der Annahme, daß zwischen den einzelnen touristischen Leistungsträgern eine Kooperation mit der regionalen Fremdenverkehrsorganisation besteht, deren Aufgabenfeld den gemeinsamen Vertrieb umfaBt, ist allerdings die zuordnung des Vertriebs über regionale Fremdenverkehrsorganisationen zum direkten Absatz gerechtfertigt.

Der Vorteil des direkten Absatzes von originären touristischen Leistungen über Fremdenverkehrsorganisationen wird in Literatur und Praxis darin gesehen, daB Reisevermittler der Erklärungsbedürftigkeit des touristischen Angebots im besonderen Maße Rechnung tragen können. 56 so stehen den Fremdenverkehrsorganisationen i.d.R. alle Angebotsalternativen einer Fremdenverkehrsregion zur Verfügung, die den nachfragenden Touristen gemäB ihren spezifischen Bedürfnissen als Angebotsvorschläge unterbreitet werden können. Allerdings erweist sich hierbei die räumliche Distanz zwischen dem Wohnort des potentiellen Besuchers und dem Reiseort als problematisch. Zwar stehen den Fremdenverkehrsorganisationen das gesamte Instrumentarium des Direct Marketing (Direct Mailing, Telefonmarketing, BTX, etc.) auch für distributionspolitische Aktivitäten zur Verfügung. Häufig sucht der Tourist jedoch das persönliche Vermittlungsgespräch. Ein solches Gespräch kann mit der regionalen Fremdenverkehrsorganisation i.d.R. nur erfolgen, wenn sich der Tourist bereits in der Fremdenverkehrsregion aufhält. 57 Bezogen auf

Vgl. Bleile, G., Marketing-strategien im Fremdenverkehr, a.a.o., s. 78 .

57 Alternativ könnten beispielsweise seitens der regionalen Fremdenverkehrsorganisation mobile "Verkaufsstellen" eingesetzt werden, die eine Akquisition potentieller Touristen auch auBerhalb der Fremdenverkehrsregion ermöglichen. Allerdings ist $z u$ vermuten, daß diesem Ver- 
die fur die Untersuchungsregion identifizierten zielgruppen bedeutet dies, daB mit Ausnahme der "sportaktiven Familienurlauber" bei allen zielgruppen angesichts ihrer geringen Besuchsabsichtsnennungen nur eine unterdurchschnittliche Erstansprache gewährleistet ist und auf die eigentliche Aufenthaltsdauer beschränkt ist. Um auch in der Phase der Besuchsentscheidung akquisitorisch aktiv zu werden, ist der direkte Vertrieb über Fremdenverkehrsorganisationen um den indirekten Vertrieb über Absatzmittler zu ergänzen.

Der indirekte vertriob erfolgt im Fremdenverkehr von Regionen über Absatzmittler, die als wirtschaftlich selbständige Unternehmungen zwischen den eigentlichen Fremdenverkehrsproduzenten oder den regionalen Fremdenverkehrsorganisationen und den Touristen geschaltet sind (Kanal III und Kanal IV). Die wichtigsten Absatzmittler im Fremdenverkehr sind Reisebüros und Reiseveranstalter 58 sowie Busreiseunternehmen, sofern diese selbst als Reiseveranstalter tätig werden. Generell ist $z u$ beachten, daB für kleinere Fremdenverkehrsunternehmen nur stark eingeschränkte Möglichkeiten bestehen, in das Sortiment von Reiseveranstaltern oder Reisebüros aufgenommen $\mathrm{zu}$ werden. 59 Dies ist einerseits darauf zurückzuführen, daB Reisebüros und -veranstalter mit Angebotsvermitt-

triebsweg Wirtschaftlichkeitsüberlegungen entgegenstehen.

Reiseveranstalter sind Fremdenverkehrsbetriebe, die überwiegend Leistungen Dritter zur Befriedigung des zeitweiligen ortsveränderungsbedürnisses und damit zusammenhängender anderweitiger Bedürfnisse $\mathrm{zu}$ einer neuen, eigenständigen Leistung verbinden und dies im $\mathrm{Na}-$ men und auf Rechnung des Reiseveranstalter-Unternehmens anbieten. Vgl. Freyer, W., Tourismus, a.a.0., S. 166. Reisemittler hingegen verkaufen touristische Teilleistungen oder Pauschalreisen im Auftrag von touristischen Leistungsanbietern oder Reiseveranstaltern an den Endverbraucher bzw. Touristen. Dabei sind die bekannteste Form dieses Zwischenhandels die Reisebüros. Vgl. ebenda, S. 186 .

59 Vgl. Bleile, G., Marketing-Strategien im Fremdenverkehr, a.a.0., s. 78 . 
lungen aus dem Deutschlandtourismus nur geringe Ertragspotentiale realisieren können und daher vor allem Angebote des "Outgoing-Tourismus" in ihrem sortiment präferieren. Andererseits nehmen Reiseveranstalter häufig nur touristische Angebote $a b$ einer bestimmte GröBenordnung (z.B. Hotelangebote mit einer Kapazität von mehr als 100 Betten) in ihr Sortiment auf. 60 somit stehen den einzelnen Fremdenverkehrsanbietern faktisch wichtige Vertriebsmöglichkeiten (Kanal III) nicht zur Verfügung.

Werden hingegen die Einzelangebote durch die regionale Fremdenverkehrsorganisation $z u$ Angebotsbündeln zusammengefaßt, so erleichtert sich, angesichts des hieraus resultierenden größeren Angebotsumfangs, eine Aufnahme in das Sortiment von Reiseveranstaltern und -büros (Kanal IV). Dies gilt insbesondere dann, wenn es den Fremdenverkehrsorganisationen durch eine zielgruppenspezifische produktbündelung gelingt, absatzmittlerbezogene Beratungsanforderungen $z u$ senken. Der potentielle Akquisitionserfolg läßt sich zusätzlich erhöhen, wenn von der Fremdenverkehrsorganisation gezielt solche Reisemittler angesprochen werden, die sich mit zielgruppenspezifischen Sortimentsschwerpunkten am Markt positionieren. Beispielsweise könnten Programmpakete, die an die "sportaktiven Familienurlauber" gerichtet sind, vor allem durch Absatzmittler vertrieben werden, die sich auf Familienreisen spezialisiert haben. Demgegenüber erscheint es angebracht, für die "land- und kulturorientierten Urlauber" auf Reisebüros mit Angebotschwerpunkten im Bereich "Bildungsreisen" zuzugehen.

Bei dem Vertrieb über Reisevermittler ist $z u$ beachten, daß der indirekte Absatz touristischer Leistungen nur für diejenigen Zielgruppen zweckmäBig ist, die für eine längere Auf-

60 Vgl. Bleile, G., Marketing-strategien im Fremdenverkehr, a.a.0., s. 78 . 
enthaltsdauer in der Fremdenverkehrsregion in Betracht kommen. Offensichtlich "rechnet" sich nur bei entsprechendem Ausgabenbudget die indirekte Distribution. Ferner lohnt sich der indirekte Vertrieb nur dann, wenn trotz der anfallenden Provisionskosten an Reisevermittler eine insgesamt effektivere zielgruppenerreichung realisiert werden kann. 61 Für die Untersuchungsregion bietet es sich daher schwerpunktmäBig an, Reisevermittler anzusprechen, deren Einzugsgebiet in der Entfernungsgruppe II liegt. Demnach wären die segmente "sportaktive Familienurlauber" 62 und "regenerations- und genuBorientierte Urlauber" über Reisemittler anzusprechen. Bezogen auf die Zielgruppen der Entfernungsgruppe I, d.h. des Tagesausflugs- bzw. Naherholungsverkehrs kommt demgegenüber der Vertrieb über Reisevermittler eine nur stark untergeordnete Bedeutung $\mathrm{zu}$. Hier haben andere empirische Analysen gezeigt, daß im Naherholungsverkehr Touristen in den seltensten Fällen Reisemittler aufsuchen. ${ }^{63}$ Aussichtsreicher erscheint hier, die zielgruppenangebote der Fremdenverkehrsregion ubber kommunikative Maßnahmen im Vorfeld $z u$ bewerben, um damit eine Buchung und letztlich eine Inanspruchnahme der Fremdenverkehrsregion $\mathrm{zu}$ erreichen.

\subsection{Ansatzpunkte für zielgruppenspesifische MaBnahmen im Rahmen der Rommunikationspolitik}

Die Aufgabe der Kommunikationspolitik besteht darin, den potentiellen Touristen über die nicht transportfähigen touristischen Leistungen $z u$ informieren und Präferenzen für eine

61 So lohnt sich ein indirekter Absatz nur, wenn ein ausreichendes Nachfragevolumen bei den Reisemittlern vorliegt.

62 In diesem Fall handelt es sich nur um die sportaktiven Familienurlauber der Entfernungsgruppe I.

63 Vgl. Bleile, E., Marketing-strategien im Fremdenverkehr, a.a.0., S. 78 . 
Inanspruchnahme der Fremdenverkehrsregion aufzubauen. Die wesentlichen Instrumente der Kommunikationspolitik im Fremdenverkehr sind die klassische Werbung, öffentlichkeitsarbeit, Verkaufsförderung und vor allem das Direct Mailing. 64

Im Rahmen der klassischen werbung werden im Fremdenverkehr die Imagewerbung und die Angebotswerbung unterschieden.65 ziel der Imagewerbung ist es, das allgemeine Vorstellungsbild potentieller und aktueller Besucher und damit ihre Einstellungen über eine Fremdenverkehrsregion zu beeinflusssen, um dadurch Präferenzen für eine Region aufzubauen. 66 Die Imagewerbung ist in diesem sinne als kommunikatives Rahmenkonzept zu verstehen, an welchem sich alle anderen kommunikativen Maßnahmen orientieren sollten. Sie dient vor allem dazu, die jeweiligen Einstellungen der zielgrupppen in ein integriertes Image $\mathrm{zu}$ vereinen. Gleichwohl sollte die Imagewerbung in Teilaspekten zielgruppenspezifisch ausgestaltet werden.

Bezogen auf die vorliegende Untersuchungsregion hat die empirische Analyse beispielsweise gezeigt, daß in einigen segmenten Fehlwahrnehmungen und wissensdefizite bezüglich der Untersuchungsregion bestehen. Zum Abbau dieser Fehlwahrnehmungen bietet es sich beispielsweise an, bei den "traditionellen spaziergängerfreunden" die touristische, sportunabhängige Infrastruktur und bei den "regenerations- und genußorientierten Urlaubern" zusätzlich das natürliche Angebot

Vgl. Roth, P., Grundlagen des Touristik-Marketing, a.a.0., S. 165 ff.; Krippendorf, J., Marketing im Fremdenverkehr, a.a.O.; Wölm, D., Marketing für Deutschlandreisen, a.a.0., S. 100 ff. Ferner können auch Sonderformen, wie zum Beispiel sponsoring oder Product Placement im Fremdenverkehr eingesetzt werden. Eine detaillierte Auseinandersetzung mit diesen Instrumenten soll an dieser stelle unterbleiben.

Vgl. ebenda.

Vgl. Wölm, D., Marketing im Tourismus, a.a.0., S.233 f. 
im Rahmen der Imagewerbung verstärkt kommunikativ herauszustellen. Ferner sollte die Botschaftsgestaltung eine hohe Anmutungsqualität haben, um positive stimmungen auszulösen und somit positive Assoziationen mit der Fremdenverkehrsregion aufzubauen. Aufgrund der übergeordneten Funktion der Imagewerbung im Kommunikationsmix und der daraus abzuleitenden Notwendigkeit eines konsistenten Werbeauftritts ist unmittelbar einsichtig, daB die Imagewerbung von der regionalen Fremdenverkehrsorganisation übernommen werden muB.

während die Imagewerbung vornehmlich auf den Aufbau eines übergreifenden positiven Regionenimages ausgerichtet sein sollte, bedarf es zusätzlich einer generellen Ansprache einzelner Segmente. 67 Diese Aufgabe fält vornehmlich der Angebotswerbung zu. Die Angebotswerbung hat zum ziel, das konkrete touristische Leistungsprogrammm uber geeignete Medien bekannt $z u$ machen. Dabei sollten die Werbeinhalte auf die unterschiedlichen Bedürfnisse der zielgruppen abgestimmt werden und einen Bezug $z u$ den für diese segmente ausgestalteten Programmpaketen aufweisen. Demnach sind für die "familienorientierten Urlauber" beispielsweise Familienpackages kommunikativ herauszustellen, während bei den "kultur- und landorientierten Urlaubern" die Besichtigungspackages im vordergrund stehen sollten.

Die Fremdenverkehrspraxis zeigt, daB die Imagewerbung überwiegend von der regionalen Fremdenverkehrsorganisation und die Angebotswerbung vor allem durch die einzelnen touristischen Leistungsträger vorgenommen wird. ${ }^{68}$ Diese Vorgehens-

67 Vgl. Freter, H., Marktsegmentierung, a.a.o., S. 141.

68 Wölm schlägt vor diesem Hintergrund vor, nach der Imagewerbung eine Anschlußwerbung durch die einzelnen touristischen Leistungsanbieter vorzunehmen. Dabei sollte die Anschlußwerbung Werbeaussagen über die einzelnen Angebote enthalten. Vgl. Wölm, D., Marketing für Deutschlandreisen, a.a.0., S. 102 ff. Es besteht hierbei jedoch die Gefahr, daß sich die Angebotswerbung von der 
weise erscheint jedoch insbesondere in Fällen kooperativer, durch verschiedene Leistungsträger erstellter Fremdenverkehrsleistungen diskussionswürdig. Für derartige Leistungskombinationen bietet sich eine trägerübergreifend abgestimmte Gemeinschaftswerbung an.

Im Hinblick auf die Auswahl geeigneter werbemedien für die Image- und Angebotswerbung kommen vor allem Zeitungen, Zeitschriften und Hörfunkwerbung in Betracht. 69 Bei diesen Medien besteht auf der Grundlage der identifizierten soziodemographischen Merkmale und der ermittelten Werthaltungen der zielgruppen die vergleichsweise einfache Möglichkeit, in Verbindung mit Informationen aus Media-Analysen eine geeignete zielgruppenspezifische Werbeträgerauswahl vorzunehmen. 70 Beispielsweise kommen für die "traditionellen spaziergängerfreunde" angesichts des recht hohen Alters und der ausgeprägten Traditions- und sicherheitsorientierung andere Printmedien in Frage als für das junge Publikum der "Badeurlauber".

Als weiterer bedeutsamer Werbeträger im Fremdenverkehr von Regionen sind Prospekte und Rataloge zu nennen. I.d.R. erstellen sowohl die einzelnen Leistungsträger als auch die

Imagewerbung in formaler und inhaltlicher Hinsicht entfernt.

69 Als weiteres Werbemedium ist das Fernsehen zu nennen. Angesichts überproportional hoher Schaltungskosten ist jedoch davon auszugehen, daß dieses Medium für Fremdenverkehrsregionen kaum in Frage kommt.

70 Vgl. zum Beispiel das Programm zur Medienauswahl MediaDialog-System (MDS), Version 3.1, auf der Grundlage der Verbraucheranalyse 92 und das dazugehörige Handbuch für den Benutzer, Hamburg 1992, s. $28 \mathrm{ff}$. Vgl. ferner die Ergebnisse der Media-Analyse 1992 für Publikumszeitschriften, Supplements, Tageszeitungen, Kongress, Lesezirkel, Kino, Hörfunk, Fernsehen, Hrsg.: Arbeitsgemeinschaft Media-Analyse, Frankfurt 1992. Zu der theoretischen Vorgehensweise bei der Medienauswahl vgl. Böcker, F., Gierl, H., Die Beurteilung einer Zeitschrift als Werbeträger, Berlin 1986, S. 22 ff. 
regionalen Fremdenverkehrsorganisationen Prospekte. Bei den auf die Fremdenverkehrsregion bezogenen Prospekten erscheint eine redaktionelle Ausgestaltung sinnvoll, in deren Rahmen die Programmangebote zielgruppenspezifisch aufbereitet werden. Je nach Größe des Segments und des Umfangs an zielgruppenspezifischen Angeboten bzw. Angebotsmodulen erscheint es darüber hinaus zweckmäBig, pro segment einen eigenständigen Zielgruppenprospekt $z u$ erstellen. 71 Dabei sollte der Name der Fremdenverkehrsregion als Markierung verwendet und mit dem Namen des Programmangebots als zusatz versehen werden. Diese Vorgehensweise empfiehlt sich jedoch nicht nur für die Prospekte der regionalen Fremdenverkehrsorganisation, sondern in gleichem MaBe für die Prospekte der touristischen Einzelanbieter. Nur so ist ein über das gesamte regionale touristische Leistungsprogramm einheitliches Erscheinungsbild und damit eine Unique Advertising Proposition (UAP) realisierbar.

Wichtige Erfolgsvoraussetzung für eine langfristige zielgruppenspezifische Profilierung ist die inhaltliche und formale Abstimmung der von der regionalen Fremdenverkehrsorganisation und den einzelnen Entscheidungsträgern angesetzten Kommunikationsaktivitäten. Eine integrierte Rommunikation

71 Diese Vorgehensweise ist bei Reiseveranstaltern üblich. So hat TUI zum Beispiel Prospekte für Zielgruppen erstellt, die spezielle Kundenbedürfnisse berücksichtigen: z.B. Prospekte über Schiffsreisen, clubreisen, Jugendreisen etc. Vgl. Goebel, M., Reisemarkt im strukturwandel - strategische Neuausrichtung eines Touristikkonzerns, in: Meffert, H., Wagner, H., Backhaus, K. (Hrsg.), Marketing in der Freizeit- und Dienstleistungsgeselischaft, Dokumentation des 20. Münsteraner Führungsgesprächs vom 21./22. Februar 1991, Dokumentationspapier Nr. 62 der Wissenschaftlichen Gesellschaft für Marketing und Unternehmensführung e.v., Münster 1991, s. $73 \mathrm{f}$. In Fremdenverkehrsregionen findet sich solch eine zielgruppenspezifische Prospekterstellung nur ansatzweise. In der vorliegenden Untersuchungsregion wäre als Beispiel ein Prospekt zu "Ferien auf dem Bauernhof" zu nennen. 
führt zu einer Erhöhung des wiedererkennungseffektes der Fremdenverkehrsregion, wenn auf einzelne, standardisierte Kommunikationsmodule zurückgegriffen werden kann. Zudem können damit erhebliche Kosteneinsparungen verbunden sein.

Im engen Zusammenhang mit der Imagewerbung stehen Maßnahmen der öffentlichkeitsarbeit (Public Relations). Mit der öffentlichkeitsarbeit soll eine systematische Meinungspflege erreicht werden, durch die in der gesamtem öffentlichkeit Vertrauen zur Fremdenverkehrsregion aufgebaut und die Bildung eines positiven Regionenimages unterstützt wird.72 Hierzu müssen die regionalen Fremdenverkehrsorganisationen aktiv auf die Medien zugehen, um zum Beispiel über Pressekonferenzen, Pressemappen, Empfänge, Gesprächsrunden, etc. ein größtmögliches $M a B$ an Publizität zu gewinnen. Da vor allem kleine touristische Leistungsanbieter kaum die Möglichkeit für eine breite öffentlichkeitsarbeit haben, bietet es sich an, durch gemeinschaftliche PR-Aktionen unter Federführung der regionalen Fremdenverkehrsorganisation öffentlichkeitsarbeit zu betreiben.

Im Zusammenhang mit der öffentlichkeitsarbeit ist $z u$ beachten, daß dieses Instrument sich an eine vielzahl relevanter Gruppen der öffentlichkeit richtet, die vielfach nicht mit den zielsegmenten übereinstimmen. Eine Verbesserung der zielgruppenspezifität kann allerdings über themenspezifische Aktionen und deren informatorische Umsetzung in der entsprechenden Presse erreicht werden. Als Beispiel können hier gemeinsame Wandertage mit entsprechenden "Öffentlichkeiten" angeführt werden, die neben der Unterstützung eines positiven Regionenimages auch darauf abzielen, zum Beispiel bei dem segment "kultur- und landorientierte Urlauber", ein positives Meinungsbild zu erzeugen.

72 Vgl. zur Definition von öffentlichkeitsarbeit Meffert, H., Marketing, a.a.0., S. 493 . 
Eine weitere wichtige Funktion kommt im Rahmen der Kommunikation von Fremdenverkehrsangeboten den verkaufsförderungsmaBnahmen zu. Sie enthalten jene Kommunikationsmaßnahmen, die der Unterstüzung und Erhöhung der Effizienz der Absatzorgane, d.h. der Reisemittler, und oder der Beeinflussung der Verwender bei der Beschaffung und Nutzung von Fremdenverkehrsleistungen dienen. ${ }^{73}$ Konsumentengerichtete Verkaufsförderunganaßnahmen sind beispielsweise befristete Preissenkungen $z u$ bestimmten Reiseterminen oder von einzelnen Angebotsteilen, aber auch Couponanzeigen, Preisausschreiben, etc.. Ein zielgruppenspezifischer Einsatz der Verkaufsförderung kann zum Beispiel dadurch erreicht werden, daB eine segmentspezifische Angebotswerbung mit einer Couponanzeige kombiniert wird. Am Point of Sale, d.h. dem Verkaufsbüro der Reisemittler kommt der Verkaufsförderung die Aufgabe $z u$, durch surrogatspräsentationen wie Plakate, Filme und DiaDisplays dem potentiellen Besucher ein visuelles Vorleben des Urlaubs oder der Reise zu ermöglichen. Adressaten dieser Kommunikationsform sind, im vorliegenden Untersuchungsfall vor allem die zielgruppen der Entfernungsgruppe II, da nur hier ein Vertrieb über Reisemittler zweckmäBig ist. 74

Die distributionsgerichtete Verkaufsförderung hat zum $\mathrm{Ziel}$, die Aufnahme des eigenen Angebots in das Programm der Distributionsorgane $z u$ erreichen bzw. den dortigen Absatz zu steigern. 75 Ferner soll darauf hingewirkt werden, daB die Verkaufstätigkeit sachkundig und engagiert ausgeübt wird. Geeignete Maßnahmen sind z.B. die Einladung zu Präsentationen und Vorträgen, Besichtigungen, Schulungen, Prämierung von Reisemittlern, Sonderprovisionen für bestimmte Programmbestandteile, Verkaufswettbewerbe etc.. Für die vorliegende

73 Vgl. Wölm, D., Marketing für Deutschlandreisen, a.a.o., S. $109 \mathrm{ff}$.

74 Vgl. die Ausführungen in Kapitel c.22.

75 Vgl. Meffert, H., Marketing, a.a.o., s. $490 \mathrm{f}$. 
Untersuchungsregion bieten sich solche Aktivitäten ebenfalls überwiegend für Reisemittler an, die ihren Firmensitz in der Entfernungsgruppe II haben. Von diesen erweisen sich für entsprechende Aktivitäten vor allem solche Reiseveranstalter und -mittler als interessant, die als spezialanbieter hohe Affinitäten mit den identifizierten zielgruppen aufweisen.

Eine zentrale Bedeutung im Fremdenverkehr von Regionen ist schlieblich dem Direct-Mailing zuzusprechen. Mit diesem Instrument sollen die einzelnen zielgruppen direkt angesprochen und mit zielgruppenspezifischen Werbebotschaften bearbeitet werden. ${ }^{76}$ Voraussetzung ist jedoch, daB deren Adressen bekannt sind. Folglich kommt vor allem dem Direct-Mailing bei denjenigen Touristen eine besonders hohe Bedeutung $z u$, die schon einmal mit touristischen Institutionen oder Anbietern der Fremdenverkehrsregion Kontakt aufgenommen haben. Damit auch die regionale Fremdenverkehrsorganisation eine gezielte Ansprache vornehmen kann, sollten die Adressen in einer gemeinsamen Datenbank gesammelt werden. Angesichts der hohen Quote an wiederholungsbesuchern sowohl in Deutschland als auch in der Untersuchungsregion ist das Instrument des Direct-Mail besonders für die Erhöhung der zielgebietstreue geeignet. Dabei müssen allerdings die Informationen - wie im Rahmen der Angebotswerbung - zielgruppenspezifisch übermittelt werden.

Letztlich werden diejenigen Fremdenverkehrsregionen den größten Erfolg aufweisen, die entlang aller Maßnahmen ein eindeutiges, auf die Anforderungen der einzelnen Zielgruppen abgestimmtes Profil aufweisen. Die Hauptschwierigkeit bei der Umsetzung von Marketingkonzepten ist allerdings, da $B$ innerhalb der Entscheidungsträger einer Fremdenverkehrsregion nur bedingt Einigkeit über die Gestaltung der Marketingin-

76 Vgl. Roth, P., Grundlagen des Touristik-Marketing, a.a.O., S. 184 . 
strumente besteht. Nur dann, wenn die einzelnen Maßnahmen aufeinander abgestimmt sind und die einzelnen Fremdenverkehrsträger dem generellen Handlungsrahmen entsprechend agieren und kommunizieren, erscheint eine Profilierung einer gesamten Fremdenverkehrsregion erfolgversprechend.

Die notwendige und für ein zielgruppenspezifisches Marketing charakteristische Focussierung auf spezifische segmente kann demnach nicht darüber hinwegtäuschen, daB es einer "integrativen Klammer" für alle vorgeschlagenen Maßnahmen bedarf. So ist für die Umsetzung zielgruppenspezifischer Markbearbeitungskonzepte die Verankerung eines gemeinsam getragenen selbstverständnisses bei allen Interessengruppen einer Fremdenverkehrsregion eine Grundvoraussetzung. 77 Insbesondere geht es darum, daB die einzelnen touristischen Leistungsträger und die ansässige Bevölkerung sich auf einen gemeinsamen Identitätskern beziehen, in dem die tatsächlichen stärken und Schwächen der Fremdenverkehrsregion zum Ausdruck kommen. Nur wenn es gelingt, die ziele und Handlungen der einzelnen touristischen Leistungsträger als auch der Bevölkerung entsprechend dieser identifikatorischen Grundlage aufeinander abzustimmen und damit eine Bündelung der Aktivitäten $z u$ erreichen, kann auch ein zielgruppenmarketing-Konzept für eine Fremdenverkehrsregion erfolgreich sein. Für ein integriertes Gesamtbild einer Fremdenverkehrsregion ist somit ein einheitliches Erscheinungbild (Corporate Design, Corporate Communication) und ein einheitliches, zielgruppenorientiertes Verhalten (Corporate Behaviour) notwendig. 78

77 Vgl. Meffert, H., u.a., Regionenmarketing Münsterland, a.a.0., S. 180 .

78 Vgl. Meffert, u.a. Regionenmarketing Münsterland, a.a.0., S. 205. Vgl. zu diesen strukturelementen der Corporate Identity und zu entsprechenden Gestaltungsalternativen Birkigt, K., Stadler, M.M., Fuck, H.J., Corporate Identity. Grundlagen, Funktionen, Fallbeispiel, 5. Aufl., Landsberg a.L. 1992, S. $15 \mathrm{ff}$. und S. $39 \mathrm{ff}$. 
3. Ansatzpunkte für die Durchsetzung zielgruppenspezifischer Marktbearbeitungskonzepte

Die vielschichtigen Einzelinteressen und komplexen Zuständigkeitsstrukturen innerhalb von Fremdenverkehrsregionen machen die Notwendigkeit der Entwicklung von Durchsetzungskonzepten für eine erfolgreiche Umsetzung zielgruppenspezifischer Marktbearbeitungskonzepte deutlich. Aufgabe der regionalen Fremdenverkehrsorganisation muß es daher sein, die Unterstützung aller Leistungträger der Fremdenverkehrsregion als auch der Bevölkerung bei der Gestaltung und Vermarktung des Produktes "Fremdenverkehrsregion" sicherzustellen. 79 Da die Angebotskomponenten einer Fremdenverkehrsregion von einer Vielzahl rechtlich selbständiger Fremdenverkehrsunternehmen und -institutionen erstellt werden, kommt der koordination der Einzelaktivitäten durch die regionale Fremdenverkehrsorganisation eine herausragende Bedeutung $\mathrm{zu}$.

Nach Kolks sind bei der Durchsetzung von Marketingkonzepten und -strategien zwei Arbeitsstufen zu differenzieren: 80

- In einer ersten stufe sind die Konzeptinhalte den betroffenen Entscheidungs- und Handlungsträgern sowie den sonstigen Betroffenen zu kommunizieren.

- In einer zweiten Stufe sind im Rahmen eines Konfliktmanagements mögliche widerstände einzelner Gruppen gegen die

79 Vgl. Reppel, R., Innenmarketing - eine strategie für Fremdenverkehrsorte, in: Wolf, J., seitz, E. (Hrsg.), Tourismusmanagement und -marketing, a.a.0., s. 31 .

80 Kolks unterteilt dabei den Implementierungsprozeß generell in eine Umsetzungs- und eine Durchsetzungsphase. Erstere beinhaltet die Festlegung aller Maßnahmen, die der Spezifizierung von strategien und Konzepten dienen, wie dies im vorangegangenen Teilkapitel dargestellt wurde. Vgl. Kolks, U., Strategieimplementierung - Ein anwendungsorientiertes Konzept, Wiesbaden 1990. 
Strategie und die spezifizierten MaBnahmen zu eliminieren.

Als bedeutende Entscheidungsträger im Fremdenverkehr von Regionen wurden die touristischen Einzelanbieter und Fremdenverkehrsinstitutionen der kommunalen Ebene identifiziert. 81 In einem weiteren "Betroffenheitskreis" ist zusätzlich die einheimische Bevölkerung $z u$ sehen, die teilweise selbst Bestandteil des Fremdenverkehrsproduktes ist. Beide Gruppen gilt es im Rahmen eines innengerichteteten Regionenmarketing $^{82}$ für die Durchsetzung des zielgruppenkonzeptes zu gewinnen.

Auf der Bbene der Eingelanbieter ist es für eine erfolgreiche zielgruppenspezifische Marktbearbeitung notwendig, daB möglichst alle touristischen Leistungsanbieter ihre Marketingaktivitäten auf die Bearbeitung einzelner oder aller von der regionalen Fremdenverkehrsorganisation ermittelten zielgruppen abstimmen. In der ersten Durchsetsungsstufe sind daher die Vertreter der verschiedenen touristischen Leistungsträger z.B. in Form von Seminaren oder Fachtagungen zusammenzuführen und über das zielgruppenspezifische Marktbearbeitungskonzept, sowie dessen Inhalte und Erfolgserwartungen $\mathrm{zu}$ informieren.

Eine erste Durchsetzungsbarriere, die vielfach in Fremdenverkehrsregionen anzutreffen $i s t^{83}$, liegt in Interessengegensätzen der Leistungs- und Entscheidungsträger. Diese Interessengegensätze ergeben sich vor allem aus den Egoismen

81 Vgl. Kapitel A.2.

82 Zur Bedeutung des innengerichteten Marketing vgl. auch die Ausführungen bei Meffert u.a., Regionenmarketing Münsterland, a.a.0., S. 170 ff.; Meffert, H., u.a, Marketing für das Münsterland, a.a.0., s. 23 .

83 Vgl. Tschiderer, F., Ferienortplanung, a.a.0., S. 207 f. 
der Leistungsträger. Dabei ist bei einer vielzahl von touristischen Leistungsträgern davon auszugehen, daB ihr Interesse am Gemeinwohl der Fremdenverkehrsregion schwach ausgeprägt ist, solange individuelle ökonomische ziele zufriedenstellend erreicht werden. 84

Eine solche Einschätzung kann daraus resultieren, daB die Leistungsträger generell den Vorteil einer zielgruppenspezifischen Marktbearbeitung in Abrede stellen oder die vorgestellten Segmente in ihrer spezifischen Bedeutung anders gewichten als dies von der regionalen Fremdenverkehrsorganisation vorgeschlagen wird. Im Extremfall legen die Einzelanbieter ihrem Marketing eine andere Zielgruppentypologie zugrunde, die in ihrer Bearbeitung den übergreifend ermittelten zielgruppen zuwiderläuft. Beispielhaft wäre anzuführen, daB ein marktstarker Anbieter von Ferienparks die Untersuchungsregion als belebtes Verkehrszentrum positioniert und sich damit im Hinblick auf die segmentpositionierung der "traditionellen spaziergängerfreunde" kontraproduktiv verhält.

Vor diesem Hintergund sind in der zweiten Durchsetzungsstufe zielgruppenspezifischer Marktbearbeitungskonzepte, die touristischen Entscheidungsträger innerhalb der Fremdenverkehrsregion zur Kooperation zu motivieren. Dabei muß die regionale Fremdenverkehrsorganisation gegenseitiges Verständnis zwischen den touristischen Leistungsanbietern fördern und den individuellen Nutzen eines gemeinschaftlichen Vorgehens herausstellen. Dies macht unmittelbar deutlich, daB eine frühzeitige Einbindung aller Entscheidungsträger in der Durchsetzungsphase notwendig ist. Im Rahmen dieses Dialogs ist die konkrete Umsetzung der zuvor von der Fremdenver-

84 Vgl. Krippendorf, J., Marketing im Fremdenverkehr, a.a.0., s. 97 ff. 
kehrssorganisation entwickelten Maßnahmen unter Einbindung der Leistungsträger einzuleiten.

Dazu erscheint es zweckmäßig, Projektteams einzurichten, in denen eine detaillierte Ausarbeitung der zielgruppenspezifischen Marktbearbeitungskonzepte vorgenommen wird. Hierbei bietet es sich an, wie beim Aufbau des Projektmanagment ${ }^{85}$ in der Konsumgüterindustrie für die einzelnen zielgruppen jeweils eigenständige Projektteams zu bilden. Als Mitglieder sind Vertreter von denjenigen touristischen Leistungsträgern einzubinden, die in besonderem Maße Angebote für diese zielgruppen bereitstellen. So könnte für die vorliegende Untersuchungsregion das Projektteam für "Kultur- und landorientierte Urlauber" aus Vertretern der behördlichen Kulturdezernate, der Naturschutzverbände, Museumsleitern, Theaterintendanten, etc. gebildet werden.

Durch die Einbindung der Entscheidungsträger kann ihr Engagement gesichert, das vorhanden know how genutzt und der leistungsträgerspezifische Erfolgsbeitrag für das zielgruppenspezifische Marktbearbeitungskonzept : systematisch erhöht werden.

Insbesondere bei $\mathrm{zu}$ erwartenden Konflikten innerhalb der Projektteams, z.B. aufgrund von wahrgenommmenen Konkurrenzverhältnissen, ist es sinnvoll, vertreter der regionalen Fremdenverkehrsorganiation als Moderator in das Projektteam aufzunehmen. ${ }^{86}$ ziel muß es hierbei sein, Einzelinteressen in den Hintergrund zu stellen, auf persönlichen und politischen

85 Projekte sind umfangreiche, aber zeitlich begrenzte Aufgaben. Zu den unterschiedlichen Ausgestaltungsalternativen des Projektmanagement vgl. Kieser, A., Kubicek, H., Organisation, 3. Aufl., Berlin, New York 1992, S. 138 ff.; Frese, E., Grundlagen der Organisation. Die Organisationsstruktur der Unternehmung, Wiesbaden 1987, S. 460 ff.

Vgl. Reppel, R., Innenmarketing, a.a.0., s. 35 . 
Gegensätzen beruhende Interessengegensätze zu schlichten und den Dialog sachlich zu führen. Zentrale Bedeutung kommt in diesem Durchsetzungsschritt der Auswahl eines adäquaten Argumentationsstils $\mathrm{zu} .^{87}$ Wesentliche Erfolgsvoraussetzung einer Projektteamkoordination ist die Akzeptanz durch die touristischen Leistungsanbieter. Bei zu geringer Akzeptanz kann auf externe Berater als Moderatoren zurückgegriffen werden. 88

Neben der kommunikativen Überzeugungsarbeit können auch Beratungsleistungen und finanzielle Anreize seitens der Fremdenverkehrsorganisation eingesetzt werden. 89

Mit Blick auf die regionale Bevölkerung ist es erforderlich, frühzeitig über die zielgruppenspezifischen Marktbearbeitungsmaßnahmen $z u$ informieren, um möglichen widerständen frühzeitig entgegenzutreten. Widerstände auf der seite der Bevölkerung bei der Durchsetzung zielgruppenspezifischer Marktbearbeitungskonzepte bestehen zunächst in einer vielfach zu beobachtenden generellen Fremdenverkehrsverdrossenheit der ansässigen Bevölkerung. 90 Dies kann sich zum Beispiel in einem unfreundlicheren Verhalten gegenüber Touristen, hier im besonderen gegenüber spezifischen zielgruppen, äußern. Da aber der Kontakt zwischen Touristen und der ansässigen Bevölkerung unmittelbarer Bestandteil des Produktes "Fremdenverkehrsregion" ist, sind direkte Wechselwirkungen zwischen dem Verhalten der Bewohner und der wahrgenommenen Besuchsqualität offensichtlich. Ferner können sich Bevölkerungsgruppen herausbilden, die den Fremdenverkehr generell als bürgerfeindlich und landschaftszerstörend empfinden. Es

Vgl. Kolks, U., strategieimplementierung, a.a.o.

Vgl. Reppel, R., Innenmarketing, a.a.0., S. 35 .

Vgl. Reppel, R., Innenmarketing, a.a.0., S. 39.

Vgl. Reppel, R., Innenmarketing, a.a.o., S. 34 . 
besteht die Gefahr, daß diese über die Bildung von Interessengemeinschaften aktiv einer Durchsetzung von Infrastrukturprojekten entgegentreten, die aus einer zielgruppenspezifischen Marktbearbeitung resultieren kann (z.B. die Anlage von Wanderpfaden für "land- und kulturorientierte Urlauber").

Damit ist die aktive Einbindung der ansässigen Bevölkerung bei der Durchsetzung zielgruppenspezifischer Marktbearbeitungskonzepte erforderlich. Geeignete Instrumente sind sowohl Informationsveranstaltungen (z.B. Tag der offenen Tür) seitens der regionalen Fremdenverkehrsorganisation, Bürgerversammlungen, die Einrichtung von Interessengemeinschaften als auch eine aktive und breit angelegte Informationspolitik über geplante Marketing-Aktivitäten.91

Zur sicherstellung eines $z u$ jedem Zeitpunkt koordinierten Vorgehens erweist sich die klare Festschreibung von verantwortlichkeiten der regionalen Fremdenverkehrsorganisation als weitere Erfolgsvoraussetzung. Die Gestaltung der Fremdenverkehrsorganisation als Koordinationsstelle mit eindeutig umrissenen Kompetenz- und Verantwortungsbereichen ist in diesem zusammenhang unabdingbar. 92 Dabei sollten sich die Weisungsrechte jedoch nicht nur - wie vielfach in der Praxis üblich - auf die Kommunikationspolitik beschränken. Entsprechend den aufgezeigten Ansatzpunkten der zielgruppenspezifischen Marktbearbeitung gilt es vielmehr eine Kompetenzausweitung der Fremdenverkehrsorganisation, anzustreben. Eine solche Institution sollte neben Informations- und Mitwirkungsrechten auch die Budgetverantwortung und Entscheidungskompetenz für die Umsetzung des Konzepts des zielgruppenmarketing erhalten.

91 Vgl. Reppel, K., Innenmarketing, a.a.0., S. 39.

92 Vgl. Meffert, H. U.a., Regionenmarketing Münsterland, a.a.o., S. 205 . 


\section{Zusammenfassung und Ausblick}

\section{1. zusammenfassende würdigung der ontersuchungsergebnisse}

Die bisherige theoretische und empirische Auseinandersetzung mit dem zielgruppenmarketing im Fremdenverkehr von Regionen weist insgesamt einen nur wenig befriedigenden Forschungsstand auf. Dies gilt insbesondere für die Markterfassung und zielgruppenspezifische Marktbearbeitung aus sicht regionaler Fremdenverkehrsorganisationen. Entsprechend wurde in der vorliegenden Untersuchung versucht, eine theoretische Fundierung und umfassende empirische Analyse dieses Untersuchungskomplexes $z u$ leisten. Die empirische Analyse erfolgte anhand einer Fremdenverkehrsregion aus dem norddeutschen Raum.

Im Mittelpunkt stand dabei die Fragestellung, unter Berücksichtigung welcher Markterfassungsansätze und segmentierungskriterien im Fremdenverkehr eine zielgruppenabgrenzung vorzunehmen ist und wie die identifizierten zielgruppen segmentspezifisch bearbeitet werden können. Die Untersuchung erfolgte dabei in zwei zentralen Arbeitsschritten.

In einem ersten Schritt wurden die Möglichkeiten der Markterfassung einer tiefergehenden Analyse unterzogen. Auf der Grundlage eines empirischen Bezugsrahmens wurde eine hypothesengestützte Analyse soziodemographischer und ausgewählter psychographischer Merkmale (Werte, Motive und Einstellungen) hinsichtlich ihrer Besuchsverhaltensrelevanz durchgeführt. Dabei dienten als abhängige Variable die sich in der Besuchsabsicht und -dauer unterscheidenden Verhaltenssegmente, die innerhalb von zwei auf der Basis der Entfernung zum zielgebiet gebildeten Entfernungsgruppen abgegrenzt werden konnten. Auf der Grundlage der identifizierten Bestimmungsfaktoren des Besuchsverhaltens wurde in beiden Entfernungsgruppen eine psychographische Marktsegmentierung 
durchgeführt. Im zweiten Arbeitsschritt wurden Ansatzpunkte einer zielgruppenspezifischen Marktbearbeitung von Fremdenverkehrsregionen aufgezeigt und Möglichkeiten der Begegnung von widerständen bei ihrer Durchsetzung erarbeitet.

Unter Berücksichtigung der eingangs formulierten zielsetzungen können die folgenden zentralen Erkenntnisse der Untersuchung auf der Basis der empirischen Analysen und den darauf aufbauenden konzeptionellen Überlegungen zur zielgruppenspezifischen Marktbearbeitung zusammengefaBt werden:

(1) Die gewählte operationalisierung des Besuchsverhaltens auf der Basis der Besuchsabsicht und -dauer hat sich als tragfähige Grundlage einer differenzierten Analyse der Bestimmungsfaktoren des regionenspezifischen Besuchsverhaltens erwiesen. Dabei konnte gezeigt werden, daß der Verhaltensparameter "Anreisedauer" über die Entfernung zum zielgebiet erfaßt werden kann und diese sinnvollerweise nicht als abhängige Verhaltensvariable, sondern als Bestimmungsfaktor des Besuchsverhaltens zu interpretieren ist. Der vermutete Zusammenhang zwischen der Entfernung zum zielgebiet und der Besuchsabsicht konnte nicht bestätigt werden. Demgegenüber wurde ein starker Zusammenhang zwischen der zielgebietsentfernung und der Besuchsdauer festgestellt. Die aufgrund dieses Ergebnisses durchgeführte Makrosegmentierung auf der Basis der Zielgebietsentfernung ermöglichte, im Naherholungsverkehr (Entfernungsgruppe I) die Verhaltenssegmente "Tagesausflügler" und "Besucher mit Ubbernachtung" und in der Entfernungsgruppe II die "Kurzurlauber" und "Urlauber" zu unterscheiden. Darüber hinaus wurden die Besucher und Nicht-Besucher entfernungsgruppenspezifisch erfaßt.

(2) Die Analyse des Einflusses soziodomographischer Merkmale auf die Besuchsabsicht und -dauer zeigte, daß vor 
allem in der Entfernungsgruppe I die in den Verhaltenssegmenten zum Ausdruck kommenden Unterschiede im Besuchsverhalten nur unzureichend anhand soziodemographischer Merkmale erklärt werden können (vgl. Abbildung 46). Während die Besuchsabsicht durch das Haushaltseinkommens und die schichtzugehörigkeit erklärt werden kann, besteht zwischen soziodemographischen Merkmalen und der Besuchsdauer kein Zusammenhang. In der Entfernungsgruppe II kann die Besuchsabsicht und-dauer durch die Kinderanzahl und vor allem durch sozioökonomische Merkmale beschrieben werden. Wichtige Merkmale wie Alter und Bildung bringen aber auch hier keinen Erklärungsbeitrag. Diese Ergebnisse machen deutlich, daß das regionenspezifische Besuchsverhalten offenbar vor allem von dem Haushalts-Einkommen der Besucher abhängt.

Gleichzeitig wird deutlich, daß die entfernungsgruppenspezifischen Verhaltenssegmente nur eingeschränkt die soziodemographische Identifizierbarkeit gewährleisten, so daß die verhaltensorientierte Marktsegmentierung im Fremdenverkehr von Regionen nur bedingt als geeigneter segmentierungsansatz anzusehen ist.

(3) Die Analyse der Identifizierbarkeit der psychographischen Merkmale "Werte", "Motive" und "Einstellungen" erfolgte auf der Grundlage der verdichteten Werte-, Motiv- und Einstellungsfaktoren. Es zeigte sich, daß diese gut anhand soziodemographischer Merkmale beschrieben werden konnten und daher als geeignete segmentierungskriterien angesehen werden können. Einen zusammenfassenden Überblick über die Zusammenhänge zwischen soziodemographischen und psychographischen Merkmalen findet sich in Abbildung 47. Es wird deutlich, daß die Werteausprägungen besonders gut anhand der Merkmale "Alter", "Lebenszyklusphase", "Bildungsgrad" und "Schichtzugehörigkeit" erklärt werden können. Ur- 


\begin{tabular}{|c|c|c|c|c|c|c|c|c|c|c|c|c|c|}
\hline \multirow{3}{*}{\multicolumn{2}{|c|}{$\begin{array}{c}\text { Soziodemo- } \\
\text { graphische } \\
\text { Merkmale }\end{array}$}} & \multicolumn{6}{|c|}{ Besuchsabsicht } & \multicolumn{6}{|c|}{ Besuchsdauer } \\
\hline & & \multirow{2}{*}{\multicolumn{3}{|c|}{$\begin{array}{l}\text { Entfernungsgruppe I } \\
\begin{array}{c}\text { Hypo- EinfluB B/N } \\
\text { these }\end{array}\end{array}$}} & \multirow{2}{*}{\multicolumn{3}{|c|}{$\begin{array}{l}\text { Entfernungsgruppe II } \\
\begin{array}{l}\text { Hypo- EinfluB } B / N \\
\text { these }\end{array}\end{array}$}} & \multicolumn{3}{|c|}{ Entfernungsgruppe I } & \multicolumn{3}{|c|}{ Entfernungsgruppe I } \\
\hline & & & & & & & & \begin{tabular}{|c} 
Hypo- \\
these
\end{tabular} & EinfluB & $B / N$ & $\begin{array}{l}\text { Hypo- } \\
\text { these }\end{array}$ & EinfluB & $\mathrm{B} / \mathrm{N}$ \\
\hline \multirow{4}{*}{ 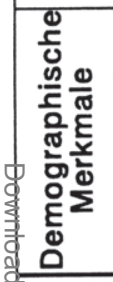 } & Geschlecht & $\mathrm{H}_{\text {sozlo3 }}$ & 0 & B & $\mathrm{H}_{\mathrm{soz} 103}$ & 0 & B & $\mathrm{H}_{\text {sozlos }}$ & 0 & B & $\mathrm{H}_{\text {sozlos }}$ & 0 & B \\
\hline & Durchschnittsalter & $\mathrm{H}_{\text {sozlo1 }}$ & 0 & B & $\mathrm{H}_{\mathrm{s0z} 101}$ & 0 & B & $\mathrm{H}_{\mathrm{s02102}}$ & 0 & $\mathrm{~N}$ & 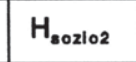 & 0 & $\mathbf{N}$ \\
\hline & Kinderanzahl & $\mathrm{H}_{802104}$ & 0 & $\mathrm{~N}$ & $\mathrm{H}_{\mathrm{s0z105}}$ & + & B & & 0 & & $\mathrm{H}_{\mathbf{s 0 z 1 0 5}}$ & + & B \\
\hline & Lebenszyklusphase & $\mathrm{H}_{\text {soz108 }}$ & 0 & $\mathrm{~N}$ & $\mathrm{H}_{\mathrm{s02108}}$ & 0 & $\mathrm{~N}$ & $\mathrm{H}_{\mathrm{s} 0 \mathrm{z} 107}$ & 0 & $\mathrm{~N}$ & $\mathrm{H}_{\mathrm{s} 02107}$ & 0 & $\mathbf{N}$ \\
\hline \multirow{5}{*}{ 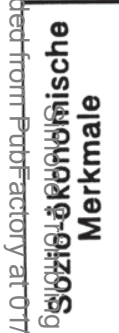 } & Bildung & $\mathrm{H}_{\mathrm{s0z108}}$ & 0 & $\mathrm{~N}$ & $\mathrm{H}_{\text {sozio8 }}$ & 0 & $\mathbf{N}$ & $H_{\text {sozios }}$ & 0 & $\mathrm{~N}$ & $\mathrm{H}_{\text {soziog }}$ & 0 & $\mathbf{N}$ \\
\hline & Beruf & $\mathrm{H}_{\mathrm{soz1013}}$ & 0 & B & $\mathrm{H}_{\mathrm{soz1013}}$ & 0 & $\mathrm{~N}$ & $\mathrm{H}_{\mathrm{s} 021014}$ & 0 & B & $\mathrm{H}_{\mathrm{soz1014}}$ & $\bullet$ & $\mathbf{N}$ \\
\hline & $\begin{array}{l}\text { HH-Nettoein- } \\
\text { kommen }\end{array}$ & $\mathrm{H}_{\mathrm{soz} 1010}$ & + & B & $\mathrm{H}_{\mathrm{s} 021010}$ & $\bullet$ & (B) & $\mathrm{H}_{\text {sozlo11 }}$ & 0 & $\mathrm{~N}$ & $\mathrm{H}_{\text {sozio11 }}$ & $\bullet$ & (B) \\
\hline & $\begin{array}{l}\text { Persōnliches Netto- } \\
\text { einkommen }\end{array}$ & $\mathrm{H}_{\mathrm{s} 0 \mathrm{z} 1010}$ & 0 & $\mathrm{~N}$ & $\mathrm{H}_{\mathrm{s} 021010}$ & 0 & (B) & 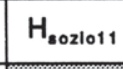 & 0 & $\mathrm{~N}$ & $\mathrm{H}_{\text {sozio11 }}$ & 0 & $\mathbf{N}$ \\
\hline & Schicht & $\mathrm{H}_{\mathrm{s} 021012}$ & + & B & $\mathrm{H}_{\mathrm{soz} 1012}$ & 0 & $\mathrm{~N}$ & & 0 & & & 0 & \\
\hline \multicolumn{14}{|c|}{ 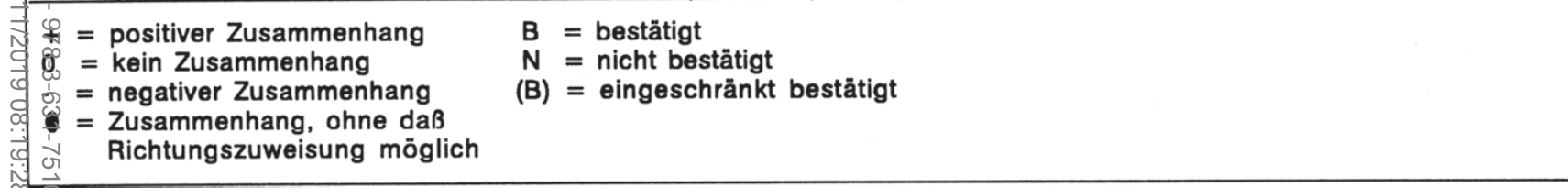 } \\
\hline
\end{tabular}

Abb. 46: Zusammenfassende Ubersicht zur Prüfung der Untersuchungshypothesen zum EinfluB soziodemographischer Merkmale auf das Besuchsverhalten 


\begin{tabular}{|c|c|c|c|c|c|c|c|c|c|c|c|c|c|c|c|}
\hline & \multicolumn{5}{|c|}{ Werte } & \multicolumn{5}{|c|}{ Urlaubsmotive } & \multicolumn{3}{|c|}{ Realeinstellungen } \\
\hline & & & 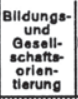 & $\begin{array}{l}\text { Iodonis- } \\
\text { musts }\end{array}$ & 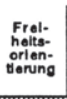 & 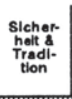 & 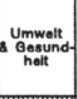 & 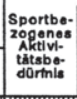 & 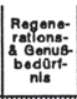 & 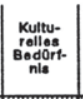 & 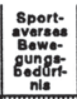 & 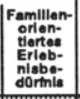 & 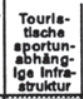 & $\begin{array}{l}\text { Netor: } \\
\text { Alchos: } \\
\text { Angobot }\end{array}$ & $\begin{array}{l}\text { Sport- } \\
\text { anoobot }\end{array}$ \\
\hline \multirow{12}{*}{ 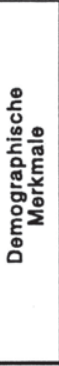 } & & $\begin{array}{l}\text { Hypo- } \\
\text { Hingos:-... }\end{array}$ & & & & & & & & & & & & & \\
\hline & Geschlecht & Elnfue & 0 & - & 0 & - & - & - & 0 & 0 & 0 & 0 & 0 & 0 & 0 \\
\hline & & $\mathrm{B} / \mathrm{N}$ & & & & & & & & & & & & & \\
\hline & & $\begin{array}{l}\text { Hypo: } \\
\text { Hybs:- }\end{array}$ & & & $\mathrm{H}_{\text {wertiote }}$ & & & $H_{\text {molturte }}$ & & & $H_{\text {motivit }}$ & & & & \\
\hline & Alter & Einfue & O & 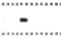 & - & + & 0 & $-\cdots$ & - & $(+)]$ & + & 0 & $(+)$ & + & - \\
\hline & & $\mathrm{B} / \mathrm{N}$ & & & B & & & $B$ & & & B & & & & \\
\hline & & $\begin{array}{l}\text { Hypo- } \\
\text { thyose.... }\end{array}$ & & & $\mathrm{H}_{\text {monote }}$ & & & & & & & $\mathrm{H}_{\text {wallvi: }}$ & & & \\
\hline & Kinderanzah! & Elentue & 0 & 0 & 0 & $(+)$ & $(+)$ & + & 0 & - & O & + & 0 & $(-)$ & 0 \\
\hline & & $\mathrm{BN}$ & & & $\mathbf{N}$ & & & & & & & B & & & \\
\hline & & $\begin{array}{l}\text { Hyyo- } \\
\text {.theose }\end{array}$ & & & & & & & & & & & & & \\
\hline & \begin{tabular}{|l|} 
Lebebnszy- \\
klusphase
\end{tabular} & Ennues & $(-)$ & - & - & + & O & - & - & O & + & $(+)]$ & $(+)$ & + & - \\
\hline & & $B N$ & & & & & & & & & 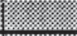 & & & & \\
\hline \multirow{15}{*}{ 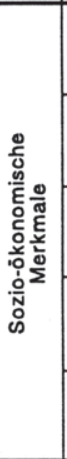 } & & $\begin{array}{l}\text { Hypo- } \\
\text { thesese }\end{array}$ & & & $\mathrm{H}_{\text {werterte }}$ & & & $H_{\text {Motivi: }}$ & & $\mathrm{H}_{\text {mottute }}$ & & & & & \\
\hline & Bildung & EInAuB & + & - & + & - & 0 & + & 0 & $\mid(+)$ & $(-)$ & - & $(-)$ & - & + \\
\hline & & $\mathrm{BN}$ & & & B & & & B & & $\mathrm{N}$ & & & & & \\
\hline & & $\begin{array}{l}\text { Hypo- } \\
\text { Hhass... }\end{array}$ & & & & & & & & & & & & & \\
\hline & Beruf & Elnnue & 0 & 0 & O & 0 & 0 & 웅 & ? & 0 & 0 & e & e & e & O \\
\hline & & $\mathrm{BN}$ & & & & & & & & & & & & & \\
\hline & & $\begin{array}{l}\text { Hypo: } \\
\text { Hyose:- }\end{array}$ & & & & & & & & & & & & & \\
\hline & $\begin{array}{l}\text { HH-Nettoein- } \\
\text { kommen }\end{array}$ & EInfue & + & $(-)$ & + & + & O & $(+)$ & O & $\mathrm{O}$ & O & $(+)$ & $(+)$ & $(+)$ & + \\
\hline & & $\mathrm{B} / \mathrm{N}$ & & & & & & & & & & & & & \\
\hline & Persönliches. & $\begin{array}{l}\text { Hypo- } \\
\text { Hyose... }\end{array}$ & & & & & & & & & & & & & \\
\hline & $\begin{array}{l}\text { Netto- } \\
\text { einkommen }\end{array}$ & Einfus & + & 0 & 0 & O & 0 & 0 & O & $(+)$ & $(+)$ & 0 & 0 & + & O \\
\hline & & $\mathrm{BN}$ & & & & & & & & & & & & & \\
\hline & & $\begin{array}{l}\text { Hypo- } \\
\text { theses: }\end{array}$ & & & $\mathrm{H}_{\text {mortote }}$ & & & & & $H_{\text {Motivide }}$ & & & & & \\
\hline & $\begin{array}{l}\text { Schichtzu- } \\
\text { gehörigkeit }\end{array}$ & Elnnus & + & - & + & 0 & 0 & + & O & $(+)$ & O & $(-)$ & 0 & 0 & + \\
\hline & & $\mathrm{B} / \mathrm{N}$ & . & & B & & & 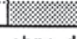 & . & $\mathrm{N}$ & 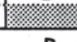 & 11 & & & \\
\hline
\end{tabular}

Abb. 47: Zusammenfassende Übersicht zur Prüfung der Untersuchungshypothesen zum EinfluB psychographischer Merkmale auf das Besuchsverhalten 
laubsmotive stehen in einem engen zusammenhang mit dem "Alter", der "Kinderanzahl", der "Lebenszyklusphase" und dem "Bildungsgrad". Demgegenüber können Einstellungen weitaus schlechter anhand soziodemographischer Merkmale erklärt werden.

(4) Der Einfluß der untersuchten psychographischen Kerkmale auf das regionenspezifische Besuchsverhalten ist zusammenfassend in Abbildung 48 wiedergegeben. Dabei wurde deutlich, daß sich alle Konstruktebenen im Rahmen univariater Analysen als geeignet erwiesen, einen Erklärungsbeitrag zum regionenspezifischen Besuchsverhalten zu liefern.

Die Analyse des Einflusses von Werten auf das regionenspezifische Besuchsverhalten läßt den Schluß zu, daß diese vor allem zur Erklärung der Besuchsdauer im Naherholungsverkehr (Entfernungsgruppe I) geeignet sind. Allerdings konnte die Besuchsabsicht nur eingeschränkt anhand der Wertefaktoren erklärt werden, so daß eine Segmentierung ausschlieBlich auf der Basis von Wertefaktoren nicht erfolgversprechend ist. orlaubsmotive sind besonders dazu geeignet, die Besuchsabsicht und -dauer im Naherholungsverkehr zu beschreiben. Den Einstellungen kommt vor allem zur Erklärung der Besuchsabsicht und -dauer in der Entfernungsgruppe II eine hohe Bedeutung zu.

Die Einzelergebnisse machen unmittelbar deutlich, daß die drei psychographischen Merkmale dazu geeignet sind, das regionenspezifische Besuchsverhalten zu erklären. Ferner verdeutlichen die Analyseergebnisse die ZweckmäBigkeit, die Ermittlung von Bestimmungsfaktoren des Besuchsverhaltens auf der Grundlage von entfernungsgruppenspezifischen Verhaltenssegmenten durchzuführen. Denn innerhalb der Entfernungsgruppen konnten jeweils unter- 


\begin{tabular}{|c|c|c|c|c|c|c|c|c|c|c|c|c|c|}
\hline & \multicolumn{6}{|c|}{ Besuchsabsicht } & \multicolumn{6}{|c|}{ Besuchsdauer } \\
\hline & & $\begin{array}{c}\text { Entfernu } \\
\text { Hypo- } \\
\text { these }\end{array}$ & $\begin{array}{l}\text { ungsgru } \\
\text { Einfluß }\end{array}$ & $\begin{array}{l}\text { ppe I } \\
\mathrm{B} / \mathrm{N}\end{array}$ & \multicolumn{3}{|c|}{ Entfernungsgruppe II } & \multicolumn{3}{|c|}{ Entfernungsgruppe I } & $\begin{array}{c}\text { Entfernur } \\
\begin{array}{c}\text { Hypo- } \\
\text { these }\end{array}\end{array}$ & $\begin{array}{l}\text { Ingsgrup } \\
\text { Einfluß|}\end{array}$ & $\begin{array}{l}\text { ppe II } \\
B / N\end{array}$ \\
\hline \multirow{5}{*}{ 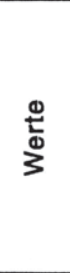 } & \begin{tabular}{|l|} 
Bildungs- und \\
Gesellschaftsorientierung
\end{tabular} & $\mathrm{H}_{\text {Werte28 }}$ & + & B & $\mathrm{H}_{\text {Worte2s }}$ & 0 & $\mathrm{~N}$ & $\mathrm{H}_{\text {Worte3a }}$ & + & B & & 0 & \\
\hline & Hedonismus & & 0 & & & \multirow{2}{*}{$\begin{array}{l}0 \\
0\end{array}$} & & & + & & & 0 & \\
\hline & Freiheitsorientierung & $H_{\text {worte2b }}$ & 0 & $\mathrm{~N}$ & $\mathrm{H}_{\text {Worte 2b }}$ & & $\mathrm{N}$ & & - & & & - & \\
\hline & $\begin{array}{l}\text { Sicherheit \& } \\
\text { Tradition }\end{array}$ & & 0 & & & \multirow[t]{2}{*}{0} & & $\mathrm{H}_{\text {Worte3b }}$ & - & B & $\mathrm{H}_{\text {Wortesc }}$ & 0 & $\mathrm{~N}$ \\
\hline & $\begin{array}{l}\text { Umwelt \& } \\
\text { Gesundheit }\end{array}$ & & 0 & & & & & & + & & & - & \\
\hline \multirow{5}{*}{ 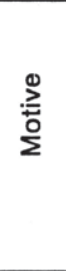 } & $\begin{array}{l}\text { Sportbezogenes } \\
\text { Aktivitätsbedürrnis }\end{array}$ & $\mathrm{H}_{\text {Motivec }}$ & + & B & $\mathrm{H}_{\text {Motiv2c }}$ & 0 & $\mathrm{~N}$ & $\mathrm{H}_{\text {Motive }}$ & 0 & N & & 0 & \\
\hline & $\begin{array}{l}\text { Regenerations- \& } \\
\text { GenuBbedúrtnis }\end{array}$ & $\mathrm{H}_{\text {Motlv2a }}$ & - & B & & 0 & & $\mathrm{H}_{\text {Motiv3s }}$ & 0 & $\mathrm{~N}$ & $\mathrm{H}_{\text {Motlv3b }}$ & 0 & $\mathrm{~N}$ \\
\hline & $\begin{array}{l}\text { Kulturelles } \\
\text { Bedürfnis }\end{array}$ & $\mathrm{H}_{\text {Motiv2b }}$ & + & B & $\mathrm{H}_{\text {Motlv2b }}$ & 0 & $\mathrm{~N}$ & $\mathrm{H}_{\text {Motivac }}$ & + & B & $\mathrm{H}_{\text {Motiv3d }}$ & 0 & N \\
\hline & $\begin{array}{l}\text { Sportaverses Bewe- } \\
\text { gungsbedürnis }\end{array}$ & & 0 & & & (2) & : & & : & & & 0 & \\
\hline & $\begin{array}{l}\text { Familienorientiertes } \\
\text { Erlebnisbedúrfnis }\end{array}$ & & 0 & & & 4. & & & 8 & & & + & \\
\hline \multirow{3}{*}{ 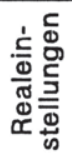 } & $\begin{array}{l}\text { Touristische sport- } \\
\text { unabhanngige Intrastruktur }\end{array}$ & $\mathrm{H}_{\text {Elnst2c }}$ & + & B & $\mathrm{H}_{\text {Elnat2c }}$ & + & B & $\mathrm{H}_{\text {Elnot3c }}$ & 0 & $\mathrm{~N}$ & $\mathrm{H}_{\text {Elnot3c }}$ & 0 & $\mathrm{~N}$ \\
\hline & $\begin{array}{l}\text { Natūrliches } \\
\text { Angebot }\end{array}$ & $\mathrm{H}_{\text {Elnst2a }}$ & 0 & $\mathrm{~N}$ & $\mathrm{H}_{\text {Elnst2b }}$ & + & B & $\mathrm{H}_{\text {Elnst3e }}$ & + & B & $\mathrm{H}_{\text {Elnot3e }}$ & + & B \\
\hline & Sportangebot & $\mathrm{H}_{\text {Elnstab }}$ & 0 & $\mathrm{~N}$ & $\mathrm{H}_{\text {Elnst2b }}$ & + & B & $\mathrm{H}_{\text {Elnat } 3 \mathrm{~b}}$ & - & $N$ & $\mathrm{H}_{\text {Elnatsb }}$ & 0 & $\mathrm{~N}$ \\
\hline \multicolumn{14}{|c|}{$\begin{array}{l}+=\text { positiver Zusammenhang } \\
0=\text { kein Zusammenhang } \\
-\quad=\text { negativer Zusammenhang }\end{array}$} \\
\hline
\end{tabular}

Abb 48: Zusammenfassende Übersicht zur Prüfung der Untersuchungshypothesen zum Einfluß psychographischer Merkmale auf das Besuchsverhalten 
schiedliche Bestimmungsfaktoren des Besuchsverhaltens identifiziert werden.

(5) Unter Zugrundelegung der identifizierten Bestimmungsfaktoren der entfernungsgruppenspezifischen Besuchsabsicht und -dauer wurde ein multivariates Analyseverfahren eingesetzt, um den gesamten Erklärungsbeitrag paychographischer Merkmale auf das Besuchsverhalten zu ermitteln. Hierdurch konnten die zentralen Bestimmungsfaktoren des regionenspezifischen Besuchsverhaltens identifiziert werden. In der Entfernungsgruppe I erwies sich die zielgebietserfahrung als dominanter Bestimmungsfaktor der Besuchsabsicht. Die Besuchsdauer ist hingegen gleichermaßen von Werten, Urlaubsmotiven und Realeinstellungen abhängig. In der Entfernungsgruppe II konnten die Realeinstellungen als zentrale Bestimmungsfaktoren der Besuchsabsicht ermittelt werden. Demgegenüber kommt der zielgebietserfahrung, den Werten, den Motiven und den Einstellungen zur Erklärung der Besuchsdauer eine relativ gleich hohe Bedeutung $\mathrm{zu}$.

Aufgrund dieser Ergebnisse ist die in der Fremdenverkehrsforschung vorherrschende einseitige Ausrichtung auf Urlaubsmotive zur Erklärung des Besuchsverhaltens als nicht ausreichend $z u$ würdigen. Erst die Kombination von Werten, Motiven, Einstellungen und Zielgebietserfahrung ermöglicht eine hinreichend präzise Erklärung des Besuchsverhaltens bzw. der Besuchsabsicht und -dauer.

Einschränkend ist jedoch festzuhalten, daB die Erklärungsbeiträge in der integrierten Analyse darauf hinweisen, daß neben den hier untersuchten Konstrukten das Besuchsverhalten von weiteren Einflubfaktoren wie z.B. zielgebietspräferenzen abhängt. 
(6) Die auf der Basis der zentralen Bestimmungsfaktoren durchgeführte psychographische Marktsegmentierung führte in der Entfernungsgruppe $I$ zu vier und in der Entfernungsgruppe II $z u$ drei deutlich voneinander $a b-$ grenzbaren Zielsegmenten. Diese zielsegmente lassen sich gut anhand soziodemographischer Merkmale beschreiben. Damit ist im vorliegenden Untersuchungskontext die psychographische Marktsegmentierung im Vergleich zur verhaltensorientierten Marktsegmentierung besser dazu geeignet, die soziodemographische Identifizierbarkeit zu gewährleisten. Angesichts der signifikanten Unterschiede im Besuchsverhalten erfüllt die psychographische Marktsegmentierung ferner in hohem Maße die Anforderung der Besuchsverhaltensrelevanz, wobei sich die identifizierten segmente jedoch nicht in ihrer Besuchsdauer unterscheiden.

Die sich anschließende Homogenitätsanalyse der zielgruppen der beiden Entfernungsgruppen zeigt, daB sich jeweils zwei der zielgruppen in beiden Entfernungsgruppen in ihren Motivstrukturen stark ähneln. Daher wurden diese für eine zielgruppenspezifische Marktbearbeitung zusammengefaßt. Vor diesem Hintergund konnten für die Untersuchungsregion folgende 5 zielgruppen identifiziert werden:

- "Traditionelle Spaziergängerfreunde" im Naherholungsverkehr

- "Land- und kulturorientierte Urlauber" im Naherholungsverkehr

- "Sportaktive Familienurlauber" auf dem Gesamtmarkt

- "Regenerations- und genußorientierte Urlauber" in der Entfernungsgruppe II

- "Bade-Urlauber" auf dem Gesamtmarkt 
(7) Unter Berücksichtigung der identifizierten Zielgruppen wurden Ansatzpunkte einer zielgruppenspezifischen Marktbearbeitung herausgestellt. Hierbei zeigte sich, daß vor allem Motive bei der Ableitung zielgruppenspezifischer Marketingmaßnahmen Ansatzpunkte für die Ausgestaltung des Marketingprogramms liefern. Die Einstellungen geben - unter der Annahme, daB es sich bei negativen Einstellungen vor allem um Wahrnehmungsdefizite handelt - vor allem Hinweise auf eine verstärkte Informationspolitk und auf spezifische Werbeinhalte zum Abbau von Wahrnehmungsverzerrungen. Werte geben bei ihrer Interpretation für den zielgruppenspezifischen Maßnahmeneinsatz vor allem Hilfestellungen bei der Medienauswahl.

Insgesamt wurde hinsichtlich der zielgruppenspezifischen Marktbearbeitung deutlich, daB der regionalen Fremdenverkehrsorganisation in der Umsetzungsphase vor allem die Funktion eines Koordinators zukommt. Hierfür konnten Maßnahmen erarbeitet werden, die in der derzeitigen Praxis von regionalen Fremdenverkehrsorganisationen kaum Anwendung finden, aber für eine zukünftige Marktbearbeitung erfolgversprechend sein dürten. Als Grundvoraussetzung für ein erfolgreiches zielgruppenmarketing im Fremdenverkehr von Regionen erweist sich dabei generell der Aufbau eines regionalen selbstverständisses.

(8) Als wesentliche Erfolgsvoraussetzung für ein erfolgreiches zielgruppenmarketing im Fremdenverkehr von Regionen wurden Ansatzpunkte für die einheitliche Durchsetzung der entwickelten Konzepte durch die eigentlichen touristischen Leistungsträger aufgezeigt. In diesem $\mathrm{Zu}-$ sammenhang wurden vor allem Durchsetzungsmaßnahmen herausgearbeitet, mit deren Hilfe mögliche Durchsetzungs- 
widerstände in der Fremdenverkehrsregion abgebaut werden können.

In einer zusammenfassenden würdigung ist festzuhalten, daB mit der empirischen Analyse der Bestimmungfaktoren des regionenspezifischen Besuchsverhaltens und der darauf aufbauenden Ansatzpunkte einer zielgruppenspezifischen Marktbearbeitung der vorliegenden Arbeit der Charakter einer Basisuntersuchung zukommt. Diese zeigt den Gesamtrahmen des Zielgruppenmarketing im Fremdenverkehr von Regionen am Beispiel einer Untersuchungsregion exemplarisch auf. Während die empirischen Ergebnisse zum Zusammenhang zwischen soziodemographischen und psychographischen Merkmalen aufgrund des Untersuchungsdesigns durchaus allgemeingültigen Anspruch erheben können, gelten die Detailergebnisse zum regionenspezifischen Besuchsverhalten nur für die Untersuchungsregion. Darüber hinaus wurden Aussagen zur regionenspezifischen Marktbearbeitung abgeleitet, die unmittelbar auf andere Fremdenverkehrsregionen übertragen werden können.

Im Rahmen einer möglichen Replizierung der Untersuchung sind folgende Aspekte in besonderem Maße zu berücksichtigen.

Die vorgenommene operationalisierung des Besuchsverhaltens über die Besuchsabsicht und -dauer weist einen direkten Bezug zu den von Fremdenverkehrsregionen verfolgten zielen einer Erhöhung der Übernachtungs- und Gästezahlen auf. Demgegenüber wird mit dieser operationalisierung den Ertragszielen einer Fremdenverkehrsregion nur unzureichend Rechnung getragen. Es erscheint daher für zukünftige Untersuchungen sinnvoll, im Rahmen der Erfassung des Besuchsverhaltens zusätzlich die Ausgabebereitschaft und -fähigkeit der Touristen $z u$ berücksichtigen.

Ferner wurde im Rahmen dieser Arbeit das Besuchsverhalten nur nominalskaliert erfaßt. Daher konnte der Einfluß der 
psychographischen Konstrukte nur mit Hilfe von T-Tests analysiert werden. Auf der Grundlage von signifikanten Unterschieden $z$ wischen den Verhaltenssegmenten wurden Rückschlüsse auf Beziehungen zwischen den Ausprägungsformen der Konstrukte und dem Besuchsverhalten geschlossen. Eine Verbesserung der Aussagefähigkeit der Analyseergebnisse erscheint demnach uber eine metrische Erfassung der Verhaltenskomponenten denkbar. Zum einen wäre hierduch eine Quantifizierung des Einflusses psychographischer Merkmale auf das Besuchsverhalten in Form von Erklärungsbeiträgen möglich. Zum anderen wird hierdurch die Anwendbarkeit weitergehender multivariater Analysemethoden gewährleistet.

Darüber hinaus deuten die im Rahmen der integrierten Analyse ermittelten Ergebnisse darauf hin, daB zur Erklärung des Besuchsverhaltens neben Werten, Motiven, Einstellungen und der Zielgebietserfahrung weitere psychographische Konstrukte heranzuziehen sind. Neben den schon erwähnten Präferenzen für andere Fremdenverkehrsregionen könnten darüber hinaus insbesondere das mit einer Reise in Verbindung stehende wahrgenommene Risiko sowie das spezifische Involvement, das Urlauben und Reisen entgegengebracht wird zusätzlich in die empirische Analyse einbezogen werden. Ferner erscheint es zweckmäBig, neben der Einstellung zur Untersuchungsregion generelle Einstellungen $z u$ Deutschlandreisen, differenziert nach Tagesausflügen, Kurzurlaubs- und Urlaubsreisen als erklärende Größe heranzuziehen.

Zudem zeigen die Analyseergebnisse, daB sich die im Rahmen der psychographischen Marktsegmentierung identifizierten Segmente nicht in ihrer beabsichtigten Besuchsdauer unterscheiden. Dies ist darauf zurückzuführen, daß die Bestimmungsfaktoren der Besuchsdauer und -absicht simultan zur zielgruppenbildung herangezogen wurden. Daher ist es für Forschungsstudien überlegenswert, auf der Basis gröBerer stichproben eine zweistufige psychographische segmentierung 
durchzuführen. Hierbei wäre auf der ersten stufe auf Basis der Bestimmungsfaktoren der Besuchsabsicht eine Segmentierung vorzunehmen, um anschließend die ermittelten segmente auf Basis der Bestimmungsfaktoren der Besuchsdauer weiter zu differenzieren. Um dabei der Gefahr einer zu tiefen zielgruppenbildung vorzubeugen, die eine wirtschaftliche Bearbeitung der segmente nicht mehr gewährleistet, bietet es sich an, in Anlehnung an die hier vorgestellte Homogenitätsanalyse die identifizierten Zielgruppen im Hinblick auf die Marktbearbeitung auf mögliche Überschneidungen $z u$ überprüfen.

Mit der Ermittlung der wirtschaftlich optimalen segmentanzahl ist auch die Frage aufgeworfen, ob die ermittelten Marktsegmente angesichts zunehmend heterogener Kundenwünsche, spontaneren Reiseentscheidungen etc. ausreichende zeitliche stabilität aufweisen. Die zunehmende Individualisierung von Konsumwünschen und hybrider werdenden Konsumenten zeigen hier generelle Grenzen einer zielgruppenspezifischen Marktbearbeitung auf. ${ }^{1}$ Dem kann allerdings abschwächend gegenübergehalten werden, daß auf der Individualebene die Prognostizierbarkeit des Kauf- bzw. Urlaubs-oder Reiseverhaltens immer schwieriger wird, während auf der Ebene von Segmenten aufgrund der generellen Urlaubs- und Reiseneigungen ausreichende Ansatzpunkte für eine zielgruppenspezifische Marktbearbeitung abgeleitet werden können.

Die aufgezeigten Einzelergebnisse sowie die aufgezeigten Verbesserungsvorschläge im Rahmen der kritischen würdigung leiten unmittelbar auf zukünftige Forschungsgebiete über.

1 Vgl. Meffert, H., Erfolgreiches Marketing in den neunziger Jahren, a.a.O., S. 19; Wiswede, G., Der "Neue Konsument" im Lichte des Wertewandels, in: Szallies, R., Wiswede, G. (Hrsg.), Wertewandel und Konsum, Landberg a.L., 1990, S. $11 \mathrm{ff}$. 


\section{Implikationen für weiterführende Forschungen}

Angesichts der vorliegenden Untersuchungsergebnisse und den aufgezeigten Ansatzpunkten einer zielgruppenspezifischen Marktbearbeitung sind für weiterführende empirische studien und konzeptionelle Forschungsüberlegungen zum zielgruppenmarketing im Fremdenverkehr von Regionen folgende Ansatzpunkte aufzuzeigen:

(1) Unter Berücksichtigung der Tatsache, daB die in der vorliegenden Arbeit ermittelten Beziehungszusammenhänge zum Besuchsverhalten nur für eine Untersuchungsregion gelten, erscheint es in erster Linie interessant, diese Analyse für andere Fremdenverkehrsregionen zu replizieren. Darüber hinaus bietet es sich an, eine entsprechende Untersuchung nicht nur auf den Deutschlandtourismus zu beschränken, sondern auch das europäische Ausland oder zumindest Nachbarländer in die Analyse einzubeziehen. Dabei sollten die aufgezeigten Verbesserungsvorschläge im Rahmen der empirischen Analyse berücksichtigt werden.

(2) Zunehmende Individualisierung und "hybrider" werdende Konsumstrukturen deuten auf die Notwendigkeit hin, die vorliegende studie für die gleiche Untersuchungsregion zu replizieren. Hierbei ist im sinne einer Längsschnittanalyse zu untersuchen, welche zeitliche stabilität den Zielgruppen im Fremdenverkehr zugesprochen werden kann. Neben der fremdenverkehrsspezifischen Relevanz dieses Untersuchungsaspekts kann damit ein genereller Beitrag $z u$ der in Theorie und Praxis viel diskutierten Problemstellung der zeitlichen stabilität von zielgruppen geleistet werden.

(3) Angesichts der in dieser Untersuchung vorgenommenen Eingrenzung auf den Erholungstourismus erscheint es sinn- 
voll, Ansatzpunkte zur Markterfassung und zielgruppenspezifischen Marktbearbeitung auch im Geschäfts- und Kongreßtourismus $z u$ analysieren. In diesem Zusammenhang könnte auf die Erkenntnisse der Marktsegmentierung im Investitionsgütermarketing zurückgegriffen und auf den Geschäftstourimus übertragen werden.

(4) Weiterführende Forschungsarbeiten sind ferner auf dem Gebiet der Erfolgswirksamkeit zielgruppenspezifischer Marktbearbeitungsmaßnahmen im Fremdenverkehr von Regionen zu initiieren. Hier ist es zum einen notwendig, den differenzierten Erfolgsbeitag einzelner Marketingmaßnahmen, z.B. der klassischen werbung im Fremdenverkehrskontext zu analysieren. Zum anderen ist es von Bedeutung, entsprechende Erfolgsgrößen auf der Basis objektiver Daten (Umsatzentwicklung, Gästezahlen, etc.) zu erheben und zu untersuchen.

(5) Angesichts der aufgezeigten Probleme bei der Durchsetzung von zielgruppenspezifischen Marktbearbeitungskonzepten im Fremdenverkehr von Regionen bietet sich ein breites Forschungsspektrum in der Analyse und strukturierung möglicher widerstände seitens unterschiedlichster Interessengruppen in Fremdenverkehrsregionen. Unter Berücksichtigung dieser widerstände erscheint es zweckmäßig, Ansatzpunkte für ein Konfliktmanagment im Fremdenverkehr von Regionen zu erarbeiten, im Rahmen dessen alle Anspruchsgruppen einer Fremdenverkehrsregion und ihre Beziehungsstrukturen Berücksichtigung finden müssen.

(6) Ferner stellt sich die Frage, welche organisationsformen von regionalen Fremdenverkehrsorganisationen besonders dazu geeignet sind, in einer Fremdenverkehrsregion eine einheitliche Ausrichtung der Marketingmaßnahmen der touristischen Leistungsanbieter und das oben dargestellte 
Konfliktmanagement $z u$ gewährleisten. Dabei ist insbesondere von Interesse, welche Kompetenzen dieser regionalen Fremdenverkehrsorganisation zweckmäBigerweise zuzuordnen sind.

Insgesamt wird deutlich, daß von einer konsequenten Umsetzung des Zielgruppenmarketing im Fremdenverkehr von Regionen erhebliche Effektivitätssteigerungen in der Marktbearbeitung $\mathrm{zu}$ erwarten sind. Die Marketing-Wissenschaft ist ebenso wie die Fremdenverkehrsforschung aufgerufen, ihrer entscheidungsunterstützenden Funktion gegenüber Fremdenverkehrsregionen durch verstärkte Berücksichtigung dieses noch in weiten Teilen unzureichend erforschten Untersuchungsbereichs gerecht zu werden. 
A $\mathbf{N}$ H A N G I

TABELLEN DER EMPIRISCHEN UNTERSUCHUNG 


\section{Verzeichnis des Anhangs I}

Abb. 1: Faktorenanalytische Verdichtung der werteindikatoren

Abb. 2: Faktorenanalytische Verdichtung der Motivindikatoren

Abb. 3: Faktorenanalytische Verdichtung der Real-Einstellungsindikatoren

Abb. 4: Zusammenhänge zwischen Werten, Motiven, Einstellungen und Zielgebietserfahrung

Abb. 5: Standardisierte Diskriminanzfunktion für die nach der Besuchsabsicht abgegrenzten Verhaltenssegmente in der Entfernungsgruppe I

Abb. 6: Standardisierte Diskriminanzfunktion für die nach der Besuchsdauer abgegrenzten Verhaltenssegmente in der Entfernungsgruppe I

Abb. 7: Standardisierte Diskriminanzfunktion für die nach der Besuchsabsicht abgegrenzten Verhaltenssegmente in der Entfernungsgruppe II

Abb. 8: Standardisierte Diskriminanzfunktion für die nach der Besuchsdauer abgegrenzten Verhaltenssegmente in der Entfernungsgruppe II

Abb. 9: Psychographische Zielgruppenbildung und -beschreibung in der Entfernungsgruppe I

Abb. 10: Psychographische Zielgruppenbildung und -beschreibung in der Entfernungsgruppe II

Abb. 11: Zielgruppenbeschreibung anhand der Werte in der Entfernungsgruppe I

Abb. 12: Zielgruppenbeschreibung anhand der Urlaubsmotive in der Entfernungsgruppe I 
Abb. 13: Zielgruppenbeschreibung anhand der Realeinstellungen in der Entfernungsgruppe I

Abb. 14: Zielgruppenbeschreibung anhand der Werte in der Entfernungsgruppe II

Abb. 15: Zielgruppenbeschreibung anhand der Urlaubsmotive in der Entfernungsgruppe II

Abb. 16: Zielgruppenbeschreibung anhand der Realeinstellungen in der Entfernungsgruppe II 
ROTATED FACTOR MATRIX:

\begin{tabular}{|c|c|c|c|c|c|}
\hline & FACTOR 1 & FACTOR 2 & FACTOR 3 & FACTOR & FACTOR \\
\hline WERTEIA & .75269 & -.00390 & .30682 & -.01252 & -.01882 \\
\hline MERTELIS & .74978 & -.07565 & 14484 & .03376 & .23038 \\
\hline WERTE I3 & .72506 & .09272 & -.11530 & .03939 & .17101 \\
\hline MERTEL & .52684 & .30204 & .02571 & .14934 & .03463 \\
\hline MERIEA & -.02953 & .79973 & .10356 & .14302 & -.01064 \\
\hline WERTEII & .26566 & .77518 & -.00225 & -.08991 & -.01811 \\
\hline MERTET & -.03472 & .71573 & .34690 & .09650 & .06958 \\
\hline WERTEG & .06391 & .23516 & .75888 & .06889 & .03547 \\
\hline WERTE3 & .26565 & .09040 & .73120 & -.01611 & .15429 \\
\hline WERTEI2 & .28824 & .17918 & -.32835 & .71827 & .02283 \\
\hline MERTEB & .02505 & -.11259 & .07625 & .70988 & .31092 \\
\hline WERTES & -.05570 & .23479 & .39697 & .64922 & -.05041 \\
\hline WERTEQ & .02646 & -.06307 & .05505 & .33212 & .76419 \\
\hline WERTE? & .25355 & -.06394 & .33763 & .12626 & .65343 \\
\hline WERTEIO & .28738 & .34196 & -.13435 & -.26975 & .64962 \\
\hline
\end{tabular}

Abb. 1: Faktorenanalytische Verdichtung der Werteindikatoren 
ROTATEO FACTOR MATRIX:

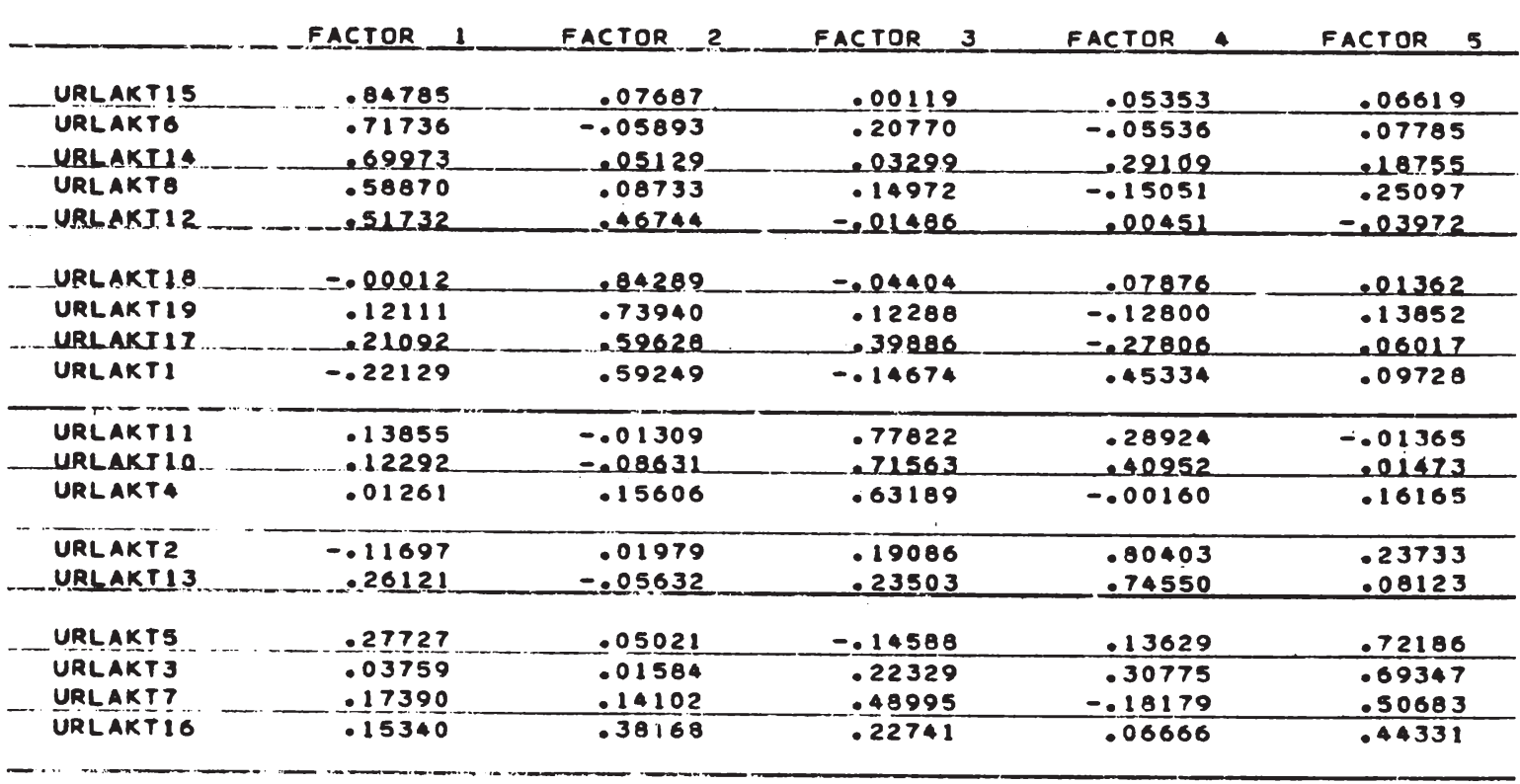

Abb. 2: Faktorenanalytische Verdichtung der Motivindikatoren 
ROTATEO FACTOR MATRIX:

\begin{tabular}{|c|c|c|c|}
\hline & FACTOR & FACTOR & FACTOR \\
\hline ZUTMSLS & .80671 & .22832 & .09441 \\
\hline ZUTMSLG & .75270 & .02043 & .17224 \\
\hline ZUTMSL 1 & .70430 & .30663 & .16633 \\
\hline zUTMSL & .05410 & .34784 & .20620 \\
\hline $\begin{array}{l}\text { ZUTMSL9 } \\
\text { ZUTMSL? }\end{array}$ & $\begin{array}{r}.59230 \\
.46168 \\
\end{array}$ & $\begin{array}{r}25812 \\
.31477 \\
\end{array}$ & $\begin{array}{r}38254 \\
-34382 \\
\end{array}$ \\
\hline ZUTMSLI1 & .11867 & .79809 & .25978 \\
\hline ZUTMSL 10 & .14779 & .75260 & -.00979 \\
\hline ZUTMSL 12 & .31834 & .69997 & .14156 \\
\hline ZUTMSL 13 & .46504 & .61930 & .14616 \\
\hline $\begin{array}{l}\text { ZUTMSLB } \\
\text { ZUTMSL }\end{array}$ & $\begin{array}{r}.11326 \\
.17400\end{array}$ & $\begin{array}{r}-.00507 \\
.29124\end{array}$ & $\begin{array}{r}.89177 \\
.74049\end{array}$ \\
\hline ZUTMSL 7 & .30580 & .10370 & .62298 \\
\hline
\end{tabular}

Abb. 3: Faktorenanalytische Verdichtung der Real-Einstellungsindikatoren 


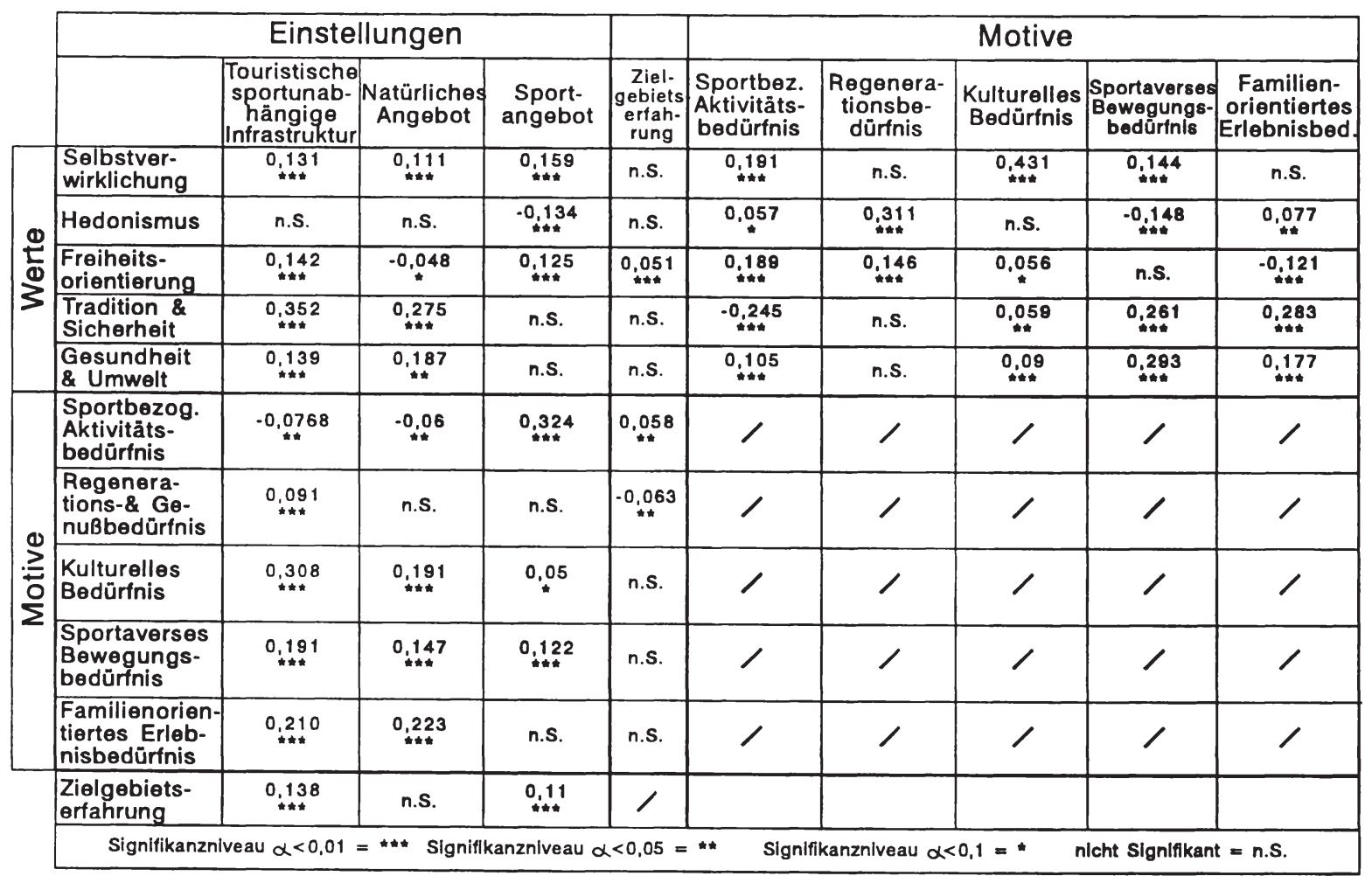

Abb. 4: Zusammenhänge zwischen Werten, Motiven, Einstellungen und Zielgebietserfahrung 
CANONICAL DISCRIMINANT FUNCTIONS

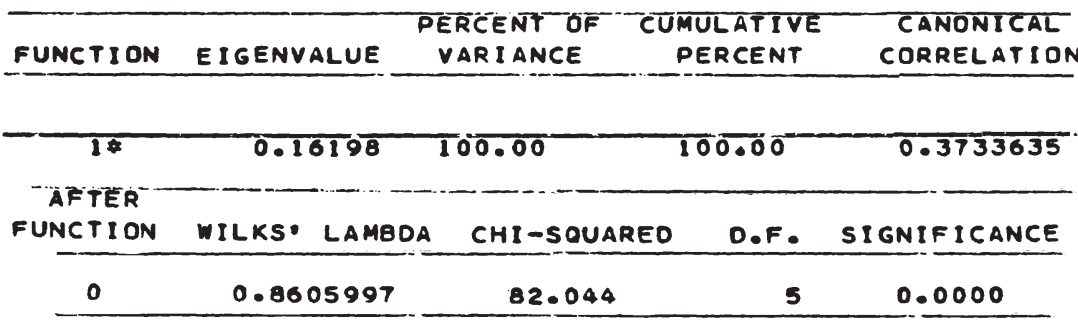

STAFOAROTZEO CANONT CAL OTSCRTIINATN FUNCTION COEFFICIENTS

\begin{tabular}{ll}
\hline & FUNC \\
MOTIVFI & -0.17573 \\
MOTIVF2 & 0.14454 \\
TOTIVF3 & -0.228085 \\
REALF1 & -0.25241 \\
BESHAUF & 0.85574
\end{tabular}

Abb. 5: Standardisierte Diskriminanzfunktion fur die nach der Besuchsabsicht abgegrenzten Verhaltenssegmente in der Entfernungsgruppe I

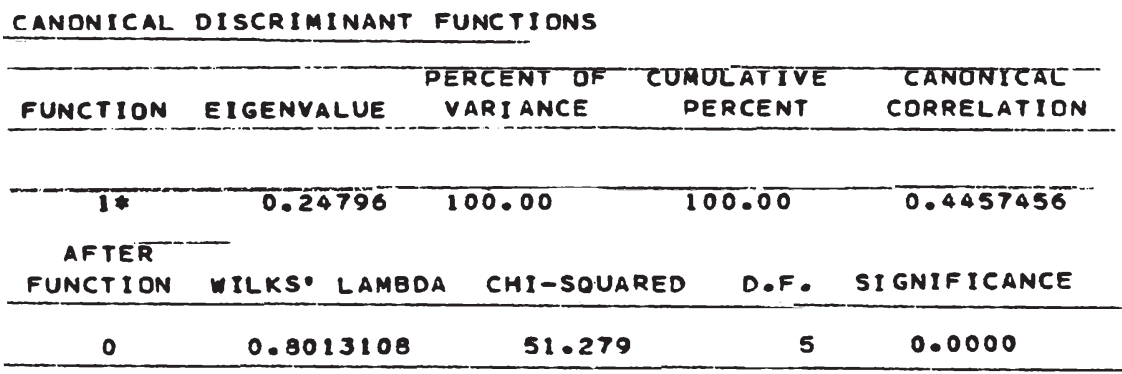

STANOXRDTZEO CANONTCAL OTSCRIATNATIT FUNCTION COEFFICTENTS

$\begin{array}{ll} & \text { FUNC } \\ \text { MERTEFS } & -0.61260 \\ \text { MOTIVF3 } & 0.46642 \\ \text { MOTIVF4 } & 0.027115 \\ \text { REALF2 } & 0.65352 \\ \text { WERTEF3 } & -0.19276\end{array}$

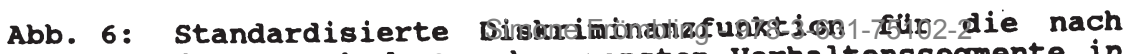

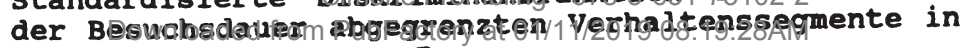
der Entfernungsgruppe I 


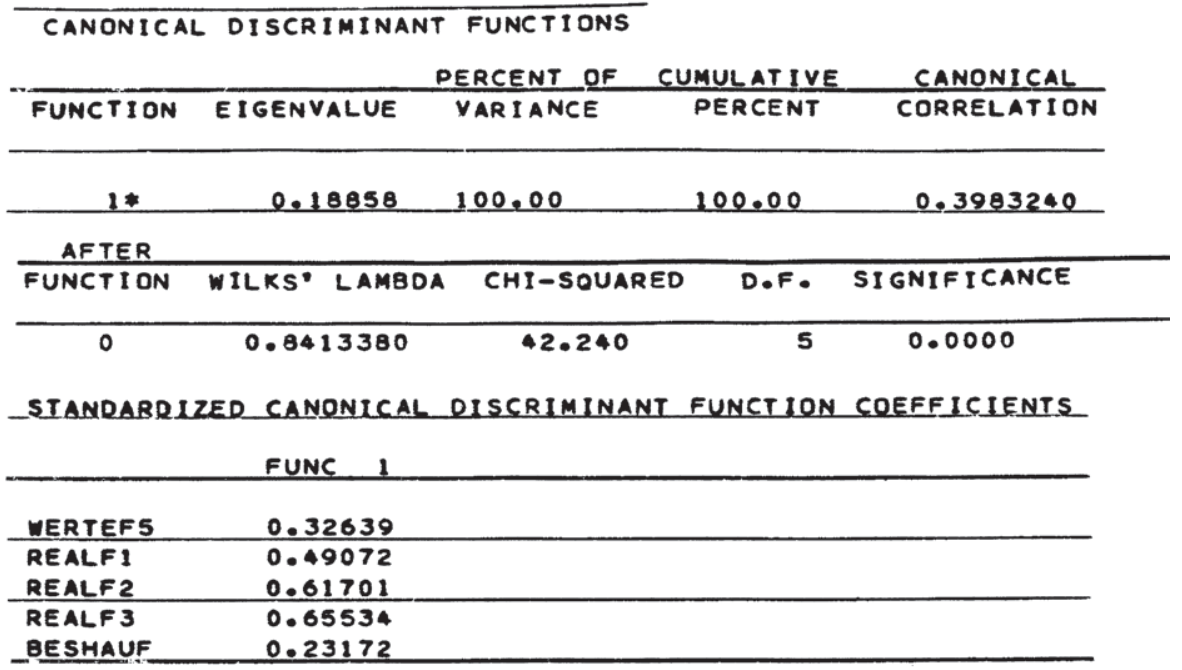

Abb. 7: Standardisierte Diskriminanzfunktion für die nach der Besuchsabsicht abgegrenzten Verhaltenssegmente in der Entfernungsgruppe II

CANONICAL DISCRIMINANT FUNCTIONS

\begin{tabular}{|c|c|c|c|c|}
\hline & & PERCENT OF & CUMULATIVE & CANONICAL \\
\hline FUNCTION & EIGENVALUE & VAR I ANCE & PERCENT & CORRELATION \\
\hline $1 \%$ & 0.50112 & 100.00 & 100.00 & 0.5777805 \\
\hline
\end{tabular}

AFTER

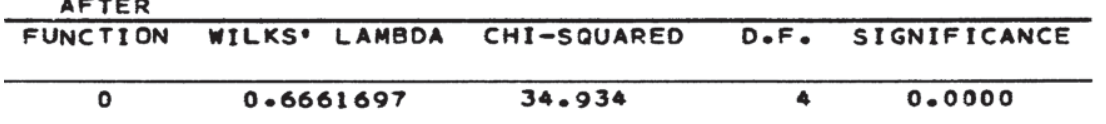

STANDAROIZED CANONICAL DISCRIMINANT FUNCTION COEFFICIENTS FUNC 1

\begin{tabular}{lr}
\hline & \\
MERTEFS & 0.54314 \\
\hline MOTIVF5 & -0.74450 \\
BEALE2 & -0.44410 \\
BESHAUF & 0.66144
\end{tabular}

Abb. 8: Standardisierte Diskriminanzfunktion -631-75102-2 die nach der Besuchsabuer abgegrenzen verhaltenssegmente in der Entfernungsgruppe II 


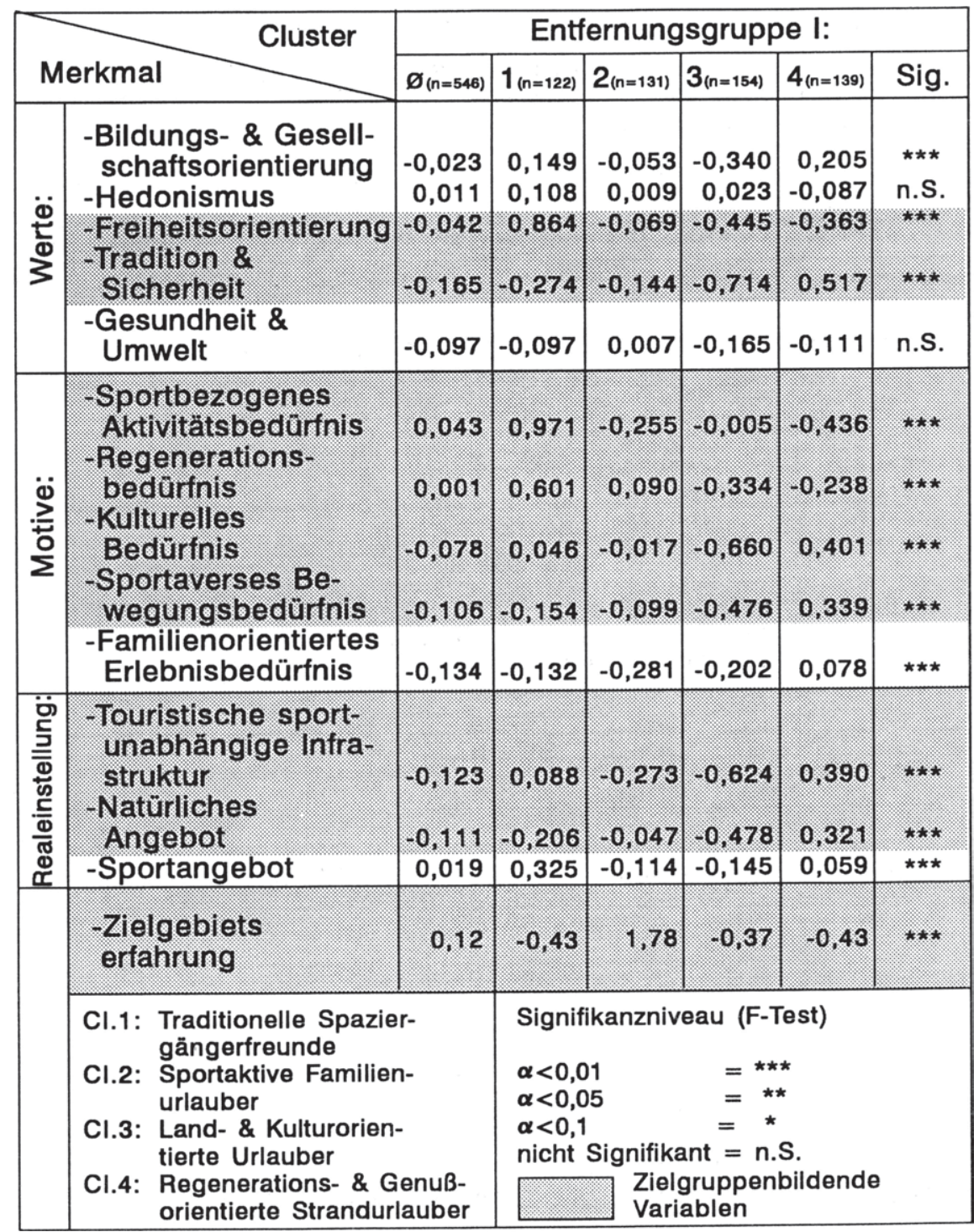

Abb. 9: Psychographische zielgruppenbildung und -beschreibung in der Entfernungsgruppe I 


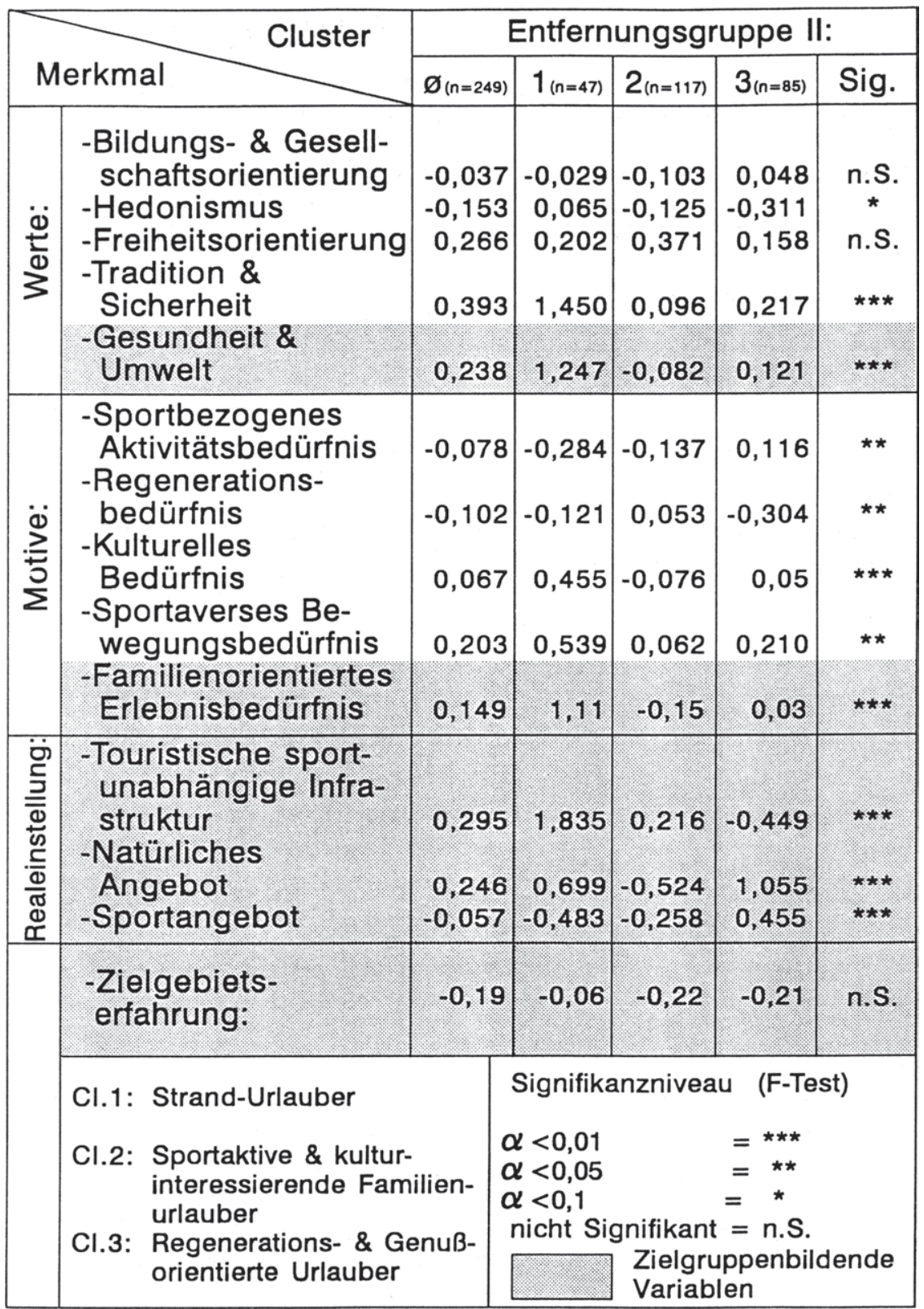

Abb. 10: Psychographische Zielgruppenbildung und -beschreibung in der EntfernungegrüppealinI- 978-3-631-75102-2 


\begin{tabular}{|c|c|c|c|c|c|c|c|}
\hline & \multirow{2}{*}{ Werte } & \multicolumn{6}{|c|}{ Entfernungsgruppe I: } \\
\hline & & $\varnothing$ & Cl. 1 & Cl. 2 & Cl. 3 & Cl. 4 & Sig. \\
\hline 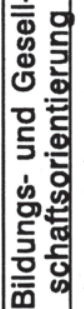 & $\begin{array}{l}\text { Hoher Bildungsstand } \\
\text { Kulturelles Interesse } \\
\text { Aufgaben für die Gesell- } \\
\text { schaft übernehmen } \\
\text { Gesellschaftliche } \\
\text { Anerkennung }\end{array}$ & $\begin{array}{l}2,37 \\
2,35 \\
2,72 \\
2,21\end{array}$ & $\begin{array}{l}2,83 \\
2,55 \\
2,73 \\
2,32\end{array}$ & $\begin{array}{l}2,22 \\
2,39 \\
2,80 \\
2,21\end{array}$ & $\begin{array}{l}2,17 \\
1,86 \\
2,49 \\
2,04\end{array}$ & $\begin{array}{l}2,33 \\
2,66 \\
2,89 \\
2,30\end{array}$ & $\begin{array}{l}\star \star \star \\
\star \star \star \\
\star \star \\
\star\end{array}$ \\
\hline 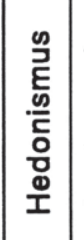 & $\begin{array}{l}\text { Ein bequemes Leben } \\
\text { führen } \\
\text { Ein genußreiches Leben } \\
\text { führen } \\
\text { Viel Freizeit }\end{array}$ & $\begin{array}{l}2,46 \\
2,77 \\
1,99\end{array}$ & $\begin{array}{l}2,54 \\
3,03 \\
2,30\end{array}$ & $\begin{array}{l}2,54 \\
2,66 \\
1,97\end{array}$ & $\begin{array}{r}2,36 \\
2,69 \\
1,86\end{array}$ & $\begin{array}{l}2,42 \\
2,73 \\
1,89\end{array}$ & $\begin{array}{l}\text { n.S. } \\
\star \star \\
\star \star \star\end{array}$ \\
\hline 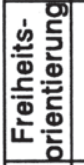 & $\begin{array}{l}\text { Persönliche Freiheit } \\
\text { Sich selbst entfalten } \\
\text { können }\end{array}$ & $\begin{array}{l}1,53 \\
1,73\end{array}$ & $\begin{array}{l}1,98 \\
2,32\end{array}$ & 1,52 & $\begin{array}{l}1,21 \\
1,42\end{array}$ & $\begin{array}{l}1,51 \\
1,55\end{array}$ & 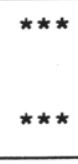 \\
\hline 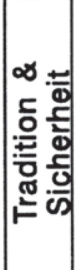 & $\begin{array}{l}\text { Sich an "Bewährtem" } \\
\text { orientieren } \\
\text { Ein ausgeprägtes Familien- } \\
\text { leben führen } \\
\text { Persönliche und finanzielle } \\
\text { Sicherheit }\end{array}$ & $\begin{array}{l}2,29 \\
1,83 \\
1,59\end{array}$ & $\begin{array}{l}1,93 \\
1,87 \\
1,89\end{array}$ & $\begin{array}{l}2,35 \\
1,76 \\
1,63\end{array}$ & $\begin{array}{l}1,82 \\
1,40 \\
1,22\end{array}$ & $\begin{array}{l}3,09 \\
2,35 \\
1,71\end{array}$ & $\star \star \star \star$ \\
\hline 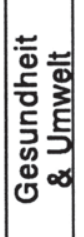 & $\begin{array}{l}\text { Gesundheitsbewußtes } \\
\text { Leben } \\
\text { Umwelt- und energie- } \\
\text { bewußtes Leben } \\
\text { "Alternativ" leben }\end{array}$ & $\begin{array}{l}1,69 \\
1,75 \\
2,92\end{array}$ & $\begin{array}{l}1,70 \\
2,00 \\
2,93\end{array}$ & $\begin{array}{l}1,73 \\
1,86 \\
2,96\end{array}$ & $\begin{array}{l}1,50 \\
1,46 \\
3,03\end{array}$ & $\begin{array}{l}1,85 \\
1,76 \\
2,75\end{array}$ & $\begin{array}{l}\star \star \star \\
\star \star \star \\
\text { n.S. }\end{array}$ \\
\hline & $\begin{array}{l}\text { Cl.1: Traditionelle Spaziergän } \\
\text { Cl.2: Sportaktive Familienurla } \\
\text { Cl.3: Land- \& Kulturorientierte } \\
\text { Cl.4: Regen.- \& GenuBorientie }\end{array}$ & $\begin{array}{l}\text { erfreu } \\
\text { per } \\
\text { Jrlaub } \\
\text { e Stre }\end{array}$ & aub & & $\begin{array}{l}\text { gnifika } \\
<0,01 \\
<0,05 \\
<0,1 \\
\text { cht Sig }\end{array}$ & $\begin{array}{r}\text { niveau } \\
= \\
= \\
= \\
\text { ikant }=\end{array}$ & . \\
\hline
\end{tabular}

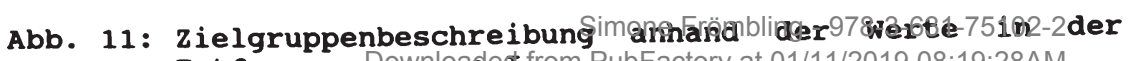
Entfernungsopupped from PubFactory at 01/11/2019 08:19:28AM 


\begin{tabular}{|c|c|c|c|c|c|c|c|}
\hline & \multirow{2}{*}{ Urlaubsmotive } & \multicolumn{6}{|c|}{ Entfernungsgruppe I: } \\
\hline & & ø & Cl. 1 & Cl. 2 & Cl. 3 & Cl. 4 & Sig. \\
\hline 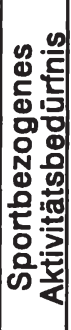 & $\begin{array}{l}\text { Sport treiben } \\
\text { Aktiv sein } \\
\text { Radfahren } \\
\text { Hobbies betreiben } \\
\text { Schwimmen, baden, am } \\
\text { Strand liegen }\end{array}$ & $\begin{array}{l}3,18 \\
2,55 \\
2,84 \\
2,94 \\
2,30\end{array}$ & $\begin{array}{l}4,11 \\
3,52 \\
3,69 \\
3,64 \\
3,31\end{array}$ & $\begin{array}{l}2,92 \\
2,26 \\
2,48 \\
2,87 \\
2,15\end{array}$ & $\begin{array}{l}3,10 \\
2,28 \\
2,62 \\
2,87 \\
2,11\end{array}$ & $\begin{array}{l}2,68 \\
2,27 \\
2,70 \\
2,54 \\
1,76\end{array}$ & $\begin{array}{l}\star \star \star \\
\star \star \star \\
\star \star \star \\
\star \star \star\end{array}$ \\
\hline 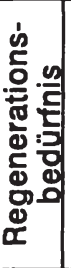 & $\begin{array}{l}\text { Faulenzen } \\
\text { Schlemmen } \\
\text { Gaststätten/ Kneipen } \\
\text { besuchen } \\
\text { Sich ausruhen }\end{array}$ & $\begin{array}{l}2,10 \\
2,48 \\
2,71 \\
1,89\end{array}$ & $\begin{array}{l}2,70 \\
3,16 \\
3,52 \\
1,93\end{array}$ & $\begin{array}{l}2,21 \\
2,54\end{array}$ & $\begin{array}{l}1,71 \\
2,03 \\
2,33 \\
1,68\end{array}$ & $\begin{array}{l}1,91 \\
2,32 \\
2,48 \\
1,96\end{array}$ & 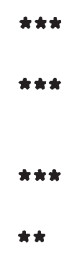 \\
\hline 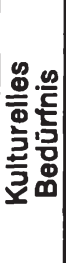 & $\begin{array}{l}\text { Kulturelle Veranstaltungen } \\
\text { besuchen } \\
\text { Sehenswũrdigkeiten } \\
\text { besichtigen } \\
\text { Begegnung mit Ein- } \\
\text { heimischen }\end{array}$ & $\begin{array}{l}2,46 \\
2,06\end{array}$ & $\begin{array}{l}2,67 \\
2,22 \\
2,34\end{array}$ & $\begin{array}{l}2,49 \\
2,11\end{array}$ & $\begin{array}{l}1,86 \\
1,48 \\
1,74\end{array}$ & $\begin{array}{l}2,93 \\
2,51 \\
2,63\end{array}$ & $\star \star \star \star$ \\
\hline \multirow{3}{*}{ 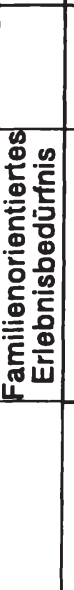 } & $\begin{array}{l}\text { Spaziergänge machen } \\
\text { Wandern }\end{array}$ & $\begin{array}{l}1,99 \\
2,22\end{array}$ & $\begin{array}{l}1,91 \\
2,45\end{array}$ & $\begin{array}{l}2,01 \\
2,10\end{array}$ & $\begin{array}{l}1,53 \\
1,69\end{array}$ & $\begin{array}{l}2,58 \\
2,71\end{array}$ & 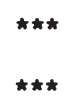 \\
\hline & $\begin{array}{l}\text { Mit den Kindern spielen } \\
\text { Tier-, Freizeitparks besuchen } \\
\text { Volksfeste, Veranstaltungen } \\
\text { besuchen } \\
\text { Einkaufen gehen }\end{array}$ & $\begin{array}{l}3,28 \\
2,43 \\
2,65 \\
2,72\end{array}$ & $\begin{array}{l}3,71 \\
2,57 \\
2,97 \\
3,23\end{array}$ & $\begin{array}{l}2,92 \\
2,37 \\
2,61 \\
2,56\end{array}$ & $\begin{array}{l}3,22 \\
2,01 \\
2,25 \\
2,47\end{array}$ & $\begin{array}{l}3,32 \\
2,83 \\
2,85 \\
2,72\end{array}$ & $\star \star \star \star$ \\
\hline & \multicolumn{3}{|c|}{$\begin{array}{l}\text { Cl.1: Traditlonelle Spazlergăngerfreunde } \\
\text { Cl.2: Sportaktive Familienurlauber } \\
\text { Cl.3: Land- \& Kulturorientierte Urlauber } \\
\text { Cl.4: Regen.- \& GenuBorientlerte Strandurlauber }\end{array}$} & \multicolumn{4}{|c|}{ 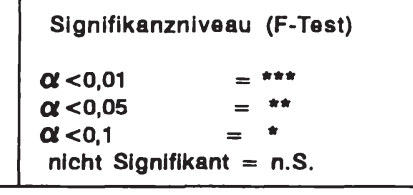 } \\
\hline
\end{tabular}

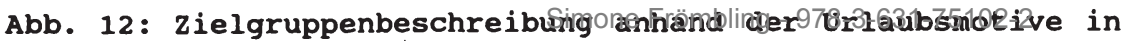
der Ent fermunigsignduippePIbFactory at 01/11/2019 08:19:28AM 


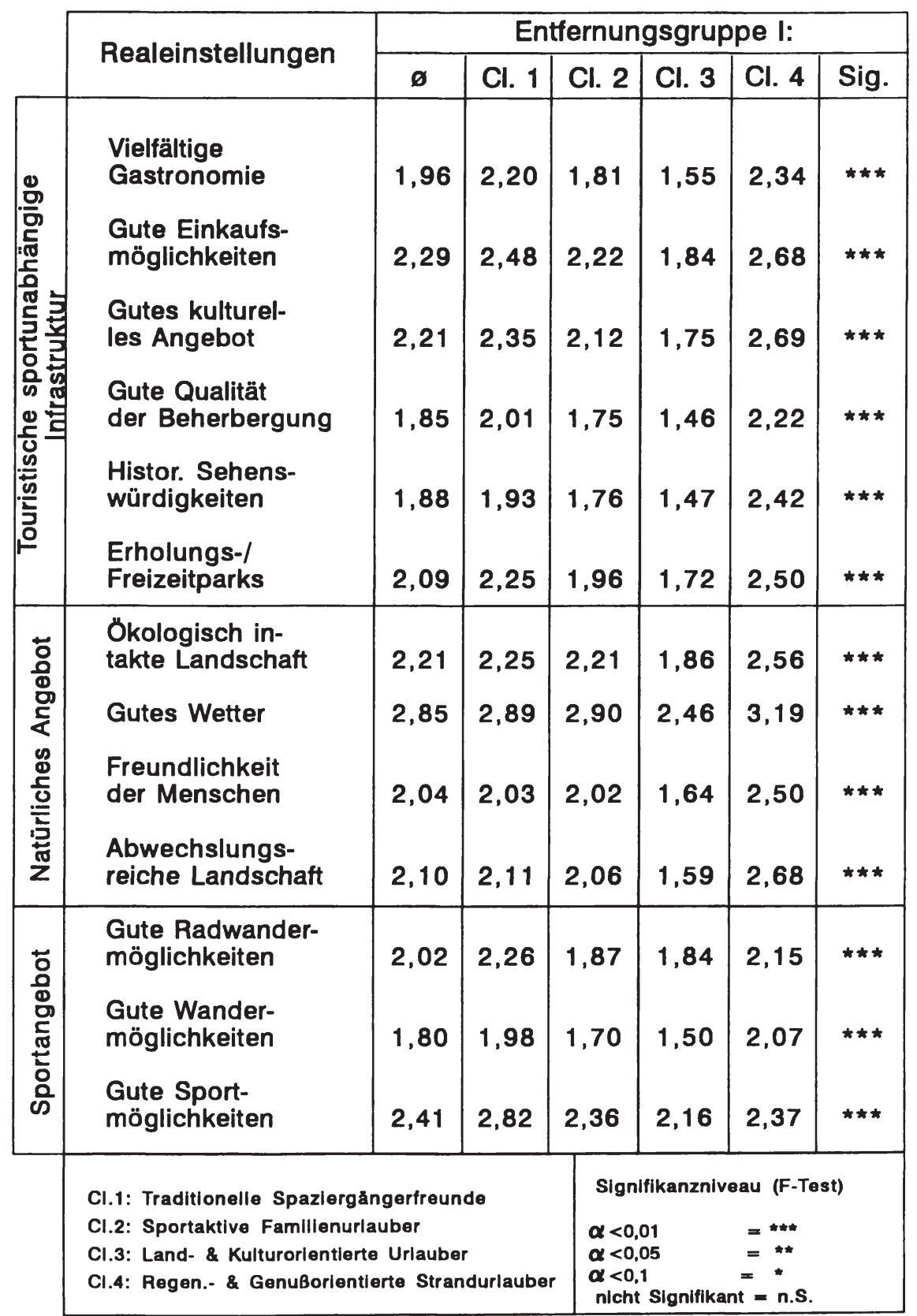

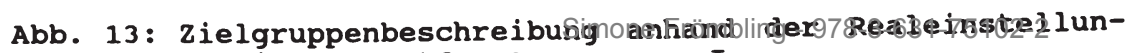
gen in deboEntberenungsempaperyIat 01/11/2019 08:19:28AM 


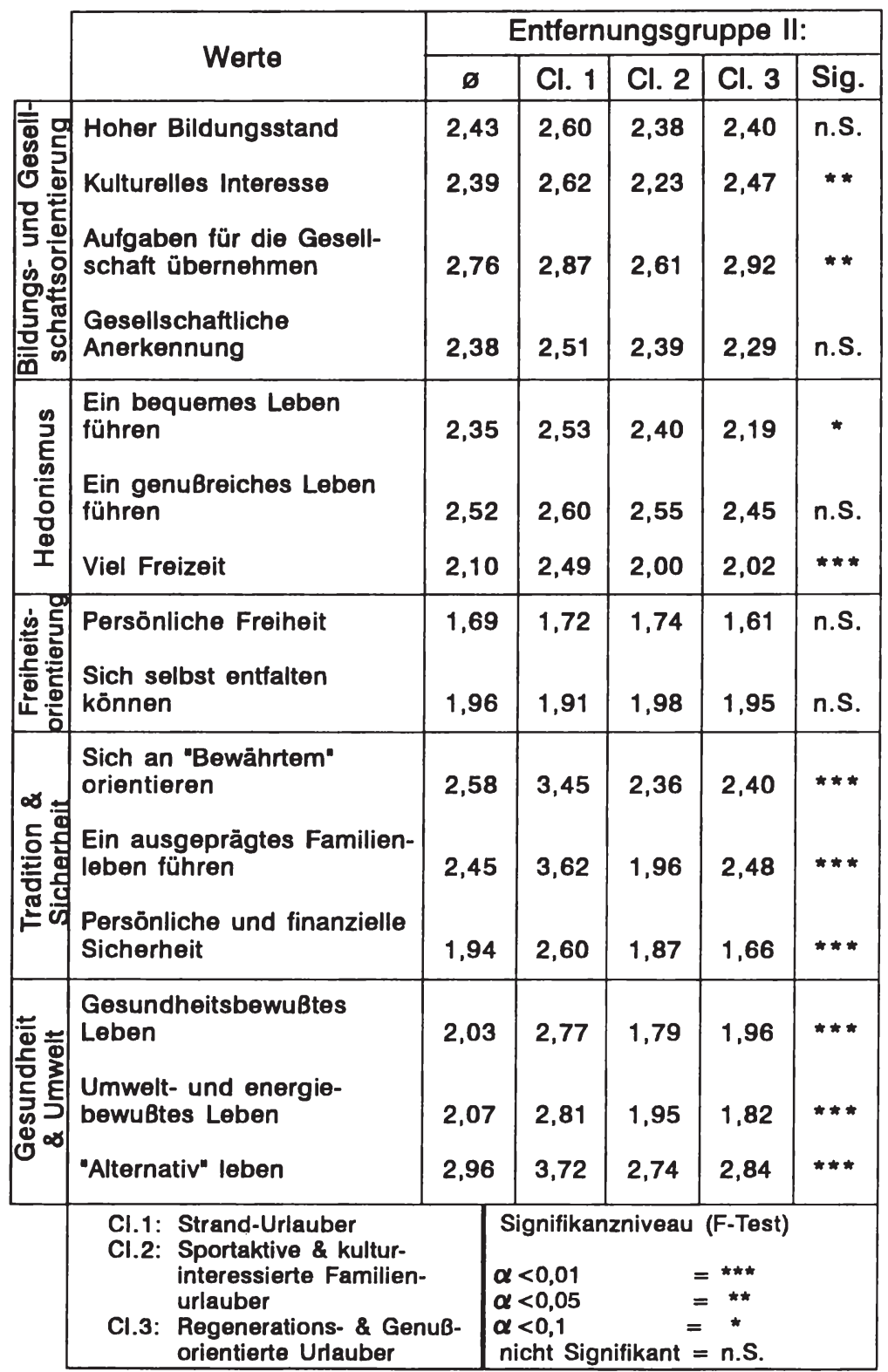

Abb. 14: Zielgruppenbeschreibung anhand der werte in der Entfernungsgruppe II Simone Frömbling - 978-3-631-75102-2 


\begin{tabular}{|c|c|c|c|c|c|c|}
\hline & \multirow{2}{*}{ Urlaubsmotive } & \multicolumn{5}{|c|}{ Entfernungsgruppe II: } \\
\hline & & $\varnothing$ & Cl. 1 & Cl. 2 & Cl. 3 & Sig. \\
\hline \multirow{5}{*}{ 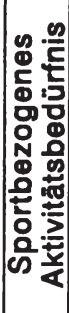 } & Sport trei & 2,98 & 94 & .85 & 3,20 & ^ \\
\hline & Aktiv sein & 2,46 & 2,47 & 2,22 & 2,79 & 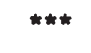 \\
\hline & Radfahren & 2,86 & 2,96 & 2,79 & 2,89 & n.s. \\
\hline & Hobbies betreiben & 2,86 & 3,09 & 2,72 & 2,92 & n.s. \\
\hline & $\begin{array}{l}\text { Schwimmen, be } \\
\text { Strand liegen }\end{array}$ & & 2,00 & 2,61 & 2,31 & *\# \\
\hline \multirow{4}{*}{ 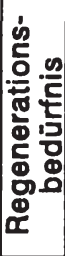 } & Faulenzen & 2,03 & 2,00 & 13 & 1,92 & n.s. \\
\hline & Schlemmen & 2,24 & 2,36 & 2,29 & 2,11 & n.S. \\
\hline & besuchen & 2,59 & 2,77 & 2,65 & 2,41 & n.s. \\
\hline & Sich ausruhen & 1,97 & 2,15 & 1,97 & 1,88 & n.s. \\
\hline \multirow{5}{*}{ 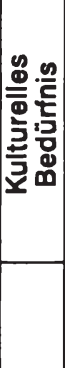 } & $\begin{array}{l}\text { Kulturelle Vers } \\
\text { besuchen }\end{array}$ & 2,54 & 3,00 & 2,38 & 2,49 & 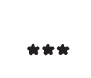 \\
\hline & $\begin{array}{l}\text { Sehensw } \\
\text { besichtig }\end{array}$ & 2,43 & 2,46 & 2,26 & 2,56 & * \\
\hline & $\begin{array}{l}\text { Begegnu } \\
\text { heimisch }\end{array}$ & 2,40 & & 2,32 & 2,28 & *** \\
\hline & Spazier & 2.31 & 3,04 & 06 & 2.26 & $\# * *$ \\
\hline & Wandern & 2,57 & 3,15 & 2,32 & 2,61 & *** \\
\hline \multirow{5}{*}{ 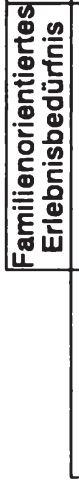 } & Mit den Kindern : & 3,68 & 4,53 & 38 & .63 & \#** \\
\hline & Tier-, Freizeitparks besuchen & 2,73 & 3,62 & 2.44 & 2,64 & $\star \star \star \star$ \\
\hline & $\begin{array}{l}\text { Volksfeste, } \\
\text { besuchen }\end{array}$ & 2,78 & 3,47 & 2,45 & 2,84 & $\star \star \star *$ \\
\hline & Einkaufen gehen & 2,99 & 3,83 & 2,87 & 2,69 & *** \\
\hline & \multicolumn{2}{|c|}{$\begin{array}{l}\text { Cl.1: Strand-Urlauber } \\
\text { Cl.2: Sportaktive \& kultur- } \\
\text { interessierte Familien- } \\
\text { urlauber } \\
\text { CI.3: } \begin{array}{l}\text { Regenerations- \& GenuB- } \\
\text { orientierte Urlauber }\end{array}\end{array}$} & \multicolumn{4}{|c|}{ Signifikanzniveau (F-Test) } \\
\hline
\end{tabular}

Abb. 15: Zielgruppenbeschreibung anhand der yplaubsmatiye in der Ent fernungsgruppe IY 


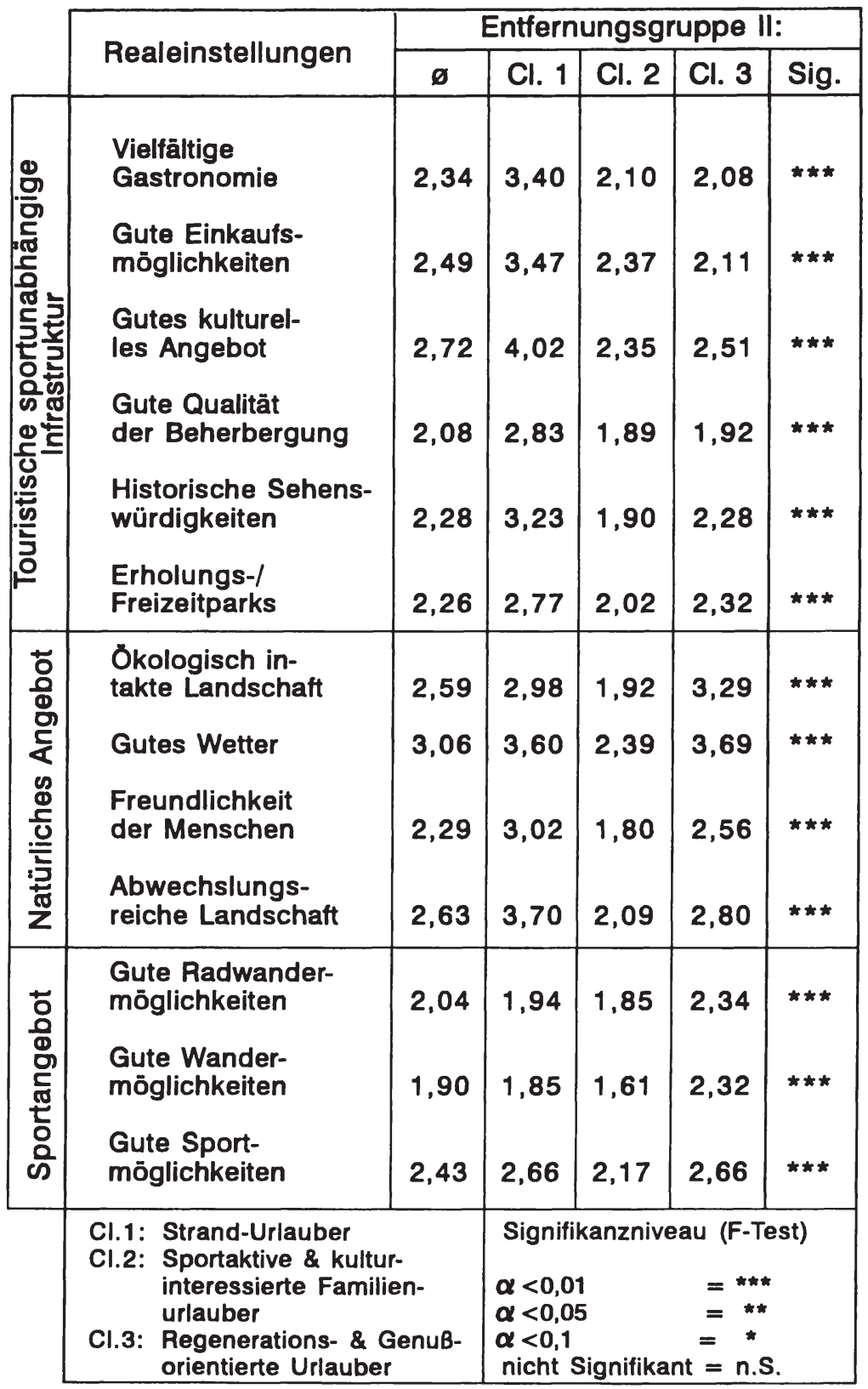
gen in der Entfernungsgruppe $\mathrm{II}^{1}$ 
Simone Frömbling - 978-3-631-75102-2

Downloaded from PubFactory at 01/11/2019 08:19:28AM

via free access 


\section{A $\quad \mathrm{N}$ H A $\mathrm{N}$ G II}

\section{FRAGEBOGEN}


Simone Frömbling - 978-3-631-75102-2

Downloaded from PubFactory at 01/11/2019 08:19:28AM

via free access 
211. In dieser bundeswelten Uhfrage geht os - das Münsterland. Ich molne dealt dos Cebiot nürdl leh des Ruhrgebletes zwisehen dor hollizndischen Grenze und den Teutoburger Wald wit Munster und den Londkrol sen Borken, Coesfeld, Stelnfurt und Werendorf.

Wie hiuflo haben Sie inmerhalb der letzten

2 Jahre das Munsterland besuche?

प, mehrere Mole

ए]? eImel Jedes Johr Imal in den letzten 2 Jahren

Oborhoupt nleht $222 / 5.3$

212. Aus wolchem Grund haben S10 das Münsterland in den lotzten 2 Jahren besucht? Sagen Sle alr das bltte anhand dieser Ll ste. LISTE 1 vorlegen

\begin{tabular}{|l|l|l|l|l|}
11 & 12 & 13 & 14 & 15 \\
\hline & & & & \\
\hline
\end{tabular}

213. Als Sle das Munsterland besuche haben, ind sle dann tiberwlogend alleine oder in Bogleltung gokommen? 2 In Begleitung $\longrightarrow 214-16$
214-216. Mit wem haben sie das Münsterland besucht?

MUR FORS IMSTITUT :

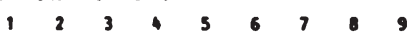

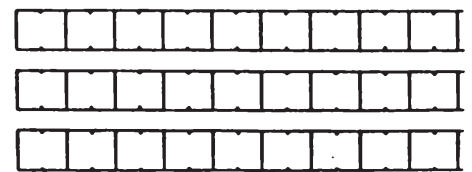

217. Als Sle das Münaterland besuche haben, haben Sle dann Oberwiegend nur oln oinziges Relsezlel (z. B. eI one bestimate Stedt/Comelnde) gohabt, wo Sle sleh whreand ihres dufonthalts ousschlleslich oufhlelten, oder haben Sle die Högllehkelt genutzt, ouch das Ebrige Müsterland naher kennenzulernen?

$\square$ nur eln Zlel 2 mehrere 2lele 


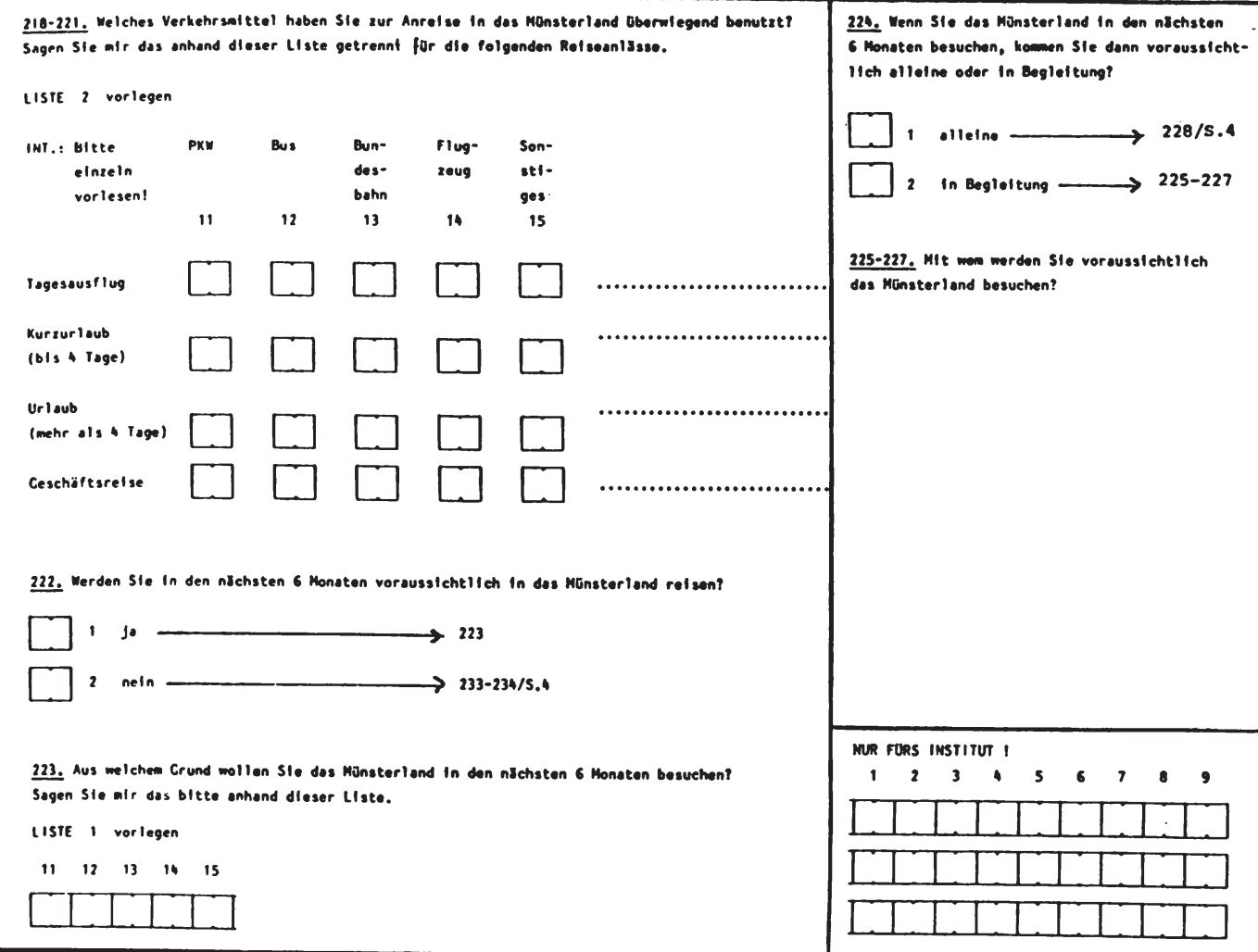


228. Wenn Sle in den nāchsten 6 Monaten das Münsterland besuchon, haben Sle dann nur eln elnalges Relseziel (2. B. el ne bestimete Stadt/Cem de), mo sle slch withrend Ihros Aufenthalts ausschliesifich aufhalten, oder nutzen Sle die Maglichkelt, auch das bbrige Münsterland kennenzulernen?

$[\cdots]$, nur ein zlel

[], mehrere ziele

229-232. Welches Verkehrsmlttel werden S10 vorsusslchtlleh zur Anrelso In des Munsterland benutzen? Sagen Sie mir das anhand dieser Liste getrennt für die folgenden Rol seanlasse In den nächsten 6 Monaten.

LISTE 2 vorlegen

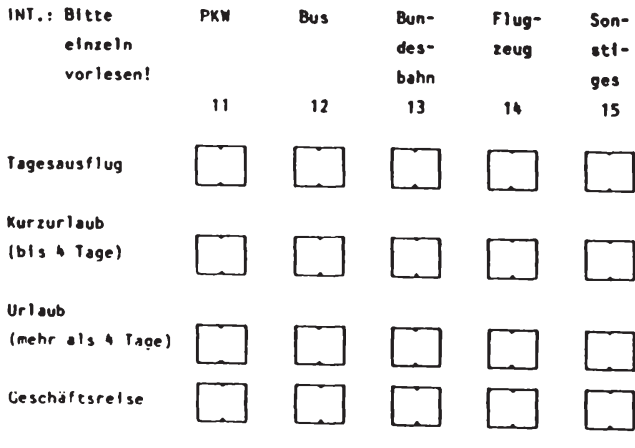

233-234. Bitte sagen Sie wir anhand dieser Liste, welehe der oufgof Ghrten Stidte Ihrer Molnung noch im Münsterland llegen.

LISTE 3 vorlegen

\begin{tabular}{|l|l|l|l|l|l|l|l|l|}
11 & 12 & 13 & 14 & 15 & 16 & 17 & 18 & 19 \\
\hline & & & & & & & & \\
\hline
\end{tabular}

21

$\square$

235. Ich komme Jetzt ouf Tagessusfloge, Kurzurlaub ( 2 b/s Tage) und Urlaub (5 Tage und IJnger) zu sprechen.

Zuerst zum Tagesurlaub. Stellen Sie slch el mal vor, Slo mollten elnen Tagosausflug machen. Wolche der auf dleser Liste aufgeführten Ziele kJmen für Sle für el Inen Tagesausflug sleher oder vielleicht in Botracht?

LISTE vorlegen

$\begin{array}{llllllll}11 & 12 & 13 & 14 & 15 & 16 & 17 & 18\end{array}$

\begin{tabular}{|l|l|l|l|l|l|l|l|}
\hline & & & & & & & \\
\hline
\end{tabular}




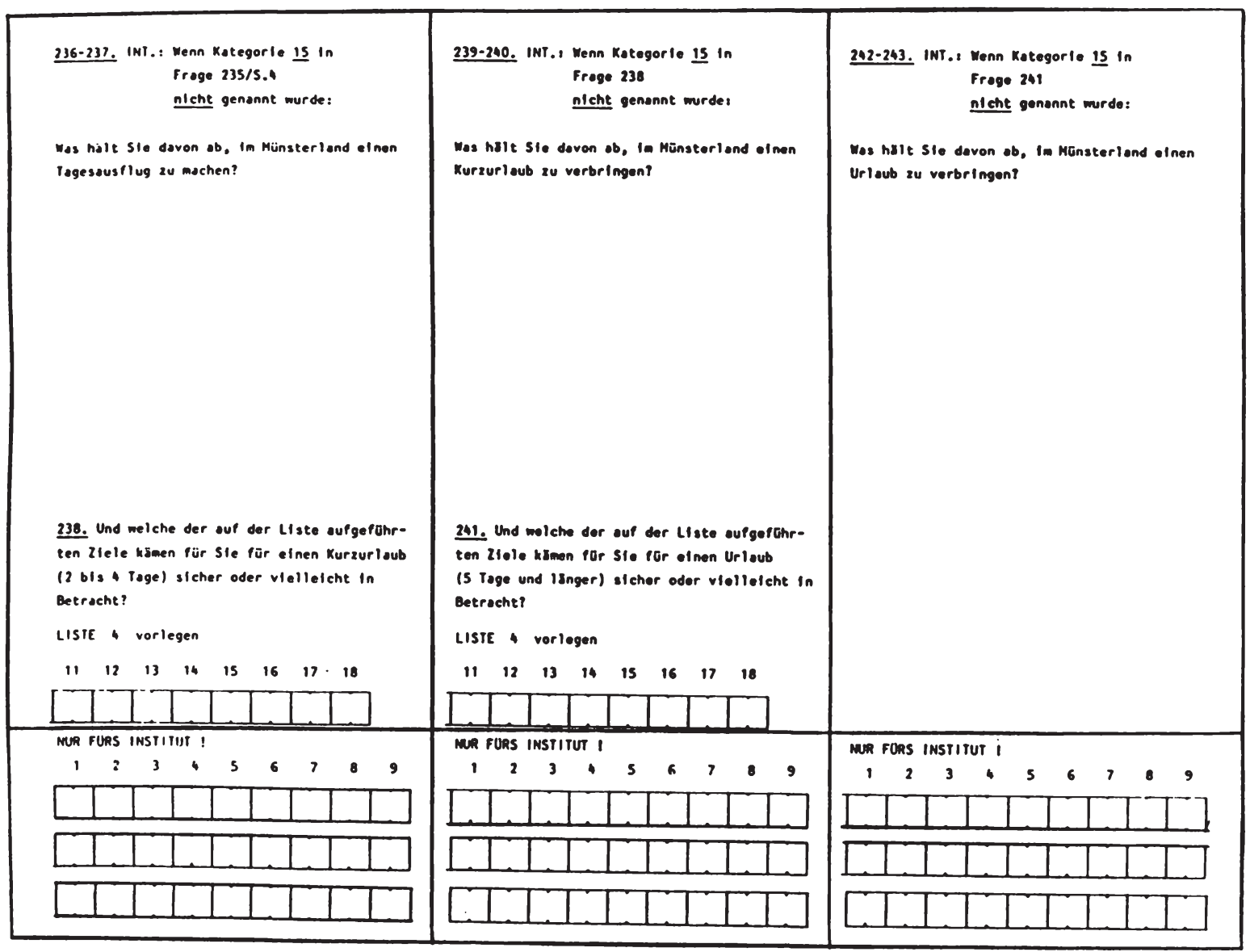


Welche Aktivitäten sind für Sle im Urlaub wichtig? Sagen Sie mir das bitte anhand dieser Liste.

Liste 6 vorlegen

ilicte sehr slem- otwas haum gar

wich- niche

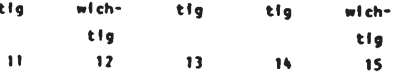

sich susruthen $\square \quad \square \quad \square \quad \square$

Sporlergänge machen

$\square \square \square \square$

Nier-, freizelt-.

llaturparks besuchen

ए $\square \square \square \square$

mit Menschen der

Kegion ins Cesprych

komen

$\square \square \square \square \square$

mit den kindern

spielen

oktiv sein

Volksfeste, Veran-

stoltungen besuchen

Hobbles betrelben

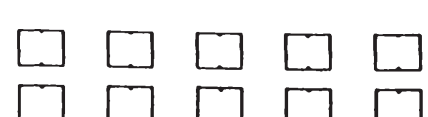

$\square \quad \square \quad \square \quad \square$

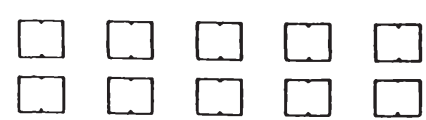

Sengmirdigkelten, Boumerke, Museen besichtigen

enlturelle Veran-

stal tungen besuchen

\section{ictaring beden,}

en Strand llegen

mondern

redf ehren

Sport trelben

(allgemen $(n)$

ohne Zettdruck

Elnkaufen gethen

Coststztton/Kneipen

besuchen

foulenzen

schlemmen

fernsohen

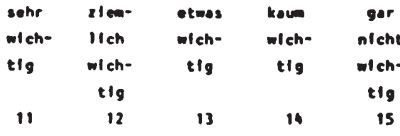

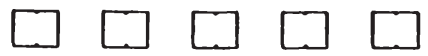

$\square \quad \square \quad \square \quad \square$

$\square \square \square \square \square$

$\square \square \square \square \square$

$\square \square \square \square \square$

$\square \square \square \square \square$

$\square \square \square \square \square$

$\begin{array}{llllll}\square & \square & \square & \square & \square\end{array}$

$\begin{array}{lllll}\square & \square & \square & \square & \square\end{array}$

$\begin{array}{lllll}\square & \square & \square & \square & \square\end{array}$ 


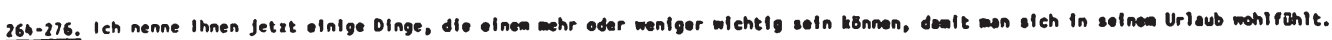
Segen Sle mir bltte anhand dleser Llste zu Jeder der folgenden Aussogen, wle sohr dle Aussag quf sie solbst sutrifft.

LISIE 6 vorlegen

INT.: BItte

$\begin{array}{lccccc}\text { elnzeln } & \text { sehr } & \text { tem- } & \text { etwas } & \text { kam } & \text { gar } \\ \text { vorlesent } & 20 & \text { lich } & 20 & 20 & \text { nlcht } \\ & & 20 & & & 20 \\ & 11 & 12 & 13 & 14 & 15\end{array}$

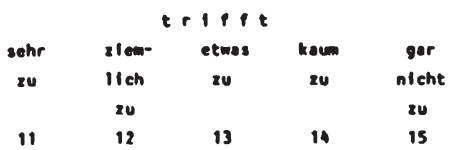

Ich fühle alch in

Urlaub wohl megen ...

der guten Redwander-

Urlaub wohl megen ...

mogilichkelten

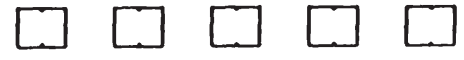

des guten kulturellen

Angebots

\section{ए dor historischen}

Sehenawurdigkelten

$\square$

dos guten Wetters

Ner Erholungs- und

Frelzeleparks

der guten Mander-

migllchielten

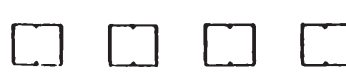

$\square$

dor Bkologlseh

Intekten Lendscheft

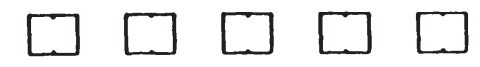

der guten Qualltat

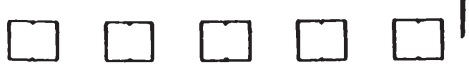

der Freundlichkelt

der Beherbargungstztten
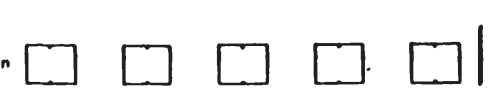

der vielfäligen

Costronomie
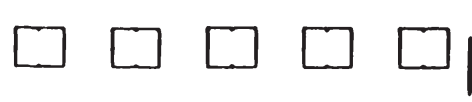

der abwechslungs:relction Landschoft

der guten Elnkaufs-

mölichiclten

der guten sport-

nigllenkeltien

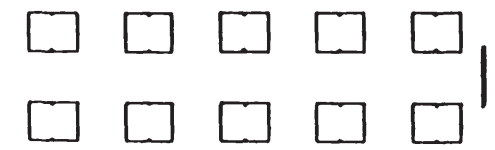


311-323. Ich lese Ithen die Punkte aus der vorhergehenden frage noch el mall vor

Sagen Sle mir bltte Jewells wieder anhand der Liste Imwiowelt die Punkte Ihrer Molnung nach ouf das Münsterland zutreffen.

LISTE 6 vorlegen

InT.: Bitte

einzeln

vorlesen!

gutes kulturelles Angebot

frholungs- und frelzeltparks

gute Mandermiglichkelten

gute Qualität der Beherbergungs-

statten

vielfältige Castronomle

gute Einkoufsmögl ichkeiten

gute Sportmöglichketten

gute Radwandermögll chkeiten

hlstorische Sehenswürdigkelten

gutes Wetter

ökolngisch intakte Landschaft

Freundlichkelt der Menschen

abrechslungsreiche Landschoft
Informleren sie sich über das Münsterland

bevor sle dorthin fahren bzw. gefahren sind?

sagen sle mir dae bitte anhand der Liste.

LISTE, vorlegen

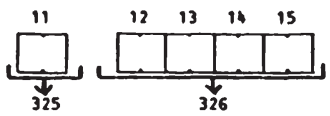

325. Wo bzw. bel wen wirden Sle in erster LInle Informotionen Gber dos Münsterland suchen?

Mennon Sle mir aus dieser Liste bltto die zwel wlchtigsten Punkte.

LISTE 8 vorlegen

\begin{tabular}{|l|l|l|l|l|l|l|}
\hline 11 & 12 & 13 & 14 & 15 & 16 & 17 \\
\hline & & & & & & \\
\hline
\end{tabular}

Sonstiges:

326. We bewerten Sie das Ihnen zur Verfügung stehende Inforationsangebot ober das Münsterl and insgesamt?

Sagen sle mir das bitte anhand der Noten von 1 - sehr gut bls 5 = mangelhaft.

$\square$, sehr gut
$\square$, gut
$\square$, befriedigend
$\square$, eusrelchend
$\square$, mongelhoft


327-328. Sagen Sle mir bltte onhand der folgenden Liste, wodurch slch das Münsterl and Ihrer Meinung nach besonders auszelchnet.

Was trifft zu?

Liste 9 vorlegen

$\begin{array}{lllllllll}11 & 12 & 13 & 14 & 15 & 16 & 17 & 18 & 19\end{array}$ \begin{tabular}{|l|l|l|l|l|l|l|l|l|}
\hline & & & & & & & & \\
\hline
\end{tabular} $21 \quad 22 \quad 23$

T.. T.. T.

Sonstiges:

329. Auch wenn Sie den Flughafen MönsterOsnabrück selbst noch nicht benutzt haben, was meinen Sle: Hat slch das Lel stungsangebot des Flughafens Münster-0snabrück in den letzten 5 Jahren stark verbessert - zlealfch etwas - kaum - oder gar nicht verbessert?

$\square$, stork verbessert

I Iiemlich verhessert

3 etwas verbessert

प kaum verbessert

T gar nicht verbessert
330-341. Welche der folganden El genschaften sind Ihror Melnung nach typlsch for die Mensehen In Münsterland? Murkleren Sie bitte einas dep Folder, Je nachdem, ob eher die linke oder die rechte Elgenschaft ouf dle Menschen Im Mönsterland zutrifft.

INT.: Fragebogon bitte dom Bofragten zum Markleren Obergeben

(mit. Minlstift und sentrochten LInten markforen lassen)l

fletsig

gründlich

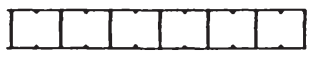

foul

sparsem

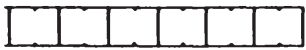

nechlassig

sporsom

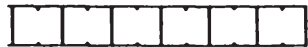

verschwenderi sch

schl app

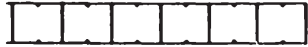

eusdouernd

stur

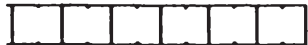

offon

beschel den

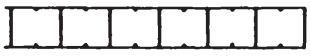

protzig

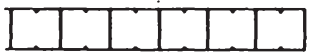

morrlsch

woltoffon

versehlossen

abmel send

unzuver $133 s$ /g

handfest

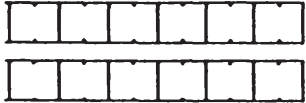

felnsinnig

BITTE CEBEN SIE DEN FRACEBOCEN WIEDER AN DEM INTERVIEWER ZUROCK 
342-356. Zum Abschlub etwas all gemelnes: Es wird oft derüber gesprochen, welchen Stellenwert bestimate Merte und ziele für die el gene Lebensführung el Innehmen. Für wie wichtig halten sie persönlleh d folgenden Merte und zlele?

Hiltie sagen sie mir das anhand der folgenden Liste.

LISTE 10 vorlegen

$\begin{array}{lccccc}\text { INT.: Eitte } & \text { sehr } & \text { zlem- } & \text { etwas } & \text { kaum } & \text { gar } \\ \text { elnzeln } & \text { wich- } & \text { lich } & \text { wich- } & \text { wich- } & \text { nicht } \\ \text { vorlesen! } & \text { tig } & \text { tig } & \text { tig } & \text { tig } & \begin{array}{r}\text { wich } \\ \text { tig }\end{array} \\ & 11 & 12 & 13 & 14 & 15\end{array}$

\begin{tabular}{|c|c|c|c|c|}
\hline sehr & $21 \mathrm{em}$ & etwes & koum & gor \\
\hline wich- & Ilch & wich- & wich- & nicht \\
\hline glt & tIg & tig & tig & $\begin{array}{l}\text { Wich- } \\
\text { tig }\end{array}$ \\
\hline 11 & 12 & 13 & 14 & 15 \\
\hline
\end{tabular}

gesell schaftliche

Anerkennung

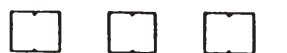

$\square$

umelt- und energle-

$\square$

bewultes Leben

slch selbst entfalten

können
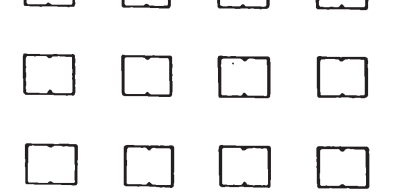

gosunchel tsbemultes Leben

ein bequemes Leben

führen

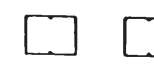

persünliche und finan-

zielle sicherhelt.
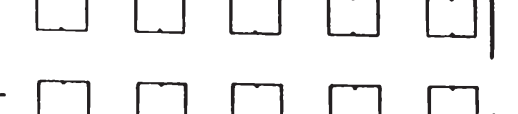

"alternativ" loben

$\square[\square$

$\square[$

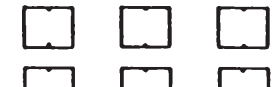

- In gonubrol ches Laben

fohren

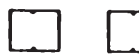

persönliche Freiheit

viel frelzelt

$\left[\begin{array}{c}0 \\ 0\end{array}\right]$

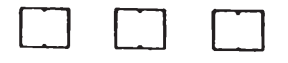

$\square$

alch on "Bowlhrtem"

Aufgaben für die

eln susgeprägtes

$\square \square$

$\square$ notmen

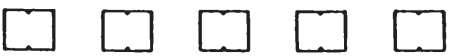

familienleben führen

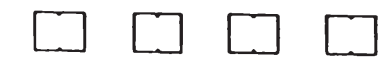

$\square$ kulturelles interesse

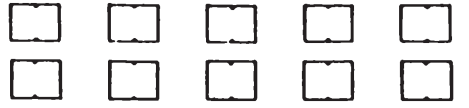


112: Sind Sle Im Münsterland geboren?

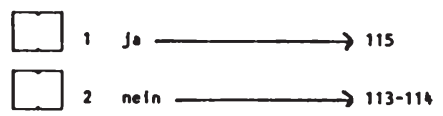

110-111. Darf ich Sie fragen, wie alt sie sind?

\section{Jahre}

$1 \square$

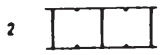

$3 \square$

- $\square$.

$5 \square$

$6 \square$

$7 \square$

- $\square$.

$9 \square$

$0 \square$.

113-114. Wo sind sie geboren?

Sagen Sie das bitte anhond dieser Liste.

LISTE 11 vorlegen

$\begin{array}{lllllllll}11 & 12 & 13 & 14 & 15 & 16 & 17 & 18 & 19\end{array}$

$\begin{array}{llll}21 & 22 & 23 & 24\end{array}$

\begin{tabular}{|l|l|l|l|}
\hline & & & \\
\hline
\end{tabular}

115. Haben S10 zwischenzeltilich in einew ondoren Ort ols heute gowohnt?

$\square, 1$

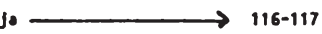

$\square 2$ nein $\rightarrow 120$

116-117. Wo haben Sie zuletze gewohnt, bevor Sle in dlesen Ort gezogen sind?

LISTE 11 vorlegen

$\begin{array}{lllllllll}11 & 12 & 13 & 14 & 15 & 16 & 17 & 18 & 19\end{array}$
118-119. Wie viele Jahre haben Sie dort gowohnt?

$\lfloor\perp$ Jahre

\begin{tabular}{|l|l|l|l|l|l|l|l|l|}
\hline & & & & & & & & \\
\hline
\end{tabular}

$1 \square$

$=\square . T$

3 ए.T.

- $\square$.

$5 \square$

$6 \square$

, प.

- $\square$

, $\square$

- ए.

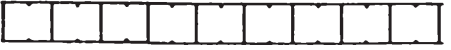

$\begin{array}{llll}21 & 22 & 23 & 24\end{array}$

\begin{tabular}{|l|l|l|l|}
\hline & & & \\
\hline
\end{tabular} 
120. Wie viele Kinder unter 18 Jahren leben In Ihrem Haushalt?

$\square, 1$ kind

$[-] 2$ kinder

[.] 3 kinder

ए, 4 kinder

ए 5 und mehr xinder

ए 6 kein KInd

121. Welche Schule haben Sle zuletzt besucht brin. welchen Schulabschlub haben S10?

LISTE 12 vorlegen

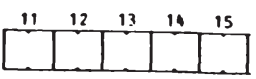

122. Welcher der Beruf sgruppen auf dieser Liste gehören Sie on?

LISTE 13 vorlegen

\begin{tabular}{|l|l|l|l|l|l|l|l|l|}
\hline 11 & 12 & 13 & 15 & 16 & 18 & 18 & 19 \\
\hline & & & & & & & & \\
\hline
\end{tabular}
123. Wohnen S1e in el genen Haus - In einer Eigantunswohnung - wohnen Slo zur Miete ols Hauptaileter - oder sind SI Untermleter?

ए, Eigantüner Haus

प, Elgentümer Wohnung

[.] 3 Houptmioter

ए. Untermleter

124. Wonn Sle mol olles zuscomennrechnen: wie hoch ist dann das monatllicho MattoEinkommon, des Ste alle zusammen hler im Haushalt haben, nach Abzug der Steuern und Sozlelversicherung? Nennen Sle bltte die entsprechende ziffer.

LISTE 14 vorlegen

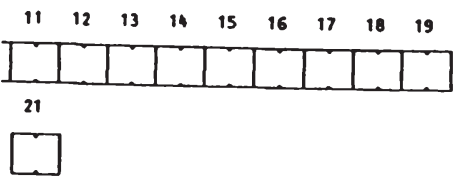

125. Und wie hoch 1st Ihr personllehes lotto-EInkomen pro Monat?

LISTE 14 vorlegen

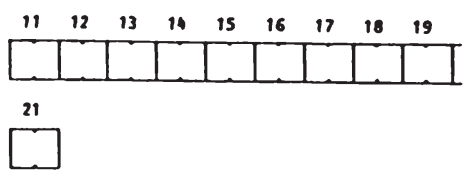


181. Dieses Interview wurde durchgeführt ons

Ich, der Unterzelchner, versichere, das leh des Intervlew entsprechend den Anmel sungen des Inati tuts durchgof thrt habe.

Cerichtsstand lat der Sitz des instituts. 
Listen für die Teilbefragung Bürger bundesweit:

\section{Liste 1:}

11 Tagesausflug (keine Übernachtung)
12 Kurzurlaub (nicht mehr als 4 Tage)
13 Urlaub (mehr als 4 Tage)
14 Geschäftsreise
15

\section{Liste 2:}

11 PKW

12 Bus

13 Bundesbahn

14 Flugzeug

15 Sonstiges, was? (bitte angeben)

\section{Liste 3 :}

11 Osnabrück

12 Bielefeld

13 Oldenburg

14 Recklinghausen

15 Warendorf

16 Coesfeld

17 Steinfurt

18 Borken

19 Gütersloh

21 Gelsenkirchen

\section{Liste 4:}

11 Niederrhein

12 Sauerland

13 Emsland

14 Teutoburger Wald

15 Münsterland

16 Harz (auch DDR)

17 Mecklenburg (ohne Ostseeküste)

18 Thüringer Wald

\section{Liste 5:}

11 sehr wichtig

12 ziemlich wichtig

13 etwas wichtig

14 kaum wichtig

15 gar nicht wichtig

\section{Liste 6:}

11 trifft sehr zu

12 trifft ziemlich zu

13 trifft etwas zu

14 trifft kaum zu

15 trifft gar nicht $\mathbf{z u}$ Simone Frömbling-978-3-631-75102-2 


\section{Liste 7:}

11 ich suche gezielt nach Informationen

12 ich überlasse des dem Zufall, etwas über das Münsterland zu erfahren

13 es ist mir gleichgültig, etwas über das Münsterland zu erfahren

14 ich informiere mich nicht vorher

15 ich fahre nicht ins Münsterland bzw. bin nicht dorthin gefahren

\section{Liste 8:}

11 über Freunde/Bekannte/Verwandte

12 Prospekte

13 Zeitungsberichte

14 Reiseliteratur

15 Reisebüro

16 Prospektanforderung bei Fremdenverkehrsverbänden des Münster-

landes

17 Sonstiges, wo/bei wem? (bitte angeben)

\section{Liste 9:}

II zahlreiche Wasserburgen und Wasserschlösser

12 Universität Münster

13 hat eigenen Flughafen

14 hohe Lebensqualität

15 liebenswerte Städte/Orte

16 sympathische Menschen

17 zentrale Lage im Bundesgebiet

18 geringe Kriminalität

19 geeigneter Altersruhesitz

21 bietet Möglichkeiten für "Ferien auf dem Bauernhof"

22 Veranstaltungen, die mit der Region verbunden sind (z.B. Wildpferdefang in Dülmen, Ballonflugwettbewerb, Hengstparade in Warendorf)

23 Sonstiges, was? (bitte angeben)

\section{Liste 10:}

$\begin{array}{ll}11 & \text { sehr wichtig } \\ 12 & \text { ziemlich wichtig } \\ 13 & \text { etwas wichtig } \\ 14 & \text { kaum wichtig } \\ 15 & \text { gar nicht wichtig }\end{array}$

\section{Liste 11:}

$\begin{array}{ll}11 & \text { Berlin (West) } \\ 12 & \text { Schleswig-Holstein } \\ 13 & \text { Hamburg } \\ 14 & \text { Bremen } \\ 15 & \text { Niedersachsen } \\ 16 & \text { Nordrhein-Westfalen } \\ 17 & \text { Hessen }\end{array}$




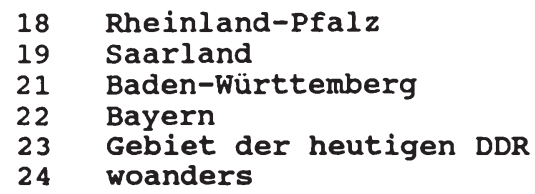

\section{Liste 12:}

11 Volks-, Hauptschule ohne abgeschlossene Lehre

12 Volks-, Hauptschule mit abgeschlossener Lehre

13 weiterbildende Schule ohne Abitur

14 Abitur, Hochschulreife, Fachhochschulreife

15 Studium (Universität, Akademie, Fachhochschule)

\section{Liste 13:}

$\begin{array}{ll}11 & \text { Selbständiger } \\ 12 & \text { Angestellter } \\ 13 & \text { Beamter } \\ 14 & \text { Arbeiter } \\ 15 & \text { Hausfrau } \\ 16 & \text { Schüler/Student } \\ 17 & \text { Auszubildender/Lehrling } \\ 18 & \text { nicht erwerbstätig } \\ 19 & \text { Rentner }\end{array}$

\section{Liste 14:}

\begin{tabular}{|c|c|c|c|c|c|}
\hline 11 & & & unter & 500 & DM \\
\hline 12 & 500 & bis & unter & 1.000 & DI \\
\hline 13 & 1.000 & bis & unter & 1.500 & DI \\
\hline 14 & 1.500 & bis & unter & 2.000 & DM \\
\hline 15 & 2.000 & bis & unter & 2.500 & $D$ \\
\hline 16 & 2.500 & bis & unter & 3.000 & DII \\
\hline 17 & 3.000 & b. & un & 500 & 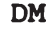 \\
\hline 18 & 00 & 5 & $r$ & 000 & \\
\hline 1 & 00 & & & 00 & \\
\hline & 5.000 & & & & \\
\hline
\end{tabular}




\section{Literaturverzeichnis}

Aaker, D.A.

Aderhold, P.

Ajzen, $J$.

Fishbein, $M$.

Ajzen, J.

Fishbein, M.

Akademie für Raumforschung und Landesplanung (Hrsg.)

Andritzky, $K$.

Angehrn, 0 .

Arbeitsgemeinschaft Media Analyse (Hrsg.)

Arndt, $\mathrm{H}$.

Backhaus, $\mathrm{K}$.
Strategisches Marketing-Management. Wettbewerbsvorteile erkennen, Märkte erschließen, Strategien entwickeln, Wiesbaden 1989

Das Marketing von städtekurzreisen. Rahmenbedingungen und Ansatzpunkte einer Fremdenverkehrspolitik unter beispielhafter Berücksichtigung der Verhältnisse in Kopenhagen, Kopenhagen 1976

Attitudinal and Normative Variables as Predictors of Specific Behaviour, in: Journal of Personality and Social Psychology, 1973, S. 4157

The Prediction of Behaviour from Attitudinal and Normative Variables, in: Journal of Experimental Social Psychology, 1970, Heft 6 , S. 466-487

Fremdenverkehr und Regionalpolitik, Forschungs- und sitzungsberichte, Bd. 172, Hannover 1988

Die Operationalisierbarkeit von Theorien zum Konsumentenverhalten, Berlin 1976

Marktsegmentierung als Absatzmethode, in: GFM, 1968, Heft 2, S. $38-42$

Media Analyse 1992 für Publikumszeitschriften, Supplements, Tageszeitungen, Kongress, Lesezirkel, Kino, Hörfunk, Fernsehen, Frankfurt 1992

Definitionen des Begriffs "Fremdenverkehr" im Wandel der Zeit, in: Jahrbuch für Fremdenverkehr, 1978/ 79 , S. 160-174

Investitionsgütermarketing, 3 . Aufl., München 1992 
Backhaus, K., u.a.

Backhaus, $K$. Piltz, K.

Backhaus, $\mathrm{K}$. Piltz, K. (Hrsg.)

Backhaus, $\mathrm{K}$. Weiber, $R$.

Bänsch, A.

Balderjahn, I.

Banning, T.E.

Bass, F.M.

Talarzyk, W.W.

Bauer, E.

Bauer, F.

Bauer, R.A.

Becker, u.a. (Hrsg.)
Multivariate Analysemethoden, 6 . Aufl., Berlin u.a. 1990

Strategische Allianzen - eine neue Form kooperativen Wettbewerbs?, in: Backhaus, K., Piltz, K. (Hrsg.), Strategische Allianzen, Sonderheft Nr. 27 der ZfbF, Düsseldorf, Frankfurt 1989 , S. 1-11

Strategische Allianzen, Sonderheft Nr. 27 der ZfbF, Düsseldorf, Frankfurt 1989

Entwicklung einer Marketing-Konzeption mit SPSS/PC+, Berlin u.a. 1989

Käuferverhalten, 4. Aufl., München 1989

Das umweltbewußte Konsumentenverhalten. Eine empirische studie, Berlin 1986

Lebensstilorientierte MarketingTheorie. Analyse und Weiterentwicklung modelltheoretischer und methodischer Ansätze der Lebensstil-Forschung im Marketing, Heidelberg 1987

An Attitude Model for the study of Brand Preference, in: Journal of Marketing Research, Vol. 9, 1972, S. 93-96

Markt-Segmentierung als Marketingstrategie, Berlin 1976

Datenanalyse mit sPSS, Berlin u.a. 1984

Konsumentenentscheidungen als Risikoverhalten, in: Specht, K.G., wiswede, G. (Hrsg.), Marketing-Soziologie - soziale Interaktionen als Determinanten des Marktverhaltens, Berlin 1976, S. 209-217

Freizeitverhalten in verschiedenen Raumkategorien, Trier 1979 
Becker, J.

Behrens, F.

Berekoven, L. Eckert, w. Ellenrieder, P.

Bergler, R.

Berneker, P.

Berry, L.L. Parasuraman, A. Zeithaml, V.A.

Birkigt, $\mathrm{K}$. Stadler, M.M. Funck, H.J.

Bleile, G.

Bleile, G.

Böcker, F. Gierl, $\mathrm{H}$.

Böhler, H.
Marketing-Konzeption. Grundlagen des strategischen Marketing-Management, 4. Aufl., München 1992

ZIN - Zusammenarbeit ist nötig, in: städte- und Gemeinderat, 1990, S. 63-67.

Marktforschung. Methodische Grundlagen und praktische Anwendung, 4. Aufl., Wiesbaden 1989

Einstellungen und Verhalten als theoretisches Problem, in: Hartmann, K.D., Koeppler, K.F. (Hrsg.), Fortschritte der Marktpsychologie, Bd. 2, Grundlagen - Methoden - Anwendungen, Frankfurt a.M. 1977

Die Wandlungen des Fremdenverkehrsbegriffes, in: Jahrbuch für Fremdenverkehr, 1952/53, Heft 1, S. 3138

Moving Forward in Quality Research. Presentation Handouts for Marketing Science Institute Conference, Supporting Service Quality, Boston 1992 (unveröffentlichtes Manuskript)

Corporate Identity. Grundlagen, Funktionen. Fallbeispiel, 5. Aufl., Landsberg a.L. 1992

Gemeinsam in den Wettbewerb. Fremdenverkehrsgemeinschaften - Vorteile und Erfolgsbedingungen, in: Der Fremdenverkehr, 1983, Heft 6, S. 14-16

Marketing-strategien im Fremdenverkehr, in: Der Fremdenverkehr, 1978, Heft 11 , S. 18-28

Die Beurteilung einer Zeitschrift als Werbeträger, Berlin 1986

Methoden und Modelle der Marktsegmentierung, stuttgart 1977 
Böhler, H.

Bosshart, M.

Frey, $C$.

Lombardi, D.

Schönfeld, B.

Brisoux, J. Laroche, M.

Brosius, G.

Brosius, G.

Bruhn, M.

Büker, B.
Beachtete Produktalternativen und ihre relevanten Eigenschaften im Kaufentscheidungsproze $B$ von Konsumenten, in: Meffert, H., Steffenhagen, H., Freter, H. (Hrsg.), Konsumentenverhalten und Information, Wiesbaden 1979, S. 261-289

Tagestourismus in Basel. Ergebnisse einer empirischen Studie, erstellt im Rahmen des Marketingsseminars des Instituts für Betriebswirtschaft der Universität Basel, Schriften zum Basler Tourismus Nr. 4, Hrsg.: Wirtschaftswissenschaftliches Zentrum der Universität Basel, Basel 1990

A proposed Consumer strategy of Simplification for Categorizing Brands, in: Summey, J.D., Taylor, R.D. (Hrsg.), Evolving Marketing Thoughts for 1980. Proceedings of the Annual Meeting of the Southern Marketing Association, Carbandale, 1980, Heft 11, S. 112-114

SPSS/PC+. Basics and Graphics. Einführung und praktische Beispiele, Hamburg, New York u.a. 1988

SPSS/PC+. Advanced statistics and Tables. Einführung und praktische Beispiele, Hamburg, New York u.a. 1989

Das soziale Bewußtsein von Konsumenten. Erklärungsansätze und Ergebnisse einer empirischen Untersuchung in der Bundesrepublik Deutschland, Wiesbaden 1978

Qualitätsbeurteilung investiver Dienstleistungen. Operationalisierungsansätze an einem empirischen Beispiel zentraler EDV-Dienste, Frankfurt a.M. 1991 
Bürklin, w.

Dahlhoff, H. -D.

Dahlhoff, H. -D.

Datzer, R.

Datzer, R.

De la Beaumelle, s.s.

Dichtl, E.

Dichtl, E.

Müller-Heumann, G.

Diller, H.
Wertewandel oder zyklische Wertaktualisierung?, in: Luthe, H.O., Meulemann, H. (Hrsg.), Wertewandel - Faktum oder Fiktion? Bestandsaufnahmen und Diagnosen aus kultursoziologischer sicht, Frankfurt, New York 1988, S. 193-216

Wertorientierungen von Verbrauchern, in: Haase, H., Molt, w. (Hrsg.), Handbuch der angewandten Psychologie, Bd. 3, Markt und Umwelt, Landsberg a.L. 1981, S. 130152

Individuelle Wertorientierungen. Analyse und Aussagewert personenspezifischer Werthierarchien im Marketing, Arbeitspapier $\mathrm{Nr} .23$ des Instituts für Marketing, Hrsg.: H. Meffert, Münster 1980

Informationsverhalten von Urlaubsreisenden. Ein Ansatz des verhaltenswissenschaftlichen Marketing, starnberg 1983

Ein Ưberblick über Ansätze der psychologischen und soziologischen Tourismusforschung, in: Reisemotive - Länderimages - Urlaubsverhalten, studienkreis für Tourismus e.V. (Hrsg.), Starnberg 1981, S. 7-39

Contribution to Market-Segmentation in Tourism Research, in: ESOMAR-Bericht, 1976, S. 249-267

Die Bildung von Konsumententypen als Grundlage der Marktsegmentierung, in: wirtschaftswissenschaftliches Studium, 1974, Heft 2, S. 55-59

Konsumententypologie und produktorientierte Marktsegmentierung, in: Jahrbuch für Absatz- und Verbrauchsforschung, 1972, Heft 4, S. 249-269

Preispolitik, stuttgart u.a. 1985 
Dundler, F.

Dundler, F.

DWIF (Hrsg.)

Ender, w., u.a.

Engel, J.F.

Blackwell, R.D.

Miniopd, P.W.

European Travel Commission (ETC) (Hrsg.)

Fazio, R.N.

Fehrlin, P.

Fingerhuth, C., u.a.
Urlaubsreisen 1954-1988. 35 Jahre Erfassung des touristischen Verhaltens der Deutschen durch soziologische stichprobenuntersuchungen, in: studienkreis für Tourismus e.v. (Hrsg.), Starnberg 1989

Urlaubsreisen 1987. Einige Ergebnisse der Reiseanalyse 1987. Kurzfassung. Studienkreis für Tourismus e.V. (Hrsg.), Starnberg 1988

Markt- und Motivstudie für den Fremdenverkehrsmarkt Westfalens, München 1989

Von der Hotelbetriebslehre zur Management Science des Tourismus? ZeitgemäBe Aufgaben einer Betriebswirtschaftslehre des Fremdenverkehrs, in: Der Markt, 1983, S. 3646

Consumer Behaviour, 6. Aufl., Chicago u.a. 1986

Market Research Study: Attitude Segmentation of the American Market for Travel to Europe, Implications for a Market in Transition, Dublin, New York 1974

Direct Experience and Attitude-Behaviour-Relationship, in: Journal of Experimental Social Psychology, 1981 , S. 398-408

Marketing-Konzeption für einen $\mathrm{Ba}-$ dekurort am Beispiel von Bad Ragaz, Heerbrugg 1983

Arbeitsmethode zur Bewertung der Erholungseignung eines landschaftlichen Angebots für verschiedene Typen von Erholungssuchenden, in: Landschaft und Stadt, 1973, Heft 4, S. 161-171 
Fishbein, M.

Frank, R.E.

Massay, W.F. wind, $Y$.

Frese, E.

Freter, H.

Freter, H.

Freter, H.

Freyer, w.

Fürst, D.

Klemmer, $P$.

Zimmermann, $\mathrm{K}$.

Geiger, $\mathrm{H}$.

Gilbrich, M.
A Behaviour Theory Approach to the Relations between Beliefs about an object and the Attitude towards the object, in: Readings in Attitude Theory and Measurement, M. Fishbein (Hrsg.), New York u.a. 1967

Market Segmentation, Englewood Cliffs, N.J. 1972

Grundlagen der organisation. Die organisationsstruktur der Unternehmung, Wiesbaden 1987

Mehrdimensionale Einstellungsmodelle im Marketing. Interpretation, Vergleich und Aussagewert, Arbeitspapier Nr. 12 des Instituts für Marketing, Hrsg.: H. Meffert, Münster 1976

Interpretation und Aussagewert mehrdimensionaler Einstellungsmodelle im Marketing, in: Meffert, H., Steffenhagen, H., Freter, H. (Hrsg.), Konsumentenverhalten und Information, wiesbaden 1979, S. $163-184$

Marktsegmentierung, stuttgart u.a. 1983

Tourismus. Einführung in die Fremdenverkehrsökonomie, 3. Auf 1., München, wien 1991

Regionale Wirtschaftspolitik, Tübingen, Düsseldorf 1976

Interessen und Verhaltensweisen von Urlaubsreisenden, in: studienkreis für Tourismus e.v. (Hrsg.), Motive - Meinungen - Verhaltensweisen, Bericht über eine Tagung des studienkreises für Tourismus, starnberg 1969 , S. 119-131

Urlaubsreisen 1991, Kurzfassung der Reiseanalyse 1991, studienkreis für Tourismus e.v. (Hrsg.), starnberg 1992 
Goebel, M.

Goodall, B.

Gröne, A.

Gruner + Jahr AG \& Co (Hrsg.)

Hachmann, H. Haedrich, H.

Haedrich, G. Kaspar, C.

Kleinert, $\mathrm{H}$.

Klemm, K. (Hrsg.)

Hänni, H.D.

Häussler, X.
Reisemarkt im strukturwandel Strategische Neuausrichtung eines Touristikkonzerns, in: Meffert, H., Wagner, H., Backhaus, K. (Hrsg.), Marketing in der Freizeit- und Dienstleistungsgesellschaft, Dokumentation des 20. Führungsgesprächs vom 21./22. Februar 1991, Dokumentationspapier Nr. 62 der Wissenschaftlichen Gesellschaft für Marketing und Unternehmensführung e.V., Münster 1991, S. 66-76

How Tourists choose their Holidays: An Analytical Framework, in: Goodall, B., Ashworth, G. (Hrsg.), Marketing in the Tourism Industry. The Promotion of Destination Regions, New York 1988, S. 1-17

Marktsegmentierung bei Investitionsgütern. Analyse und Typologie des industriellen Einkaufsverhaltens als Grundlage der Marketingplanung, wiesbaden 1977

Profile: Medien - Märkte - Konsumenten. Repräsentativ-Erhebung über Medienverhalten, Einstellungen zu Reisen und Tourismus. Einzelband: Marktprofil Reisen und Tourismus, Hamburg 1975

Reisebefragung 1977, Starnberg 1978

Angebotspolitik, in: G. Haedrich, C. Kaspar, H. Kleinert, K. Klemm (Hrsg.), Tourismus-Management. Tourismusmarketing und Fremdenverkehrsplanung, Berlin, New York 1983 , S. 241-261

Tourismus-Management. Tourismusmarketing und Fremdenverkehrsplanung, Berlin, New York 1983

Regionales Tourismus-Marketing. Am Beispiel regionaler Verkehrsverbände in der Schweiz, Zürich 1985

Der Fremdenverkehr, Leipzig 1930 
Hahn, H.

Hartmann, K.D.

Haneklaus, w.

Hartmann, K.D.

Hartmann, K.D.

Hartmann, K.D.

Koeppler, K. (Hrsg.)

Haseloff, W.

Hebestreit, D.

Heidenreich, $\mathrm{K}$.

Heinemann, G.

Hilke, R.

Hochreiter, $\mathrm{R}$.

Arndt, $U$.
Reiseinformationen, Reiseentscheidung, Reisevorbereitung, Starnberg 1973

Regionalpolitik in der Europäischen Gemeinschaft. ziele, Kompetenzen und Instrumente von Gemeinden, Bund und Ländern nach Inkrafttreten der Einheitlichen Europäischen Akte, Munster 1991

Urlaubsland Schleswig-Holstein. Eine motiv- und meinungspsychologische Untersuchung, Kurzfassung, studienkreis für Tourismus e.V. (Hrsg.), Starnberg 1979

Urlaubertypen und Urlauberlandschaften, in: praktische Psychologie, 1974, Heft 4, S. 1-10

Fortschritte der Marktpsychologie, Bd. 1, Grundlagen, Methoden, Anwendungen, Frankfurt 1977

Urlaubstests, Testbogen zu einem im Zusammenhang mit dem Institut für Freizeitpsychologie GmbH für Quelle-Reisen-International durchgeführten Urlaubstest, Tübingen 1969

Touristik-Marketing. Ziele, strategien, Instrumentarium, organisation und Planung des Marketing für Reiseveranstalter, Berlin 1979

Grundbegriffe der MeB- und Testtheorie, in: Sozialwissenschaftliche Methoden, E. Roth (Hrsg.), Munchen, Wien 1984, S. 351-384

Betriebstypenprofilierung und Erlebnishandel. Eine empirische Analyse am Beispiel des textilen Facheinzelhandels, wiesbaden 1989

Grundlagen normorientierter und kriterienorientierter Tests, Bonn 1980

Die Tourismusindustrie - eine Markt- und Wettbewerbsanalyse, Frankfurt, Bern, Las Vegas 1978 
Hörning, K.H.

Holm, K.

Howard, J.A.

Sheth, J.N.

Hünerberg, $\mathbf{R}$.

Hummer, $\mathrm{H}$.

Hunziker, w. Krapf, K.

Inglehart, $R$.

Jacob, P.E.

Singh, K.K.

Jacob, P.E.

u.a. (Hrsg.)

Kaiser, A.

Kannacker, V.A.

Karmasin, H.

Kaspar, C.
Sozialökonomische schichtdifferenzierung und Konsumstandards, in: Specht, K.G., Wiswede, G., Marketing Soziologie. Soziale Interaktionen als Determinanten des Marktverhaltens, Berlin 1976, S. 111-131

Die Gültigkeit des sozialwissenschaftlichen Messens, in: $K$. Holm (Hrsg.), Die Befragung 4, München 1976

The Theory of Buyers Behaviour, New York u.a. 1969

Zur segmentierungsproblematik im Rahmen der Marketingpolitik, in: Marktforschung, 1981, Heft 2, s. 50-57

Psychologische Marktsegmentierung am Beispiel: Urlaubsreisen, in: Marketing Journal, 1972, Heft 1, S. 63-67

GrundriB der allgemeinen Fremdenverkehrslehre, Zürich 1942

The silent Revolution, Princeton 1977

Measuring Values, in: Jacob, P.E. u.a. (Hrsg.), The International studies in Politics. Values and the Active Community, New York 1971

The International studies in Politics. Values and the Active community, New York 1971

Die Identifikation von Marktsegmenten, Darmstadt 1978

Habitualisiertes Kaufverhalten von Konsumenten, München 1982

Das Image von Österreich als Reiseland, in: studienkreis für Tourismus, 1972, S. 53-69

Fremdenverkehrslehre im GrundriB, 2. Aufl., Bern, stuttgart 1982 
Kaspar, C.

Kaspar, C. Kunz, B.R.

Katona, G.

Kieser, A. Kubicek, H.

Kirchgeorg, $\mathbf{M}$.

Kirsch, K.

Klemz, $W$.

Kluckhohn, C.

Knappe, H. -J.
Die Entwicklung des modernen Tourismus - Ursachen, Erscheinungsformen und wirkungen, in: statistisches Bundesamt (Hrsg.), Tourismus in der Gesamtwirtschaft, Ergebnisse des 4. Wiesbadener Gesprächs am 28./29. März 1990, Schriftenreihe Forum der Bundesstatistik, Bd. 17, Stuttgart 1991, S. 17-25

Unternehmensführung im Fremdenverkehr. Eine Grundlage für das Management von Hotels und Restaurants, Sportbahnen und -anlagen, Reisebüros, Kur- und Verkehrsbüros, Bern, Stuttgart 1982

Das Verhalten der Verbraucher und Unternehmer, Tübingen 1968

Organisation, 3. Aufl., Berlin, New York 1992

ökologieorientiertes Unternehmensverhalten. Typologien und Erklärungsansätze auf empirischer Grundlage, Wiesbaden 1990

Schwerpunktförderung als Instrument regionaler Wirtschaftspolitik unter besonderer Berücksichtigung des Fremdenverkehrs, Diss., Reichenberg 1980

Interkommunale Zusammenarbeit im Fremdenverkehrsbereich. Zugleich ein Beitrag zur ökonomischen Analyse des Zweckverbandes, Frankfurt a.M. U.a. 1989

The study of Values, in: Values in America, Barret, D.N. (Hrsg.), Notre Dame, Indiana 1961, S. 17-46

Informations- und Kaufverhalten unter Zeitdruck, Frankfurt a.M. 1981 
Koch, A.

Zeiner, $M$.

Koerdt, w.

Kollat, D.T. Blackwell, R.D. Robenson, J.F.

Konert, F.-J.

Kolks, U.

Kols, P.

Kook, w.

Kopp, M.

Kotler, $\mathrm{Ph}$.

Kotler, $\mathrm{Ph}$.

Bliemel, F.

Krautter, J .
Wirtschaftliche Wirkungen des Urlaubsreiseverkehrs in der Bundesrepublik Deutschland, in: Akademie für Raumforschung und Landesplanung (Hrsg.), Fremdenverkehr und Regionalpolitik, Forschungs- und sitzungsberichte, Bd. 172, Hannover 1988 , S. 37-62

Das Anspruchsniveau von Urlaubsreisenden. Ein empirischer Beitrag zur Konsumentenforschung, Heidelberg 1988

Strategic Marketing, New York 1972

Vermittlung emotionaler Erlebniswerte, Heidelberg 1986

Strategieimplementierung - Ein anwendungsorientiertes Konzept, Wiesbaden 1990

Bedarfsorientierte Marktsegmentierung auf Produktivgütermärkten, Frankfurt a.M. 1986

Einstellungen zur Universität. Operationalisierung und empirische Prüfung verhaltenswissenschaftlicher Hypothesen am Beispiel der stadtbevölkerung von Münster, Frankfurt 1980

Hypothesenformulierung in der $\mathrm{Ab}-$ satzforschung, Berlin 1972

Marketing Management. Analysis, Planing, Implementation, and Control, 6. Aufl., Englewood Cliffs 1988

Marketing-Management. Analyse, Planung, Umsetzung und steuerung, 7 . Aufl., stuttgart 1992

Zum Problem der optimalen Marktsegmentierung, in: ZfB, 1975, Heft 2, S. 109-128 
Kreilkamp, E.

Krippendorf, J .

Kroeber-Riel, w.

Lehmann, D.R. O'Shaughnessy, J.

Lessing, P.V.

Levine, F.M. (Hrsg.)

Lohmann, M. Wohlmann, $R$.

Luthe, H.O.

Meulemann, H. (Hrsg.)

Maier, J.

Maier, J.

Troeger-Weise, G.
Strategisches Managment und Marketing. Markt- und Wettbewerbsanalyse, strategische Frühaufklärung, Portfolio-Management, Berlin, New York 1987

Marketing im Fremdenverkehr, 2 . Aufl., Bern 1980

Konsumentenverhalten, 5. Aufl., München 1992

Difference in Attribute Importance for Different Industrial Products, in: Journal of Marketing, Vol. 38, 1974, Heft 2, S. 36-38

A Measurement of Dependencies between Values and other Levels of the Consumer's Belief Space, in: Journal of Business Research, 1975, Heft 5, S. 227-240

Theoretical Readings in Motivation. Perspectives on Human Behaviour, Chicago 1975

Urlaub in Deutschland. Eine Grundlagenuntersuchung über Verhaltensweisen, Meinungen und Einstellungen von Urlaubern, Studienkreis für Tourismus e.V. (Hrsg.), Starnberg 1987

Wertewandel - Faktum oder Fiktion? Bestandsaufnahmen und Diagnosen aus kultursoziologischer sicht, Frankfurt a.M., New York 1988

Bewertung eines regionalen Entwicklungsfaktors, in: Akademie für Raumforschung und Landesplanung (Hrsg.), Fremdenverkehr und Regionalpolitik, Forschungs- und sitzungsberichte, Bd. 172, Hannover 1988 , S. 111-118

Marketing in der räumlichen Planung. Ansätze und Wege zu einer marktorientierten Regional- und Kommunalplanung, in: Beiträge der Akademie für Raumforschung und Landesplanung, Beitrag 117, Hannover 1990 
Maleri, $\mathbf{R}$.

Marks, L.J.

Kamins, M.A.

Marplan-Forschungsgesellschaft

Maslow, A.H.

Mazanec, J.

Mazanec, J.

McDougall, G.H.G. Munro, H.

McIntosh, R.W.

Meffert, $\mathrm{H}$.
Grundlagen der Dienstleistungsproduktion, 2. Aufl., Berlin, Heidelberg 1991

The Use of Product Sampling and Advertising: Effects of Sequence of Exposure and Degree of Advertising Claim Exaggeration on Consumer's Belief Strength, Belief Confidence and Attitudes, in: Journal of Marketing Research, 1983, Heft 4, S. 266-281

Urlaubsreisen 1976. Psychologische Typologiestudie, Starnberg 1977

Motivation and Personality, in: Levine, F.M. (Hrsg.), Theoretical Readings in Motivation: Perspectives on Human Behaviour, Chicago 1975 , S. 358-379

Voraussetzungen rationaler Entscheidungsvorbereitung im Fremdenverkehrs-Marketing unter Rücksicht auf neuere Analysemethoden, in: Der Markt, 1979, Heft 7, S. 190-204

Zielgruppenplanung im Fremdenverkehrs-Marketing: Empirische Ergebnisse einer segmentationsanalyse des österreichischen Urlaubsreisenden, in: Journal für Betriebswirtschaft, 1979, S. 176-193

Scaling and Attitude Measurement in Tourism and Travel Research, in: Ritchie, J.R.B., Goeldner, C.R., Travel, Tourism and Hospitality Research. A Handbook for Managers and Researchers, New York U.a. 1987, S. 87-100

Tourism. Principles, Practices, Philosophies, 3. Aufl., Columbus 1980

Grundlagen der strategieimplementierung, in: Marketingmanagement. Analyse, Strategie, Umsetzung, wiesbaden 1993 (im Druck) 
Meffert, $\mathrm{H}$.

Meffert, $\mathrm{H}$.

Meffert, $H$.

Meffert, $H$.

Meffert, $\mathrm{H}$.

Meffert, $\mathrm{H}$.

Meffert, $H$.

Meffert, $H$.

Meffert, $H$.
Marketingforschung und Käuferverhalten, 2. Auf1., Wiesbaden 1992

Erfolgreiches Marketing in den neunziger Jahren, in: Marketing im Umbruch. Chancen und Gefahren für den Unternehmer, Referate anläBlich der Dolder-Tagung vom 23. Mai 1991, Zürich 1991, S. 7-43

Städtemarketing - Pflicht oder Kür?, in: Symposium stadtvisionen 1989, Dokumentation, Ideenschmiede Münster (Hrsg.), Münster 1989

Änderungen und Entwicklungstendenzen im Konsumentenverhalten, in: Meffert, H., Strategische Unternehmensführung und Marketing. Beiträge zur marktorientierten Unternehmenspolitik, wiesbaden 1988, S. 297-312

Markenstrategien im Wettbewerb, in: Meffert, H., Strategische Unternehmensfuhrung und Marketing. Beiträge zur marktorientierten Unternehmenspolitik, Wiesbaden 1988 , S. 115-138

Marketing. Grundlagen der Absatzpolitik, 7. Aufl., Wiesbaden 1986

Stellenwert der Werteforschung im Marketing, in: Wertewandel und Konsumentenverhalten, Dokumentation des Workshops vom 29. Juni 1984, Arbeitspapier Nr. 20 der Wissenschaflichen Gesellschaft für Marketing und Unternehmensführung e.V., Hrsg.: Meffert, H., Wagner, H., Münster 1984, S. 1-8

Der Kundendienst als Marketinginstrument - Einführung in die Problemkreise des Kundendienst-Management, in: Meffert, H. (Hrsg.), Kundendienst-Management. Entwicklungsstand und Entscheidungsprobleme der Kundendienstpolitik, Frankfurt 1982 , S. 1-30

Marktsegmentierung und Marktwahl im internationalen Marketing, in: DBW, 1977, Heft 3, S. 433-446 
Meffert, $\mathbf{H}$.

Meffert, H., u.a.

Meffert, $\mathrm{H}$. Birkelbach, R.

Meffert, H., u.a.

Meffert, $H$. Althans, J.

Meffert, $H$. Steffenhagen, $\mathrm{H}$. Freter, H.

Meffert, $H$. Wagner, H. (Hrsg.)

Meffert, H. (Hrsg.)

Meffert, H. (Hrsg.)
Modelle des Käuferverhaltens und inr Aussagewert für das Marketing, in: Zeitschrift für die gesamte Staatswissenschaft, 1971, Heft 2, S. 327-353

Regionenmarketing Münsterland. Ansatzpunkte auf der Grundlage einer empirischen Untersuchung, Münster 1991

Freizeitgesellschaft und marktorientierte Unternehmensführung. Thesen $z u$ den Auswirkungen des Freizeitphänomens auf die Marktbearbeitung, in: Marketing-ZFP, 1991, Heft 4, S. 265- 269

Marketing für das Münsterland. Eine Bestandsaufnahme auf der Grundlage sekundärstatistischer Analysen, Münster 1989

Internationales Marketing, stuttgart, u.a. 1982

Entwicklungen und Schwerpunkte der Käuferverhaltensforschung, in: Meffert, H., Steffenhagen, H., Freter, H. (Hrsg.), Konsumentenverhalten und Information, Wiesbaden 1979, S. 23-35

Wertewandel und Konsumentenverhalten, Dokumentation des Workshops vom 29. Juni 1984, Arbeitspapier $\mathrm{Nr}, 20$ der Wissenschaflichen Gesellschaft für Marketing und Unternehmensführung e.V., Münster 1984

Kundendienst-Management. Entwicklungsstand und Entscheidungsprobleme der Kundendienstpolitik, Frankfurt 1982

Das Meinungsbild einer stadtbevölkerung über öffentliche Institutionen - Empirische Studie von Einstellungen der Münsteraner gegenüber Universität, Stadttheater und Landesmuseum, Münster 1979 
Meffert, $H$. Steffenhagen, $\mathrm{H}$. Freter, H. (Hrsg.)

Meffert, $H$. Wagner, $\mathrm{H}$. Backhaus, K. (Hrsg.)

Meuter, $\mathrm{H}$.

Meyer, w.

Meyer, w.

Middleton, V.T.C.

Mill, R.Ch.

Morrison, A.M.

Monhemius, K.Ch.
Konsumentenverhalten und Information, Wiesbaden 1979

Marketing in der Freizeit- und Dienstleistungsgesellschaft, Dokumentation des 20. Múnsteraner Führungsgesprächs vom 21./22. Februar 1991, Arbeitspapier Nr. 62 der Wissenschaftlichen Gesellschaft für Marketing und Unternehmensführung e.V., Münster 1991

Ein Ansatz zur Typisierung von Wochenendausflügen auf der Basis von Freizeitaktivitäten, in: Becker, u.a. (Hrsg.), Freizeitverhalten in verschiedenen Raumkategorien, Trier 1979 , S. 101-124

Aktivität im Urlaub. Ergebnisse und Probleme der psychologischen Tourismusforschung zum Problem "Aktiver Urlaub", in: Hartmann, K.D., Koeppler, K. (Hrsg.), Fortschritt der Marktpsychologie. Grundlagen, Methoden, Anwendungen, Bd. 1, Frankfurt 1977, S. 259-291

Typologie der Touristen unter dem Aspekt des sozialen Kontaktes beim Auslandsurlaub, in: Zeitschrift für Kulturaustausch, 1978, Heft 3, S. 41-48

Meyer-Schwickerath, M. Perspektiven des Tourismus in der Bundesrepublik Deutschland. Zur Notwendigkeit eines wirtschaftspolitischen Konzepts, Münster 1990

Marketing in Travel \& Tourism, Oxford u.a. 1988

The Tourism System. An Introducing Text, Englewood Cliffs 1985

Umweltbewustes Kaufverhalten von Konsumenten. Ein Beitrag zur operationalisierung, Erklärung und TYpologie des Verhaltens in der Kaufsituation, Münster 1992 
Mühlbacher, H.
Botschen, G.
Müller, w.-H.
Mủller, K.
Goldberger, E.
Müller-Hagedorn, L.

Naether, E.A.

Nieschlag, R.

Dichtl, E.

Hörschgen, H.

Norusis, M.J.

Opaschowski, H.w.

Opaschowski, H.W.

Opaschowski, H.W.

Opaschowski, H.W. Raddatz, G.

o.v.
Benefit-segmentierung von Dienstleistungsmärkten, in: Marketing ZFP， 1990, Heft 3, S. 159-168

Der Fremdenverkehr im kommunalen Marketing, in: Der städtetag, 1990, Heft 3, S. 225-232

Unternehmens-Kooperation bringt Wettbewerbsvorteile. Notwendigkeit und Praxis zwischenbetrieblicher Zusammenarbeit in der schweiz, Zürich 1986

Das Konsumentenverhalten. Grundlagen für die Marktforschung, Wiesbaden 1986

Ist Hamburg eine Kurzreise wert? Eine psychologische Grundlagenstudie im Auftrag der Arbeitsgemeinschaft Hamburg-Information e.V., Hamburg 1971

Marketing, 15. Aufl., Berlin 1988

SPSS/PC+. Advanced Statistics 4.0, Chicago 1990

Urlaub 1991/92 - Trendziele und Trendsetter im Tourismus der 90er Jahre, B.A.T. Freizeit-Forschungsinstitut (Hrsg.), Hamburg 1992

Tourismusforschung, in: Freizeitund Tourismusstudien, Bd. 3, Opladen 1989

Urlaub 1986/87 - Was Reiseziele bieten müssen, B.A.T. Freizeit-Forschungsinstitut (Hrsg.), Hamburg 1987

Freizeit im Wertewandel. Die neue Einstellung $z u$ Arbeit und Freizeit, B.A.T. Freizeit-Forschungsinstitut (Hrsg.), Bd. 4 der Schriftenreihe zur Freizeitforschung, Hamburg 1982

Marken - Profile 4: Touristik, Fluggesellschaften, Mietwagen, in: Die Stern Bibliothek, Hamburg 1992 


$$
\begin{aligned}
& \text { o.v. } \\
& \text { o.v. }
\end{aligned}
$$

Parasuraman, A. Berry, L.L.

Parasuraman, A. Zeithaml, V.A. Berry, L.L.

Perreault, w.D. Darden, D.K. Darden, W.R.

Raaij, van, W.T.

Rehorn, J.

Reppel, R.

Richter, B.

Richtie, J.R.B. Goeldner, C.R.

Rosenberg, M.J.
Regionales Entwicklungsprogramm Münsterland, März 1991

Urlaub '74, in: Für Sie, 1974, Heft 3 , S. $2-26$

Service Marketing, Frankfurt a.M. 1992

A Conceptual Model of Services Quality and its Implications for Future Research, in: Journal of Marketing, Vol. 49, 1985, Heft 4, s. 41-50

A Psychographic Classification of Vacation Life Styles, in: Journal of Leisure Research, 1977, Heft 3, S. 208-224

Consumer Research on Tourism. Mental and Behavioural Constructs, in: Annals of Tourism Research, Vol. 13,1986 , S. 1-9

Marktsegmentation - Modeerscheinung oder orientierungshilfe?, in: Markenartikel, 1973, Heft 8, s. 402408

Innenmarketing - eine strategie für Fremdenverkehrsorte, in: Wolf, J., Seitz, E. (Hrsg.), Tourismusmanagement und -marketing, Landsberg a.L. 1991, S. 29-39

Anmerkungen zur Marktsegmentierung, in: Jahrbuch der Absatz- und Verbrauchsforschung, 1972, Heft 1, S. 37-42

Travel Tourism and Hospitality Research. A Handbook for Managers and Researchers, New York u.a. 1987

Cognitive structure and Attitudional Affect, in: Journal of Abnormal and Social Psychology, 1956, $S$. 367-372 
Rosenberg, M.J.

RosmeiB-Stracke, F.

RosmeiB-Stracke, F.

Roth, P.

Roth, P.

Schrand, A. (Hrsg.)

Russo, J.E.

Johnson, E.J.

Schanz, G.

Scheuch, F.
Inconsistency Arousal and Reduction in Attitude Change, in: Steiner, I.D., Fishbein, M. (Hrsg.), Current Studies in Social Psychology, New York 1965

Die Veränderungen der gesellschaftlichen Rahmenbedingungen für die Touristik, in: Roth, P., Schrand, A. (Hrsg.), Touristik-Marketing. Das Marketing der Tourismus-organisationen, Verkehrsträger, Reiseveranstalter und Reisebüros, München 1992 , S. 21-28

Zukunftsperspektiven für den Tourismus. Möglichkeiten und Grenzen einer Freizeitgesellschaft, in: statistisches Bundesamt (Hrsg.), Tourismus in der Gesamtwirtschaft, Ergebnisse des 4 . Wiesbadener Geprächs am 28./29. März 1990, Schriftenreihe Forum Bundesstatistik, Bd. 17, Wiesbaden 1991, S. 26-34

Grundlagen des Touristik-Marketing, in: Roth, P., Schrand, A. (Hrsg.), Touristik-Marketing. Das Marketing der Tourismus-organisationen, Verkehrsträger, Reiseveranstalter und Reisebüros, München 1992, S. 111192

Touristik-Marketing. Das Marketing der Tourismus-organisationen, Verkehrsträger, Reiseveranstalter und Reisebüros, München 1992

What do Consumers know about Familiar Products? in: olson, J.C. (Hrsg.), Advances in Consumer Research, Vol. 7, Ann Arbor, 1980, S. 417-423

Zwei Arten von Empirismus, in: ZfbF, 1975, S. 324-326

Dienstleistungsmarketing, München 1982 
Scheuch, F.

Schmid, J.-M.

Schmidt-Atzert, L.

Schmidt-Scherzer, R. Rudinger, G.

Schnetkamp, G.

Scholz, J.

Schrand, A.

Schrattenecker, G.

Schubö, W.

Uehlinger, H.M. U.a.

Schulte-Frankenfeld, H.
Logische strukturen und pragmatische Bedeutung der Marktsegmentierung, in: Die Unternehmung, 1974, Heft 3, S. 213-230

Kundenstamm-Marketing - aus der Sicht des Reiseveranstalters, Gais 1989

Emotionspsychologie, stuttgart, Berlin u.a. 1981

Motive - Erwartungen - Wünsche in bezug auf Urlaub und Verreisen, in: Studienkreis für Tourismus (Hrsg.), Motive - Meinungen - Verhaltensweisen. Einige Ergebnisse und Probleme der psychologischen Tourismusforschung, Starnberg 1969, S. 9-19

Einstellungen und Involvement als Bestimmungsfaktoren des sozialen Verhaltens. Eine empirische Analyse am Beispiel der organspendebereitschaft in der Bundesrepublik Deutschland, Frankfurt 1982

Wertewandel und wirtschaftskultur, München 1987

Tourismus 2000: Der strukturwandel auf den Touristik-Märkten, in: Roth, P., Schrand, A., TouristikMarketing. Das Marketing der Tourismus-organisationen, Verkehrsträger, Reiseveranstalter und Reisebüros, München 1992, S. 1-20

Die Beurteilung von Urlaubsländern durch Reisekonsumenten. Die Anwendung von Konsumverhaltensmodellen in der Tourismusforschung, wien 1984

SPSS-X. Handbuch der Programmversion 4.0 und SPSS-X 3.0, stuttgart, New York 1991

Vereinfachte Kaufentscheidungen von Konsumenten. Erklärung psychischer Prozesse kognitiv limitierten Entscheidungsverhaltens von Konsumenten, Frankfurt, Bern, New York 1985 
Seitz, E.

Wolf, J. (Hrsg.)

seyfarth, C.

Sheth, J.N.

Sheth, J.N.

Talarzyk, W.W.

Simon, H.

Specht, K.G.

wiswede, G. (Hrsg.)

Statistisches Bundesamt (Hrsg.)

Steinecke, A.

Steinhausen, D. Zörkendörfer, D.
Tourismusmanagement und -marketing, Landsberg a.L. 1991

Wertewandel und gesellschaftliche Rationalisierung: Eine theoretische Diskussion aktueller Tendenzaussagen, in: Luthe, H.O., Meulemann, H. (Hrsg.), Wertewandel - Faktum oder Fiktion? Bestandsaufnahmen und Diagnosen aus kultursoziologischer Sicht, Frankfurt a.M. , New York 1988 , S. 163-192

Marktsegmentierung als relevante Planungshilfe des Marketing, in: Jahrbuch für Absatz- und Verbrauchsforschung, 1972, Heft 2, S. 129-144

Perceived Instrumentality and Value Importance as Determinants of Attitudes, in: Journal of Marketing Research, Vol. 9, 1972, S. 6-9

Preismanagement. Analyse - Strategie - Umsetzung, 2. Aufl., Wiesbaden 1992

Marketing-Soziologie - Soziale Interaktionen als Determinanten des Marktverhaltens, Berlin 1976

Tourismus in der Gesamtwirtschaft, Ergebnisse des 4. Wiesbadener Gesprächs am 28./29. März 1990, Schriftenreihe Forum der Bundesstatistik, Bd. 17, stuttgart 1991

Gesellschaftliche Grundlagen der Fremdenverkehrsentwicklung, in: Haedrich, G., Kaspar, C., Kleinert, H., Klemm, K. , Tourismus-Management. Tourismusmarketing und Fremdenverkehrsplanung, Berlin, New York 1983, S. 37-55

Statistische Datenanalyse mit dem Programmsystem SPSS-X, Software-Information 13 des Universitätsrechenzentrums, Universität Münster, 4. Aufl., Münster 1987 
storbeck, D.

storbeck, D.

struck, U.

Strumpe1, B. Scholz-Ligma, J .

specht, $\mathbf{G}$.

Specht, K.G.

Wiswede, G. (Hrsg.)

Studienkreis für Tourismus e.V. (Hrsg.)

Studienkreis für Tourismus e.v. (Hrsg.)

Studienkreis für Tourismus e.v. (Hrsg.)
Die Entwicklung der Rahmenbedingungen für den Fremdenverkehr in der Bundesrepublik Deutschland, Arbeitsberichte "Regional- und Raumplanung", Heft 9, Bielefeld 1987

Die Entwicklung der Rahmenbedingungen für den Fremdenverkehr in der Bundesrepublik Deutschland, in: Akademie für Raumforschung und Landesplanung (Hrsg.), Fremdenverkehr und Regionalpolitik, Forschungsund sitzungsberichte, Bd. 172, Hannover 1988, S. 81-110

Marketingprobleme der seetouristik, in: Marketing im Tourismus, studienkreis für Tourismus (Hrsg.), Bericht über eine Tagung des Studienkreises für Tourismus, starnberg 1970, S. 65-69

BewuBtseins- und sozialer Wandel: wie erleben Menschen die wirtschaft?, in: Luthe, H.O., Meulemann, H. (Hrsg.), Wertewandel Faktum oder Fiktion? Bestandsaufnahmen und Diagnosen aus kultursoziologischer sicht, Frankfurt, New York 1988, S. 21-47

Distributionsmanagement, stuttgart, u.a. 1988

Marketing-Soziologie - Soziale Interaktionen als Determinanten des Marktverhaltens, Berlin 1976

Konzepte und strategien furr heute und morgen. Bericht über eine Fachtagung des studienkreises für Tourismus am 7. März 1990 im Rahmen der Internationalen Tourismus-Börse (ITB) in Berlin, starnberg 1991

Tourismus und Umwelt. Bericht uber eine Fachtagung des studienkreises für Tourismus e.v. im Rahmen der 21. Internationalen Tourismus-Börse (ITB) am 11. März 1987 in Berlin, Starnberg 1987

Reisemotive - Länderimages - Urlaubsverhalten, Starnberg 1981 
Studienkreis für

Tourismus e.V. (Hrsg.)

Summey, J.D.

Taylor, R.D. (Hrsg.)

Szallies, R.,

Wiswede, G. (Hrsg.)

Tostmann, Th.R.

Thomae, H. (Hrsg.)

Triandis, H.C.

Trommsdorff, $V$.

Trommsdorff, v.

Tschiderer, F.

Uebele, $\mathrm{H}$.

Ulrich, H.

Uttitz, P.
Motive - Meinungen - Verhaltensweisen. Einige Ergebnisse und Probleme der psychologischen Tourismusforschung, Starnberg 1969

Evolving Marketing Thought for 1980. Proceedings of the Annual Meeting of the Southern Marketing Association, Carbandale, No. 11, 1980

Wertewandel und Konsum, Landsberg a.L. 1990

Konsument und Kaufentscheidung. Untersuchung zur praktischen Brauchbarkeit von KaufprozeBmodellen, Bielefeld, Köln 1982

Enzyklopädie der Psychologie, Bonn 1983

Einstellungen und Einstellungsänderungen, Weinheim, Basel 1975

Konsumentenverhalten, stuttgart, Berlin, Köln 1989

Die Messung von Produktimages für das Marketing - Grundlagen und operationalisierung, Köln, u.a. 1975

Ferienortplanung. Eine Anwendung unternehmensorientierter Planungsmethodik auf den Ferienort, Bern, stuttgart 1980

Marktsegmentierung im Investitionsgüter-Bereich, in: $\mathrm{ZfbF}$, 1984, Heft 2 , S. $158-170$

Die Unternehmung als produktives System. Grundlagen der allgemeinen Unternehmenslehre, Bern, stuttgart 1968

Von den vertikalen spannungslinien zu den horizontalen Gegensätzen. wandel des Freizeitverhaltens als Indikator, Köln 1984 
Veen, van, W.M.O. Verhallen, T.W.M.

Venth, 0 . Freyer, M.

Vershoven, w.

Vinson, D.E. Scott, J.E. Lamont, L.M.

Wachenfeld, H. Waltermann, B.

Weinberg, $P$.

Weinberg, $P$.

Werner, J.

wind, $Y$.

Cardozo, R.N.

windhorst, K. -G.
Vacation Market Segmentation. A Domain. Specific Value Approach, in: Annals of Tourism Research, Vol. 13,1986, S. 37-58

Lebensziele - Umweltbewußtein - Urlaubsverhalten, in: studienkreis für Tourismus (Hrsg.), Tourismus und Umwelt. Bericht über eine Fachtagung des studienkreises für Tourismus e.V. im Rahmen der 21. Internationalen Tourismus-Börse (ITB) am 11. März 1987 in Berlin, starnberg 1987 , S. 9-26

Die Marktentnahme als Kernstück der wirtschaftsforschung, Berlin 1959

The Role of Personal Values in Marketing and Consumer Behavior, in: Journal of Marketing, 1977, Heft 4, S. 44-50

Freizeitverhalten und Marketing. Grundlagen des Marketing für Freizeitangebote, Heidelberg 1987

Internationale Markenpolitik und Produktpositionierung. Markenpolitische Entscheidungen im europäischen Automobilmarkt, wien 1989

Die Produkttreue der Konsumenten, Wiesbaden 1977

Das Entscheidungsverhalten der Konsumenten, Paderborn, u.a. 1981

Marktsegmentierung für eine erfolgreiche Marktbearbeitung, in: Jahrbuch für Absatz- und Verbrauchsforschung, 1987, Heft 4, S. 396-405

Industrial Market Segmentation, in: Industrial Marketing Management, 1974, Heft 3, S. 153-166

Wertewandel und Konsumentenverhalten. Ein Beitrag zur empirischen Konsumrelevanz individueller Wertvorstellungen in der Bundesrepublik Deutschland, Münster 1985 
winkler, A.

Wiswede, G.

Wiswede, G.

wöhler, K.

Wölm, D.

Wölm, D.

Wolf, $K$.

Jurczek, P.

Wolf, J.

Seitz, E.

Zolles, H.

Ferner, F.K.

Müller, R.

Zucker, W.H.
Praktische Anwendungen der Marktsegmentierung. Marktsegmentierung als Methode der Marktforschung, in: Jahrbuch der Absatz- und Verbrauchsforschung, 1972, Heft 1 , S. 101-120

Der "Neue Konsument" im Lichte des Wertewandels, in: szallies, R., Wisewede, G. (Hrsg.), Wertewandel und Konsum, Landsberg a.L. 1990

Motivation und verbraucherverhalten. Grundlagen der Motivforschung, München 1973

Zielgruppenorientiertes Marketing, in: studienkreis für Tourismus. Konzepte und strategien für heute und morgen. Bericht über eine Fachtagung des studienkreises für Tourismus am 7. März 1990 im Rahmen der Internationalen Tourismusbörse (ITB) in Berlin, starnberg 1991, $\mathrm{S}$. 71-86

Marketing für Deutschlandreisen unter besonderer Berücksichtigung der strategie der Marktsegmentierung, Bamberg 1980

Marketing im Tourismus, in: Marketing ZFP, 1979, Heft 4, s. 229-236

Geographie der Freizeit und des Tourismus, stuttgart 1986

Tourismusmanagement und -marketing, Landsberg a.L. 1991

Marketingpraxis für den Fremdenverkehr, Wien 1981

Urlaubsreisen 1985. Kurzfassung einiger Ergebnisse der Reiseanalyse 1985, Studienkreis für Tourismus (Hrsg.), Starnberg 1986 
Simone Frömbling - 978-3-631-75102-2

Downloaded from PubFactory at 01/11/2019 08:19:28AM

via free access 


\section{SCHRIFTEN ZUM MARKETING}

Band 1 Friedrich Wehrle: Strategische Marketingplanung in Warenhäusern. Anwendung der Portfolio-Methode. 1981. 2. Auflage. 1984.

Band 2 Jürgen Althans: Die Übertragbarkeit von Werbekonzeptionen auf internationale Mărkte. Analyse und Exploration auf der Grundlage einer Befragung bei europaweit tătigen Werbeagenturen. 1982.

Band 3 Günter Kimmeskamp: Die Rollenbeurteilung von Handelsvertretungen. Eine empirische Untersuchung zur Einschätzung des Dienstleistungsangebotes durch Industrie und Handel. 1982.

Band 4 Manfred Bruhn: Konsumentenzufriedenheit und Beschwerden. Erklärungsansätze und Ergebnisse einer empirischen Untersuchung in ausgewăhlten Konsumbereichen. 1982.

Band 5 Heribert Meffert (Hrsg.): Kundendienst-Management. Entwicklungsstand und Entscheidungsprobleme der Kundendienstpolitik. 1982.

Band 6 Ralf Becker: Die Beurteilung von Handelsvertretern und Reisenden durch Hersteller und Kunden. Eine empirische Untersuchung zum Vergleich der Funktionen und Leistungen. 1982.

Band 7 Gerd Schnetkamp: Einstellungen und Involvement als Bestimmungsfaktoren des sozialen Verhaltens. Eine empirische Analyse am Beispiel der Organspendebereitschaft in der Bundesrepublik Deutschland. 1982.

Band 8 Stephan Bentz: Kennzahlensysteme zur Erfolgskontrolle des Verkaufs und der MarketingLogistik. Entwicklung und Anwendung in der Konsumgüterindustrie. 1983.

Band 9 Jan Honsel: Das Kaufverhalten im Antiquitätenmarkt. Eine empirische Analyse der Kaufmotive, ihrer Bestimmungsfaktoren und Verhaltenswirkungen. 1984.

\section{SCHRIFTEN ZU MARKETING UND MANAGEMENT}

Band 10 Matthias Krups: Marketing innovativer Dienstleistungen am Beispiel elektronischer Wirtschaftsinformationsdienste. 1985.

Band 11 Bernd Faehsler: Emotionale Grundhaltungen als Einflußfaktoren des Käuferverhaltens. Eine empirische Analyse der Beziehungen zwischen emotionalen Grundhaltungen und ausgewählten Konsumstrukturen. 1986.

Band 12 Ernst-Otto Thiesing: Strategische Marketingplanung in filialisierten Universalbanken. Integrierte Filial- und Kundengruppenstrategien auf der Grundlage erfolgsbeeinflussender Schlüsselfaktoren. 1986.

Band 13 Rainer Landwehr: Standardisierung der internationalen Werbeplanung. Eine Untersuchung der Prozeßstandardisierung am Beispiel der Werbebudgetierung im Automobilmarkt. 1988.

Band 14 Paul-Josef Patt: Strategische Erfolgsfaktoren im Einzelhandel. Eine empirische Analyse am Beispiel des Bekleidungsfachhandels. 1988. 2. Auflage. 1990.

Band 15 Elisabeth Tolle: Der Einfluß ablenkender Tätigkeiten auf die Werbewirkung. Bestimmungsfaktoren der Art und Höhe von Ablenkungseffekten bei Rundfunkspots. 1988.

Band 16 Hanns Ostmeier: Ökologieorientierte Produktinnovationen. Eine empirische Analyse unter besonderer Berücksichtigung ihrer Erfolgseinschätzung. 1990.

Band 17 Bernd Büker: Qualitätsbeurteilung investiver Dienstleistungen. Operationalisierungsansätze an einem empirischen Beispiel zentraler EDV-Dienste. 1991.

Band 18 Kerstin Ch. Monhemius: Umweltbewußtes Kaufverhalten von Konsumenten. Ein Beitrag zur Operationalisierung, Erklärung und Typóiogiecdesđ̉ethialthensininder 
Band 19 Uwe Schürmann: Erfolgsfaktoren der Werbung im Produktlebenszyklus. Ein Beitrag zur Werbewirkungsforschung. 1993.

Band 20 Ralf Birkelbach: Qualitătsmanagement in Dienstleistungscentern. Konzeption und typenspezifische Ausgestaltung unter besonderer Berücksichtigung von Verkehrsflughăfen. 1993.

Band 21 Simone Frömbling. Zielgruppenmarketing im Fremdenverkehr von Regionen. Ein Beitrag zur Marktsegmentierung auf der Grundlage von Werten, Motiven und Einstellungen. 1993. 